Göttinger Schriften zum Medizinrecht Band 3

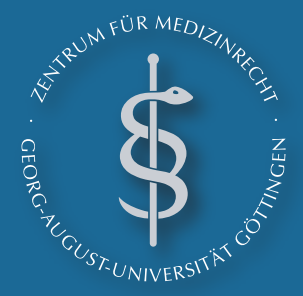

Gisela Bockenheimer-Lucius, Petra Thorn, Christiane Wendehorst (Hrsg.)

\title{
Umwege zum eigenen Kind
}

Ethische und rechtliche Herausforderungen an die Reproduktionsmedizin 30 Jahre nach Louise Brown 

Gisela Bockenheimer-Lucius, Petra Thorn, Christiane Wendehorst (Hrsg.) Umwege zum eigenen Kind

This work is licensed under the Creative Commons License 2.0 "by-nc-nd", allowing you to download, distribute and print the document in a few copies for private or educational use, given that the document stays unchanged and the creator is mentioned. Commercial use is not covered by the licence.

\begin{tabular}{|c|}
\hline C. \\
SORIE RIGHIS RESERVED
\end{tabular} 
erschienen als Band 3 in der Reihe „Göttinger Schriften zum Medizinrecht” im Universitätsverlag Göttingen 2008 
Gisela Bockenheimer-Lucius,

Petra Thorn,

Christiane Wendehorst (Hrsg.)

\section{Umwege zum eigenen Kind}

Ethische und rechtliche

Herausforderungen an die

Reproduktionsmedizin

30 Jahre nach Louise Brown

Göttinger Schriften zum

Medizinrecht

Band 3

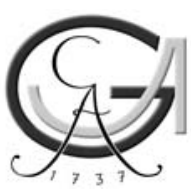

Universitätsverlag Göttingen 2008 


\section{Bibliographische Information der Deutschen Nationalbibliothek}

Die Deutsche Nationalbibliothek verzeichnet diese Publikation in der Deutschen Nationalbibliographie; detaillierte bibliographische Daten sind im Internet über $<$ http://dnb.ddb.de $>$ abrufbar.

Herausgeber der Reibe

Zentrum für Medizinrecht

Juristische Fakultät der Georg-August-Universität Göttingen

Geschäftsführender Direktor : Prof. Dr. Gunnar Duttge

Dieses Buch ist auch als freie Onlineversion über die Homepage des Verlags sowie über den OPAC der Niedersächsischen Staats- und Universitätsbibliothek

(http://www.sub.uni-goettingen.de) erreichbar und darf gelesen, heruntergeladen sowie als Privatkopie ausgedruckt werden. Es gelten die Lizenzbestimmungen der

Onlineversion. Es ist nicht gestattet, Kopien oder gedruckte Fassungen der freien

Onlineversion zu veräußern.

Umschlaggestaltung: Kilian Klapp und Margo Bargheer

(C) 2008 Universitätsverlag Göttingen

http:/ / univerlag.uni-goettingen.de

ISBN: 978-3-940344-46-5

ISSN: $1864-2144$ 


\section{Vorwort}

Die Geburtshilfe hat in den letzten 50 Jahren zweifellos einen außerordentlichen Wandel durchgemacht, der entsprechend tiefgreifende Folgen für Paare und ihren Kinderwunsch mit sich gebracht hat. Von der Hilfe beim Gebären wurde sie erweitert um die Pränatalmedizin, die Perinatalmedizin und die Reproduktionsmedizin. Jedes dieser Handlungsfelder hat seinerseits wiederum vielfältige Herausforderungen und Veränderungen erfahren. So ist es nicht verwunderlich, dass das Verhältnis zur eigenen Nachkommenschaft durch Planbarkeit, technologische Möglichkeiten und eine neue Art von Konflikthaftigkeit geprägt ist.

Wenn man davon ausgeht, dass in Deutschland 1,4 Mio. Frauen und Männer zwischen 25 und 59 Jahren aus medizinischen Gründen von ungewollter Kinderlosigkeit betroffen sind, ${ }^{1}$ so ist der unerfüllte Wunsch nach einem Kind durchaus ein gesellschaftlich wie medizinisch bedeutsames Problem. Kommt das ersehnte Kind nicht, so gerät für viele Paare die Lebensplanung durcheinander, und die nächste Zukunftsperspektive wird fraglich. Auch im Jahr 2008 - 30 Jahre nach der Geburt von Lonise Brown - bleibt für viele Paare der Wunsch nach einem eigenen Kind ein vergeblicher, und die Reproduktionsmedizin soll die erhoffte Lösung bringen.

Der vorliegende Band vereint im Wesentlichen Beiträge zweier Symposien, die vom Forum für Ethik in der Medizin Frankfurt am Main e.V. in Zusammenarbeit mit der Arbeitsgruppe „Reproduktionsmedizin und Embryonenschutz" in der Akademie für Ethik in der Medizin, Göttingen, im März 2007 in Frankfurt am Main und vom Zentrum für Mediquinrecht der Georg-August-Universität Göttingen im Juni 2007 in Göttingen durchgeführt wurden.

${ }^{1}$ Institut für Demoskopie Allensbach, Bericht 11/2007 („Unfreiwillig Kinderlos“). 
Beide Veranstaltungen nahmen neben derzeit so intensiv diskutierten Problemen wie dem der Mehrlingsschwangerschaften oder der Inkaufnahme überzähliger Embryonen (Michelmann) bewusst Fragestellungen auf, die in der deutschen Diskussion bisher noch wenig Beachtung gefunden haben. Wie geht man mit dem Kinderwunsch körperlich schwer behinderter Menschen um (Crawshaw)? Welche Erfahrungen machen lesbische Frauen, wenn sie ihren Kinderwunsch erfüllen möchten (Herrmann-Green)? Welche ethischen Herausforderungen stellen sich, wenn bei todbringenden Krankheiten des männlichen Partners eine Kryokonservierung des Samens möglich wäre (Bockenheimer-Lucius)? Wie weit vergessen und vernachlässigen ethische Theorien mit Blick auf den Embryo die Bedeutung menschlicher Beziehungen (Wiesemann)? Aber auch Grundsatzfragen spielen immer wieder eine wichtige Rolle. Letztlich zeigen die Kontroversen, dass es in Deutschland keinen Konsens zum Stellenwert der assistierten Reproduktion gibt: Wie viel „Kinderwunsch“ ist eigentlich in den Augen der Öffentlichkeit akzeptiert (Krones)? Hat ein unerfüllter Kinderwunsch Krankheitswert, und muss die Solidargemeinschaft reproduktionsmedizinische Leistungen entsprechend finanziell mittragen (Rauprich)? Welche Bedeutung haben die rechtlichen Schranken, die das Embryonenschutzgesetz und die Richtlinien zur IVF in Deutschland aufgebaut haben, aus verfassungsrechtlicher Sicht (Heun)? Was bringt es an Chancen und/oder Gefahren mit sich, wenn Wunscheltern Behandlungen im Ausland durchführen, wo reproduktionsmedizinische Möglichkeiten vielfältiger, aber womöglich auch für Patienten undurchsichtiger sind (Knoll)?

Einen besonderen Schwerpunkt beider Tagungen - insbesondere der Göttinger Veranstaltung - bildeten donogene Techniken assistierter Reproduktion. Denn auch wenn der medizinische Fortschritt es ermöglicht, dass zur IVF taugliche Keimzellen aus immer unreiferen Vorstadien von Spermatozoen und Eizellen gewonnen werden und wenn sich der Blick längst darauf richtet, mittels induzierter Haploidisierung der Chromosomensätze somatischer Zellen „künstliche“ Keimzellen zu generieren, bleiben Samenspende, Eizellspende und Embryospende doch immer noch vergleichsweise einfache, sichere (Zoll) und kostengünstige Methoden mit bislang unübertroffener Erfolgswahrscheinlichkeit. Diesen offenkundigen Vorteilen stehen natürlich auch gewichtige Probleme gegenüber. Bei der Spendersamenbehandlung, die auch in Deutschland mittlerweile eine lange Tradition hat (Katzorke), treten vor allem Fragen der sozialen Stigmatisierung (Thorn) und die Dilemmata anonymer Vaterschaft (Blyth) in den Vordergrund. Bei der Eizellspende sind es zusätzlich die gesundheitlichen und psychischen Risiken für die Spenderinnen (Graumann), die sorgsam gegen die Chancen für die von Kinderlosigkeit betroffenen Frauen (Berg) abgewogen werden wollen. Angesichts dieser Vielfalt unterschiedlicher Aspekte stellt sich akut die Frage, ob die derzeitige gesetzliche Regelung donogener Techniken (Wendehorst) zeitgemäß ist.

Die moderne Reproduktionsmedizin bietet demnach auch 30 Jahre nach der Geburt des ersten „Retortenbabys“ eine Fülle neuer Herausforderungen, die erheb- 
liche moralische und rechtliche Implikationen haben. Ihr Spektrum ist groß geworden, und bisher unübliche Formen der Familienbildung werfen erneut die Frage auf, wie eine verantwortungsvolle und bestmögliche medizinische wie psychologische Betreuung von Paaren mit unerfülltem Kinderwunsch, die körperliche Integrität der betroffenen Frauen und das Wohl des Kindes zugleich zu gewährleisten sind. Die Methoden sind nach wie vor umstritten. Für viele sind es Technologien, deren Zugriff auf die Frauen mit scharfer Kritik kommentiert wird. So führten ein Beitrag von Magda Telus ${ }^{2}$ zur IVF im Deutschen Ärzteblatt im Jahr 2001 und ein ergänzender Bericht von Ina Zuber-Jerger $2002^{3}$ zu heftigen Vorwürfen und einer anschließenden Kontroverse. Telus und Zuber-Jerger beklagten eine Verschleierung der Risiken und des Schadenspotenzials der Reproduktionsmedizin durch die behandelnden Ärzte und die Ausblendung der Gefahren für Frauen im öffentlichen Diskurs. Für andere Teilnehmende an der Debatte, nicht zuletzt für viele Frauen, sind die unterschiedlichen Methoden der Reproduktionsmedizin längst etablierte Techniken, die vielen Paaren weltweit die Geburt des gewünschten Kindes ermöglicht haben.

Vorläufig hat uns die medizinisch unterstützte (assistierte) Reproduktion eine Fülle an Chancen für unfreiwillig kinderlose Paare gegeben. Diese Chancen sind aber auch mit Risiken verbunden, über die wir uns redlich austauschen und denen wir den rechten Stellenwert geben müssen, um vermeidbaren Schaden rechtzeitig abzuwehren. Dazu sollen die vorliegenden Auseinandersetzungen einen Beitrag leisten.

Alle Aufsätze des vorliegenden Bandes befassen sich mit Facetten dieser aktuellen Debatten um die assistierte Reproduktion und ihre Möglichkeiten. Dabei kann und soll es nicht um Vollständigkeit gehen und werden viele drängende Fragestellungen - etwa verbunden mit der Herstellung künstlicher Gameten oder mit dem Kinderwunsch homosexueller Männer - noch ausgespart bleiben müssen.

An dieser Stelle darf nicht vergessen werden, dass ein solcher Band viele sehr engagierte Helfer braucht. Wir haben allen Autorinnen und Autoren für ihre Bereitschaft zur Mitarbeit und eine unkomplizierte Zusammenarbeit herzlich zu danken! Vor allem gilt unser Dank aber Herrn Ass. Mag. Johannes Kehrer und Frau stud. jur. Sarah Trautmann (Wien) für ihre Geduld und Sorgfalt bei der redaktionellen Bearbeitung der Texte.

Frankfurt am Main, Mörfelden und Wien, September 2008

Gisela Bockenheimer-Lucius Petra Thorn Christiane Wendehorst

2 Telus, Reproduktionsmedizin: Zwischen Trauma und Tabu, Dt. Ärzteblatt 2001, 3430 ff.

${ }^{3}$ Zuber-Jerger, Zu hohe Risikobereitschaft, Dt. Ärzteblatt 2002, 617 ff. 



\section{Inhaltsverzeichnis}

Vorwort I

Teil I: Kinderwunsch im Jahr 2008 allgemeine ethische und rechtliche Herausforderungen

Reproduktionsmedizin im Jahre 2008:

Probleme - Wünsche - Lösungsansätze 1

Hans-Wilhelm Michelmann

Der Kinderwunsch - wie viel ist in

den Augen der Öffentlichkeit zulässig?

Tanja Krones

Sollen Kinderwunschbehandlungen

von den Krankenkassen finanziert werden?

Ethische und rechtliche Aspekte

Oliver Rauprich

Restriktionen assistierter

Reproduktion aus verfassungsrechtlicher Sicht.

Werner Heun

So weit gehen für ein Kind:

Reproduktionstourismus als grenzüberschreitender Umweg.

Eva-Maria Knoll

Der Embryo im Kontext: Warum die Biopolitik

die menschlichen Beziehungen nicht vergessen darf.

Claudia Wiesemann

\section{Teil II: Chancen und Probleme donogener Techniken}

Entstehung und Entwicklung

der Spendersamenbehandlung in Deutschland

Thomas Katzorke

Die rechtliche Regelung donogener ART

in Deutschland und Österreich.

Christiane Wendehorst 
Genetische Risiken durch Keimzellspende

Barbara Zoll

Samenspende und Stigmatisierung -

ein unauflösbares Dilemma?

Petra Thorn

Donor insemination

and the dilemma of the "unknown father"

Eric Blyth

Eizellspende und Eizellhandel -

Risiken und Belastungen für die betroffenen Frauen

Sigrid Graumann

\section{Teil III: Kinderwunsch in besonderen Situationen}

"What about my right to choose?"

Young people with physical impairments

exercising reproductive choice

Marilyn Crawshaw

Wenn der Partner an einer progredienten,

potenziell lebensbedrohlichen Erkrankung leidet -

Fragen zur Entscheidungsfindung für eine

In-vitro-Fertilisation mit ICSI

Gisela Bockenheimer-Lucius, Timo Sauer, Falk Ochsendorf,

Inka Wiegratz, Aglaja Stirn, Yasar Toraman

Lesben mit Kinderwunsch:

Eine ethische Herausforderung für die Reproduktionsmedizin?

Lisa Herrmann-Green

Die Eizellspende - eine Chance für wen?

Giselind Berg

Anhang: Autorenverzeichnis 


\section{Abkürzungsverzeichnis:}

a.

Abb.

Abs.

AID

allg.

ANOVA

ANZICA

ART

Art.

ASRM

AußStrG

Aufl.

Az.

BBC

$\mathrm{Bd}$.

BGB

BGer

$\mathrm{BGH}$

BGHZ

BICA

Blg.

BSG

BT-Drs.

BVerfG

BVerfGE

bzw.

ca.

col.

cols.

d.h.

DI

DIR auch

Abbildung

Absatz

Artificial Insemination by Donor

allgemein, -e, -er, -es

Analysis of Variance

Australian and New Zealand Infertility Counsellors

Association

Assisted Reproductive Techniques

Artikel

American Society for Reproductive Medicine

Österr. Außerstreitgesetz

Auflage

Aktenzahl

British Broadcasting Corporation

Band

Bürgerliches Gesetzbuch

Schweizer Bundesgericht

Deutscher Bundesgerichtshof

Entscheidungen des Bundesgerichtshofes in Zivilsachen

British Infertility Counselling Association

Beilage

Deutsches Bundessozialgericht

Bundestagsdrucksache

Bundesverfassungsgericht

Entscheidungen des Bundesverfassungsgerichtes

beziehungsweise

circa

column

columns

das heißt

Donogene Insemination/Donor Insemination

Deutsches IVF-Register 


\begin{tabular}{ll} 
DJT & Deutscher Juristentag \\
DNA & Desoxyribonukleinsäure/Deoxyribonucleic Acid \\
DSM & Diagnostic and Statistical Manual \\
dt. & deutsch, -e, -er, -es \\
e.g. & exempli gratia \\
e.V. & eingetragener Verein \\
ed. & editor \\
edn. & edition \\
eds. & editors \\
EMRK & Europäische Menschenrechtskonvention \\
Erstb. & Erstbearbeitung \\
ESchG & Embryonenschutzgesetz \\
ESCO & Europäischer Sterilitätskongress \\
eSET & elektiver Single Embryo Transfer \\
ESHRE & European Society for Human Reproduction and \\
etc. & Embryology \\
EuGRZ & et cetera \\
EU & Europäische Grundrechte-Zeitschrift \\
evtl. & Europäische Union \\
f. & eventuell \\
FamRZ & folgend, -e, -er, -es \\
FAP & Zeitschrift für das gesamte Familienrecht \\
ff. & familiäre adenomatöse Poliposis Coli \\
FMedG & fortfolgend, -e, -er, -es \\
Fußn. & Fortpflanzungsmedizingesetz \\
GebFra & Fußnote \\
GG & Zeitschrift für Geburtshilfe und Frauenheilkunde \\
ggf. & Grundgesetz für die Bundesrepublik Deutschland \\
GMG & gegebenenfalls \\
GP & Gesundheitsmodernisierungsgesetz \\
GSOEP & Gesetzgebungsperiode \\
GUS & German Socio Economic Panel \\
HFEA & Gemeinschaft Unabhängiger Staaten \\
HIV & \\
& Humandefizienz-Virus \\
\hline &
\end{tabular}


HLA Leukozyten-Antigen, Histokompatibilitäts-Typisierung

Hrsg. Herausgeber

i.e.

id est

idF.

in der Fassung

ICD

International Classification of Diseases

ICSI

Intracytoplasmic Sperm Injection

IVF

In-vitro-Fertilisation

JuS

Juristische Schulung

$\mathrm{JZ}$

Juristenzeitung

Kap.

Kapitel

$\mathrm{KI}$

Künstliche Insemination

m.w.N.

mit weiteren Nachweisen

MedR

Zeitschrift für Medizinrecht

n.

note

n.Chr.

nach Christus

NJW

Neue Juristische Wochenschrift

no.

number

$\mathrm{Nr}$.

Nummer

NSW

New South Wales

NZZ

Neue Züricher Zeitung

o.a.

oben angeführte

OHSS

Ovarian Hyperstimulation Syndrome

op.cit.

opus citatum

p.

page

pp.

pages

PGD

Preimplantation Genetic Diagnosis

PGS

Preimplantation Genetic Screening

PID

Präimplantationsdiagnostik

PND

Pränataldiagnostik

Rdn.

Randnummer

RG

Reichsgericht

RV.

Regierungsvorlage

S.

Seite

s. siehe

SET

Single Embryo Transfer 


$\begin{array}{ll}\text { SGB } & \text { Sozialgesetzbuch } \\ \text { sig. } & \text { sigma } \\ \text { SMA } & \text { spinale Muskelatrophie } \\ \text { sog. } & \text { sogenannt, -e, -er, -es } \\ \text { SPD } & \text { Sozialdemokratische Partei Deutschlands } \\ \text { sSET } & \text { selektiver Single Embryo Transfer } \\ \text { suppl. } & \text { supplement } \\ \text { Tab. } & \text { Tabelle } \\ \text { TESE } & \text { Testikuläre Spermatozoenextraktion } \\ \text { TPG } & \text { Transplantationsgesetz } \\ \text { u.a. } & \text { unter anderem } \\ \text { UK } & \text { United Kingdom } \\ \text { USA } & \text { United States of America } \\ \text { v. } & \text { von, van, versus } \\ \text { v.a. } & \text { vor allem } \\ \text { v.d. } & \text { van der, van den } \\ \text { vgl. } & \text { vergleiche } \\ \text { vol. } & \text { volume } \\ \text { WA } & \text { Western Australia } \\ \text { WHO } & \text { World Health Organisation } \\ \text { Z. } & \text { Ziffer } \\ \text { z.B. } & \text { zum Beispiel } \\ \text { z.T. } & \text { zum Teil } \\ \text { zit. } & \text { zitiert } \\ \text { ZRP } & \text { Zeitschrift für Rechtspolitik } \\ \end{array}$




\title{
Reproduktionsmedizin im Jahre 2008: Probleme - Wünsche - Lösungsansätze
}

\author{
Hans-Wilhelm Michelmann
}

Bis zur Einführung der IVF waren Behandlungen bei ungewollter Kinderlosigkeit extrem eingeschränkt. Es gab nur wenige medizinische Therapien bei tubarer Sterilität oder bei männlicher Unfruchtbarkeit. Erst durch die Möglichkeit, über die IVF die nicht funktionstüchtigen Eileiter mit Hilfe des Reagenzglases zu umgehen, ist seit 1978 weltweit für unzählige Paare ein gangbarer Weg eröffnet, doch noch zu einem eigenen Kind zu kommen. Es ist eine Substitutionstherapie im Rahmen der assistierten Reproduktion, bei der die Befruchtung und die ersten Schritte der frühembryonalen Entwicklung in vitro, d.h. im Reagenzglas außerhalb des mütterlichen Körpers stattfinden. Effektive Hilfe bei männlicher Subfertilität gibt es erst seit 1992 durch die Technik der Mikroinjektion (ICSI), bei der eine Samenzelle in die Eizelle injiziert wird. Selbst bei blockierten Samenleitern können Spermatozoen nach einer Hodenbiopsie aus dem Testesgewebe ausgewaschen werden (Testikuläre Spermatozoenextraktion; TESE) und für die ICSI benutzt werden. Für die Ergebnisse der ICSI-Technik ist es belanglos, ob die eingesetzten Spermatozoen von fertilen Männern stammen oder von Männern mit schwersten andrologischen Störungen. Alle diese Techniken der assistierten Reproduktionstechnologien (ART) gehören heute zum akzeptierten und auch häufig angewandten Repertoire medizinischer Behandlung, lösen aber immer noch kontroverse Debatten aus. So sind viele Probleme nicht gelöst, wie z.B. die hohe Rate an Mehrlingsschwangerschaften, die Bewertung der Präimplantationsdiagnostik (PID) und die Diskussion 
Hans-Wilhelm Michelmann

um das durch das Embryonenschutzgesetz (ESchG) vorgegebene Verbot einer elektiven Embryokultur.

In Deutschland gelten 10 - 15\% aller Paare als ungewollt kinderlos. ${ }^{1}$ Dieser Wert beruht auf Schätzungen und kann nicht mit harten Daten untermauert werden. So wundert es nicht, dass andere Angaben weit unter 10\% liegen. ${ }^{2}$ Diese Unterschiede kommen dadurch zustande, dass man bei kinderlosen Paaren zwischen temporärer und dauerhafter Kinderlosigkeit unterscheiden muss. Paare mit temporärer Sterilität stellen das Klientel in der Reproduktionsmedizin und fließen deshalb auch in die Schätzwerte mit ein, während für demografische Erhebungen nur Paare mit dauerhafter Kinderlosigkeit gezählt werden. Die ungewollte Kinderlosigkeit hat sich in Deutschland zu einem bevölkerungspolitischen Problem entwickelt, das dadurch zustande kommt, dass immer mehr Paare immer später heiraten und immer mehr Frauen ihren Kinderwunsch in einen späteren Lebensabschnitt verschieben. Der biologisch eindeutige Zusammenhang zwischen Alter und abnehmender Fruchtbarkeit der Frau durch das deutlich gestiegene Erstgraviditätsalter bzw. den aufgeschobenen Kinderwunsch hat sich besonders in den letzten Jahrzehnten demografisch bemerkbar gemacht: Noch im Jahr 1970 lag das Durchschnittsalter der Erstgebärenden bei 24,3 Jahren und stieg bis zur Jahrtausendwende auf 29 Jahre. $^{3}$ Parallel dazu stieg auch das durchschnittliche Alter von Frauen in der Kinderwunschbehandlung. So lag es 1997 bei 32,6 Jahren, um 2006 auf 34,5 Jahre anzusteigen. ${ }^{4}$ Der Effekt des Alters auf die weibliche Fruchtbarkeit ist in erster Linie auf chromosomale Aberrationen in der älteren Oozyte zurückzuführen. In einer Untersuchung mit Hilfe der PID zeigten 63\% der Embryonen von Frauen über 35 Jahren chromosomale Aberrationen und sogar bei 81\% der Embryonen von 43 -jährigen Frauen. ${ }^{5}$

Parallel zum ansteigenden Alter von Frauen mit Kinderwunsch und der dadurch zunehmenden Zahl von Kinderwunschpatienten entwickelte die Reproduktionsmedizin immer mehr Möglichkeiten, solche Patienten zu behandeln. Auch wuchsen die Zahl der Reproduktionszentren sowie die Zahl der Behandlungen. ${ }^{6}$ Allein in den Jahren 1997 bis 2004 wurden in Deutschland über 380.000 Behandlungen durchgeführt, die pro Embryotransfer im Durchschnitt zu 28\% in einer klinischen Schwangerschaft mündeten. Ist eine Frau jünger als 36 Jahre, so steigt ihre Schwangerschaftswahrscheinlichkeit auf 32\%. Die Verfahren der ART tragen

${ }^{1}$ Keck/Neulen/Behre/Breckwoldt, Praxis der Frauenheilkunde: Endokrinologie, Reproduktionsmedizin, Andrologie, 2002.

2 Templeton/Fraser/Thompson, The epidemiology of infertility in Aberdeen, British Medical Journal 1990/301, 148 ff.; Bräbler/Stöbel-Richter, Zum Wandel im Reproduktionsverhalten in Deutschland und im europäischen Vergleich, Zeitschrift für Ärztliche Fortbildung und Qualitätssicherung 2002/96, $459 \mathrm{ff}$.

3 Strauß/Beyer, Ungewollte Kinderlosigkeit. Gesundheitsberichterstattung des Bundes 2004/20.

${ }^{4}$ Felberbaum/Bübler/v.d.Ven, Das deutsche IVF-Register 1996-2006, 2007.

${ }^{5}$ Gianaroli/Magli/Fiorentino/Baldi/Ferraretti, Clinical value of preimplantation genetic diagnosis, Placenta 2003/24, Suppl. B, 77 ff.

${ }^{6}$ Felberbaum/Bübler/v.d.Ven (Fußn. 4). 
immerhin zu mehr als 2\% aller Geburten in Deutschland bei. Unter diesen Tatsachen sind gesundheitspolitische Rahmenbedingungen inakzeptabel, die durch die Maßgaben des Gesundheitsmodernisierungsgesetzes (GMG vom 1.1.2004) zu einem signifikanten Rückgang der Behandlungszahlen geführt haben.7 Durch eine nun 50\%ige Selbstbeteiligung an den Behandlungskosten hat der Gesetzgeber dafür gesorgt, dass zahlreiche Paare aufgrund ihrer finanziellen Voraussetzungen von den Behandlungen ausgeschlossen werden. Dass die Möglichkeit, sich fortpflanzen zu dürfen, von der finanziellen Situation der betroffenen Paare abhängig gemacht wird, widerspricht der Antrittsrede unseres Bundespräsidenten, Horst Köhler, vom Mai 2004. Dort beklagte er die mangelhafte Unterstützung für diejenigen, die Verantwortung übernehmen wollen, um Deutschland zum „Land der Kinder“ zu machen.

\section{Behandlungsergebnisse}

Die Verfahren der ART sind heute neben der Inseminationsbehandlung sehr effektive Methoden. So wurden 2006 in Deutschland 10.935 Paare mit Hilfe der IVF und 27.633 Paare mit Hilfe der ICSI behandelt. Die Rate an klinischen Schwangerschaften lag bei beiden Verfahren zwischen $28 \%$ und $30 \%$ pro Embryotransfer. Damit steht Deutschland in Konkurrenz zu Ländern wie Spanien (34,8\%), den Niederlanden $(33,6 \%)$ und Polen $(31,4 \%)$. Ein Vergleich der durchschnittlichen Schwangerschaftsraten ist aber nur dann aussagefähig, wenn gleichzeitig Aussagen zum Alter der behandelten Patientinnen gemacht werden. So kann eine Patientin in Deutschland, die jünger als 36 Jahre ist, pro Transfer nach Angabe des Deutschen IVF-Registers (DIR) zu über 34\% damit rechnen, schwanger zu werden. Frauen unter 30 Jahren haben sogar eine Chance von 37\% - 41\%. Durchschnittliche Schwangerschaftsraten von derzeit 28 bis $30 \%$ pro Embryotransfer sind zwar ein großer Erfolg, doch zählt für Patienten nur die Geburt pro Behandlungszyklus. Diese Rate liegt zurzeit bei unter 20\%. Das bedeutet, dass mit über $80 \%$ Wahrscheinlichkeit Kinderwunschpaare in einem Behandlungszyklus keinen Erfolg haben.

\section{Das Embryonenschutzgesetz}

Die Art und Weise, wie mit menschlichen Gameten und Embryonen in Deutschland umgegangen werden darf, wird zum größten Teil im Embryonenschutzgesetz von 1991 geregelt. So sind, mit Ausnahmen, Drittpersonen im Reproduktionsprozess nicht erlaubt. Weiterhin wird die Zahl der entstehenden Embryonen auf drei limitiert, ihre Verwendung nur auf das homologe System beschränkt und ihr Gebrauch nur zur Initiierung einer Schwangerschaft gestattet. Trotz dieser zwei-

${ }^{7}$ Felberbaum/Bübler/ v.d.Ven (Fußn. 4). 
felsfrei positiven Aspekte, die das Embryonenschutzgesetz seit 1991 auf die Arbeit innerhalb der Reproduktionsmedizin hat, kann nicht verschwiegen werden, dass die Vorgaben dieses Gesetzes die Dilemmasituation der deutschen Reproduktionsmedizin hervorrufen und in gewisser Weise Misserfolge vorprogrammieren. So sagt $\ 1$ ESchG, dass maximal drei Embryonen transferiert werden dürfen und dass eine Fertilisation von mehr Eizellen als übertragen werden sollen, nicht statthaft ist. Diese, als Dreier-Regel bezeichnete Einschränkung, verlangt vom Laborpersonal, aus allen befruchteten Eizellen (= Vorkernstadien) maximal drei zur Weiterkultur auszuwählen und alle anderen zu verwerfen oder zu kryokonservieren. Da es im Vorkernstadium unmöglich ist, diejenigen Zygoten zu erkennen, die die Entwicklungspotenz haben, sich zu implantieren und eine Schwangerschaft zu initiieren, werden fast immer nach zwei- bis dreitägiger Kultur mindestens zwei Embryonen in der Hoffnung übertragen, dass wenigstens einer sich implantieren und zu einer Schwangerschaft bis zur Geburt führen wird. Dies ist der Grund dafür, dass, bedingt durch die Vorgaben im ESchG, durch ART eine nicht zu akzeptierende hohe Rate an Mehrlingsgeburten entsteht. Während die spontane Rate für Zwillinge bei 1,2\% und für Drillinge bei 0,013\% liegt, haben Frauen nach ART gemäß des DIR ein Zwillingsrisiko von 22\% und ein Drillingsrisiko von 1,9\%.

Prognostische Aussagen über die Möglichkeit des Eintretens einer Schwangerschaft können nicht nur aufgrund der Zahl gewonnener und befruchteter Oozyten, sondern auch aufgrund der Entwicklungsgeschwindigkeit und der Morphologie der einzelnen Embryonalstadien gemacht werden. ${ }^{9}$ Dabei ist es für die Diskussion von größter Wichtigkeit zu wissen, dass nur etwa $40 \%$ aller befruchteten Eizellen das Blastozystenstadium erreichen. Wird durch eine Kultur von fünf bis sechs Tagen in speziellen Medien dieses Stadium erreicht und dann transferiert, lassen sich Schwangerschaftsraten von über 50\% erreichen. Das hat weiterhin zur Folge, dass die normalerweise hohen Mehrlingsraten nach IVF/ICSI signifikant reduziert werden können. Das in zahlreichen Ländern routinemäßig praktizierte Labor-Verfahren ist deshalb die selektive Embryokultur: Aus der Gesamtheit aller über drei bis sechs Tage kultivierten Embryonen wird der am weitesten entwickelte und morphologisch am unauffälligsten aussehende Embryo selektiert und transferiert (Single Embryo Transfer; SET). Als günstigstes Entwicklungsstadium wird die nach einer Kulturdauer von fünf bis sechs Tagen entstandene, expandierte Blastozyste angesehen, die, nach dem heutigen Stand der Wissenschaft beurteilt, eine gute Chance hat, sich zu implantieren. Diese selektive Embryokultur kann man von der elektiven Embryokultur (electivus = die Wahl lassend) unterscheiden. Zur Nomenklatur der „elektiven Embryokultur“ ist zu erklären, dass man darunter die Labor-Kultur von mehreren Embryonalstadien versteht, bei der man lediglich die

\footnotetext{
8 Felberbaum/Bübler/v.d.Ven (Fußn. 4).

9 Templeton/Morris, Reducing the risk of multiple births by transfer of two embryos after in vitro fertilization, The New England Journal of Medicine 1998/339, 573 ff.
} 
von der Natur selbst durch Entwicklungsarrest ausgesonderten Embryonen nicht mehr berücksichtigt, d.h. nicht transferiert und eliminiert. So tauchen in der Literatur sowohl die Begriffe sSET (selektiver single Embryo Transfer) als auch eSET (elektiver single Embryo Transfer) auf. In den meisten Fällen wird im Ausland der sSET praktiziert, wobei davon ausgegangen wird, dass der übertragene Embryo derjenige mit der vermeintlich „höchsten Entwicklungsfähigkeit“ ist.

Der wichtigste reproduktionsmedizinische und therapeutische Vorteil des Transfers nur eines Embryos ist die Vermeidung von Mehrlingsschwangerschaften. Obwohl der SET in jedem Fall die Mehrlingsschwangerschaften begrenzen würde, sind sowohl die selektive als auch die elektive Embryokultur in Deutschland derzeit in ihrer rechtlichen Zulässigkeit strittig und werden in deutschen IVFZentren nicht angewendet. Das ESchG untersagt nicht nur einen Transfer von mehr als drei Embryonen, sondern bei der bisherigen Lesart auch eine Kultur von mehr Embryonen als „einzeitig“ übertragen werden sollen („Dreier-Regel“). Es sind in den letzten Jahren zahlreiche Artikel publiziert worden, die sich mit der Möglichkeit einer auf den aktuellen wissenschaftlichen Kenntnisstand beruhenden Auslegung des ESchG intensiv auseinander gesetzt haben und zu dem Schluss kommen, dass eine liberalere Auslegung erlaubt sei. ${ }^{10}$ Danach zeigt sich, dass im ESchG ein individueller Spielraum im Rahmen eines austarierten Algorithmus gegeben ist. ${ }^{11}$

Als Alternative zur elektiven Embryokultur wurden in den letzten Jahren von deutschen Zentren Verfahren eingesetzt, um im Rahmen des ESchG solche Vorkernstadien zu erkennen, die das größte Entwicklungspotenzial besitzen. Leider hat die Vorkernanalyse die in sie gesetzten Erwartungen, trotz anders lautender Aussagen in der Literatur, nicht erfüllt. ${ }^{12}$ So bleibt zusammenfassend festzuhalten, dass sichere Klassifikationsschemata zur Beurteilung der Eizellqualität und ihrer Entwicklungsfähigkeit im Rahmen der IVF/ICSI bis heute fehlen. Es wäre zu wünschen, dass in einem zukünftigen Embryonenschutzgesetz die sog. „Dreier-Regel“ aufgehoben wird und eine Kultur aller entstandenen Zygoten, auch mit anschließender Kryokonservierung, möglich ist.

\footnotetext{
${ }^{10}$ Frommel, Deutscher Mittelweg in der Anwendung des Embryonenschutzgesetzes (ESchG) mit einer an dem aktuellen wissenschaftlichen Kenntnisstand orientierten Auslegung der für die Reproduktionsmedizin zentralen Vorschrift des $₫ 1$, Abs. 1, Nr. 5 ESchG, Journal für Reproduktionsmedizin und Endokrinologie 2007, $27 \mathrm{ff}$.

${ }^{11}$ Geisthövel/Beier/Ochsner/Frommel/Taupitz, A novel algorithm of ART in Germany based on an adequate interpretation of the German Embryo Protection Act (Deutsches Embryonenschutzgesetz, ESchG), Human Reproduction 2007/22, Suppl. 1, 505 ff.

$12 \mathrm{Scott} /$ Ruben/Leondires/Miller, The morphology of human pronuclear embryos is positively related to blastocyst development and implantation, Human Reproduction 2000/15, 2394 ff.
} 


\section{Kryokonservierung von Embryonen}

In ihrer frühembryonalen Entwicklung erreichen bis zum Tag 5 im Durchschnitt nur etwa 40\% der entstandenen Embryonen das Blastozystenstadium. Nach einer elektiven Embryokultur sollte dann von diesen Blastozysten eine, im Ausnahmefall zwei, für den Embryotransfer ausgewählt werden. Nur dadurch könnte eine gute Schwangerschaftsrate erreicht, die Mehrlingsrate aber signifikant gesenkt werden. Alle zusätzlich entstandenen Embryonen müssten kryokonserviert werden. Sie stünden dann für einen weiteren Transfer bei der Frau zur Verfügung, von der die Eizellen, aus denen sie entstanden sind, stammen. Verzichtet eine Frau auf eine Übertragung ihrer kryokonservierten Embryonen, so sollten sie einem anderen Paar kostenfrei zur Verfügung gestellt werden. All das müsste unter der Regelung eines Gesetzes, ähnlich dem des Adoptionsgesetzes, ablaufen. Eine Embryonenspende gegen Honorar bzw. Aufwandsentschädigung sowie die Leihmutterschaft werden strikt abgelehnt.

\section{Präimplantationsdiagnostik}

Die Dilemmasituation, in der sich die Reproduktionsmedizin in Deutschland befindet, wird besonders deutlich bei der Diskussion um die Präimplantationsdiagnostik (PID). Beide, Befürworter und Gegner dieser Art frühembryonaler genetischer Analyse mit anschließender Selektion, führen überzeugende Argumente ins Feld. Bisher aufgrund des ESchG nicht in Deutschland erlaubt, fordern die Befürworter eine Änderung dieser Regel. Nur die PID als früheste Form der Pränataldiagnostik verhindert den späteren Schwangerschaftsabbruch bei Frauen, die aufgrund eines Risikofaktors (Alter; genetische Disposition; vorhergehende Geburt eines fehlgebildeten Kindes) in jedem Fall eine Pränataldiagnose durchführen lassen würden. Die PID erlaubt es, noch vor dem Entstehen einer Schwangerschaft den genetisch kranken Embryo zu erkennen, ihn nicht zu übertragen und so der Frau die Not und die Belastung eines Abbruchs zu ersparen.

Kritiker hingegen argumentieren, dass die Erlaubnis zur Durchführung dieser Art von Diagnostik zu einer Art reproduktionsbiologischem Dammbruch führt, der dem zukünftigen Missbrauch Tür und Tor öffnet. Dadurch, dass sich das betroffene Paar noch vor dem Eintritt einer Schwangerschaft nicht in einer Konfliktsituation für oder gegen das Leben eines Feten befindet, würden Entscheidungen gegen den Embryo viel leichter getroffen und immer größere Ansprüche an den „perfekten“ Embryo gestellt werden - von einer Geschlechtsselektion ganz zu schweigen.

Würde die PID auch in Deutschland erlaubt, so sollte sie nur an speziell ausgewählten Zentren durchgeführt werden. Bei ihrer Indikation muss dann die humangenetische Diagnostik und Beratung an erster Stelle stehen. Ein Einsatz der PID zur Auswahl von Embryonen nach gesundheitsirrelevanten Parametern sollte absolut verboten bleiben. 


\section{Forschung an Embryonen}

Erst seit Einführung der ART sind menschliche Embryonen verfügbar geworden. Sie können für reproduktionsmedizinische und biotechnologische Verfahren benutzt werden. Dies wirft die Frage auf, wie mit werdendem menschlichem Leben umgegangen werden darf. Wo liegen die Grenzen zwischen Heilung und Verbesserung, und sollte der Technisierung der Medizin im Bereich der Reproduktionsmedizin eine Grenze gezogen werden? Nach welchen Rechten, Pflichten, Werten soll man sich dabei richten? Die Frage nach dem moralischen Status des menschlichen Embryos ist ein besonders umfangreiches und schwieriges Thema. Die Idee der unverletzlichen Integrität des menschlichen Embryos hat viele Gremien veranlasst, die Instrumentalisierung frühester Formen des Menschseins zum Zweck zukünftiger Hilfsmöglichkeiten zu verurteilen. Dennoch ist zu berücksichtigen, dass die Debatte über den moralischen Status des menschlichen Embryos nicht abgeschlossen ist. In dieser Diskussion um den Schutz des menschlichen Embryos müssen der mögliche Erkenntnisgewinn einer Embryonenforschung und damit sich in Zukunft vielleicht ergebende Heilungschancen abgewogen werden gegen die Verletzung des Lebensrechtes ungeborenen menschlichen Lebens.

Diese Wertung kann entweder nur jeder für sich selber vornehmen, oder aber es findet eine politische Willensbildung statt, so wie sie auch im Stammzellgesetz zum Tragen kommt. Es ist meine persönliche Meinung, dass jegliche „Verwendung von Embryonen" (Definition des Embryos nach dem jetzigen ESchG) weiterhin verboten bleiben sollte. Das schließt sämtliche Forschung einschließlich der Keimbahnmanipulation, des reproduktiven sowie therapeutischen Klonens und der Forschung an menschlichen embryonalen Stammzellen ein. Ein eindrucksvolles Beispiel für den ungleichen Umgang mit frühen Embryonen in Deutschland ergibt sich aus dem Konflikt zwischen den in Deutschland gültigen Regelungen durch das Embryonenschutzgesetz und der internationalen Forschungs- bzw. Anwendungspraxis. Der Tatsache, dass in Deutschland die Zeugung von Embryonen zu anderen Zwecken als der Empfängnis und Geburt durch die Eizellen spendende Frau verboten ist, begegnen deutsche Wissenschaftler damit, embryonale Stammzellen (von „überzähligen“ Embryonen aus der IVF/ICSI) aus dem Ausland zu importieren, wo diese ganz legal kultiviert und gehandelt werden dürfen. In der Zukunft sollten die Anstrengungen dahin gehen, ausschließlich adulte, somatische Stammzellen in der Forschung einzusetzen.

\section{Diskussion}

Die Geburt von Louise Brown nach IVF im Jahre 1978 muss als reproduktionsmedizinischer Durchbruch angesehen werden. Seit dieser Zeit durchläuft die Reproduktionsmedizin eine beispiellose Entwicklung, deren Auswirkungen und deren Ende nicht abzusehen sind. Heute wird die Behandlung der ungewollten Kinderlosigkeit durch demografische Entwicklungen, technologische Innovationen sowie 
Hans-Wilhelm Michelmann

ethisch-juristische Normen beeinflusst. So hat die Änderung des reproduktiven Verhaltens durch die Verschiebung des Alters bei Erstgravidität zu einem altersbedingten Rückgang der Fertilität sowie der Kinderzahl geführt. Parallel dazu haben sich die ART entwickelt und in immer mehr Kliniken und Praxen etabliert. Trotz der zweifellos großen Erfolge der Reproduktionsmedizin müssen aber auch Probleme diskutiert werden:

- Die in Deutschland praktizierte Dreier-Regel ermöglicht nur eine durchschnittliche Schwangerschaftsrate von ca. 30\%. Deshalb sind mehrere Behandlungszyklen bis zum Eintreten einer Schwangerschaft notwendig.

- Die Dreier-Regel hat ebenfalls eine nicht akzeptabel hohe Rate von Mehrlingsschwangerschaften zur Folge.

- Durch das Gesundheitsmodernisierungsgesetz müssen Patienten die Hälfte aller Behandlungskosten selbst tragen.

Da die ART für ein bestimmtes Patientenkollektiv die einzige Möglichkeit darstellt, ein Kind zu zeugen, sollten im Interesse der Patienten alle drei Problemfelder entschärft und Lösungen gefunden werden. Die unbefriedigende Situation in Deutschland zwingt Paare zu der Entscheidung, entweder durch den Transfer von nur einem Embryo geringere Chancen für eine Schwangerschaft zu haben und eventuell viele Behandlungszyklen zu durchlaufen oder aber ein erhöhtes Mehrlingsrisiko in Kauf zu nehmen. Es bieten sich aber Lösungsvorschläge an, die die Mehrlingsrate signifikant senken und gleichzeitig die Schwangerschaftsrate steigern könnten. Die elektive Blastozystenkultur, d.h. die natürliche Selektion qualitativ schlechter Embryonen während einer In-vitro-Kultur bis zur expandierten Blastozyste wäre durch eine Änderung des ESchG ein Weg in die richtige Richtung. Als Folge dieser Änderung müsste aber auch die Kryokonservierung überzähliger Embryonen und bei nicht weiterer Verwendung dieser Embryonen die Weitergabe an solche Paare erlaubt werden, bei denen es nicht zum Entstehen von Embryonen kommt. Die Übertragung eines fremden Embryos gäbe diesen Paaren gute Chancen auf eine Schwangerschaft.

In der Diskussion um die Forschung an embryonalen Stammzellen hat der Molekularbiologe Jens Reich in einem Spiegelinterview (Der Spiegel 2000/50) wörtlich gesagt: „Der Sündenfall ist dadurch geschehen, dass die menschliche Fortpflanzung vor 25 Jahren durch die In-vitro-Fertilisation in die Petrischale ausgelagert worden ist". Das bedeutet für ihn, dass jegliche negative Entwicklung (reproduktives Klonen; verbrauchende Embryonenforschung; gespaltene Mutterschaft etc.) der Fortpflanzungsmedizin angelastet werden muss. Auch wenn diese Meinung sicher nicht repräsentativ ist, so ist es Zeit, trotz aller positiven Aspekte, die „neue“ Reproduktionsmedizin kritisch zu hinterfragen und sich mit den Ängsten und Befürchtungen einer verunsicherten Bevölkerung auseinander zu setzen. Es wird sicher nicht möglich sein, die Entwicklung zurückzuschrauben, wohl aber, auch im internationalen Konsens, Rahmenbedingungen zu schaffen, die von allen Teilen der Gesellschaft akzeptiert werden. 


\title{
Der Kinderwunsch - wie viel ist in den Augen der Öffentlichkeit zulässig?
}

\author{
Tanja Krones
}

„Sie fahren links und essen Würstchen zum Frühstück. Dass in England die Uhren anders gehen als auf dem Kontinent, gehört zur europäischen Vielfalt. Die Sympathie für die Extravaganzen der Briten schlägt jedoch in Unverstand und Entsetzen um, wenn das Inselvolk wieder eine Nachricht aus dem Reich der Biomedizin produziert. Gerade den Deutschen kommt das Land dann vor, wie die Heimstatt des biomedizinischen Grauens."

„(...) Ich (sehe) persönlich das entscheidende moralische Argument gegen die Embryonenforschung nicht darin, dass sie in irgendwelche vorgegebenen „Rechte“ des menschlichen Embryos eingreift (...) sondern einfach in der gesellschaftlichen Nichtakzeptanz dieser Forschung aufgrund starker und ersichtlich stabiler Gefühle von Abwehr und Angst.“2

In diesen beiden Aussagen zweier prominenter Protagonisten der bioethischen und biopolitischen Debatte, eines Journalisten und eines Philosophen, werden Aussagen zur gesellschaftlichen Auffassung gegenüber Reproduktionsmedizin und Gentechnologie in Deutschland und in Großbritannien getroffen. In Deutschland

\footnotetext{
1 Spiewak, Der große Unterschied. Die Briten sind im Umgang mit Genforschung und Reproduktionsmedizin das liberalste Volk Europas. Ihr Antrieb ist pragmatische Neugier, Die Zeit 2008/23.

${ }_{2}$ Birnbacher, Bioethik zwischen Natur und Interesse, 2006.
} 
besteht demnach eine „Nichtakzeptanz“ dieser Forschung, wohingegen „die Briten“ und ihre „Extravaganzen“ bei „den Deutschen“ Grauen, Abwehr und Angst auslösen. Nun sollte man aus verschiedenen sozialwissenschaftlichen wie philosophischen Überlegungen heraus gegenüber Pauschalisierungen über „die Gesellschaft", „die Briten“ oder „die Deutschen“ skeptisch sein, ebenso gegenüber ethischen Argumentationen oder politischen Entscheidungen, die allein auf solchen Annahmen oder „Fakten“ beruhen, obgleich natürlich die Auffassung in der Gesellschaft zu bioethischen Fragen in säkularen Demokratien ein wichtiges Entscheidungskriterium sein sollte. Stimmen diese Vermutungen jedoch? Sind „die Briten“ liberal und „die Deutschen“ skeptisch? Öffentliche Diskurse sind immer auch eine Konstruktion. Wer wird wie gefragt, was wird aus Meinungsumfragen und Forschungsprojekten wie aufgenommen und diskutiert?

Habermas unterscheidet in seinen gesellschaftsphilosophischen und diskursethischen Analysen zwei wesentliche Bereiche: Den der „informelle(n) Meinung von Privatleuten obne Publikum" und den der ,formellen Meinungen der publizistisch wirksamen Institutionen. “3 Diese können durchaus voneinander abweichen. In diesem Artikel werden einige zentrale Ergebnisse von sozialwissenschaftlichen Studien unserer Arbeitsgruppe (AG Bioethik-Klinische Ethik, Zentrum für Konfliktforschung, Marburg) und von weiteren Umfragen zu Auffassungen der bundesdeutschen Bevölkerung, von Experten und Betroffenen gegenüber neueren reproduktionsmedizinischen Technologien zusammengetragen und mit Ergebnissen aus internationalen Studien verglichen. Hierbei wird sich zeigen, dass der Skeptizismus in Deutschland weniger in der von Habermas erstgenannten Öffentlichkeit, den ,informellen Meinungen von Privatleuten ohne Publikum", sondern wesentlich in den „formellen Meinungen der publizistisch wirksamen Institutionen“ besteht. Dies kommentiere ich kurz im letzten Abschnitt.

\section{Einstellungen zur Kinderlosigkeit}

Ein demografisches Schreckgespenst droht: Die Deutschen, so liest man in den Feuilletons der Tageszeitungen, wie der ZEIT und der FAZ, bekämen immer weniger Kinder. „Das Scblimmste an der Entwicklung" sei, so schreibt die FAZ am 5.4.2004, „daß man so gut wie nichts mehr daran ändern kann. Wenn nicht ein Wunder geschieht und jedes Pärchen in Deutschland demnächst mindestens zwei Kinder in die Welt seťt, drohen langfristig gesellschaftliche Konflikte unbekannten Ausmaßes." Weiter wird berichtet, so in der ZEIT vom 15.1.2004, vor allem Akademikerinnen hätten „Angst vorm Kinderkriegen"; über 40\% der Akademikerinnen blieben kinderlos. Die Aussage, dass Menschen in Deutschland im internationalen Vergleich weniger Kinder bekommen, ist durchaus richtig. Allerdings beruht diese Aussage, dass vor allem

\footnotetext{
${ }^{3}$ Habermas, Strukturwandel der Öffentlichkeit, 1990, S. 356.
} 
Akademikerinnen keine Kinder bekommen, im Wesentlichen auf den Analysen des Mikrozensus, einer Querschnittsstudie an in Deutschland lebenden Menschen, die der deutschen Sprache weitgehend mächtig sind. Aus diesen Daten wurden vor allem die Frauen und hier jene im Alter bis zu 37 oder 39 Jahren analysiert. Hierbei ergibt sich jedoch das methodische Problem, dass über die „wahre“ Kinderlosigkeit aus Querschnittsstudien nur bedingt Aussagen getroffen werden können, da bei einer Tendenz zur späten Erstgeburt viele Frauen aus den Statistiken herausfallen. Und - warum nur Frauen? Wie steht es mit der Kinderlosigkeit der Männer?

Die bisher valideste Kohortenanalyse aus Daten des German Socio Economic Panels (GSOEP), einer großen Befragungsbatterie, die im Längsschnitt durchgeführt wird, zeigt nach der Analyse von Christian Schmitt und Ulrike Winkelmann ein anderes Bild. ${ }^{4}$ Danach liegt der reale Anteil der Kinderlosen sowohl von Akademikerinnen als auch von Akademikern bei unter $25 \%$, da viele erst sehr spät Kinder bekommen. Diejenigen, die am wahrscheinlichsten kinderlos bleiben, sind nach diesen Analysen der Längsschnittdaten Männer mit niedrigem Bildungsabschluss.

Dieses Beispiel verdeutlicht einiges: Es geht nicht nur um die Frage der Kinderlosigkeit und des Kinderwunsches, sondern auch darum, wer Kinder kriegen kann, darf und soll.

Sowohl wissenschaftliche Analysen als auch öffentliche Debatten greifen bestimmte Fakten auf, die bestimmten moralischen Vorstellungen von einem guten Leben entsprechen. Die blinden Flecken und Verzerrungen (dass es auch um männliche Kinderlosigkeit geht und augenscheinlich am meisten befürchtet wird, dass sich die „geistigen Eliten“ nicht „,reproduzieren“) sind bei Fragen der menschlichen Fortpflanzung offenbar sehr deutlich. Dies ist auch verständlich. Geht es hierbei doch auch um Fragen von höchster Privatheit einerseits, und von höchster gesellschaftspolitischer Relevanz andererseits.

Wir haben in den Studien unserer Arbeitsgruppe sowohl eine Vielzahl qualitativer, offener Interviews, als auch große standardisierte Befragungen von Menschen aus der Bevölkerung, Paaren ohne Sterilitätsproblematik und ohne ein bekanntes erhöhtes Risiko für die Vererbung von Erkrankungen oder Behinderungen, Paaren in der IVF Behandlung, Paaren mit einem bekannten Risiko für genetisch bedingte Erkrankungen (sowohl deutsch- als auch türkischstämmige Paare) und verschiedener Expertengruppen (Hebammen, Gynäkologen/Reproduktionsmediziner, Humangenetiker, Pädiater und Ethiker) durchgeführt.

In den qualitativen Interviews zeigten sich bereits sehr divergente Auffassungen, als was der Kinderwunsch anzusehen sei und was Kinderlosigkeit bedeute. Die Aussagen der Experten reichten von „der Kinderwunsch ist ein wesentlicher, zentraler Aspekt des Lebens", über Ansichten, er sei vorwiegend „sozial strukturiert", „manchmal

4 Schmitt/Winkelmann, Wer bleibt kinderlos? Was sozialstrukturelle Daten über Kinderlosigkeit bei Frauen und Männern verraten, Feministische Studien 2005/1, 9 ff. 
pathologisch“ bis zu der Vorstellung eines Kinderwunsches als einer ,natürlichen, biologischen Determination“. Ungewollte Kinderlosigkeit stellt für manche ein „Schicksal dar, mit dem sich Betroffene abfinden sollten", oder auch einen "therapierbaren Zustand" oder einen durchaus krankhaften, leidvollen Zustand.

Wir haben gemäß den Aussagen aus den qualitativen Interviews und der Literatur die Instrumente für unsere standardisierten Befragungen erstellt. Die Meinungen der Experten bezüglich des Status ungewollter Kinderlosigkeit klaffen hierbei deutlich auseinander.

Von den befragten Expertengruppen sehen Hebammen und auch in geringerem Maße die befragten Ethiker in den standardisierten Befragungen den Kinderwunsch und die Kinderlosigkeit am ehesten als sozial konstruiertes Phänomen an. 4,5\% der Hebammen (gegenüber 0\% der Gynäkologen und Reproduktionsmediziner, $0,6 \%$ der Pädiater, $1 \%$ der Humangenetiker und 2,4\% der Ethiker) meinen, ungewollte Kinderlosigkeit sei ein "Schicksal, dem sich die Betroffenen fügen sollten". Knapp 20\% der Hebammen und Ethiker, 12\% der Pädiater, 6\% der Humangenetiker und 1,4\% der Gynäkologen/Reproduktionsmediziner meinen, ungewollte Kinderlosigkeit sei ein „Lebensumstand, mit dem sich die Betroffenen im Normalfall arrangieren sollten." Die quantitativ meisten Befragten aller Gruppen sind der Auffassung, die ungewollte Kinderlosigkeit sei zwar eine Funktionsstörung, die aber „nicht grundsätzlich behandlungsbedürftig" sei. Allein Gynäkologen sehen mit über 40\% die ungewollte Kinderlosigkeit als eine behandlungsbedürftige Krankheit an.

IVF-Paare unterscheiden sich ebenfalls hinsichtlich ihrer Ansichten zum Kinderwunsch. Die Wichtigkeit eines leiblichen Kindes von beiden Partnern ist für fast alle befragten deutsch- und türkischstämmigen IVF Paare „extrem wichtig“. Während sich jedoch manche ein Leben ohne eigenes Kind überhaupt nicht vorstellen können, sehen andere durchaus Alternativen in der Lebensplanung als gleichwertig an. Jeweils 20\% der IVF Paare können sich ein Leben ohne Kind überhaupt nicht, $20 \%$ sehr gut vorstellen. Interessanterweise hängt die geschätzte Erfolgsquote der IVF Behandlung nach unseren Analysen weniger von „objektiven" Faktoren, wie Alter, Dauer der Behandlung und Anzahl der durchgeführten Zyklen, sondern von den genannten ,subjektiven“ Faktoren, vor allem der Wichtigkeit eines leiblichen Kindes ab. Die genannten Erfolgsquoten der Behandlung reichen von $0-100 \%$, was möglicherweise an einer inadäquaten Aufklärung, oder aber auch daran liegt, dass unabhängig von den genannten Zahlen die Vorstellung über den Erfolg der Behandlung, wie viele kognitionspsychologische Untersuchungen zeigen, von psychologischen Faktoren, Wünschen und Sehnsüchten abhängen, da Menschen weniger „logisch“ als „psycho-logisch“ denken. 


\section{Einstellungen zu neuen reproduktionsmedizinischen Technologien}

Auf dem Feld der Reproduktionsmedizin haben sich in den letzten 20 Jahren seit Verabschiedung des Embryonenschutzgesetzes viele neue Entwicklungen ergeben. Durch die Präimplantationsdiagnostik (PID) wurde erstmals die genetische Untersuchung von in vitro erzeugten Embryonen ermöglicht; die Felder der Molekulargenetik und der Reproduktionsmedizin überschneiden sich nun in einem neuen Feld der „Reprogenetik“. Während die ersten Überlegungen dahin gingen, die PID für in der Regel nicht-sterile Paare einzusetzen, die ein bekanntes genetisches Risiko für die Vererbung von genetisch (mit-)bedingten Erkrankungen oder Behinderungen haben, wurde die PID recht schnell auf Indikationen in der Routine-IVF übertragen. Nach den letzten veröffentlichten Daten des ESHRE PGD (European Society for Human Reproduction preimplantation genetic diagnosis) Konsortiums ${ }^{5}$ wurden von den insgesamt bis 2003 berichteten 6.200 durchgeführten PID Zyklen nur 46,3\% aufgrund eines vorher genau bekannten genetischen Risikos (monogenetischen Erkrankungen, monogenetischen, geschlechtsgebundenen Erkrankungen, bekannten Translokationen/Chromosomenfehlverteilungen der Keimzellen, einzelne Fälle von HLA-Typisierungen) durchgeführt. Bei weiteren 49,7\% wurde die PID in der IVF angewendet, das heißt, bei Frauen, die sich aufgrund der eigenen Infertilität oder der Infertilität ihres Partners einer IVF unterzogen haben, und die PID zusätzlich angewendet haben. Diese Frauen waren älter und/oder hatten wiederholt ein Implantationsversagen in der IVF, d.h. sie haben nicht erfolgreiche IVF Zyklen erlebt. In diese Zahlen gehen auch die wenigen Fälle von Paaren ein, die wiederholt Fehlgeburten erlitten haben, nicht steril sind, und die PID aufgrund dieser Fehlgeburten durchgeführt haben.

Die Rate dieses Präimplantationsscreenings (PGS; preimplantation genetic screening, dem routinemäßigen Untersuchen von zahlenmäßigen Chromosomenfehlverteilungen) ist in den letzten Jahren zunehmend gestiegen. Der Anteil des PGS lag in den Zyklen 2003 bereits bei 57,7\%. 2007 erschien jedoch in der renommiertesten Zeitschrift der Medizin, dem New England Journal of Medicine, das Ergebnis des ersten randomisierten, doppelblinden, kontrollierten Versuchs zum PGS bei Altersindikation, d.h. bei Frauen, die sich einer IVF unterziehen und über 35 Jahre alt sind, ohne dass es häufiger zu Implantationsversagen oder habituellen Fehlgeburten gekommen war. ${ }^{6}$ Hierbei zeigte sich, dass es durch das PGS

\footnotetext{
${ }^{5}$ Das ESHRE PGD Konsortium ist ein Zusammenschluss vorwiegend europäischer Zentren, die alle durchgeführten PID Zyklen dokumentieren und deren Ausgang an das Steering Committee senden. Waren dies zu Beginn der Datenerhebung 1999 noch 16 Zentren, so stieg die Zahl der teilnehmenden Zentren in der letzten Datenerhebung auf 50. Hierbei nehmen auch einige nicht europäische Zentren, wie z.B. indische Zentren, an der Datenerhebung teil. Vgl. zu den Daten: ESHRE PGD Consortium Steering Committee, ESHRE PGD Consortium Data Collection VI: cycles from January to December 2003 with pregnancy follow up to October 2004, Human Reproduction 2007/22, $323 \mathrm{ff}$.

${ }^{6}$ Die Studie wurde in den Niederlanden durchgeführt, vgl. Mastenbroek/Moniek/Twisk/ v.Echten-Arends/Sikkma-Raddatz/Korevaar/Verboeve/Vogel/Eus/d.Vries/Bosuyt/Charles/Buys/Heineman/ Reping/v.d.Veen, In Vitro Fertilization with Preimplantation Genetic Screening, The New England
} 
zu weniger Schwangerschaften und Lebendgeburten kam als bei der Durchführung einer Routine IVF ohne PGS. Die Autoren erklären dies im Wesentlichen mit möglichen Auswirkungen der Biopsie auf die Implantationsmöglichkeit des Embryos, auf die geringere Anzahl von Embryonen, die nach Biopsie zur Verfügung stehen. Durch diese Ergebnisse ist zu vermuten, dass die große Gruppe derer, die ein PGS allein aufgrund ihres erhöhten Eintrittsalters in die IVF haben durchführen lassen („Routine-IVF ab 35“) deutlich geringer werden wird. Diese Ergebnisse sind allerdings nicht direkt auf die weiteren oben beschriebenen Gruppen übertragbar, die ein PGS durchführen lassen (wiederholtes Implantationsversagen, habituelle Aborte). In den ESHRE PGD Daten sind zudem für alle Zyklen bis 2003 4,1\% als Zyklen zum ,social sexing“ gekennzeichnet (2003: 2,7\%), d.h. die Bestimmung des Geschlechts wurde nicht aufgrund einer geschlechtsgebundenen Erkrankung, sondern aufgrund des Wunsches der Eltern durchgeführt, ein Kind eines bestimmten Geschlechts zu bekommen. Welche Zentren weltweit social sexing durchführen, ist nicht genau bekannt. Als 2002 zum ersten Mal durch das ESHRE Konsortium von social sexing berichtet wurde, bekannte sich ein teilnehmendes Zentrum aus Australien zu dieser Praxis, ebenso etwas später ein Zentrum aus Indien. Vermutet wurde, dass social sexing in Einzelfällen auch in den USA durchgeführt wurde. Zu diesem Zweck wird dort häufiger als die PID die sogenannte Microsort Technik angewendet, durch welche Spermien mit weiblichen und männlichen Chromosom durch Zentrifugation voneinander getrennt werden, und so die Wahrscheinlichkeit, ein Kind des gewünschten Geschlechts zu bekommen, deutlich ansteigt, wenn die erwünschte Spermienfraktion zur Befruchtung (häufig nicht durch IVF, sondern durch Insemination, d.h. Einbringen der Spermien zum Zeitpunkt des Eisprungs in die Scheide) verwendet wird. In Europa wird social sexing mit hoher Wahrscheinlichkeit bisher nicht durchgeführt; in der Debatte gibt es jedoch durchaus einige Stimmen, die dies auch für Europa befürworten. ${ }^{7}$

Die PID zur HLA (humane Leukozyten-Antigen/Histokompatibilitäts-Typisierung) wurde erstmals im Jahr 2000 in den USA durchgeführt. In einigen europäischen Ländern, wie England oder Belgien, wurde in Einzelfällen, teils begleitet von öffentlichen Debatten, eine HLA-Typisierung in Familien durchgeführt, in denen ein Geschwisterkind an einer tödlich verlaufenden Erkrankung leidet, die durch die Transplantation von Stammzellen aus dem Nabelschnurblut geheilt wer-

Journal of Medicine 2007/1, 9 ff. Randomisiert und doppelblind bedeutet, dass die Zuteilungen von Frauen über 35 einem Zufallsprinzip entsprachen. Hierdurch versucht man, bekannte und unbekannte Confounder (beeinflussende Variablen) auf die Interventionsgruppe (PGS) und die Kontrollgruppe (IVF ohne PGS) gleich zu verteilen. Doppelblind bedeutet, dass weder den Ärzten, noch den Patientinnen bekannt war, ob ein PGS bei ihren Embryonen durchgeführt wurde. Dadurch wird der Effekt des Wissens über Behandlung und Nicht-Behandlung auf das Studienergebnis (Placebo Effekt) ausgeschlossen, und das Ergebnis hängt mit einer großen Wahrscheinlichkeit von der Intervention (hier: PGS) ab. Die Durchführung eines solchen Versuchs erfüllt damit den höchsten Standard der evidenzbasierten Medizin.

7 Vgl. Savulescu/Dahl, Sex selection and preimplantation diagnosis: a response to the Ethics Committee of the American Society of Reproductive Medicine, Human Reproduction 2000/15, $1879 \mathrm{ff}$. 
den können, u.a. verschiedene Formen der Thalassämie und erbliche Leukämieformen.

Wie auch in dem Beitrag von Michelmann geschildert, hat sich die Praxis der Reproduktionsmedizin jedoch vor allem dahingehend geändert, dass sich international ein neuer Goldstandard der IVF Behandlung durchsetzt: der sogenannte Single Embryo Transfer, d.h. einer Blastozystenkultivierung (Entwicklung aller Embryonen bis zum mehrzelligen Stadium), die Auswahl des sich morphologisch am besten entwickelnden frühen Embryos und dessen Einsetzung in die Gebärmutter, in der Regel ohne zusätzliche genetische Diagnostik. ${ }^{8}$ Hierdurch werden insgesamt deutlich höhere Schwangerschaftsraten erzielt, darüber hinaus kommt es zu weniger Mehrlingsschwangerschaften und -geburten, die eines der größten Risiken der IVF Behandlung für die Mutter und die Kinder darstellen. Nach Mehrheitsauffassung des deutschen Parlamentes (vgl. Deutscher Bundestag, , Nationaler Ethikrat, ${ }^{10}$ nicht aber unbedingt der Verfassungsrichter $)^{11}$ ist die Präimplantationsdiagnostik und die Blastozystenkultivierung („Dreier-Regel“) in Deutschland verboten. ${ }^{12}$

Hinsichtlich der Ansichten zu neuen reproduktionsmedizinischen Technologien haben wir verschiedene Optionen von den Betroffenen, den Experten und der Bevölkerung bewerten lassen, wobei nicht alle Gruppen zu allen Möglichkeiten gleichermaßen gefragt wurden. Während wir alle Gruppen zur PID befragt haben, wurden Blastozystenkultivierung und verschiedene Möglichkeiten der Forschung an Embryonen durch unsere Studien nur von IVF Paaren und Experten bewertet. $\mathrm{Zu}$ dieser Thematik liegen jedoch auch Befragungen anderer Forschungsgruppen, unter anderem aus Leipzig vor, die an dieser Stelle ebenfalls dargestellt und diskutiert werden.

Wir haben jedoch nicht nur die oben beschriebenen Gruppen befragt, sondern auch eine Printmedienanalyse der Debatte in einem Forschungsseminar am Zent-

\footnotetext{
8 Michelmann, Reproduktionsmedizin im Jahre 2008: Probleme - Wünsche - Lösungsansätze, in diesem Band.

${ }^{9}$ Enquete-Kommission „Recht und Ethik der modernen Medizin“, Schlussbericht, 2002 (BT-Drs.14/9020).

10 Nationaler Ethikrat, Genetische Diagnostik vor und während der Schwangerschaft, 2003; Online unter: www.ethikrat.org/stellungnahmen/pdf/Stellungnahme_Genetische_Diagnos-ik.pdf.

11 Als weitere interessante Diversifizierung der öffentlichen Debatte vgl. die Analysen der Verfassungsrechtlerin Frommel, Auslegungsspielräume des Embryonenschutzgesetzes, Journal für Reproduktionsmedizin und Endokrinologie 2004, $104 \mathrm{ff}$.

${ }^{12}$ Ebenfalls verboten ist die Herstellung von embryonalen Stammzellen aus den dennoch wenigen vorhandenen ,überzähligen“ Embryonen, nicht aber, wie weitgehend bekannt, der Import embryonaler Stammzellen, die vor einem bestimmten Stichtag entstanden sind (seit April 2008 gilt der 1. Mai 2007 als Stichtag). An diese Stammzellen sind gemäß dem deutschen Stammzellgesetz und seiner jüngsten Novellierung neben dem Entstehungsdatum vor dem Stichtag einige weitere Bedingungen geknüpft: Spenderinnen dürfen keine geltwerten Vorteile erhalten, die Embryonen dürfen nicht aus Gründen gespendet worden sein, die in den Eigenschaften des Embryos selbst liegen (z.B. bei Vorhandensein einer Anlage für Erkrankungen). Nicht explizit vorgeschrieben ist jedoch, dass die Spender/Paare wirksam in die Spende eingewilligt haben. Davon wird zwar ausgegangen, es wird jedoch nicht im Gesetzestext erwähnt.
} 
rum für Konfliktforschung durchgeführt. Hinsichtlich der in den Medien geäußerten Ansichten zur Präimplantationsdiagnostik und Stammzellforschung haben wir eine systematische Analyse aller Artikel aus der Frankfurter Allgemeinen Zeitung, der TAZ, der WELT, der Süddeutschen Zeitung und der Oberhessischen Presse als Lokalzeitung der Jahre 2000 und 2001 durchgeführt. Hierbei war zum einen ein Schwenk zu beobachten. Während die Präimplantationsdiagnostik als reproduktionsmedizinische Möglichkeit nach dem Diskussionsentwurf der Bundesärztekammer 2000 in den Medien breit diskutiert wurde, schwenkte die Debatte Ende 2000 auf die Diskussion um die Zulässigkeit, Möglichkeiten und Grenzen der Stammzellforschung um. Die PID (wie auch die Möglichkeit der Blastozystenkultivierung) werden seitdem eher am Rande diskutiert, die Debatte um die embryonale Stammzellforschung reißt jedoch nicht ab. Im Hinblick auf die embryonale Stammzellforschung kam es im Jahre 2002 zu gesetzlichen Regulierungen und jüngsten Nachregulierungen, während sich auf dem Feld der Reproduktionsmedizin auf Gesetzesebene in den letzten 8 Jahren seit dem Diskussionsentwurf der Bundesärztekammer keine Neuerungen ergeben haben.

Die Printmedienanalyse der Jahre 2000 und 2001 erbrachte einige spannende Ergebnisse. So zeigte sich, dass die meisten Artikel über das gesamte Spektrum hinweg Präimplantationsdiagnostik und Stammzellforschung eher negativ beschrieben (vgl. Abb. 1, die die wertende Darstellung der Präimplantationsdiagnos-

\section{Darstellung der PID im}

Gesamtartikel (Jahresvergleich)

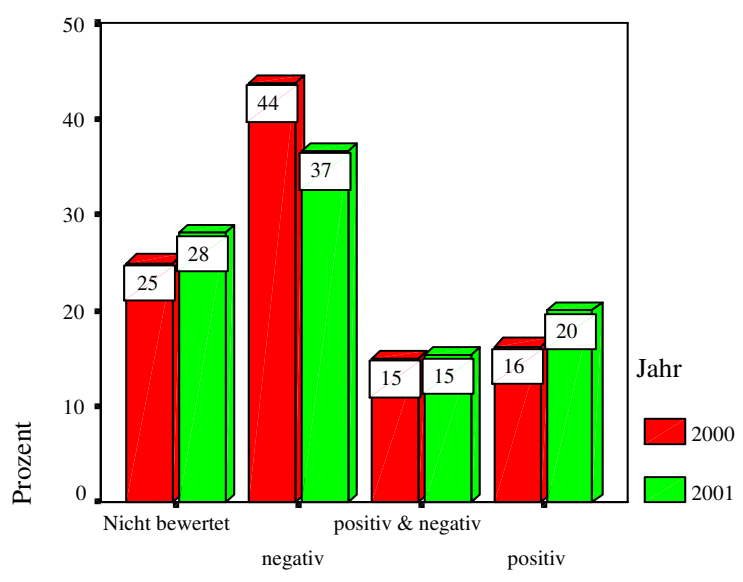

tik in den Printmedien veranschaulicht). Wenn eine Forderung durch die Autoren erhoben wurde, dann eher diejenige nach dem Verbot der Techniken. Verschiedene Akteure kamen zu Wort: Während die Meinung von Experten auch unkommentiert mitaufgenommen oder stehen gelassen wurde, wurde die Erfahrung von Betroffenen (IVFPaaren, Paaren mit einem bekannten

genetischen Risiko) meist als Aufhänger benutzt, um eigene Ansichten oder die Ansichten von Experten zu schildern. Wir haben zusätzlich die Leserbriefe an die Zeitungen analysiert. In diesen sprachen sich interessanterweise die meisten $\mathrm{Zu}$ schriften für eine Legalisierung der Präimplantationsdiagnostik aus. Dass sich be- 
züglich der Auffassung zur Präimplantationsdiagnostik die „formelle“ (journalistische, intellektuelle und politische) Öffentlichkeit von der ,informellen“ Öffentlichkeit (Privatpersonen, Praktiker und direkt Betroffene) tatsächlich deutlich unterscheidet, zeigte sich sowohl in den Umfragen, die unsere Arbeitsgruppe durchgeführt hat, als auch in den zeitgleichen Umfragen der Leipziger Arbeitsgruppe. ${ }^{13}$ Dass die Zustimmung zu einer Legalisierung der PID bei Hochrisikopaaren (89\% der deutschstämmigen Hochrisikopaare), aufgrund der unmittelbaren Betroffenheit eher hoch sein würde, hatten wir erwartet. Diese Befragung wurde von unserer Arbeitsgruppe bereits 2001 durchgeführt. Mit einer ähnlich hohen oder noch höheren Zustimmung hatten wir dann auch bei deutschstämmigen IVF Paaren und türkischstämmigen Paaren gerechnet, die zusammen mit allen weiteren Gruppen zwischen 2002 und 2004 befragt wurden. Dass sich jedoch in allen Gruppen, sowohl in der repräsentativ befragten Bevölkerung, als auch in den repräsentativ befragten Expertengruppen keine Mehrheit für ein Verbot der PID finden würde, sondern im Gegenteil eine derart überwältigende Zustimmung zu einer Legalisierung der PID in Deutschland existiert, hatten wir nicht erwartet.
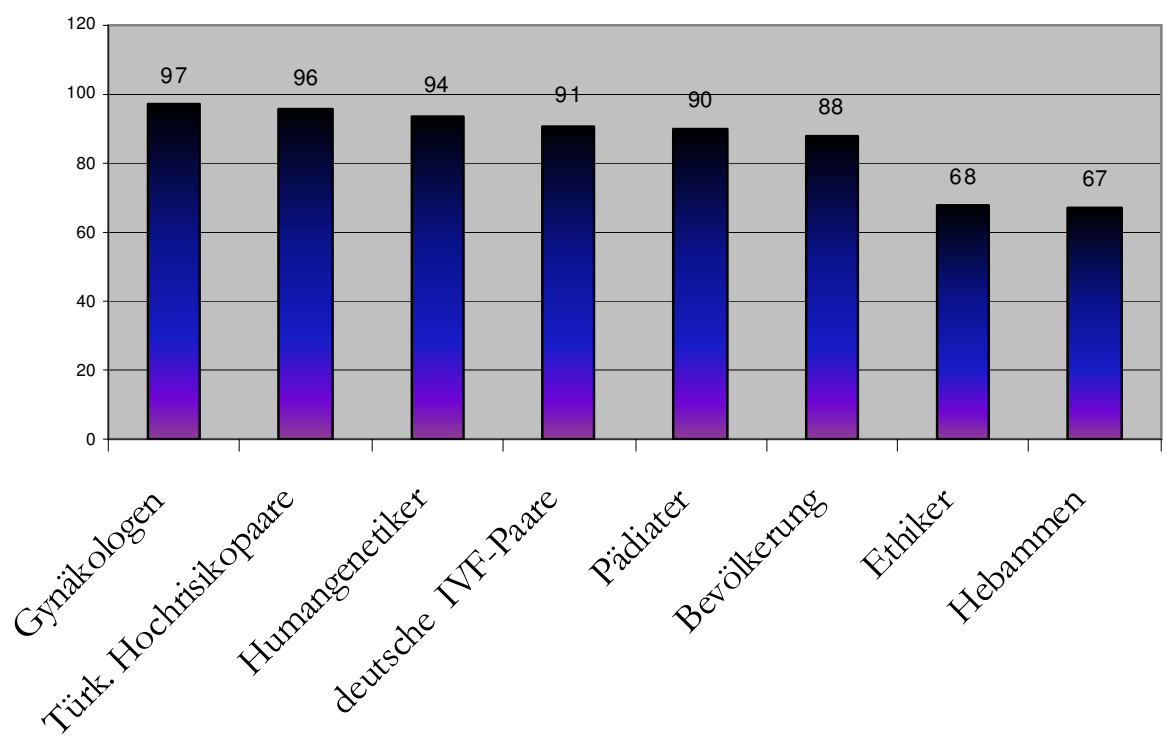

Abb. 2: Zustimmung zur Legalisierung der PID in allen Stichproben, Angaben in Prozent.

Wir haben jedoch nicht nur danach gefragt, ob und, wenn ja, in welchem Maße die Präimplantationsdiagnostik legalisiert werden solle, sondern auch dezidiert nach der Bewertung von verschiedenen Möglichkeiten gefragt, die PID anzuwenden

13 Meister/Finck/Stäbel-Richter/Schmutzer/Bräbler, Knowledge and attitudes towards preimplantation genetic diagnosis in Germany, Human Reproduction 2005/20, $231 \mathrm{ff}$. 
und dies auch mit Ansichten zu Indikationen zur Pränataldiagnostik und zum Schwangerschaftsabbruch verglichen. Dabei wurde in der Experten- und Bevölkerungsstichprobe abgefragt, für welche der Erkrankungen/Eigenschaften eine PID (sowie eine PND und ein Schwangerschaftsabbruch) zulässig sein sollten und inwiefern die Befragten selbst über eine Nutzung nachdenken würden. Die Kategorien wurden in der Expertengruppe durch Beispielerkrankungen erfragt (z.B. früher Tod: Werdnig-Hoffmannsche Muskelatrophie (SMA Typ I), chronische Krankheit, die früher zum Tod führt: Mukoviszidose). Die moralische Bedenklichkeit der PID für verschiedene weitere Indikationen (u.a. Aneuploidiescreening, Verbesserung des Genpools und Steigerung von bestimmten Eigenschaften, sog. Enhancement) wurde sowohl in der Experten- als auch in den Paar-Stichproben erhoben. Geschlechtsselektion und Enhancement wurden in der Expertengruppe nicht in den Indikationskatalog aufgenommen, sondern ausschließlich deren moralische Bedenklichkeit erfragt, da in den Pretests deutlich wurde, dass die Erfragung von Geschlechtsauswahl und Eigenschaften als mögliche PID Indikation zu stärkerem non-response bei einigen Experten führt.

Abb. 3 zeigt die Ansichten der Bevölkerung zu verschiedenen Möglichkeiten der PID im Vergleich zu der Auffassung der Experten (Tab. 1). Die Säulen in Abb. 3 stellen prozentuale Angaben der Gesamtbevölkerung dar.

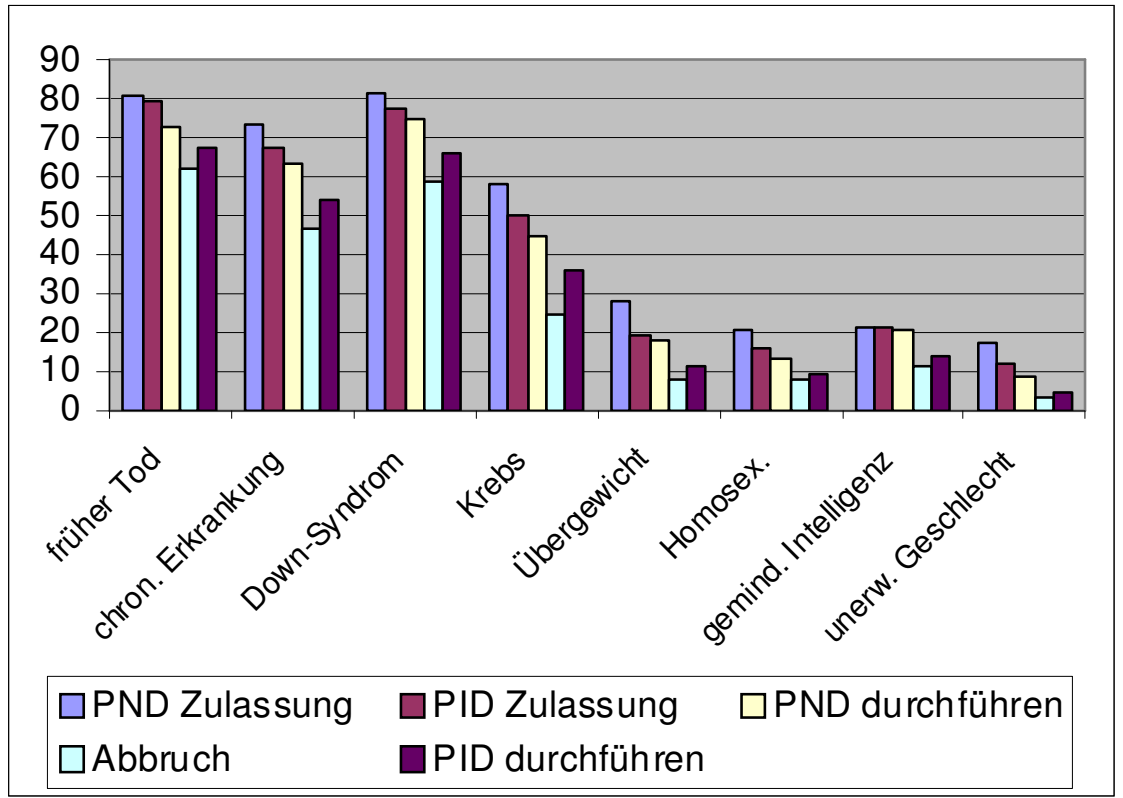

Abb. 3: PND-, PID- und Abbruchsindikationen, Ansichten der Bevölkerung ( $\mathrm{n}=1017)$, Angaben in Prozent.

Zwar stimmen geringfügig weniger Befragte der Bevölkerung für eine allgemeine Verfügbarkeit der PID im Verhältnis zur PND für alle Indikationen (linke zwei Balken) zu, selbst würden jedoch mehr Befragte bei Antizipation der Situation eine 
PID als einen Schwangerschaftsabbruch durchführen lassen (rechte zwei Balken). Experten halten die PID ebenfalls in allen Fällen für zulässiger als einen Schwangerschaftsabbruch, insbesondere für spätmanifestierende Erkrankungen, wie Tabelle 1 zeigt.

Eine Ausnahme bildet die Beurteilung der Trisomie 21 durch Humangenetiker, die bei Vorliegen eines Down Syndroms eher eine PND mit möglichem Abbruch als eine PID für angemessen erachten. Dies resultiert vermutlich aus dem humangenetisch-medizinischen Wissen, dass in der Regel das Down Syndrom nicht aufgrund einer Translokation des Chromosoms 21 vererbt wird, für die eine PID möglich ist, sondern durch die nicht erbliche Ursache entsteht, dass sich die Chromosomen nicht voneinander trennen, was zufällig, mit steigendem Alter jedoch häufiger passiert und so nur mittels PND ermittelt werden kann. Vergleicht man die Ansichten der Bevölkerung (Abb. 3) mit denjenigen der Experten (Tab. 1), so zeigt sich, dass diese bei schweren Erkrankungen sowie beim Down Syndrom nicht sehr stark differieren. Die Gruppe der Hebammen und der Ethiker lehnt jedoch auch bei schweren Erkrankungen sowohl Schwangerschaftsabbruch als auch PID häufiger ab und würde nach eigenen Angaben signifikant weniger häufig selbst einen Schwangerschaftsabbruch in diesen Situationen vornehmen lassen. Für spät manifestierende Erkrankungen oder möglicherweise unerwünschte Eigenschaften war sich der Großteil aller Experten darin einig, dass diese weder eine Indikation für einen Schwangerschaftsabbruch noch für eine PID darstellen sollten. Geschlechtsselektion und Enhancement wurden von allen Experten als extrem moralisch bedenklich eingestuft (Daten hier nicht gezeigt). ${ }^{14}$ Die Bevölkerung ist in diesen Fällen bereiter, eine PID oder einen Abbruch für zulässig zu erachten.

Tab. 1: Zulässige Indikationen für Schwangerschaftsabbruch und PID, Beurteilung durch Experten:

\begin{tabular}{|l|c|c|c|}
\hline $\begin{array}{l}\text { Krankheiten/ } \\
\text { Behinderungen/Eigenschaft +* }\end{array}$ & $\begin{array}{l}\text { Spätab- } \\
\text { bruch } \\
\text { ethisch } \\
\text { zulässig }\end{array}$ & $\begin{array}{l}\text { PID ethisch } \\
\text { zulässig }\end{array}$ & $\begin{array}{l}\text { Würde selbst } \\
\text { Abbruch } \\
\text { durchführen }\end{array}$ \\
\hline Früher Tod im Kleinkindalter (gesamt) & $\mathbf{5 7 . 2} * * *$ & $\mathbf{6 7 . 6} * * *$ & $\mathbf{5 3 . 6 * * *}$ \\
Humangenetiker & 87.5 & 89.4 & 79.8 \\
Gynäkologen & 79.6 & 87.8 & 74.1 \\
Pädiater & 65.1 & 79.5 & 66.9 \\
Hebammen & 40.5 & 51.4 & 31.6 \\
Ethiker & 40.5 & 53.0 & 44.6 \\
\hline Schwere chron. Erkrankung, Tod in & & & \\
frühem Erwachsenenalter (gesamt) & $\mathbf{3 2 . 1 * * *}$ & $\mathbf{5 5 . 4 * * *}$ & $\mathbf{2 8 . 7 * * *}$ \\
\hline
\end{tabular}

${ }^{14}$ Krones/Schlüter/Manolopoulos/Bock/Tinneberg/Kocb/Lindner/Hoffmann/Mayatepek/Huels/Neunohner/El Ansari/Wissner/Richter, Public expert and patients opinions on preimplantation genetic diagnosis (PGD) in Germany, Reproductive Biomedicine online 2005/10, $116 \mathrm{ff}$. 


\begin{tabular}{|l|l|l|l|}
\hline Humangenetiker & 72.1 & 80.8 & 55.8 \\
Gynäkologen & 50.3 & 80.3 & 44.9 \\
Pädiater & 34.3 & 68.1 & 37.3 \\
Hebammen & 13.6 & 35.7 & 8.8 \\
Ethiker & 21.4 & 39.9 & 23.8 \\
\hline Downsyndrom (gesamt) & $41.0 * * *$ & $\mathbf{5 2 . 1} * * *$ & $\mathbf{3 5 . 5} * * *$ \\
Humangenetiker & 84.6 & 71.2 & 59.6 \\
Gynäkologen & 68.7 & 83.0 & 63.9 \\
Pädiater & 42.2 & 65.1 & 41.0 \\
Hebammen & 22.4 & 35.1 & 15.6 \\
Ethiker & 20.8 & 29.8 & 25.0 \\
\hline Hohe Disposition für Krebs im & & & \\
Erwachsenenalter (gesamt) & $\mathbf{1 2 . 7}$ & $\mathbf{3 5 . 7} *$ & $\mathbf{1 3 . 1}$ \\
Humangenetiker & 15.4 & 34.6 & 11.5 \\
Gynäkologen & 12.9 & 46.3 & 16.3 \\
Pädiater & 11.4 & 44.0 & 17.5 \\
Hebammen & 10.9 & 29.3 & 8.8 \\
Ethiker & 15.4 & 30.4 & 14.3 \\
\hline Übergewicht (gesamt) & $\mathbf{0 . 6}$ & $\mathbf{4 . 2}$ & $\mathbf{0 . 7}$ \\
Humangenetiker & $\mathrm{n}=1$ & 3.8 & $\mathrm{n}=0$ \\
Gynäkologen & $\mathrm{n}=2$ & 8.2 & $\mathrm{n}=2$ \\
Pädiater & $\mathrm{n}=1$ & 3.6 & $\mathrm{n}=1$ \\
Hebammen & $\mathrm{n}=0$ & 3.1 & $\mathrm{n}=1$ \\
Ethiker & $\mathrm{n}=1$ & 3.6 & $\mathrm{n}=2$ \\
\hline
\end{tabular}

${ }^{*} \mathrm{p}<.05 ; * * * \mathrm{p}<.0001$ für den Intergruppenvergleich

* Indikatorerkrankungen der Expertenbefragung:

- Tod im frühen Kindesalter: Werdnig-Hoffmannsche Muskelatrophie

- Schwere chronische Erkrankung: Mukoviszidose

- Spätmanifestierender Krebs: Familiäre adenomatöse Poliposis Coli (FAP)

Interessant ist hinsichtlich der Grenzen der Legalisierung die Tatsache, dass über 10\% der Hebammen und Ethiker, die eine PID selbst bei frühem Tod des Kindes, z.B. bei der Werdnig-Hoffmannschen Muskelatrophie, nicht für moralisch gerechtfertigt halten, dennoch einer Legalisierung zustimmten (Abb. 2). Wir gehen davon aus, dass beide Gruppen hoch differenziert urteilen und daher diejenigen Ethiker und Hebammen, die der PID sehr skeptisch gegenüber stehen, dennoch nicht der Auffassung sind, ihre persönlichen Werte sollten sich in allgemeinen Normen niederschlagen und die PID verboten bleiben. Die meisten Befragten der Bevölkerung und der genetischen Hochrisikopaare waren der Ansicht, die PID solle für alle genetischen Erkrankungen zugelassen werden und die Paare sollten über die Rechtmäßigkeit selbst entscheiden. Die relative Mehrheit der Experten (bis auf die Gynäkologen) war der Ansicht, die PID solle eher nur für schwere genetische Erkrankungen zulässig sein. Gynäkologen meinten, die PID solle regelhaft auch in der IVF zur Verfügung stehen, und IVF Paare meinten mehrheitlich, die PID solle allen zur Verfügung stehen, die die Diagnostik nutzen möchten. Die Experten wurden darüber hinaus gefragt, wie denn, falls sie einer Legalisierung zustimmen würden, die rechtliche Regelung konkret aussehen solle. Die relative Mehrheit aller Expertengruppen war der Auffassung, die PID sollte analog zu \218 geregelt wer- 
den. Sie solle verbunden sein mit einer verpflichtenden Beratung, die Paare sollten aber selbst darüber entscheiden, ob in ihrem Fall eine PID angemessen wäre. Wie die Subanalysen in der Bevölkerung zeigten, spielte beispielsweise die Parteienpräferenz in der Gesamtbevölkerung für die Bewertung der Legalisierung der PID kaum eine Rolle. Wählerinnen und Wähler der Grünen stimmen einer Legalisierung der PID ohne jegliche Beschränkungen z.B. eher zu als Befragte mit anderen Parteipräferenzen. Im Bundestag macht sich die Fraktion der Grünen dagegen insgesamt für ein Verbot der PID stark. Personen, die sich im politischen Spektrum links einordnen, neigen eher zu einem Verbot der PID als diejenigen, die sich eher rechts einordnen, die Differenzen sind jedoch nicht signifikant.

Im Hinblick auf Auffassungen zur PID gibt es einige vergleichbare Studien, beispielsweise aus England und den USA. In Tabelle 2 sind einige dieser Ergebnisse zusammengefasst.

Tab. 2: Ansichten zur PND, Schwangerschaftsabbruch und PID in Deutschland (eigene Daten), den USA und UK:

\begin{tabular}{|l|c|c|c|}
\hline \multicolumn{1}{|c|}{ Zustimmung zu: } & Deutschland & USA $^{1,2}$ & UK $^{3,4,5}$ \\
\hline $\begin{array}{l}\text { PND bei schweren, u.a. früh tödlichen geneti- } \\
\text { schen Erkrankungen }\end{array}$ & $74-81$ & $66^{1}$ & $41-71^{3,4}$ \\
\hline $\begin{array}{l}\text { PID bei schweren, u.a. früh tödlichen geneti- } \\
\text { schen Erkrankungen }\end{array}$ & $68-79$ & $74^{1}$ & $35-37^{3}$ \\
\hline $\begin{array}{l}\text { PID bei spätmanifestierenden erblichen } \\
\text { Krebs-Erkrankungen }\end{array}$ & 58 & - & $34^{4}$ \\
\hline $\begin{array}{l}\text { PID bei spätmanifestierenden erblichen } \\
\text { Krebs-Erkrankungen }\end{array}$ & 50 & $60^{1}$ & - \\
\hline $\begin{array}{l}\text { Würde Abbruch bei spätmanifestierenden } \\
\text { Krebs-Erkrankungen durchführen }\end{array}$ & 25 & - & $16^{4}$ \\
\hline PND zur Geschlechtsbestimmung & 11 & $5^{1}$ & $9^{4}$ \\
\hline PID für Geschlechtsauswahl & 3 & $5^{2}$ & $8-18^{4,5}$ \\
\hline $\begin{array}{l}\text { Würde selbst Abbruch aufgrund eines nicht } \\
\text { erwünschten Geschlechts durchführen }\end{array}$ & & $3^{4}$ \\
\hline
\end{tabular}

Harris 2002: Repräsentative US-Stichprobe (Princeton Survey Research Associates), Alter 18+. Singer 1991: Repräsentative US-Stichprobe (Gallup survey), Alter 18+..$^{15}$

Stratford 2001: Repräsentative britische Stichprobe (British Social Attitudes survey), Alter 18+. ${ }^{16}$

Marteau 1995: Repräsentative britische Stichprobe (Gallup survey) Alter 18-45.17

HFEA 2003: Repräsentative britische Stichprobe (MORI survey), Alter 16+. ${ }^{18}$

15 Singer, Public attitudes towards genetic testing, Population Research and Policy Review 1991/10, $235 \mathrm{ff}$.

16 Stratford/Marteau/Bobrow, Genetic research: friend or foe?, in: Park/Curtice/Thomson (eds.), British Social Attitudes: Public policy, Social ties, The 18 ${ }^{\text {th }}$ report, 2001.

${ }_{17}$ Marteau/Michie/Drake/Bobrow, Public attitudes towards the selection of desirable characteristics in children, Journal of Medical Genetics 1995/32, 796 ff. 
Obgleich der Vergleich natürlich Schwierigkeiten bereitet, da die Fragen etwas unterschiedlich sind und sich auch die Stichproben etwas unterscheiden (wir haben lediglich Personen im Alter zwischen 18 und 50 Jahren befragt), zeigen die Angaben dennoch, dass bisher nichts dafür spricht, dass „die Briten“ oder auch „die US Amerikaner“" wesentlich liberaler seien als „die Deutschen“. Im Gegenteil - in der Tendenz stimmten in der deutschen Bevölkerungsstichprobe mehr Befragte den vergleichbaren Möglichkeiten zu Pränataldiagnostik und Schwangerschaftsabbruch zu. In der antizipierten Durchführung eines Schwangerschaftsabbruchs aufgrund eines nicht erwünschten Geschlechts sind die genannten Raten eher vergleichbar, liegen jedoch immerhin bei $3-5 \%$.

\section{Einstellungen zum Umgang und zur Forschung an Embryonen}

Hinsichtlich der weiteren Möglichkeiten, mit Embryonen umzugehen, haben wir uns damals entschlossen, diesen Fragenkomplex nur in die betroffenen Paarstichproben und den Expertengruppen mit hineinzunehmen. Um die Bevölkerung zu Thematiken zu befragen, die nicht zum Alltagswissen gehören, bedarf es ausgefeilter und gründlicher Instrumente und Überlegungen zur Kurzinformation für diejenigen, die noch nie etwas von der jeweiligen Technik gehört haben. Beispielsweise hatten vor der Befragung lediglich 30\% bereits einmal von der PID gehört (USA in der Umfrage von Harris zum Vergleich 24\%). Wir haben über mehrere Monate und mittels verschiedener Pretests die Kurzinformationen für die Bevölkerung, die Paarstichproben und die Experten entwickelt, um diese möglichst informativ und ausgewogen zu gestalten. Um die Bevölkerungsbefragung nicht zu überfrachten, haben wir uns gegen die Aufnahme des Fragenkomplexes entschieden, zumal wir wussten, dass eine große Forschungsgruppe im Rahmen des Eurobarometers eine Studie zu diesen Ansichten zeitgleich durchführte. ${ }^{19}$ Die Ergebnisse dieser Studie, ebenso wie die einer weiteren Umfrage der Stiftung „Ja zum Leben“ werden ebenfalls kurz zusammengefasst, bevor ich zu einem kurzen Resümee komme.

Die Tabellen 3 und 4 geben die Ansichten der IVF-Paare, sowie der befragten Experten zu Blastozystenkultivierung, Eizell- und Embryonenspende, die Möglichkeit des Kostenerlasses bei Spende, zur Verwendung von Embryonen für verschiedene Forschungszwecke wieder. Alle angegebenen Möglichkeiten wurden auf einer Skala von 1 (moralisch nicht bedenklich) bis 6 (moralisch sehr bedenklich) bewertet. Danach wurden Experten und IVF-Paare gebeten mitzuteilen, ob die jeweilige Möglichkeit in Deutschland zugelassen oder verboten bleiben sollte. IVF-

18 HFE A, Sex Selection: Public Consultation, 2003; Online at: www.hfea.gov.uk.

19 Solter/Beyleveld/Friele, Embryo Research in Pluralistic Europe, 2003. 
Paare wurden darüber hinaus gefragt, ob sie die jeweilige Möglichkeit selbst nutzen bzw. einer Verwendung von eigenen Eizellen oder überzähligen Embryonen für die angegebenen Zwecke zustimmen würden und ob sie auch selbst eine Eizellbzw. Embryonenspende eines anderen Paares annehmen würden.

Im Gegensatz zur hohen Zustimmung für die Zulassung der PID war die Bewertung unterschiedlicher Möglichkeiten, präimplantive Embryonen zu nutzen, bei Experten und auch bei den befragten Paaren eher von Skepsis geprägt. IVF-Paare stimmten jedoch im Gegensatz zu den Experten fast allen Möglichkeiten mehrheitlich zu. Die höchste Zustimmung fand sich bei IVF-Paaren für die Zulassung der Blastozystenkultivierung (80\%), 74\% würden diese nutzen wollen. Die Zustimmung zur Eizellspende lag mit 77\% ebenfalls hoch, wobei nur 33\% sich vorstellen konnten, eine Eizellspende zu nutzen, 37\% würden selbst Eizellen spenden. $70 \%$ der IVF-Paare waren für die Zulassung der Embryonenspende, selbst nutzen würden diese lediglich 16\%, selbst Embryonen spenden dagegen 30\%. Der Anteil der IVF Paare, welche die Forschung an Embryonen für verschiedene Möglichkeiten zulassen würden, liegt zwischen 55\% für die Grundlagenforschung und 75\% für die Forschung zur Verbesserung der IVF Behandlung und zum besseren Verständnis von Erbkrankheiten. Für eine Zulassung der Stammzellforschung und -herstellung aus Embryonen sind $71 \%$ der Paare. Die Hälfte der IVF Paare würde die eigenen Embryonen für die Stammzellherstellung und für IVF-/genetische Erkrankungsforschung zur Verfügung stellen, ein gutes Drittel auch für die Grundlagenforschung.

Tab. 3: Moralische Bedenklichkeit der Eizellspende, Embryonenspende, Embryonenforschung, Stammzellproduktion, Blastozystenkultivierung und der Spende gegen kostenlose IVF-Behandlung:

\begin{tabular}{|l|c|c|c|c|c|c|c|}
\hline $\begin{array}{l}\text { Moralische Akzep- } \\
\text { tanz von: }\end{array}$ & $\begin{array}{c}\text { IVF- } \\
\text { Paare } \\
(\mathrm{n}=202)\end{array}$ & $\begin{array}{c}\text { Ethiker } \\
(\mathrm{n}=168)\end{array}$ & $\begin{array}{c}\text { Hebam- } \\
\text { men } \\
(\mathrm{n}=298)\end{array}$ & $\begin{array}{c}\text { Pädiater } \\
(\mathrm{n}=166)\end{array}$ & $\begin{array}{c}\text { Human- } \\
\text { genetiker } \\
(\mathrm{n}=104)\end{array}$ & $\begin{array}{c}\text { Gynä- } \\
\text { kologen } \\
(\mathrm{n}=147)\end{array}$ & $\mathrm{P}$ \\
\hline $\begin{array}{l}\text { Blastozystenkul- } \\
\text { tivierung }\end{array}$ & $2.5(1.7)$ & $4.2(1.8)$ & $4.8(1.5)$ & $3.9(1.8)$ & $2.6(1.6)$ & $2.2(1.7)$ & $<.0001$ \\
\hline Eizellspende & $3.1(1.8)$ & $4.1(1.6)$ & $4.1(1.8)$ & $3.8(1.8)$ & $3.4(1.6)$ & $3.0(1.9)$ & $<.0001$ \\
\hline Embryonenspende & $3.7(1.9)$ & $4.6(1.5)$ & $5.0(1.4)$ & $4.5(1.7)$ & $4.2(1.6)$ & $3.7(1.9)$ & $<.0001$ \\
\hline $\begin{array}{l}\text { Spende gegen kos- } \\
\text { tenlose IVF Behand- } \\
\text { lung }\end{array}$ & $4.1(1.9)$ & $5.2(1.5)$ & $5.5(1.1)$ & $5.4(1.2)$ & $5.3(1.2)$ & $4.9(1.5)$ & $<.0001$ \\
\hline Stammzellproduktion & $3.1(1.7)$ & $4.0(1.8)$ & $4.4(1.6)$ & $3.4(1.7)$ & $3.4(1.7)$ & $3.3(1.7)$ & $<.0001$ \\
\hline $\begin{array}{l}\text { Forschung zur Ver- } \\
\text { besserung der IVF } \\
\text { Behandlung }\end{array}$ & $3.3(1.8)$ & $4.4(1.7)$ & $5.1(1.4)$ & $4.3(1.7)$ & $4.0(1.7)$ & $3.8(1.8)$ & $<.0001$ \\
\hline $\begin{array}{l}\text { Forschung zum } \\
\text { besseren Verständnis } \\
\text { von Erbkrankheiten }\end{array}$ & $3.1(1.7)$ & $4.0(1.8)$ & $4.6(1.6)$ & $3.8(1.7)$ & $3.6(1.8)$ & $3.4(1.7)$ & $<.0001$ \\
\hline Grundlagenforschung & $3.8(1.9)$ & $4.4(1.8)$ & $5.0(1.4)$ & $4.1(1.8)$ & $3.9(1.8)$ & $3.8(1.8)$ & $<.0001$ \\
\hline
\end{tabular}

Die Zahlen geben die Mittelwerte und Standardabweichungen der Likertskalen wieder:

$1=$ moralisch völlig angemessen, $6=$ moralisch völlig unangemessen.

$\mathrm{p}=$ sig. F der Varianz, Einfaktorielle ANOVA. 
Tab 4.: Einstellung zur Legalisierung der Eizellspende, Embryonenspende, Embryonenforschung, Stammzellproduktion, Blastozystenkultivierung und der Spende gegen kostenlose IVF-Behandlung:

\begin{tabular}{|l|c|c|c|c|c|c|c|}
\hline $\begin{array}{l}\text { Zustimmung zur } \\
\text { Legalisierung von: }\end{array}$ & $\begin{array}{c}\text { IVF- } \\
\text { Paare } \\
(\mathrm{n}=202)\end{array}$ & $\begin{array}{c}\text { Ethiker } \\
(\mathrm{n}=168)\end{array}$ & $\begin{array}{c}\text { Hebam- } \\
\text { men } \\
(\mathrm{n}=298)\end{array}$ & $\begin{array}{c}\text { Pädiater } \\
(\mathrm{n}=166)\end{array}$ & $\begin{array}{l}\text { Human- } \\
\text { genetiker } \\
(\mathrm{n}=104)\end{array}$ & $\begin{array}{c}\text { Gynäko- } \\
\text { logen } \\
(\mathrm{n}=147)\end{array}$ & $\mathrm{p}$ \\
\hline $\begin{array}{l}\text { Blastozystenkul- } \\
\text { tivierung }\end{array}$ & 80.4 & 37.7 & 23.2 & 44.1 & 72.9 & 78.4 & $<.0001$ \\
\hline Eizellspende & 77.2 & 35.4 & 40.5 & 46.4 & 58.8 & 64.9 & $<.0001$ \\
\hline Embryonenspende & 69.6 & 23.8 & 16.1 & 33.1 & 35.3 & 45.0 & $<.0001$ \\
\hline $\begin{array}{l}\text { Spende gegen } \\
\text { kostenlose IVF } \\
\text { Behandlung }\end{array}$ & 50.0 & 11.5 & 7.7 & 10.0 & 7.1 & 18.5 & $<.0001$ \\
\hline $\begin{array}{l}\text { Stammzellproduk- } \\
\text { tion }\end{array}$ & 71.3 & 41.1 & 32.2 & 65.5 & 63.1 & 68.0 & $<.0001$ \\
\hline $\begin{array}{l}\text { Forschung zur } \\
\text { Verbesserung der } \\
\text { IVF Behandlung }\end{array}$ & 75.3 & 30.9 & 16.8 & 38.1 & 46.3 & 49.6 & $<.0001$ \\
\hline $\begin{array}{l}\text { Forschung zum } \\
\text { besseren Ver- } \\
\text { ständnis von } \\
\text { Erbkrankheiten }\end{array}$ & 75.0 & 38.9 & 28.3 & 58.6 & 56.5 & 64.8 & $<.0001$ \\
\hline $\begin{array}{l}\text { Grundlagenfor- } \\
\text { schung }\end{array}$ & 55.0 & 29.1 & 17.5 & 35.5 & 38.1 & 46.0 & $<.0001$ \\
\hline
\end{tabular}

Die Zahlen geben gültige Prozentwerte der Subgruppen an.

$\mathrm{p}=$ Signifikanz chi-Quadratwerte der Kreuztabellen Subgruppe/Verfahren, df=5

Das moralische Urteil der Experten über alle Formen des Umgangs mit Embryonen fällt sehr unterschiedlich aus. Gynäkologen standen allen Formen der weiteren Nutzung von Embryonen eindeutig am positivsten gegenüber. Die Auffassung der Humangenetiker im Hinblick auf die Forschung an Embryonen gleicht derjenigen der Pädiater, welche die Forschung an Embryonen zum besseren Verständnis von Erbkrankheiten und die Verwendung von Embryonen für die Stammzellherstellung sogar positiver als die Humangenetiker beurteilen. 59\% der Kinderärzte würden die Forschung an Embryonen zulassen, um ein besseres Verständnis für genetische Erkrankungen zu erreichen, 66\% stimmen einer Verwendung zur Herstellung von Stammzellen zu, ebenso wie die Mehrheit der Gynäkologen/Reproduktionsmediziner und Humangenetiker. Der Blastozystenkultivierung und Eizell-/Embryonenspende stehen Pädiater jedoch skeptischer gegenüber als Humangenetiker und insbesondere als Gynäkologen, wobei sich für die Eizellspende nur die Mehrheit der Gynäkologen und Humangenetiker ausspricht. Für die Spende von Eizellen oder Embryonen an andere Paare findet sich weder bei Pädiatern, noch bei Humangenetikern oder in den weiteren Expertengruppen eine Mehrheit. Ethiker stehen der Forschung an Embryonen, sowie allen weiteren Verfahren zum Teil signifikant positiver gegenüber als Hebammen, beide Gruppen lehnen jedoch alle Verfahren mit deutlicher Mehrheit ab. Am ehesten positiv be- 
werten Ethiker zu 40\% die Verwendung überzähliger Embryonen zur Stammzellherstellung, gefolgt von der Zulassung der Forschung an Embryonen zum besseren Verständnis von Erbkrankheiten (39\%) und der Blastozystenkultivierung $(38 \%)$.

Ebenfalls erfragt wurde in diesem Zusammenhang, ob die Kosten der IVFBehandlung erlassen werden sollten, wenn Paare Eizellen oder Embryonen spenden, wie dies teilweise in England praktiziert wird. Dies wurde mehrheitlich sowohl von den IVF-Paaren, als auch mit überwältigender Mehrheit von allen Expertengruppen abgelehnt und moralisch mit Werten zwischen 4,1 (IVF-Paare) und 5,1 (Hebammen) als höchst bedenklich eingestuft. IVF-Paare sahen die „Bezahlung“ einer Spende jedoch hochsignifikant als weniger problematisch an als alle befragten Expertengruppen. Die Gruppe der Gynäkologen sah die ethische Problematik des finanziellen Anreizes zur Spende dabei als statistisch signifikant weniger bedenklich an als Hebammen und Pädiater, lehnte die Zulassung einer „Bezahlung der IVF durch Embryonen“ jedoch ebenfalls mit großer Mehrheit ab.

Zur Frage danach, was die deutsche Bevölkerung über Forschung an Embryonen denkt, liegen, wie bereits oben beschrieben, zwei Studien aus den letzten Jahren vor: eine große, nach sozialwissenschaftlichen Standards durchgeführte umfangreiche internationale Studie, sowie eine von der Stiftung "Ja zum Leben“ finanzierte Umfrage, in welcher in einer Mehrthemenbefragung ${ }^{20}$ drei Fragen zu Ansichten über die embryonale Stammzellforschung gestellt wurden. In der Studie von Solter wurde beschrieben, dass es um die moralische Bewertung der Verwendung von aus der IVF gespendeten überzähligen, wenige Tage alten mehrzelligen Embryonen oder aber an für die Forschung erzeugten Embryonen im gleichen Stadium geht, um ,in Zukunft möglicherweise einmal andere Behandlungsmöglichkeiten für schwere Erkrankungen zu finden. “21 Korreliert und kontrolliert wurde unter anderem für die Variablen Religiosität, Wissen, Bildung und Geschlecht. Tabelle 5 gibt das Ergebnis der Studie von Solter zu dieser Frage wieder.

Tab. 5: Ansichten zu Stammzellherstellung aus gespendeten überzähligen Embryonen oder eigens für die Forschung erzeugten Embryonen:

\begin{tabular}{|l|c|c|c|c|c|c|}
\hline & \multicolumn{3}{|c|}{$\begin{array}{c}\text { Forschung an gespendeten über- } \\
\text { zähligen Embryonen aus der IVF }\end{array}$} & \multicolumn{3}{|c|}{$\begin{array}{c}\text { Forschung an eigens für die For- } \\
\text { schung erzeugten Embryonen }\end{array}$} \\
\hline Land & $\begin{array}{c}\text { Ab- } \\
\text { lehnung }\end{array}$ & Neutral & $\begin{array}{c}\text { Zustim- } \\
\text { mung }\end{array}$ & $\begin{array}{c}\text { Ab- } \\
\text { lehnung }\end{array}$ & Neutral & $\begin{array}{c}\text { Zustim- } \\
\text { mung }\end{array}$ \\
\hline Dänemark & 19,1 & 17,5 & 63,4 & 28,8 & 21,9 & 49,8 \\
\hline Frankreich & 22,1 & 23,4 & 54,4 & 48,1 & 23,1 & 28,8 \\
\hline Niederlande & 24,8 & 25,0 & 50,2 & 50,9 & 25,7 & 23,2 \\
\hline Italien & 25,2 & 30,7 & 44,2 & 44,4 & 27,6 & 27,9 \\
\hline Spanien & 27,4 & 27,6 & 44,9 & 37,9 & 29,8 & 32,9 \\
\hline England & 28,0 & 25,0 & 47,0 & 41,1 & 27,3 & 31,6 \\
\hline
\end{tabular}

20 Mehrthemenbefragungen sind Befragungen, in welchen Meinungsforschungsinstitute die Fragen verschiedener Auftraggeber mit sehr unterschiedlichen Themen (z.B. Konsumverhalten, Meinungen, Wissen) erfragen.

21 Solter/Beyleveld/Friele (Fußn.19). 


\begin{tabular}{|l|c|c|c|c|c|c|}
\hline Deutschland & 33,1 & 26,3 & 40,7 & 63,7 & 19,0 & 17,1 \\
\hline Polen & 39,7 & 21,6 & 38,7 & 50,9 & 20,7 & 28,3 \\
\hline Österreich & 52,4 & 27,6 & 20,1 & 63,0 & 23,8 & 13,2 \\
\hline
\end{tabular}

Angaben in Prozent, Ablehnung: Werte von 0-3, Neutral: Werte von 4-6, Zustimmung: Werte 7-10 auf der Likert-Skala.

Die Tabelle ist nach dem Grad der Zustimmung zur Herstellung und Forschung an embryonalen Stammzellen sortiert. Die dänische Bevölkerung ist im Vergleich am „liberalsten“ und stimmt als einzige mehrheitlich der Erzeugung und Verwendung von Forschungsembryonen zu. Die deutsche und englische Bevölkerung unterscheidet sich kaum. Beide Gruppen liegen im internationalen europäischen Vergleich eher am skeptischen Ende, stimmen der Verwendung von überzähligen Embryonen für die Herstellung von Stammzellen eher zu, als dass sie diese ablehnen, und lehnen andererseits die Erzeugung und Nutzung von Forschungsembryonen eher (England) oder recht deutlich (Deutschland) ab.

Anders die Ergebnisse der Stiftung „Ja zum Leben“, die in den Jahren 2007 und 2008 die Meinung der deutschen Bevölkerung in drei Fragen ermittelt hat. Zunächst wurde eine Wissensfrage gestellt, ob man embryonale oder adulte Stammzellen kenne. Die zwei Meinungsfragen wurden durch folgenden Satz eingeleitet:

Zur medizinischen Forschung können sowobl menschliche embryonale Stammzellen verwendet werden als auch adulte Stammzellen, die jedem erwachsenen Menschen entnommen werden können. Heilungserfolge sind bisher nur mit adulten Stammzellen erzielt worden. Es gibt aber auch Forscher, die sich Erfolge von der Forschung an embryonalen Stammzellen erboffen. Um embryonale Stammzellen zur Forschung zu gewinnen, müssen menschliche Embryonen erzengt und zerstört werden. Was befürworten Sie?

Als Hauptantwortmöglichkeiten waren angegeben:

- Es sollte nur an adulten Stammzellen, die jedem, auch Erwachsenen, entnommen werden können, geforscht werden, nicht aber an Stammzellen aus Embryonen.

- Es sollte an beiden Zellarten, also auch an embryonalen Stammzellen geforscht werden können.

Hierbei wählten 56,3\% der Befragten die Antwort a), 32,9\% die Antwort b), der Rest gab „weiß nicht“ oder „keine Angabe“ an.

Die zweite Frage lautete wie folgt:

Deutschland hat ein klares Embryonenschutzgesetz, das es verbietet, einen menschlichen Embryo für einen wissenschaftlichen oder medizinischen Zweck zu verwenden, der nicht dem Leben des Embryos dient. Was balten sie von dieser Regelung?

- Halte ich für richtig, in Deutschland sollen keine menschlichen Embryonen zu Forschungszwecken erzeugt und zerstört werden.

- Halte ich nicht für richtig, in Deutschland sollen Embryonen zu Forschungszwecken verwertet werden dürfen.

Hier stimmten 66,5\% der Antwort a), 28,8\% der Antwort b) zu. 
Valide, sozialwissenschaftlich fundierte Umfragen durchzuführen ist sehr schwer. Im Soziologie-Studium lernt man, wie viele Fallstricke es gibt, wie sorgfältig Fragen gestellt und getestet werden müssen. So sind vor Umfragen verschiedene, u.a. so genannte kognitive Pretests unabdingbar, in welchen die Fragen vorgelegt werden und gebeten wird, zu erzählen, wie die Fragen verstanden werden. Menschen neigen dazu, im Sinne der Fragestellung und der Meinung des Interviewers gemäß zu antworten. In Einleitungen zu Fragen sollten auf keinen Fall Formulierungen in Richtung der positiveren oder negativeren Bewertung einer Antwortmöglichkeit gewählt werden. Es sollten neutrale, klar voneinander unterscheidbare Antwortmöglichkeiten gegeben sein, möglichst in skalierter Form, wertende Ausdrücke vermieden werden und insbesondere Vorinformationen auf Verständlichkeit und Verständnis hin geprüft werden. Fundamentale sozialwissenschaftliche Kriterien wurden bei der Umfrage der Stiftung ,Ja zum Leben“ nicht erfüllt. Die Befragung wurde nicht pregetestet. Hätten die Auftraggeber dies getan, wäre herausgekommen, was sich in unseren Studien und in vielen anderen qualitativen Studien zu Einstellungen gegenüber frühem menschlichen Leben fand: Spricht man allein von Embryonen, ohne anzugeben, dass es sich dabei um frühe Embryonen aus mehreren Zellen handelt, denken die Befragten an späte Embryonen, die bereits menschliche Gestalt haben. Wie die Studie von Solter zeigte, macht es zudem einen fundamentalen Unterschied, ob Forschung an Embryonen bewertet wird, die eigens für die Forschung erzeugt wurden, oder ob (was momentan wesentlich häufiger der Fall ist) Embryonen verwendet werden, die IVF Paare gespendet haben. Gemäß der Formulierungen der Stiftung „Ja zum Leben“ mussten die Befragten davon ausgehen, dass es sich um Forschungsembryonen handelt. Gegenüber dieser Art der Erzeugung und Verwendung von Embryonen sind, so zeigte auch die Studie von Solter nicht nur Deutsche, sondern - bis auf die Dänen - alle anderen europäischen Bevölkerungen eher skeptisch eingestellt.

\section{$4 \quad$ Fazit}

Nach bisherigen Ergebnissen sozialwissenschaftlicher Studien im Bereich der Reprogenetik zeigt sich, dass die Auffassungen über die eingangs erwähnten Differenzen zwischen „den Briten“ und „den Deutschen“, sowie zur grundsätzlich ,skeptischen Haltung“ der deutschen Bevölkerung nicht zutreffend sind. Skeptisch(er) oder liberal(er) ist nicht die Bevölkerung, sondern sind die gesetzgebenden Organe, die Medienöffentlichkeit und einige Expertengruppen. Während die Umfragen unserer Arbeitsgruppe, der Arbeitsgruppe von Elmar Brähler sowie die Ergebnisse der internationalen Studie von Solter in die Parlamentsdebatten zur Stammzellforschung keinen Eingang fanden, wurde die Studie der Stiftung ,Ja zum Leben" nicht nur in den Medien breit diskutiert, sondern auch im Parlament als „Auffassung der Bevölkerung zur Stammzellforschung“ in der aktuellen Debatte zitiert. Dies lag nicht nur daran, dass die Fragen nochmals nach einem Jahr 
kurz vor der Parlamentsdebatte erfragt wurden und eine große Pressekampagne der Stiftung dazu erfolgte. Die Studie wurde auch von dem CDU Abgeordneten Hubert Hüppe vor der Debatte an alle Parlamentarier versendet.

Warum aber werden diese offensichtlichen Probleme der Studie von den Abgeordneten und deren Beratern nicht beachtet, gerade in Debatten, wo es wirklich ums Ganze, um neue Strafgesetzgebungen geht? Es scheint nach unseren Analysen vieles dafür zu sprechen, dass in Fragen der Reprogenetik die formelle und die informelle Öffentlichkeit, also diejenige der Parlamentarier, Journalisten und „offiziellen intellektuellen Diskutanten“ auf der einen Seite und diejenige der Bevölkerung, der Betroffenen und direkt involvierten professionellen Gruppen auf der anderen Seite unterschiedliche Auffassungen vertreten. Hinzu kommt, dass zudem die „formelle Öffentlichkeit“ über die „informelle Öffentlichkeit“ nicht zutreffende Ansichten vertritt - oder wahrnimmt - oder wahrnehmen will: So insbesondere diejenige, dass die Deutschen grundsätzlich skeptisch (und „die Briten“ grundsätzlich liberal) sind und die Bevölkerung dem Parlament in der strengen Auslegung des Embryonenschutzgesetzes folgt. Dies führt momentan immer noch dazu, dass die Mehrheit des Parlamentes der Auffassung ist, weder PID noch Blastozystenkultivierung seien zulässig oder sollten zulässig sein.

Wir haben uns viele Gedanken zu diesen Befunden gemacht und uns die Frage gestellt, warum in der Enquetekommission Recht und Ethik der modernen Medizin sogar eine enge Zulassung der PID mit 3 zu 16 Stimmen (und damit überschlagen zu über $80 \%$ ) abgelehnt wurde, während nicht nur Betroffene, sondern auch die Bevölkerung und verschiedene, darunter durchaus auch eher skeptische Expertengruppen mit ebenso deutlicher Mehrheit der Auffassung waren, die PID sollte zugelassen werden. Oder aber, warum in den Parlamentsdebatten vielfach erwähnt wurde, dass man sich darüber einig sei, dass das Embryonenschutzgesetz nicht angetastet werden dürfe, sowie dass die Herstellung von embryonalen Stammzellen in Deutschland auf gar keinen Fall zur Debatte stehe. Ich möchte dies an dieser Stelle nicht diskutieren, sondern nur zwei abschließende Bemerkungen dazu machen:

Ich weiß, dass es aufgrund der geschilderten Ergebnisse auch und gerade im biopolitischen und bioethischen Diskurs Rezipienten gibt, die diese als Beweis für die Unzulänglichkeit von sozialwissenschaftlichen Daten und von der nur relativen Wichtigkeit von Auffassungen von Betroffenen und der Bevölkerung im Hinblick auf Präskriptionen (Wertungen, Gesetzgebungen) im Feld der Reprogenetik nehmen. Hier hilft nur, die Kollegen zu bitten, die Fachexpertise der soziologischen Wissenschaft ernst zu nehmen. Meinungsumfragen sind nicht per se schlecht, nur schlechte Meinungsumfragen sind schlecht.

Und zum zweiten: Ich habe die Befürchtung, dass durch die Rezeption von derart schlechten Studien im Parlament und die Negierung der unterschiedlichen Auffassungen der formellen und informellen Öffentlichkeit - womöglich noch mit Verweis auf Argumente, wie die des relativistischen Schreckgespenstes oder des naturalistischen Fehlschlusses - allein ethische Expertise und die Auffassung der 
„Experten“ und der „Stellvertreter“ in die Entscheidungsfindung einbezogen werden. Auch Habermas' eigene Äußerungen zur Problematik gehen in die letztere Richtung. Über die Ansichten von Betroffenen wird nicht nur spekuliert, sondern diese werden auch als tendenziell verzerrt, problematisch und/oder nicht relevant für die politische Entscheidungsfindung gekennzeichnet. ${ }^{22}$

Es gibt durch die bestehende Gesetzgebung und die geschilderten Divergenzen Probleme, die wir ernst nehmen müssen. Wenn diese ignoriert werden, werden nicht nur Bedürfnisse von Betroffenen nicht beachtet, sondern durchaus Grundfesten der Demokratie in diesem Bereich in Frage gestellt.

22 Vgl. Habermas, Die Zukunft der menschlichen Natur: Auf dem Weg zu einer liberalen Eugenik?, 2001, S. 118: „Der Umstand, dass wir für andere eine folgenreiche Unterscheidung zwischen lebenswertem und lebensunwertem Leben vornehmen, bleibt (...) beunruhigend. Verfehlen die Eltern, die sich um ihres eigenen Kindeswunsches willen zur Selektion entschließen, eine klinische, am Ziel des Heilens orientierte Einstellung? Oder verhalten sie sich doch, wenn auch unüberprüfbar fiktiv, zum Ungeborenen wie zu einer zweiten Person - in der Annahme, dass diese selbst zu einer in bestimmter Weise eingeschränkten Existenz Nein sagen würde. Ich weiß es nicht.“ - Warum fragt der Diskursethiker denn diese Eltern nicht selbst? Dann wüsste er, dass beide von ihm vorgeschlagenen Beschreibungen nicht die Situation, nicht die Frage des guten Lebens treffen, die diese Eltern umtreiben, da er das Verhältnis zwischen Eltern/Paaren und ihren Embryonen als Gerechtigkeitsdiskurs der öffentlichen Sphäre konstruiert. Er ist leider schon lange kein Empiriker mehr. 



\title{
Sollen Kinderwunschbehandlungen von den Krankenkassen finanziert werden? Ethische und rechtliche Aspekte ${ }^{1}$
}

\author{
Oliver Rauprich
}

Der erste mit Hilfe einer In-Vitro-Fertilisation (IVF) gezeugte Mensch, Louise Brown, kam 1978 in England zur Welt. In Deutschland wurde vier Jahre später erstmals ein IVF-Kind geboren. ${ }^{2}$ Waren die ersten „Retortenbabys“ noch eine Sensation, sind heutzutage künstliche Befruchtungen zur Routinebehandlung von Paaren mit unerfülltem Kinderwunsch geworden. Nach Schätzungen leben weltweit mittlerweile ca. 3,5 Millionen IVF-Kinder, davon über 100.000 in Deutschland. ${ }^{3}$ Im Jahr 2003 wurden hierzulande 2,6\% aller Kinder nach einer IVF-Behandlung geboren. ${ }^{4}$

Generell ist eine wachsende Nachfrage nach Kinderwunschbehandlungen zu verzeichnen. Dies liegt zum einen an einer zunehmenden technischen Etablierung

\footnotetext{
${ }^{1}$ Dieser Beitrag entstand im Rahmen der BMBF-Nachwuchsgruppe „Gerechtigkeit in der modernen Medizin“ (FKZ: 01GP0590). Für verallgemeinernde Personenbezeichnungen werden im Folgenden die üblichen generischen Formen verwendet. Dabei sind stets beide Geschlechter gleichermaßen gemeint.

2 Vgl. Rauprich/Siegel, Der Natur den Weg weisen. Ethische Aspekte der Reproduktionsmedizin, in: Ley/Ruisinger (Hrsg.), Von Gebärhaus und Retortenbaby. 175 Jahre Frauenklinik Erlangen, 2003, S. 152.

3 DIR, Deutsches IVF-Register Jahrbuch 2006, abrufbar unter http://www.meb.uni-bonn.de/frau en/DIR_downloads/dirjahrbuch2006.pdf (letzter Zugriff am 18.09.2006).

${ }^{4}$ DIR (Fußn. 3); Statistisches Bundesamt, Geborene und Gestorbene, abrufbar unter: www.destatis.de (letzter Zugriff am 18.9.2006).
} 
und sozialen Akzeptanz der künstlichen Befruchtung. Zum anderen gibt es Hinweise auf einen steigenden Anteil von Paaren mit Fruchtbarkeitsstörungen. Die Ursachen sind in zunehmenden Risikofaktoren wie Umweltbelastungen, Übergewicht und urogenitalen Infektionen zu suchen, vor allem aber in der zunehmenden Verschiebung des Kinderwunsches in eine spätere Lebensphase, in der die natürliche Fruchtbarkeit bereits deutlich gesunken ist. Insgesamt gelten etwa $3-9 \%$ aller Paare mit Kinderwunsch als ungewollt kinderlos. ${ }^{5}$

Die Kosten für künstliche Befruchtungen sind beachtlich, sie betragen in Deutschland für eine Standard-IVF-Behandlung inklusive Medikamenten etwa 3.000 Euro. Wird zusätzlich eine Intracytoplasmatische Spermieninjektion (ICSI) durchgeführt, was bei männlicher Subfertilität angezeigt ist, so erhöht sich der Betrag auf etwa 3.600 Euro. Die Erfolgswahrscheinlichkeit der Behandlungen schwankt mit dem Alter der Frau und anderen Faktoren; durchschnittlich wird in Deutschland eine Lebendgeburtenrate pro begonnenem Behandlungszyklus von $18 \%$ erreicht. ${ }^{6}$ Daraus errechnen sich Kosten von ungefähr 15.000 Euro pro lebend geborenem Kind. Volkswirtschaftlich bedeutet dies bei etwa 20.000 IVFKindern, die 2003 in Deutschland geboren wurden, einen Betrag in der Größenordnung von 300 Millionen Euro. Vergleicht man dies mit den Gesamtausgaben der Gesetzlichen Krankenversicherung (GKV) in Höhe von 145 Milliarden Euro, so würde sich bei voller Kostenübernahme ein Anteil von etwa $0,2 \%$ für künstliche Befruchtungen ergeben. Umgelegt auf die etwa 50 Millionen beitragszahlenden Mitglieder der GKV würde dies durchschnittlich etwa 50 Cent und im GKVHöchstsatz etwa 1 Euro pro Monat und Mitglied ausmachen. Diese Zahlen dürften in anderen europäischen Ländern variieren, jedoch in der gleichen Größenordnung liegen.

Hinsichtlich der Finanzierung reproduktionsmedizinischer Behandlungen bietet Europa ein komplexes und heterogenes Bild. ${ }^{7}$ So werden z.B. in Spanien die Kosten vom öffentlichen Gesundheitssystem zu 100\% übernommen, in Österreich zu 70\%, in Deutschland zu 50\%, in der Schweiz gar nicht und in England regional unterschiedlich. Länder, die keine oder eine geringe explizite Begrenzung der Kostenübernahme vornehmen, rationieren vielfach implizit, indem die Behandlungen nur in öffentlichen Zentren nach langen Wartezeiten erhältlich sind. Unter diesen Umständen entschließen sich viele Paare, die es sich leisten können, für eine pri-

\footnotetext{
${ }^{5}$ Wischmann, Ungewollte Kinderlosigkeit und Reproduktionsmedizin - Einige grundlegende Daten, in: Kleinschmidt/Thorn/Wischmann (Hrsg.), Kinderwunsch und professionelle Beratung. Das Handbuch des Beratungsnetzwerkes Kinderwunsch Deutschland (BKID), 2008, S. 31.

${ }^{6}$ Vgl. DIR (Fußn. 3). Demnach bleiben rechnerisch etwa $80 \%$ aller Paare nach dem ersten Versuch ohne Erfolg, 64\% nach dem zweiten, 50\% nach dem dritten und 40\% nach dem vierten. Die individuellen Erfolgswahrscheinlichkeiten der Paare variieren stark.

7 Übersichten bieten Beckhove, Künstliche Befruchtung. Ein juristischer Ratgeber, 2008, S. 170 ff.; Dostal, Ethical and Legal Aspects of Assisted Reproduction. Situation in the Member States that Joined the European Union in 2004, 2007, pp. 47 ff.; Dill, Consumer Perspectives, in: Vayena/Rowe/ Griffin (eds.), Current Practises and Controversies in Assisted Reproduction. Report of a WHOMeeting, 2002, pp. $263 \mathrm{ff}$.
} 
vate Behandlung. Andere, die es sich nicht leisten können, erhalten weniger Behandlungsversuche angeboten als sie ohne lange Wartezeiten in Anspruch nehmen würden. Diese implizite Rationierung kann dazu führen, dass in einem Land die Krankenkassen einen höheren Anteil der Kosten für Kinderwunschbehandlungen übernehmen als in einem anderen, jedoch erstens weniger Kinderwunschbehandlungen durchgeführt werden und zweitens der Anteil der von den Krankenkassen finanzierten Kinderwunschbehandlungen geringer ist. Auch hinsichtlich der Zulassung und Erstattungsfähigkeit einzelner Verfahren und Techniken (z.B. Eizellspende, Kryokonservierung) sowie der Leistungsvoraussetzungen (z.B. Begrenzung der erstattungsfähigen Versuche, Altersgrenzen, Familienstand) bestehen vielfältige Unterschiede zwischen verschiedenen europäischen Ländern. Häufig existieren spezielle Finanzierungsmodelle und Regelungen, die sich von der allgemeinen Finanzierung des Gesundheitswesens unterscheiden.

Fast immer ist die öffentliche Finanzierung reproduktionsmedizinischer Behandlungen politisch umstritten. Von den Befürwortern werden zwei zentrale Argumente vorgebracht: Erstens wird darauf hingewiesen, dass Unfruchtbarkeit eine Krankheit sei und daher, wie bei jeder anderen Krankheit auch, ein Anspruch auf Kostenübernahme von den Krankenkassen bestünde. Zweitens wird auf die prekäre demographische Situation verwiesen, in der sich Deutschland und andere europäische Länder befinden. Angesichts der sinkenden Geburtenraten und der damit verbundenen Herausforderungen für die sozialen Sicherungssysteme könne man es sich nicht leisten, an der Geburtenförderung zu sparen und auf potenzielle IVF-Kinder zu verzichten. Kritiker bezweifeln den Krankheitswert von Reproduktionsstörungen und argumentieren, die Erfüllung von Kinderwünschen gehöre nicht zu den Aufgaben von Sozialversicherungen, sondern sei primär eine private Angelegenheit.

Im Folgenden werden zunächst die Finanzierungsregelungen und deren Auswirkungen in Deutschland näher dargestellt, um anschließend die Aspekte zu thematisieren, die für die Frage, ob Kinderwunschbehandlungen von den Krankenkassen aus ethischer Sicht übernommen werden sollten, wesentlich erscheinen. Ziel dieses Beitrages ist es, einen fokussierten Aufriss der Problematik zu liefern.

\section{Der juristische Hintergrund in Deutschland}

Während die Gesetzliche Krankenversicherung (GKV) dem Sozialrecht unterliegt und insbesondere im Fünften Buch Sozialgesetzbuch (SGB V) geregelt wird, hat die Private Krankenversicherung (PKV) zivilrechtliche Grundlagen. Daraus ergibt sich die rechtliche Möglichkeit einer unterschiedlichen Ausgestaltung der Leistungen beider Versicherungsarten.

Für die PKV ist die Rechtslage wie folgt: Sofern in Krankenversicherungsverträgen spezielle Vereinbarungen zur Reproduktionsmedizin getroffen wurden, gelten diese unmittelbar. Ansonsten, und dies ist der Regelfall, gelten die Muster- 
bedingungen für die Krankheitskostenversicherungen des Verbandes der privaten Krankenversicherung von 2008. Dort gibt es keine explizite Regelung für reproduktionsmedizinische Behandlungen. Vielmehr gewährt nach $\ 1$ der Versicherer allgemein „Ersatz von Aufwendungen für $[\ldots]$ medizinisch notwendige Heilbehandlungen einer versicherten Person wegen Krankheit oder Unfallfolgen". 8 Demnach sind künstliche Befruchtungen von der PKV zu erstatten, wenn erstens Fertilitätsstörungen Krankheiten und zweitens künstliche Befruchtungen anerkannte und notwendige medizinische Maßnahmen zur Heilung oder Linderung dieser Krankheiten sind.

Bereits 1986 hat der Bundesgerichtshof entschieden, dass die organisch bedingte Unfähigkeit, eigene Nachkommen zu erzeugen, eine Krankheit im Sinne der Versicherungsbedingungen der PKV ist. ${ }^{9}$ Dabei berief er sich auf die Definition von Krankheit als ein „objektiv nach ärztlichem Urteil bestehender anomaler Körper- oder Geisteszustand“. In demselben Urteil erkannte der Bundesgerichtshof die IVF als eine erstattungsfähige Heilbehandlung von Sterilität an, weil sie die Krankheit zwar nicht heilen, jedoch lindern könne, indem sie die gestörte Reproduktionsfunktion ersetzt. Dementsprechend werden die Kosten für künstliche Befruchtungen genau wie für alle anderen medizinisch anerkannten Behandlungen von Krankheiten zu 100\% von der PKV erstattet.

Im Bereich der GKV werden zwei verschiedene Leistungsarten unterschieden: „Leistungen zur Herstellung der Zeugungs- oder Empfängnisfähigkeit" heilen eine Fruchtbarkeitsstörung dergestalt, dass eine Fortpflanzung auf natürlichem Wege (wieder oder erstmalig) möglich wird (z.B. operative Öffnung verschlossener Eileiter, Hormonbehandlungen, Psychotherapie). Diese Leistungen werden im SGB V explizit als reguläre, erstattungsfähige Krankenbehandlungen anerkannt und vollständig übernommen ( $\$ 27$ Satz 4 SGB V). Davon unterschieden werden künstliche Befruchtungen als „medizinische Maßnahmen zur Herbeiführung einer Schwangerschaft". Sie führen zu keiner Heilung, sondern zu einer Linderung von Fruchtbarkeitsstörungen im Sinne einer Substitution der gestörten Funktion. Diese Leistungen wurden im Zuge des KOV-Anpassungsgesetzes von 1990 in den Katalog der GKV eingeführt. ${ }^{10}$ Rechtsgrundlage ist allerdings nicht die Krankenbehandlung nach \27 SGB V sondern \27a SGB V, der als Spezialregelung für künstliche Befruchtungen in das Sozialgesetzbuch aufgenommen wurde.

In dieser Vorschrift wurde der Leistungsanspruch auf Ehepaare im homologen System (d.h. unter Verwendung von Ei- und Samenzellen der Ehepartner und nicht eines Spenders) beschränkt, die Leistungspflicht der GKV auf in der Regel

\footnotetext{
8 Verband der privaten Krankenversicherung, Musterbedingungen 2008 für die Krankheitskosten- und Krankenhaustagegeldversicherung, abrufbar unter: http://www.pkv.de/recht/musterbedingungen_ 2008/krankheitskosten_und_krankenhaustagegeldversicherung_mb_kk2008_pdf.pdf (letzter Zugriff am 7.8.2008).

${ }^{9}$ BGH, Urteil v. 17.12.1986, BGHZ 99, 228.

${ }^{10}$ Gesetz über die neunzehnte Anpassung der Leistungen nach dem Bundesversorgungsgesetz sowie zur Änderung weiterer Sozialrechtlicher Vorschriften (KOV-Anpassungsgesetz 1990) vom 26.6.1990 (BGBl. I S. 1211).
} 
Tab.1: Vergleich der Regelungen zur künstlichen Befruchtung bei PKV und GKV. Erläuterungen im Text.

\begin{tabular}{|lll|}
\hline Versicherungsfall & \multicolumn{1}{c|}{ PKV } & \multicolumn{1}{c|}{ GKV } \\
Behandlungszweck & Fertilitätsstörung als Krankheit & Unerfüllter Kinderwunsch \\
Leistungsumfang & $\begin{array}{l}\text { Linderung der Fruchtbarkeits- } \\
\text { störung beim Versicherten }\end{array}$ & $\begin{array}{l}\text { Erfüllung des Kinderwunsches } \\
\text { eines Ehepaares }\end{array}$ \\
Leistungsvoraussetzungen & $\begin{array}{l}\text { Verursacherprinzip } \\
\text { keine strikten Altersgrenzen } \\
\text { keine strikte Begrenzung der } \\
\text { Anzahl erstattungsfähiger } \\
\text { Behandlungsversuche } \\
\text { kein Ehegattenerfordernis (?) }\end{array}$ & $\begin{array}{l}\text { Körperprinzip } \\
\text { strikte Altersgrenzen } \\
\text { strikte Begrenzung der Anzahl } \\
\text { übernahmefähiger Behand- } \\
\text { lungsversuche }\end{array}$ \\
& \begin{tabular}{l} 
Ehegattenerfordernis \\
\hline
\end{tabular} & \\
\hline
\end{tabular}

nicht mehr als vier Behandlungsversuche begrenzt, die Durchführung der Behandlungen zertifizierten Zentren übertragen und eine umfassende Unterrichtung der Paare über die medizinischen und psychosozialen Aspekte der Behandlung durch den überweisenden Arzt zur Leistungsvoraussetzung gemacht. Durch das GKV-Modernisierungsgesetz von 2003 wurden mit Wirkung zum 1.1.2004 die Leistungen deutlich eingeschränkt. ${ }^{11}$ Seitdem werden nach $\ 27$ a SGB V nur noch 3 Behandlungsversuche von den Krankenkassen übernommen. Begründete Ausnahmen von dieser Begrenzung sind nicht mehr möglich. Die Kosten für diese Versuche werden nicht mehr voll, sondern nur noch zu 50\% getragen. Zudem wurden Altersgrenzen eingeführt. Frauen müssen zwischen 25 und 40 Jahren alt sein, Männer zwischen 25 und 50 Jahren (vgl. Tab. 1). Weitere Details werden in Richtlinien über künstliche Befruchtung des Gemeinsamem Bundesausschusses dem zentralen Gremium der gemeinsamen Selbstverwaltung von Ärzten, Krankenhäusern und Krankenkassen - festgelegt. ${ }^{12}$

Wie ist die Sonderstellung der künstlichen Befruchtung innerhalb des Leistungssystems der GKV begründet? Zunächst einmal wird, ähnlich wie im PKV-

11 Gesetz zur Modernisierung der gesetzlichen Krankenversicherung (GKV-ModernisierungsgesetzGMG) vom 14.11.2003 (BGBl. I S. 2190).

${ }^{12}$ Gemeinsamer Bundesausscbuss (G-BA), Richtlinien über ärztliche Maßnahmen zur künstlichen Befruchtung („Richtlinien über künstliche Befruchtung“), idF. vom 14.8.1990, zuletzt geändert am 15.11.2007, in Kraft getreten am 6.2.2008, abrufbar unter: http://www.g-ba.de/downloads/62-492246/RL-Befruchtung-2007-11-15.pdf (letzter Zugriff am 1.9.2008). 
Bereich, durchaus anerkannt, dass Fruchtbarkeitsstörungen Krankheiten im Sinne des Rechts sind oder sein können. Dies ergibt sich unter anderem daraus, dass die „Leistungen zur Herstellung der Zeugungs- oder Empfängnisfähigkeit“ als normale Krankenbehandlung eingestuft werden ( $(27$ Abs. 1 Satz 4 SGB V). Künstlichen Befruchtungen wird demgegenüber nicht der Status einer Krankenbehandlung im Sinne des Sozialgesetzbuchs eingeräumt. Damit wird nicht der Krankheitswert von Reproduktionsstörungen geleugnet, sondern der politische Wille zum Ausdruck gebracht, diese medizinischen Behandlungen in einer eigenständigen Vorschrift abweichend von Krankenbehandlungen nach \27 SGB V zu regeln.

Nach der Interpretation des Bundessozialgerichts wurde mit dem \27a SGB V vom Gesetzgeber ein neuer Versicherungsfall geschaffen. Im Rahmen dieser Vorschrift käme es gar nicht darauf an, dass Fertilitätsstörungen als Krankheiten im Sinne des Rechts der gesetzlichen Krankenversicherung gewertet werden können. Künstliche Befruchtungen würden auch dann finanziert, wenn Fertilitätsstörungen keine Krankheiten in diesem Sinne wären. Denn die Vorschrift knüpfe die Leistungspflicht der Krankenkasse nicht an einen regelwidrigen Körper- oder Geisteszustand ihres Mitglieds, sondern an die ungewollte Kinderlosigkeit des Ehepaares: „Nicht die Krankheit, sondern die Unfähigkeit des Paares, auf natürlichem Wege Kinder zu zeugen und die daraus resultierende Notwendigkeit einer künstlichen Befruchtung bildet den Versicherungsfall “. ${ }^{13}$

Demnach werden also im Bereich der PKV künstliche Befruchtungen zum Zwecke der Linderung von Krankheiten erbracht, die eine Fortpflanzung auf natürlichem Wege verhindern, wohingegen im Bereich der GKV die Leistungen zum Zwecke der Erfüllung von Kinderwünschen erbracht werden, die auf natürlichem Weg nicht erfüllt werden können, unabhängig davon, ob die Unfähigkeit auf einer Krankheit beruht oder nicht.

Dieser Unterschied ist nicht nur eine juristische Spitzfindigkeit, sondern begründet die weit reichenden Unterschiede in den Leistungsvoraussetzungen und im Leistungsumfang zwischen PKV und GKV (vgl. Tab. 1). Würden künstliche Befruchtungen auch im SGB V als reguläre Krankenbehandlungen eingestuft, müssten sie im Regelfall auch von den Krankenkassen zu 100\% getragen werden. Zwar hat der Gesetzgeber durchaus Spielraum bei der Ausgestaltung der gesetzlichen Gesundheitsversorgung und bereits in einigen anderen Fällen Leistungskürzungen beschlossen (z.B. bei Sehhilfen, Zahnersatz, Kuren), jedoch bedarf es besonderer Begründungen, warum die jeweiligen Kürzungen sachgerecht und nicht willkürlich sind. Werden künstliche Befruchtungen dagegen als Leistungen zur Erfüllung von Kinderwünschen eingestuft, so ist es wesentlich leichter zu rechtfertigen, die Kosten nur zu 50\% zu übernehmen. Denn medizinische Maßnahmen, die anderen Zwecken als der Behandlung von Krankheiten dienen, können prinzipiell auch eine andere Behandlung im Leistungskatalog der Krankenkassen erfahren. Aus

${ }^{13}$ BSG, Urteile v. 3.4.2001, Az. B 1 KR 22/00 R und Az. B 1 KR 40/00 R. 
diesem Grund hat das Bundessozialgericht die reduzierte Kostenübernahme für künstliche Befruchtungen durch die Krankenkassen als verfassungskonform eingeschätzt. ${ }^{14}$

Es gibt auch Nachteile für privat versicherte Paare: Können keine körperlichen Ursachen für eine Reproduktionsstörung festgestellt werden (sog. idiopathische Sterilität), besteht kein Nachweis einer Krankheit und mithin keine Leistungspflicht der PKV. Die GKV übernimmt demgegenüber auch die Kosten für Behandlungen von idiopathischen Fruchtbarkeitsstörungen, weil der Nachweis einer Krankheit keine Leistungsvoraussetzung ist. Liegt die Ursache nachweislich bei einem privat versicherten Ehepartner, so ist die PKV verpflichtet, die gesamten Kosten einschließlich der extrakorporalen Maßnahmen sowie der Behandlungen am Körper des anderen Partners zu erstatten. ${ }^{15}$ Für die GKV gilt hingegen nicht das Verursacher- sondern ein „Körperprinzip“; sie übernimmt - unabhängig davon, bei welchem Partner die Fertilitätsstörung ursächlich vorliegt - die (Hälfte der) Kosten für die Behandlung am Körper ihres Versicherten sowie die (Hälfte der) Kosten für extrakorporale Maßnahmen, nicht jedoch die (Hälfte der) Kosten für die Maßnahmen am Körper des Partners. ${ }^{16}$

Für die GKV gilt ein Mindestalter von 25 Jahren, um nicht „durch fehlende Geduld" von Paaren oder Ärzten die Chance auf eine Spontanschwangerschaft zu vertun, wie es in der Gesetzesbegründung heißt. ${ }^{17}$ Die Obergrenzen von 40 bzw. 50 Jahren werden mit dem Kindeswohl begründet (ebd.). Nach Ansicht des Bundessozialgerichts bezieht sich die männliche Obergrenze auf die politische Maßgabe, keine künstlichen Befruchtungen zu finanzieren, bei denen die potenziellen Väter in der Regel vor dem Schul- und Ausbildungsabschluss der Kinder sterben. ${ }^{18}$ Auch diese Ausführungen sind (wenn überhaupt) nur vor dem Hintergrund nachvollziehbar, dass es sich bei künstlichen Befruchtungen im Bereich der GKV um

\footnotetext{
${ }^{14}$ BSG, Urteil v. 19.9.2007, Az. B 1 KR 06/07 R; vgl. LSG Baden Württemberg, Urteil v. 14.2.2007, Az. L 5 KR 973/06.

${ }^{15}$ So ist es, um eine künstliche Befruchtung durchführen zu können, auch bei männlicher Subfertilität notwendig, Behandlungen am Körper der Frau vorzunehmen (Hormonstimulierung, Punktion der Eizellen, Embryotransfer). Ist der Mann privat versichert, hat seine Versicherung auch die Kosten für diese Behandlungen zu erstatten, weil er aufgrund seiner Subfertilität der alleinige „Verursacher“ der Gesamtbehandlung ist. Vgl. BGH, Urteil v. 3.3.2004, Az. IV ZR 25/03.

${ }_{16}$ BSG, Urteil v. 22.3.2005, Az. B 1 KR 11/03 R. Sind beide Partner gesetzlich versichert, so übernimmt die Kasse der Frau die Kosten für die Behandlung an ihrem Körper und die Kosten für die extrakorporalen Maßnahmen, die Kasse des Mannes die Kosten für die Maßnahmen an seinem Körper, aber keine Kosten für extrakorporale Maßnahmen. Vgl. BSG (Fußn. 13); Gemeinsamer Bundesausschuss (G-BA) (Fußn. 12). Je nachdem, ob einer oder beide Partner bei der GKV oder der PKV versichert sind und bei wem die Ursache der Fruchtbarkeitsstörung liegt, resultiert eine Übernahme bzw. Erstattung der Behandlungskosten von weniger als 50-100\%. Beamten und ihren Angehörigen wird Beihilfe zu künstlichen Befruchtungen nach Maßgabe des $₫ 27$ a SGB V gewährt, sie sind also diesbezüglich den GKV-Versicherten gleichgestellt. Zusammen mit privaten Zusatzversicherungen ergeben sich spezielle Konstellationen der Kostenübernahme. Für Details siehe Beckhove (Fußn. 7), S. 65 ff., $72 \mathrm{ff}$.

17 Vgl. den Entwurf eines Gesetzes zur Modernisierung der gesetzlichen Krankenversicherung (GKV-Modernisierungsgesetz - GMG), BTDrs. 15/1525, S. 83.

18 BSG, Urteil v. 24.5.2007, Az. B 1 KR 10/06 R.
} 
Sozialleistungen zur Erfüllung eines Kinderwunsches und nicht um Krankenbehandlungen handelt. Denn von einer Krankenbehandlung darf niemand aus Altersgründen ausgeschlossen werden oder um nicht durch Ungeduld die Möglichkeit einer Spontanheilung zu vertun oder um nicht die Chance zu erhalten, im höheren Alter noch Kinder zu bekommen. In der PKV gibt es dementsprechend keine festen Altersgrenzen.

Indirekt sind jedoch auch bei der PKV Altersgrenzen relevant. Der Bundesgerichtshof hat die Leistungspflicht der privaten Versicherer auf Paare begrenzt, deren Chance auf Herbeiführung einer Schwangerschaft durch eine künstliche Befruchtung nicht unter 15\% sinkt. ${ }^{19}$ Solche Mindestanforderungen an die Erfolgswahrscheinlichkeit sind auch bei Krankenbehandlungen rechtens. Diese Schwelle wird bei Frauen durchschnittlich im Alter von etwa 40 Jahren erreicht. Daher orientiert sich auch die PKV an dieser Altersgrenze, sie darf jedoch ältere Frauen nicht pauschal ausschließen, sondern muss deren individuelle Prognose anhand von objektiven Kriterien (z.B. Ergebnisse früherer Versuche, Hormonspiegel nach Stimulation etc.) würdigen. Aus dem gleichen Grund kann die PKV die Anzahl der Versuche nicht pauschal begrenzen, sondern ist zur wiederholten Leistungserbringung verpflichtet, solange die Erfolgsaussichten bei mindestens 15\% pro Versuch liegen. Für Behandlungen zum Zwecke der Erfüllung eines Kinderwunsches nach $\int 27$ a SGB V wird demgegenüber in pauschalisierender Betrachtungsweise festgelegt, dass eine hinreichende Erfolgsaussicht nach drei erfolglosen Versuchen und bei Frauen ab 40 Jahren nicht mehr besteht.

Die Leistungspflicht der GKV beschränkt sich auf Paare, die miteinander verheiratet sind. Nach Ansicht des Bundesverfassungsgerichts wäre dieses so genannte Ehegattenerfordernis unwirksam, wenn künstliche Befruchtungen den Status einer Krankenbehandlung hätten. Denn eine derartige Benachteiligung unverheirateter Personen in der Gesundheitsversorgung würde gegen den Gleichheitsgrundsatz nach Art. 3 Abs. 1 des Grundgesetzes verstoßen. ${ }^{20}$ Die Beschränkung von Sozialleistungen zur Erfüllung von Kinderwünschen auf Verheiratete ist demgegenüber nach Ansicht des Bundesverfassungsgerichts grundgesetzkonform, weil in der Ehe höhere rechtliche Verpflichtungen gegenüber dem Ehepartner und den Kindern bestehen als in einer nichtehelichen Lebensgemeinschaft und dies günstigere Vorraussetzungen für das Kindeswohl sowie für die gemeinsame Bewältigung der physisch und psychisch belastenden Behandlungen böte. ${ }^{21}$ Diese Rechtssprechung des Bundesverfassungsgerichts stützt Auffassungen, nach denen die PKV im Gegensatz zur GKV auch ihren unverheirateten Mitgliedern die Kosten für künstliche Befruchtungen zu erstatten habe, da ihre Behandlung als Kran-

\footnotetext{
${ }^{19}$ BGH, Urteil v. 21.9.2005, Az. IV ZR 113/04.

${ }^{20}$ BVerfG, Urteil v. 28.2.2007, Az. 1 BvL 5/03.

${ }^{21}$ BVerfG (Fußn. 20).
} 
kenbehandlung zählt und somit dem Gleichheitsgebot unterliegt. Eine diesbezügliche Entscheidung des Bundesgerichtshofs steht jedoch noch aus. ${ }^{22}$

Unter der Voraussetzung, dass medizinische Maßnahmen zur Herbeiführung einer Schwangerschaft in der GKV nicht als Krankenbehandlungen, sondern als ein eigenständiger Versicherungsfall gehandhabt werden, erscheinen die abweichenden Regelungen grundsätzlich als juristisch nachvollziehbar, auch wenn man sicher Kritik im Detail anbringen kann. Die grundlegende Frage ist, ob es grundgesetzkonform ist, künstliche Befruchtungen aus der regulären Krankenbehandlung auszuklammern und eigenständig zu regeln. Für viele andere medizinische Behandlungen wäre dies sicher nicht der Fall. So hätte vermutlich eine Vorschrift, die den Ersatz von Hüftgelenken nicht mehr als eine Krankenbehandlung, sondern als „medizinische Maßnahme zur Herbeiführung einer Beweglichkeit“ regelt und mit entsprechenden Leistungseinschränkungen versieht, vor dem Grundgesetz keinen Bestand. Die letztendliche Begründung für die Sonderregelung von künstlichen Befruchtungen kann nur sein, dass Reproduktionsstörungen zwar das Kriterium einer Krankheit im Sinne der Krankenversicherungen erfüllen mögen, ihr Krankheitswert jedoch so gering ist, dass ihr Ausschluss aus den regulären Leistungen zur Krankenbehandlung einer juristischen Prüfung standhält. In obersten Rechtsprechungen wurde dies so gesehen. So liegen nach Ansicht des Bundesverfassungsgerichts künstliche Befruchtungen „,in einem Grenzbereich zwischen Krankheit und solchen körperlichen und seelischen Beeinträchtigungen eines Menschen, deren Beseitigung oder Besserung durch Leistungen der gesetzlichen Krankenversicherung nicht von vornherein veranlasst ist" ${ }^{\text {“. }}{ }^{23}$ Der Gesetzgeber überschreite sein Gestaltungsermessen nicht, wenn er zur finanziellen Sicherung der Behandlung schwerer Krankheiten Leistungsansprüche in weniger dringlichen Fällen wie der künstlichen Befruchtung beschränkt oder gar nicht erst vorsieht. ${ }^{24}$ Die Ungleichbehandlung von Versicherten der GKV und PKV sei hinzunehmen, denn es läge ebenfalls im Ermessen des Gesetzgebers, sich für zwei Systeme der Krankenversicherung zu entscheiden, in denen es zu einzelnen Leistungsunterschieden komme..$^{25}$

\section{Folgen für die Reproduktionsmedizin}

Die mit dem GKV-Modernisierungsgesetz zum 1.1.2004 eingeführte Beschränkung der Kostenübernahme auf 50\% hat zu einem deutlichen Rückgang der Nachfrage nach künstlichen Befruchtungen geführt (Abb. 1).

Bis 2003 war die Anzahl der behandelten Frauen, der durchgeführten Behandlungszyklen und der nach einer Behandlung geborenen IVF-Kinder stetig

\footnotetext{
22 Vgl. Beckhove (Fußn. 7), S. 51 ff.

23 BVerfG (Fußn. 20).

${ }^{24}$ BVerfG, Urteil v. 6.12.2005, Az. 1 BvR 347/98; BSG (Fußn. 14).

${ }^{25}$ BSG, Beschluss v. 2.11.2006, Az. B 1 KR 111/06 B.
} 


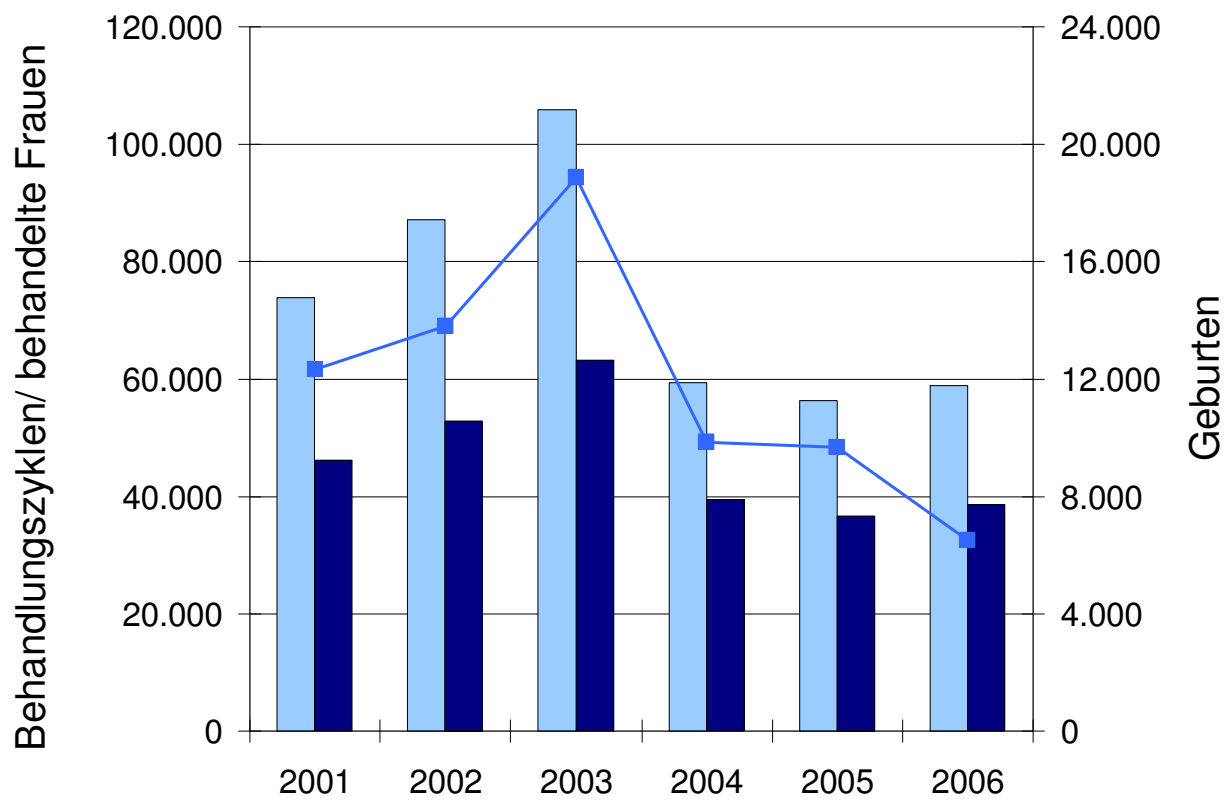

Behandlungszyklen

behandelte Frauen

-Geburten

Abb. 1: Die Entwicklung medizinischer Behandlungen zur Herbeiführung einer Schwangerschaft in Deutschland 2001-2006. ${ }^{28}$

gestiegen. Der Anstieg in 2003 war besonders groß, weil aufgrund der bevorstehenden Leistungskürzung viele Paare noch einen „kostenlosen“ Behandlungsversuch durchführen ließen, den sie ansonsten erst im folgenden Jahr in Anspruch genommen hätten. Im Vergleich zu 2002 ist die Anzahl der behandelten Frauen 2004 um etwa 25\% (von 53.000 auf 39.000) und die Anzahl der Behandlungszyklen (von 87.000 auf 59.000) sowie der geborenen IVF-Kinder (von 14.000 auf 10.000) um etwa 30\% gesunken und seitdem in etwa konstant geblieben. ${ }^{26}$

Diese Preiselastizität der Nachfrage zeigt, dass sich viele Paare mit unerfülltem Kinderwunsch eine Behandlung zumindest nicht in dem Umfang leisten können oder wollen, den sie bei voller Kostenübernahme durch die GKV in Anspruch nehmen würden. Es ist auch zu beobachten, dass die Paare länger warten, bevor sie sich in reproduktionsmedizinische Behandlung begeben. Dadurch ist das Durchschnittsalter der behandelten Frauen um etwa 1 Jahr gestiegen, was der Erfolgswahrscheinlichkeit der Behandlungen nicht zuträglich ist. ${ }^{27}$

\footnotetext{
${ }^{26}$ Für 2006 sind noch nicht alle Geburten erfasst, die Zahl wird daher noch nach oben korrigiert.

${ }^{27}$ DIR (Fußn. 2)

${ }^{28}$ Nach DIR (Fußn. 3).
} 
Diese Entwicklung war vorhersehbar und politisch zum Zwecke der Beitragssatzstabilisierung der GKV gewollt; sie ist somit vom Gesetzgeber zu verantworten. Auch wenn die aktuellen Regelungen im \27a SGB V nicht gegen das Grundgesetz verstoßen, so ist doch zu fragen, ob es sich um eine gute Politik handelt. Es wäre ebenso vom Grundgesetz gedeckt, künstliche Befruchtungen von der GKV gänzlich übernehmen zu lassen. Gibt es überzeugende Gründe für eine Rückkehr zur alten Politik?

\section{Das demographische Argument}

Das in der öffentlichen Diskussion - und insbesondere unter politisch aktiven Reproduktionsmedizinern - wohl am häufigsten genannte Argument bezieht sich auf den Beitrag, den künstliche Befruchtungen zur Geburtenförderung machen können. So wurde in einer gemeinsamen Stellungnahme der Wissenschaftlichen Gesellschaften und des Bundesverbandes Reproduktionsmedizinischer Zentren geschrieben: „Unter dem Eindruck der derzeitigen demographischen Entwicklung und der ökonomischen Perspektive des deutschen Sozialsystems sollten ferner alle Maßnahmen unterstützt werden, die einer Geburtenförderung in Deutschland dienlich sind. Es darf nicht vergessen werden, dass auch der größte Teil der nach der so genannten künstlichen Befruchtung geborenen Kinder später mit ihren Beiträgen das soziale System der Solidargemeinschaft unterstützen““.29

Dieses Argument ist insofern untypisch für eine ärztliche Sichtweise, als die Medizin sich üblicherweise primär dem Wohl ihrer Patienten verpflichtet fühlt und nicht dem Nutzen für das Sozialsystem. Jedenfalls würde man die medizinische Notwendigkeit einer Behandlung üblicherweise nicht davon abhängig machen, ob sie sich bevölkerungspolitisch oder volkswirtschaftlich rechnet. Aus ärztlicher Sicht kann dieser Aspekt also nicht wesentlich für die Übernahme künstlicher Befruchtungen in den Leistungskatalog der GKV sein, sondern bestenfalls ein zusätzliches, über den medizinischen Aufgabenbereich hinaus gehendes Argument.

Doch welche bevölkerungspolitische Bedeutung kann die künstliche Befruchtung haben? Nach den optimistischsten Prognosen könnten bei voller Kostenübernahme durch die GKV 10.000 Kinder jährlich mehr in Deutschland geboren werden. Das Geburtendefizit (die Anzahl mehr Gestorbener als Geborener) belief sich in den vergangenen Jahren auf rund 140.000.30 Dies allein verdeutlicht, dass wir unser demographisches Problem mit den Mitteln der Reproduktionsmedizin bestenfalls um wenige Prozentpunkte abmildern können.

29 Wissenschaftliche Gesellschaften und Bundesverband reproduktionsmedizinischer Zentren (BRZ), Gemeinsame Stellungnahme zur geplanten Veränderung des SGB V \28a - Maßnahmen der Künstlichen Befruchtung, abrufbar unter: http://www.repromed.de/downloads/plaene-gmg.pdf (letzter Zugriff am 2.9.2008).

30 Statistisches Bundesamt (Fußn. 4). 
Nun mag man der Ansicht sein, dass auch kleine Steigerungen der Geburtenrate unterstützt werden sollten. Wenn man dies jedoch im Rahmen volkswirtschaftlicher Argumente vorbringt, dann muss auch die Kosteneffektivität bedacht werden. Wie oben kalkuliert, belaufen sich die Kosten für reproduktionsmedizinische Behandlungen in Deutschland auf eine Größenordnung von etwa 15.000 Euro pro lebend geborenem Kind. Hierbei sind Folgekosten, z.B. für die entstehenden Mehrlingsschwangerschaften mit ihren häufigen Komplikationen, noch nicht eingerechnet. Manche Untersuchungen kommen daher auf deutlich höhere Kostenwerte. ${ }^{31}$ Auf jedem Fall sind künstliche Befruchtungen zunächst einmal eine recht teure Weise, Kinder in die Welt zu setzen. Sie „lohnen“ sich volkswirtschaftlich nur, wenn es keine kostengünstigeren Maßnahmen gibt, um wieder mehr Menschen, die nicht unter Reproduktionsstörungen leiden, zu einer natürlichen Fortpflanzung zu motivieren - etwa durch eine bessere Vereinbarkeit von Beruf und Familie. Für eine abschließende Bewertung des Demographie-Arguments bedürfte es einer wissenschaftlich fundierten Kosten-Nutzen-Analyse von künstlichen Befruchtungen im Vergleich mit klassischen familienpolitischen Maßnahmen. Solange eine solche Studie nicht vorliegt, erscheint die Suggestion mancher Interessensvertreter, die Übernahme reproduktionsmedizinischer Maßnahmen durch die GKV sei eine gute und ökonomisch geradezu zwingend gebotene Investition in die Nachhaltigkeit unserer sozialen Sicherungssysteme, etwas voreilig.

\section{Das Argument des Krankheitswertes}

Wie oben erläutert, basieren die Regelungen der künstlichen Befruchtung im Bereich der GKV letztlich auf dem Argument, Reproduktionsstörungen hätten einen hinreichend geringen Krankheitswert, um Leistungseinschränkungen zu legitimieren. Dem mag man entgegenhalten, dass viele andere Beschwerden wie vorübergehende Kopfschmerzen, moderater Heuschnupfen, Schürfwunden etc. auch geringen Krankheitswert haben und die Behandlungen trotzdem von der GKV übernommen werden. Zudem ist das Leiden an einem unerfüllten Kinderwunsch für viele Paare eine geradezu existenzielle Krise, die, wie bereits 1986 der Bundesgerichtshof anmerkte, mit erheblichen Einschränkungen des Selbstwertgefühls einhergehen und bis zu einer seelischen Erkrankung führen kann. ${ }^{32}$ Wissenschaftliche Untersuchungen belegen ein häufig traumatisches Erleben von ungewollter Kinderlosigkeit. ${ }^{33}$ Von daher mag man sich fragen, ob eine Fertilitätsstörung durch

31 Vgl. Neumann/Gharib/Weinstein, The Cost of Successful Delivery with In Vitro Fertilization, The New England Journal of Medicine 1994/331, p. 239; Ryan, Ethics and Economics of Assisted Reproduction. The Cost of Longing, 2001, pp. $17 \mathrm{ff}$.

32 BGH (Fußn. 9).

33 Groß, Psychosomatik und Reproduktionsmedizin, in: Beckermann/Perl (Hrsg.), Frauen-Heilkunde und Geburts-Hilfe. Integration von Evidence-based Medicine in eine frauenzentrierte Gynäkologie 2004, S. 922. 
Tab. 2: Expertenmeinungen zum Status ungewollter Kinderlosigkeit. ${ }^{34}$

\begin{tabular}{lccccc}
\hline $\begin{array}{l}\text { Status ungewollter Kinder- } \\
\text { losigkeit }\end{array}$ & $\begin{array}{l}\text { Gynäkol./ } \\
\text { Repro.med }\end{array}$ & Pädiater & $\begin{array}{l}\text { Human- } \\
\text { gentiker }\end{array}$ & $\begin{array}{l}\text { Hebam- } \\
\text { men }\end{array}$ & Ethiker \\
$\begin{array}{l}\text { Schicksal/Lebensumstand, } \\
\text { i.d.R. hinzunehmen }\end{array}$ & $1,4 \%$ & $12,6 \%$ & $6,9 \%$ & $22 \%$ & $19,3 \%$ \\
$\begin{array}{l}\text { Funktionsstörung, nicht } \\
\text { grundsätzlich behandlungs- } \\
\text { bedürftig }\end{array}$ & $27,6 \%$ & $48,2 \%$ & $44,1 \%$ & $48,8 \%$ & $51,2 \%$ \\
$\begin{array}{l}\text { Funktionsstörung, häufig } \\
\text { behandlungsbedürftig }\end{array}$ & $24,1 \%$ & $21,7 \%$ & $31,4 \%$ & $13,4 \%$ & $13,9 \%$ \\
$\begin{array}{l}\text { Krankheit, } \\
\text { behandlungsbedüftig }\end{array}$ & $42,1 \%$ & $9,6 \%$ & $12,7 \%$ & $6,5 \%$ & $9,0 \%$ \\
\hline
\end{tabular}

den als Leiden wahrgenommenen unerfüllten Kinderwunsch nicht doch einen signifikanten Krankheitswert erhält.

Die Ansichten hierüber gehen auseinander, nicht nur im Alltag, sondern auch unter Experten. In einer Studie waren befragte Reproduktionsmediziner und Gynäkologen zwar mehrheitlich der Meinung, eine ungewollte Kinderlosigkeit habe den Status einer behandlungsbedürftigen Krankheit, für deren Behandlung die Gesellschaft Ressourcen zur Verfügung stellen müsse; immerhin fast 30\% von ihnen sahen sie jedoch als eine nicht grundsätzlich behandlungsbedürftige Funktionsstörung an. Andere Berufsgruppen tendierten mehrheitlich zu dieser weniger pathologischen Charakterisierung, zeigten jedoch ihrerseits eine auffällige Breite in ihren Ansichten (Tab. 2).

In der philosophischen Debatte unterscheidet man zwei gängige Typen von Krankheitsbegriffen. So genannte naturalistische Krankheitsmodelle knüpfen an objektive Struktur- und Funktionsstörungen an. Der bekannteste Vertreter, Christopher Boorse, definiert Krankheit als Einschränkung spezies- und alterstypischer Körper- oder Geistesfunktionen zur biologischen Überlebens- und Reproduktionsfähigkeit. ${ }^{35}$ In dieser Definition sind Reproduktionsstörungen explizit eingeschlossen. Auf ein ähnliches naturalistisches Modell stützt sich die deutsche Rechtssprechung: Krankheit als objektiv regelwidriger Körper- oder Geisteszustand. Wie oben erläutert, wird anerkannt, dass Fruchtbarkeitsstörungen Krankheiten im diesem Sinne sind.

\footnotetext{
${ }^{34}$ Nach Krones/Nemwohner/El Ansari/Wissner/Richter, Kinderwunsch und Wunschkinder. Möglichkeiten und Grenzen der In-vitro-Fertilisations-Behandlung, Ethik in der Medizin 2006, $51 \mathrm{ff}$.

35 Boorse, On the Distinction between Disease and Illness, Philosophy and Public Affairs 1975/5, 49 ff.; idem, Concept of Health, in: V.d.Veer/Regan (eds.), Health Care Ethics. An Introduction, 1987, p. 351.
} 
Der zweite gängige Typus umfasst so genannte normativistische Krankheitsmodelle. Sie knüpfen daran an, dass es stets eine negative Wertung beinhaltet, wenn man etwas als Krankheit bezeichnet. Ein Hauptvertreter dieser Richtung ist Lennart Nordenfelt. Er definiert Krankheit als Einschränkung der Fähigkeit einer Person, unter normalen Umständen alle wesentlichen Ziele zu erreichen. ${ }^{36}$ Unterstellt man, dass die Erfüllung des Kinderwunsches für die meisten Paare in reproduktionsmedizinischer Behandlung ein wesentliches Ziel in ihrem Leben ist, so sind auch nach dieser Definition Fertilitätsstörungen als Krankheiten anzusehen.

Nach anderen Überlegungen können Reproduktionsstörungen als eine Art von Behinderung aufgefasst werden. ${ }^{37}$ Diese Ansätze knüpfen daran an, dass es sich um körperliche Funktionsausfälle handelt, die zu eingeschränkten Lebensmöglichkeiten führen. Ähnlich wie Blindheit, Sprachverlust oder Lähmungen die Teilhabe an einem normalen Leben in vielfacher Hinsicht behindern, behindern Reproduktionsstörungen die Teilhabe an einer Familienbildung.

Es gibt also vielfältige Anknüpfungspunkte für die Begründung eines Krankheitswertes von Reproduktionsstörungen. Doch damit ist die Sache noch nicht entschieden. Denn es gibt auch gegenteilige Anknüpfungspunkte. ${ }^{38}$ Unfruchtbarkeit ist weder schmerzhaft noch gefährlich. Ein verschlossener Eileiter oder eine schlechte Spermienqualität reduziert nicht die Lebenserwartung von Personen und ist nicht unmittelbar mit körperlichen oder seelischen Schmerzen verbunden. Dies unterscheidet sie von typischen Krankheiten. Sie behindern auch keine Tätigkeiten. Alle normalen motorischen, sprachlichen, akustischen, visuellen, geistigen und in der Regel auch sexuellen Handlungen sind möglich. Dies unterscheidet sie von typischen Behinderungen. Reproduktionsstörungen werden erst zu einem Leiden, wenn ein Kinderwunsch hinzutritt. Das Leiden an einem unerfüllten Kinderwunsch hat den Charakter des seelischen Schmerzes und der Trauer über ein (bislang) gescheitertes Lebensziel. Es ist vergleichbar mit dem Leiden an einem unerfüllten Berufs-, Karriere- oder Partnerwunsch. Das gleiche Leiden erfüllt viele Menschen, die kinderlos bleiben, weil sie keinen geeigneten Partner gefunden haben. Da diese Menschen wohl kaum als krank bezeichnet werden können, kann das Leiden an einem unerfüllten Kinderwunsch per se kein Krankheitssymptom sein. Andere Leiden sind aber bei Menschen mit einer Fruchtbarkeitsstörung nicht ersichtlich. Aus dieser Sicht dienen künstliche Befruchtungen somit eher einer Wunsch erfüllenden als einer Krankheit behandelnden Medizin.

Letztlich sind Reproduktionsstörungen wohl als ein Zweifels- oder Grenzfall anzusehen, der in manchen Hinsichten Krankheiten oder Behinderungen gleicht und in anderen nicht. Wie immer man zu dieser Statusfrage steht: Ohne Zweifel haben Paare mit unerfülltem Kinderwunsch eine Reihe von Belastungen und Her-

\footnotetext{
36 Nordenfelt, On the Nature of Health: An Action-Theoretic Approach, 1988, 2. edn. 1995.

${ }^{37}$ Ryan (Fußn. 31), pp. 67 ff.

38 Vgl. Rauprich, Unfruchtbarkeit als „Sonderfall“ von Krankheit?, Erwägen Wissen Ethik 2007, $121 \mathrm{ff}$.
} 
ausforderungen zu bewältigen, die sehr ähnlich zu denen von Patienten mit schweren chronischen Krankheiten sind. Die Diagnose zwingt sie dazu, ihre persönlichen Ziele und Beziehungen neu zu überdenken und auszurichten. Sie erfahren vielfach das Gefühl der Machtlosigkeit, der Kränkung und des Kontrollverlustes. Sie müssen sich mit der inneren Annahme oder Ablehnung ihres Leidens auseinander setzen. Und die Medizin macht ihnen das Angebot, ihr Leiden als medizinisches Problem wahrzunehmen und zu behandeln.

Vor diesem Hintergrund ist die Frage nach dem Krankheitsstatus von Fertilitätsstörungen vielleicht gar nicht so wichtig. Entscheidend ist vielleicht vielmehr, sich darüber klar zu werden, welcher gesellschaftliche Wert der Möglichkeit zugemessen werden soll, Paaren mit Fertilitätsstörungen auf medizinischem Wege zu einem Kind zu verhelfen. ${ }^{39}$ Die Fragen, die wir uns als Gesellschaft stellen sollten, sind: Welchen Charakter hat das Leiden von Menschen mit unerfülltem Kinderwunsch? Was sollte der Zweck unseres öffentlichen Gesundheitssystems sein? Wie passt die Behandlung von Reproduktionsstörungen zu diesem Zweck?40 Mit diesen Fragen kommen wir zum dritten Argument.

\section{Das Argument der Bedürftigkeit}

Reproduktionsstörungen mögen Krankheiten sein oder nicht. Davon unabhängig kann argumentiert werden, dass der Wunsch nach Kindern in der Regel nicht den Charakter eines Konsum- oder Selbstverwirklichungswunsches hat, sondern eines Bedürfnisses. Menschen wünschen sich nicht Kinder so wie sie sich ein neues Auto wünschen oder gerne einmal in die Karibik reisen würden. Der Wunsch nach Kindern drückt auch nicht primär eine Leidenschaft aus, so wie man leidenschaftlich ein Hobby pflegt. Er ist auch nicht zu vergleichen mit dem Streben nach Selbstverwirklichung, etwa in einer beruflichen Karriere. Er ist vielmehr Ausdruck eines Bedürfnisses, so wie wir ein Bedürfnis nach einem Partner, nach Sozialkontakten, nach Anerkennung, nach persönlichen Freiheiten und Privatsphäre haben. Dies gilt auch dann, wenn heutzutage eine Reihe von Menschen keinen Kinderwunsch hat. Sofern ein Kinderwunsch vorliegt, ist er in der Regel ein Bedürfnis und kein bloßer Wunsch.

Bedürfnisse begründen einen stärkeren Anspruch auf gesellschaftliche Unterstützung als bloße Wünsche. Sie sind zentraler Gegenstand von Erwägungen des Wohlergehens und der sozialen Gerechtigkeit. Sofern es zutrifft, dass der Wunsch nach Kindern als ein Bedürfnis gelten kann, ist eine Gesellschaft verpflichtet, Menschen mit diesen Bedürfnissen genauso zu beachten, wie Menschen mit anderen Bedürfnissen von gleichem Rang. Welcher Art diese Beachtung zu sein hätte und

\footnotetext{
${ }^{39} \mathrm{Vgl}$. Rauprich/Siegel (Fußn. 2).

${ }^{40}$ Ryan (Fußn. 31), pp. 66 ff.
} 
woran sich die soziale Gerechtigkeit bemisst, wäre in Diskussionen geeigneter ethischer Theorien zu ermitteln.

Zumindest zwei Typen ethischer Theorien bieten hier unmittelbare Anknüpfungspunkte: Eine Reihe von Ansätzen bezieht sich auf die Idee, dass jeder Mensch die gleichen grundlegenden Chancen in seinem Leben haben sollte und unverdiente Nachteile ungerecht sind. Norman Daniels beispielsweise, einer der prominentesten Vertreter von Theorien der Chancengleichheit im Bereich der Medizinethik, argumentiert, es sei Aufgabe des Gesundheitswesens dazu beizutragen, jedem Mitglied der Gesellschaft eine faire Chance auf die Verwirklichung von normalen, der Gesellschaft entsprechenden Lebensplänen zu ermöglichen. ${ }^{41} \mathrm{Da}$ die biologische Fortpflanzung und Gründung einer Familie zu den normalen Lebensplänen in jeder Gesellschaft gehört, verhindern Fruchtbarkeitsstörungen eine faire Chancengleichheit für die Betroffenen und begründen somit einen Anspruch auf gesellschaftliche Unterstützung zur Überwindung ihres unverdienten Nachteils.

Ein anderer Typus ethischer Theorien bezieht sich auf die ethische Verpflichtung einer Gesellschaft, allen Mitgliedern die grundlegenden Möglichkeiten für ein gutes, würdiges Lebens zu verschaffen. Mittelpunkt des Anliegens ist hier weniger die Schaffung gleicher als vielmehr binreichender Lebensmöglichkeiten für jeden. Prominente Vertreter von Theorien dieses Typs sind z.B. Amartya Sen und Martha Nussbaum. ${ }^{42}$ Sie beziehen sich nicht auf Chancen zur Realisierung von Lebensplänen, sondern auf effektive Fähigkeiten und Möglichkeiten, bestimmte Dinge zu tun oder zu sein (so genannte „capabilities"). Entscheidend ist, dass der Fokus auf grundlegende Fähigkeiten gelegt wird, die jeder Mensch haben sollte. Dazu gehört nach Nussbaum vor allem die Möglichkeit der Befriedigung grundlegender menschlicher Bedürfnisse. Sofern der Wunsch nach Kindern zu einem solchen grundlegenden Bedürfnis gerechnet werden kann, folgt ein ethischer Anspruch gegenüber der Gesellschaft, Menschen mit Reproduktionsstörungen zur Erfüllung dieses Wunsches zu befähigen.

Diese Skizze kann einen plausiblen Anfangsverdacht begründen, dass im Rahmen verschiedener Theorien der Gerechtigkeit eine öffentliche Finanzierung von künstlichen Befruchtungen gerechtfertigt oder sogar geboten erscheint. Grundlage ist die Charakterisierung von Kinderwünschen als Bedürfnisse sowie einer Zweckbestimmung des Gesundheitswesens, über die Versorgung von Krankheiten hinaus zur sozialen Gerechtigkeit beizutragen. Das Argument der Bedürftigkeit wird in der öffentlichen Diskussion eher selten verwendet. Es wendet die Perspektive von der harten Einforderung eines Rechts auf Krankenbehandlung auf die weichere, aber vielleicht nicht weniger eindringliche Forderung, die Bedürfnisse von Paaren mit unerfülltem Kinderwunsch im Lichte gesellschaftlicher Chancengleichheit und Mindeststandards zu betrachten. Gerade vor dem Hintergrund der Tatsache, dass

\footnotetext{
${ }^{41}$ Daniels, Just Health Care, 1985; idem, Just Health: Meeting Health Needs Fairly, 2008.

42 Sen, Equality of What?, The Tanner Lectures on Human Values, 1980; idem, Inequality Reexamined, 1992; Nussbaum, Gerechtigkeit oder Das gute Leben, 1999.
} 
der Krankheitswert von Reproduktionsstörungen notorisch umstritten ist und wohl auch bleibt, sollte man der weiteren Erkundung dieses Argumentes besondere Aufmerksamkeit schenken. 



\title{
Restriktionen assistierter Reproduktion aus verfassungsrechtlicher Sicht
}

\author{
Werner Heun
}

Fortpflanzung ist seit Längerem nicht mehr auf natürliche Methoden beschränkt, assistierte Reproduktion ist in vielfältigen Formen möglich und weit verbreitet. Wenn eine Frau auf natürliche Weise kein Kind empfangen oder austragen oder ein Mann kein Kind zeugen kann, sind verschiedene Wege künstlicher, d.h. medizinischer Fortpflanzungshilfe denkbar. ${ }^{1}$ Zum einen kommt eine künstliche Besamung bzw. Insemination, zum anderen eine künstliche Befruchtung, die sog. Invitro-Fertilisation (IVF) oder auch extrakorporale Befruchtung, mit anschließender Übertragung des so erzeugten Embryos in den Körper der sog. Wunschmutter (Embryo-Implantation) in Betracht. Sowohl die Insemination als auch die In-vitroFertilisation kann mit dem Sperma des Ehepartners, also homolog, oder mit dem Sperma eines anderen, meist unbekannten Mannes erfolgen, was als heterolog oder auch donogen bezeichnet wird. Weiterhin ist die homologe oder heterologe Befruchtung der Eizelle einer anderen Frau, einer Eizellspenderin, im Wege der IVF möglich. Von diesen Fällen der homologen oder heterologen Insemination und Fertilisation sind die Konstellationen der Embryo-Transplantation, der Leihmut-

${ }^{1}$ Für einen Überblick über Formen und Terminologie vgl. z.B. Trotnow/Coester-Waltjen, Möglichkeiten, Gefahren und rechtliche Schranken befruchtungstechnischer und gentechnischer Eingriffe unter besonderer Berücksichtigung des Entwurfs eines Embryonenschutzgesetzes, 1990, S. 1 ff.; Harder, Wer sind Vater und Mutter? - Familienrechtliche Probleme der Fortpflanzungsmedizin, JuS 1986, 505 ff.; Kamps, Das Recht der Reproduktionsmedizin - Ein Überblick, MedR 1994, 339 ff.; Bundesärætekammer, (Muster-) Richtlinie zur Durchführung der assistierten Reproduktion, Novelle 2006, Dt. Ärzteblatt 2006, 1392 ff. 
terschaft und der Ersatzmutterschaft zu unterscheiden. ${ }^{2}$ Bei der EmbryoTransplantation wird eine Eizelle im Körper einer anderen Frau - homolog oder heterolog - befruchtet und dieser Embryo in den Körper der Wunschmutter übertragen, die das Kind dann austrägt. Von einer Embryospende spricht man, wenn Eizellspende und Samenspende, also beide Formen der heterologen Befruchtung, zusammenkommen. ${ }^{3}$ Im Fall der Leih- oder Tragemutterschaft ist die Frau nicht in der Lage, das Kind selbst auszutragen, sodass der - homolog oder heterolog befruchtete - Embryo in den Körper einer anderen Frau, der Leihmutter, implantiert wird, die das Kind unter dem Versprechen austrägt, es nach der Geburt der Wunschmutter bzw. den Eltern zu übergeben. Ist die Wunschmutter steril, kann bei einer anderen Frau, der Ersatzmutter, eine Insemination oder Fertilisation mit dem Sperma des Wunschvaters vorgenommen werden, die dann ebenfalls unter dem Versprechen späterer Herausgabe das Kind austrägt. ${ }^{4}$

Medizinisch sind alle diese Formen, wenngleich mit unterschiedlichen Risiken und Erfolgsraten, durchführbar. Die Fälle heterologer Insemination sind seit 1993 auf die Hälfte zurückgegangen, da die intrazytoplasmatische Spermieninjektion (ICSI) im Rahmen extrakorporaler Befruchtung die Möglichkeiten erfolgreicher homologer Zeugung drastisch verbessert hat. ${ }^{5}$ Auch die In-vitro-Fertilisationen sind in den Jahren 2004-2006 auf fast die Hälfte reduziert worden, nachdem sie zuvor von anfänglich 742 im Jahr 1982 auf 105.854 im Jahr 2003 gestiegen waren. ${ }^{6}$

Die verschiedenen Formen assistierter Reproduktion werden rechtlich sehr unterschiedlich behandelt. Während die Fülle homologer Insemination und Fertilisation innerhalb stabiler Ehe zwar von entsprechenden medizinischen Indikationen abhängen ${ }^{7}$, ansonsten jedoch rechtlich weitgehend zulässig sind, unterliegen heterologe Insemination und Fertilisation einigen Restriktionen. Eizellspende, Embryo-Transplantation, Leih- und Ersatzmutterschaft sind durch das Embryo-

\footnotetext{
${ }^{2}$ Hier ist die Terminologie am umstrittensten: Differenzierung wie hier bei Harder (Fußn. 1), 506; Begriff der Tragemutterschaft bei Bernat, Rechtsfragen medizinisch assistierter Zeugung, 1989, S. 244; Hieb, Die gespaltene Mutterschaft im Spiegel des deutschen Verfassungsrechts, 2005, S. 10; weitere Begriffe bei Keller, in: Keller/Günther/Kaiser (Hrsg.), Embryonenschutzgesetz, 1992, \& 1 Nr. 7 Rdn. 15.

${ }^{3}$ Die Embryospende ist daher ein Unterfall der Embryo-Transplantation.

${ }^{4}$ Ersatz- und Leih-/Tragemutterschaft werden teilweise unter dem Begriff der Ammenmutterschaft zusammengefasst, s. etwa Bernat (Fußn. 2), S. 243 ff.

${ }^{5}$ Katzorke, Donogene Insemination, Gynäkologische Endokrinologie 2003, 85 ff.; grundlegend für die Einführung, Palermo/Joris/Devroey/v.Steirteghem, Pregnancies after intracytoplasmic injection of single spermatozoon into an oocyte, The Lancet 1992/340, $17 \mathrm{ff}$.

${ }^{6}$ Deutsches IVF Register, DIR Jahrbuch 2006, S. 6; allgemeine Darstellung der Entwicklung in: EnqueteKommission Recht und Ethik in der modernen Medizin, Schlussbericht, Zur Sache 2/2002, S. 65 ff. (= BT-Drs. 14/9020).

${ }^{7}$ Vgl. Bundesärztekammer (Fußn. 1), Z. 2.
} 
nenschutzgesetz sogar verboten. ${ }^{8}$ Außerdem sind die Leistungen der gesetzlichen Krankenversicherung für eine künstliche Befruchtung auf Ehepaare beschränkt. ${ }^{9}$

Diese Restriktionen und insbesondere die unterschiedliche Behandlung weiblicher und männlicher Infertilität werfen daher die Frage nach der verfassungsrechtlichen Einordnung und den gesetzgeberischen Spielräumen auf.

\section{Grundrechtliche Fundierung eines Rechts auf Fortpflanzung}

Das Grundgesetz kennt kein explizites Recht auf Fortpflanzung, das Wunscheltern in Anspruch nehmen könnten. Selbstverständlich umfasst das Auffanggrundrecht der allgemeinen Handlungsfreiheit gem. Art. 2 Abs. 1 GG die Freiheit zur autonomen Lebensgestaltung, die auch die Entscheidungen über eine eigene Nachkommenschaft oder überhaupt eine Lebensführung mit Kindern etwa durch Adoption einschließt. Man wird aber ein Recht auf Fortpflanzung zusätzlich im allgemeinen Persönlichkeitsrecht verankern können, ${ }^{10} \mathrm{zu}$ dem nicht nur die sexuelle Selbstbestimmung, ${ }^{11}$ sondern ebenso die Entscheidung für eigene Kinder gehört. ${ }^{12}$ Angesichts des engen Bezugs zur eigenen Persönlichkeitsentfaltung und zur privaten Lebensgestaltung ist die Realisierung von Kinderwünschen ein elementarer Teil individueller Selbstverwirklichung und wesentlicher Ausdruck der eigenen Persönlichkeit, der von den engeren Persönlichkeitssphären erfasst wird und Mann und Frau gleichermaßen zusteht, ${ }^{13}$ ohne dass der unantastbare Kernbereich des allgemeinen Persönlichkeitsrechts erreicht würde. ${ }^{14}$ Selbstverständlich ist dieses Recht nicht als Anspruch auf Fortpflanzung zu verstehen, sondern im Sinne der Entscheidungsfreiheit für Kinder und entsprechender Abwehransprüche gegenüber Behinderungsversuchen und staatlichen Eingriffen. ${ }^{15}$ Schließlich wird das

8 Vgl. $\int 1$ Abs. 1 Z. 1, 2 ESchG (Eizellspende), \ 1 Abs. 1 Z. 6 ESchG (Embryo-Transplantation), $\int 1$ Abs. 1 Z. 7 ESchG (Ersatz- und Leihmutterschaft, die dort einheitlich als Ersatzmutterschaft bezeichnet werden).

9 \27a Abs. 1 Z. 3 SGB V; das BVerfG hat diese Regelung für verfassungsmäßig erklärt, NJW 2007, 1343; demgegenüber ist die künstliche Befruchtung als Heilbehandlung aber steuerlich absetzbar, s. Rüsken, Künstliche Befruchtung als Heilbehandlung. Zur steuermindernden Berücksichtigung von Kosten homo- und heterologer Befruchtung, NJW 1998, $1745 \mathrm{ff}$.

${ }^{10}$ Vgl. zur Konstituierung dieses Rechts Ramm, Die Fortpflanzung - ein Freiheitsrecht?, JZ 1989, 861 ff. (870); Günther, Der Kindeswohlbegriff als Zulässigkeitskriterium für die In-vitro-Fertilisation, 1996, S. 58 ff.; Hieb (Fußn. 2), S. 15 ff.; Gröschner, in: Dreier (Hrsg.), Grundgesetz-Kommentar, Bd. I, 2. Aufl. 2004, Art. 6, Rdn. 65.

11 BVerfGE 47, 46 (73).

12 BGHZ 97, 372 (379).

13 Vgl. a. BVerfGE 96, 56 (61) zur Sexualsphäre; s. ferner Hieb (Fußn. 2), S. 21 f.

${ }^{14}$ Hieb (Fußn. 2), S. 22 f.; anders Balz, Heterologe künstliche Samenübertragung beim Menschen, 1980, S. 20: „Kernbereich der menschlichen Persönlichkeit und damit der Menschenwürde“.

15 Vgl. a. Coester-Waltjen, Elternschaft außerhalb der Ehe, in: Bundesministerium für Gesundheit (Hrsg.), Fortpflanzungsmedizin in Deutschland, 2001, S. 158 ff. (159). 
Werner Heun

Recht auf Fortpflanzung zusätzlich durch Art. 6 Abs. 1 GG verstärkt, ${ }^{16}$ der die Familie als geschlossenen, eigenständigen Lebensbereich ${ }^{17}$ und dabei insbesondere die Familiengründung unmittelbar schützt. ${ }^{18}$ Für verheiratete Eltern tritt die Ehegestaltungsfreiheit noch hinzu. ${ }^{19}$ Im Übrigen steht der Entschluss, Kinder haben zu wollen, auch unter dem Schutz des Art. 8 EMRK. ${ }^{20}$

Die medizinisch assistierte Reproduktion ist in den Schutzbereich dieses Rechts auf Fortpflanzung jedenfalls prinzipiell einzubeziehen. Allenfalls kann im Hinblick auf die notwendige Beteiligung Dritter im Fall der medizinisch assistierten Reproduktion der Staat zu ausgestaltender Regelung befugt sein. ${ }^{21}$ Das gilt zumindest für alle Fälle homologer Insemination und Fertilisation. ${ }^{22}$ Zweifelhafter ist dies jedoch für die Fälle heterologer Reproduktion, wobei noch einmal zwischen der heterologen Reproduktion, bei der nur eine Spende heterolog erfolgt, und der Embryonenspende, bei der Eizelle und Samen von Dritten stammen, abgestuft werden muss. Irrelevant ist dagegen für die Frage, ob das Recht auf Fortpflanzung überhaupt berührt ist, die Unterscheidung zwischen Eizell- und Samenspende. Auch hier wird man prinzipiell den Kinderwunsch in den Schutzbereich des „Rechts auf Fortpflanzung“ fallen lassen müssen. Angesichts des zunehmenden Sozialbezugs durch die Beteiligung fremder Spender steigen indes in diesen Fällen die Ingerenzrechte des Gesetzgebers, der Schutz des grundrechtlich gesicherten Fortpflanzungsrechts schwächt sich ab. Die Embryospende und Ersatzmutterschaft stellen im strengen Sinn gar keine Fortpflanzung mehr dar, sondern realisieren die Freiheit zu sozialer Elternschaft wie bei der Adoption. ${ }^{23}$

\footnotetext{
16 BVerfGE 42, 234 (236); 57, 170 (178); 66, 84 (94).

${ }_{17}$ BVerfGE 24, 119 (135).

${ }^{18}$ Gröschner (Fußn. 10), Rdn. 65.

${ }^{19} \mathrm{Vgl}$. a. Hieb (Fußn. 2), S. 24 f.

${ }^{20}$ Right to respect for the decision to have a child, so ECHR, Evans v. United Kingdom, no. 6339/05, \ 71 ECHR 2007; E.B. v. France, no. 43546/02, \ 41 ECHR 2008; Maraubn/Meljnik, in: Grote/Marauhn (Hrsg.), Konkordanzkommentar zum europäischen und deutschen Grundrechtschutz, 2006, Kap. 16, Rdn. 52; Fabrenborst, Fortpflanzungstechnologien und Europäische Menschenrechtskonvention, EuGRZ 1988, 125 ff.; s. auch Österr. Verfassungsgerichtshof, MedR 2000, 389 (390) mit Anm. Bernat, zur Schweiz s. Schweiz. BGer., EuGRZ 1994, 223 (228); EuGRZ 1989, 370 (374); zur Anerkennung des Rechts auf Elternschaft durch die Nahmani Entscheidung des Israel Supreme Court s. Sina, Recht auf Elternschaft?, FamRZ 1997, 862 ff.

${ }^{21}$ Nur insoweit ist eine Differenzierung zwischen natürlicher und assistierter Reproduktion verfassungsrechtlich gerechtfertigt. Eine Differenzierung im Hinblick auf die Einbeziehung in den Schutzbereich überhaupt ist dagegen abzulehnen, insoweit zutreffend Hieb (Fußn. 2), S. 25 ff.

22 Vgl. In re Baby M, New Jersey Supreme Court, 537 A.2d 1227, 109 N.J. 396 (N.J. 2. 3. 1988).

${ }^{23}$ Anders insoweit Hieb (Fußn. 2), S. 31 f.
} 


\section{Verfassungsrechtliche Rechtfertigungen der Restriktionen assistierter Reproduktion und ihre Problematik}

Die Restriktionen des Embryonenschutzgesetzes werden regelmäßig mit verfassungsrechtlichen Argumenten begründet, deren Validität nicht unzweifelhaft ist.

\subsection{Menschenwürde des Kindes}

Nicht ganz selten wird als Einwand vor allem gegen die Formen assistierter Reproduktion, die eine gespaltene Mutterschaft bewirken, die Menschenwürde des Kindes ins Spiel gebracht. Die Unantastbarkeit der Menschenwürde ${ }^{24}$ und ihre Unabänderbarkeit gem. Art. 79 Abs. 3 GG $^{25}$ sichern einen absoluten Schutz, der schon im Ansatz die betreffende Fortpflanzungshilfe ausschließen würde. Indes lassen schon die Begründungsversuche die Fragwürdigkeit dieses Arguments erkennen, wenn die „widernatürliche Aufspaltung“ der Mutterschaft ${ }^{26}$ oder ganz pauschal Menschenwürdeverletzungen angeführt werden. ${ }^{27}$ Abgesehen davon, dass hier ein einseitiges, tradiertes Mutterbild ganz unverstellt in den Menschenwürdebegriff eingeschrieben wird, ist diese These aus mehreren Gründen unhaltbar. Da in diesen Fällen ohne Fortpflanzungshilfe das Kind gar nicht geboren werden könnte und würde, verdankt es den konkreten Entstehungsbedingungen überhaupt sein Leben und seine Existenz, sodass dadurch nicht seine Menschenwürde verletzt sein kann. ${ }^{28}$ Der Entstehungsvorgang selbst kann bei heterologer Samen- und Eizellspende zudem nicht als Würdeverletzung konstruiert werden, da dieser sogar der Kernverschmelzung vorausliegt und der Würdeschutz frühestens mit diesem Vorgang beginnen kann. Es fehlt damit an einem verletzungsfähigen Subjekt. Die Embryospende geht denknotwendig der Nidation voraus, sodass auch hier nach zutreffender Auffassung noch gar kein Träger der Menschenwürde angenommen werden kann, da kein individuelles menschliches Leben vorliegt und die Grundrechtsträgerschaft ein Individuum voraussetzt. ${ }^{29}$ Allein bei Ersatz- und Leihmutterschaft ist theoretisch eine Menschenwürdeverletzung denkbar, sofern ein verletzungsfähiges Subjekt zumindest in den späten Phasen der Schwangerschaft existiert, sie scheitert aber daran, dass diese Vorgänge dem Kind erst zum Leben verhelfen. Auch ein vorwirkender Menschenwürdeschutz kann daher nicht greifen.

${ }^{24}$ Vgl. hier nur Dreier, in: Dreier (Fußn. 10), Art. 1 I, Rdn. 131 ff.

${ }^{25}$ Dreier (Fußn. 24), Rdn. 43.

${ }^{26}$ Benda, Humangenetik und Recht - eine Zwischenbilanz, NJW 1985, 1730 ff. (1733).

27 Vgl. z.B. Kamps (Fußn. 1), 341; BT-Drs. 11/5460, S. 6 f.; Keller, Fortpflanzungstechnologie und Strafrecht, in: Günther/Keller (Hrsg.), Fortpflanzungsmedizin und Humangenetik - Strafrechtliche Schranken, 2. Aufl. 1991, S. 193 (202); früh schon Dürig, in: Maunz/Dürig (Hrsg.), Grundgesetz, Art. 1 I (Erstb. 1958), Rdn. 39.

28 Coester-Waltjen, Gutachten zum 56. DJT 1986, S. 46 (47); Starck, Gutachten zum 56. DJT 1986, S. 37; Dreier (Fußn. 24), Rdn. 93 m.w.N.

${ }^{29}$ Zur Begründung näher Heun, Embryonenforschung und Verfassung - Lebensrecht und Menschenwürde des Embryos, JZ 2002, 517 ff. 
Ferner wird man kein „Recht auf die biologische Mutter“ anerkennen können, ${ }^{30}$ das nicht nur schwerlich konkretisierbar ist, sondern auch Adoptionen verbieten müsste, solange noch die biologische Mutter lebt. Die Weggabe des Kindes an die Wunscheltern durch die Ersatz- oder Leihmutter, die gem. S 1591 BGB rechtlich die Mutter des Kindes ist, ist nämlich rechtlich als Adoptionsvorgang konstruiert. ${ }^{31}$ Die rechtliche Elternstellung kann von den Wunscheltern somit lediglich durch eine Adoption erlangt werden. ${ }^{32}$ Die Adoption des Kindes durch die Wunscheltern ist aber keinesfalls eine Menschenwürdeverletzung.

\subsection{Recht des Kindes auf Kenntnis der eigenen Abstammung}

Nicht unmittelbar dem Grundsatz der Menschenwürde, aber dem allgemeinen Persönlichkeitsrecht, das aus Art. 2 Abs. 1 GG in Verbindung mit Art. 1 Abs. 1 GG abgeleitet wird, wird vielfach ein generelles Recht auf Kenntnis der eigenen Abstammung entnommen, das seinerseits den Formen heterologer Insemination und Fertilisation wie auch Embryospende und Leih- bzw. Ersatzmutterschaft entgegengehalten wird. ${ }^{33}$ Vor einer allzu pauschalen Inanspruchnahme eines solchen Rechts muss aber gewarnt werden. Als Einwand gegen assistierte Reproduktion taugt es nur sehr begrenzt.

Das Recht auf Kenntnis der eigenen Abstammung wird man nicht allein deswegen verneinen können, weil bei Samen- oder Eizell- wie Embryospenden noch kein geeignetes Grundrechtssubjekt vorliegt, da das Recht ohnehin erst dem späteren Kind zusteht und die Rechtsgarantie insoweit prinzipiell Vorwirkungen entfalten kann. Gegebenenfalls muss daher gesichert werden, dass später die betreffende Kenntnis erlangt werden kann. Damit wird bereits eine erste Begrenzung dieses Rechts deutlich. Es schließt in keiner Weise eine dieser Formen assistierter Reproduktion aus, sondern allenfalls die Anonymität der Spende oder der gespaltenen Mutterschaft. ${ }^{34}$ Aber selbst dieser Ausschluss anonymer Spenden oder biologischer Mutterschaft ist äußerst zweifelhaft, weil die Reichweite des Rechts auf Kenntnis der eigenen Abstammung damit überdehnt wird.

Meist wird ohne weitere Einschränkung ein allgemeines Recht auf Kenntnis der eigenen Abstammung behauptet und dafür das Bundesverfassungsgericht in

\footnotetext{
30 So der Sache nach Vatikanische Kongregation für die Glaubenslehre, Instruktion über die Achtung vor dem beginnenden menschlichen Leben und die Würde der Fortpflanzung, 1987, S. 22; Wolkinger, Lebensbeginn durch Menschenhand?, in: Bernat (Hrsg.), Lebensbeginn durch Menschenhand, 1985, S. $89 \mathrm{ff}$. (104).

31 Vgl. zur familienrechtlichen Zuordnung Coester-Waltjen (Fußn. 15), S.83 ff.; Harder (Fußn. 1), 510 f.

32 S.a. Hieb (Fußn. 2), S. 104.

33 Vgl. z.B. Laufs, Arztrecht, 5. Aufl. 1993, Rdn. 371 ff.; Kleineke, Das Recht auf Kenntnis der eigenen Abstammung, 1976, S. 292 ff.; gänzlich verfehlt die Reduktion auf ein „Recht auf genetische Abstammung“" bei Donhauser, Das Recht auf Kenntnis der genetischen Abstammung, 1996, S. 53 ff.; Mansees, Jeder Mensch hat ein Recht auf Kenntnis seiner genetischen Herkunft, NJW 1988, 2984 ff.; dagegen zutreffend Coester-Waltjen (Fußn. 15), S. 64; Hieb (Fußn. 2), S. 126 f.

${ }^{34}$ Vgl. Starck (Fußn. 28), S. 23 ff. (29).
} 
Anspruch genommen. Das ist jedoch allenfalls partiell gerechtfertigt. Das Bundesverfassungsgericht betont zwar abstrakt die Bedeutung dieser Kenntnis für die eigene Persönlichkeitsentfaltung und schließt diese Darlegungen mit dem Satz: „Daher umfasst das Persönlichkeitsrecht auch die Kenntnis der eigenen Abstammung “'.35 Indes werden die modifizierenden und qualifizierenden folgenden Sätze des Gerichts meist nicht mehr zur Kenntnis genommen, wonach das Recht sich gerade nicht auf unaufklärbare Fälle erstreckt, sondern vielmehr nur einen von vornherein begrenzten Anspruch vermittelt. ${ }^{36}$ Das Persönlichkeitsrecht ,verleiht kein Recht auf Verschaffung von Kenntnissen der eigenen Abstammung, sondern kann nur vor der Vorenthaltung erlangbarer Informationen schützen“.37 Dieses vom Verfassungsgericht bejahte Recht steht damit selbst anonymen Formen der assistierten Reproduktion nicht entgegen.

Das schließt die Annahme eines Rechts auf positive Kenntniserlangung jedoch noch nicht definitiv aus. ${ }^{38}$ Indes lässt sich ein derartiges Recht auch sachlich nicht aufrechterhalten. Selbst in Fällen natürlicher Zeugung ist Aufschluss über den biologischen Vater nicht immer zu gewinnen, wenn etwa die Mutter mit mehreren Männern und zudem noch anonym sexuellen Verkehr hat. ${ }^{39}$ Es dürfte schwerfallen, in den Vorgängen der anonymen Spende oder der sexuellen Selbstbestimmung der Frau Menschenwürdeverletzungen für das Kind zu erblicken, zumal auch hier der Gesichtspunkt zu beachten ist, dass das Kind erst dank dieser Vorgänge überhaupt seine Existenz erlangt. Erst die Anonymität ermöglicht vielfach die Bereitschaft zur Spende. Allgemeines Persönlichkeitsrecht und Menschenwürde sind daher für die assistierte Reproduktion weder positiv noch negativ ausschlaggebend.

\subsection{Das Kindeswohl als verfassungsrechtliches Argument}

Der Gesetzgeber hat die Verbotsnormen des Embryonenschutzgesetzes zentral mit dem Kindeswohl begründet ${ }^{40}$, das im Familienrecht fest verankert ${ }^{41}$, aber auch verfassungsrechtlich fundiert ist. Es stellt zwar kein eigenständiges Grundrecht des Kindes $\mathrm{dar}^{42}$, findet jedoch wiederum im allgemeinen Persönlichkeitsrecht des Kindes eine grundrechtliche Stütze. ${ }^{43}$ Zugleich dient es als „Richtpunkt für den

\footnotetext{
35 BVerfGE 79, 256 (269).

36 Enders, Das Recht auf Kenntnis der eigenen Abstammung, NJW 1989, 881 ff.; Dreier (Fußn. 24), Rdn. 93 mit Fußn. 297.

${ }^{37}$ BVerfGE 79, 256 (269).

${ }^{38}$ Vgl. Hieb (Fußn. 2), S. 110 ff. m.w.N.

${ }^{39}$ Vgl. Dreier (Fußn. 24), Rdn. 93; dagegen mit dem Argument, es handele sich dabei um eine „schicksalhafte Situation“"Starck (Fußn. 28), S. 24; unklar insoweit Hieb (Fußn. 2), S. 133.

40 BT-Drs. 11/5460, S. 6.

${ }^{41}$ Vgl. Coester, Das Kindeswohl als Rechtsbegriff, 1983, S. 134 ff.; Günther (Fußn. 10), S. 103 ff.

42 Jestaedt, in: Bonner Kommentar zum Grundgesetz, Art. 6 II u. III (1995), Rdn. 35, 146.

${ }^{43}$ Vgl. Jestaedt (Fußn. 42), Rdn. 33, 146; Jeand'Heur, Verfassungsrechtliche Schutzgebote zum Wohl des Kindes und staatliche Interventionspflichten aus der Garantienorm des Art. 6 Abs. 2 Satz 2 GG, 1993, S. 18 f.; vgl. zum allg. Persönlichkeitsrecht des Kindes BVerfGE 24, 119 (144); 75, 201 (218).
} 


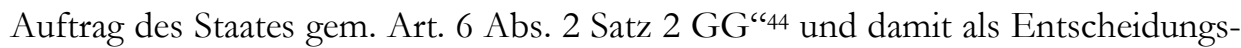
maßstab und Eingriffslegitimation für das staatliche Handeln, bleibt aber als Grund und Grenze des Elternrechts auf die Eltern-Kind-Beziehung beschränkt. ${ }^{45}$ In dieser Bedeutung scheidet es daher als Maßstab für die elterliche Entscheidung über den Kindeswunsch und die Inanspruchnahme assistierter Reproduktionsformen von vornherein aus. ${ }^{46}$ Lediglich Gesichtspunkte des Kindeswohls, die unmittelbar aus dem allgemeinen Persönlichkeitsrecht des Kindes selbst erwachsen, können folglich für die verfassungsrechtliche Beurteilung assistierter Reproduktionstechniken herangezogen werden.

Das lässt erneut Zweifel an der Tauglichkeit dieses Arguments für die Frage der Verfassungsmäßigkeit jeglicher Fortpflanzungshilfe aufkommen. Im Zeitpunkt der Anwendung der Reproduktionstechniken fehlt es an einem verletzungsfähigen Subjekt, und im Hinblick auf den künftigen Grundrechtsträger unterliegt das Kindeswohl demselben Einwand wie die Menschenwürde, dass es schwerlich der eigenen Existenz entgegengehalten werden kann. ${ }^{47}$ Darüber hinaus sind die unter dem Mantel des Kindeswohls vorgetragenen, vorwiegend sozialpsychologischen Argumente kaum geeignet, ein Verbot zu tragen. Sie sind allenfalls für die Fälle einer Leih- oder Ersatzmutterschaft überhaupt ernsthaft zu erwägen.$^{48}$ Eine erschwerte Identitätsfindung ist selbst bei einer unmittelbar nach der Geburt erfolgenden Weggabe an die Wunscheltern kaum zu befürchten, sondern stellt allenfalls bei mehrfachem Wechsel der Bezugspersonen eine reale Gefährdung dar. Etwaige Interessenkonflikte der sozialen und biologischen Mütter sind vielleicht ein Problem bei der Entscheidung über die Freigabe zur Adoption, nach diesem Zeitpunkt sind Konflikte jedoch kaum zu erwarten. Sie unterscheiden sich jedenfalls nicht von anderen Konflikten in unzweifelhaft verfassungsmäßigen Fällen der Adoption. Die Beziehung zur sozialen Mutter - und zum Vater - ist für die Persönlichkeitsentwicklung ausschlaggebend. Das Kindeswohl steuert die rechtliche Ausgestaltung der sozialen Eltern-Kind-Beziehung, steht aber keiner Form der assistierten Reproduktion dem Grunde nach entgegen.

\subsection{Recht der Spender, Spenderinnen sowie Leih- oder Ersatzmütter}

Einschränkungen assistierter Reproduktion werden teilweise auch mit den betroffenen Rechten der jeweiligen Spender und Tragemütter begründet. Es kann kaum verwundern, dass selbst in diesem Fall die Menschenwürde mobilisiert wird, wenn-

\footnotetext{
${ }^{44}$ BVerfGE 24, 119 (144); 60, 79 (88); 72, 122 (134); Jeand'Heur (Fußn. 43), S. 17. ff.

45 BVerfG, NJW 2008, 1287 (1288); s.a. Coester-Waltjen, in: Münch/Kunig (Hrsg.), GrundgesetzKommentar, 5. Aufl. 2000, Art. 6, Rdn. 81.

46 Vgl. a. Hieb (Fußn. 2), S. 141.

47 Im vorliegenden Zusammenhang vgl. Bernat, Fortpflanzungsmedizin und Recht, MedR 1991, 308 (310); Hieb (Fußn. 2), S. 146 f.

48 Vgl. zum Folgenden auch Bernat (Fußn. 2), S. 91 ff.; Hieb (Fußn. 2), S. 147 ff.; Dietrich, Mutterschaft für Dritte, 1989, S. 274 ff.
} 
gleich eine Verletzung der Menschenwürde des Samenspenders nirgends behauptet wird, wohl aber vor allem der Leih- und Ersatzmütter. Der Vorwurf der Menschenwürdeverletzung wird hier inhaltlich mit dem ebenso beliebten wie beliebigen Instrumentalisierungsverbot begründet, ${ }^{49}$ weil die Ersatz- und Leihmutter zur „Ge-

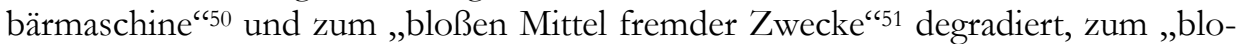
Ben Brutobjekt" herabgewürdigt würde. ${ }^{52}$ Das lässt sich selbst gegen eine Eizellspenderin wenden, die ,als Produktionsstätte von Eizellen“ instrumentalisiert werden könnte. ${ }^{53}$ Sieht man davon ab, dass diese Argumente eher die völlige Beliebigkeit und Fragwürdigkeit der Objektformel erweisen als eine substanzielle Menschenwürdeverletzung, ist die Unterstellung einer Beeinträchtigung der Menschenwürde schon deswegen nicht haltbar, weil die betreffenden Frauen sich freiwillig zur Verfügung stellen und ein Verhalten, das Ausdruck der Selbstbestimmung der jeweiligen Frauen ist, prinzipiell keine Menschenwürdeverletzung begründen kann. ${ }^{54}$ Das Bundesverwaltungsgericht lässt sich zwar nicht davon abhalten, eine objektive Menschenwürde gegen die Betroffenen selbst in Stellung zu bringen $^{55}$, stößt damit aber zu Recht fast einhellig auf „,vernichtende Kritik“ “. 56

Plausibler sind demgegenüber die Einwände, die auf die Gefährdung der Gesundheit der Betroffenen hinweisen. Die Samenspende führt allerdings zu keiner nennenswerten physischen oder psychischen Belastung des männlichen Spenders. Gesundheitsgefährdungen treten lediglich bei den beteiligten Frauen auf, und zwar in unterschiedlichem Maß. Bei der Wunschmutter sind mit der künstlichen Befruchtung nicht unerhebliche Belastungen und Gefahren verbunden, auch die Entnahmeverfahren bei der Übertragung auf eine Leihmutter sind nicht völlig unbedenklich. Die notwendige Einwilligung in die Körperverletzung unterstellt, welche insoweit aufgrund eines ,informed consent" gerechtfertigt ist, ${ }^{57}$ sind die freiwillig eingegangenen Risiken jedoch Ausdruck des Willens zum eigenen Kind und damit der Selbstbestimmung. Demgegenüber tritt die gesundheitliche Selbstgefährdung vollständig zurück. Ein Verbot der assistierten Reproduktion im Verhältnis allein zur Mutter wäre in keiner Weise gerechtfertigt. ${ }^{58}$

\footnotetext{
${ }^{49}$ Vgl. dazu allg. hier nur Dreier (Fußn. 24), Rdn. 53.

${ }^{50}$ So die Vorsitzende des Deutschen Ärztinnenbundes, zit. nach Hieb (Fußn. 2), S. 152.

${ }^{51}$ Balz (Fußn. 14), S. 30.

52 Kienle, Künstliche Befruchtung und artifizielles Recht, ZRP 1995, $201 \mathrm{f}$.

53 Hieb (Fußn. 2), S. 153.

54 Vgl. hier nur Geddert-Steinacher, Menschenwürde als Verfassungsbegriff, 1990, S. 86 ff.; Dreier (Fußn. 24), Rdn. 151 ff.; Hillgruber, Der Schutz des Menschen vor sich selbst, 1992, S. 104 ff.; Steiner, Der Schutz des Lebens durch das Grundgesetz, 1992, S. 15 f.; Starck (Fußn. 28), S. 42.

55 Vgl. BVerwGE 64, 274 (279 f.) - Peepshow; 113, 340 (341) - Nacktfoto.

56 Discher, Die Peep-Show-Urteile des BVerwG, JuS 1991, 642 (649); s.a. Dreier (Fußn. 24), Rdn. 152 m.w.N.

${ }^{57}$ Zur Notwendigkeit des informed consent bei Eingriffen in die körperliche Integrität vgl. hier nur Deutsch/Spieckhoff, Medizinrecht, 6. Aufl. 2008, S. $162 \mathrm{ff.}$

$58 \mathrm{Vgl}$ a. Hieb (Fußn. 2), S. 160.
} 
Meist werden die Einschränkungen daher auch mit den Gesundheitsgefahren für die Spenderinnen und Leih- bzw. Ersatzmütter begründet. Selbst die Eizellspende bedarf eines invasiven Eingriffs, ebenso wie die Embryospende. Die mit dem Embryotransfer und einer Schwangerschaft verbundenen gesundheitlichen Risiken und Belastungen für die Ersatz- und Leihmutter sind noch gravierender. Auch diese Eingriffe in die körperliche Unversehrtheit erfolgen jedoch mit Zustimmung der betroffenen Frauen und sind ebenfalls Ausdruck ihrer individuellen Selbstbestimmung. Dadurch wird häufig das Ziel verfolgt, die Kinderwünsche anderer zu unterstützen. Gefahren für unbeteiligte Dritte entstehen nicht. Im Hinblick auf den hohen Rang der Autonomie der beteiligten Frauen sind ernsthafte Rechtfertigungsgründe für ein Verbot freiwilliger Spenden und der Formen der Ersatz- und Leihmutterschaft wegen der Beeinträchtigung der Rechte der Frauen nicht erkennbar. ${ }^{59}$

\subsection{Schutz von Ehe und Familie}

Schließlich wird der Schutz von Ehe und Familie gem. Art. 6 Abs. 1 GG den Formen heterologer assistierter Reproduktion entgegengehalten. ${ }^{60}$ Die Verwendung „ehefremder“ Keimzellen erweckt hier erstaunliche Bedenken. Samen- oder Eizellspender würden als fremde „Dritte in die Ehe eindringen“ und das herkömmliche Familienbild einer Gemeinschaft verheirateter Eltern mit ehelich gezeugten Kindern zerstören. ${ }^{61}$ Vereinzelt versteigt sich diese Auffassung sogar dazu, die heterologe Insemination in Anlehnung an die strafrechtliche Terminologie als „Ehebruch in mittelbarer Täterschaft“ zu qualifizieren, bei welcher der Arzt als Werkzeug der Frau und der Ehemann als Teilnehmer handelt. ${ }^{62}$

Diese Auffassung ist aus mehreren Gründen schwer nachvollziehbar. Betrachtet man zunächst isoliert die Ehegarantie des Art. 6 Abs. 1 GG, ist eine Beeinträchtigung kaum erkennbar. Unbeschadet der hier irrelevanten Kontroversen um die Geschlechtsverschiedenheit ${ }^{63}$ und die Einehe ${ }^{64}$ als Wesensmerkmale ist die Ehe „die Vereinigung eines Mannes und einer Frau zur grundsätzlich unauflösbaren Lebensgemeinschaft" ${ }^{\text {" } 65}$, die auf gegenseitigem Konsens beruht. ${ }^{66}$ Inwieweit eine auf dem Konsens der Ehepartner basierende medizinische Fortpflanzungs-

\footnotetext{
${ }^{59} \mathrm{Vgl}$. Hieb (Fußn. 2), S. 160 f.

60 Z.B. Starck (Fußn. 28), S. 19 (28).

${ }^{61} \mathrm{Vgl}$. Balz (Fußn. 14), S. 22; Kongregation (Fußn. 30), S. 20 ff.

62 Keller, Fortpflanzungstechnologie. Ein Gesamtkonzept staatlichen Regelungsbedarfs, MedR 1988, 59 ff. (62).

63 NJW 1993, 3958 ff.; BVerwGE 100, 287 (294); Coester-Waltjen (Fußn. 45), Rdn. 9; Gröschner (Fußn. 10), Rdn. 43 ff.

${ }^{64}$ Coester-Waltjen (Fußn. 45), Rdn. 8; Robbers, in: v.Mangoldt/Klein/Starck (Hrsg.), Kommentar zum Grundgesetz, Bd. I, 5. Aufl. 2006, Art. 6, Rdn. 42.

65 BVerfGE 10, 59 (66).

${ }^{66}$ BVerfGE 53, 224 (250); vgl. a. Coester-Waltjen (Fußn. 45), Rdn. 6; Gröschner (Fußn. 10), Rdn. 40; die auch das Moment des formalisierten Eheschlusses bei freier Willensübereinstimmung hervorheben.
} 
hilfe in diesen Schutzbereich eingreifen soll, ist nicht recht ersichtlich. Meist wird deswegen auch bloß eine Gefährdung der Ehe als Institution, ${ }^{67}$ die mit abstrakten überkommenen Ehebildern gleichgesetzt wird, behauptet. ${ }^{68}$ Empirisch ist dies haltlos, da Untersuchungen ganz im Gegenteil in Fällen gelungener assistierter Reproduktion auf eine Stabilisierung der betreffenden Ehen deuten. ${ }^{69}$ Jedenfalls ist der Ehebegriff des Grundgesetzes nicht mit den Vorstellungen der Vatikanischen Kongregation für die Glaubenslehre identisch.

Es ist zudem verfehlt, den Begriff der Ehe mit demjenigen der Familie gem. Art. 6 Abs. 1 GG zu identifizieren und die Ehe gegen die Familie auszuspielen. Der grundgesetzliche Familienbegriff umfasst zwar in erster Linie die Gemeinschaft zwischen verheirateten Eltern und ihren Kindern, ${ }^{70}$ aber ebenso das Verhältnis zwischen unverheirateten Eltern und ihren Kindern. ${ }^{71}$ Auch Stief-, Adoptiv- und Pflegekinder unterfallen dem Familienbegriff. ${ }^{72}$ Deshalb zählen Kinder, die durch die modernen Fortpflanzungshilfen entstehen, zur Familie im Sinne des Art. 6 Abs. 1 GG. Assistierte Reproduktion ermöglicht in diesen Fällen regelmäßig überhaupt erst eine Familiengründung und fördert damit nachhaltig und positiv die individuelle Familie und ebenso die Institution als solche. ${ }^{73}$ Die Überwindung ungewollter Kinderlosigkeit stabilisiert und erhält damit Ehe und Familie sowohl in individueller als auch in sozialer und institutioneller Hinsicht. Als individuelles Grundrecht wie als Institutsgarantie steht Art. 6 Abs. 1 GG der assistierten Reproduktion damit nicht entgegen, sondern rechtfertigt ihre Unterstützung vielmehr positiv. ${ }^{74}$ Das gilt nicht nur für die heterologe Insemination und Fertilisation, sondern ebenso für die Formen der Leih- und Ersatzmutterschaft, jedenfalls hinsichtlich der Wunscheltern. Die Anstrengung einer Schwangerschaft durch die Ersatzoder Leihmutter innerhalb einer Ehe dürfte im vorauszusetzenden Fall eines Konsenses der Ehepartner ebenfalls kaum eine Beeinträchtigung darstellen und ist dann gleichfalls durch die Einwilligung gerechtfertigt.

\section{Gleichheitsprobleme}

Die verschiedenen Regelungen des Embryonenschutzgesetzes mit den Verboten von Eizell- und Embryonenspende sowie Ersatz- und Leihmutterschaft laufen auf ein prinzipielles Verbot aller Formen sog. gespaltener Mutterschaft hinaus, wäh-

${ }^{67}$ Zur Ehe als Institutsgarantie Coester-Waltjen (Fußn. 45), Rdn. 12 ff.

${ }^{68}$ Kongregation (Fußn. 30), S. 20 ff.; Loeffler, Die künstliche Insemination beim Menschen, in: Ranke/Dombois (Hrsg.), Probleme der künstlichen Insemination, 1960, S. 22 (32); Wolkinger (Fußn. 30), S. $104 \mathrm{ff}$.

${ }^{69}$ Vgl. Coester-Waltjen (Fußn. 28), S. 46; Bernat (Fußn. 2), S. 91; Hieb (Fußn. 2), S. 172 f. m.w.N.

${ }^{70}$ BVerfGE 48, 327 (339); Coester-Waltjen (Fußn. 45), Rdn. 11.

${ }^{71}$ BVerfGE 18, 97 (105 f.); 45, 104 (123); 79, 203 (211).

72 BVerfGE 68, 176 (187); 80, 81 (90).

${ }^{73} \mathrm{Vgl}$. Hieb (Fußn. 2), S. 173 (175).

74 Vgl. a. Coester-Waltjen (Fußn. 28), S. 46, 82. 
rend die Samenspende als einzig denkbare Form medizinisch assistierter, gespaltener Vaterschaft nicht erfasst wird und damit erlaubt bleibt. ${ }^{75}$

Unbeschadet der unterschiedlichen Auffassungen zum Verhältnis zwischen den Gleichberechtigungsregelungen der Art. 3 Abs. 2 und 3 Abs. 3 GG $^{76}$ besteht Einigkeit, dass der dort verankerte Grundsatz der Gleichberechtigung von Mann und Frau ein prinzipielles Differenzierungsverbot nach dem Geschlecht begründet. ${ }^{77}$ Ebenfalls unabhängig von der Frage, ob dieses Differenzierungsverbot ein Anknüpfungs- oder richtigerweise ein Begründungsverbot beinhaltet, ${ }^{78}$ stellt die unterschiedliche Behandlung von infertiler Frau und infertilem Mann eine Differenzierung nach dem Geschlecht dar, die von dem Differenzierungsverbot erfasst wird. Mit der Anknüpfung der unterschiedlichen Behandlung an die Eizelle einerseits und die Samenzelle andererseits lässt sich das Begründungsverbot nicht umgehen. Es handelt sich in beiden Fällen um Keimzellen. ${ }^{79}$ Außerdem ist der identische Ausgangpunkt, nämlich die biologische Infertilität, für die Anwendung des Differenzierungsverbots maßgebend.

Die Durchbrechung des Differenzierungsverbots ist daher außerordentlich rechtfertigungsbedürftig. Es reicht gerade nicht aus, dass es überhaupt sachliche Differenzierungsgründe ${ }^{80}$ gibt und kein Verstoß gegen das Willkürverbot vorliegt, denn die Anforderungen an eine Rechtfertigung nach Art. 3 Abs. 2 und 3 GG sind wesentlich schärfer als bei einer Ungleichbehandlung gem. Art. 3 Abs. 1 GG. ${ }^{81}$ Umso mehr muss erstaunen, dass der Gesetzgeber beim Embryonenschutzgesetz auf jede Begründung der Ungleichbehandlung verzichtet hat. ${ }^{82}$ Sachlich muss zwischen den Fällen der Ersatz- und Leihmutterschaft einerseits und der Eizellspende andererseits unterschieden werden. Bei den Fällen der Austragung durch eine andere Frau als die vorgesehene soziale Mutter knüpft das Embryonenschutzgesetz an die Schwangerschaft selbst an. Das Bundesverfassungsgericht hat insoweit vertreten, dass das Differenzierungsverbot in Fällen, in denen der betreffende Sachverhalt überhaupt nur in einem Geschlecht verwirklicht werden kann, wofür die Schwangerschaft als Paradebeispiel gilt, das Differenzierungsverbot ins Leere laufe und damit gar nicht anwendbar sei. ${ }^{83}$ Das ist allerdings nicht unkritisiert geblieben. ${ }^{84}$ Immerhin lassen sich insoweit noch Gründe für ein Verbot von Ersatz- und

\footnotetext{
75 Vgl. Keller/Günther/Kaiser (Fußn. 2), Einführung, B. V Rdn. 12; Hieb (Fußn. 2), S. 178 f.

${ }^{76}$ Vgl. näher Heun, in: Dreier (Fußn. 10), Art. 3, Rdn. 100 ff. m.w.N., 126.

${ }^{77}$ Heun (Fußn. 76), Rdn. 107, 118.

${ }^{78} \mathrm{Vgl}$. Heun (Fußn. 76), Rdn. 122, 124.

${ }^{79}$ Vgl. Trotnow/Coester-Waltjen (Fußn. 1), S. 8; s.a. Katzorke, Keimzellspende - Medizinische, soziale und juristische Aspekte, in: Bundesministerium für Gesundheit (Hrsg.), Fortpflanzungsmedizin in Deutschland, 2001, S. 122 ff. (128).

80 Verfehlt insoweit daher Hieb (Fußn. 2), S. 197 ff.

${ }^{81}$ BVerfGE 3, 225 (240); Heun (Fußn. 76), Rdn. 107 m.w.N.

82 BT-Drs. $11 / 5460$.

${ }^{83}$ BVerfGE 6, 389 (422 f.); 52, 369 (374).

${ }^{84}$ Heun (Fußn. 76), Rdn. 110 m.w.N.
} 
Leihmutterschaft finden, die eine Durchbrechung jedenfalls unter Zugrundelegung der Rechtsprechung des Bundesverfassungsgerichts rechtfertigen.

Das gilt jedoch nicht für die Untersagung der Eizellspende. Die Aussage, dass sich die Eizellspende nur bei einem Geschlecht verwirklichen lasse, mag zwar äuBerlich und begrifflich richtig sein, ist aber im Hinblick auf die biologische Gleichartigkeit und Gleichwertigkeit von männlicher und weiblicher Keimzelle nicht aufrechtzuerhalten. Insofern kann zur Rechtfertigung ebenso wenig auf ausschließlich biologische Unterschiede zwischen Mann und Frau rekurriert werden, die eine verschiedene Behandlung zwingend erfordern würden, wie Bundesverfassungsgericht und Literatur für eine zulässige Differenzierung nach dem Geschlecht einhellig verlangen. ${ }^{85}$ Gerade daran fehlt es. Zwar bedarf die Eizellspende eines höheren medizinischen Aufwandes bei der Entnahme als die Samenspende, aber das genügt hier nicht, zumal weniger invasive Methoden wie das egg-sharing zur Verfügung stehen und die Eingriffe mit Zustimmung der Betroffenen stattfinden. Die angeblich drohenden Identitätskonflikte des Kindes und Konflikte zwischen sozialer und biologischer Mutter stellen im Vergleich zur Parallelproblematik bei Vätern in keiner Weise derartige zwingende, allein biologisch fundierte Rechtfertigungsgründe dar, die Eizellspende ist in dieser Hinsicht sogar wegen der Bindung zwischen austragender Mutter und Kind weniger problematisch. ${ }^{86}$

Deswegen wird man im Ergebnis festhalten können, dass allenfalls das Verbot von Leih- und Ersatzmutterschaft zu rechtfertigen ist, während das Verbot der Eizell- und Embryospende gegen das absolute Differenzierungsverbot des Art. 3 Abs. 2 und 3 GG verstößt und deshalb verfassungswidrig ist. ${ }^{87}$

\section{Resümee}

Nach der geltenden Rechtslage sind nur wenige Formen assistierter Reproduktion zulässig. Während homologe Insemination und Fertilisation als unproblematisch gelten, sind heterologe Formen der Fortpflanzungshilfe lediglich hinsichtlich der Samenspende gesetzlich freigegeben. Demgegenüber werden alle Formen einer sog. gespaltenen Mutterschaft zur Überwindung der Infertilität der Frauen unterschiedslos untersagt, von der Eizell- über die Embryospende bis zur Leih- und Ersatzmutterschaft. Dieses undifferenzierte Verbot ist verfassungsrechtlich nicht haltbar. Jedenfalls die Eizellspende und wohl auch die Embryospende sind von Verfassungs wegen nicht zu untersagen. Allenfalls für Leih- und Ersatzmutterschaft wird man dem Gesetzgeber einen Entscheidungsspielraum zubilligen müssen, den er freilich bisher allzu selbstverständlich in Anspruch genommen hat.

\footnotetext{
85 BVerfGE 84, 9 (18); Heun (Fußn. 76), Rdn. 111 m.w.N.

86 Bernat (Fußn. 2), S. 221.

87 Darin ist sich das Schrifttum weitgehend einig vgl. z.B. Zumstein, Keimzellspende - Juristische Thesen, in: Bundesministerium für Gesundheit (Fußn. 79), Fortpflanzungsmedizin in Deutschland, 2001; Coester-Waltjen (Fußn. 15), S. 160; Hieb (Fußn. 2), S. 201.
} 



\title{
So weit gehen für ein Kind: Reproduktionstourismus als grenzüberschreitender Umweg
}

\author{
Eva-Maria Knoll
}

„In contrast to illicit flows across borders (such as alcohol, arms, illegal immigrants) a pregnancy cannot be arrested any more than a person's acquired knowledge. This is the crux of the matter."

- Marit Melhuus (2003)

Im April 2007 wurde Maria M. aus der Steiermark zur ältesten gebärenden Mutter Österreichs und damit zu einer Sensationsmeldung mit Kuriositätswert in diversen Tageszeitungen. ${ }^{1}$ Eine In-vitro Fertilisation (IVF) mit gespendeten Eizellen einer jüngeren Frau verhalf ihr zum späten Mutterglück mit 66 Jahren, lange nach ihrem Klimakterium. Eines Tages wird ihr aufgewecktes, gesundes Mädchen ein Teenager sein - ein Teenager mit einer 80-jährigen Mutter. Für die österreichischen Medien war Maria M. nicht nur als „Omi-Mutti“ von besonderem Interesse, sondern auch als Reisende in Sachen Kinderkriegen. Der Weg zum Wunschkind führte Maria M. ins Ausland, denn die Spende von Eizellen und deren Verwendung ist in Österreich verboten.

Im Frühjahr 2005 wurde Elodie als erstes Schweizer und auch als erstes europäisches „Designer Baby“ zur medialen Berühmtheit. ${ }^{2}$ Ihr sechs Jahre älterer Bru-

\footnotetext{
1 Tageszeitung ÖSTERREICH am 1.4.2007.
}

${ }^{2} \mathrm{NZZ}$ am 29.5.2006; BAZ am 3.6.2006. 
der Noab leidet an einer tödlich verlaufenden Erkrankung des Immunsystems, die ausschließlich in der männlichen Linie vererbt wird. Eine erfolgreiche Knochenmarkspende stellt die letztmögliche Hilfe und seine einzige Überlebenschance dar - eine geeignete Spenderperson konnte aber über Jahre hinweg nicht gefunden werden. Schließlich wandten sich Noabs Eltern an eine US-amerikanische Fertilitätsklinik, die sich auf die Selektion von Spermien spezialisiert hat. In dieser USKlinik wurden aus der Samenflüssigkeit von Noabs Vater nur weibliche Spermien ausgewählt, jene mit XX-Chromosomen, die nicht von der Immunerkrankung betroffen sein können, an der Noab leidet. Diese wurden tiefgefroren und zu einer anderen Fertilitätsklinik nach Brüssel in Belgien verschickt. Die Eizellen von Noahs Mutter wurden in der Brüsseler Klinik mit den aufgetauten weiblichen Spermien seines Vaters befruchtet und entwickelten sich im Brutkasten. Im embryonalen Achtzellstadium wurde jedem der entstandenen Embryonen eine Zelle entnommen, um sie gentechnisch zu untersuchen. Mittels dieser Prä-ImplantationsDiagnostik (PID) wurde jener Embryo ermittelt, dessen Gewebestruktur und genetische Charakteristik hervorragend mit jenen von Noab kompatibel wäre. Der ausgewählte Embryo wurde in den Uterus von Noabs Mutter transferiert, entwickelte sich tatsächlich auch zu einer Schwangerschaft und schließlich wurde Elodie geboren - eine perfekte Knochenmarkspenderin für Noah. Da sowohl die Geschlechtsselektion als auch die PID in der Schweiz verboten sind, haben simple, geradezu alltägliche Reisetechnologien gleichermaßen zu Elodies Entstehung als so genanntes „Rescuer-Baby“ (Retter-Baby) beigetragen, ${ }^{3}$ wie auch hochkomplexe und ethisch-moralisch höchst umstrittene Reproduktionstechnologien. ${ }^{4}$

Die Medienberichte über die österreichische „Omi-Mutti“ Maria M. und über das Schweizer Rescuer-Baby Elodie weisen über ihren medialen Sensationswert hinaus weitere Gemeinsamkeiten auf: Erstens waren diese beiden außergewöhnlichen Geschichten vor der Geburt von Lonise Brown als einem historischen Wendepunkt in der Entstehung menschlichen Lebens undenkbar. ${ }^{5}$ Zweitens haben beide Fälle ethisch-moralische Debatten ausgelöst: um die Abwägung der Wahlfreiheit der künftigen Eltern gegenüber dem Persönlichkeitsrecht und Wohl der

\footnotetext{
${ }^{3}$ Deech/Smajdor, Saviour Siblings, Designer Babies, and Sex Selection, in: Deech/Smajdor (eds.), From IVF to Immortality. Controversy in the Era of Reproductive Technology, 2007, pp. $53 \mathrm{ff}$.

${ }^{4}$ Einen ausgezeichneten aktuellen und Beispiel bezogenen Überblick zu den umstrittenen Segnungen der Reproduktionstechnologie, zu den diesbezüglichen ethisch-moralischen Reibflächen und zu den zentralsten Kontroversen geben: Deech/Smajdor, From IVF to Immortality. Controversy in the Era of Reproductive Technology, 2007.

${ }^{5}$ Die Sozialanthropologin Sarah Franklin bezeichnete Louise Brown als "historical watershed" und betonte mit Verweis auf die Arbeiten von Strathern, After nature. English kinship in the late twentieth century, 1995 (1992) und Strathern, Reproducing the Future. Essays on anthropology, kinship and the new reproductive technologies, 1992, dass natürliche Fakten im westlichen Denken das Fundament für andere Bedeutungen darstellen (wie etwa Sexualität für Verwandtschaftsbeziehungen) und dass die In-vitro-Fertilisation (IVF) dieses Fundament und die anhängigen nachhaltigen Bedeutungen verändert hat. Mit der Geburt von Louise 1978 ist nicht nur die revolutionäre IVF-Innovation als Zugriff und Gestaltbarkeit menschlichen Lebens verbunden, sondern ebenso ein tiefgreifender sozialer Wandel Franklin, Konferenz „IVF as a Global Form“, Humboldt-Universität Berlin, 2008.
} 
künftigen Kinder etwa, oder um die altersspezifische „Natürlichkeit“ von Mutterschaft, um ein Menschenrecht auf Fortpflanzung und Elternschaft sowie um Menschen nach $\mathrm{Maß}$ und zu Heilmitteln instrumentalisierte Kinder. ${ }^{6}$ Drittens verbindet die Geschichten von Maria M. und Elodie ein zentrales Moment von Mobilität. Die postmenopausale Mutter und das Rescuer-Baby entstanden durch fortpflanzungsorientierte Reisetätigkeiten, die als grenzüberschreitende Umwege zum Kind im Zentrum dieses Beitrags stehen sollen. ${ }^{7}$

\section{Eigenwillige Formen touristischer Beweglichkeit}

Eine gesteigerte Mobilität und die weltweite Verfügbarkeit von Technologien sind die wahrscheinlich markantesten Eckpfeiler der Globalisierung. ${ }^{8}$ Durch die viel zitierte Raum-Zeit-Kompression erscheinen die Weiten des Globus als enger zusammengerückt: Große geographische Entfernungen werden in wenigen Flugstunden überwunden und Menschen sind allerorts in Bewegung: als Migranten ${ }^{9}$ und Flüchtlinge, als Konferenzbesucher und Forscher - und natürlich auch als Touristen. Das Mobilitätsmoment unserer global vernetzen Welt hat in den letzten Jahrzehnten eine bunte Vielzahl von z.T. sehr eigenwilligen Formen von Tourismus hervorgebracht: Neben Pauschal-, Individual-, Low-Budget-, Club-, Abenteuer-, Städte- oder sanftem Tourismus wurde 2001 mit dem ersten zahlenden Milliardär Dennis Tito der Weltraumtourismus etabliert. Kriegsschauplätze, Erdbeben- und Tsunamiregionen wurden und werden vom so genannten „dark tourism“, den dunklen Seiten des Tourismus heimgesucht, vom Gewalt und Katastrophentourismus.

Fortpflanzungstourismus kann meiner Ansicht nach diesen „eigenwilligen“ Formen von zielgerichteter und organisierter touristischer Beweglichkeit zugerechnet werden. Zielgerichtet meint dabei jene Tourismusformen, die auf einen persönlichen Vorteil hin ausgerichtet sind: Im Juni 2008 wurde beispielsweise durch eine Entscheidung des Europäischen Gerichtshofs der Führerscheintourismus unterbunden, der bis dahin Verkehrssündige zum Erwerb einer Fahrerlaubnis ins benach-

\footnotetext{
${ }^{6}$ Zur ethischen Debatte um postmenopausale Schwangerschaften siehe Pennings, Postmenopausal Women and the Right of Access to Oocyte Donation, Journal of Applied Philosophy 2001/2, 171 ff.; zu jener um Retter-Babies siehe Pennings/Liebaers, Creating a child to save another: HLA matching of siblings through Preimplantation Genetic Diagnosis, in: Shenfield/Sureau (eds.), Ethical Dilemmas in Reproduction, 2002, pp. 51 ff.; Pennings, Saviour Siblings: Using Preimplantation Genetic Diagnosis for Tissue Typing, International Congress Series 2004/1266, pp. $311 \mathrm{ff}$.

${ }^{7}$ Nicht nur Omi-Mütter und Retter-Babies erwecken das mediale Interesse, auch über Reproduktionstourismus an sich wird sehr gerne berichtet (v.a. um die Dimension der Gesetzesumgehung). Einige Journalisten haben sich sogar auf das Thema Reproduktionstourismus spezialisiert, wie etwa Martin Spiewak, der mehrfach zur Thematik in Die Zeit publizierte oder Helen Briggs in BBC News.

8 Kreff/Knoll/Gingrich, Handbuch Globalisierung. Sozialanthropologische und sozialwissenschaftliche Zugänge zur Praxis, 2009, derzeit in Druck.

${ }_{9}$ Sofern im nachfolgenden Text der generische Maskulin Verwendung findet, so ist bei Entsprechung stets auch die weibliche Form inkludiert. Eine durchgehend geschlechtersensible Schreibweise war aus redaktionellen Gründen leider nicht möglich.
} 
barte Ausland führte, nachdem ihnen diese in ihrem Heimatland entzogen wurde. Mit Blick auf das Auto ist in Österreich immer wieder der Tanktourismus im Gespräch, dessen Ziel es ist, das Auto jenseits der Staatsgrenze kostengünstiger mit Treibstoff zu füllen: in der Tschechischen Republik etwa, oder in der Slowakei, in Ungarn oder in Slowenien. Jüngsten Zeitungsmeldungen zufolge schwappt ein sogenannter e-card-Tourismus nach Österreich herein. Dieser Neologismus beschreibt die vorteilsorientierte Mobilität von Menschen aus östlichen Nachbarländern, die sich angeblich mit gestohlenen e-cards (Krankenversicherungskarten) in österreichischen Krankenhäusern und bei niedergelassenen Ärzten gratis behandeln lassen. Österreichische Staatsbürger sind aber auch ihrerseits medizintouristisch mobil. Im grenzüberschreitenden Dentaltourismus besuchen tausende jährlich eine der zahllosen, auf deutschsprachiges Klientel ausgerichteten Zahnkliniken in Ungarn und bezahlen dort bis zu 60\% weniger für ihre Füllungen und Implantate. Im Frühjahr 2008 kam aus aktuellem Anlass der Sterbetourismus zunehmend ins mediale Blickfeld, da sich unheilbar kranke Österreicher und Deutsche immer öfter auf einen letzten Weg begeben, der sie nach Belgien, in die Niederlande oder in die Schweiz führt; in jene Länder, deren Gesetze eine Beihilfe zur Selbsttötung nicht unter Strafe stellen und wo einschlägige Vereine den nötigen Service anbieten.

Unterschiedlichste Formen von Mobilität boomen vor allem im medizinischen Feld, denn der Gesundheitsmarkt entwickelt sich zunehmend von einem nationalen Verteilungssystem, das eine ausreichende, flächendeckende und kostenlose oder zumindest leistbare Versorgung sicherstellen sollte, hin zu einem global orientierten Nachfragesystem. Dieses bedürfnis- und konsumorientierte Nachfragesystem, in dem nicht Basisversorgung, sondern Wahlmöglichkeit im Vordergrund steht, hat eine neue Spezies von Patienten hervorgebracht, schreibt das Magazin Gesundheitswirtschaft am 13.2.2007: die informierten und mobilen „Selbstzahler“, die über die nötigen Mittel verfügen und es verstehen, ein globales Angebot vorteilsorientiert auszuloten und zu nutzen. Im transnationalen Gesundheits-, Spitalsoder Patiententourismus und in vielen weiteren Spielarten solch medizinisch motivierter Reisetätigkeit managen diese mobilen Selbstzahlerpatienten sowohl Angebote als auch Distanzen. Das Internet als wertvolle Informationsquelle und alle erdenklichen Fortbewegungstechnologien, vom Auto über Bus und Bahn bis hin zum Flugzeug, spielen dabei zentrale Rollen. Fortpflanzungstouristen können diesem spezifischen, transnational agierenden Klientel von mobilen Patienten im Medizinbereich zugerechnet werden. ${ }^{10}$

\footnotetext{
${ }^{10}$ Verschiedenartige Technologien werden im Reproduktionstourismus zu dem, was Callon, Elements of a sociology of translation: Domestication of the Scallops and the Fishermen of St Brieuc Bay, in: Law (ed.), Power, Action and Belief: A New Sociology of Knowledge?, 1986, pp. 196 ff.; als "obligatory passage point" bezeichnete. Mit einem solch obligatorischen Korridor oder Durchgang meint Callon eine Knotenposition in einem Netzwerk, an der die meisten Akteure einfach nicht vorbei können. Für die postmenopausale Mutter aus der eingangs erwähnten Geschichte und für die Eltern von Elodie, dem Rescuer-Baby, gab es mehrere Technologie-bezogene obligatorische Passagepunkte: diese beiden Entstehungsgeschichten menschlichen Lebens wären zuallererst ohne die moderne
} 
Fortpflanzungstourismus als eigenwillige Form touristischer Beweglichkeit ist aber nicht nur auf einen Vorteil hin zielorientiert, sondern er ist auch organisiert. Das Ausmaß dieses stillen und höchst mobilen Geschäftszweiges und auch dessen Umsätze und Gewinne lassen sich nicht beziffern oder auch nur abschätzen, was eine Beforschung und Einschätzung des Phänomens nicht einfach macht. Gesichert ist jedoch, dass es - wie in jeder anderen Spielart des modernen Tourismus auch - ein entsprechendes Angebot für die vorhandene Nachfrage grenzüberschreitender Serviceleistungen im Fortpflanzungsmedizinbereich gibt und auch spezifische Umschlagplätž, wie etwa das Internet, wo sich Angebot und Nachfrage treffen.

\section{IVF-Holiday - Pauschalangebote für den zugfreudigen Klapperstorch}

Es gibt bereits erste reproduktionsmedizinische Pauschalangebote, was eine zunehmende Verschränkung der beiden Dienstleistungssegmente Tourismus und Fertilitätsbehandlung anzeigt. Einschlägige IVF-Reise-Pakete umfassen neben der eigentlichen repromedizinischen Behandlung die Organisation der Reise und nach Bedarf auch die Abwicklung von Visaprozeduren. Auch sind oft eine Betreuung vor Ort in der Landessprache der Patienten sowie das Angebot oder zumindest die Auskunft über touristische Highlights der Zieldestination in Form von Kulturangeboten und/oder Bademöglichkeiten inkludiert. Reproduktionsmedizinische Pauschalpakete werden (noch) nicht von Reiseagenturen zusammengestellt und in Katalogform angeboten wie im regulären Pauschaltourismus, sondern bislang direkt von Fertilitätskliniken geschnürt und offeriert, oder das Angebot steht zumindest in einer direkten und engen Beziehung zu einer spezifischen Klinik, was die folgenden drei Beispiele zeigen:

Das „Barbados Fertility Center“" vermarktet gezielt die Verknüpfung einer IVF mit entspannter Urlaubsatmosphäre im luxuriösen Zimmer oder Apartment am Palmenstrand für das Übersee-Klientel wird der Flug aus New York, Miami, London oder Toronto als „IVF holiday package“ angeboten, dazu gehören die 14-tägige Unterbringung im Hotel der Wahl mit hauseigenem Strand, die Transfers vom und zum Flughafen und auch jene zu den Behandlungsterminen in der IVF-Klinik sowie ein Mobiltelefon, damit die Klinik jederzeit kostenlos kontaktiert werden kann. ${ }^{11}$ Die Pauschalangebote sind preisgestaffelt als Comfort-, Luxus-, Premiumoder Elite-Paket und offerieren eine Bandbreite, die vom inkludierten Economy bis hin zum Erste-Klasse-Flug und vom self-catering- bis hin zum Luxus-Hotel reicht und darüber hinaus in den höchsten Preisklassen auch Akupunktur, Massage, Yoga und weitere Wellness-Angebote umfasst. Nur um die Reiseversicherung

Reproduktionstechnologie nicht möglich gewesen, aber darüber hinaus wären sie ohne Informationsund Reisetechnologien nicht realisierbar gewesen.

11 Siehe www.barbadosivf.org/holidays.htm (letzter Zugriff: 10.9.2008). 
müssen sich die Patienten/Konsumenten selbst kümmern, wenn sie eines dieser „IVF holiday packages“ direkt bei der karibischen Fertilitätsklinik buchen.

Ein anderes Beispiel einer repro-touristischen Rundumversorgung ist das kleine Unternehmen „IVF Travel Solutions“, das seine Dienste Fortpflanzungstouristen aus Großbritannien mit der Zieldestination CERAM Klinik in Marbella, Spanien anbietet. ${ }^{12}$ Gegründet wurde das hoch spezialisierte Nischenunternehmen vom Ehemann einer Angestellten dieser Fertilitätsklinik mit dem Ziel, die Fortpflanzungsreise so angenehm und stressfrei wie nur möglich zu gestalten und damit zu einem besseren Behandlungsergebnis beizutragen. "Less stress equals more success", besagt das Firmenmotto; umgesetzt wird es in Form einer Rundumbetreuung: Die Flüge werden von IVF Travel Solutions recherchiert und gebucht, ein Britischer Chauffeur (besagter Ehemann) holt die Britischen Reprotouristen vom Flugplatz ab, steht für die Fahrten zwischen Hotel und Klinik bereit und chauffiert die Patienten darüber hinaus zu guten Restaurants und touristisch interessanten Plätzen der Umgebung.

Das dritte Beispiel der touristischen Angebotseite für ungewollt Kinderlose ist „IVF-holiday“. Dieses Unternehmen bietet mit dem Slogan „eine Reise zu neuem Leben" seit 2007 Hotelbuchungen für Patienten an, die Fertilitätskliniken in Bregenz und Salzburg (Österreich) sowie in den beiden grenznahen Städten Plzeň und Karlovy Vary (Pilsen und Karlsbad in der Tschechischen Republik) aufsuchen. Die in rosa und blauen Pastelltönen gehaltene Homepage hat eine zweiteilige Seitengestaltung: Ein Teil ist einem ärztlichen Ratschlag in Form einer persönlich gehaltenen Mitteilung des jeweiligen Klinikleiters vorbehalten, der zweite Teil behandelt Wissenswertes aus Geschichte und Gegenwart der jeweiligen Stadt. ${ }^{13}$ IVF-holiday wurde gegründet, um das Personal einer Bregenzer IVF-Klinik von den zahlreichen Anfragen nach empfehlenswerten Unterkünften in der Umgebung zu entlasten und die Patienten vom Organisationsstress. Der Buchungsservice wird vor allem von Repro-Touristen beansprucht, die einen längeren Anfahrtsweg mit dem eigenen PKW haben, was aufgrund der allgegenwärtigen Staugefahr die Einhaltung der vereinbarten Kliniktermine und damit letztlich auch die gesamte Behandlung gefährden könnte. Der Service von IVF-holiday wird den Patienten nicht nur kostenlos offeriert, diese bezahlen für die Nächtigungen sogar auch noch weniger als bei einer direkten Buchung, da IVF-holiday über die im Tourismusgeschäft üblichen Sonderkonditionen verfügt.

Es gibt also erste Geschäftsideen zur Verschränkung von Tourismus und Fertilitätsbehandlung, aber es gibt keine Katalogangebote für reproduktive Pauschalreisen, keinen Massentourismus in diesem Bereich und keine last-minute-Schnäppchen. Ganz im Gegenteil: Fortpflanzungstourismus ist zumeist ein geheimes und tabuisiertes Unterfangen. Ungewollte Kinderlosigkeit ist ein schmerzliches Tabuthema, das oft nicht einmal im engsten Familien- und Freundeskreis angesprochen

\footnotetext{
12 Siehe www.ivftravelsolutions.com (letzter Zugriff: 10.9.2008).

${ }^{13}$ Siehe www.ivf-holiday.de (letzter Zugriff: 10.9.2008).
} 
wird. Kommt noch eine Zeugungsreise hinzu, vielleicht gar auch Behandlungsformen, die im Heimatland gesetzlich verboten und moralisch umstritten sind, so potenziert sich dieses Tabu. Demgemäß bezeichnet Martin Spiewak die ,grenzüberschreitende Zeugungshilfe“ als „ein stilles Geschäft, das weder Patienten noch Ärzte publik machen". ${ }^{14}$ Dennoch unterscheidet sich die Tabuisierung des Fortpflanzungstourismus erheblich von etwa jener des Abtreibungstourismus. Gerade die beispielhaft aufgezeigten repromedizinischen Pauschalangebote der IVF-Klinik auf Barbados, von IVF Travel Solutions oder von IVF-holiday bringen „ein gewisses Mehr" an Service, Kulturangebot, Entspannung oder Badeurlaub in die Fortpflanzungsreise ein. Mit der semi-Professionalisierung organisierter Pauschalangebote tritt der Fortpflanzungstourismus zunehmend aus einem tabuisierten Graubereich heraus und wird vom Kuriosum mit medialem Sensationswert zu einem seriösen und verbindenden Segment des ökonomisch durchaus bedeutsamen Geschäfts mit der Hoffnung und der Tourismuswirtschaft. ${ }^{15} \mathrm{Da}$ das pauschalierte Repro-Reise-Angebot aber noch sehr wenig entwickelt und nur vereinzelt vorhanden ist, dominieren nach wie vor die mobilen Selbstzahler den Reproduktionstourismus. Sie vergleichen Kliniken und Flüge und nehmen die Terminorganisation und Buchung in die eigene Hand, sie organisieren sich eine zur Klinik verkehrsgünstig gelegene Unterkunft und bereichern ihren Repro-trip oft auch mit ein wenig Sightseeing, mit Städte- oder Strandurlaub in Eigeninitiative. Schließlich fressen reproduktionsmedizinische Behandlungen im fernen Ausland auch schnell den gesetzlichen Jahresurlaub auf, sodass eine kreative Verbindung von Urlaub und Behandlung nahe liegt. ${ }^{16}$

\section{Wie soll es heißen?}

Das relativ rezente Phänomen mobiler Selbstzahler, die außerhalb der eigenen Nationalstaatlichkeit medizinische Fertilitätsbehandlungen durchführen, wurde in wissenschaftlichen Vorträgen und Publikationen sowie im Medienbereich zwar immer wieder im Nahbereich des Gesundheits-, Patienten-, oder Medizintourismus verortet, aber mit unterschiedlichsten Termini bezeichnet: Der reproduktive Grenzübertritt wurde etwa als Reproduktions-, Fertilitäts- oder Fortpflanzungstourismus bezeichnet; als Befruchtungs-, Schwangerschafts-, Baby-, Kinderwunsch- oder gar Wunschkindtourismus; oder aber als transnationales Baby shopping, als outsourcing Geschäft, als medizinische Pilgerreise oder repromedizinische

\footnotetext{
14 Spiewak, Schwanger um jeden Preis. Im Ausland kaufen deutsche Paare Eizellen und lassen Embryonen testen. Fortpflanzungstouristen umgehen die strengen deutschen Gesetze, Die Zeit 2002/20, 35.

15 Spar, The Baby Business. How money, science, and politics drive the commerce on conception, 2006.

${ }^{16}$ Knoll, Rituale der künstlichen Befruchtung: Dimensionen eines Erfolgsdiskurses und dessen Schattenseiten, in: Sauer/Knoll (Hrsg.), Ritualisierungen von Geschlecht, 2006, S. 69 ff.
} 
Odyssee, als IVF-trafficking oder reproduktives Globetrotten, als grenzüberschreitende Fortpflanzungshilfe und als therapeutischer Transnationalismus oder gar als biomedizinischer Grenzverkehr in ein gewähltes Fortpflanzungsexil. Diese eigenwillige Form von Patienten-Mobilität wurde auch als moralischer Pluralismus in Bewegung und als Export ethischer Dilemmata angesehen und folglich als Sicherheitsventil, das erst ein friedliches Zusammenleben im Angesicht dieser ethischen Dilemmata ermöglicht.

Im Laufe meiner mehrjährigen Befassung mit der Thematik Reproduktionstourismus begegneten mir all diese Bezeichnungen in deutscher und englischer Sprache in Büchern, Journalen, Zeitungen, Filmen, Broschüren und auf Internetseiten. Der Reproduktionstourismus ist aber nicht nur hinsichtlich seiner Bezeichnungen und Beschreibungen von einer bestechenden Vielfalt gekennzeichnet, vielmehr spiegelt diese Heterogenität bloß die Komplexität des Phänomens wieder: auch die Reisetätigkeit selbst, sowie die Motivationen, Ziele, Perspektiven, Strategien und Praktiken der unterschiedlichsten involvierten Akteure im grenzüberschreitenden Geschäft mit der Hoffnung, zeichnen sich durch Mannigfaltigkeit, Kreativität, Planung und Improvisation aus. Eine griffige Bezeichnung und eine hinlänglich weitreichende Definition, die der perspektivenreichen und schon fast Kaleidoskop-artigen Vielfalt und Unterschiedlichkeit reproduktiver Reisetätigkeit gerecht werden, sind tatsächlich nur schwer zu entwickeln.

\section{Reproduktives Globetrotten - Gesetzesumgehung und mehr}

In der journalistischen Aufarbeitung, aber auch in Analysen und Ansätzen der Theoriebildung zum Reproduktionstourismus wird zumeist das Moment einer Gesetzesumgehung in den Vordergrund gestellt. ${ }^{17}$ Das hat einerseits disziplinäre Hintergründe, denn zuallererst wurden neben dem Sensationsjournalismus vor allem Juristen, Rechtswissenschafter und Ethiker auf die Praxis der Umgehung restriktiver, einschränkender oder nachteiliger gesetzlicher Rahmenbedingung für die Nutzung von Reproduktionstechnologien mittels Grenzübertritt aufmerksam. ${ }^{18}$ Mit einem dementsprechenden Fokus entwickelte der Bioethiker Guido Pennings sein Konzept des Fortpflanzungstourismus als einem „moralischen Pluralismus in Bewegung“, der zwischen dem „Erlaubt“ und „Verboten“ in einer moralischen Landschaft navigiert und eigentlich eine Art Sicherheitsventil darstellt ${ }^{19}$, das letztlich moralische Konflikte reduziert und damit einen Beitrag zur Anerkennung von

\footnotetext{
17 Spiewak, Dann gehen wir eben ins Ausland. In Deutschland verbotene Methoden, in: Spiewak (Hrsg.), Wie weit gehen wir für ein Kind? Im Labyrinth der Fortpflanzungsmedizin, 2002, S. 147 ff.

${ }^{18}$ Koch, Fortpflanzungsmedizin im europäischen Rechtsvergleich, Politik und Zeitgeschichte 2001/27, 44 ff.; Benöhr-Laqueur, Sollten Frauen jenseits der Menopause Mutter werden können? Eine rechtspolitische Analyse, Die Hebamme 2008, derzeit in Druck.

19 Pennings, Reproductive Tourism as Moral Pluralism in Motion, Journal of Medical Ethics 2002/28, $337 \mathrm{ff}$.
} 
moralischer Autonomie leistet. ${ }^{20}$ Andererseits stellt Reproduktionstourismus als Phänomen, in dem die Patienten die Dinge einfach selbst in die Hand nehmen, eine gewisse Bedrohung des Einflusses der nationalen Rechtstaatlichkeit dar, handelt es sich doch mehr oder weniger um ein informelles ökonomisches Segment, das in den Randzonen der Legalität operiert, wenn hierzulande per Gesetz verbotene Behandlungsformen wenige Kilometer weiter, jenseits der Landesgrenze durchgeführt werden. Dementsprechend stellt Stefan Beck fest, dass die Frage nach den realen Zahlen im Reproduktionstourismus nicht beantwortbar, aber eigentlich auch nebensächlich sei, denn viel interessanter ist, welch große Irritation und Bedrohung für den Rechts- und Nationalstaat die legislativen Umwege der mobilen Selbstzahler zum eigenen Kind offensichtlich darstellt. ${ }^{21}$

Die Ansicht, dass Reproduktionstourismus zuallererst eine legislative Umgehungsstrategie sei, ist aber auch aus einem weiteren Grund durchaus legitim: Bei ungewollt Kinderlosen, die häufig einen bereits mehrjährigen, aber fruchtlosen Leidensweg assistierter Reproduktion hinter sich haben, stellt sich rasch ein schmerzliches Gefühl von nicht nachvollziehbarer Ungerechtigkeit ein, wenn vielversprechende Behandlungsmöglichkeiten für eine lang ersehnte Schwangerschaft hierzulande verboten, aber nur wenige Auto- oder Flugstunden entfernt erlaubt sind. Viele ungewollt Kinderlose erleben die jeweiligen gesetzlichen Rahmenbedingungen für Fruchtbarkeitsbehandlungen als skandalöse und schikanöse Ungerechtigkeit. Für diese Klientel lebt die Hoffnung auf eine Schwangerschaft und auf ein Kind daher gleich hinter dem nächsten Grenzbalken weiter. Bis zum tatsächlichen Grenzübertritt ist es dann oft nur mehr ein kleiner Schritt, sind Grenzbalken innerhalb der Europäischen Union doch einerseits für den freien Personenverkehr immer unbedeutsamer geworden, markieren aber andererseits in der Fortpflanzungsmedizin eine tiefe, jedoch nicht unüberwindbare Kluft zwischen reproduktiver Option und auswegsloser Exklusion.

Die Umgehung gesetzlicher Einschränkungen der assistierten Fortpflanzung bringt als Motivationsfaktor folglich sicher eine große Zahl - vielleicht sogar eine deutliche Mehrheit - der reproduktiven Globetrotter hervor. Den Aspekt der rechtlichen Umgehung hervorzustreichen, ist daher durchaus notwendig, wichtig und richtig; als alleinige Erklärung für das Phänomen Fortpflanzungstourismus ist diese Motivation aber nicht hinreichend. Denn Fortpflanzungstouristen überwinden nicht nur juristische Grenzen und Barrieren, sie begeben sich darüber hinaus auch durchaus aus ganz anderen Gründen auf eine repromedizinische Reise.

\footnotetext{
20 Pennings, Legal Harmonization and Reproductive Tourism in Europe, Human Reproduction 2004/19, $2689 \mathrm{ff}$.

${ }^{21}$ Beck, Globalisierte Reproduktionsregimes: Anmerkungen zur Emergenz biopolitischer Handlungsräume, in: Beck/Cil/Hess/Klotz/Knecht (Hrsg.), Verwandtschaft machen. Reproduktionsmedizin und Adoption in Deutschland und der Türkei, Berliner Blätter. Ethnographische und ethnologische Beiträge 2007/42, 124 ff. (129).
} 


\section{UMdeinetWEGen gewünschtes Kind - zur Vielfalt von Grenz-Überschreitungen}

In der Verquickung von Reproduktionstechnologien und touristischen Aktivitäten werden neben den besonders augenscheinlichen Gesetzesumgehungen eine ganze Reihe von weiteren Grenzen und Limits überschritten sowie Begrenzungen und Barrieren überwunden. Das, was Charis Thompson als „ontologische Choreographie“ der assistierten Reproduktion bezeichnet, die technologische, rechtliche, moralische, verwandtschaftliche, geschlechtsspezifische, emotionale, politische und finanzielle Aspekte koordiniert und orchestriert, erstreckt sich im Fortpflanzungstourismus über verschiedenartige Grenzen, Hürden und Distanzen hinweg: Die mobilen Selbstzahler der grenzüberschreitenden assistierten Reproduktion überbrücken erstens geographische Distanz̧en, wobei eine Repro-Reise sehr unterschiedliche Reichweiten aufweisen kann: Sie kann den nächstgelegenen Grenzübergang ansteuern und in die Fertilitätsklinik eines benachbarten Staates führen, die nur wenige Kilometer und Autostunden entfernt ist. ${ }^{22}$

Oft sind es Fertilitätskliniken selbst, die eine geographische Nähe zu Nachbarländern geschickt nutzen und über die Gründung von Partnerkliniken jenseits der Staatsgrenze ein bi- oder gar multi-nationales, regionales Netzwerk von Kliniken etablieren. Als österreichische Beispiele dafür können das „Kinderwunschzentrum der Privatklinik Goldenes Kreuz“ im Osten und das „Institut für Reproduktionsmedizin und Endokrinologie“ im Westen des Landes angeführt werden: Das Kinderwunschzentrum in Wien hat mit der IVF Klinik „Ferticent Bratislava“ eine Tochterklinik in der Slowakei eingerichtet. Die beiden Kliniken sind nur 70 Kilometer von einander entfernt und verweisen auf ihren Homepages prominent auf die jeweils andere Institution. Per Mausklick kann zwischen zwei verschiedenen rechtlichen Rahmenbedingungen und zwischen zwei unterschiedlichen Preisniveaus für IVF Behandlungen gewechselt werden. ${ }^{23}$ Die Homepage der Wiener Klinik wird in Deutsch und Englisch angeboten, jene der Klinik in Bratislava in Slowakisch, Deutsch und Englisch, was Rückschlüsse auf einen Patientenstrom zulässt, der eher von West nach Ost als umgekehrt verläuft.

Das Institut für Reproduktionsmedizin und Endokrinologie in Bregenz wurde 1985 gegründet und zählt heute mit sechs Tochterkliniken in Salzburg, Nideruzwil (Schweiz), Meran (Italien), Pilsen (Tschechische Republik) und München (Deutschland) zu den größten Kliniknetzwerken in Europa (eine siebente Klinik in Brüssel (Belgien) ist im Entstehen). ${ }^{24}$ Die Homepage umfasst alle Institute, wird in

\footnotetext{
22 Thompson, Making Parents. The Ontological Choreography of Reproductive Technologies, 2005.

${ }^{23}$ vgl. www.kinderwunschzentrum.com (letzter Zugriff: 10.9.2008).

24 Beck, in: Beck/Cil/Hess/Klotz/Knecht (Fußn. 21); hat die unterschiedlichen Geschäftsmodelle transnationaler Kooperationen und Klinikgründen am Beispiel dreier türkischer Kliniken mit Verbindungslinien zu Deutschland als ,joint reproductive ventures“ und als „knowlege franchising“ analysiert. Ich habe mit dem Argument, dass Fortpflanzungstourismus nicht nur durch die Mobilität von Patienten charakterisiert ist, sondern ebenso durch die expandierende Mobilität von Kliniken und von ambitionierten medizinischen Persönlichkeiten, ein Beispiel grenzüberschreitender, österrei-
} 
sechs Sprachen angeboten und enthält neben einer Fülle verschiedenartigster Information auch Straßenkarten mit den markierten Anfahrtswegen und einen prominent platzierten Linkbutton zu „IVF-holiday“, jener zuvor diskutierten touristischen Einrichtung, die zur Entlastung des Klinikpersonals auf Initiative des Leiters gegründet wurde. Bei beiden fertilitätsklinischen Netzwerken werden eine regionalgeographische Nähe, ein Rechts- und Kostenpluralismus sowie der hohe Stellenwert des Autos als Fortbewegungsmittel geschickt zu einer äußerst erfolgreichen Geschäftsstrategie gebündelt. ${ }^{25}$

Neben einem solch regional orientierten Grenzverkehr, in dem die mobilen reproduktiven Selbstzahler meist auch zu Selbstfahrern im eigenen PKW werden und in dem nur geringe geographische Distanzen zu überwinden sind, nehmen andere ungewollt Kinderlose für eine Behandlung einige Flugstunden in Kauf und wieder andere bereisen durchaus auch einen anderen Kontinent: Solch reproduktive Langstreckenflüge bringen Europäer in die USA, das Land der nahezu unbegrenzten Repro-möglichkeiten, oder aus Kostengründen wird die entgegengesetzte Richtung eingeschlagen; eine Klinik in Südafrika hat sich auf deutschsprachiges Klientel spezialisiert und israelische Frauen reisen nach Zypern, um Eizellen zu empfangen, die jüngeren, aus Russland oder der Ukraine eingeflogen Frauen entnommen wurden.

$\mathrm{Ob}$ sie nun kürzere Distanzen mittels Auto oder Langstreckenflüge absolvieren, Reproduktionstouristen überschreiten zweitens immer auch Landesgrenzen, also geo-politische Grenzen von Nationalstaaten. Sie wechseln von einer nationalstaatlichen Souveränität in eine andere, mit mehr oder weniger ausgeprägten Formen von Grenzbalken, Pass- und Zollkontrollen sowie Einreisebestimmungen.

Drittens übertreten diese Reisenden in Sachen Nachwuchs auch moralische Grenzen. Sie durchstreifen eine moralische Landschaft, die von grundlegenden Fragen sowie von Konsens und Konflikt in Beantwortung dieser Fragen gezeichnet ist: Was macht „richtige Eltern“ aus? Wer darf und soll sich vermehren, und wer nicht? Wie sind die Interessen der künftigen Eltern gegenüber denen ihrer künftigen Kinder abzuwägen? Welche Art von Forschung und Behandlung darf die Medizin durchführen, und in welchen Bereichen soll aber nicht alles, was technisch möglich wäre, auch tatsächlich getan werden? Akteure des Reproduktionstourismus der Nachfrage- und auch der Angebotseite navigieren in dieser moralischen Landschaft, die aus umstrittenen Themen, kontroversen Ansichten und Interessenskonflikten zusammengewürfelt ist. Die Reibungsflächen reichen von geteilter,

chisch-ungarischer Kooperation im IVF Bereich diskutiert. Knoll, Transnationale AkteurInnen im Geschäft mit der Hoffnung. Österreichische Momente globaler Verflechtungen der In-VitroFertilisation, in: Riegler (Hrsg.), Kulturelle Dynamik der Globalisierung. Ost- und Westeuropäische Transformationsprozesse aus sozialanthropologischer Perspektive, 2005, S. $203 \mathrm{ff}$.

25 Der besondere Faktor Auto im regional-medizinischen Grenzverkehr wird von Sven Bergmanns Untersuchungen bestätigt. Er arbeitet vergleichend zur grenzüberschreitenden Eizellenspende in Spanien und der Tschechischen Republik. Deutsche Paare, die zur Behandlung in eine Klinik in der Tschechische Republik fuhren, gaben meist als Begründung für ihre Klinikwahl an, dass sie hierhin mit dem Auto fahren können (persönliche Mitteilung 15.6.2008). 
postmenopausaler und Leih-Mutterschaft, über die Kryokonservierung, Spende und Adoption von Embryonen sowie deren Vernichtung durch Forschungsaktivitäten bis hin zur Frage, ob sich nur verheiratete, heterosexuelle Paare im richtigen Alter, mit der richtigen Staatsbürgerschaft und mit gutem finanziellen Background, aber ohne Erbkrankheiten im Stammbaum einer künstlichen Befruchtung unterziehen dürfen, oder auch alleinstehende, schwule, lesbische, und transgender Personen; Schwerkranke, Behinderte, Arbeitslose, Migranten und Asylanten ${ }^{26}$ Soll den künftigen Kindern das Recht auf Kenntnis ihrer biologischen und genetischen Abstammung zugesichert werden, und sollen Gameten und Embryonen nur an ungewollt Kinderlose gespendet werden, oder sollen diese auch für die wissenschaftliche Forschung genutzt werden? Wenn Eizellen, Samenzellen und Embryonen der Forschung zur Verfügung gestellt werden, sollen die wissenschaftlichen Experimente dann vorrangig dazu dienen, die Erfolgsraten von IVF-Behandlungen zu erhöhen, oder auch um an der Behandlung und Heilung verbreiteter Alterserkrankungen zu forschen wie etwa Alzheimer oder Parkinson? Oder sollen diese Substanzen auch für Experimente der Klon-, Ektogenese- und Chimärenforschung $^{27}$ herangezogen werden dürfen? Dreißig Jahre nach der Geburt von Lonise Brown hat sich der Einsatzbereich der IVF vervielfacht, und sie wurde zur unabdingbaren Basistechnologie vieler ethisch umstrittener Hoffnungs- und Innovationsbereiche, wie etwa der Stammzellenforschung, und damit auch zu einem wesentlichen Investitionsfaktor.

Die moralische Landschaft der Reproduktionsmedizin wurde somit immer stärker zu einer ökonomischen. Neben dem Anwendungsbereich hat sich auch das Spektrum der Technologie-Nutzer ausgeweitet. Die jeweiligen moralischen Normen und Wertvorstellungen spielen eine zentrale Rolle bei der Ausgestaltung des Handlungsspielraums, der festlegt, welche Anwendungsgebiete der assistierten Fortpflanzung, unter welchen Voraussetzungen und Rahmenbedingungen für welche Personen zugänglich sein sollen. Damit stehen die moralischen Normen und Wertvorstellungen einer Gesellschaft in eng verzahnter Wechselwirkung mit gesetzlichen und auch mit religiösen Grenzen. Im Reproduktionstourismus werden viertens diese Grenzen des „Erlaubt" versus „Verboten“ überschritten, seien diese nun rechtlich in

\footnotetext{
26 Single Wunschmamas als weibliche Fortpflanzungstouristinnen habe ich diskutiert in: Knoll, Fortpflanzungsmedizin ohne Sexualität als gesellschaftliche Irritation: Diskurse über „JungfrauenGeburten“, in: Pethes/Schicktanz (Hrsg.), Sexualität als Experiment. Identität, Lust und Reproduktion zwischen Science und Fiction, 2008, S. 331 ff.; lesbische Frauen in: Knoll, Wo knutschen die Humanic-Lesben?, Politix 2005/19, 4 ff.; der fortpflanzungsmedizinisch reisende Single Mann wird von de Jong, Intimität auf dem Prüfstand. Neue Verwandtschaftsverhältnisse durch assistierte Reproduktionstechnologien, in: Egli/Tomkowiak (Hrsg.), Intimität, derzeit in Druck, thematisiert.

${ }^{27}$ Im April 2008 wurden in Großbritannien erstmals Embryonen aus einer Kombination von Erbinformationen des Menschen und der Kuh erschaffen. Die Mensch-Tier-Hybride wurden aufgrund der großen Nachfrage nach Stammzellen erzeugt, da tierische im Gegensatz zu menschlichen Eizellen in unbeschränkter Anzahl zur Verfügung stehen. Die Chimären-Stammzellen sollen in der Forschung zur Behandlung schwerer Krankheiten zum Einsatz kommen.
} 
Form von Gesetzen und Richtlinien definiert, oder in Form religiöser Handlungsanleitungen gesetzt. ${ }^{28}$

Eine fünfte Dimension von Grenzen, die im Fortpflanzungstourismus überschritten werden, sind Körpergrenzen. Die IVF hat Eizellen außerhalb der weiblichen Fortpflanzungsorgane verfügbar gemacht, und auch die Befruchtung sowie die ersten Tage der Embryonalentwicklung finden nicht tief im Inneren, sondern auBerhalb des Frauenkörpers statt. Gespendete Eizellen wandern von einem Frauenkörper in einen anderen und etablieren in einer Art von ,kollaborativer Reproduktion“29 auch grenzüberschreitende Verwandtschaftsbande. ${ }^{30}$ Eine fortpflanzungsmedizinische Behandlung überschreitet Körpergrenzen, geht tief unter die Haut, vor allem oder sogar primär unter die begrenzende Haut des Frauenkörpers.

Mit einer Eizellspende erscheint sechstens auch die zeitliche Begrenzung der reproduktiven Lebensphase nicht mehr als unüberwindbar, sondern kann ausgedehnt werden, und die späte, die menopausale oder gar die postmenopausale Schwangerschaft wird zur Option. Eine fundierte Ausbildung und ein erfolgreicher Karriereweg mit einem dementsprechenden Verdienst in jungen Jahren entsprechen den Anforderungen unserer gegenwärtigen Gesellschaft, sodass ein allfälliger Kinderwunsch schon fast auf später verschoben werden muss. Heutzutage erscheint eine Familiengründung geradezu ideal ins „Alter 40 plus“ einer Frau zu passen, wenn sie zufrieden auf einen beruflichen Erfolg zurückblicken kann, gut situiert ist und dem Kind finanziell und auch zeitlich ,etwas bieten“ kann. Soll folglich die geradezu epidemische Ausbreitung dieser Form Lebensstil-bedingter Subfertilität tatsächlich nur der Selbstsüchtigkeit von Frauen zur Last gelegt werden? ${ }^{31}$ Können die realen gesellschaftlichen Lebensbedingungen außer Acht gelassen werden und die medizintechnisch realisierten späten Mutterschaften und zweifelhaften Wettbewerbe hervorbringen? Adriana Iliescu wurde 2005 als damals 66-jährige und älteste Gebärende der Welt zum globalen Medienereignis. Nachdem in den späten 70er und frühen 80er Jahren ein globaler Wettlauf stattfand, in dem sich die verschiedenen Nationen eine gute Platzierung mit ihrem ersten IVF-Baby erhofften,

\footnotetext{
28 Zum Reproduktionstourismus muslimischer Kulturen siehe Inhorn, Reproductive Distuptions and Assisted Reproductive Technologies in the Muslim World, in: Inhorn (ed.), Reproductive Disruptions. Gender, Technology, and Biopolitics in the New Millenium, Fertility, Reproduction and Sexuality, 2007, vol. 11, pp. 183 ff.; Inhorn, Making Muslim Babies: IVF and Gamete Donation in Sunni versus Shi'a Islam, Culture, Medicine and Psychiatry, 2006/4, 427 ff.; Inhorn, Religion and Reproductive Technologies: IVF and Gamete Donation in the Muslim World, Anthropology News 2005/2, 10 ff.; Inhorn, Middle Eastern Masculinities in the Age of New Reproductive Technologies: Male infertility and stigma in egypt and lebanon, Medical Anthropology Quarterly 2004/2, 162 ff.; Inhorn, Local Babies, Global Science. Gender, Religion and In-vitro Fertilization in Egypt, 2003, pp. 114 ff.; Einblicke in reproduktive Reisetätigkeiten im jüdischen Kontext gibt Kabn, Reproducing Jews, A Cultural Account of Assisted Conception in Israel, 2000, pp. $131 \mathrm{ff}$.

${ }^{29}$ Bergmann, Kollaborative Reproduktion \& reproduktiver Tourismus. Kommentierte Bibliographie und Materialsammlung zu Ökonomie(n) von Keimzellen in nationalen und transnationalen Räumen, unveröffentlichte Bibliographie, 2006.

${ }^{30}$ Beck (Fußn. 21).

${ }^{31}$ Deech/Smajdor (Fußn. 4), pp. 81 ff.
} 
ist das internationale Omi-Mutti-Ranking weit weniger mit nationalem Stolz verbunden, sondern hat Dank der Sensationspresse den Status einer traurigen Berühmtheit. Mittlerweile wurde Frau Illiescu als zweifelhafte Rekordhalterin von einer 67 Jährigen abgelöst, und selbst die kleine Alpenrepublik Österreich hat - wie eingangs dargelegt - eine reproduktionstouristische 66-jährige Omi-Mama. Die Überschreitung und Expansion der reproduktiven Lebensphase zeigt die moralischen Konflikte als einen wichtigen Dreh- und Angelpunkt im Fortpflanzungstourismus besonders deutlich. ${ }^{32}$

Mit solchen Schwangerschaften nach der Menopause werden auch die Grenzen klassischer Dichotomien wie Natur/Kultur unscharf und die Frage „ist das noch normal“", steht im Raum. Auch die Grenze zwischen öffentlich/privat wird auf besondere Art brüchig und übertreten: Es mag argumentiert werden, dass es sich um eine völlig private und als solche zu respektierende Angelegenheit handelt, wenn eine Grazerin jenseits der 60 unbedingt ein Kind haben will, ins Ausland fährt und dort sehr viel Geld für eine repromedizinische Behandlung mit einer Eizellenspende bezahlt, die in Österreich verboten ist. Es mag aber auch argumentiert werden, dass es triftige Gründe für dieses Verbot gibt: Nämlich dass für die Spende von Eizellen völlig gesunde Frauen einer hochdosierten Hormonbehandlung und einem invasiven Eingriff unterzogen werden und dass es sich bei den Empfängerinnen um Hochrisikoschwangerschaften und auch um Hochrisikogeburten handelt, die einen längeren Aufenthalt der Neugeborenen auf Neonatalstationen nach sich ziehen können. All diese Kosten hat das österreichische Gesundheitswesen zu tragen, und es betrifft darüber hinaus auch die breitere Öffentlichkeit, wenn sich ein 14-jähriger Teenager um eine 80jährige Mutter zu kümmern hat. Andererseits mag argumentiert werden, dass jedes Neugeborene ein zukünftiger Steuerzahler ist und der Staat und die Gemeinschaft angesichts einer rückläufigen Geburtenrate daher gewiss profitieren, unabhängig vom Alter der Mutter. All das könnte argumentiert werden; auf jeden Fall sollte aber reflektiert werden, dass in dieser ganzen Debatte und medialen Aufregung um altersbezogene reproduktive Grenzüberschreitungen die Frage nach dem Alter der Väter niemals gestellt wird selbst dann nicht, wenn eine reduzierte oder unzureichende Spermienqualität als Ursache der Kinderlosigkeit feststeht. ${ }^{33}$

Eine siebente wesentliche Grenze, die Fortpflanzungstouristen auf ihren weitschweifigen Umwegen zum Kind überwinden, ist die Grenze des Angebots. Dabei geht es nicht nur um das, was in einem Land erlaubt ist und angeboten wird, in einem anderen aber verboten ist und daher im reproduktiven Angebot fehlt. Auch hinsichtlich Standard und Qualität weist der globale Repromedizinmarkt ein höchst ausdifferenziertes Angebot auf, das daher auch zum Anreiz für eine Fertilitätsreise werden kann. ${ }^{34}$ Und selbst das Preis-, Leistungs- und Qualitätsmoment individuel-

32 Pennings (Fußn. 6); Benöbr-Laqueur (Fußn. 18).

${ }^{33}$ Zur sexistischen Betrachtung später Elternschaft vgl. Deech/Smajdor (Fußn. 4), pp. 81 ff.

${ }^{34}$ Siehe etwa die Reiseaktivitäten Russischer Fertilitätspatienten nach Österreich bei Knoll (Fußn. 24). 
ler Kliniken kann gegeneinander abgewogen werden. Manchmal ist es gar eine charismatische Persönlichkeit der Reproduktionsmedizin, die gezielt aufgesucht wird. ${ }^{35}$ In vielen Ländern gibt es darüber hinaus lange Wartelisten für IVF Behandlungen, die verzweifelte Patienten ins Ausland treiben, oder einen Mangel an Samen- und vor allem an Eizellspenderinnen, wie beispielsweise in Großbritannien.

Einige der reproduktiven Globetrotter sind achtens unterwegs, weil sie von attraktiven Preisen angezogen werden. Ein Grenzübertritt kann mitunter bis zu 50\% der Behandlungskosten sparen, und letztlich ist die Durchführung einer assistierten Fortpflanzung immer auch eine Einkommensfrage. Die neue Patienten-Spezies der mobilen Selbstzahler im Gesundheitssektor gehört wohl mehrheitlich einer einkommensstärkeren, gebildeten Mittel- und Oberschicht oder gar der wohlhabenden Elite an - das ist grundsätzlich auch im Bereich der Repro-Reiseklientel so. Es sind aber auch Durchschnittsverdiener unter diesen fortpflanzungswillig Mobilen zu finden, die es mit ihren letzten Ersparnissen in billigere Kliniken Osteuropas zieht, gerade weil sie die Kosten für eine Behandlung im Heimatland noch schwerer auftreiben könnten. Diese Reprotouristen navigieren nicht zwischen „erlaubt“ und „verboten“; sie reisen nicht aus Gründen einer Gesetzesumgehung, sondern sie versuchen die Grenze des Leistbaren zu überwinden. Es gibt aber auch Kliniken, wie jene in Albanien etwa, die von albanischen Arbeitsmigranten frequentiert werden. Diese ungewollt Kinderlosen könnten sich von ihren Hilfsarbeitslöhnen die Behandlung in ihren Migrationsländern nur schwer leisten. ${ }^{36}$

Das albanische Beispiel zeigt eine neunte, ethnische Grenze auf, die im Reproduktionstourismus ebenfalls eine Rolle spielt. Manchmal führt ein fertilitätsbezogener Grenzübertritt nämlich nicht ins Ausland, sondern paradoxerweise „nach Hause“. ${ }^{37}$ Wenn Migranten, Kosmopoliten und flexible Staatsbürger für reproduktive Behandlungszwecke in das Land ihrer ethnischen Herkunft reisen, so hat das einerseits sprachliche Gründe. Denn die assistierte Reproduktion erfordert ein hohes $\mathrm{Ma}$ an compliance, an aktivem Zutun der Patienten zum Behandlungsverlauf, was wiederum vom guten Verständnis der Erklärungen zu komplexen Prozeduren, verschiedenen Medikamenten und einem straffen Zeitablauf abhängig ist. „Zur IVF nach Hause zu fahren" hat aber auch eine ethnische Dimension. Diese Form des Nationalismus war im globalen Wettlauf um frühe Geburtserfolge Ende der 70er bzw. anfangs der 80er Jahre sichtbar und wird heute an der Nachfrage nach

\footnotetext{
35 Die Tatsache, dass zunehmend Reisen „zum absoluten Experten“ eines medizinischen Fachbereichs unternommen werden, oder zur „Koryphäe“ einer spezifischen Erkrankung oder einer speziellen Therapieform, ist ein charakteristisches Merkmal der zunehmenden Ausgestaltung eines Konsumund Nachfrage-orientierten, kompetitiven und global agierenden Medizinmarktes.

${ }^{36}$ Heinrich Boell Foundation, European Biopolitics Conference. Connecting Civil Society - Implementing Basic Values, 17.-19.3.2006 Berlin, Workshop "IVF Trafficking and Egg Cell Donation", Konferenz Dokumentation, 2006.

37 Knoll, Reproducing Hungarians - Reflections on Fuzzy Boundaries in Reproductive Medicine, Vortragsmanuskript zur Konferenz "IVF as Global Form. Ethnographic Knowledge and the Transnationalization of Reproductive Technologies", 12.-14.6.2008, 2008 (Publikation in Vorbereitung).
} 
ethnisch „eigenen“ Gameten oder einer Laborzeugung in einer „eigenen Klinik“ deutlich.

Die Grenze des Wissens ist eine zehnte Dimension repromedizinischer Überschreitungen. Der Wunsch nach Kenntnis einer Spenderidentität oder das genaue Gegenteil, der Wunsch nach absoluter Anonymität, kann als Motivationsfaktor für eine reproduktionsmedizinische Reise fungieren.

Während die Geburt von Louise Brown 1978 sensationell die Grenzen der menschlichen Reproduktionsfähigkeit überschritten hat, werden im gegenwärtigen Fortpflanzungstourismus weitläufige Umwege zum erwünschten Kind in Kauf genommen und eine ganze Reihe weiterer Grenzen und Hindernisse überwunden, was ich mit den hier aufgelisteten zehn Dimensionen der Grenzüberschreitung zu verdeutlichen versucht habe.

\section{Conclusio}

Drei Jahrzehnte nach der IVF-Sensationsgeburt in England ist die assistierte Fortpflanzung nicht nur in nahezu alle Winkel der Welt vorgestoßen, sondern hat auch eine besondere Form von Mobilität hervorgebracht, den vielschichtigen Reproduktionstourismus: ein Mobilitätsphänomen, das dieser Beitrag als zielgerichtete und organisierte touristische Bewegung beschreibt, die Angebote des globalisierten Medizinmarktes vorteilsorientiert auszuloten und zu nutzen vermag. Der Beitrag betont die Schnittflächen der repromedizinischen und touristischen Sphäre und spricht sich daher für die Verwendung des teilweise umstrittenen Begriffs „Reproduktionstourismus" ${ }^{\text {"38 }}$ aus: Erstens wird die zunehmende Verschränkung mit der Tourismuswirtschaft aufgezeigt und zweitens wird argumentiert, dass reproduktionstouristisches Reisen nicht ausschließlich mit der politischen Motivation einer Gesetzesumgehung gleichzusetzen ist. Beim fertilitätsbezogenen Reisen geht es zwar in vielen Fällen durchaus um unterschiedliche Umgehungsstrategien nationaler Rechtsstaatlichkeit, in anderen Fällen geht es jedoch eindeutig über rein rechtliche Bezugspunkte des „erlaubt“ versus „,verboten“ hinaus. Maria M., die OmiMutti aus Österreich hat neben der Verbotsgrenze der Eizellspende auch Staats-, Alters- und ethnische Grenzen überschritten. Der Geburt des Rescuer-Babies Elodie ging auch die Übertretung eugenischer Grenzen voraus. In beiden Fällen wurden nicht nur geographische Distanzen überwunden, sondern auch Körper- und Angebotsgrenzen sowie die moralische Grenze des „erlaubt/verboten“ überschritten. Ein Preisgefälle war für diese beiden Reproreisen nicht die Triebfeder, ist allerdings ein in anderen Fällen relevanter Faktor.

\footnotetext{
38 Zur ablehnenden Diskussion um die Angemessenheit des Begriffs "Reproduktionstourismus" siehe die Argumente von Matorras, Reproductive Exile Versus Reproductive Tourism, Letters to the Editor, Human Reproduction 2005/20, 3571; Pennings, Reply: Reproductive Exile Versus Reproductive Tourism, Letters to the Editor, Human Reproduction 2005/20, 3571 ff.
} 
Reproduktionstourismus kann daher meiner Ansicht nach nicht mit einer bloßen Umgehungsstrategie gleichgesetzt werden, sondern muss breiter gefasst werden.

Die weitreichendste Arbeitsdefinition zum Fortpflanzungstourismus hat bislang die Sozialanthropologin ${ }^{39}$ Marcia Inhorn vorgelegt. Im Kontext ihrer Forschungsarbeiten zu Globalisierung und Reproduktionstourismus in der muslimischen Welt charakterisiert sie Reproduktionstourismus als "travel in the pursuit of assisted reproductive technologies (...), usually from one country to another, hypothetically caused by religious prohibitions, lack of expertise and supplies, safety, discrimination against certain categories of individuals, shortages and waiting lists, and costs". ${ }^{40}$ In Anlehnung an Inhorns Beschreibung möchte ich Reproduktionstourismus vereinfacht als grenzüberschreitende Reisetätigkeit definieren, die darauf abzielt, Reproduktionstechnologien unter anderen Rahmenbedingungen zu nutzen, als sie zu Hause gegeben sind. Die breite Formulierung von „anderen Rahmenbedingungen“ geht über einen bloßen Dualismus von „erlaubt“ versus „verboten“ hinaus, denn beim Fortpflanzungstourismus handelt es sich nicht ausschließlich um ein Rechtssystem, das mit einem Grenzübertritt gewechselt wird. Reproduktives Globtrotting ist zumeist mit weitläufigen Umwegen und vielgestaltigen Grenzüberschreitungen verbunden. Wenn grenzüberschreitende assistierte Reproduktion nicht als bloße „Gesetzesumgehung“ erachtet wird, nicht allein als „klassische(r) Konflikt zwischen Gemeinschaftsethik und individuellen Wünschen, zwischen staatlicher Moral und der privaten Sehnsucht nach einem Kind, der durch den sich globalisierenden Medizinmarkt nun noch verstärkt wird“"41 sondern auch als touristische Bewegung, die nicht nur das Verbotene umgeht und das Erlaubte in der Ferne sucht, dann erweitert sich der Blick für einen Facettenreichtum von Motivationen, Strategien und Praktiken.

\footnotetext{
${ }^{39}$ In Deutschland wird diese Wissenschaftsdisziplin mit dem Begriff „Ethnologie“ bezeichnet. Einige Institutionen in Österreich und der Schweiz haben sich in „Kultur- und Sozialanthropologie“ umbenannt, um sich bewusst von der älteren regionalen Völkerkunde abzugrenzen, die der Begriff „Ethnologie" impliziert.

${ }^{40}$ Vgl. Pounds, NSF Funds New Research, Anthropology News 2007/9, 30; Marcia Inhorn's Arbeiten zum Fortpflanzungstourismus in der Arabischen Welt in Inhorn (Fußn. 28); sind - wie auch meine, Europa-bezogenen Forschungen zur Thematik - inspiriert durch jüngere Globalisierungstheorien und Einsichten aus der Medical Anthropology, den Gender und den Science and Technology Studies. ${ }^{41}$ Spiewake (Fußn. 14).
} 



\title{
Der Embryo im Kontext: Warum die Biopolitik die menschlichen Beziehungen nicht vergessen darf $^{1}$
}

\author{
Claudia Wiesemann
}

Die Fortpflanzungsmedizin stellt eine besondere Herausforderung für die Bioethik dar. Entscheidungen betreffen nie nur ein Individuum, sondern Menschen in besonderen, herausgehobenen Beziehungen zu anderen. ${ }^{2}$ Wenngleich Schlüsseltechnologien wie die In-vitro-Fertilisation primär die biologischen Prozesse der Reproduktion betreffen, haben sie doch Einfluss auf bedeutsame soziale und moralische Institutionen, vor allen Dingen auf Elternschaft. Die Eltern-Kind-Beziehung wird gewollt oder ungewollt zum Zentrum fortpflanzungsmedizinischer Aktivitäten, sie begründet deren gesellschaftliche Bedeutung wie auch deren Problempotenzial. Doch die rechtliche und ethische Debatte in der Fortpflanzungsmedizin weist eine strukturelle Blindheit für die involvierten sozialen Beziehungen und deren moralische Bedeutung auf. ${ }^{3}$ Dieser Aufsatz geht einigen Ursachen für diese Blindheit

\footnotetext{
${ }^{1}$ Der Aufsatz beruht in Teilen auf zwei bereits erschienenen, hier zusammengefassten sowie gründlich überarbeiteten Artikeln: Wiesemann, Der Embryo und die Ethik der Elternschaft. Eine Antwort auf Anton Leist, Zeitschrift für Evangelische Ethik 2007/51, 58 ff.; Wiesemann, Fortpflanzungsmedizin und die Ethik der Elternschaft, Journal für Reproduktionsmedizin und Endokrinologie 2007, $189 \mathrm{ff}$.

2 Irreführend ist aus diesem Grund die Einstufung der in diesem Feld verhandelten Fragen als "Ethische Probleme am Lebensanfang", so als würden diese sich einzig und allein aus der Perspektive des zu gebärenden Kindes stellen.

${ }^{3}$ Ethnologinnen hingegen haben schon früh die Bedeutung der reproduktionsmedizinischen Techniken für die Konzeptualisierung von Verwandtschaft untersucht. Franklin, Embodied progress. A cultural account of assisted conception, 1997; Hauser-Schäublin/Kalitzkus/Petersen/Schröder, Der geteilte
} 
nach und begründet, warum und auf welche Weise Elternschaft als moralisches Konzept in der Ethik der Fortpflanzungsmedizin berücksichtigt werden sollte.

\section{Ethik des Fremden}

Die meisten ethischen Theorien, die in der Bioethik Anwendung finden, kann man als eine Ethik des Fremden bezeichnen. Sie fokussieren auf Individuen und gehen davon aus, dass Menschen, die sich in einem moralischen Konflikt befinden, Interessen oder Rechte haben, die es gegeneinander abzuwägen oder auszugleichen gilt. Das Ideal solcher Ethiken ist erfüllt, wenn es gelingt, eine möglichst einfache Regel oder Formel zu finden, die für alle unter allen Umständen gültig ist. Diese Regeln definieren das ethische Minimum, mit dem ein Zustand hinreichender Moralität innerhalb der Gesellschaft erreicht wird: Ein solches ethisches Minimum ist z.B. der Respekt vor der Menschenwürde des anderen, die wechselseitige Nicht-Einmischung bei der Verfolgung individueller Ziele oder die Einnahme einer vernunftgeleiteten, neutralen Position in Diskursen über moralische Konflikte. Je größer die soziale Gemeinschaft, desto wichtiger die Einfachheit und Überschaubarkeit dieser moralischen Maximen.

Diese Ethiken wurden in den letzten 250 Jahren im Zuge der Überwindung feudaler und anderer autoritärer politischer Systeme entwickelt und haben sich als Grundlage demokratischer Gesellschaften bewährt, in denen Konflikte über Lebensweisen oder Überzeugungen zwischen den Mitgliedern eines größeren Gemeinwesens einvernehmlich, doch ohne Kompromittierung individueller Freiheit zu regeln sind.

Sie haben so erfolgreich dazu beigetragen, unsere demokratischen politischen Systeme zu etablieren, zu lenken und zu stabilisieren, dass wir dazu tendieren, sie für allgemeingültig zu halten und auf alle moralischen Konflikte unserer Zeit anzuwenden. Doch so entsteht ein Problem, das vor allen Dingen die Medizinethik betrifft, denn in der Medizin geht es oft nicht um einander neutral gegenüberstehende, fremde Menschen, wie die Bürger eines Staates, sondern um Menschen in sehr engen sozialen Beziehungen, wie die Eltern eines Kindes. Es geht nicht um eine große Zahl von einander gleichen, unabhängigen Individuen, sondern um wenige, herausgehobene Wesen, denen man eine besondere Verantwortung schuldet, wie einem kranken Ehepartner. Dies sind auch Beziehungen, in denen der eine auf fundamentale Weise abhängig ist vom anderen, wie der Patient auf der Intensivstation von den ihn behandelnden Personen, in denen der eine eng leiblich mit dem anderen verbunden ist, wie der Embryo und die schwangere Frau, oder in denen eine umfassende, langfristige und persönliche Verantwortung im Mittelpunkt steht, wie bei Eltern und ihren Kindern.

Leib. Die kulturelle Dimension von Organtransplantation und Reproduktionsmedizin in Deutschland, 2001. 


\section{Ethik menschlicher Beziehungen}

Der Unterschied zwischen der Ethik des Fremden und einer Ethik, die für enge menschliche Beziehungen nötig ist, wird besonders deutlich, wenn man das jeweils zu erwartende ethische Minimum betrachtet. Für zwei Bürger eines Staates gilt: Es reicht, wenn sie Autonomie und Menschenwürde des anderen respektieren und sich in der Verfolgung ihres Lebensglücks nicht behelligen. Für Eltern und Kinder gilt aber: Mindestens gefordert ist die Bereitschaft zu einer langfristigen und weit reichenden persönlichen Verantwortung und einer liebevollen Beziehung. Verantwortungs- und Liebesbereitschaft sind in diesem Fall keine zusätzlichen, „supererogatorischen“, also zwar besonders löblichen, aber moralisch nicht geforderten Leistungen. ${ }^{4}$ Sie charakterisieren vielmehr die Eltern-Kind-Beziehung auf fundamentale Weise.

Dem Züricher Philosoph Anton Leist zufolge sollte sich Ethik stärker an solchen Beziehungen ausrichten, weil Moral „eine Qualität der menschlichen sozialen Beziehungen"5 und kein „Selbstbegrenzungsprogramm für Egoisten"6 sei. Die moralischen, werthaften Einstellungen für unser Handeln, so Leist, finden Menschen in der Art und Weise ihres Zusammenlebens. Zum besseren Verständnis der Güter, die unser Handeln lenken, können wir uns an den empirisch vorgefunden menschlichen Beziehungen orientieren, ohne dabei deren kritische Weiterentwicklung aus dem Auge zu verlieren. ${ }^{7}$

Dies gilt in besonderem Maße für die Fortpflanzungsmedizin. Mehr als alle anderen Bereiche der modernen Medizin greift die Fortpflanzungsmedizin mit ihren Techniken in menschliche Nahbeziehungen, vor allen Dingen die ElternKind-Beziehung, ein. Und dennoch hat man versucht, sich mit der Ethik des Fremden zu arrangieren und diese für Nahbeziehungen passend zu machen. ${ }^{8}$

\footnotetext{
${ }^{4}$ S. z.B. Elisabeth Conradis Kritik an Onora O'Neills Position: O'Neill, The 'Good Enough Parent' in the Age of the New Reproductive Technologies, in: Haker/Beyleveld (eds.), The Ethics of Genetics in Human Procreation, 2000, pp. 33 ff. (37); Conradi, Take Care. Grundlagen einer Ethik der Achtsamkeit, 2001, S. 75 ff.

${ }^{5}$ Leist, Ethik der Beziehungen. Versuche über eine postkantianische Moralphilosophie, 2005, S. 89.

${ }^{6}$ Leist (Fußn. 5), S. 121.

7 Auf ähnliche Weise versuchen sozialethische Ansätze oder die "kontextsensitive Ethik" den Menschen als soziales Wesen in den Mittelpunkt zu stellen. Sie versuchen so, einige grundlegende Schwächen der vorherrschenden Ethik zu kompensieren. Vgl. die Stellungnahmen theologischer Ethikerinnen und Ethiker zu Konflikten in der Fortpflanzungsmedizin wie z.B. $A n$ selm/Fischer/Frey/Körtner/Kreß/Rendtorff/Rössler/Schwarke/Tanner, Starre Fronten überwinden. Eine Stellungnahme evangelischer Ethiker zur Debatte um die Embryonenforschung, Frankfurter Allgemeine Zeitung 2002; Haker, Ethik der genetischen Diagnostik, 2002; Dabrock/Klinnert/Schardien, Menschenwürde und Lebensschutz. Herausforderungen theologischer Bioethik, 2004; sowie die Arbeiten vorwiegend empirisch arbeitender Ethikerinnen und Ethiker, wie z.B. Krones/Ricbter, Kontextsensitive Ethik am Rubikon, in: Düwell/Steigleder (Hrsg.), Bioethik. Eine Einführung, 2003, S. 238 ff. Für eine ausführliche und kritische Übersicht s. Biller-Andorno, Gerechtigkeit und Fürsorge. Zur Möglichkeit einer integrativen Medizinethik, 2001.

8 Für eine ausführliche Kritik s. Wiesemann, Von der Verantwortung, ein Kind zu bekommen. Eine Ethik der Elternschaft, 2006.
} 
Bei moralischen Konflikten in der Schwangerschaft fördert die Ethik des Fremden jedoch eine einseitige und in ihrer Einseitigkeit verzerrende Betrachtungsweise: Die ethischen Auseinandersetzungen drehen sich dann um die Frage, $\mathrm{ob}$ und $\mathrm{ab}$ wann der Embryo wie ein autonomes Individuum zu behandeln sei, dessen Rechte und Interessen gegen die der Frau in Anschlag zu bringen sind. Beide werden als jeweils für sich stehende moralische Individuen betrachtet. Als Konsequenz erlaubt es beispielsweise das deutsche Gesetz zum Schwangerschaftsabbruch der schwangeren Frau nur, auf sich selbst bezogene Argumente für einen Schwangerschaftsabbruch geltend zu machen ( $\left(218 \mathrm{a}\right.$ Abs. 2 StGB). ${ }^{9}$ Geht man aber davon aus, dass Eltern schon während der Schwangerschaft bemüht sind, dem Ideal der Elternschaft gerecht zu werden, so wird klar, dass sich ihnen ganz andere moralische Fragen stellen. Die Schwangere wird z.B. überlegen, wie sie der Verantwortung für dieses Kind gerecht werden kann und wie sich eine gute ElternKind-Beziehung realisieren lassen wird. Dies sind Überlegungen, die auf die Beziehung zum Kind und das Gewicht der besonderen Verantwortung ausgerichtet sind und nicht in das Schema einer individualistischen Ethik des Fremden passen.

\section{Zur Problematik einer Ethik des Fremden}

Was ist das Problem der Ethik des Fremden? Sie bringt den ganzen Reichtum moralischer Beziehungen, der sich über die Jahrtausende menschlicher Sozialisation entwickelt hat, nicht zur Geltung, da sie alle moralischen Fragen unter dem Gesichtspunkt der Beziehungen von Bürgern in einem demokratischen Gemeinwesen betrachtet. Es kommt zu einer unangemessenen Reduzierung von menschlichen Beziehungsformen und deren moralischer Bedeutung - so als ob man versuchte, eine besonders bunte Blumenwiese auf einem Schwarz-Weiß-Foto abzubilden. Dies lässt sich besonders deutlich an der Definition des Embryos im deutschen Embryonenschutzgesetz zeigen.

Dort heißt es:

„Als Embryo im Sinne dieses Gesetzes gilt bereits die befruchtete, entwicklungsfähige menschliche Eizelle vom Zeitpunkt der Kernverschmelzung an, ferner jede einem Embryo entnommene totipotente Zelle, die sich bei Vorliegen der dafür erforderlichen weiteren Voraussetzungen zu teilen und zu einem Individuum zu entwickeln vermag." (ESchG $₫ 8$ Abs. 1)

Dies ist eine problematische, wenn nicht gar irreführende Formulierung. Ohne Implantation in die Gebärmutter einer Frau vermag sich keine befruchtete Eizelle in ein menschliches Individuum zu entwickeln. Das hier verwendete substantivierte deutsche Verb „vorliegen“ aber unterstellt, bei den „erforderlichen Voraussetzungen" handele es sich um rein materiale Gegebenheiten wie Nährlösung oder Umgebungstemperatur. Tatsächlich müssen sich komplexe soziale Prozesse ereig-

${ }^{9}$ Heinemann, Frau und Fötus in der Prä- und Perinatalmedizin aus strafrechtlicher Sicht, 2000. 
nen, die auf den Entscheidungen und Handlungen von Menschen beruhen und höchst verantwortungsgeladene Beziehungen initiieren.

Motiv für die vorliegende Definition des Embryos im Embryonenschutzgesetz war es, eine möglichst von den wechselnden Manipulationskontexten der befruchteten Eizelle unabhängige Beschreibung des schützenswerten frühen menschlichen Lebens zu finden. ${ }^{10}$ Dies muss man als eine Reaktion auf die Verbreitung der In-vitro-Fertilisation Ende der achtziger Jahre verstehen, welche nicht nur die Vorstellung von einer „natürlichen“ Entwicklung des Menschen konterkarierte, sondern auch die künstliche Gebärmutter wieder als wahrscheinlicher erscheinen ließ. Der Wunsch, das Wesen des Menschlichen an seinem Ursprung in einer von diesen technologischen Entwicklungen unabhängigen Weise zu definieren, war verständlicherweise groß.

Doch heute zeigt sich, dass diese ethisch-rechtliche Konzeptualisierung eines zentralen Aspekts der Fortpflanzungsmedizin problematische Folgen hat. ${ }^{11}$ Sie führt zu einer strukturellen Blindheit für menschliche Beziehungen. Sie blendet die soziale Wirklichkeit von Elternschaft und Schwangerschaft aus. Eine Definition, die bewusst von sämtlichen sozialen Bedingungen der menschlichen Fortpflanzung abstrahiert, kann zudem nicht mehr verständlich machen, warum wir uns überhaupt um den Umgang mit befruchteten menschlichen Eizellen so viele Sorgen machen. Die Instrumentalisierung des Menschen durch den biologisch-technischen Fortschritt lässt sich eben nicht dadurch verhindern, dass man dessen Wesen ausschließlich biologisch-technisch definiert. Dies führt im Gegenteil dazu, den ursprünglichen moralischen Impetus ins Leere laufen zu lassen. Der Kontext von Menschwerdung und Elternschaft, der dem Ganzen doch zunächst seine soziale Bedeutung verliehen hat, geht verloren.

\section{Das moralische Ideal der Elternschaft}

Wie könnte man stattdessen vorgehen? Zunächst einmal sollte man sich vergegenwärtigen, welche höchst wertvoll und bedeutsam erscheinenden sozialen Sachverhalte durch das Recht geschützt und bewahrt oder zumindest jedenfalls nicht leichtfertig aufs Spiel gesetzt werden sollten. Über solche Sachverhalte lässt sich womöglich auch im internationalen Kontext leichter Einigung erzielen als über die konkrete gesetzliche Regelung des Klonens oder der Forschung mit humanen embryonalen Stammzellen. Eine Analyse der sozialen Bedeutung der Embryogenese und der moralischen Bedeutung von Elternschaft könnte gemeinsame kultu-

${ }^{10}$ Keller/Günther/Kaiser, Embryonenschutzgesetz. Kommentar zum Embryonenschutzgesetz, 1992.

11 Jedes neue Verfahren zur Reprogrammierung von Keim- oder Körperzellen lässt es als fragwürdiger erscheinen, in einer nur vermuteten, aber unter konkreten praktischen Bedingungen nicht überprüfbaren Entwicklungsfähigkeit, das allein entscheidende Kriterium für den Beginn menschlichen Lebens zu sehen. 
relle Wertvorstellungen zu Tage fördern und damit die Verständigung über nationale Grenzen hinweg erleichtern.

Kritiker einer solchen beziehungsorientierten Ethik der Fortpflanzungsmedizin werden fragen, ob man die Moral davon abhängig machen darf, was einzelne Eltern ihren Kindern gegenüber empfinden, oder gar was eine einzelne Schwangere dem Embryo gegenüber empfindet? Die beziehungsorientierte Ethik würde allerdings gründlich missverstanden, wenn man ihr unterstellte, sie rede nur einem persönlichen „Laissez-faire“ das Wort. Es handelt sich nicht um eine besonders raffinierte Rechtfertigung von persönlicher Willkür! Im Gegenteil: Eltern müssen ihr Verhalten an einer allgemeinen moralischen Bedeutung von Elternschaft messen lassen. Sie werden sich bemühen, dem kulturell verankerten Ideal von Elternschaft gerecht zu werden. Anders als in der Ethik des Fremden führt das jedoch nicht zur Übernahme von ein paar einfachen und abstrakten Pflichten. Es führt zu einer komplexen, lebenslangen, persönlichen Verantwortung. Wie die Eltern dieser Verantwortung angemessen gerecht werden, müssen sie persönlich unter den jeweils konkreten Lebensumständen immer wieder neu entscheiden. Verantwortung ohne diese persönliche Freiheit ist nichts. Sie ist aber keinesfalls mit individueller Willkür gleichzusetzen.

Welche Konsequenzen hätte es, wenn wir das moralische Ideal der Elternschaft zum Ausgangspunkt einer Ethik der Fortpflanzungsmedizin nähmen? Dies hieße, einer auf Beziehung fokussierenden Ethik den Vorrang vor einer ausschließlich Individuen bzw. biologische Entitäten berücksichtigenden Ethik zu geben. Der extrakorporale Embryo wäre nicht mehr primär Objekt staatlicher Schutzinteressen und Gegenspieler seiner potenziellen Eltern, sondern Subjekt elterlicher Verantwortung. Wir dürfen dabei nicht vergessen, dass diese Verantwortung in Ausmaß und Intensität in der sozialen Welt ohne gleichen ist. Eine derart gewichtige Beziehung entsteht in einem Prozess. Sie fällt den Eltern nicht wie eine staatlich verordnete Pflicht zu und ist auch nicht angeboren, sondern muss, gerade weil auf Liebe zum Kind fußend, aus freier Entscheidung übernommen werden.

Natürlich gehört es zu den besonders problematischen Seiten der Fortpflanzungsmedizin, dass die bedeutsame, aber fragile Phase der Elternwerdung in den ersten Tagen und Wochen nach der Befruchtung heute dem menschlichen Zugriff offen steht. Für uns alle ist es noch neu und ungewohnt, Eltern das Stadium der allmählichen Übernahme von Verantwortung und der leiblichen Realisierung von Elternschaft quasi öffentlich durchlaufen zu sehen. Eine ehemals sehr persönliche und intime Zeit des Abwartens, Zögerns, Vergewisserns muss nun vor den Augen von Dritten durchlebt werden. Die in der Öffentlichkeit zelebrierte Zeugung eines Kindes - ihr Sinnbild das Foto einer Pipette mit männlichem Samen, welche die Hülle der Eizelle durchsticht - gewinnt eine hohe symbolische Bedeutung. Sie stilisiert den Embryo zum öffentlichen Gut. Doch das ist er nicht und das sollte er auch nicht sein. 
Umso wichtiger ist es, die Akteure dieses Geschehens, die zukünftigen Eltern, nicht aus den Augen zu verlieren, und dafür zu sorgen, dass sie diesen Teil ihrer sehr persönlichen Lebensgeschichte zurückerhalten. Zwar bedürfen die Methoden der Fortpflanzungsmedizin durchaus beständiger öffentlicher Kontrolle. Es sollten die Eltern und ihre Maßstäbe dabei jedoch nicht vergessen werden. Und für sie ist dies zweifellos eine Übergangszeit, eine Phase, in der sich Gewissheiten erst allmählich einstellen, in der Elternschaft erst in Gestalt der gesicherten Schwangerschaft real erfahrbar wird. Aus der Perspektive der zukünftigen Eltern - das haben die empirischen Untersuchungen von Tanja Krones und Gerd Richter gezeigt - ist die befruchtete Eizelle in moralischer Hinsicht mehr als ein Zellhaufen, aber weniger als ein Kind. ${ }^{12}$ Eine Ethik, die diese Phasen ignoriert und jede Eizelle von der Befruchtung an moralisch wie ein geborenes menschliches Individuum behandelt, bürdet den potenziellen Eltern ein absurdes Ausmaß an Verantwortung auf.

\section{$5 \quad$ Fazit}

Definitionen des menschlichen Embryos, derer das Recht wohl auch in Zukunft bedarf, sollten die Perspektive der Eltern nicht unterschlagen. Es sind soziale, am Ethos der Elternschaft ausgerichtete Bedingungen, die aus einer befruchteten Eizelle einen Embryo und aus einem Embryo einen Menschen werden lassen. So verstehen wir auch, warum befruchtete Eizellen zwar nicht geborenen Menschen moralisch gleichzusetzen sind, aber dennoch nicht wie Ware oder ausschließlich wie ein technisches Produkt behandelt werden sollten. Extrakorporale Verfahren sollten kein Einfallstor für die Instrumentalisierung und Kommerzialisierung menschlichen Lebens werden. Aus der Beziehung zwischen den zukünftigen Eltern und dem Kind im Werden würde sonst ein Verhältnis von Produzent und Produkt.

Schließt das die Spende von überzähligen befruchteten Eizellen aus der Invitro-Fertilisation für sinnvolle gemeinnützige Zwecke, wie zum Beispiel die Forschung an embryonalen Stammzellen, aus? Ich meine: nein. Das Netzwerk familiärer Beziehungen ist nicht hermetisch gegen die Außenwelt abgeschlossen. Elternschaft ist nur der Prototyp fürsorglicher und verantwortungsvoller menschlicher Beziehungen, ein Vorbild für eine Reihe anderer altruistischer Lebensverhältnisse. Es kann eine sinnvolle Interpretation des Ethos der Elternschaft sein, wenn überzählige befruchtete Eizellen, die nicht zur Implantation vorgesehen sind, aus altruistischen Gründen für gemeinnützige Projekte in Forschung und Therapie freigegeben werden.

Aus diesen Überlegungen wird deutlich, warum auch Techniken wie das Reprogrammieren von Eizellen und der Kerntransfer beim therapeutischen Klonen, die nicht eigentlich als Befruchtungstechniken gelten, uns dennoch wegen ihrer

12 Krones/Richter, Preimplantation Genetic Diagnosis (PGD): European Perspectives and the German Situation, Journal of Medicine and Philosophy 2004/29, 623 ff. 
großen Nähe zu Prozessen der Fortpflanzung nicht gleichgültig sein können. Wegen der Ähnlichkeit dieser Verfahren mit den Abläufen bei der Befruchtung und weil das Potenzial dieser Zellen noch in vieler Hinsicht unerforscht ist, scheint es sinnvoll, diesen Bereich besonderer gesellschaftlicher Kontrolle zu unterziehen. Erst dann lässt sich rechtzeitig erkennen, wo daraus möglicherweise eine Gefahr für unser Verständnis der Eltern-Kind-Beziehung erwachsen könnte.

Doch die äußerst bedeutsamen praktischen Unterschiede zwischen den beiden Techniken - hier Zellreprogrammierung, dort Befruchtung, damit ein Kind geboren wird - dürfen nicht unterschlagen werden. Die Definition des menschlichen Embryos sollte noch einen Eindruck davon vermitteln, um welcher sozialer Beziehungen willen wir uns Sorgen um eine befruchtete Eizelle machen. Nur dann können wir einen sinnvollen Embryonenschutz betreiben. Dies jedenfalls wäre dann ein Embryonenschutz, der nicht Gefahr läuft, mit jeder neuen zellbiologischen Technik wieder ad absurdum geführt zu werden. 


\title{
Entstehung und Entwicklung der Spendersamenbehandlung in Deutschland ${ }^{1}$
}

\author{
Thomas Katzorke
}

Seit der ersten dokumentierten instrumentellen Spendersamenübertragung (Philadelphia 1884) bei männlicher Zeugungsunfähigkeit ist diese Behandlungsmethode Gegenstand äußerst kontrovers geführter Diskussionen: ethische, moralische, juristische, religiöse und viele andere Bereiche werden tangiert. Trotzdem wird die Samenspende heute als eine allgemein akzeptierte, wissenschaftlich begründete und zulässige Therapieform in sämtlichen Industrieländern betrachtet. Es gibt bisher keinen Anhalt für eine Einschränkung der Lebensqualität der späteren Eltern und der so gezeugten Kinder. Lediglich die Praxis der Anonymisierung ist ungeklärt. Konkretisiert werden muss z.B. besonders das deutsche Recht im Hinblick auf das Erbrecht. Weiterhin müssten im Zuge der Revision des bestehenden Embryonenschutzgesetzes oder durch Erlass eines neuen Fortpflanzungsmedizingesetzes den veränderten gesellschaftlichen Entwicklungen Rechnung getragen werden. Hier muss von der Gesellschaft besonders zu der Frage der Behandlung von ledigen Frauen und gleichgeschlechtlichen Paaren Stellung genommen werden.

1 Dieser Beitrag ist ursprünglich im Journal für Reproduktionsmedizin und Endokrinologie 2008, 14 ff. erschienen. Der Nachdruck erfolgt mit freundlicher Genehmigung des Verlages. 


\section{Historische Anfänge}

Die künstliche Insemination (KI) beim Menschen geht auf Dokumente aus der jüdischen Geschichte aus dem 2. - 3. Jahrhundert n. Chr. zurück. Diese bekunden, dass man sich im jüdischen Kulturkreis mit der Möglichkeit der Schwängerung der Frau ohne sexuellen Verkehr befasst hat. Die ersten, denen die Insemination beim Menschen bekannt war und welche theoretisch über die fecundatio sine concubito diskutierten, waren somit die alten Talmud-Juristen.

Seit dem 14. Jahrhundert war die Methode der KI in der Pferdezucht, vor allem im arabischen Raum, bekannt und üblich. Der italienische Arzt und Naturwissenschaftler Lazzaro Spallanzani (1729 - 1799) bewies, dass neues Leben nicht nur durch den Geschlechtsverkehr, sondern auch durch das Einführen von Sekret, welches dem Geschlechtsorgan des männlichen Tieres entnommen wurde, ein weibliches Tier entstehen kann. Er experimentierte hierzu erfolgreich am Hund und an Fröschen. Die besondere Bedeutung der in diesem Sekret gefundenen „kleinen Lebewesen“ (Spermien) blieb ihm jedoch unklar.

Es ist nicht ganz sicher, wer die erste erfolgreiche KI beim Menschen durchgeführt hat. 1799 berichtet der englische Experimentalchirurg John Hunter in einer Veröffentlichung über eine homologe (vom Ehemann stammende) KI, welche bei der Frau eines Tuchhändlers zu einer künstlichen Schwängerung führte.

Nach diesem Bericht wurde dem Ehemann, welcher wegen einer hochgradigen Hypospadie steril war, von Hunter der Rat gegeben, sein Sperma auf ein Schwämmchen aufzufangen bzw. mit einer Spritze aufzunehmen und diese möglichst tief in die Vagina seiner Frau zu deponieren bzw. zu injizieren. Dies war der erste bekannte Fall einer sogenannten „homologen“ (vom Ehemann stammenden) Insemination.

1866 berichtete der amerikanische Gynäkologe J. M. Sims über 55 künstliche Inseminationen bei 6 Frauen, wobei in jedem Fall der Samen des Gatten benutzt und intrauterin injiziert wurde. Eine Patientin wurde schwanger und hatte später eine Fehlgeburt; dies war die erste erfolgreiche KI in den Vereinigten Staaten von Amerika.

Sims meisterte die Methode zuerst, doch später verurteilte er sie und seinen Anteil an ihr, weil es ein unmoralisches medizinisches Verfahren sei. Sims hat auch als erster die Lehre von der künstlichen Insemination beim Menschen wissenschaftlich unterbaut, indem er die Ursachen der Sterilität bei der Frau und die physiologischen Bedingungen der Zeugung sowie ihre pathologischen Hemmnisse genau erforschte.

Die erste überlieferte „donogene Insemination“ (Donor: der Spender, fälschlicherweise besonders im juristischen Schrifttum als „,heterologe Insemination“ bezeichnet), also die Verwendung von Sperma eines anderen Mannes, wurde 1884 durch Dr. William Pancoast am Jefferson Medical College, USA, durchgeführt. Er half einem Ehepaar, bei dem der Mann durch eine Geschlechtskrankheit unfrucht- 
bar geworden war. Hierbei inseminierte er der in der Ehe bisher kinderlos gebliebenen Frau die Samenflüssigkeit eines Medizinstudenten.

Die Insemination wurde unter Chloroform-Anästhesie durchgeführt, der Spender blieb anonym (,... the woman was never told“). Neun Monate später gebar sie einen Sohn.

Ab 1890 wurde die donogene Insemination (DI) von Dr. Robert L. Dickinson (ebenfalls in Philadelphia) durchgeführt, der somit als „Pionier der donogenen künstlichen Insemination" gilt.

In der 2. Hälfte des 19. Jahrhunderts wuchs auch in Frankreich das Interesse an der künstlichen Insemination. 1838 teilte Girault eine erfolgreiche KI durch unmittelbare Einspritzung von männlicher Samenflüssigkeit in den äußeren Muttermund mit und berichtete 1868 bereits über 10 erfolgreich behandelte Fälle. Nach anderen Mitteilungen scheint Girault jedoch der erste Arzt gewesen zu sein, welcher den Samen in das Corpus uteri einbrachte. 1865 veröffentlichte Dehaut die erste wissenschaftliche Arbeit über die Möglichkeit der künstlichen Insemination, welche sich zunächst mit den wissenschaftlichen Grundlagen beschäftigte. Es folgten mit praktischen Erfahrungen Lesuer und Gigon (1867), Rimbaud (1872 mit einer umfangreichen wissenschaftlichen Abhandlung), Lajatre (nennt 1876 die Zahl von 567, davon 504 nach dieser Methode erfolgreich behandelten Frauen), Gaillard, Nicola de Nancy, Gautier (1872), Garnier (1881), Bossi (1881) und Lutaud (1890).

In der französischen Ärzteschaft fand die KI damals Gegner und Verteidiger. Im Jahr 1880 wurde eine Gesellschaft gegründet, welche den Namen „Do Vitam“ trug und sich mit Problemen der künstlichen Insemination befasste; doch im gleichen Jahr erklärte die Pariser Medizinische Fakultät die KI für „,unnatürlich“ und „unmoralisch“. Die Pariser Gerichtsmedizinische Gesellschaft hielt 1884 die homologe KI nur als letzten Versuch zur Zeugung ehelicher Kinder bei Einverständnis der Ehegatten für zulässig. Es ging vor allem um die Frage der rechtlichen Erlaubtheit sowie der sozialen und sittlichen Tragweite der künstlichen Insemination, wodurch in der Öffentlichkeit eine leidenschaftliche Kontroverse entstand. Die Pariser Medizinische Fakultät hatte 1871 eine Dissertation von P. F. Gigon zu dieser Problematik angenommen; eine gleiche These von Gerard wurde aber 1895 durch die Fakultät aus sittlichen und moralischen Gründen abgelehnt. Kurz vorher 1884 (1889?) hatte das Tribunal von Bordeaux ein erstes, dieses neue Fortpflanzungsverfahren betreffendes Urteil gesprochen, den Arzt Lajatre wegen einer künstlichen Insemination bestraft und diese Methode als standesunwürdig erklärt. Demgegenüber erklärte die Medizinische Gesellschaft von Frankreich unter Brouardet 1883 den Eingriff für korrekt und als letzte Möglichkeit, die Fortpflanzung zu sichern.

In Italien berichteten Mantegazza 1887 und Sosiac 1897, in Spanien Manaut 1882 und Martin 1885, in England Harley 1880 über erfolgreiche künstliche Inseminationen; in der Schweiz Fürbringer und Meyer-Ruegg 1916. ${ }^{2}$

2 Guttmacher, Artificial insemination, Human Fertility 1946/1, 16 ff.; Major, History of Medicine, vol. 2. 1956; Schellen, Artificial Insemination in the Human, 1957; Guttmacher, The role of artificial 
Schon am Ende des 19. Jahrhunderts konzentrierte sich auch das Interesse der deutschen Ärzte auf die homologe KI. Durch Döderlein wurde seit 1912 die KI beim Menschen zu einem streng wissenschaftlich orientierten Bestandteil der Sterilitätstherapie. ${ }^{3} 1912$ haben Döderlein und Hirsch über einen erfolgreich verlaufenen Fall berichtet, jedoch wurde auch vor den Gefahren (z. B. Übertragung einer gonorrhoischen Infektion) gewarnt. Erfolgreiche homologe künstliche Inseminationen wurden ferner 1912 von Robleder, 1914 von Fraenkel, 1915 von Prochownik, 1917 von Bumm und 1924 von Sellheim durchgeführt.

Das Oberlandesgericht Köln hatte sich 1905 zum ersten Mal in Deutschland mit künstlichen Inseminationen befasst und aufgrund eines gynäkologischen Gutachtens von Fritsch die Möglichkeit dieses Verfahrens zunächst abgelehnt. Auch das Deutsche Reichsgericht RG hatte sich schon 1908 mit der familienrechtlichen Problematik der homologen künstlichen Insemination beim Menschen zu befassen.

Die erste Publikation über die KI erschien in der deutschen Fachliteratur 1909 von Fraenkel. 4

Wann die donogene KI in Europa bzw. in Deutschland übernommen wurde, ist nicht sicher; sie hat hier immer eine wesentlich kleinere Rolle als in den USA gespielt, aber auch dort blieb sie lange Zeit auf wenige Fälle beschränkt. Bis 1918 wurden im deutschen Schrifttum von Hofstätter über 117 künstliche Inseminationen (mit 44 Erfolgen) berichtet; aber auch in Deutschland gab es zahlreiche Ärzte, welche die KI ablehnten, da es nie sicher war, dass die Kinder, welche nach einer künstlichen Insemination geboren würden, wirklich durch diese Methode gezeugt wurden. ${ }^{5}$ Lange Zeit blieb die KI eine Einzelerscheinung, erst durch die Lehre von den zyklischen Schwankungen der Fruchtbarkeit der Frau zu Beginn der 1930erJahre durch die beiden Gynäkologen Knaus (Österreich) und Ogino (Japan) wird die $\mathrm{KI}$ in größerem Umfang erfolgreich durchgeführt.

\section{Die Zeit der nationalsozialistischen Herrschaft (1933-1945)}

Die KI während der Zeit des Nationalsozialismus wird meistens mit der Organisation Lebensborn e.V. in Verbindung gebracht.

Die Organisation Lebensborn e.V. wurde von 10 namentlich nicht bekannten SS-Führern am 12. Dezember 1935 in Berlin gegründet. Das allgemeine Wissen darüber ist spekulativ, gemischt mit einer Prise Sensationslust, manchmal sogar mit einer gewissen Faszination. So behauptet $A$. Mayer. Einen weiteren Auftrieb bekam

insemination in the treatment of sterility, Obs.Gyn.Sur. 1946/15, 767 ff.; Finegold, in: Thomas (ed.), Artificial Insemination, 1964; Heiss, Die künstliche Insemination der Frau, 1972.

${ }^{3}$ Döderlein, Über künstliche Befruchtung, Münchener Medizinische Wochenschrift 1912, 1081 ff.

${ }_{4}^{4}$ Fraenkel, Über die künstliche Befruchtung beim Menschen und ihre gerichtsärztliche Bedeutung, Ärztliche Sachverständigen Zeitung 1909/15, 169 ff.

${ }^{5}$ Hofstätter, Künstliche Befruchtung, in: Marcuse (Hrsg.), Handwörterbuch der Sexualwissenschaft, 1926. 
das Verfahren der künstlichen Befruchtung durch die Bevölkerungspolitik nach 1933. ${ }^{6}$ Es gibt kaum fundierte Literatur; es existierten 9 Entbindungsheime des Lebensborn auf dem Gebiet des Deutschen Reiches und 13 in den usurpierten Ländern, davon allein 8 in Norwegen - wegen seiner „rassisch hochwertigen“ (blond/blauäugig) Bevölkerung. Viele der Kollaborations-Kinder wurden zur Adoption freigegeben.

Es ranken sich viele Legenden um den Lebensborn: ,pervers schicke Edelbordelle, in denen stramme Zuchtbullen der SS mit ausgesuchten deutschen Mädels Nachwuchs für den ,arischen’ Adel zeugten“. Dass der Lebensborn keine derartige Praxis beschrieben hat, ist längst nachgewiesen. ${ }^{7}$

Die Anziehungskraft von Lebensborn beruhte vor allem auf den Angeboten, die es unverheirateten Müttern machte, indem es sie vor der gesellschaftlichen Diskriminierung bewahrte. Diese praktizierte die katholische Kirche ebenso wie die Nationalsozialisten in unterschiedlicher Couleur. Uneheliche Schwangerschaft galt als „Sünde“ bzw. als Beschmutzung des „sauberen“ Familienadels, die per Gesetz bestraft wurde. So wurden unverheiratete Beamtinnen, die ein Kind erwarteten, entlassen. Der Lebensborn reagierte auf derlei Probleme, jedoch nicht unter sozialen, sondern unter „rassenpolitischen“ Vorzeichen: Waren Mutter und Vater gesund und nachgewiesen ,arisch“, bot die Organisation „frühzeitige Heimunterkunft, Geheimhaltung der Geburt, Übernahme der Vormundschaft, wenn das Kind den „Elite“- Anforderungen entsprach, Heranziehung der Väter zur Alimentenzahlung, längerfristige Unterbringung des Kindes, Hilfe bei der Suche nach einer Arbeitsstelle, manchmal sogar eine Anstellung beim Lebensborn selbst und Vermittlung von Pflegeeltern und Adoptivfamilien."

Aber noch weit in die Nachkriegszeit wird den Nationalsozialisten hier fälschlicherweise eine aktive Bevölkerungspolitik unter Zuhilfenahme der künstlichen Befruchtung unterstellt. ${ }^{8}$

\section{Die Zeit nach dem 2. Weltkrieg - Einführung der Kryokonservierung}

Erste Beobachtungen über die Reaktion menschlicher Spermatozoen bei Temperaturen unter dem Gefrierpunkt sind von dem italienischen Physiologen Spallanzani aus dem Jahr 1776 überliefert, der Spermatozoen im Schnee einfror.

Nähere Erkenntnisse über die Gefrierfähigkeit von Sperma begann man im Zeitraum nach 1938 besonders durch die Untersuchungen von Jabnel zu gewinnen.

Die Kryokonservierung von Sperma beruhte auf einer zufälligen Entdeckung, die rund 70 Jahre zurückliegt und sich in München abgespielt hat. Damals, im Jahr

\footnotetext{
${ }^{6}$ Mayer, Über juristische und psychologische Fragen der künstlichen Befruchtung, Münchener Medizinische Wochenschrift 1954, $393 \mathrm{ff}$.

7 Schmitz-Köster, Deutsche Mutter, bist du bereit...: Alltag im Lebensborn, 1997.

${ }^{8}$ Mayer (Fußn. 6).
} 
1938, befasste sich Jabnel, der Leiter des Instituts für Spirochätenforschung an der Deutschen Forschungsanstalt für Psychiatrie (Kaiser-Wilhelm-Institut) mit der Resistenz von Syphilisspirochäten bei extrem tiefen Temperaturen. Nach Einfrieren von syphilitischem Hodengewebe eines Kaninchens in flüssigem Stickstoff bei $-196{ }^{\circ} \mathrm{C}$ und dem Auftauen beobachtete Jabnel zufällig, dass einige Spermatozoen beweglich geblieben waren.

Bei gleichartigen Versuchen mit Humansperma, das er Temperaturen von $196{ }^{\circ} \mathrm{C}$ und $-269^{\circ} \mathrm{C}$ (also nur noch etwa $3,7^{\circ} \mathrm{C}$ vom absoluten Nullpunkt entfernt in flüssigem Helium) aussetzte, stellte er ebenfalls ein Wiederauftreten der Beweglichkeit der Spermatozoen fest. Jahnel gab damals die Anregung, „Abkühlungs- und Befruchtungsversuche an Samenzellen aller Tierklassen anzustellen“. Niemand auch nicht der Autor - konnte ahnen, welche weltweiten Auswirkungen diese zufällige Entdeckung haben würde.

Allerdings war der Weg zur Praxisreife der Samenkonservierung mittels Tiefgefrierung sehr weit. Experimente mit tierischem Sperma, und zwar von Ratte, Maus, Meerschweinchen, Kaninchen und Bullen, verliefen zunächst völlig negativ. $\mathrm{Zu}$ einem Wendepunkt der Forschungsarbeiten führte die wiederum zufällige Feststellung von Polge, Smith und Parkes (1949), dass Glyzerin die Spermatozoen vor den schädlichen Wirkungen des Gefrier- und Auftauprozesses schützen kann. 1952 gaben Polge und Rowson eine praxisreife Methode zur Tiefgefrierung von Bullensperma bekannt. Das 1964 von Nagase und Niwa publizierte Pellet-Verfahren vereinfachte nicht nur die Langzeitkonservierung von Bullensperma, sondern ermöglichte die erfolgreiche Anwendung bei anderen Tierarten; es wurde ebenfalls mit Erfolg bei Humansperma angewendet. ${ }^{9}$

1954 berichtet Sherman erstmals über eine Schwangerschaft mit konserviertem Sperma auf Trockeneis, 1963 erfolgt dann die Anwendung der Kryokonservierung in flüssigem Stickstoff.

In den USA entstehen 1960 die ersten Samenbanken: Pioniere sind Sherman, Kleegman, Guttmacher, Behrman, Finegold. ${ }^{10}$

In der Bundesrepublik sollen bis 1954 1.000 Kinder nach donogener Insemination geboren worden sein. Diese Zahlenangabe findet sich allerdings auch noch für die Jahre 1955 bis 1960 unverändert in der Fachliteratur. Demgegenüber rechnet man seit dem Jahr 1954 mit jährlich 100 Geburten, die der Ausgangszahl von 1.000 hinzuzurechnen seien. Das würde bedeuten, dass die Bundesrepublik im Jahr 1967 insgesamt 2.300 Geburten dieser Art aufweisen konnte.

In der Nachkriegszeit fand allerdings eine beispiellose Polemik und Hetzkampagne gegen die donogene künstliche Insemination statt.

Hatte schon der Nestor der deutschen Gynäkologie, Geheimrat Prof. Walter Stöckel, in seinem Lehrbuch der Gynäkologie ${ }^{11}$ die Vornahme der donogenen In-

\footnotetext{
9 Bunge/Keetel/Sherman, The clinical use of frozen semen, Fertility and Sterility 1954/5, 520 ff.; Sherman, Research on frozen sperm, Fertility and Sterility 1963/1, 49 ff.

10 Behrmann, Artificial Insemination, International Journal of Fertility 1961/6, 291 ff.; Kleegmann, Therapeutic donor insemination, Fertility and Sterility 1954/5, 7 ff.
} 
semination als eine „Perversität des Denkens, des Fühlens und des Handelns“ bezeichnet und die Ärzteschaft eindringlich gewarnt, sich damit ihre Hände zu beschmutzen, so fand er in dem späteren Direktor der Universitätsfrauenklinik Tübingen, Prof. August Mayer, einen Nachfolger, der allein in 15 Publikationen zwischen 1954 und 1962 polemisch gegen die donogene Insemination zu Felde zog. ${ }^{12}$

Laut Gutachten der Deutschen Gesellschaft für Psychiatrie und Tiefenpsychologie entspringt der Wunsch „Mutter zu werden“, ohne den Vater zu kennen oder ihn zu heiraten, in der überragenden Mehrzahl der Fälle aus einer ,,schweren neurotischen Persönlichkeitsstörung“. Die Behandlung soll zu einer alsbaldigen Hasseinstellung des Ehemannes gegen das fremde Kind führen. Der Tübinger Jurist Dölle (zitiert bei $A$. Mayer) spricht von einem grundsätzlichen Verstoß gegen die Idee von Ehe, Vaterschaft und Familie, also gegen Dinge, auf die ein Kulturstaat und seine Rechtsordnung angewiesen sind. Bundesrichter Geiger erblickt in der Einmischung eines Dritten in den Fortpflanzungsvorgang eine Störung der natürlichen Ordnung und hält die donogene künstliche Besamung nach Artikel 1 des Grundgesetzes der Bundesrepublik Deutschland „mit dem Rechtsgebot der Achtung und Wahrung der Menschenwürde“ nicht für vereinbar.

Es verwundert daher nicht, dass der 62. Deutsche Ärztetag 1959 in Lübeck die donogene Insemination aus sittlichen Gründen für standesunwürdig erklärte: Die künstliche donogene Insemination widerspricht der Ordnung der Ehe. Der Bund der Deutschen Ärztinnen beantragte ein Strafverbot und hauptsächlich wegen des Unrechtes am Kind wurde in einem Entwurf zum \ 203 des Strafgesetzbuches die Pönalisierung der ärztlich vorgenommenen donogenen Samenübertragung gefordert. Der Deutsche Juristentag verlangte gesetzliche Strafandrohung gegen den beteiligten Arzt und die inseminierte Frau.

1970 siegte jedoch ärztliches Denken über weltanschauliche Voreingenommenheit, als der 73. Deutsche Ärztetag in Stuttgart das Verdikt von Lübeck aufhob und erklärte, dass die donogene Insemination nicht mehr standesunwürdig sei, jedoch mit zahlreichen Problemen belastet, sodass der Deutsche Ärztetag die Vornahme der donogenen Insemination nicht empfehlen konnte. Trotz der Strafandrohung des Deutschen Ärztetages hatte es offensichtlich Ärzte gegeben, die die Behandlung durchführten: besonders sei hier das Institut von Dr. Gerhard Schaad in Bad Pyrmont genannt. Diese Klinik war in den 1960er- und den frühen 1970erJahren die Anlaufstelle für Paare mit androgen bedingter Sterilität. Der Arzt berichtet auf internationalen Kongressen über seine Behandlungen und gab 1977 an, dass durch seine Klinik mehr als 4300 Schwangerschaften gezeugt worden seien. ${ }^{13}$ Pioniere während dieser Zeit, in der hierfür Berufsverbot drohte, waren neben

11 Stöckel, Lehrbuch der Gynäkologie, erschienen in den Jahren 1931, 1940, $1943,1947$.

12 Mayer, Die heterologe künstliche Insemination in psychologisch-ethischer Sicht, Ärzte Magazin $1959 / 11,341 \mathrm{ff}$.

13 Schaad, Vortrag II. Europäischer Sterilitätskongress (II. ESCO), 8.-11.Oktober 1969. Dubrovnik, Jugoslavia, Nr. 267: Die therapeutische heterologe Insemination in Deutschland. 
Schaad (Bad Pyrmont) Hellmann (Hamburg), Ockel (Frankfurt), Mutke (München) und Lübke (Berlin).

Im Zuge der Liberalisierung nahmen auch viele Kliniken und Universitätskliniken in der Bundesrepublik Deutschland die donogene Inseminationsbehandlung mit in ihr Programm auf, stellten dies jedoch 1984 besonders an den Universitätskliniken wegen der ungeklärten Rechtslage wieder ein. Unter der Leitung von F. Lübke (Berlin) versuchte im Jahr 1979 ein Expertengremium (Teilnehmer: H. J. Heite [Freiburg], H. G. Mutke [München], J. Kunze [Berlin], M. Stauber [Berlin] und $H$. W. Vasterling [Hannover]) anlässlich eines Kongresses in Kiel, allgemein verbindliche Maßstäbe für die Indikation und Durchführung der donogenen Insemination aufzustellen. ${ }^{14}$

Das Symposium wurde als erster Schritt auf dem Wege einer allgemeinen, das heißt schließlich auch juristischen, Anerkennung der donogenen Insemination als ärztliche Behandlungsmaßnahme verstanden. Nach der Übereinkunft und gemeinsamen Anerkennung medizinischer Behandlungskriterien steht der Versuch, über die Fachgesellschaften (Deutsche Gesellschaft zum Studium der Fertilität und Sterilität, Deutsche Gesellschaft für Geburtshilfe und Gynäkologie) eine Lösung der juristischen Problematik anzustreben, wie sie im Ausland bereits gehandhabt wird (Lübkee).

Von Katzorke, Propping und Tauber wurde 1980 und 1981 über größere Erfahrungen bei der Behandlung mit Spendersamen an der Universitätsfrauenklinik Essen in der nationalen und internationalen Literatur berichtet. ${ }^{15} 1984$ führte Schweden ein Verbot der anonymen Spendersamenbehandlung ein. Aufgrund der Möglichkeit einer HIV-Infektion durch Samenübertragung wurde notgedrungen seit 1984 vermehrt die Anwendung von Kryosperma erforderlich.

Im Zuge der neuen Erkenntnisse um die In-vitro-Fertilisation erfolgte seit 1984 eine erneute Medienkampagne gegen die donogene Insemination.

Auch in der Deutschen Demokratischen Republik wurde die donogene Insemination durchgeführt. E. Günther berichtete 1985 über das nationale ADI-Register der Deutschen Demokratischen Republik (ADI - Artifizielle Donogene Insemination). ${ }^{16}$ Hier war es zu 1.590 Schwangerschaften gekommenen, von denen 1.373 ausgetragen wurden. Die Behandlung wurde in insgesamt 12 Einrichtungen der Deutschen Demokratischen Republik durchgeführt und war rechtlich geregelt.

1986 erklärte der 56. Deutsche Juristentag die heterologe Inseminationsbehandlung als nicht sittenwidrig und nicht rechtswidrig. 1989 wurde der Artikel 7 der UNO-Konvention über die Rechte der Kinder beschlossen, der das Recht der

\footnotetext{
14 Schirren/Mettler/Semm (Hrsg.), Fortschritte der Fertilitätsforschung Bd. 8, Kongreßbericht 1979.

15 Katzorke/Propping/Tauber/Ludwig, Artifizielle Insemination mit Spendersamen (AID) - 140 Schwangerschaften bei 290 Ehepaaren, Frauenarzt 1980/21, 405 ff.; Katzorke/Propping/Tauber, Results of Donor Artificial Insemination (AID) in 415 couples, International Journal of Fertility 1981/26, $260 \mathrm{ff}$.

16 Günther, The national ADI (insemination artificial donor) "Sterility in Marriage" Register 1985, Zentralblatt für Gynäkologie 1987/109, 1255 ff.
} 
Kenntnis der genetischen Herkunft festschrieb. Gleichlautende Entscheidungen wurden vom Bundesverfassungsgericht 1987, 1989, 1990 und 1994 festgestellt.

Begründet wird dieses Recht auf Kenntnis der genetischen Abstammung gemeinhin damit, dass die genetische Abstammung ein wesentlicher Faktor bei der Identitätsfindung eines Individuums bildet. Bei Unkenntnis ist eine negative Persönlichkeitsentwicklung und damit Beeinträchtigung des Kindeswohls zu befürchten. Aus diesem Grund kann der Reproduktionsmediziner in Deutschland keine Anonymität (mehr) garantieren. Er ist gut beraten, mit dem Spender eine Vereinbarung zu treffen, dass er dem gezeugten Kind auf Verlangen den genetischen Erzeuger zu nennen hat. Die Dokumentationspflicht regelt $\$ 11,1$ der Berufsordnung für Ärzte. Im Zuge der Vorbereitungen für das am 1.1.1991 in Kraft getretene Embryonenschutzgesetz, das die In-vitro-Fertilisation und verwandte Techniken regelt, sollte auch die donogene Insemination mit geregelt werden. Wegen der Vielfalt der Probleme wurde der Problemkreis donogene Insemination jedoch später wieder ausgeklammert.

Auch die SPD-Fraktion hat noch im Jahre 1990 während der Beratungen zum Embryonenschutzgesetz einen Änderungsantrag eingebracht, mit dem die DI unter Strafe gestellt werden sollte. Der Änderungsantrag lautete in $\ 1$ : „Verbotene Samenübertragung. Wer auf eine Frau Samen eines Mannes, der nicht mit dieser Frau verheiratet ist, oder nicht mit ihr in einer auf Dauer angelegten Lebensgemeinschaft lebt, künstlich überträgt, wird mit Freiheitsstrafe bis zu einem Jahr oder Geldstrafe bestraft." 17

Initiiert von Prof. E. Günther aus Jena konstituierte sich 1995 ein Arbeitskreis für donogene Insemination (e.V.), dem die Mehrzahl der diese Behandlung in Deutschland durchführenden Ärzte mittlerweile angehört. ${ }^{18}$

Rechtlich fanden einige Konkretisierungen statt. 1998 brachte das Kindschaftsreformgesetz gewisse rechtliche Sicherheit. 2002 wurde im Kinderrechteverbesserungsgesetz \1600 Abs. 2 BGB am 9.4.2002 festgestellt: „Ist das Kind mit Einwilligung des Mannes und der Mutter durch KI mittels Samenspende eines Dritten gezeugt worden, so ist die Anfechtung der Vaterschaft durch den Mann und die Mutter ausgeschlossen", so dass damit eine relative Rechtssicherheit vorhanden ist.

Mit dem neuen $\int 1600$ Abs. 2 BGB ist die Wahrscheinlichkeit der Feststellung eines Samenspenders als Vater deutlich herabgesetzt worden.

In mehreren Entscheidungen hat das Bundesverfassungsgericht seit 1989 erkannt, dass jeder Mensch aus dem Recht auf Wahrung seiner Menschenwürde und aus dem allgemeinen Persönlichkeitsrecht das Recht auf Kenntnis seiner genetischen Abstammung hat. Dieses Recht ist allerdings ein Recht, das wie jeder Auskunftsanspruch zwar klagbar, aber nicht durchsetzbar ist, wenn derjenige, der über die Daten verfügt, diese nicht herauszugeben bereit ist. Demjenigen, der verfügt

\footnotetext{
17 Vgl. Beschlussempfehlung und Bericht des Rechtsausschusses, BT-Drs. 11/8057, S. 13.

18 Arbeitskereis für donogene Insemination e.V., Richtlinien zur Qualitätssicherung der Behandlung mit Spendersamen in Deutschland, 2006; http://www.donogene-insemination.de/ (letzter Zugriff am 15.2.2008).
} 
oder verfügen könnte, drohen deshalb ausschließlich Zwangsgelder oder Schadenersatzansprüche des Abkömmlings aus der Verletzung des Persönlichkeitsrechtes.

Eine zwingende und zweifellose gesetzliche Regelung zur Aufbewahrung der Spenderdaten gibt es zurzeit nicht.

\section{$4 \quad$ Wo stehen wir heute?}

Seit 1970 sind in der Bundesrepublik Deutschland schätzungsweise mehr als 100.000 Kinder nach Behandlung mit Spendersamen geboren worden. Der jährliche Zuwachs lag früher bei ca. $1.500-2.000$ Geburten. Seit 1993, der Einführung der intrazytoplasmatischen Spermatozoeninjektion im Rahmen der In-vitro-Fertilisation, hat sich die Zahl mehr als halbiert. Man geht heute von ca. 1.000 Geburten nach Spendersamenbehandlung pro Jahr aus.

Im Jahr 2000 fanden erste systematische Untersuchungen zur Praxis der donogenen Insemination in der Bundesrepublik Deutschland statt. ${ }^{19}$ Dieselbe Autorin veranstaltet regelmäßig sogenannte „Vorbereitungsseminare“ unter Mitwirkung von Ärzten, Juristen und Sozialtherapeuten. ${ }^{20}$

Katzorke fasst den Stand der Behandlung in der Bundesrepublik Deutschland zusammen. ${ }^{21}$ Der Arbeitskreis für donogene Insemination gab 2006 aktualisierte Richtlinien heraus. ${ }^{22}$ Erstmals für die Bundesrepublik Deutschland wurden auch Befragungen bei Samenspendern durchgeführt. ${ }^{23}$

Die Bundesärztekammer hat 2006 auf Empfehlung des wissenschaftlichen Beirates die Richtlinien zur Durchführung der assistierten Reproduktion aktualisiert. ${ }^{24}$ Erstmalig sind hier Vorschläge zur Durchführung der donogenen Insemination gemacht worden. Donogene Insemination und donogene In-vitro-Fertilisation sind bei verheirateten und unverheirateten heterosexuellen Paren unproblematisch. Die Behandlung von Frauen ohne Partnerschaft oder in gleichgeschlechtlicher Partnerschaft soll nach Inhalt der Musterrichtlinie 2006 ausgeschlossen sein.

Die Auswirkungen der Musterrichtlinie zur Durchführung der assistierten Reproduktion unter Verwendung von fremdem Samen im Rahmen der Reproduktionsmedizin sind von Keiper und Kentenich dargestellt worden..$^{25}$ Es erscheint fraglich,

19 Thorn/Daniel, Die Praxis der donogenen Insemination in Deutschland, GebFra 2000/60, $630 \mathrm{ff}$.

20 Daniel/Thorn/Westerbrooke, Confidence in the use of donor insemination: an evaluation of the impact of participating in a group preparation programme, Human Fertility 2007/10, 13 ff.

${ }^{21}$ Katzorke, Donogene Insemination. Gegenwärtiger Stand der Behandlung in der BRD, Gynäkologische Endokrinologie 2003, 85 ff.; Katzorke, Donogene Insemination, Gynäkologe 2007, 807 ff.

22 Arbeitskreis für donogene Insemination e.V. (Fußn. 18).

${ }^{23}$ Katzorke/Thorn/Werdehausen/Daniels (eds.), Attitudes of semen donors in Germany - is it possible to recruit identifiable donors in a society where secrecy prevails? 63rd Annual Meeting of the ASRM, October 13.-17., 2007, oral presentation, 0-223.

24 Bundesärztekammer, (Muster-)Richtlinie zur Durchführung der assistierten Reproduktion - Novelle 2006, Dt. Arrzteblatt 2006, 1392 ff.

${ }^{25}$ Keiper/Kentenich, Die Verwendung von fremdem Samen im Rahmen der Reproduktionsmedizin, Journal für Reproduktionsmedizin und Endokrinologie 2007, 34 ff. 
ob die Landesärztekammern befugt sind, die Vorschläge der Bundesärztekammer in Bezug auf die Behandlung lediger und homosexueller Frauen in Berufsrecht umzusetzen. Regelungen in diesem Bereich stehen nach Meinung vieler Juristen nur dem Bundesverfassungsgericht zu. Auch andere Regelungspunkte, die den Umgang mit Spendersamen regeln sollen, erscheinen problematisch.

Insbesondere wird in den neuen Richtlinien festgestellt, dass im Falle einer Kooperation mit einer Samenbank die Dokumentation nicht an diese delegiert werden kann. Dadurch soll der Gefahr begegnet werden, dass die behandelnde Ärztin/ der behandelnde Arzt einem berechtigten Auskunftsverlangen nicht entsprechen kann, weil die Samenbank nicht mehr existiert, keine ausreichende Dokumentation vorgenommen hat oder die Auskunftserteilung verweigert. Nicht mehr erläutert wurde, ab welchem Zeitpunkt die eigene ärztliche Dokumentation zu gewährleisten ist, ob schon bei Einsatz des Spermas oder erst bei Eintritt einer Schwangerschaft.

Unbeschadet einer klaren gesetzlichen Regelung empfiehlt sich eine Dokumentationsdauer von 30 Jahren für Samenbanken bzw. Ärzte.

Mit Erlass des Gewebegesetzes gibt es klare gesetzliche Regelungen zumindest zur Aufbewahrung der Spenderdaten. Sie sind zum Zwecke der Rückverfolgbarkeit 30 Jahre lang aufzubewahren ( $\$ 15$ Abs. 2 TPG).

Sinnvoll wäre die Einrichtung einer zentralen (staatlichen) Dokumentationsstelle, bei der die Daten bei Eintritt einer Schwangerschaft hinterlegt werden müssten (wie z. B. in der Schweiz). Hier müssten auch verbindliche Zugangsregelungen für das auskunftsberechtigte Kind hinterlegt werden.

Bei einem Auskunftsverlangen des Kindes sollen die Personalien des Spenders bekannt gegeben werden. Dieses sollte auch bei einem Auskunftsverlangen von nur einem der künftigen Elternteile gelten. Leider fehlen zu diesem Problemkreis weitere Ausführbestimmungen. Wie sensibel dieser Bereich ist, zeigen erste Spenderbefragungen in Großbritannien nach Veränderung der Gesetzeslage 2005 und Elternbefragungen in Schweden 20 Jahre nach Veränderung der Gesetzeslage. $\mathrm{Ab}$ diesem Zeitpunkt waren Behandlungen in anonymisierter Form in Schweden nicht mehr zulässig; 89\% der Eltern hatten ihre Kinder noch nicht aufgeklärt! Die Aufhebung der Spenderanonymität führt zunächst unweigerlich zu einem Mangel an Spendern.

Ob durch bessere Aufklärung und Freistellung der Spender mittelfristig eine ausreichende Rekrutierung von Spendern möglich sein kann, ist ungewiss.

Wichtigster Punkt bei deutschen Spendern und Spendern im Ausland ist die Freistellung von sämtlichen (wechselseitigen!) finanziellen Verpflichtungen, auch im Falle einer Vaterschaftsfeststellung. Dies ist in fast allen Nachbarländern gegeben. Eine eindeutige gesetzliche Regelung hierzu wurde wohl vom deutschen Gesetzgeber schlicht vergessen.

Auch müssen Fragen des „Fertilitätstourismus“ oder des Bezugs von Spermaproben aus dem Ausland geklärt werden. 
Die Beschaffung von Spermaproben aus dem Ausland stellt für das betroffene Paar meistens kein Problem dar, da durch das Internet weltweit international tätige Samenbanken (z. B. in Dänemark oder Kalifornien) entsprechendes Material anbieten. Da die deutschen Vorschriften aber dadurch teilweise umgangen werden, ergeben sich Haftungsprobleme für die inseminierenden Ärzte. ${ }^{26}$

So erfreulich die Stellungnahme der Bundesärztekammer und die teilweise Übernahme in das Berufsrecht durch die Landesärztekammern sind, so mangelhaft sind die konkreten Ausführbestimmungen.

Durch die Bekanntgabe der Spenderdaten soll eine genetische Verwandtschaft zum Anlass genommen werden, im Nachhinein eine menschliche Beziehung herzustellen.

Genetische Dispositionen existieren, aber Bindungen zwischen Menschen, ob genetisch unterlegt oder nicht, sind stets die Folge sozialer Prozesse, die ihre Zeit gedauert haben und von Gefühlen begleitet gewesen sein müssen. Familie ist eine soziale Konstellation; die sogenannten Blutsbande wurden in der Vergangenheit als Harmoniespender überschätzt und mystisch überhöht. Das wachsende Verständnis vom Einfluss der Umweltfaktoren auf die Entwicklung der Persönlichkeit relativiert den Wert der Verwandtschaft. Für das gedeihliche Heranwachsen eines Kindes ist ein stabiles soziales Netz, eine Familie, das Beste. Genetische Verwandtschaft ist keine Garantie für die Dauerhaftigkeit und Stabilität des Zusammenseins und die Verträglichkeit von Personen. Von daher muss es erlaubt sein, wirklich zu hinterfragen, ob im Nachhinein eine Beziehung zwischen DI-Kind und Spender zulässig sein soll.

Auch die Sphäre des Spenders ist schützenswert! Seine familiären Verhältnisse können sich gegenüber jenen aus der Zeit der Spende drastisch verändert haben. Vielleicht will der Spender gegenüber seinen Kindern die Exklusivität seiner Vaterschaft aufrechterhalten und wünscht keine Kontaktaufnahme durch das DI-Kind.

Viele Autoren (nicht die Ärzte) glauben, dass Kinder Kenntnisse über ihre biologischen Eltern haben müssen und dass die Geheimhaltung die familiären Beziehungen untergraben könnte.

Die soziopsychische Entwicklung des Kindes hängt von der Beziehung zu den (sozialen) Eltern ab, nicht aber von biologischen Gemeinsamkeiten.

Andere Wege scheinen möglich: Im Sinne eines zweigleisigen Vorgehens sollte Ehepaaren die Möglichkeit eingeräumt werden, von vornherein zu entscheiden, wie sie mit der Tatsache der DI-Behandlung umgehen wollen: $80 \%$ der Eltern wollen den Kindern ihre wahre Herkunft verheimlichen, nur $20 \%$ sind von vornherein für eine Aufklärung. Dem könnte durch eine „double track policy“ Rechnung getragen werden, durch die für beide Möglichkeiten (anonym und nicht-anonym) Vorsorge getragen wird. Das Angebot der Offenlegung der Anonymität an die Patienten mit Ja/Nein-Entscheidung kommt bei einem Mangel an Konsens dem Pluralismus der verschiedenen moralischen Gesichtspunkte am nächsten. So

26 Breitinger, Der Babymacher aus dem hohen Norden, Die Weltwoche 2000. 
lauten auch ähnliche Vorschläge der „American Society for Reproductive Medicine“. .27

Intensivere psychologische Begleitung von DI-Kindern in der Bundesrepublik ist erforderlich, bevor gesetzliche Regelungen zu einer Zementierung führen.

Die Einrichtung einer zentralen Kommission, ähnlich der HFEA („Human Fertilisation and Embryology Authority") in Großbritannien erscheint dringend notwendig, um zeitnah Fragen im Zusammenhang mit der Fortpflanzung zu regeln bzw. ein neues Fortpflanzungsmedizingesetz vorzubereiten.

Weiterhin besteht aber die Kollision zweier Rechtsansprüche:

- Recht des Kindes auf das Wissen um die Abstammung

- Recht des Spenders auf Anonymität

Die Gesellschaft muss hier möglichst unter Einbeziehung vieler Gruppierungen entscheiden, welcher Weg eingeschlagen werden soll.

27 Ethics Committee of the American Society for Reproductive Medicine, Informing offspring of their conception by gamete donation, Fertility and Sterility 2004/81, $527 \mathrm{ff}$. 



\title{
Die rechtliche Regelung donogener ART in Deutschland und Österreich
}

\author{
Christiane Wendehorst
}

Mit Entscheidung vom 15. November $2007^{1}$ hat der Europäische Gerichtshof für Menschenrechte (ECHR) die Beschwerde von vier österreichischen Staatsbürgern für zulässig erklärt, die sich durch $₫ 3$ Abs. 1 und 2 des österreichischen Fortpflanzungsmedizingesetzes (FMedG) ${ }^{2}$ in ihren Rechten aus Artikel 8 EMRK (Achtung des Privat- und Familienlebens), alleine und in Verbindung mit Artikel 14 (Diskriminierungsverbot), verletzt fühlen. Nach den genannten Regelungen des FMedG dürfen für eine medizinisch unterstützte Fortpflanzung nur Eizellen und Samenzellen derjenigen Ehegatten oder Lebensgefährten verwendet werden, die das Kind gemeinsam erziehen wollen. Allein die Behandlung mit Spendersamen ist ausnahmsweise zulässig, wenn der männliche Partner fortpflanzungsunfähig ist, aber nur für eine Insemination. Ausgeschlossen ist dagegen die Verwendung der Eizelle einer Spenderin ebenso wie die Verwendung von Spendersamen im Rahmen einer künstlichen Befruchtung in vitro. Der Vorgang beweist, dass die rechtliche Regelung donogener Techniken zur assistierten Reproduktion (assisted reproductive techniques, ${ }^{3}$ ART) auch in Zeiten, in denen wir längst über „künstliche Keimzellen“ und ähnliche Methoden der Zukunft sprechen, ${ }^{4}$ kontrovers beurteilt wird.

\footnotetext{
${ }^{1}$ EGMR, S. H. and OTHERS v. Austria, 15.11.2007, Application No. 57813/00.

${ }^{2}$ Fortpflanzungsmedizingesetzes (FMedG), BGBl. I Nr. 275/1992, idF v. BGBl. I Nr. 49/2008.

3 Auch: assisted reproductive technology, artificial reproductive technology.

${ }^{4}$ Vgl. z.B. Pearson, Making babies: The next 30 years, Nature 2008/454, 260 ff.
} 


\section{Strukturierung der gegenwärtigen Debatte}

\subsection{Donogene Techniken in der Debatte um die moderne Reproduktionsmedizin}

Bei der gegenwärtigen ethischen und rechtlichen Diskussion über assistierte Reproduktion geht es um drei sich überlappende, aber dennoch voneinander zu unterscheidende Problembereiche.

Der erste dieser Bereiche betrifft die Techniken zur assistierten Herbeiführung einer Schwangerschaft (ART) ganz allgemein, also etwa Insemination, intratubarer und intrauteriner Gametentransfer (GIFT) oder In-vitro-Fertilisation (IVF) bzw. Interzytoplasmatische Spermatozoeninjektion (ICSI) mit anschließendem Embryotransfer (ET). Dazu gehört auch bereits die technische Isolierung und weitere Behandlung der Keimzellen, also beispielsweise von Eizellen mittels hormoneller Stimulation und anschließender Follikelpunktion oder von Spermatozoen mittels natürlicher Stimulation oder testikulärer Spermatozoenextraktion (TESE). Bereits diese diversen Techniken werfen eine Reihe von Fragen auf, die von ihrer ethischen Legitimität bis hin zu den Bedingungen ihrer sicheren und kunstgerechten Durchführung reichen. Im Großen und Ganzen bewegen wir uns hier freilich noch in einem Gebiet, das wesentlich durch den Stand der medizinischen Entwicklung bestimmt wird und das möglicherweise durch religiöse Vorbehalte - etwa seitens der römisch-katholischen Kirche ${ }^{5}$ - aber nur am Rande durch rechtliche Vorgaben geprägt erscheint.

Der zweite dieser Bereiche betrifft den Embryonenschutz, also die assistierte Erzeugung von und den Umgang mit menschlichen Embryonen in vitro. Er hat in den letzten Jahren für die intensivsten Debatten gesorgt und steht momentan ganz im Zentrum des öffentlichen Interesses. Enge Berührungspunkte mit dem Bereich der ART allgemein bestehen insofern, als etwa die Blastozystenkultur mit anschlieBendem Transfer nur eines (SET) oder maximal zweier (DET) Embryonen die Behandlungschancen nur dann wirklich erhöht, wenn sie mit einer Selektion von Embryonen in vitro einhergeht, ${ }^{6}$ was dann seinerseits die Frage nach dem Umgang mit den überzähligen Embryonen, mithin also eine Frage des Embryonenschutzes aufwirft. Ganz im Kontext des Embryonenschutzes sind auch die Diskussionen um die Zulässigkeit der Präimplantationsdiagnostik (PGD) oder der Forschung an embryonalen Stammzellen zu sehen.

Der dritte große Problembereich schließlich betrifft die Elternschaft, also die Herkunft des zur assistierten Reproduktion verwendeten genetischen Materials

\footnotetext{
${ }^{5}$ Vgl. etwa die Vatikanische Kongregation für die Glaubenslebre, Instruktionen über die Achtung vor dem beginnenden menschlichen Leben und die Würde der Fortpflanzung, abrufbar unter: http://www.vatican.va/roman_curia/congregations/cfaith/documents/rc_con_cfaith_doc_1987022 2_respect-for\%20human-life_ge.html (letzter Zugriff: 27.8.2008).

${ }^{6}$ Vgl. dazu den Beitrag von Michelmann, Reproduktionsmedizin im Jahre 2008: Probleme - Wünsche - Lösungsansätze, in diesem Band.
} 
bzw. die Zuordnung erzeugter Nachkommen zu bestimmten Angehörigen einer vorangehenden Generation. Besonders spektakulär ist in diesem Zusammenhang die Diskussion um die Zulässigkeit reproduktiven Klonens, das ja weniger wegen der angewendeten speziellen ART an sich (etwa Kerntransfer aus somatischen Zellen, SCNT), sondern vor allem wegen seiner genetischen Implikationen in der Kritik steht. Auch und gerade in diesem Zusammenhang zu nennen sind jedoch die Leihmutterschaft bzw. Ersatzmutterschaft sowie donogene Techniken im engeren Sinn, also ART, bei denen das für die assistierte Reproduktion verwendete genetische Material ganz oder teilweise nicht von einem Wunschelternpaar stammt.

\subsection{Formen donogener ART}

Nach gegenwärtigem Stand der reproduktionsmedizinischen Entwicklung geht es bei donogenen Techniken vor allem um die Gametenspende (Keimzellspende), die ihrerseits wieder in die Eizellspende und die Samenspende zerfällt, je nachdem, ob die Keimzelle der (sozialen) Wunschmutter oder die des (sozialen) Wunschvaters durch eine Keimzelle außen stehender Dritter ersetzt wird. Statt von Gametenspende als Oberbegriff über Samenspende und Eizellspende spricht man auch gerne von assistierter Reproduktion im heterologen System. Stammt weder die Eizelle von der Wunschmutter noch die Samenzelle vom Wunschvater liegt an sich schlicht eine Kombination aus Eizellspende und Samenspende vor. Hat allerdings die Kernverschmelzung schon außerhalb der konkret betrachteten Kinderwunschbehandlung stattgefunden, sollte terminologisch eher von „Embryospende ${ }^{\text {“7 }}$ gesprochen werden, auch wenn hier erhebliche Grauzonen bestehen. ${ }^{8}$

Nicht unmittelbar in diesen Zusammenhang gehört dagegen die „Spende“ von Keimzellen zu anderen als reproduktiven Zwecken, etwa für die Forschung oder die Industrie. Zwar mag insbesondere die Gewinnung von Eizellen für forschungsbezogene oder industrielle Zwecke zu Recht juristische und gesellschaftspolitische Aufmerksamkeit erregen, doch ist damit die Debatte um ART eindeutig verlassen und bewegt man sich eher im Diskussionsfeld um Probandenschutz bzw. um Organ- und Gewebehandel.

\subsection{Rechtliche Vorgaben}

Abgesehen von den alles umspannenden verfassungsrechtlichen Rahmenbedingungen ${ }^{9}$ sind für die donogenen ART vor allem von Bedeutung das die Zulässig-

\footnotetext{
7 Auch: Embryoadoption, pränatale Adoption. Hierzu Wendeborst, Zur Möglichkeit der Embryoadoption in einem künftigen Fortpflanzungsmedizingesetz, Reproduktionsmedizin 2003, 147 ff., sowie umfassend A.Hübner, Die Embryoadoption (im Erscheinen).

8 Als Beispiel sei der Transfer imprägnierter Eizellen im Vorkernstadium, die von einem anderen Paar stammen, genannt.

9 Zum deutschen Verfassungsrecht vgl. umfassend den Beitrag von Heun, Restriktionen assistierter Reproduktion aus verfassungsrechtlicher Sicht, in diesem Band.
} 
keit und Durchführung von Methoden regelnde Fortpflanzungsmedizinrecht, das eher auf Sicherheitsaspekte konzentrierte Geweberecht und das Abstammungsrecht (Familien- und Personenstandsrecht). Daneben steht das ärztliche Berufsrecht, das die gesetzlichen Maßgaben teilweise reflektiert, teilweise aber auch in wesentlichen Aspekten präzisiert und ergänzt.

\section{Fortpflanzungsmedizinrecht}

Die Zulässigkeit ärztlicher Behandlungsmaßnahmen ist grundsätzlich nicht davon abhängig, dass der Gesetzgeber sie explizit ausspricht. Vielmehr ist sowohl die Erbringung ärztlicher Leistungen als auch ihre Inanspruchnahme von diversen Freiheitsrechten gedeckt, so dass alles, was nicht von Gesetzes wegen untersagt ist und sich im Rahmen allgemeiner rechtlicher Mindestanforderungen (Schädigungsverbot, informed consent usw.) hält, auch als erlaubt gelten darf. ${ }^{10}$ Das Schweigen der einschlägigen fortpflanzungsmedizinischen Spezialgesetze zu einer bestimmten Methode kommt daher der Zulassung der Methode gleich. Das gilt umso mehr, als es sich bei den einschlägigen Gesetzen entweder von vorneherein um reines Strafrecht handelt, wie beim deutschen Embryonenschutzgesetz (ESchG) von 1990, ${ }^{11}$ oder aber, wie beim österreichischen FMedG, um Rechtsvorschriften, deren Durchsetzung ganz wesentlich auf akzessorischen Straftatbeständen beruht: Da im Strafrecht durch den Grundsatz nulla poena sine lege (vgl. Art. 103 Abs. 2 GG, Art. 7 EMRK) ein verschärftes Analogieverbot gilt, kann nicht vom Verbot bestimmter Methoden auf das Verbot ähnlicher Methoden geschlossen werden.

\subsection{Verbot der Eizellspende}

Die Eizellspende ist in einer Reihe europäischer Staaten ${ }^{12}$ gesetzlich verboten, darunter vor allem in Deutschland ( $\$ 1$ Abs. 1 Nr. 1 und 2, Abs. 2 ESchG) und in Österreich ( $\$ 3$ Abs. 3 FMedG).

Nach $₫ 1$ Abs. 1 ESchG etwa wird mit Freiheitsstrafe oder mit Geldstrafe bestraft, wer 1. auf eine Frau eine fremde unbefruchtete Eizelle überträgt oder 2. es unternimmt, eine Eizelle zu einem anderen Zweck künstlich zu befruchten als eine Schwangerschaft der Frau herbeizuführen, von der die Eizelle stammt. Bestraft

\footnotetext{
${ }^{10} \mathrm{Vgl}$. Heun (Fußn. 9).

${ }^{11}$ Embryonenschutzgesetz vom 13.12.1990 (BGBl. I S. 2746), geändert durch Artikel 22 des Gesetzes vom 23.10.2001 (BGBl. I S. 2702). Dass das ESchG ausschließlich Strafvorschriften enthält, ist darauf zurückzuführen, dass zum Zeitpunkt seines Erlasses die konkurrierende Bundeskompetenz in Art. 74 Abs. 1 Nr. 26 GG noch nicht bestand und eine bundeseinheitliche Regelung daher nur auf Art. 74 Abs. 1 Nr. 1 GG (Strafrecht) gestützt werden konnte.

12 Rechtsvergleichende Betrachtungen z.B. bei Koch, Fortpflanzungsmedizin im europäischen Rechtsvergleich, Aus Politik und Zeitgeschichte 2001/27, 44 ff. Zu den rechtlichen Regelungen zur Fortpflanzungsmedizin in europäischen Ländern vgl. zudem die tabellarische Darstellung des MaxPlanck-Instituts für ausländisches und internationales Strafrecht, abrufbar unter: http://www.cueno.de/medr/show_all.asp (letzter Zugriff am 27.8.2008).
} 
wird im Ergebnis der Mediziner, der die Maßnahmen durchführt, und ebenso wer ihn zur Maßnahme anstiftet oder ihm behilflich ist, ${ }^{13}$ sogar wenn es sich um Beihilfe zu einer Eizellspende handelt, die im Ausland durchgeführt wird und dort nicht mit Strafe bedroht ist. ${ }^{14}$ Nicht strafbar ist allerdings die Spenderin oder Empfängerin der Eizelle ( $\mathbb{1}$ Abs. 3 Nr. 1 ESchG). Infolge dieses umfassenden Verbots wird eine Eizellspende in Deutschland nicht praktiziert. Wer medizinisch auf sie angewiesen ist, muss auf eine Schwangerschaft verzichten oder aber auf eigene Faust den Weg ins Ausland antreten. ${ }^{15}$

Ähnliches gilt in Österreich, wo der Arzt gemäß $\ 23$ Abs. 1 Nr. 1 a) FMedG eine Verwaltungsübertretung begeht.

\subsection{Bedingungen einer zulässigen Samenspende}

Im Gegensatz zur Eizellspende ist die Samenspende im deutschen ESchG überhaupt nicht erwähnt und daher nach dem oben Gesagten von Gesetzes wegen erlaubt. Insbesondere ein Analogieschluss zur Eizellspende ist wegen des verfassungsrechtlich abgesicherten Grundsatzes nulla poena sine lege völlig ausgeschlossen. Die einzige diesbezügliche Maßgabe stellt $\int 4$ Abs. 1 ESchG dar, der die wissentliche künstliche Befruchtung mit dem Samen eines Verstorbenen unter Strafe stellt. ${ }^{16}$

Dementsprechend wird die donogene Insemination und IVF auch in Deutschland in größerem Umfang praktiziert. Allerdings wäre es vorschnell, anzunehmen, die Samenspende bewege sich in Deutschland derzeit noch ganz im rechtsfreien Raum, da zumindest das Berufsrecht entsprechend reagiert hat (dazu unten 4.2) und auch die Umsetzung der Geweberichtlinie durch Änderung diverser Gesetze zu einer gewissen rudimentären Regelung geführt hat (dazu unten 3.1).

In Österreich finden sich explizite Vorschriften in $\ 3$ Abs. 2 und $\$ ₫ 11$ bis 16 FMedG. Danach wird interessanterweise zunächst eine Verknüpfung hergestellt zwischen der Tatsache, dass die Samenzelle von einem Dritten stammt, und der konkret angewendeten ART: \3 Abs. 2 FMedG erklärt die Spendersamenbehandlung von vorneherein nur bei Inseminationen für zulässig, womit - in Zusammenschau mit $₫ 3$ Abs. 1 FMedG - insbesondere die Verwendung von Spendersamen

\footnotetext{
13 Vgl. \ 26 StGB, wonach das ernst gemeinte, vorsätzliche und erfolgreiche Bestimmen eines anderen zur Begehung einer vorsätzlichen und rechtswidrigen Straftat pönalisiert wird (Anstiftung); sowie $\int 27$ StGB, der jede Handlung unter Strafe stellt, die geeignet ist, eine Haupttat zu fördern (Beihilfe).

${ }^{14}$ Vgl. $\int 9$ Abs. 2 StGB, wonach selbst bei grenzüberschreitenden Delikten die Tat auch als an dem Ort begangen gilt, an dem der Teilnehmer gehandelt hat. Kritisch zur Problematik ethisch umstrittener und rechtlich von Staat zu Staat unterschiedlich bewerteter gesellschaftlicher Bereiche z.B. Valerius, Stammzellgesetz und grenzüberschreitende Forschung, NStZ 2008, $121 \mathrm{ff}$.

${ }^{15}$ Hierzu eingehend der Beitrag von Knoll, So weit gehen für ein Kind: Reproduktionstourismus als grenzüberschreitender Umweg, in diesem Band.

16 Dieser Straftatbestand war im Gesetzesentwurf der BReg (BT-Drs. 11/5460) ursprünglich gar nicht vorgesehen und fand erst auf Empfehlung des Rechtsauschusses (BT-Drs. 11/8057) Einzug in das ESchG; skeptisch zur Strafwürdigkeit der postmortalen Befruchtung weiterhin Pelchen, in: Erbs/Kohlhass, Strafrechtliche Nebengesetze, EL 144 Dezember 2001, \4, Rdn. 1.
} 
bei IVF oder ICSI verboten ist. Damit ist etwa Paaren, bei denen der Mann absolut unfruchtbar ist, die Frau dagegen nur an eileiterbedingter Sterilität leidet, der Weg zur assistierten Reproduktion abgeschnitten. ${ }^{17}$ Der Gesetzgeber hat diese Differenzierung, die vom österreichischen Verfassungsgerichtshof (VerfGH) in einer Entscheidung vom 14. 10. 1999 abgesegnet worden ist, ${ }^{18}$ mit angeblichen zusätzlichen Gefahren der IVF gegenüber der Insemination (Vermarktung, Kommerzialisierung, Zuchtwahl, überzählige Embryonen) begründet. ${ }^{19}$

Die $\int S 11$ bis 16 FMedG enthalten weitere Bedingungen hinsichtlich der durchführenden Stelle, der Sicherheit der verwendeten Zellen, der Zustimmung des Dritten, der Dokumentation persönlicher Daten und des Verbots des Keimzellhandels. Damit waren im FMedG schon immer Punkte geregelt, die in Deutschland erst seit Umsetzung der Geweberichtlinie (näher unten 3.1) Niederschlag auf gesetzlicher Ebene gefunden haben. Erwähnenswert ist ferner, dass ein Spender seinen Samen stets nur einer Krankenanstalt zur Verfügung stellen und dass der Samen nur bei höchstens drei Wunschelternpaaren zur Anwendung gelangen darf. ${ }^{20}$

Daneben unterliegt die Samenspende natürlich noch allgemeineren Anforderungen nach dem FMedG. Dazu gehört etwa, dass die Aufbewahrung von Samenzellen bei Widerruf oder nach dem Tod des Spenders zu beenden ist $(\mathbb{\$} 17$ Abs. 1). Nach $\int 18$ sind unter anderem Namen, Geburtstag und Geburtsort, Staatsangehörigkeit und Wohnort des Spenders sowie der anderen beteiligten Personen zu dokumentieren. Diese Aufzeichnungen sind von der Krankenanstalt 30 Jahre lang aufzubewahren, danach den öffentlichen Behörden zu übermitteln, die sie auf Dauer archivieren. $\ 20$ regelt das Auskunftsrecht des Kindes (vgl. unten 6.4).

\subsection{Haltung zur Embryospende}

Die oft gemachte Behauptung, die Embryospende sei in Deutschland unzulässig, ist nur teilweise zutreffend. Richtig ist, dass im Wesentlichen dieselben Vorschriften, die auch der Eizellspende entgegen stehen (oben 2.1), eine gezielte Erzeugung von Embryonen mit dem Ziel, diese auf eine andere Frau als diejenige zu transferieren, von der die Eizelle stammt, verbieten. Ebenso wird durch die berüchtigte „Dreier-Regel“ in $\ 1$ Abs. 3 Nr. 3 bis 5 ESchG - zumindest nach der Vorstellung des Gesetzgebers und nach offiziellen Darstellungen - normalerweise verhindert,

\footnotetext{
${ }_{17}$ Dies ist die Situation der ersten beiden Beschwerdeführer des in Fußn. 1 erwähnten Verfahrens.

18 VfGH, 14.10.1999, VfSlg. 15632; vgl. hierzu die Urteilsbesprechung von Bernat, MedR 2000, 389 ff.; kritisch: Coester-Waltjen, Fortpflanzungsmedizin, EMRK und österreichische Verfassung, FamRZ 2000, 598 ff.

${ }^{19}$ Vgl. die Erläuterungen zur RV. 216 Blg.NR. XVIII. GP., S. 11 ff.

${ }^{20}$ Vgl. Bernat, Rechtliche Aspekte, Journal für Fertilität und Reproduktion 2001, 40 ff.
} 
dass überzählige Embryonen entstehen. ${ }^{21}$ Das schließt es freilich nicht aus, dass Embryonen, die zunächst mit dem Ziel einer ESchG-konformen Kinderwunschbehandlung erzeugt wurden, bei denen es später aber aus irgendwelchen Gründen (Tod oder Krankheit der Wunschmutter, Trennung der Wunscheltern usw.) nicht zum Transfer kommt, einer anderen Frau übertragen werden. ${ }^{22}$ Richtig ist aber auch, dass dies wegen der rechtlichen Unsicherheiten faktisch zurzeit so gut wie nicht praktiziert wird.

In Österreich, ebenso wie in manchen anderen europäischen Ländern, ${ }^{23}$ ist die Embryospende dagegen eindeutig von Gesetzes wegen untersagt (vgl. \3 Abs. 3 FMedG).

\section{Geweberecht}

Der europäische Gesetzgeber hat zu einigen Teilfragen der Keimzellspende, die sich unter dem Oberbegriff „Qualitäts- und Sicherheitsstandards“ zusammenfassen lassen, in der Geweberichtlinie von $2004^{24}$ und einer ihrer Durchführungsrichtlinien von 200625 nähere Vorgaben gemacht. Freilich entfalten europäische Richtlinien keine unmittelbare Wirkung in den Mitgliedstaaten, sondern bedürfen der Umsetzung durch die mitgliedstaatlichen Gesetzgeber (vgl. Art. 249 Unterabs. 3 EG-Vertrag). Diese Umsetzung ist mittlerweile in Europa größtenteils erfolgt, allerdings überwiegend mit deutlicher Verspätung.

\subsection{Keimzellspende im deutschen Geweberecht}

Bei der Umsetzung der Geweberichtlinie sind Deutschland und Österreich unterschiedliche Wege gegangen. ${ }^{26}$

21 Vgl. etwa Hepp/Diedrich, Reproduktionsmedizin in der BRD, Gynäkologe 2008, 167 ff.; kritisch zur vieldiskutierten Problematik der „Dreier-Regel“ etwa Frommel, Deutscher Mittelweg in der Anwendung des Embryonenschutzgesetzes (ESchG) mit einer an dem aktuellen wissenschaftlichen Kenntnisstand orientierten Auslegung der für die Reproduktionsmedizin zentralen Vorschrift des $\ 1$ Abs. 1 Nr. 5 ESchG, Journal für Reproduktionsmedizin und Endokrinologie 2007, 27 ff.; dies, Auslegungsspielräume des Embryonenschutzgesetzes, Journal für Reproduktionsmedizin und Endokrinologie 2004, 104 ff.; Neidert, Embryonenschutz im Zwiespalt zwischen staatlichem Gesetz und ärztlicher Lex artis, ZRP 2006, $85 \mathrm{ff}$.

22 Vgl. Wendehorst (Fußn. 7); A.Hübner (Fußn. 7).

${ }^{23}$ Umfassende rechtsvergleichende Darstellung (weltweit) bei A.Hübner (Fußn. 7).

${ }^{24}$ Richtlinie 2004/23/EG des Europäischen Parlaments und des Rates zur Festlegung von Qualitätsund Sicherheitsstandards für die Spende, Beschaffung, Testung, Verarbeitung, Konservierung, Lagerung und Verteilung von menschlichen Geweben und Zellen v. 31.3.2004, Abl. Nr. L 102/48.

25 Richtlinie 2006/17/EG der Kommission zur Durchführung der Richtlinie 2004/23/EG des Europäischen Parlaments und des Rates hinsichtlich technischer Vorschriften für die Spende, Beschaffung und Testung von menschlichen Geweben und Zellen v. 8.2.2006, Abl. Nr. L 38/40.

${ }^{26}$ Dazu näher Wendehorst, Umsetzung der Geweberichtlinie in Deutschland - Bilanz und Vergleich mit Österreich, in: Kopetzki (Hrsg.), Gewebesicherheitsrecht, 2009 (im Erscheinen). 
Das deutsche Gewebegesetz ${ }^{27}$ ist ein reines Artikelgesetz, das sich auf Änderungen im Transplantationsgesetz (TPG) ${ }^{28}$, im Arzneimittelgesetz (AMG) ${ }^{29}$, im Transfusionsgesetz (TFG) ${ }^{30}$ sowie in einer Reihe weiterer Gesetze beschränkt hat. Ergänzt werden die vom Gewebegesetz bewirkten Änderungen mittlerweile durch die TPG-Gewebeverordnung (TPG-GewV), ${ }^{31}$ welche Einzelheiten festlegt, die Rahmen und Regelungsstil von TPG, AMG und TFG gesprengt hätten. Auffällig ist im vorliegenden Zusammenhang, dass das ESchG nicht zu den geänderten Gesetzen gehört. ${ }^{32}$ Damit hat der deutsche Gesetzgeber eine weitere Chance verpasst auf dem Weg zu einem einigermaßen zeitgerechten Fortpflanzungsmedizingesetz wenigstens einen kleinen Schritt weiter zu kommen. ${ }^{33}$ Gerade die Regelung hinsichtlich Keimzellen ist in hohem Maße intransparent und für Rechtsanwender, die sich nicht intensiv mit der Materie befasst haben, schwer zugänglich.

Zunächst sind gespendete Keimzellen vorrangig im TPG geregelt, wo sie allerdings nicht explizit angesprochen werden, sondern nur als „einzelne menschliche Zellen" der allgemeinen Definition von „Gewebe" in $\$ 1$ a Nr. 4 TPG unterfallen. Da schon rein faktisch nur eine Entnahme beim lebenden Spender infrage kommen dürfte, sind des Näheren die $\iint 8$ bis $8 \mathrm{c}$ TPG einschlägig. Darin finden sich etwa ein Arztvorbehalt, detaillierte Anforderungen an Aufklärung und Einwilligung, aber auch eine Pflicht der Bereitschaft zur Nachbetreuung ( $\int 8$ Abs. 3 Satz 1). $\iint 8 \mathrm{~d}$ bis $8 \mathrm{f}$ enthalten organisatorische und qualitative Anforderungen an die Gewebeeinrichtungen, und $\int \mathbb{S} 13$ bis 15 Richtlinien zur Dokumentation, Datenrückverfolgung und zum Datenschutz. Dort sind auch wichtige Paragraphen für das viel diskutierte Recht des Kindes auf Kenntnis der eigenen genetischen Abstammung (näher unten 6.4) zu suchen, obgleich sie hier auf den ersten Blick niemand vermuten würde. $\int 17$ TPG schließlich enthält ein Gebot des Gewebehandels, das auch für Keimzellen gelten muss.

\footnotetext{
27 Gewebegesetz vom 20.7.2007 (BGBl. I S. 1574). Zur Umsetzung der europarechtlichen Vorgaben vgl. Pübler/Middel/M.Hübner, Umsetzung der Geweberichtlinie 2004/23/EG und ihrer Durchführungsrichtlinien 2006/17/EG und 2006/86/EG auf untergesetzlicher Ebene; v.Auer, Die Umsetzung europäischer Richtlinien in innerstaatliches Recht am Beispiel der Geweberichtlinie 2004/23/EG, Bundesgesundheitsblatt - Gesundheitsforschung - Gesundheitsschutz 2008, 757 ff.; Kentenich, Kritische Anmerkungen zum Gewebegesetz, Gynäkologische Endokrinologie 2007, 49 ff.

${ }^{28}$ Transplantationsgesetz in der Fassung der Bekanntmachung vom 4.9.2007 (BGBl. I S. 2206).

${ }^{29}$ Arzneimittelgesetz in der Fassung der Bekanntmachung vom 12.12.2005 (BGBl. I S. 3394), zuletzt geändert durch Artikel 9 Abs. 1 des Gesetzes vom 23.11.2007 (BGBl. I S. 2631).

${ }^{30}$ Transfusionsgesetz in der Fassung der Bekanntmachung vom 28.8.2007 (BGBl. I S. 2169).

31 TPG-Gewebeverordnung vom 26.3.2008 (BGBl. I S. 512).

32 Gerade das ESchG ist seit seiner Verlautbarung massiver Kritik ausgesetzt und wird vielfach als unzeitgemäß kritisiert. So beispielsweise Elser, Rechtliche Fragen im Rahmen des Embryonenschutzgesetzes, in: Grünwaldt (Hrsg.), Was darf der Mensch? Neue Herausforderungen durch Gentechnik und Biomedizin, S. 86 ff.; Neidert (Fußn. 21); u.v.m.

33 Vgl. z.B. Wendehorst, Rechtliche Anforderungen an ein künftiges Fortpflanzungsmedizingesetz, in: Oduncu/Platzer/Henn (Hrsg.), Der Zugriff auf den Embryo. Ethische, rechtliche und kulturvergleichende Aspekte der Reproduktionsmedizin, 2005; Kreß, Embryonenstatus und Gesundheitsschutz. Reformbedarf im Rahmen eines umfassenden Fortpflanzungsmedizin- und Stammzellgesetzes, Jahrbuch für Recht und Ethik 2007, S. 25 ff.
} 
$\int 6$ der TPG-GewV trägt die viel versprechende Überschrift „Voraussetzungen für die Verwendung von Keimzellen im Rahmen von Maßnahmen einer medizinisch unterstützten Befruchtung“. Abs. 1 fordert für die Verwendung von Keimzellen generell, dass nach ärztlicher Beurteilung die Verwendung medizinisch indiziert und der gesundheitliche Schutz der Empfängerin und des Kindes gewährleistet sind. Ferner wird auf einschlägige Pflichtenkataloge hinsichtlich der erforderlichen Laboruntersuchungen und Dokumentationsverfahren verwiesen. Speziell für die heterologe Verwendung von Samenzellen fordert darüber hinaus Abs. 2, dass der Spender nach ärztlicher Beurteilung aufgrund seines Alters, seines Gesundheitszustandes und seiner Anamnese für die Samenspende geeignet ist und Gesundheitsrisiken für andere ausgeschlossen sind.

Auf den ersten Blick erhält man den Eindruck, dass Keimzellen nur dem TPG, nicht aber dem AMG unterfallen. Denn als Reaktion auf vehemente Kritik an früheren Entwürfen ${ }^{34}$ hat der Gesetzgeber unter den Begriffsbestimmungen in \4 Abs. 30 Satz 2 AMG die Klarstellung angefügt: „Menschliche Samen- und Eizellen, einschließlich imprägnierter Eizellen (Keimzellen), und Embryonen sind weder Arzneimittel noch Gewebezubereitungen." ${ }^{35}$ Wer daraus schließen will, dass das AMG für die Keimzellspende gänzlich irrelevant sei, wird freilich eines Besseren belehrt, weil etwa die in $\iint 20 \mathrm{~b}$ und 20c getroffenen Regelungen sehr wohl (auch) auf die Keimzellspende anwendbar sind. So bedarf etwa laut $\int 20 \mathrm{~b}$ Abs. 1 AMG eine Einrichtung, die „zur Verwendung bei Menschen bestimmte Gewebe im Sinne von $\ 1$ a Nr. 4 des Transplantationsgesetzes gewinnen (Entnahmeeinrichtung) oder die für die Gewinnung erforderlichen Laboruntersuchungen durchführen will,“ einer Erlaubnis der zuständigen Behörde. Das Gleiche gilt nach $\$ 20$ c für die Be- oder Verarbeitung, Konservierung, Lagerung oder das Inverkehrbringen von Gewebe. Anders als noch im ursprünglichen Entwurf ${ }^{36}$ ist eine Herstellungserlaubnis im Sinne von $₫ 13$ AMG allerdings nicht mehr erforderlich. ${ }^{37}$

\subsection{Rechtslage in Österreich}

Der österreichische Gesetzgeber hat insofern einen anderen Weg gewählt als der deutsche, als er die Umsetzung der Geweberichtlinie zum Anlass genommen hat für ein eigenes Gewebesicherheitsgesetz (GSG). ${ }^{38}$ Das FMedG, das bereits grobe

\footnotetext{
34 Vgl. etwa Bundesärztekammer, Erweiterte und aktualisierte Stellungnahme zum Regierungsentwurf für ein Gewebegesetz (BT-Drs. 16/3146) anlässlich der Anhörung des Ausschusses für Gesundheit des Deutschen Bundestages am 7. März 2007, abrufbar unter: http://www.bundesaerztekammer.de/downloads/ZRegStell20070124.pdf (letzter Zugriff: 28.8.2008); ebenso die Beschlussempfehlung des Ausschusses für Gesundheit (BT-Drs. 16/5443).

35 Nur angemerkt sei an dieser Stelle, dass durch die falsche Setzung des Beistrichs beim Leser der Eindruck erweckt wird, die Regelung definiere ausschließlich imprägnierte Eizellen als Keimzellen, was natürlich sicher nicht intendiert war.

${ }^{36}$ Vgl. BT-Drs. 16/3146, zu \13 AMG.

37 Vgl. Kentenich (Fußn. 27), 49.

${ }^{38}$ Gewebesicherheitsgesetz (GSG), BGBl. I 49/2008.
} 
Leitlinien zu Aufklärung und Einwilligung der Beteiligten, Sicherheit der übertragenen Keimzellen und zur Dokumentation enthielt (oben 2.2), wurde nur marginal geändert, indem in $\ 5$ Abs. 2 ein kleiner Hinweis auf für die Zulassung erforderliche „rechtliche Befugnisse“ aufgenommen wurde. Damit ist angespielt auf die besonderen Anforderungen an Entnahmeeinrichtungen gemäß $\int S$ 3, 19 GSG, die ihre Tätigkeit zumindest den zuständigen Behörden melden müssen, und auf Gewebebanken gemäß $\iint 8,22$ GSG, die einer Bewilligung bedürfen. Im Übrigen sind die geweberechtlichen Anforderungen nach dem GSG denen nach deutschem Recht nicht unähnlich.

Die Rechtslage hinsichtlich donogener Techniken ist auch in Österreich nicht optimal transparent, vor allem weil im FMedG ein Hinweis auf das GSG und die begleitenden Verordnungen zu vermissen ist. Beide Gesetze müssen aber nebeneinander gelesen werden, wenn man sich ein Bild von der Situation der Samenspende machen will. Insgesamt ist die Konzeption aber deutlich geglückter als die vom deutschen Gesetzgeber gewählte Lösung.

\subsection{Relevanz für die Embryospende?}

Ganz dunkel ist die geweberechtliche Situation hinsichtlich der Embryospende. Das ist für Österreich nicht weiter verwunderlich, weil dort die Embryospende ganz untersagt ist (oben 2.3). In Deutschland wäre zumindest die pränatale Adoption zufällig übrig gebliebener Embryonen rechtlich möglich, so dass der Gesetzgeber der Vollständigkeit halber entsprechende Regelungen hätte erlassen sollen. Ob der Embryo als „einzelne menschliche Zelle“ im Sinne von $\ 1$ a Nr. 4 TPG dem Gewebebegriff unterfällt, ist indessen fraglich (anders natürlich bei nicht mehr totipotenten embryonalen Zellen und Geweben, die unzweifelhaft erfasst sind). Denn es erscheint anstößig, einen potenziellen Träger von Grundrechten, möglicherweise sogar der Menschenwürde ${ }^{39}$, wie ein beliebiges Produkt Qualitäts- und Sicherheitsstandards zu unterwerfen. Auf der anderen Seite ist nicht zu übersehen, dass die Gefährdungslage hinsichtlich Spendern und Empfängern identisch ist und dass zwischen der imprägnierten Eizelle, die wohl unstreitig dem Keimzellbegriff unterfällt (vgl. Legaldefinition in $\$ 4$ Abs. 30 Satz 2 AMG), und einem Embryo ein Unterschied von nur wenigen Stunden liegt.

Noch bemerkenswerter ist, dass auch der europäische Gesetzgeber die Frage nicht geklärt hat, obgleich dieser Vorgaben für Mitgliedstaaten konzipieren musste, bei denen die Embryospende mehrheitlich zulässig ist. Da die Richtlinienbestimmungen in den Mitgliedstaaten nicht unmittelbar anwendbar sind und man dem

\footnotetext{
${ }^{39}$ Die wohl mehrheitliche Lehre vertritt, dass die Trägerschaft von Menschenwürde an den Zeitpunkt der Syngamie, also der Verschmelzung der gegengeschlechtlichen Zellen, gebunden sei. Vgl. hierzu Hillgruber, in: Epping/Hillgruber (Hrsg.), Beck'scher Online Kommentar Grundgesetz, Art. 1, Rdn. 4 m.w.N.; anderer Ansicht beispielsweise Heun (Fußn. 9), der auf den Zeitpunkt der Nidation abzielen möchte. Vgl. zudem die jüngst entbrannte Debatte im Zusammenhang mit der Reform des Stammzellgesetzes: stellvertretend v.Renesse, Reform des Stammzellgesetzes: Ein neuer Stichtag?, ZRP 2008, 161 ff.; Buchbeim, Reform des Stammzellgesetzes: Ein neuer Stichtag?, ZRP 2008, 162 ff.
} 
nationalen Gesetzgeber angesichts des Schweigens der Richtlinien auch kein richtlinienwidriges Handeln vorwerfen kann, darf die Frage aber wohl einstweilen offen bleiben. ${ }^{40}$ Sollte es tatsächlich einmal zu einem Streitfall vor Gericht kommen, wäre möglicherweise eine Vorlage an den Europäischen Gerichtshof (EuGH) angezeigt.

\section{4 Ärztliches Berufsrecht}

\subsection{Eizell- und Embryospende}

Das ärztliche Berufsrecht darf mit dem Gesetz nicht in Widerspruch stehen. Daher ist es nicht verwunderlich, dass das Verbot der Verwendung fremder Eizellen bei reproduktionsmedizinischen Maßnahmen in Deutschland durch die ärztlichen Berufsordnungen wiederholt wird. Die alten Richtlinien der Bundesärztekammer zur assistierten Reproduktion von $1998^{41}$ verwiesen dabei nicht nur auf das ESchG, sondern machten sich die Überlegungen, die zum Verbot der Eizellspende geführt hatten, ausdrücklich zu eigen. Demnach „lasse eine gespaltene Mutterschaft besondere Schwierigkeiten bei der Selbstfindung des Kindes und negative Auswirkungen auf seine seelische Entwicklung befürchten. "42 Beachtlich ist allerdings, dass die Novelle der (Muster-)Richtlinien von $2006^{43}$ diesen Passus nicht übernommen hat. Das ärztliche Berufsrecht - in Gestalt der Berufsordnungen, Richtlinien und Empfehlungen der Ärztekammern - fällt freilich in die Zuständigkeit der Länder, und die Mustervorschriften der Bundesärztekammer richten sich an die für die Umsetzung in geltendes ärztliches Berufsrecht zuständigen Landesärztekammern. Diese haben das Berufsrecht inzwischen aber fast vollständig an die novellierten Musterrichtlinien der Bundesärztekammern angepasst. ${ }^{44}$

Zur Embryospende finden sich in den Richtlinien der Ärztekammern keine Vorgaben, weil diese Technik als für deutsche Ärzte und Ärztinnen nicht relevant eingestuft wird.

Ein ähnliches, den Inhalt des geltenden Fortpflanzungsmedizinrechts mehr oder weniger wiederholendes Berufsrecht existiert in Österreich ersichtlich nicht.

\footnotetext{
40 Vgl. in diesem Zusammenhang auch Vöneky/Petersen, Der rechtliche Status des menschlichen extrakorporalen Embryos: Das Recht der europäischen Union, EuR 2006, 340 ff.

41 Bundesärztekammer, Richtlinien zur Durchführung assistierter Reproduktion, Dt. Ärzteblatt 1998, $3166 \mathrm{ff}$.

42 Vgl. Bundesäržtekammer (Fußn. 41), 3171.

43 Bundesärztekammer, (Muster-) Richtlinie zur Durchführung der assistierten Reproduktion, Novelle 2006, Dt. Ärzteblatt 2006, 1392.

${ }^{44}$ Eine Ausnahme stellen die Richtlinien zur Durchführung der assistierten Reproduktion der Ärztekammer Saarland dar, die in Anhang I. weiterhin die Wertungen des Gesetzgebers anführen, abrufbar unter: http://www.aerztekammer-saarland.de/30/01Berufsordnung2/2004012001PDF.pdf (letzter Zugriff am 28.8.2008).
} 
Es ist auch nicht notwendig, weil das FMedG die wesentlichen Handlungsmaximen für die Ärzte und Ärztinnen enthält.

\subsection{Samenspende}

Während sich das ärztliche Berufsrecht in Deutschland hinsichtlich der Eizellspende auf eine Wiedergabe der gesetzlichen Regelung beschränken konnte und musste, war es viele Jahre lang der einzige Ort, an dem überhaupt nähere Vorgaben zur Samenspende zu finden waren. Bis heute findet sich im Berufsrecht die detaillierteste Regelung des gesamten Problemkomplexes. ${ }^{45}$

$\mathrm{Zu}$ den allgemeinen Voraussetzungen für eine assistierte Reproduktion gehören nach den novellierten Muster-Richtlinien der Bundesärztekammer zur assistierten Reproduktion von 2006 neben fachlicher Qualifikation des Arztes, medizinischer Indikation, Fehlen von Kontraindikationen sowie Beratung, Aufklärung und Einwilligung der Betroffenen auch das Vorliegen einer Ehe oder zumindest die Überzeugung des Arztes, dass die Frau in einer stabilen Partnerschaft lebt und ihr Partner die Vaterschaft anerkennen wird. ${ }^{46}$ Besondere Voraussetzungen für eine Verwendung von heterologem Samen sind: Scheitern einer Reproduktion im homologen System aus medizinischen Gründen, umfassende Aufklärung über psychosoziale, ethische und rechtliche Risiken der Verwendung von Spendersamen, näher spezifizierte serologische Untersuchungen und Quarantänezeit für Spendersperma, Ausschluss der Verwendung von Mischsperma, Dokumentation der Identität des Samenspenders, Einverständniserklärung des Spenders zur Preisgabe seiner Identität im Fall eines Auskunftsersuchens des Kindes und Befreiung des Arztes von seiner Schweigepflicht für diesen Fall durch die Eltern. Ferner sollen wichtige phänotypische Merkmale wie Blutgruppe, Augenfarbe, Haarfarbe, Größe, Statur und ethnische Zugehörigkeit erfasst und soll darauf geachtet werden, dass ein Spender nicht mehr als 10 Schwangerschaften erzeugt.

Auffällig gegenüber den Richtlinien von 1998 ist, dass viel mehr Zuständigkeiten und Verantwortlichkeiten auf den Arzt bzw. die Ärztin verlagert werden. Entfallen ist auch die generelle Bindung jeder Verwendung von Spendersamen an das positive Votum einer Kommission der Landesärztekammern.

${ }^{45}$ Dazu kommen freiwillige Selbstverpflichtungen von Verbänden u. dgl., so insbesondere Arbeitskreis für donogene Insemination, Richtlinien zur Qualitätssicherung der Behandlung mit Spendersamen in Deutschland, http://www.donogene-insemination.de/downloads/Richtl_Druckfassung.pdf (letzter Zugriff am 3.10.2008).

46 Vgl. Bundesärztekammer (Fußn. 43), 1395 (Punkt 3.1.1). 


\section{Sozialrecht}

Nicht viel weniger wirkungsvoll als unmittelbare Beschränkungen von ART, und insbesondere donogener Praktiken, sind Einschränkungen bei der Kostenübernahme durch die gesetzliche Krankenversicherung (GKV). ${ }^{47}$

\subsection{Leistungen der deutschen GKV}

Diesbezüglich gilt zurzeit in Deutschland \27a des Fünften Buches Sozialgesetzbuch (SGB V). Danach ist ein Leistungsanspruch der Versicherten an eine ganze Reihe von Voraussetzungen geknüpft, gemäß \ 27a Abs. 1 insbesondere an eine medizinische Indikation, hinreichende Aussicht auf Behandlungserfolg (nur drei Behandlungsdurchgänge), bestehende Ehe der Wunscheltern, ${ }^{48}$ Reproduktion im homologen System, ärztliche Beratung und Überweisung an eine Einrichtung mit spezieller Genehmigung. Damit sind donogene Praktiken von vorneherein vom Leistungsangebot der GKV ausgenommen. Nur der Vollständigkeit halber sei angemerkt, dass ein Anspruch auf Sachleistungen gemäß \27a Abs. 3 ohnehin nur für Versicherte bestehen kann, die mindestens 25 und höchstens 39 Jahre (weibliche Versicherte) bzw. 49 Jahre (männliche Versicherte) alt sind. Selbst wenn alle Voraussetzungen erfüllt sind, übernimmt die GKV nach derzeit geltender Rechtslage nur 50 vom Hundert der mit dem Behandlungsplan genehmigten Kosten. ${ }^{49}$

\subsection{Situation in Österreich}

In Österreich ist seit 1. 1. 2000 das In-vitro-Fertilisations-Fonds-Gesetz (IVFFonds-Gesetz $)^{50}$ in Kraft. Aus diesem Fonds, der zu gleichen Teilen aus Mitteln des Ausgleichsfonds für Familienbeihilfen und der Sozialversicherungsträger gespeist wird, werden bei Vorliegen der entsprechenden Voraussetzungen $70 \%$ der Kosten einer In-vitro-Fertilisation übernommen. Insgesamt werden vier Versuche pro Paar und angestrebter Schwangerschaft unterstützt. Anspruchsberechtigt sind aber nur Paare in Ehegemeinschaft oder eheähnlicher Lebensgemeinschaft in den Fällen von tubarer, durch Endometriose oder durch polyzystisches Ovar (PCO) bedingter Sterilität bei der Frau oder von Sterilität beim Mann, wenn alle anderen Möglichkeiten zur Herbeiführung einer Schwangerschaft bereits ausgeschöpft wurden. Die Höchstaltersgrenzen entsprechen denen des deutschen Sozialrechts. Ferner muss die Maßnahme in einer Einrichtung durchgeführt werden, die Vertragspartner des Fonds ist. Bei der Lektüre des Gesetzes ist freilich zu beachten,

\footnotetext{
${ }^{47} \mathrm{Vgl}$. dazu auch den Beitrag von Rauprich, Sollen Kinderwunschbehandlungen von den Krankenkassen finanziert werden? Ethische und rechtliche Fragen, in diesem Band.

48 Diese Einschränkung in $\int 27$ a Abs. 1 Nr. 3 ist nach Maßgabe der Entscheidungsformel mit dem GG vereinbar. Vgl. die Entscheidung des BVerfG v. 28. 2. 2007, BVerfGE 117, 316.

$49 \mathrm{Zu}$ den praktischen Folgen vgl. Michelmann (Fußn. 6).

50 IVF-Fondgesetz, BGBl. I. Nr. 189/1999.
} 
dass donogene Techniken in Österreich nur in Zusammenhang mit einer Inseminationsbehandlung zulässig sind (oben 2.2), die vom IVF-Fonds-Gesetz gerade nicht erfasst ist (vgl. $\int 1$ IVF-Fonds-Gesetz, der auf $\ 1$ Abs. 2 Nr. 2 bis 4 FMedG verweist). ${ }^{51}$ Daher sind auch in Österreich donogene Techniken nicht vom Leistungskatalog der GKV erfasst.

\section{Abstammungsrecht}

\subsection{Mutterschaft}

Das deutsche Familienrecht hat für den Fall, dass die Frau, die ein Kind austrägt und von ihm entbunden wird, nicht mit dessen genetischer Mutter übereinstimmt, eine denkbar einfache Lösung parat: Mutter ist nach $\ 1591$ BGB die Frau, die das Kind geboren hat. Dabei bleibt es unter allen Umständen, und keiner der Beteiligten kann daran - außer theoretisch im Wege der Adoption - etwas ändern. ${ }^{52}$

Die gleiche Lösung wurde in $\ 137 \mathrm{~b}$ ABGB vom österreichischen Gesetzgeber gewählt.

\subsection{Vaterschaft nach deutschem Recht}

Da die Abstammungsfrage auf väterlicher Seite auch schon lange vor dem Einzug reproduktionsmedizinischer Techniken schwierig war, existiert hierzu im deutschen Familienrecht ein ausführlicher Regelungskomplex. Danach gilt zunächst, dass zur gleichen Zeit immer nur eine Person in rechtlicher Hinsicht Vater eines Kindes sein kann, mit allen unterhaltsrechtlichen, erbrechtlichen usw. Konsequenzen, die dazu gehören. Existiert ein solcher Vater im rechtlichen Sinn, muss seine Vaterschaft im Wege der Anfechtung (\$S 1599 ff. BGB) beseitigt werden, bevor ein anderer an seine Stelle rücken kann.

Um in rechtlicher Hinsicht Vater zu werden, gibt es drei Möglichkeiten, zwischen denen ein Stufenverhältnis besteht ( $\$ 1592$ BGB). In erster Linie ist Vater eines Kindes der Mann, der zur Zeit der Geburt mit der Mutter verheiratet war. Erst wenn es einen solchen Mann nicht gibt oder wenn seine Vaterschaft durch Widerlegung der Abstammung wirksam angefochten wurde, kann in zweiter Linie der Mann einrücken, der die Vaterschaft wirksam anerkannt hat. Gibt es auch einen solchen Mann nicht oder wurde seine Vaterschaft durch Widerlegung der Abstammung angefochten, ist drittens Vater der Mann, dessen genetische Vater-

\footnotetext{
51 Zeugungsunfähigkeit kann durch die künstliche Befruchtung weder in ihrer Wurzel noch symptomatisch in medizinischer Hinsicht beeinflusst werden, sodass nach ständiger Rechtsprechung eine vom Sozialversicherungsträger getragene Krankenbehandlung iSd. $\int 120$ Abs. 1 Z. 1 iVm \133 Abs. 2 Satz 2 ASVG ausscheidet. Vgl. hierzu jüngst OGH, RdA 2007, 40 mit Anm. Binder. 52 Vgl. BT-Drs. 13/4899.
} 
schaft gerichtlich festgestellt wurde. Die Vaterschaft dieses genetischen Vaters kann nicht mehr angefochten werden. ${ }^{53}$

Daraus folgt zunächst, dass bei einer Samenspende der soziale Vater, der mit der Mutter verheiratet ist oder in einer festen Beziehung lebt, qua Ehe oder durch Anerkennung zunächst Vater des Kindes im Rechtssinn ist. Daraus folgt aber auch, dass seine Vaterschaft grundsätzlich durch Anfechtung beseitigt werden kann und dass damit der Weg frei ist für eine Anerkennung durch den Samenspender oder durch dessen gerichtliche Feststellung als genetischer Vater. Dem nicht seltenen Phänomen, dass ein Partner, der zunächst mit allen Kräften die Kinderwunschbehandlung mit Spendersamen unterstützt hatte, nach einer Trennung plötzlich die Vaterschaft anficht, hat der Gesetzgeber vor einigen Jahren freilich einen Riegel vorgeschoben. ${ }^{54}$ Nach \ 1600 Abs. 4 BGB ist die Anfechtung durch den sozialen Vater oder durch die Mutter in diesem Fall ausgeschlossen. Was allerdings bleibt, ist das Anfechtungsrecht des Kindes.

Letztendliche Sicherheit, nie als Vater des Kindes herangezogen zu werden, kann einem Samenspender daher nicht zugesichert werden, wenngleich man natürlich versucht, ihn durch Verträge zwischen den Beteiligten so weit wie möglich abzusichern. Auch seine anfängliche Anonymität hilft ihm nicht unbedingt, weil das Kind ein verfassungsrechtlich anerkanntes Recht auf Kenntnis der eigenen genetischen Abstammung hat und damit auf Preisgabe der Identität durch den behandelnden Arzt ${ }^{55}$ (näher unten 6.4).

\subsection{Vaterschaft nach österreichischem Recht}

Auch \ 138 ABGB enthält eine ähnliche Stufenfolge für die Begründung der Vaterschaft wie $\int 1592$ BGB. Danach ist Vater, wer im Zeitpunkt der Geburt mit der Mutter verheiratet ist, wer die Vaterschaft anerkannt hat oder wessen Vaterschaft gerichtlich festgestellt wurde. Die so begründete Vaterschaft wirkt an sich gegenüber jedermann ( $(138$ a Abs. 1 ABGB), kann aber unter bestimmten Umständen wieder beseitigt werden.

War die Vaterschaft durch die Ehe mit der Mutter begründet, kann zunächst eine Anfechtung seitens des Mannes oder des Kindes (\$S 156, 163 ABGB) erfolgen. Der als Vater geltende Ehemann selbst kann gemäß \ 157 ABGB allerdings nicht anfechten, wenn das Kind mit Spendersamen gezeugt wurde und der Mann dem in Form eines gerichtlichen Protokolls oder eines Notariatsakts zugestimmt hat. Möglich ist ferner ein Antrag des Kindes auf Feststellung der wahren Vater-

\footnotetext{
53 Ausführlich Rauscher, in: Staudinger (Hrsg.), Kommentar zum Bürgerlichen Gesetzbuch, Neubearbeitung 2004, \ 1592, Rdn. 10 ff.; Seidel, in: Münchner Kommentar zum BGB, 5. Aufl. 2008, $\$ 1592$, Rdn. 1 ff.

${ }^{54}$ Diese Änderung erfolgte durch das Gesetz zur weiteren Verbesserung von Kinderrechten (Kinderrechtsverbesserungsgesetz - KindRVerbG) v. 9.4.2002 (BGBl. I S. 1239).

${ }_{55}$ Vgl. hierzu ausführlich Habn, in: Bamberger/Roth (Hrsg.), Beck'scher Online Kommentar zum BGB, Stand 1.1.2008, \1591, Rdn. 16 ff.
} 
schaft ( $\iint 163$ b, 163 ABGB). Dabei sind jedoch $\ 163$ Abs. 3 und 4 ABGB zu beachten, wonach im Rahmen des Vaterschafts-Feststellungs-Verfahrens nach Spendersamenbehandlung derjenige Mann, der der Behandlung in Form eines gerichtlichen Protokolls oder eines Notariatsakts zugestimmt hatte, als Vater festgestellt werden muss ( $\$ 163$ Abs. 3 ABGB), sofern nicht nachgewiesen werden kann, dass das Kind tatsächlich gar nicht aus der Behandlung hervorgegangen ist. Dagegen ist ein Samenspender, der nach dem vom FMedG vorgesehenen Verfahren gespendet und dabei zum Ausdruck gebracht hat (vgl. \13 Abs. 1 FMedG), nicht als Vater des Kindes festgestellt werden zu wollen, vor der Feststellung seiner Vaterschaft sicher (\$163 Abs. 4 ABGB). Es wird freilich vertreten, dass $\ 163$ Abs. 4 teleologisch zu reduzieren sei, wenn Samenspender und Kind gemeinsam ein Anerkenntnis wollen. ${ }^{56}$ Schließlich kann eine durch Ehe begründete Vaterschaft beseitigt werden durch ein „durchbrechendes“ Anerkenntnis nach $\ 163 \mathrm{e}$ Abs. 2 ABGB, d. h. das Anerkenntnis eines anderen Mannes als des gegenwärtigen Vaters, dem das Kind bzw. Mutter und Kind mit öffentlicher oder öffentlich beglaubigter Urkunde zustimmen.

War die Vaterschaft von vorneherein nicht durch Ehe, sondern durch ein Anerkenntnis begründet, so ist - über einen Antrag des Kindes nach $\$ \int 163 \mathrm{~b}, 163$ oder das durchbrechende Anerkenntnis nach $\$ 163 \mathrm{e}$ Abs. 2 ABGB hinaus - die Möglichkeit gegeben, dass dem Anerkenntnis gemäß \164 ABGB nachträglich widersprochen wird, was auch durch den Anerkennenden selbst geschehen kann. Auch hier ist dem Mann allerdings der Widerspruch verwehrt, wenn das Kind (tatsächlich, vgl. \164 Abs. 1 Nr. 3 a) durch eine medizinisch unterstützte Fortpflanzung mit dem Samen eines Dritten gezeugt worden ist und der Mann dem in der dafür vorgesehenen Form zugestimmt hatte ( $\mathbb{1} 164$ Abs. 1 Nr. 2).

War die Vaterschaft durch gerichtliche Feststellung begründet, so kann der so zu Unrecht festgestellte Vater einen Abänderungsantrag nach $\$ \int 72$ ff. AußStrG stellen.

\subsection{Aufdeckung der genetischen Abstammungsverhältnisse}

Das Bundesverfassungsgericht (BVerfG) hat aus dem durch Art. 2 Abs. 1 mit Art. 1 Abs. 1 GG abgesicherten allgemeinen Persönlichkeitsrecht des Kindes ein Recht auf Kenntnis der eigenen Abstammung gefolgert. ${ }^{57}$ Entsprechendes wird für Art. 8 EMRK $^{58}$ sowie für Art. 7 Abs. 1 des UN-Übereinkommens über die Rechte des Kindes vom 20.11.198959 vertreten. Freilich hat das BVerfG klargestellt, dass

\footnotetext{
56 Vgl. Stabentheiner, in: Rummel (Hrsg.), Kommentar zum ABGB, 3. Aufl. 2003, \163 Rdn. 1c; Steininger, Interpretationsvorschläge für die neuen Normierungen im ABGB über die väterliche Abstammung, ÖJZ 1995, $121 \mathrm{ff}$.

${ }^{57}$ BVerfG v. 31.1.1989, BVerfGE 79, 256. Dazu eingehend und durchaus kritisch Heun (Fußn. 9).

${ }^{58}$ Vgl. EGMR, Mikulic v. Kroatien, 7.2.2002, Application No. 53176/99.

${ }^{59}$ Vgl. Huber, in: Münchner Kommentar zum BGB, Vorbem. zu $\int S$ 1626-1699, Rdn. 8, insbesondere auch zu den weitreichenden Vorbehalten der deutschen Bundesregierung.
} 
das allgemeine Persönlichkeitsrecht auch in dieser Ausprägung nicht vorbehaltlos gewährleistet ist, sondern im Hinblick auf grundrechtlich geschützte Interessen Dritter Einschränkungen erfahren kann, ${ }^{60}$ und der Europäische Gerichtshof für Menschenrechte hat diese Einschätzung im Hinblick auf Art. 8 und 14 EMRK bestätigt. 61

Das österreichische Recht hat diesen Konflikt zwischen widerstreitenden Rechten und Interessen seit langem in \20 FMedG zu lösen versucht. Danach sind zwar die Spenderdaten vertraulich zu behandeln. Dem mit dem Samen eines Dritten gezeugten Kind ist jedoch auf dessen Verlangen nach Vollendung des vierzehnten Lebensjahrs Einsicht in die Aufzeichnungen zu gewähren und daraus Auskunft zu erteilen. Der gesetzliche Vertreter oder der Erziehungsberechtigte können zum Wohl des Kindes in medizinisch begründeten Ausnahmefällen und mit pflegschaftsgerichtlicher Genehmigung Einsicht und Auskunft verlangen. Gerichten und Behörden steht ein Einsichts- und Auskunftsrecht zu, sofern dies für die Erfüllung ihrer Aufgaben unentbehrlich ist.

In Deutschland dagegen fehlt letztlich nach wie vor eine positivgesetzliche Regelung zum Recht des Kindes auf Kenntnis seiner genetischen Abstammung. Explizit erwähnt ist dieses Recht - soweit ersichtlich - nur in \14 Abs. 3 TPG, wo aber nur ausgesagt wird, das Recht bleibe im Falle der Samenspende von den Datenschutzbestimmungen des TPG „unberührt“. Wie das Recht durchgesetzt werden kann und soll ist dagegen nach wie vor in wesentlichen Punkten unklar. Gleichsam reflexartig werden bestimmte faktische Voraussetzungen der Rückverfolgung von \15 Abs. 2 TPG geschaffen, wonach bestimmte Daten 30 Jahre nach der Übertragung lang aufzubewahren, danach allerdings zu anonymisieren oder zu löschen sind. Zu diesen Daten gehören nach $\iint 5$ Abs. 2, 6 Abs. 1 Satz 4 TPGGewV Familienname, Vorname, Tag der Geburt, Anschrift oder die von der Gewebeeinrichtung für den Spender vergebene Zuordnungsnummer. Einen weiteren Schritt hat der erst jüngst ${ }^{62}$ eingefügte $\int 1598$ a BGB gebracht, der als Reaktion auf eine Entscheidung des BVerfG ${ }^{63}$ sowie zahlreiche politische Initiativen Ansprüche zwischen Vater, Mutter und Kind (im Rechtssinne) begründet, in medizinische Untersuchungen zum Zwecke der Klärung der genetischen Abstammung einzuwilligen. Damit hat das aus einer Gametenspende hervorgegangene Kind - sofern es denn überhaupt Verdacht schöpft - zumindest die Möglichkeit, sich Gewissheit über die Nichtabstammung von einem Elternteil ${ }^{64}$ oder gar beiden zu verschaffen.

\footnotetext{
${ }^{60}$ So wird das Recht des Kindes auf Kenntnis der eigenen Abstammung insoweit relativiert, als ihm das Recht der Mutter auf Achtung ihrer Privat- und Intimsphäre entgegensteht. Vgl. BVerfG v. 6.5.1997, BVerfGE 96, 56.

${ }^{61}$ Vgl. EGMR, Odièvre v. Frankreich, 13.2.2003, Application No. 42326/98.

62 Gesetz zur Klärung der Vaterschaft unabhängig vom Anfechtungsverfahren v. 10.3.2008 (BGBl I S. 441).

${ }^{63}$ BVerfG v. 13.2.2007, NJW 2007, 753 ff.

${ }^{64}$ Dazu, dass auch die Klärung der Mutterschaft umfasst sein muss, vgl. Hahn, in: Bamberger/Roth (Hrsg.), Beck'scher Online Kommentar zum BGB, Stand 1.4.2008, \1598a, Rdn. 2; Wellenhofer, Das
} 
Weiterhin nicht geregelt ist allerdings ein Anspruch des Kindes gegen den (möglichen) leiblichen Vater bzw. die (mögliche) leibliche Mutter, so dass es, wenn die sozialen Eltern trotz bestehender Auskunftsansprüche ${ }^{65}$ schweigen, kaum positiv Gewissheit über die Abstammung von einer bestimmten Person erlangen kann. ${ }^{66}$ Umgekehrt besteht nach \1598a BGB auch kein Anspruch des (mutmaßlichen) leiblichen Vaters gegen das Kind, obgleich das BVerfG diesbezüglich recht klare Vorgaben gemacht hatte. ${ }^{67}$

Ganz unabhängig von berufsrechtlichen Verpflichtungen (dazu oben 4.2) holen die reproduktionsmedizinischen Zentren, die an Samenspenden beteiligt sind, allerdings schon aus Angst vor Haftungsansprüchen von allen Beteiligten die Einwilligung ein, dass das volljährige Kind auf Verlangen Zugang zu den Spenderdaten erhalten kann. ${ }^{68}$ Berufsrecht einerseits und drohende zivilrechtliche Schadensersatzansprüche andererseits sind damit der schwankende Boden, auf dem das Recht auf Kenntnis der eigenen Abstammung derzeit wenigstens ansatzweise durchgesetzt wird. ${ }^{69}$

\section{Resümee}

Der Überblick über die derzeitigen rechtlichen Rahmenbedingungen donogener ART in Deutschland und Österreich hat vor allem deutlich gemacht, dass Eizellspende und Samenspende eklatant unterschiedlich behandelt werden, und zwar erstens, was ihre generelle Zulässigkeit anbelangt - gänzliches Verbot der Eizellspende gegenüber grundsätzlicher Erlaubtheit der Samenspende - und zweitens, was ihre familienrechtlichen Folgen anbelangt - keine Anfechtung der Mutterschaft gegenüber grundsätzlicher Anfechtbarkeit der Vaterschaft, wobei allerdings in Österreich (oben 6.3) weitestgehend für die Vaterschaft desjenigen Mannes gesorgt ist, der als sozialer Wunschvater wirksam in die Spendersamenbehandlung eingewilligt hatte.

Weiterhin ist festzustellen, dass donogene Techniken durch Gesetz oder Berufsrecht auf heterosexuelle, stabile Partnerschaften beschränkt werden und dass

neue Gesetz zur Klärung der Vaterschaft unabhängig vom Anfechtungsverfahren, NJW 2008, 1185 ff. (1186).

65 Vgl. etwa Hahn (Fußn. 64), \1598a, Rdn. 2.

${ }^{66}$ Hierzu kritisch auch Wellenhofer, in: Münchner Kommentar zum BGB, 5. Auflage 2008, \1598a, Rdn. 5 ff.

${ }^{67}$ Vgl. BVerfG (Fußn. 63), 754 f.

${ }^{68}$ Zur Haftung bei Anonymitätszusage vgl. Seidel (Fußn. 53), \1589, Rdn. 44. Stellvertretend vgl. etwa die rechtlichen Informationen auf der Homepage der Erlanger Samenbank, abrufbar unter: http://www.erlanger-samenbank.de/spenderinfo/recht.html (letzter Zugriff am 3.9.2008). Voraussetzung ist allerdings stets, dass das Kind genügend Energie aufbringt, um die richtige Spendereinrichtung zu identifizieren.

${ }^{69}$ Vgl. in diesem Zusammenhang auch Katzorke, Entstehung und Entwicklung der Spendersamenbehandlung in Deutschland, in diesem Band, der sich mit guten Gründen für die Schaffung einer zentralen Dokumentationsstelle ausspricht. 
sie generell vom Leistungskatalog der GKV ausgeschlossen sind. Eine zusätzliche Ungleichbehandlung nimmt das österreichische Recht vor, das zwar die Insemination, nicht aber die IVF mit Spendersamen erlaubt.

All diese Differenzierungen - insbesondere aber die unterschiedliche Behandlung von Eizellspende und Samenspende, die auch Konsequenzen für die Embryospende hat - müssen sich einer verfassungsrechtlichen, ethischen und rechtspolitischen Überprüfung stellen. Eine umfassende Diskussion muss anderen Beiträgen dieses Bandes vorbehalten bleiben. ${ }^{70}$

70 Siehe hierzu insbesondere die Beiträge von Heun (Fußn. 9); Rauprich (Fußn. 47); Blyth, Donor insemination and the dilemma of the "unknown father"; Crawshaw, "What about my right to choose?" Young people with physical impairments exercising reproductive choice; Herrmann-Green, Lesben mit Kinderwunsch: Eine ethische Herausforderung für die Reproduktionsmedizin?; Thorn, Samenspende und Stigmatisierung - ein unauflösbares Dilemma?; Wiesemann, Der Embryo im Kontext: Warum die Biopolitik die menschlichen Beziehungen nicht vergessen darf; jeweils in diesem Band. 



\title{
Genetische Risiken durch Keimzellspende
}

\author{
Barbara Zoll
}

Etwa $10-15 \%$ aller Paare in Deutschland sind ungewollt kinderlos, wobei die Anzahl ungewollt kinderloser Paare in Europa tendenziell ansteigt. ${ }^{1}$ Von ungewollter Kinderlosigkeit oder Sterilität wird gesprochen, wenn in Abhängigkeit vom Alter der Frau innerhalb von ein bis zwei Jahren bei regelmäßigem Geschlechtsverkehr keine Schwangerschaft eintritt.

Die Ursachen der Sterilität liegen in jeweils $40-45 \%$ der Fälle in körperlichen Störungen der Frau bzw. des Mannes und in $10-20 \%$ der Fälle in psychischen oder in nicht näher erklärbaren idiopathischen Störungen. ${ }^{2}$ Seit der Geburt von Louise Brown im Jahr 1978, dem ersten artifiziell gezeugten Kind, ist bekannt, dass die IVF (In-vitro-Fertilisation) eine effiziente Methode zur Herbeiführung von Schwangerschaften ist. ${ }^{3}$ Die Erfolgsquote hängt dabei von der Ursache der Sterilität ab. Weniger erfolgreich als z.B. bei Verschluss der Eileiter ist die IVF bei männlicher Infertilität, z.B. reduzierter Spermienzahl und -beweglichkeit oder Anomalien der Spermienanatomie. Dies hat zur Einführung von ICSI (intrazytoplasmatische Spermatozoeninjektion) geführt, bei der ein Spermium mit einer Mikropipette

1 Hellenbroich, Genetische Beratung in der Reproduktionsmedizin. Gynäkologische Endokrinologie, 5. Aufl.2007, S. 26 ff.

${ }^{2}$ Fischl, Sterilität - Fertilität, in: Fischl (Hrsg.), Kinderwunsch - Möglichkeiten, Erfüllbarkeit und Machbarkeit im neuen Jahrtausend, 2000, S. 47.

${ }^{3}$ Edwards/Streptoe/Purdy, Establishing full-term human pregnancies using cleaving embryos, British Journal of Obstetrics and Gynaecology 1980, $737 \mathrm{ff}$. 
in die Eizelle injiziert wird. ${ }^{4}$ Vielen Paaren kann durch Anwendung dieser Methode zur Realisierung ihres Kinderwunsches verholfen werden. Voraussetzung für eine erfolgreiche IVF-/ICSI-Therapie ist jedoch eine ausreichende Funktion der Gonaden. Für einige Patienten, die z.B. keine Spermien oder Eizellen produzieren, ist IVF oder ICSI nicht geeignet. Als therapeutisches Verfahren, um den Kinderwunsch solcher Paare zu befriedigen, steht lediglich eine Samen- oder Eizellenspende zur Verfügung.

Während lt. Embryonenschutzgesetz (ESchG) die Eizellspende in Deutschland nicht erlaubt ist, wird die Samenspende geduldet, wobei die rechtlichen Beziehungen zwischen den beteiligten Personen, Samenspender, Wunscheltern und Kind nicht geklärt sind. In der folgenden Stellungnahme soll aus humangenetischer Sicht sowohl auf die Samenspende als auch die Eizellspende eingegangen werden, da in einigen europäischen Ländern die Eizellspende erlaubt ist und deutsche Paare das Angebot der Herbeiführung einer Schwangerschaft mit fremden Eizellen im Ausland wahrnehmen.

Nach Einführung der ICSI, für die nur wenige Spermien zur Befruchtung der Eizellen verfügbar sein müssen und durch die somit auch subfertilen Männern zu eigenen Kindern verholfen werden kann, ist die Geburt von Kindern nach donogenen Inseminationen deutlich von geschätzt 1.500 bis 2.000 vor Einführung der IVF/ICSI auf ca. 1.000 pro Jahr zurückgegangen. ${ }^{5}$ Über die Anzahl der Geburten nach Eizellspenden liegen keine Angaben vor. Auch wenn die Anzahl der Paare, die eine Keimzellspende wünschen, gering ist, handelt es sich um eine Gruppe von Personen, die sich von den Paaren, die eine Lebensgemeinschaft bilden, abhebt. In einer normalen Paarbeziehung kennen sich die Partner und haben die Möglichkeit, Informationen über die persönliche und familiäre Lebensgeschichte auszutauschen und Risiken für Erkrankungen/Behinderungen zukünftiger Kinder abzuschätzen. Die Keimzellspender aber bleiben anonym und die Wunscheltern müssen sich auf die Sorgfalt bei der Auswahl der Eizellspenderin bzw. des Samenspenders durch den Arzt verlassen.

Es erhebt sich daher die Frage, ob den Paaren, die eine donogene Insemination oder eine Eizellspende wünschen, eine besondere Fürsorgepflicht entgegen gebracht werden muss, welche Voraussetzungen die Keimzellspender mitbringen müssen und welche Untersuchungen den Spendern und den Wunschelternpaaren ggf. angeboten werden sollten.

In diesem Kontext hat der Arbeitskreis für donogene Insemination 2006 Empfehlungen zur Qualitätssicherung der Behandlung mit Spendersamen in Deutsch-

\footnotetext{
${ }^{4}$ Palermo/Joris/Devroi/v.Steirteghem, Pregnancies after intracytoplasmic injection of single spermatozoon into an oocyte, The Lancet 1992/340, 17 ff.

${ }^{5}$ Katzorke, Entstehung und Entwicklung der Spendersamenbehandlung in Deutschland, Journal für Reproduktionsmedizin und Endokrinologie 2008, 14 ff.
} 
land erarbeitet. ${ }^{6}$ In diesen Empfehlungen wird zunächst ein Infektionsscreening auf z.B. HIV, Hepatitis B und C und venerische Infektionen empfohlen. Im Weiteren wird angeraten, der Arzt solle den Samenspender nach eigenen genetisch bedingten und familiär vorkommenden Erkrankungen befragen. Männer, in deren Familien bestimmte Erkrankungen, die katalogisiert aufgeführt sind, vorkommen, sollten von der Spende ausgeschlossen werden. Genetische Routineuntersuchungen des Spenders sind in den Empfehlungen nicht vorgesehen.

Von Seiten der Humangenetiker erheben sich in diesem Zusammenhang mehrere Fragen, die zu Diskussionen und ggf. Handlungsbedarf Anlass geben.

- Was ist über genetisch bedingte Erkrankungen der Keimzellspender und ihrer Familien vor der Ei- oder Samenspende bekannt?

- Wie kann das Risiko für genetisch bedingte Erkrankungen bei Kindern von Ei- oder Samenspendern minimiert werden?

- Ist eine besondere Behandlung der Paare, die eine Ei- oder Samenspende wünschen, gegenüber normalen Eltern gerechtfertigt?

\section{Genetisch bedingte Erkrankungen des Samenspenders, bzw. der Eizellspenderin und deren Familienangehörigen}

Unter allen Neugeborenen leiden $3-5 \%$ an einer genetisch bedingten Erkrankung oder Fehlbildung, 0,7\% an multiplen Fehlbildungen und 0,5\% an einer schweren geistigen Behinderung. Hinzu kommen genetisch bedingte Erkrankungen und Behinderungen, die erst im späteren Kindes- oder Erwachsenenalter erkennbar werden. Die Ursachen der genetisch bedingten Erkrankungen verteilen sich zu ca. 5,5\% auf Anomalien der Chromosomen, zu 12,5\% auf monogene Erkrankungen, die durch Mutationen in nur einem Gen hervorgerufen werden und zu $82 \%$ auf polygen/multifaktorielle Erkrankungen, die durch das Zusammentreffen von $\mathrm{Mu}$ tationen in mehreren Genen und unter Beteiligung von Umweltfaktoren auftreten. ${ }^{7}$

\subsection{Chromosomenanomalien}

Der normale menschliche Chromosomensatz besteht aus 46 Chromosomen, 22 sog. Autosomenpaaren und den Geschlechtschromosomen X und Y (weiblich 2 $\mathrm{X}$-Chromosomen, männlich ein $\mathrm{X}$ - und ein Y-Chromosom, Abb. 1). Bei ca. 1,5\% der Menschen liegt eine balancierte Chromosomenanomalie (Chromosomenumbau) vor, die bei dem Träger keine negativen Auswirkungen hat, die jedoch bei den Nachkommen zu einem unbalancierten Chromosomensatz mit Verlust oder Zugewinn chromosomalen Materials führen kann. Unbalancierte Chromosomenbe-

${ }^{6}$ Hammel/Bispink/Katzorke/Schreiber/Thorn. Empfehlungen des Arbeitskreises für Donogene Insemination (DI) zur Qualitätssicherung der Behandlung mit Spendersamen in Deutschland in der Fassung vom 8.2.2006, Journal für Reproduktionsmedizin und Endokrinologie 2006, 166 ff.

7 Tariverdian/Buselmaier, Humangenetik, 3. Aufl. 2004, S. 305. 
funde äußern sich bei den Trägern in Fehlbildungen und geistiger Behinderung. Wie kann ausgeschlossen werden, dass der/die Keimzellspender/in Träger/in einer balancierten Translokation ist? Aus der Familienanamnese könnten sich durch das gehäufte Vorkommen von Fehlgeburten, Totgeburten oder körperlich und geistig behinderten Kindern Hinweise auf eine mögliche familiäre Chromosomenanomalie ergeben (Abb. 2). Um diese Hinweise auf familiäre Chromosomenanomalien zu bekommen, bedarf es einer gezielten Erstellung des Stammbaums mit expliziter Frage nach z.B. Fehlgeburten/Totgeburten und behinderten Kindern bei näheren Verwandten. Wenn sich aus der Anamnese ein Hinweis auf ein erhöhtes Risiko für eine Chromosomenanomalie ergibt, sollte der Keimzellspender chromosomal untersucht oder als Spender ausgeschlossen werden. Bei einem allgemeinen Risiko von 1,5\% für eine Chromosomenanomalie wäre zu überlegen, ob nicht grundsätzlich bei allen Keimzellspendern eine Chromosomenanalyse durchgeführt werden sollte, um das Risiko für Erkrankungen/ Behinderungen der durch sie gezeugten Kinder zu reduzieren.

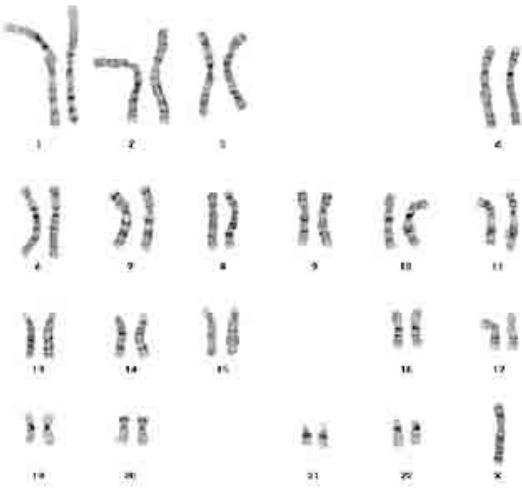

Abb.1:

Normaler Chromosomensatz 46, XY

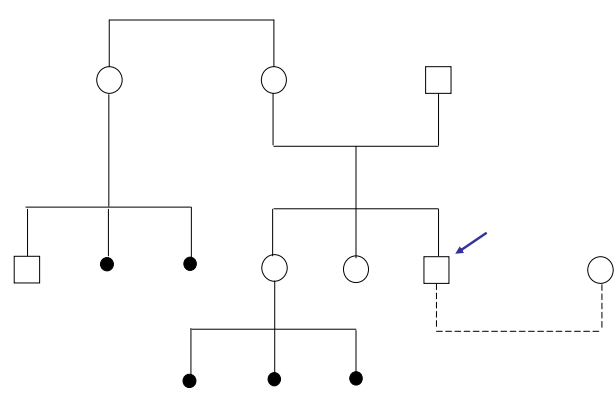

Abb. 2:

Fehlgeburten bei der Schwester und der Tante mütterlicherseits des Samenspenders

Stammbaumsymbole:

$\square$ : männlich, $\bigcirc$ : weiblich, $\quad \swarrow$ : Samenspender, $\square$ : Betroffene, $\quad$ : Fehlgeburten - - - - : Verbindung Samenspender und Empfängerin, ___ : Ehepartner/Blutsverwandte. 


\subsection{Monogene Erkrankungen}

Bei den monogenen Erkrankungen unterscheidet man autosomal dominante, autosomal rezessive und geschlechtsgebundene Erbgänge. Autosomal dominante Erbkrankheiten werden durch Mutationen in nur einer der doppelt vorliegenden Kopien eines Gens (väterlicher und mütterlicher Herkunft) hervorgerufen. Bei autosomal rezessiv erblichen Erkrankungen liegen Mutationen in beiden Kopien des Gens vor. Geschlechtsgebundene, X-chromosomal rezessiv erbliche Erkrankungen werden durch Mutationen in Genen des X-Chromosoms verursacht. Bei diesem Vererbungsmodus werden männliche Personen wegen des Vorliegens nur eines X-Chromosoms erkranken, während weibliche Mutationsträger üblicherweise gesund sind, diese Mutationen aber an ihre Söhne weitergeben können. Bei diesem Erbgang beobachtet man das sog. Überspringen von Generationen.

\subsection{Autosomal dominante Erkrankungen}

Wenn Eizellspenderinnen oder Samenspender im Kontaktgespräch mit dem Facharzt eigene autosomal dominant erbliche Erkrankungen angeben, so werden sie als Keimzellspender ausgeschlossen. Es gibt jedoch Erkrankungen, die erst im fortgeschrittenen Erwachsenenalter (z.B. Chorea Huntington, verschiedene neurologische Erkrankungen) auftreten und bei jüngeren Personen daher klinisch (noch) nicht nachweisbar sind. Wenn solche Erkrankungen in der Familie vorkommen, dem Keimzellspender aber nicht bekannt sind bzw. der entsprechende Elternteil noch nicht erkrankt ist, so hat nicht nur die/der potenzielle Ei- bzw. Samenspender ein erhöhtes Risiko, an dieser spät manifestierenden Krankheit zu erkranken, sondern auch seine Nachkommen. Das Erkrankungsrisiko kann für die Kinder bis zu 50\% betragen. Für einige Erkrankungen ist eine sog. Antizipation bekannt, d.h., dass die Symptome der Erkrankung von Generation zu Generation in jüngerem Alter auftreten. Es wäre daher nicht ausgeschlossen, dass bereits das mit fremden Keimzellen gezeugte Kind im Kindes- oder Jugendalter an einer dieser Erkrankungen leiden wird.

Einige autosomal dominant erbliche Erkrankungen verursachen nicht bei jedem Genträger eine Symptomatik. Dieses Phänomen wird als unvollständige Penetranz bezeichnet. In anderen Fällen zeigen die Patienten eine variable Manifestation der Erkrankung, die von schwerer Symptomatik bis zu nur leichten gesundheitlichen Abweichungen reichen kann. Letzteres wird als unterschiedliche Expressivität bezeichnet. Kommen solche Erkrankungen in einer Familie vor, so werden diese möglicherweise nicht wahrgenommen bzw. bagatellisiert, da der Charakter und die Bedeutung des autosomal dominanten Erbgangs dieser Erkrankungen mit ihren Risiken für den Samenspender und seine Nachkommen nicht erkannt werden. 


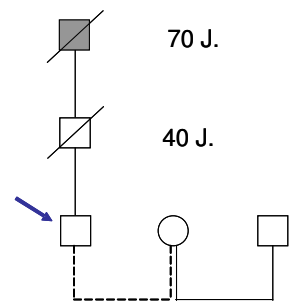

\begin{abstract}
Abb. 3:
Spät manifestierende, autosomal dominante Erkrankung des Großvaters des Samenspenders; Vater des Samenspenders vor möglichem Erkrankungsbeginn verstorben; Risiko des Samenspenders für die spät manifestierende Erkrankung rechnerisch $25 \%$.
\end{abstract}

\title{
1.4 Autosomal rezessive Erkrankungen
}

In der Familie der Eizellspenderin/des Samenspenders oder der Empfängerin kommen z.B. Personen mit einer autosomal rezessiven Erkrankung vor. In Abhängigkeit vom Verwandtschaftsgrad zu den Betroffenen haben die Keimzellspender bzw. die Wunschmutter ein deutlich erhöhtes Risiko, heterozygote gesunde Träger einer diese Erkrankung verursachenden Mutation zu sein (Abb. 4, Abb. 5). Personen, in deren Familie diese entsprechende Krankheit nicht vorkommt, haben das allgemeine Risiko der Bevölkerung, unerkannte Träger einer rezessiven krankheitsverursachenden Mutation zu sein. Bei einigen Erkrankungen liegen die Heterozygotenfrequenzen (Häufigkeit der gesunden Anlageträger in der Bevölkerung) bei $1-10 \%$ (z.B. Hämochromatose 10\%; Cystische Fibrose 4\%; Spinale Muskelatrophie ca. 3\%; Phenylketonurie 2\%). Das Erkrankungsrisiko für ein Kind ist daher bei belastender Familienanamnese deutlich höher als für ein Kind aus unbelasteter Familie.

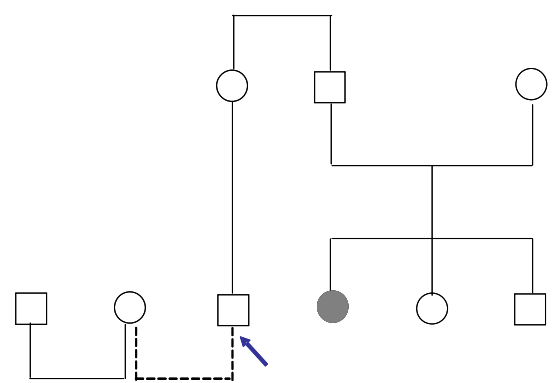

Abb. 4:

Autosomal rezessive Erkrankung bei einer Cousine des Samenspenders; Risiko des Samenspenders für Heterozygotie $25 \%$

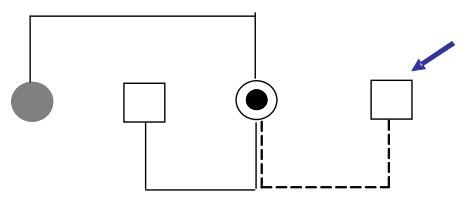

Abb. 5:

Autosomal rezessive Erkrankung der Schwester der Empfängerin des Spendersamens; Heterozygotie der Spendersamenempfängerin 
Tab. 1: Vergleich der Inzidenzen unterschiedlicher autosomal rezessiver Erkrankungen mit dem Homozygotenrisiko bei familiärer Belastung:

\begin{tabular}{|l|c|c|c|}
\hline Erkrankung & $\begin{array}{l}\text { Bevölkerungs- } \\
\text { risiko }\end{array}$ & $\begin{array}{l}\text { Heterozygoten- } \\
\text { frequenz }\end{array}$ & $\begin{array}{l}\text { Risiko bei } \\
\text { Stammbaum- } \\
\text { situation } \\
\text { Abb. 5 }\end{array}$ \\
\hline Cyst. Fibrose & $1: 2.500$ & $1: 25$ & $1: 100$ \\
\hline $\begin{array}{l}\text { Spinale Muskel- } \\
\text { atrophie }\end{array}$ & $1: 5.000$ & $1: 35$ & $1: 140$ \\
\hline Phenylketonurie & $1: 10.000$ & $1: 50$ & $1: 200$ \\
\hline
\end{tabular}

\subsection{Geschlechtsgebundene Erkrankungen}

Geschlechtsgebundene Erkrankungen spielen bei der Samenspende keine wesentliche Rolle, da ein potenzieller Samenspender mit einer X-chromosomal rezessiven Erkrankung als Spender nicht in Frage käme. Unabhängig davon hätten Kinder dieses Spenders kein erhöhtes Risiko, an der X-chromosomal rezessiven Erkrankung zu leiden. Alle Töchter wären jedoch gesunde Anlageträgerinnen des veränderten Gens. Anders verhält es sich jedoch bei der Eizellspende. Sollte in der Familie einer Eizellspenderin eine X-chromosomal rezessive Erkrankung vorkommen, so hat die Spenderin ggf. ein erhöhtes Risiko, gesunde Trägerin des krankheitsverursachenden Gens zu sein. Sollte sie heterozygote Anlageträgerin des X-chromosomalen krankheitsverursachenden Gens sein, so hätten ihre Söhne ein Risiko von 50\%, die Erkrankung zu entwickeln. Töchter wären mit 50\%iger Wahrscheinlichkeit wieder Anlageträgerinnen.

\subsection{Polygene Erkrankungen}

Polygen oder multifaktoriell bedingte Erkrankungen verhalten sich ähnlich wie autosomal dominante Erkrankungen mit reduzierter Penetranz. Wenn ein oder mehrere, auch entfernter verwandte Familienmitglieder des Keimzellspenders an einer multifaktoriellen Erkrankung leiden, wäre das Risiko für eine ebensolche Erkrankung bei Nachkommen des Spenders gegenüber dem Risiko der Durchschnittsbevölkerung erhöht. 


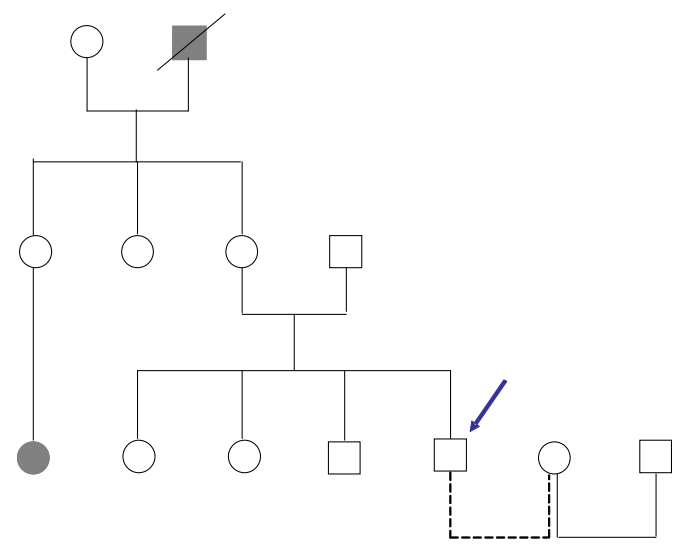

Abb. 6:

Polygen/ multifaktorielle Erkrankung des Großvaters und einer Cousine des Samenspenders; erhöhtes Wiederholungsrisiko bei Nachkommen des Samenspenders.

\subsection{Verwandtenbeziehungen}

Ein nicht zu unterschätzendes Risiko besteht darin, dass zufällig Partnerschaften von Nachkommen desselben Samenspenders, d.h. Partnerschaften von Halbgeschwistern entstehen. Halbgeschwister haben 25\% des genetischen Materials gemeinsam. Da man davon ausgeht, dass jeder Mensch $5-7$ rezessive Gene besitzt, haben Kinder aus Verbindungen von Halbgeschwistern ein Risiko von 6,5\% für mindestens eine autosomal rezessive Erkrankung. Zwar soll die Zahl der lebenden Nachkommen eines Spenders auf 15 begrenzt werden, ${ }^{8}$ wodurch die Wahrscheinlichkeit geschwisterlicher Beziehungen statistisch sehr gering wird. Jedoch sollte auch gewährleistet sein, dass die Samenproben nur an verschiedene Ärzte in unterschiedlichen Regionen abgegeben werden, um Partnerschaften von durch denselben Samenspender gezeugten Kindern möglichst zu vermeiden.

${ }^{8}$ Hammel/Bispink/Katzorke/Schreiber/Thorn (Fußn. 6), 166 ff. 


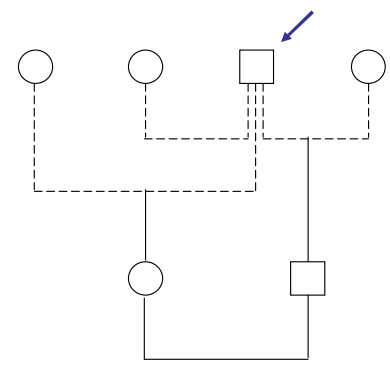

Abb. 7:

Verwandtenbeziehung unter Halbgeschwistern, die von demselben Samenspender gezeugt wurden.

\section{Diskussion}

Sind nun tatsächlich die Risiken für Erkrankungen/Fehlbildungen bei nach Keimzellspende geborenen Kindern gegenüber normal gezeugten Kindern erhöht? Grundsätzlich besteht kein erhöhtes Risiko für Erkrankungen/Fehlbildungen bei diesen Kindern gegenüber Kindern aus Partnerbeziehungen, wenn eine sorgfältige Anamnese und Stammbaumerhebung der Eizellspenderin/des Samenspenders erfolgt. Ei- oder Samenspender sind fertilen Partnern gleichzusetzen. Durch Gespräche zwischen zwei Lebensgemeinschaftspartnern wird aber zweifelsfrei mehr über Erkrankungen der Personen selbst bzw. ihrer Familienangehörigen zu erfahren sein als im Falle einer anonymen Keimzellspende.

Haben wir nicht deshalb eine größere Sorgfaltspflicht gegenüber den Wunscheltern und dem mit Spenderkeimzellen gezeugten Kind und müssen nicht Vorkehrungen getroffen werden, um eine möglichst umfassende Information über Krankheitsrisiken, die sich aus der Keimzellspende ergeben, zu erfassen? Müssen die Anstrengungen, gesunden Kindern zur Geburt zu verhelfen, größer sein als bei normalen Eltern und deren Kindern?

Von Seiten der Humangenetiker stellt sich daher die Frage, ob nicht alle Keimzellspender und alle Wunscheltern genetisch beraten werden und dabei eine sorgfältige Aufzeichnung des Stammbaums über drei Generationen durchgeführt werden sollten. Aufgrund dieser Stammbaumerhebung könnte entschieden werden, ob beim Samenspender/der Eizellspenderin weitere Untersuchungen indiziert sind, oder ob die Keimzellspender als solche überhaupt in Frage kommen. Bei der Stammbaumerhebung kann beurteilt werden, ob der potenzielle Samenspender/die potenzielle Eizellspenderin über ausreichende Informationen über ihre Verwandten verfügen, damit erhöhte Erkrankungsrisiken für das Kind ausgeschlossen werden können. In genetischen Beratungsgesprächen wird häufig sichtbar, dass ein Klient keine oder wenige Informationen über seine Verwandten hat, infolgedessen nicht ausschließen kann, dass für seine Nachkommen ein erhöhtes Risiko für genetisch bedingte Erkrankungen besteht. Potenzielle Keimzellspender mit ungenü- 
genden Kenntnissen über ihre familiäre Krankheitssituation wären demzufolge von der Spende auszuschließen.

Eine weitere Forderung könnte die bei allen Keimzellspendern durchzuführende Chromosomenanalyse sein. Vor dem Hintergrund eines 1,5\%igen Risikos für eine balancierte Chromosomenanomalie scheint diese Forderung nicht überzogen zu sein. Des Weiteren eröffnet sich die Diskussion, ob es sinnvoll ist, die potenziellen Keimzellspender auf Anlageträgerstatus hinsichtlich häufiger autosomal rezessiver Erkrankungen zu untersuchten. Was allerdings sind „häufige“ autosomal rezessive Erkrankungen, wie häufig sollten sie in der Bevölkerung vorkommen, um in das Heterozygotenscreening aufgenommen zu werden? Sollte es einen Katalog geben, der Vorgaben hinsichtlich der Untersuchungsindikationen macht? Diese Frage ist schwierig zu beantworten. Die oft geäußerten Bedenken, der Untersuchungsumfang sei zu groß, um eine Finanzierung sicherzustellen, kann in Anbetracht der kleinen Gruppe der Keimzellspender kein übergroßes Gewicht haben.

Geht man von einem klinisch gesunden Keimzellspender aus, so unterscheidet dieser sich nicht von jeder beliebigen gesunden Person. Lediglich die Information über Erkrankungen, Fehlbildungen oder geistige Behinderungen in seiner Familie kann sich von der eines Lebenspartners unterscheiden. Unterschiedlich sind aber auch die Anstrengungen, die zur Erfüllung des Kinderwunsches des infertilen Paares führen. Sind diese Unterschiede ausreichend, um die Keimzellspender umfassender zu beraten und genetisch zu untersuchen? Sollten die genetischen Untersuchungen den infertilen Paaren vorbehalten bleiben, die zur Erfüllung ihres Kinderwunsches eine Keimzellspende benötigen, oder sollten sie auf Wunsch allen interessierten potenziellen Eltern zur Verfügung stehen?

Mit einem erweiterten Angebot an genetischen Untersuchungen würden eventuell Wunschelternpaare gegenüber normalen potenziellen Eltern bevorzugt, was zu neuen Begehrlichkeiten im Gesundheitswesen Anlass geben könnte.

Eine verbindliche Empfehlung zur Lösung des Problems kann derzeit nicht gegeben werden, jedoch sollte der Beitrag ein Anstoß zu weiteren Diskussionen sein.

\section{Zusammenfassung:}

- Kinder, die nach Keimzellspende geboren werden, haben primär kein erhöhtes Risiko für Erkrankungen/Fehlbildungen gegenüber auf natürlichem Weg gezeugten Kindern.

- Informationen über Keimzellspender und ihre Familien sind wahrscheinlich geringer als bei Lebenspartnern.

- Um diesen Nachteil auszugleichen, sollte bei allen Keimzellspendern eine genetische Beratung bei Fachärzten mit Erstellung eines Stammbaums über drei Generationen erfolgen. 
- Sollte sich aus dem Stammbaum ein Hinweis auf ein erhöhtes Risiko ergeben, so sollten Chromosomenanalysen und molekulargenetische Analysen durchgeführt werden.

- Es bleibt zu diskutieren, ob prinzipiell Keimzellspendern über die bisher routinemäßig vorgenommenen Laboranalysen hinaus Chromosomenuntersuchungen und molekulargenetische Untersuchungen angeboten werden sollen. 



\title{
Samenspende und Stigmatisierung - ein unauflösbares Dilemma?
}

\author{
Petra Thorn
}

\begin{abstract}
„Wenn andere dann wüssten, dass unser Kind mit Hilfe einer Samenspende gezeugt wurde, würden sie es vielleicht ablehnen und sich hinter unserem Rücken über uns lustig machen." Solche Befürchtungen drücken viele, wenn nicht die meisten Paare aus, wenn sie sich mit der Möglichkeit auseinandersetzen, mit dem Samen eines anderen Mannes ein Kind zu zeugen. Sie zeigen damit auf, dass die Samenspende (auch donogene Insemination, im Folgenden mit „DI“ abgekürzt) einer sozialen Stigmatisierung unterliegt und dies Angst vor Ablehnung, Spott und Marginalisierung auslösen kann. Dieser Beitrag gibt einen Überblick über den aktuellen Stand der Stigmatheorie und zeigt auf, welche Faktoren auf der individuellen und familiären Ebene (der Mikroebene), der zwischenmenschlichen und professionellen Ebene (der Mesoebene) und der gesellschaftlichen Ebene (der Makroebene) diese Stigmatisierung auslösen, bzw. sie aufrecht erhalten und analysiert, ob und gegebenenfalls wie sie abgebaut werden kann.
\end{abstract}

\section{Stigmatisierung - ein Überblick über aktuelle Diskussionen}

Der amerikanische Soziologe Goffmann entwickelte Mitte des letzten Jahrhunderts theoretische Grundlagen und erste Erklärungsansätze für das Phänomen der Stigmatisierung. ${ }^{1}$ Er bezeichnete ein Stigma als eine zutiefst diskreditierende Eigen-

${ }^{1}$ Goffmann, Stigma: Über Techniken der Bewältigung beschädigter Identitäten, 3. Aufl. 1998. 
schaft, die dazu führt, dass die Person als beschädigt gilt und entwertet wird. Als Folge dieser Entwertung werden stigmatisierte Personen von nicht Stigmatisierten herabgesetzt und marginalisiert. Goffmann erklärte darüber hinaus, dass Stigmatisierung auf einem komplexen Wechselspiel bei der Kategorisierung von sozialer Interaktion beruhen kann: Es bedarf einer so genannten „virtuellen“ Identität, die basierend auf antizipierten normativen Erwartungen anderer entsteht. In jeder sozialen Situation wird dem Gegenüber häufig unbewusst und daher unreflektiert eine solche „virtuell““ Identität zugeschrieben. Eine Stigmatisierung entsteht, wenn der Gesprächspartner Qualitäten aufzeigt, die ihn von anderen unterscheiden. Es sind jedoch nicht nur die Qualitäten an sich, die die Stigmatisierung auslösen, sondern die Diskrepanz zwischen der Qualität des Gesprächspartners und den normativen Erwartungen. Der Prozess der Stigmatisierung ist daher ein relationaler: nicht stigmatisierte Personen definieren Qualitäten, die sie selbst nicht innehaben, als Stigmata, und marginalisieren diejenigen, die diese Qualitäten besitzen. Die Stigmatisierten ihrerseits akzeptieren das Stigma, empfinden sich als entwertet und verhalten sich entsprechend defensiv. Goffman unterschied zwischen sichtbaren und verborgenen Faktoren, die ein Stigma auslösen können. Er spricht von einer diskreditierten Form des Stigmas, wenn die stigmatisierende Qualität erkennbar oder offensichtlich ist (beispielsweise eine körperliche Behinderung oder die Zugehörigkeit zu einer bestimmten Ethnie). Eine diskreditierbare Stigmaform liegt vor, wenn die Abweichung nicht offensichtlich ist, beispielsweise bei Unfruchtbarkeit. Ersteres hat häufig Diskriminierung und Ausgrenzung zu Folge, letzteres zieht vor allem Gefühle von Scham und Angst vor Ausgrenzung nach sich. Personen mit einer diskreditierbaren Stigmaform entwickeln daher Techniken, das Stigma zu verdecken, beispielsweise die Geheimhaltung der stigmatisierenden Eigenschaft.

In den letzten Jahren wurden Stigmakonzepte weiterentwickelt, vor allem jedoch wurde der individuelle Fokus erweitert und Stigmatisierung als strukturelles Phänomen mit gesellschaftspolitischen Aspekten definiert; Mikro- und Mesoaspekte der Stigmatisierung wurden somit um Makroaspekte ergänzt. So haben Crocker und Kollegen stigmatisierte Personen als Menschen beschrieben, die Attribute oder Charaktereigenschaften besitzen, bzw. bei denen diese vermutet werden, die in einem bestimmten sozialen Kontext entwertet werden. ${ }^{2}$ Dies erklärt die kontextabhängige Bedeutung eines Stigmas: je nach historischem und kulturellem Zusammenhang werden unterschiedliche Eigenschaften als stigmatisierend definiert. Auch Stafford und Scott erklären, dass die Abweichung von jeweils gültigen sozialen Normen in Stigmatisierung resultieren kann. ${ }^{3}$ Link und Phelan argumentieren darüber hinaus, dass der Begriff des „,stereotypen Labellings“ besser als andere Begriffe verdeutlicht, dass Stigmatisierung ein sozialer Prozess ist und nicht die indi-

\footnotetext{
${ }^{2}$ Crocker/Major/Steele, Social stigma, in: Gilbert/Fiske/Lindzey (eds.), The handbook of social psychology, 1998, vol. 2, pp. 504 ff.

3 Stafford/Scott, Stigma deviance and social control. Some conceptual issues, in: Ainlay/Becker/Coleman (eds.), The dilemma of difference: A multidisciplinary view of stigma, 1986, pp. $77 \mathrm{ff}$.
} 
viduelle Qualität einer Person beschreibt. ${ }^{4}$ Sobald Stereotypen Fuß fassen und gesellschaftlich akzeptiert sind, müssen Personen, die diesen Stereotypen entsprechen, von einer Stigmatisierung ausgehen. Dies führt zu einem Verlust an Selbstsicherheit, zu einem Anstieg an defensivem Verhalten oder zu grundsätzlicher Vermeidung von sozialer Interaktion. Goffmann beschrieb dies als ein Dilemma, da die Mechanismen, also die stigmatisierende Eigenschaft und die negative Zuschreibung von außen, austauschbar sind und sich gegenseitig verstärken. Link und Phelan gehen davon aus, dass diese Verstärkung sowohl Stigmatisierung auf der individuellen, persönlichen und damit auf der Mikroebene als auch auf der strukturellen, politischen und somit auf der Makroebene bewirkt: Wenn eine Person mit einem Stigma nicht freiwillig ihren „niedrigeren Status“ akzeptiert, kann eine Diskriminierung dieses Stigmas auf der Makroebene ebenso zu einer Entwertung und Ausgrenzung führen. ${ }^{5}$ Auch zeigen Link und Phelan auf, dass in solchen Situationen Macht eine wichtige Komponente ist. ${ }^{6}$ Sobald diejenigen, die stigmatisieren, die Macht haben, „uns“ von „ihnen“ zu separieren und sicherzustellen, dass Unterschiede in einer Gesellschaft als allgemeingültig definiert und wahrgenommen werden und diejenigen, die stigmatisiert werden, in ihrem Zugang zu gesellschaftlichen Ressourcen (z.B. Erziehung, Arbeitsstellen, medizinische Versorgung) beschnitten werden, kann davon ausgegangen werden, dass Stigmatisierung aufgrund einer strukturell-gesellschaftlichen Komponente vorliegt.

Als Zweck bzw. Funktion der Stigmatisierung werden mittlerweile biologische, psychologische und soziale Gründe vermutet. Kurz̧ban und Leary argumentieren, dass es evolutionsbiologische Gründe für Stigmatisierung gibt.7 Ihren Überlegungen zufolge ist Stigmatisierung zumindest teilweise auf mangelnde dyadische Kooperationsfähigkeit zurückzuführen. Unter dieser Prämisse werden diejenigen, die keine Reziprozität ${ }^{8}$ leisten können oder wollen, bzw. diejenigen, bei denen Reziprozität unwahrscheinlich ist, stigmatisiert, da ihr sozialer Wert für eine Gemeinschaft geringer scheint als der Wert solcher, die uneingeschränkt Reziprozität leisten können und wollen. Neuberg und Kollegen erklären, dass Stigmatisierung hilft, Individuen zu identifizieren, die erfolgreiches Gruppenverhalten bedrohen (beispielsweise diejenigen mit einem körperlichen Makel) oder zentrale Werte einer Gruppe in Frage stellen (beispielsweise durch sexuelles Verhalten wie Homosexualität). ${ }^{9}$ Dies erklärt, weshalb Devianz, Andersartigkeit, körperliche Makel und Normabweichung zu Stigmatisierung führen können und das Maß der Stigma-

${ }^{4}$ Link/Phelan, Conceptualizing stigma, Annual Review of Sociology 2001, $363 \mathrm{ff}$.

${ }^{5}$ Link/Phelan (Fußn. 4).

${ }^{6}$ Link/Phelan (Fußn. 4).

${ }^{7}$ Kurzban/Leary, Evolutionary origins of stigmatization: The functions of social exclusion, Psychological Bulletin 2001/2, $187 \mathrm{ff}$.

8 Mit dem Begriff „Reziprozität“ soll hier die Fähigkeit beschrieben werden, von einer Gemeinschaft zu profitieren und sich im Gegenzug für die Gemeinschaft gewinnbringend einzusetzen.

9 Neuberg/Smith/Asher, Why people stigmatize: Toward a biocultural framework, in: Heatherton/Kleck/Hebl/Hull (eds.), The social psychology of stigma, 2000, pp. $31 \mathrm{ff}$. 
tisierung in den Fällen besonders hoch sein kann, wenn die stigmatisierende Qualität sichtbar ist (z.B. körperliche Behinderung), bedrohend auf soziale Interaktion wirkt (z.B. Verrat, Diebstahl) oder von dem Individuum eine Gefahr für die Gruppe ausgeht (z.B. ansteckende Erkrankung). ${ }^{10}$

Die Bemühungen, ein Stigma zu kontrollieren, werden auch als „Stigma Management" beschrieben. Goffman beschrieb das Geheimhalten der stigmatisierenden Eigenschaft als eine Möglichkeit, Außenstehende in Unkenntnis zu lassen und damit die Interaktion damit nicht zu beeinträchtigen. ${ }^{11}$ Crocker und Quinn gehen allerdings davon aus, dass sich die Stigmatisierung dennoch auf die Interaktion auswirkt. ${ }^{12}$ Sie erklären, dass die eigene Position in einer sozialen Rangordnung an konkreten oder generalisierten Einstellungen und Reaktionen anderer festgemacht wird: Individuen vermuten bestimmte Einstellungen oder beobachten die Reaktionen anderer, interpretieren diese und reagieren auf der Basis ihrer Interpretation. Es ist somit erforderlich, kollektive Bedeutungen zu verstehen, die stigmatisierte Personen verinnerlicht haben, also die Einstellungen und Reaktionen, die sie in einem bestimmten Kontext erwarten. In diesem Sinne kann eine sich selbst erfüllende Prophezeiung entstehen: Personen mit einem diskreditierbaren Stigma reagieren auf vermutete Reaktionen und Stereotypen und zeigen daraufhin beispielsweise distanziertes Verhalten auf. ${ }^{13}$ Nicht Stigmatisierte reagieren ihrerseits abweisend auf das distanzierte Verhalten und erstere fühlen sich in ihrer Vermutung bestätigt. Solche sich selbst erfüllende Prophezeiungen können auch auf der Makroebene entstehen: sozial unterdrückten Gruppen kann aufgrund politischer oder institutioneller Vorgaben der Zugang zu Bildung eingeschränkt werden, sie haben daher geringe Bildungs- und schlechtere Arbeitsmöglichkeiten und bleiben unterdrückt. Darüber hinaus kann Geheimhaltung zur Beeinträchtigung des individuellen Funktionierens führen. ${ }^{14}$ Die Bemühungen, eine Qualität geheim zu halten, nimmt die Person stark in Anspruch und führt zu Anspannungen, die besonders dann eklatant werden, wenn sie unterdrückt werden sollen. Diese innerpsychische Dynamik kann dazu führen, dass unbewusste und damit nicht kontrollierbare stigmabezogene Gedanken entstehen, die das Verhalten und die Urteilsfähigkeit beeinflussen. Die Interaktionspartner nehmen dies bewusst oder unbewusst wahr und reagieren darauf. Hebl und Kollegen haben solche Interaktionsequenzen als „unbehagliche Momente" bezeichnet, als Sequenzen, die inkongruentes oder dem

10 Jones/Farina/Hastorf/Markus/Miller/Scott, Social stigma: The psychology of marked relationships, 1984.

11 Goffmann (Fußn. 1).

12 Crocker/Quinn, Social stigma and the self: Meanings, situations, and self-esteem, in: Heatherton/Kleck/Hebl/Hull (eds.), The social psychology of stigma, 2000, pp. $153 \mathrm{ff}$.

$13 \mathrm{Jussim} /$ Palumbo/Chatman/Madon/Smith, Stigma and self-fulfilling prophecies, in: Heatherton/Kleck/Hebl/Hull (eds.), The social psychology of stigma, 2000, pp. $374 \mathrm{ff}$.

14 Smart/Wegner, The hidden costs of hidden stigma, in: Heatherton/Kleck/Hebl/Hull (eds.), The social psychology of stigma, 2000, pp. $220 \mathrm{ff}$. 
Kontext unangemessenes Verhalten andeuten. ${ }^{15}$ Diese können dazu führen, dass die Interaktion oberflächlich bleibt oder abgebrochen wird, da Außenstehende Ambivalenzen spüren und auch ihrerseits Schuld oder Scham empfinden können oder verunsichert werden.

Strategien, um Stigmatisierung abzubauen, zielen auf Veränderungen auf $M i$ kro-, Meso- und Makroebene ab. Auf der Mikroebene werden psychologische Interventionen vorgeschlagen, die das Selbstwertgefühl verbessern, Unterschiede nicht als entwertend darstellen und auf positive Persönlichkeitsmerkmale fokussieren. ${ }^{16}$ Auf der Mesoebene wird der Austausch mit anderen empfohlen, der ebenfalls zu einer Verbesserung des Selbstwertgefühls und zu einer Validierung der Gefühle führen soll. ${ }^{17}$ Auf der Makroebene können Individuen oder Gruppen versuchen, politischen Einfluss zu gewinnen, damit ihre Rechte respektiert werden, ${ }^{18}$ sie können Anwaltsfunktion übernehmen und sie können edukatives Material für die breite Öffentlichkeit entwickeln, damit gesellschaftliche Haltungen verändert. ${ }^{19}$ Einen viel versprechenden Vorschlag machen Link und Phelan. ${ }^{20}$ Sie gehen davon aus, dass die Strategien, die nur auf einer Ebene ansetzen, keine anhaltende Veränderung bewirken, sondern Gefahr laufen, durch kontextabhängige Kräfte unterminiert werden. Sie empfehlen daher Strategien parallel auf allen drei Ebenen anzugehen, da diese sich gegenseitig verstärken und den Prozess der Entstigmatisierung unterstützen können.

\section{Stigmatisierung im Kontext der donogenen Insemination}

Die Stigmatisierung im Kontext der DI ist auf drei Bereiche zurückzuführen: der „Makel“ der Unfruchtbarkeit im Allgemeinen, die stärkere Stigmatisierung männlicher Unfruchtbarkeit im Vergleich zu weiblicher Unfruchtbarkeit und letztendlich die von der Norm abweichende Zusammensetzung einer Familie nach DI. Im folgenden Teil werden die Stigma auslösenden Bedingungen für diese drei Bereiche analysiert und mit Beispielen veranschaulicht.

\footnotetext{
15 Hebl/Tickle/Heatherton, Awkward moments in interactions between nonstigmatized and stigmatized individuals, in: Heatherton/Kleck/Hebl/Hull (eds.), The social psychology of stigma, 2000, pp. $273 \mathrm{ff}$.

${ }_{16}$ Hebl/Tickle/Heatherton (Fußn. 15).

17 Corrigan/Penn, Lessons from social psychology on discrediting psychiatric stigma, American Psychologist 1999, 765 ff.; Ablon, The nature of stigma and medical condition, Epilepsy and Behavior 2002/3, 2 ff.

$18 \mathrm{Hebl} /$ Tickle/Heatherton (Fußn. 15).

19 Ablon (Fußn. 17).

${ }^{20}$ Link/Phelan (Fußn. 4).
} 


\subsection{Das Stigma der Unfruchtbarkeit}

Die meisten westlichen, wenn nicht sogar alle Kulturen sind pronatalistisch ausgerichtet, d.h. Reproduktion und Elternschaft stellen wichtige Werte dar, und von heterosexuellen Paaren, verheiratet oder (noch) nicht, wird nicht nur erwartet, dass sie sich fortpflanzen, sondern auch, dass sie sich fortpflanzen möchten. ${ }^{21}$ Paare, die sich nicht fortpflanzen können oder wollen, zeigen deviantes Verhalten auf und verstoßen gegen diese normative Erwartung. Wie stark pronatalistische Haltungen nach wie vor internalisiert sind, zeigen die nachstehenden, typischen Aussagen von ungewollt Kinderlosen auf:

\section{Eine Frau muss doch Kinder kriegen, sonst ist sie keine richtige Frau. ${ }^{22}$}

Die Diagnose ,unfruchtbar" hat meine Welt zerschmettert. Ich wollte doch nur das, was alle anderen einfach so mühelos binbekommen und was einfach normal ist und dazu gebört.

Ich hatte das Gefübl, aus der Erwachsenenwelt ausgeschlossen zu sein. Selbst kein Kind bekommen zu können, das machte mich anders, irgendwie weniger wertvoll.

Im evolutionsbiologischen Sinne dient die Stigmatisierung von Kinderlosigkeit dazu, den Fortbestand einer Gruppe zu sichern. Paare, bzw. Personen, die keine Kinder bekommen können oder möchten, werden gering geschätzt oder ausgegrenzt. Sie tragen nicht dazu bei, dass die Gruppe weiter besteht und entziehen sich damit, gewollt oder ungewollt, ihrer generativen Verantwortung. Dies erklärt beispielsweise die lange Zeit in einigen Kulturen akzeptierte Scheidung bei Unfruchtbarkeit eines Partners; der fruchtbare Partner hat dann die Möglichkeit, mit einem anderen Partner ein Kind zu zeugen. ${ }^{23}$ Auch stereotypische Zuschreibungen wie „DINK“ (double income, no kids) deuten an, dass Doppelverdiener ohne Kinder vor allem ihr individuelles Wohl berücksichtigen und das gesellschaftliche Wohl vernachlässigen. Es ist daher verständlich, dass Unfruchtbarkeit als eine Lebenskrise wahrgenommen wird, die mit depressiven Reaktionen einhergehen kann. ${ }^{24}$ Diagnoseträger berichten von Schuldgefühlen, nicht nur, weil sie gesellschaftlichen Erwartungen nicht entsprechen, sondern auch, weil ihre eigene Unfruchtbarkeit dazu führt, dass ihr Partner ebenfalls ohne Kind bleibt. ${ }^{25}$ Zwar sind Trennungen in westlichen Kulturen aufgrund von Unfruchtbarkeit mittlerweile selten, im klinischen Bereich zeigt sich dennoch, dass sich Diagnoseträger selbst

${ }^{21}$ Miall, The stigma of involuntary childlessness, Social Problems 1986/4, 268 ff.

22 Alle Zitate sind typische Aussagen, die der Autorin gegenüber im Rahmen ihrer klinischen Arbeit als Familientherapeutin gemacht wurden.

23 Ein bekanntes historisches Beispiel ist Soraya Esfandiary Bakbtiari, Kaiserin von Persien, die 1958 geschieden wurde, da sie ihrem Ehemann nicht den erhofften Thronerben schenken konnte.

24 Z.B. Covington, Psychosocial evaluation of the infertile couples: Implications for social work practice, Journal of Social Work and Human Sexuality 1987/6, 21 ff.; Kerr/Balen, The experiences of couples in the United Kingdom who have had infertility treatment: The results of a survey performed in 1997, Human Reproduction 1999/14, 934 ff.

25 Mason, Male infertility: Men talking, 1993. 
mit ihrer mangelnden Reziprozitätsfähigkeit dem Partner gegenüber auseinandersetzen und manche Paare nach Diagnosestellung ihren Willen zur Beziehung erneut explizit bekräftigen:

Meine Unfruchtbarkeit bedeutet, dass auch mein Mann keine Kinder bekommen kann. Ich hatte deswegen große Schuldgefühle, mit denen ich mich lange alleine auseinandersetate. Ich hatte schon Angst, dass mein Mann mich verlassen könnte, denn mit einer anderen Frau bätte er problemlos Kinder zeugen können. Irgendwann nabm ich meinen ganzen Mut zusammen und fragte ibn, ob er denn trotz meiner Diagnose mit mir zusammen bleiben möchte. Er bejabte dies und mir fiel ein Stein vom Herzen.

Wenn Kinderlosigkeit auf Unfruchtbarkeit zurückzuführen ist, werden zusätzlich individuelle Faktoren relevant. Wie typisch für diskreditierbare Stigmata, entstehen Angst vor Ausgrenzung und Scham ob der persönlichen Unfähigkeit, eine soziale Norm nicht erfüllen zu können. Die Geheimhaltung dient dem Schutz vor Ausgrenzung und sozialer Abwertung:

Ich habe große Angst, mit Freunden über meine Unfruchtbarkeit zu sprechen. Ich weiß genau, dass sich dann manche hinter meinem Rücken über mich lustig machen oder sie mich beim nächsten Mal komisch anschauen. Ich füble mich jetzt schon dieser fruchtbaren Welt nicht mehr zugehörig, und das würde dann noch schlimmer werden. Also sage ich nichts und tue so, als ob nichts wäre. Das hat allerdings dazu geführt, dass ich mich von vielen Freundschaften zurückgezogen habe, denn ich habe immer Angst, dass mich jemand auf die Kinderlosigkeit anspricht. Aber innerlich platze ich bald, weil ich eigentlich mit jemandem reden müsste.

Der Prozess der Marginalisierung kann wechselseitig sein und in der Tat eine sich selbst erfüllende Prophezeiung auslösen: Personen mit Furchtbarkeitsstörungen gehen von normativen Erwartungen und abwertenden Reaktionen aus und halten daher die Unfruchtbarkeit geheim. Um nicht entblößt zu werden, ziehen sie sich aus sozialen Bezügen zurück und schränken soziale Interaktion ein. ${ }^{26}$ Die Geheimhaltung belastet sie allerdings, und der soziale Rückzug wird wahrgenommen. Mit einiger Wahrscheinlichkeit löst dies auch bei Außenstehenden Unsicherheit oder Ambivalenz aus:

Seit der Diagnose babe ich mich ziemlich von Freundinnen zurückgezogen. Ich habe Angst, dass ich auf meinen Kinderwunsch angesprochen werde und dass meine Unfruchtbarkeit Thema wird. Das wäre mir peinlich. Aber ich spüre auch, dass es manche Freundinnen gar nicht verstehen und unsicher sind, wie sie mir begegnen sollen.

Die seit der Gesundheitsreform 2004 eingeschränkte Kostenübernahme für reproduktionsmedizinische Behandlung wird als zusätzliche strukturelle Diskriminierung wahrgenommen. Paare mit Kinderwunsch, die auf medizinische Unterstützung angewiesen sind, empfinden sich in einer widersprüchlichen Situation:

${ }^{26} \mathrm{Kerr} /$ Balen (Fußn. 24). 
einerseits leben sie in einer pronatalistischen Gesellschaft, deren Norm sie gerne entsprechen würden, andererseits werden finanzielle Ressourcen für medizinische Behandlungen, die dies ermöglichen könnten, limitiert, bzw. ihnen vorenthalten:

Wir möchten sehr gerne Kinder, aber können uns die medizinische Behandlung nicht erlauben, sie ist einfach zu teuer. Wir füblen uns von der Gesellschaft ziemlich alleine gelassen. Immer wieder wird in der Presse dargestellt, dass uns doch Kinder feblen in Deutschland, aber diejenigen, die sie sich sehr wünschen und Hilfe brauchen, denen wird finanziell nicht mehr geholfen. Darüber hinaus wird immer wieder diskutiert, dass Kinderlose auch noch höhere Steuern zablen sollen. Das ist doch diskriminierend, und wie sollen wir das denn stemmen?

Wir mussten heiraten, damit wir den Krankenkassenzuschuss für die medizinische Behandlung bekamen. Das war merkwürdig, andere Paare, egal ob verheiratet oder nicht, bekommen einfach ibre Kinder und kein Hahn kräht mehr danach, aber die Krankenkasse übernimmt die Teilkosten nur für verheiratete Paare. Wir wünschten uns das Geld dann von allen Verwandten und Freunden zur Hochzeit, sonst hätten wir uns die Behandlung nicht leisten können.

Aus demografischer Sicht scheint die Einschränkung der Kostenübernahme für reproduktionsmedizinische Behandlungen kontraproduktiv. Deutschland hat eine der niedrigsten Geburtenraten europaweit, und das Alter der Erstgebärenden ist in den letzten 10 Jahren deutlich angestiegen und lag bereits 2004 bei durchschnittlich 28,8 Jahren. ${ }^{27}$ Somit wäre es aus bevölkerungspolitischer Sicht sinnvoll, reproduktionsmedizinische Behandlung wieder umfassender zu finanzieren, damit zumindest mangelnde finanzielle Ressourcen kein Hindernis zur Umsetzung des Kinderwunsches darstellen. Allerdings haben möglicherweise das Tabu und die Scham dazu beigetragen, dass diese Ressourcen eingeschränkt werden konnten. Als die Gesundheitsreform in Kraft trat, gab es keine öffentlich wirksame Lobby für ungewollt Kinderlose. Zwar gab es bereits damals einen Interessenverband, der bemüht war, auf diese Diskriminierung öffentlich hinzuweisen und politisch Einfluss zu nehmen. ${ }^{28}$ Mehrere Gründe verhinderten dies jedoch. Aufgrund des Tabus waren kaum Paare bereit, ihre Unfruchtbarkeit in der Öffentlichkeit darzustellen und auf ihre individuellen Missstände hinzuweisen. Darüber hinaus verfügte der Interessenverband weder über personelle noch über finanzielle Ressourcen, eine öffentliche Kampagne durchzuführen. ${ }^{29}$

Die Stigmatisierung wird in allen drei Bereichen deutlich. Auf der Mikroebene führt Unfruchtbarkeit als diskreditierbares Stigma zu Scham und Angst. Auf der Mesoebene entstehen Schuldgefühle dem Partner gegenüber und die Geheimhaltung der Unfruchtbarkeit führt, wie von Stigmaexperten postuliert, zu innerer Anspan-

\footnotetext{
27 Ochel/ Osterkamp, Fertility policies in Germany, in: Valverde (ed.), 2050: A changing Europe. Demographic crisis and baby friendly policies. Pharmaceutical Policy and Law, 2007, vol. 9, pp. $211 \mathrm{ff}$.

${ }^{28}$ www.wunschkind.de (letzter Zugriff: 20.7.2008).

${ }^{29}$ Ziegler, mündliche Mitteilung am 3.8.2008.
} 
nung und wirkt sich auf die Interaktion mit nicht Stigmatisierten aus. Auf der Makroebene werden strukturelle Bedingungen deutlich, die Stigmatisierung von Paaren mit Fruchtbarkeitsstörungen aufrecht erhalten. Deutlich wird, dass Änderungen auf der Makroebene in der Tat durch andere kontextabhängige Mächte und Stigmatisierung auf anderer Ebene behindert werden können.

\subsection{Die stärkere Stigmatisierung männlicher Unfruchtbarkeit im Vergleich zu weiblicher Unfruchtbarkeit}

Im Vergleich zu weiblicher Infertilität wird männliche Infertilität häufig als stigmatisierter beschrieben. ${ }^{30}$ Sowohl historische als auch geschlechtsspezifische Faktoren werden als Gründe herangezogen. Lange Jahre wurde davon ausgegangen, dass die Frau für die Fähigkeit der Reproduktion alleinverantwortlich ist. ${ }^{31}$ Sie wurde schwanger bzw. nicht schwanger, trug das Kind aus und gebar es; Behandlungsangebote für Unfruchtbarkeit richteten sich folglich vor allem an Frauen. Das mangelnde Verständnis für andrologische Ursachen spiegelt sich auch in der Literatur. Frick-Bruder zeigte beispielsweise in einer Analyse auf, dass Publikationen zwischen 1935 und 1963 fast ausschließlich davon ausgingen, dass Unfruchtbarkeit durch die Frau verursacht wird. ${ }^{32}$ Die Fokussierung auf weibliche Unfruchtbarkeit setzte sich im psychosomatischen Bereich fort; auch die Bedeutung psychologischer Aspekte von Unfruchtbarkeit auf den Mann wurde in Publikationen kaum dargestellt. ${ }^{33}$ Lee beschrieb daher unser Wissen über männliche Unfruchtbarkeit Mitte der 1990er als ein Gebiet, das sich „,noch in den Kinderschuhen“ befindet. ${ }^{34}$ Dies hat sich in den letzten Jahren sicherlich deutlich verändert, doch nach wie vor werden Diagnosemöglichkeiten für Störungen männlicher Unfruchtbarkeit als eingeschränkter als für Störungen weiblicher Fruchtbarkeit empfunden. Dieser Unterschied kann bei Männern das Gefühl einer gender-spezifischen Diskriminierung auslösen:

Wir gingen beide relativ gleichzeitig zur Untersuchung und bei meiner Frau wurde recht schnell eine minimale Hormonstörung festgestellt, die dazu führte, dass sich die Gebärmutterschleimhaut nicht gut aufbaute. Auch bei mir wurde festgestellt, dass die Beweglichkeit meiner Samen schlecht ist, aber im Gegensatz zu meiner Frau konnte mir der Arat nicht sagen, was die Ursache hierfür ist. Hier scheinen die Ärzte noch im Dunkeln zu tappen und ich

\footnotetext{
${ }^{30}$ Nachtigall/Becker/Wozny, The effects of gender-specific diagnosis on men's and women's response to infertility, Fertility and Sterility 1992/57, 113 ff.; Miall (Fußn. 21); Lee, Male mysteries: Factors in male infertility, in: Jennings (ed.), Infertility Counselling in Practice, 1995, pp. 66 ff.; Keye, Medical aspects of infertility for the counsellor, in: Covington/Hammer-Burns (eds.), Infertility Counselling A comprehensive handbook for clinicians, 2006, pp. $20 \mathrm{ff}$.

31 Carrell/Urry, Male infertility, in: Burfoot (ed.), Encyclopedia of reproductive technologies, 1999, pp. $171 \mathrm{ff}$.

32 Frick-Bruder, Psychologische Gesichtspunkte der kinderlosen Ehe, in: Schirren/Leidenberger/Stoll (Hrsg.), Die kinderlose Ehe: Gynäkologische, andrologische und psychologische Aspekte, 1980, S. $217 \mathrm{ff}$

33 Strauß, Psychosomatik der Sterilität und der Sterilitätsbehandlung, 1991.

${ }^{34}$ Lee (Fußn. 30), pp. 76.
} 
füblte mich benachteiligt. Für weibliche Unfrucbtbarkeit gibt es so viele Untersuchungen, Bauchspiegelung, Ultraschall, Hormonuntersuchungen und so weiter, aber bei mir konnte der Aręt nur sagen, so ist es balt.

$\mathrm{Zu}$ dem beschriebenen historisch bedingten Erkenntnismangel kommt die Fokussierung der Gesundheitsfragen auf Frauen hinzu. So konstatieren Bräbler und Kollegen, „dass Gesundheitsförderung nunmehr auch verstärkt Zielgruppen wie Kleinkinder und ältere Menschen berücksichtigt. Gesundheitsbezogene Diskussionen über Männer haben in den letzten Jahren in der Öffentlichkeit zunehmend an Bedeutung gewonnen, unter geschlechtsspezifischen Gesichtspunkten ist dieser Themenbereich jedoch bisher kaum erforscht. Entsprechend selten findet man in der Literatur Studien, die sich explizit mit gesundheitsbezogenen Fragestellungen bei Männern beschäftigen." ${ }^{35}$ Eine von diesen Autoren durchgeführte Literaturrecherche über männliche und weibliche Gesundheit und Krankheit ergab 118 Treffer für „Männer und Krankheit“, aber 327 Treffer für „Frauen und Krankheit“. „Männer und Gesundheit“ wiesen nur 76 Treffer auf, „Frauen und Gesundheit“ jedoch 544. Sie schlussfolgern, dass die Männerbewegung weniger aktiv ist als die Frauenbewegung und dass eine differenzierte Darstellung männlicher Gesundheitsthemen nicht erforderlich scheint, da Männer als „die eigentlichen Menschen“ wahrgenommen werden. ${ }^{36}$ Darüber hinaus wird Gesundheit bei Männern so stark mit Geschlecht in Verbindung gebracht, dass eine Beschäftigung damit Männer in den Ruf mangelnder Männlichkeit bringt. Hinweise nicht nur auf Unfruchtbarkeit, sondern auf Krankheit und Störungen im Allgemeinen werden dann oft als narzisstische Kränkung empfunden und daher erstaunt es nicht, dass Fruchtbarkeit selbst mit Potenz und Virilität gleichgesetzt wird, ${ }^{37}$ Unfruchtbarkeit im Gegenzug mit sexueller Dysfunktion und mangelnder Männlichkeit. ${ }^{38}$ Dies zeigt sich deutlich, wenn Männer mit Fruchtbarkeitsstörungen beschreiben, wie sie sich wahrgenommen fühlen:

Was andere über unfruchtbare Männer denken? Das weiß ich nicht. Vielleicht denken sie, ein Mann, der keine Kinder zeugen kann, ist kein richtiger Mann. Andere denken sicherlich negativ über ibn, weil ja auch alles mit Sex zu tun hat. ... Ja, die meisten, die über Unfruchtbarkeit nachdenken, glauben, dass man keinen Sex baben kann und daber denken sie negativ darüber.

Männer müssen frucbtbar sein, das ist ibr Ideal. Männer müssen funktionieren. Und wenn das nicht geht, dann glaube ich, leidet ibr Selbstwert. Die Gesellschaft sieht Männer natürlich als Ideal. Männer müssen potent sein und fertil und sie müssen funktionieren. Und alles andere, das wird negativ gesehen.

\footnotetext{
35 Bräbler/Goldschmidt/Kupfer, Männer und Gesundheit, in: Brähler/Kupfer (Hrsg.), Mann und Medizin, 2001, S. 12.

36 Brähler/Goldschmidt/Kupfer (Fußn. 35), S. 11.

${ }^{37}$ Bräbler/Goldschmidt/Kupfer (Fußn. 35), S. 13.

${ }^{38}$ Lee (Fußn. 30).
} 
Bereits 1986 hat Miall die folgenden Strategien beschrieben, um das Stigma der männlichen Unfruchtbarkeit zu umgehen oder zumindest abzumildern: Frauen stellen sich selbst in der Öffentlichkeit als Diagnoseträger dar bzw. deuten dies an, da sie weibliche Unfruchtbarkeit als weniger stigmatisierend empfinden. ${ }^{39}$ Männer halten das Ausmaß ihrer Fruchtbarkeitsstörung geheim, um zu signalisieren, dass eine Zeugung dennoch möglich sei. Auch im klinischen Bereich werden diese Strategien geschildert:

Ich habe immer angedeutet, dass ich regelmäßige Schmerzen haben, wenn ich meine Tage habe und damit ein bisschen, na ja, angedeutet, das Ganze liegt an mir. Und ich sage immer, es dauert bei uns halt ein bisschen länger, bis ich schwanger werde, weil ich ja Endometriose habe. Aber die anderen wissen nichts von der Diagnose meines Mannes.

Ich habe meinem Freund gesagt, dass der Arzt zwar eine kleine Störung bei mir festgestellt hat, habe aber auch angedeutet, dass es durchaus einen Zufallstreffer geben könnte, dass ich also trotzdem noch irgendwie zengungsfähig bin.

Zwei weitere Faktoren sind maßgeblich dafür, dass männliche Unfruchtbarkeit stärker tabuisiert wird als weibliche Fruchtbarkeitsstörungen. Stereotypische Rollenerwartungen sehen nicht nur vor, dass Männer „männlich“ sind, sondern dass sie sich auch „männlich“ verhalten. In einigen Studien wird postuliert, dass Unfruchtbarkeit für Frauen mit größerem emotionalen Stress einher geht als für Männer. ${ }^{40}$ In diesen Studien wird beispielsweise beschrieben, dass Frauen ob der Diagnose von Unfruchtbarkeit starke Verzweiflung empfinden, Männer hingegen lediglich enttäuscht sind. Andere Autoren gehen davon aus, dass Männer und Frauen gleichermaßen unter dem unerfüllten Kinderwunsch leiden, dass jedoch geschlechtsspezifisches Rollen- und Krisenverhalten zu Unterschieden führen: Männer verleihen ihren Emotionen weniger deutlich Ausdruck und bevorzugen zielorientiertes Handeln, während Frauen Wert auf emotionales Einfühlvermögen legen und ein hohes Mitteilungsbedürfnis aufzeigen. ${ }^{41}$ Darüber hinaus werden reproduktionsmedizinische Diagnose- und Behandlungsmöglichkeiten überwiegend am Körper der Frau durchgeführt, so dass diese in der Regel körperlich belasteter sind als Männer und Männer ihre Emotionen nicht nur aufgrund ihrer

\footnotetext{
${ }^{39}$ Miall (Fußn. 21).

40 Dhillon/C.Cumming/D.Cumming, Psychological well-being and coping patterns in infertile men, Fertility and Sterility 2000/74, 702 ff.; Petok, The psychology of gender-specific infertility diagnosis, in: Covington/Hammer-Burns (eds.), Infertility Counselling - A comprehensive handbook for clinicians, 2006 , pp. $37 \mathrm{ff}$.

${ }^{41}$ Collins/Freeman/Boxer, Perceptions of infertility and treatment stress on females are compared with males entering in in vitro fertilisation treatment, Fertility and Sterility 1992/57, 350 ff.; Daniluk, Gender and infertility, in: Leiblum (ed.), Infertility: Psychological issues and counseling strategies, 1997, pp. 103 ff.; Malik/Coulson, The male experience of infertility: a thematic analysis of an online infertility support group bulletin board., Journal of Reproductive and Infant Psychology 2008/1, 18 ff.
} 
Sozialisation zurückhalten, ${ }^{42}$ sondern auch, um die Partnerin emotional unterstützen zu können: ${ }^{43}$

Einer von uns musste der Stärkere sein. Meine Frau musste die ganze Bebandlung durchstehen, die Termine wahrnehmen und sich die Spritzen setzen und die Eizellen entnehmen lassen. Wenn ich meinen Gefüblen auch noch freien Lauf gegeben bätte, bätte ich für meine Frau nicht mebr da sein können und sie unterstïtzen können. Von daber babe ich mich zurückgehalten, viel mit mir selbst ausgemacht und bin vor allem auf meine Frau eingegangen.

Möglicherweise tragen geschlechtstypische Interaktionsmuster und der frauenzentrierte Fokus im Rahmen der medizinischen Behandlung dazu bei, dass männliche Fruchtbarkeitsstörungen und die für Männer damit einhergehende emotionale Belastung lange Zeit vernachlässigt wurden und weiterhin weniger deutlich wahrgenommen werden. Das Zusammenspiel von stereotypischen Rollenerwartungen, wie das Erfordernis „seinen Mann zu stehen“ und die Geringschätzung gesundheitlicher Beeinträchtigungen bei Männern, scheinen diesen geschlechtsspezifischen Unterschied aufrecht zu erhalten.

Diese Ausführungen verdeutlichen, dass strukturelle Bedingungen auf der Makro- und Mesoebene, hier der Erkenntnismangel hinsichtlich männlicher Unfruchtbarkeit gepaart mit einer geschlechtsspezifischen Verzerrung bei Gesundheits- und Krankheitsfragen, auch bei männlicher Unfruchtbarkeit Auswirkungen auf die Stigmatisierung in der Mikroebene haben. Stereotypische männliche Rollenzuschreibungen und sog. „Labelling“ können individualpsychologischen Druck ausüben, wenn dem gesellschaftlichen Ideal nicht entsprochen werden kann. Auch hier wird die Strategie der Geheimhaltung bzw. der Delegation der Unfruchtbarkeit an die Partnerin oder der Abschwächung des Ausmaßes der Diagnose angewendet, um die Stigmatisierung zu vermeiden.

\subsection{Das Stigma der donogenen Insemination}

Die DI ist eine der ältesten Behandlungsmöglichkeiten bei männlicher Unfruchtbarkeit. ${ }^{44}$ Sie galt jedoch lange Zeit als moralisch verwerflich, da es erforderlich war, den Samen eines Dritten zur Befruchtung einer verheirateten Frau zu verwenden. Der Beitrag „Entstehung und Entwicklung der Spendersamenbehandlung in Deutschland" in diesem Band verdeutlicht, mit wie vielen sittlichen und moralischen Vorbehalten die DI in Verbindung gebracht wurde. Frauen (und ihre Ehemänner), die sich für eine DI entschieden, die also das Kind eines Mannes austrugen, mit dem sie weder in einer Beziehung oder Ehe lebten noch das Kind gemein-

\footnotetext{
42 Daniluk, in: Leiblum (Fußn. 41); Jordan/Revenson, Gender differences in coping with infertility: a meta-analysis, Journal of behavioural medicine 1999/22, $341 \mathrm{ff}$.

${ }^{43}$ Dhillon/C.Cumming/D.Cumming (Fußn. 40).

${ }^{44}$ Gregoire/Mayer, The impregnators, Fertility and Sterility 1965/16, $130 \mathrm{ff}$.
} 
sam aufzuziehen beabsichtigten, wurden pathologisiert und letztendlich wurde die DI Mitte des letzten Jahrhundert von medizinischen Berufsorganisationen als standesunwürdig erklärt. In vielen westlichen Kulturen wird die biologische bzw. genetische Verbindung als die „natürliche“ Verwandtschaft definiert"5, soziale Beziehungen hingegen als fiktive Verwandtschaft, die eigentliche, auf Blutsverwandtschaft begründete Beziehungen nur vortäuschen sollen und daher von geringerer Bedeutung sind. ${ }^{46}$ Die „biologische“ Familie, also der Familientyp, in dem Kinder mit beiden Elternteilen biologisch verwandt sind, wird in Deutschland als die übliche Familienzusammensetzung beschrieben ${ }^{47}$ und auch in den USA wird dieser als die ,einzig legitime Familie bezeichnet, die in der ,Natur' und den ,langen 'Traditionen' der Gesellschaft wurzelt; nur wenn dieses Familienmodell eingehalten wird“, so Shanley „wird das Verhalten von Individuen als ethisch erachtet.“48 Die Familienbildung mit DI und ihre Zusammensetzung fordern, wie andere Familienzusammensetzungen, in denen Kinder nicht (z.B. Adoptivfamilien) oder nicht ausschließlich (z.B. Stieffamilien) biologisch von beiden Elternteilen abstammen, diese normativen Werte heraus. ${ }^{49}$

Obgleich Soziologen wie Beck-Gernsheim inzwischen konstatieren, ${ }^{50}$ dass dieses traditionelle Familienbild mittlerweile ,sein Monopol verliert", und auch Familientherapeuten davon ausgehen, dass ehemals experimentelle Familientypen inzwischen als normale Varianten in der Familienlandschaft angesehen werden, ${ }^{51}$ trifft dies für die Familienbildung mit DI noch nicht zu. Zwar gibt es mittlerweile Studien, die u.a. die Familiendynamik, die Vater-Kind-Bindung und die Bedeutung des Samenspenders untersuchten, doch fehlen repräsentative Langzeitstudien. ${ }^{52}$ Vor allem fehlt ein sozialpsychologisches Verständnis für diesen Familientyp, das nicht nur Eltern und der Gesellschaft als Orientierung dient, sondern welches auch Fachkräfte Hinweise für ihr professionelles Vorgehen gibt. Dieser Mangel erschwert Eltern einen souveränen Umgang mit ihrer Familienzusammensetzung:

\footnotetext{
45 Schneider, American kinship: A cultural account, 2. edn. 1980, p. 34.

46 Schröder, Die kulturelle Konstruktion von Verwandtschaft unter den Bedingungen der Reproduktionstechnologien in Deutschland, 2002,

http://webdoc.sub.gwdg.de/diss/2002/schroeder_iris/schroeder_iris.pdf (letzter Zugriff: 4.8.2008).

47 Stein-Hilbers, Wem ,gehört“ das Kind? Neue Familienstrukturen und veränderte Eltern-KindBeziehungen, 1994; Krähenbübl/Jellouscheck/Kohaus-Jellousheck/Weber, Stieffamilien. Struktur - Entwicklung - Therapie, 4. Aufl. 1995.

48 Shanley, Making babies, making families: What matters most in an age of reproductive technologies, surrogacy, adoption, and same-sex and unwed parents, 2001, p. 148.

49 Wie der Beitrag von Herrmann-Green, Lesben mit Kinderwunsch. Eine ethische Herausforderung für die Reproduktionsmedizin, in diesem Band beschreibt, trifft dies in verstärktem Maß für lesbische Familien zu; die folgenden Ausführungen beziehen sich jedoch vorwiegend auf Familien mit heterosexuellen Eltern.

${ }^{50}$ Beck-Gernsheim, Was kommt nach der Familie? Einblicke in neue Lebensformen, 2000, S. 20.

51 Simon/Clement/Stierlin, Die Sprache der Familientherapie: ein Vokabular, 5. Aufl. 1999.

52 Thorn, Donor Insemination Recipient Counselling, in: Covington/Hammer-Burns (eds.), Infertility counselling: A comprehensive handbook for clinicians, 2. edn. 2006, pp. $305 \mathrm{ff}$.
} 
Wir sind im familiären Niemandsland. Wir sind keine Adoptivfamilie, keine Stieffamilie, keine normale Familie. Irgendwie sind wir von allem etwas, aber keines richtig. Wir wissen oft nicht, wie wir mit anderen darüber reden können und vor allem, wie andere vielleicht darauf reagieren. Daher sagen wir in dem meisten Fällen einfach nichts. Jeder geht ja erst einmal davon aus, dass wir eine ganz normale Familie sind.

Der Mangel hat auch dazu geführt, dass zuverlässige Informationen über DI nach wie vor spärlich sind. Bis vor kurzem war psychoedukative Ratgeberliteratur ${ }^{53}$ in Deutschland nicht verfügbar ${ }^{54}$ und auch im Ausland ist diese erst in den letzten Jahren verstärkt erhältlich. ${ }^{55}$ Das Informationsdefizit führt bei Paaren, die eine DI beabsichtigen, zu Unsicherheiten: ${ }^{56}$

Wir fragten unseren Arzt, und uns wurde gesagt, nein, die Samenspende ist in Deutschland nicht erlaubt. Wir haben dann den Arzt gewechselt, denn wir haben herausgefunden, dass es doch erlaubt ist.

Es gibt so wenig Information über die Samenspende, und wir bräuchten Bücher darüber, wie sich denn die Kinder, die aufgeklärt sind, und diejenigen, die nicht aufgeklärt sind, entwickeln. Es gibt so viele Fragen, und wir können keine Antworten finden.

Wir waren 5 Minuten bei dem Psychotherapeuten, erwähnten die Spendersamenbehandlung und er schaute uns an und sagte, ,das machen Sie besser nicht. Menschen, die so etwas machen, denken nur an sich selbst und vergessen das Wobl des Kindes'. Wenn überhaupt, konnte er uns nur ein Pflegekind empfehlen, das jederzeit wieder zu seinen Eltern zurückkehren kann. Wir waren so schockiert, dass ich nur weinte. Der nächste Psychologe versuchte auch, uns die Samenspende auszureden und das war sehr schwierig für uns.

Auf der rechtlichen Ebene wurden für einige Familientypen, die soziale Bindungen beinhalten und somit von der Norm abweichen, Sicherheiten geschaffen, beispielsweise durch das Adoptionsvermittlungsgesetz für Adoptivfamilien. Für Familien nach DI wurden in den 1950er Jahren solche juristischen Änderungen bewusst abgelehnt. Man befürchtete, dass jegliche juristische Regelung außer einem strikten Verbot das Ansehen dieses Familientyps fördern würde. ${ }^{57}$ Dies führte dazu, dass erst 2002 eine Änderung im Bürgerlichen Gesetzbuch (§ 1600d BGB)

\footnotetext{
53 Solche Ratgeberliteratur unterstützt Eltern bei der Entscheidungsfindung für oder gegen diese Familienbildung, indem relevante psychosoziale Fragestellungen aufgezeigt werden. Darüber hinaus sind inzwischen Kinderbücher publiziert worden, die Eltern für die Aufklärung über die Zeugungsart nutzen können.

${ }^{54}$ Thorn, Die Geschichte unserer Familie. Ein Buch für Familien, die sich mit Hilfe der Spendersamenbehandlung gebildet haben, 2006; Thorn, Familiengründung mit Spendersamen - ein Ratgeber zu psychosozialen und rechtlichen Fragen, derzeit in Druck.

55 Frost-Vercollone/H.Moss/R.Moss, Helping the stork: The choices and challenges of donor insemination, 1997; Lorbach, Experiences of donor conception. Parents, offspring and donors through the years, 2003; Daniels, Building a family with the assistance of donor insemination: 'It wasn't our ideal way of having a family but it is our ideal family', 2004.

56 Thorn/Daniels, Pro und Contra Kindesaufklärung nach donogener Insemination - Neuere Entwicklungen und Ergebnisse einer explorativen Studie, Geburtshilfe und Frauenheilkunde 2007/67, 993 ff. 57 Fromm, Artefizielle Insemination. Referat Dr. Fromm in der geschlossenen Sitzung des 62. Deutschen Ärztetages am 25.7.1959, 1959.
} 
dazu führte, dass zumindest die Vaterschaft dann nicht mehr angefochten werden kann, wenn sowohl die Wunschmutter als auch ihr Partner in die DI eingewilligt haben; dem Kind bleibt ein Anfechtungsrecht offen. Nach wie vor fehlt eine explizite Freistellung des Samenspenders, so dass dieser grundsätzlich vor Unterhaltsforderungen und Erbrechtansprüchen geschützt ist, einerlei, ob der Samen für ein heterosexuelles oder ein lesbisches Paar bzw. für eine allein stehende Frau verwendet wird; immerhin ist keiner dieser Gruppen der Zugang zu einer DI gesetzlich verwehrt, auch wenn die Bundesärztekammer nur die Behandlung heterosexueller Paare empfiehlt. ${ }^{58}$ Auch wurde die Dokumentation der medizinischen Unterlagen von vormals 10 auf 30 Jahre verlängert. ${ }^{59}$ Doch der Gesetzgeber hat bislang den so gezeugten Menschen - im Gegensatz zu Adoptierten - keinen gesetzlichen Anspruch eingeräumt, Zugang zu der Identität ihres Erzeugers zu erlangen. Dies widerspricht den Prinzipien von Fairness und Autonomie. Die mangelnde rechtliche Absicherung dieses Familientyps symbolisiert die geringe Wertschätzung, die ihm zugesprochen wird; sie kommt einer strukturellen Diskriminierung gleich, da sie bei allen Beteiligten zu Verunsicherung führt.

Als zusätzliche strukturelle Diskriminierung wird auch die mangelnde Finanzierung der $\mathrm{DI}^{60}$ und die Unmöglichkeit, die medizinischen Behandlungskosten steuerlich geltend zu machen erachtet. ${ }^{61}$ Die fehlende Bereitstellung gesellschaftlicher Mittel symbolisiert die Geringschätzung dieses Familientyps:

Es wäre schön gewesen, wenn unsere Krankenversicherung die Behandlung übernommen bätte. Mir macht es nichts aus zu zablen, aber ich verstehe nicht, weshalb ich für eine Methode zahlen muss und für eine andere nicht. Und dann konnten wir die Kosten, die recht hoch waren, noch nicht einmal wie andere Gesundheitskosten beim Finanzamt einreichen. Das ist sehr unfair.

Die Abweichung vom Familienideal und die mangelnde juristische Sicherheit, die nicht nur in Deutschland, sondern in vielen Ländern vorherrschte, führte dazu dass Ärzte und Patienten über lange Zeit dachten, ,das Beste sei, nicht über das zu

\footnotetext{
58 Bundesärztekammer, (Muster-) Richtlinie zur Durchführung der assistierten Reproduktion, Dt. Ärzteblatt 2006, $1392 \mathrm{ff}$.

${ }^{59}$ Richtlinie 2004/23/EG des Europäischen Parlaments und des Rates vom 31.3.2004 zur Festlegung von Qualitäts- und Sicherheitsstandards für die Spende, Beschaffung, Testung, Verarbeitung, Konservierung, Lagerung und Verteilung von menschlichen Geweben und Zellen, Amtsblatt Nr. L 201 vom 7.4.2004, S. 48 ff, abrufbar unter:

http://eur-lex.europa.eu/LexUriServ/site/de/oj/2004/1_102/1_10220040407de00480058.pdf (letzter Zugriff: 4.8.2008); Bundesäratekammer (Fußn. 58); Hammel/Bispink/Katzorke/Scbreiber/Thorn, Empfehlungen des Arbeitskreises für Donogene Insemination zur Qualitätssicherung der Behandlung von Spendersamen in Deutschland in der Fassung vom 8.2.2006, Journal für Reproduktionsmedizin und Endokrinologie 2006, 166 ff.; Transplantationsgesetz (TPG) v. 1997, neugefasst durch Bek. v. 4.9.2007: Gesetz über die Spende, Entnahme und Übertragung von Organen und Geweben; http://www.gesetze-im-internet.de/bundesrecht/tpg/gesamt.pdf (letzter Zugriff: 4.8.2008).

${ }^{60}$ Gemäß \ 27 a SBG V werden Kosten für reproduktionsmedizinische Behandlung nur übernommen, wenn die Gameten eines mit einander verheirateten Ehepaars verwendet werden.

${ }^{61}$ Bundesfinanzhof, Bundesfinanzhof Nr. 10, Urteil v. 18.5.1999, III R 46/97, 1999.
} 
sprechen, was sie taten“.62 Ärzte befürchteten, dass die Wahrheit zu unüberwindbaren sozialen und psychologischen Schwierigkeiten für das Kind und die Familie führen könne, dass also sowohl Kinder als auch deren Eltern stigmatisiert und marginalisiert werden könnten. ${ }^{63}$ Eltern ihrerseits waren selbstverständlich bemüht, nicht in Verbindung mit einer moralisch verwerflichen Handlung gebracht zu werden und sich selbst und ihre Kinder zu schützen. ${ }^{64}$ Auch Ärzte hatten Interesse, dass Eltern verschwiegen mit der DI umgingen. Immerhin wurde damit sichergestellt, dass auch ihre öffentliche Reputation gewahrt blieb, denn sie wurden nicht in Verbindung mit einer sozial geächteten Behandlung gebracht. ${ }^{65}$ Aufgrund der mangelnden Rechtssicherheit gewährleistete die Geheimhaltung auch, dass die Wahrscheinlichkeit von Unterhaltsansprüchen an Samenspender bzw. Ersatzansprüche an Ärzte selbst, wenn sie die Identität der Spender nicht preisgeben konnten oder wollten, gering blieb. ${ }^{66} \mathrm{Da}$ ärztliche Standards Normen repräsentierten, die gesellschaftlich akzeptiert wurden, befolgten Patienten ihren Rat. ${ }^{67}$ Darüber hinaus hatten Ärzte eine zentrale Rolle inne: sie waren diejenigen, die zu allen Beteiligten Kontakt hatten und konnten somit die Information bestens kontrollieren. ${ }^{68}$

Obgleich die DI dazu führt, dass Paare Kinder zeugen und damit den pronatalistischen Erwartungen entsprechen können, ist die Stigmatisierung der DI gewissermaßen potenziert. Die Zusammensetzung dieser Familie entspricht nicht der Normvorstellung der „biologischen“ Familie und bei den meisten heterosexuellen Paaren ist männliche Unfruchtbarkeit der Grund für eine DI. Die Geheimhaltung dient daher nicht nur der Verdeckung der Familienzusammensetzung, sondern auch dem Schutz des männlichen Partners vor einer Bloßstellung ob seiner Zeugungsunfähigkeit. Gleichzeitig bietet sich die Geheimhaltung der DI sozusagen an, denn - im Gegensatz zu einer Adoption - wird die Mutter schwanger und gebiert das Kind; der Beitrag des Samenspenders ist nach außen nicht wahrnehmbar. Als diskreditierbares Stigma ist dies mit Scham aufgrund der Abweichung und Angst vor Entblößung verbunden: ${ }^{69}$

Ich babe mit niemandem über die Samenspende gesprochen, denn dann würde jeder wissen, dass ich unfruchtbar bin und dass es nicht mein Samen war, der zur Befruchtung geführt hat. Meine Frau wurde ganz normal schwanger und jeder kann davon ausgehen, dass es eine

\footnotetext{
62 Snowdon, Sharing information about DI in the UK, Politics and the Life Sciences 1993/2, $194 \mathrm{ff}$.

63 Snowdon/Mitchell, The artificial family, 1981.

${ }^{64}$ Daniels/Taylor, Secrecy and openness in donor insemination, Politics and the Life Sciences 1993/12, $155 \mathrm{ff}$.

65 Haimes, Do clinicians benefit from gamete donor anonymity?, Human Reproduction 1993/8, $1518 \mathrm{ff}$.

66 Annas, Fathers anonymous: Beyond the best interest of the sperm donor, Family Law Quarterly 1980/14, 1 ff.; Haimes (Fußn. 65).

${ }^{67}$ Triseliotis, Donor insemination and the child, Politics and the Life Sciences 1993/12, $195 \mathrm{ff}$.

${ }^{68}$ Back/Snowdon, The anonymity of the gamete donor, Journal of Psychosomatic Obstetrics and Gynaecology 1988/9, $191 \mathrm{ff}$.

${ }^{69}$ Thorn/Daniels (Fußn. 56).
} 
ganz normale Schwangerschaft ist und wir dann auch eine normale Familie. Dabei werden wir es auch erst einmal belassen, auch wenn ich Angst habe, dass irgendwann alles aufgedeckt wird.

Die von der Stigmatheorie beschriebenen „unbehaglichen Momente“ in der zwischenmenschlichen Interaktion, die aufgrund stereotypischer Erwartungshaltungen und entsprechendem Verhalten zustande kommen, werden inzwischen in der Literatur zum Thema DI angedeutet. ${ }^{70}$ Erfahrungsberichte von mit DI gezeugten Erwachsenen geben erste Hinweise, dass sie inkongruentes Verhalten wahrnehmen. Sie deuten an, dass sie vor der Aufklärung Familiengeheimnisse vermuteten und beschreiben beispielsweise, dass Eltern bestimmte Themen aussparten oder dass das Verhalten des Vaters zu ihnen und der restlichen Familie distanziert war. ${ }^{71}$ Auch eine qualitative Studie zeigt auf, dass Kinder Unstimmigkeiten im Verhalten ihrer Eltern wahrnehmen, welches sie als Erwachsene auf die elterliche Bemühung, ihre Zeugungsart geheim zu halten, zurückführten. ${ }^{72}$ Es liegen keine Studien vor, die Interaktionsverhalten von Paaren in Behandlung, bzw. Eltern nach DI mit Außenstehenden untersuchen, diese selbst äußern im klinischen Kontext jedoch die Befürchtung, dass die Geheimhaltung sie selbst in ihrem Verhalten beeinträchtigen könnte:

Eigentlich ist es kaum möglich, die Samenspende immer zu verbeimlich. Wir müssten uns immens anstrengen, dass wir uns nie etwas anmerken lassen und immer genau überlegen, wem wir was gesagt haben und ob dies schlüssig ist. $\mathrm{Ob}$ dies auf Dauer tatsächlich möglich ist, wissen wir nicht.

Wir mussten immer aufpassen, was wir anderen sagen. Wir sagten zum Beispiel immer, dass wir eine Insemination macbten, um möglichst nahe an der Wabrheit zu bleiben und bielten innerlich den Atem an, dass sich niemand gut damit auskennt und fragt, welche Art von Insemination wir durchfïhren lassen.

Deutlich wird, dass die Familienbildung mit DI auf allen Ebene Stigmatisierung erfährt. Die von dem Ideal einer „biologischen“ Familie abweichende Zusammensetzung dieses Familientyps führt zu einer Verunsicherung der Eltern und dazu, dass die DI auf der Makroebene in einem rechtlich unzureichend geregelten Bereich verbleibt. Im Mesobereich angesiedelte ärztliche Empfehlungen zur Geheimhaltung verstärken die Scham und das Tabu und damit die Angst vor Stigmatisierung. Im Mikrobereich wird das Tabu nicht herausgefordert, da männliche Unfruchtbarkeit, der häufigste Grund für eine DI, mit Scham besetzt ist. Ob und

${ }^{70}$ Hebl/Tickle/Heatherton (Fußn. 15).

${ }^{71}$ Baran/Pannor, Lethal secrets: The shocking consequences and unsolved problems of artificial insemination, 1989; Lorbach, Let the offspring speak. Georges Hall, NSW: The Donor Conception Support Group of Australia, 1997; Frost-Vercollone/H.Moss/R.Moss (Fußn. 55); Cordray, An open letter to the HFEA, Donor Conception Network Journal 1999/2000, 3 ff.

72 Turner/Coyle, What does it mean to be a donor offspring? The identity experiences of adults conceived by donor insemination and the implications for counselling and therapy, Human Reproduction 2000/15, $2041 \mathrm{ff}$. 
gegebenenfalls wie eine Entstigmatisierung dieses Familientyps erreicht werden kann, soll im letzten Abschnitt analysiert werden.

\section{Entstigmatisierungspotenzial}

Strategien, die eine Entstigmatisierung voranbringen können, sind auf Maßnahmen im Mikro-, Meso- oder Makrobereich zurückzuführen. Auf der Mikroebene wird mittlerweile in vielen westlichen Ländern psychologische Unterstützung angeboten, die u.a. dazu beitragen soll, dass sich Paare mit Kinderwunsch von normativen Erwartungen lösen können, dass sie sich als kinderloses Paar nicht als weniger wertvolles Mitglied der Gesellschaft erachten, sondern ihr Selbstwert gestärkt wird. ${ }^{73}$ Auch wird, wie Hebl und Kollegen vorschlagen, ${ }^{74}$ auf positive Persönlichkeitsmerkmale fokussiert, und es werden Copingstrategien entwickelt, damit Paare mit Kinderwunsch damit souverän umgehen lernen. In einer aktuellen Studie kritisieren Malik und Coulson jedoch, dass sich viele dieser Angebote primär an Frauen richten und Männer sich hiervon ausgeschlossen fühlen. ${ }^{75}$ Sie fordern daher, dass Unterstützungsangebote adaptiert und auf die Bedürfnisse von Männern ausgerichtet werden. Dies zeigt auf, dass hier nach wie vor Handlungsbedarf besteht. Ähnliches gilt für die Beratung vor oder im Rahmen der medizinischen Behandelung mit DI. In einigen Ländern, beispielsweise in Australien, ${ }^{76}$ in Großbritannien ${ }^{77}$ und in Deutschland ${ }^{78}$ wird mittlerweile von Fachverbänden eine Beratung vor Behandlungsbeginn empfohlen. In vielen Ländern gibt es jedoch keine Empfehlung zur Beratung und kaum Beratungskonzepte. ${ }^{79}$ Auch gibt es Länder wie Kanada, die eine solche Beratung gesetzlich vorschreiben. ${ }^{80} \mathrm{Ob}$ eine verpflichtende Beratung mit dem ethischen Prinzip der Autonomie in Einklang gebracht werden kann, wird allerdings kontrovers diskutiert. ${ }^{81}$ Mit einiger Wahrscheinlichkeit wird eine Beratung auch ohne Verpflichtung als hilfreiches Angebot wahrgenommen, wenn im medizinischen Bereich, also auf der Mesoebene, dies entsprechend formuliert wird und ausreichend niedrigschwellige Beratungsangebote vorhanden sind.

73 Covington/Hammer-Burns, Infertility Counselling - A comprehensive handbook for clinicians, 2006; $B K i D$, Richtlinien psychosoziale Beratung bei Kinderwunsch, 2007;

http://www.bkid.de/richtlinien.pdf (letzter Zugriff: 3.6.2008).

${ }^{74} \mathrm{Hebl} /$ Tickle/Heatherton (Fußn. 15).

${ }^{75}$ Malik/Coulson (Fußn. 41).

76 ANZICA, Guidelines for professional standards of practice infertility counselling, 2003.

77 BICA, Guidelines for good practice in fertility counselling, BICA publications, 2006.

78 Thorn/Wischmann, Leitlinien für die psychosoziale Beratung bei Gametenspende, Journal für Reproduktionsmedizin und Endokrinologie 2008, $147 \mathrm{ff}$.

${ }^{79} \mathrm{Blyth} /$ Landau, Third party assisted conception. Social, legal and ethical perspectives, 2004.

80 Statutes of Canada, Bill C-6, 2004.

${ }^{81}$ Daniels, Donor Insemination, in: Boivin/Kentenich (eds.), Guidelines for Counselling in Infertility. ESHRE Monographs, 2002, pp. 31 ff. 
Auch die auf der Mesoebene vorgeschlagenen Selbsthilfegruppen bzw. -organisationen existieren für ungewollt Kinderlose seit über 20 Jahren. ${ }^{82}$ Gerade der Austausch mit anderen Betroffenen führt in der Tat zu einer Normalisierung der Affekte und Validierung der Einstellungen, nicht nur bei Paaren mit Fruchtbarkeitsstörungen, ${ }^{83}$ sondern auch bei solchen, die eine DI beabsichtigen. ${ }^{84}$ Vor allem jedoch können sich Paare, bzw. Personen in diesen Kreisen austauschen, ohne Abwertung befürchten zu müssen; die Teilnehmer sind sozusagen kompatibel in ihrer Interaktion und daher keiner potenziellen Diskriminierung ausgesetzt. ${ }^{85}$ Zwar gehen Forscher davon aus, dass die öffentliche Darstellung von Unfruchtbarkeit, den Nöten Betroffener sowie politische Forderungen durch Selbsthilfegruppen bzw. organisationen besonders geeignet sind, um auf deren Situation aufmerksam zu machen, um edukative Prozesse zu fördern und um Barrieren abzubauen. Allerdings setzt dies voraus, dass die Teilnehmer dieser Gruppen ausreichend Selbstbewusstsein haben, um sich in der Öffentlichkeit darzustellen. Wie in dem Beispiel oben anhand des Interessensverbands „Wunschkind“ aufgezeigt, können Faktoren aus dem Mikrobereich wie mangelnde Selbstsicherheit durchaus Aktivitäten auf dem Mesobereich einschränken oder unmöglich machen. Andererseits kann eine solche „gemischte Interaktion“, also eine Interaktion zwischen Stigmatisierten und nicht Stigmatisierten, vieles dazu beitragen, dass Bedürfnisse Stigmatisierter anerkannt und wertgeschätzt werden und vermeiden helfen, dass sie aufgrund ihrer Andersartigkeit diskreditiert werden. ${ }^{86}$ In Australien hat beispielsweise die dortige Selbsthilfeorganisation ACCESS mit dazu beigetragen, dass die Kostenübernahme für reproduktionsmedizinische Behandlungen durch das Krankenkassensystem umfassender wurde und gleichzeitig diese Gruppe als aktiver Stakeholder in der öffentlichen Diskussion wahrgenommen wurde..$^{87}$ In Großbritannien hat sich die dortige Selbsthilfeorganisation für Familien nach Gametenspende, das Donor Conception Network ${ }^{88}$, und ihre Mitglieder immer wieder für eine Identifizierbarkeit der Spender ausgesprochen und auch in Deutschland gibt es mittlerweile einen (noch ano-

\footnotetext{
82 Dill, International treatment differences: Policy, politics, partnerships and ART, in: Valverde (ed.), 2050: A changing Europe. Demographic crisis and baby friendly policies. Pharmaceutical Policy and Law, 2007, vol. 9, pp. 147 ff.

83 Covington, Group approaches to infertility counselling, in: Covington/Hammer-Burns (eds.), Infertility Counselling - A comprehensive handbook for clinicians, 2006, pp. $156 \mathrm{ff}$.

${ }^{84}$ Thorn/Daniels, A group work approach in family building by donor insemination - Empowering the marginalized, Human Fertility 2003, 46 ff.

85 Miller/Major, Coping with stigma and prejudice, in: Heatherton/Kleck/Hebl/Hull (eds.), The social psychology of stigma, 2000, pp. $243 \mathrm{ff}$.

86 Hebl/ Tickle/Heatherton (Fußn. 15).

87 Dill, Consumer Perspectives, in: Vayena/Rowe/Griffin (eds.), Current practices and controversies in assisted reproduction. Report of a meeting on "Medical, Ethical and Social Aspects of Assisted Reproduction" held at the WHO Headquarters in Geneva, Switzerland, 17-21.9.2001. World Health Organisation, Geneva, 2001, pp. 255 ff.

${ }^{88}$ www.dcnetwork.org (letzter Zugriff: 20.7.2008).
} 
nymen) Zusammenschluss von jungen mit DI gezeugten Menschen, die umfassende gesetzliche Regelungen fordern. ${ }^{89}$

Auch auf der Makroebene wurden in den letzten Jahren Veränderungen vorgenommen. In einigen Ländern wie Schweden, ${ }^{90}$ Victoria (Australien), ${ }^{91}$ Großbritannien, ${ }^{92}$ Kanada $^{93}$ und der Schweiz ${ }^{94}$ wurden für den Bereich der Samenspende (bzw. Gametenspende in den Ländern, in denen auch die Eizellspende erlaubt ist) Gesetze erlassen, die den so gezeugten Menschen das Recht einräumen, die Identität des Spenders zu erfahren. Parallel dazu wurden Gametenspender von finanziellen Ansprüchen der Menschen, zu deren Zeugung sie beigetragen haben, freigestellt. Diese Änderungen beruhen vor allem auf einer umfassenden Diskussion um das Kindeswohl: Kinder nach Gametenspende sind, im Gegensatz zu Eltern, Spender und Ärzten, die einzigen Akteure, denen eine aktive Mitentscheidung unmöglich ist. Nicht zuletzt um ethische Prinzipien wie Fairness und Autonomie einzuhalten, wurden diesen Kindern in den o.a. Ländern ähnliche Rechte wie Adoptierten eingeräumt. Damit wurde ihnen die Möglichkeit eines selbst bestimmten Umgangs mit der Frage gegeben, ob und wann sie Information über ihre biologischen Wurzeln erlangen möchten. ${ }^{95}$ Allerdings lösten diese Änderungen viele kontroverse Diskussionen aus: Vor allem wurde sowohl von Ärzten als auch von Selbsthilfeorganisationen die Befürchtung geäußert, dass eine Identifizierbarkeit der Spender dazu führen könnte, dass deutlich weniger Männer bereit wären, Samen zu spenden. ${ }^{96} \mathrm{Ob}$ dies tatsächlich der Realität entspricht, wird jedoch auch hinterfragt. ${ }^{97}$ Ebenso kontrovers wurde diskutiert, ob eine Gesetzgebung das Stigma dahingehend verändert, dass die Aufklärungsrate von Kindern steigt. In Schweden, wo die Gesetzgebung bereits 1985 geändert wurde, war man schlichtweg davon ausgegangen, dass Eltern nach der Gesetzesänderung ihre Kinder aufklären werden. Die Paare wurden über die Gesetzesänderung informiert und führten ein Gespräch mit einer Sozialarbeiterin durch, die die Aufklärung des Kindes empfahl. Tatsächlich stiegen die Aufklärungsrate und auch die Anzahl von Eltern, die mit Außenstehenden über die DI sprachen, allerdings schätzten die Autoren dieser Studie die Aufklärungsrate von 52\% insgesamt als niedrig ein. Sie schlussfolgerten, dass die Gesetzgebung alleine nicht ausreichte, um Haltungen in einer

${ }^{89}$ www.spenderkinder.de (letzter Zugriff: 20.7.2008).

${ }^{90}$ Lag om Insemination, Schweden No, 1140/1984, 1985.

${ }^{91}$ Infertility Treatment ACT, Victoria/Australia, 1994.

${ }^{92}$ HFE A, Disclosure of Donor Information Regulations, 2004.

${ }^{93}$ Statutes of Canada (Fußn. 80).

${ }^{94}$ Schweizer Fortpflanzungsmedizingesetz (FMedG) vom 18.12.1998 (Stand am 13.6.2006).

95 Gottlieb/Lalos/Lindblad, Disclosure of donor insemination to the child: The impact of Swedish legislation, Human Reproduction 2000/15, 2052 ff.; Blyth/Farrand, Anonymity in donor-assisted conception and the UN Convention on the Rights of the Child, The International Journal of Children's Rights 2004/12, 89 ff.

${ }^{96}$ Blyth, Donor insemination and the dilemma of the "unknown father", in diesem Band.

${ }^{97}$ Daniels, Anonymity and openness and the recruitment of gamete donors. Part I: semen donors, Human Fertility 2007, 151 ff. 
Bevölkerung zu ändern und schlugen zusätzliche ethische Diskussion mit allen beteiligten Fachkräften vor. ${ }^{98}$ Mit einiger Wahrscheinlichkeit ist es ebenso erforderlich, dass Eltern nicht nur die Aufklärung empfohlen wird, sondern sie konkrete Unterstützung für eine entwicklungspsychologisch angemessene Aufklärung erhalten und auch ihre Ängste und Vorbehalte ausführlich exploriert werden. Auch dies zeigt, dass Änderungen auf einer Ebene durch Änderungen auf anderen Ebenen konsolidiert werden müssen.

Diese Analyse verdeutlicht, dass Veränderungen auf allen Ebenen erforderlich sind, um Haltungen und Glaubenssätze dauerhaft zu transformieren und die DI als eine andere, jedoch vertretbare Form der Familienbildung anzuerkennen. Auf der Mikroebene wäre es erforderlich, Paare, die eine DI beabsichtigen, psychologisch zu unterstützen, damit sie souverän mit der Diagnose „Unfruchtbarkeit“ umgehen können, Aspekte männlicher Unfruchtbarkeit gewürdigt werden und sie sich mit den Fragestellungen, die die Familiebildung mit DI nach sich zieht, auseinander setzen. Auf der Mesoebene können Selbsthilfegruppen und -zusammenschlüsse gefördert werden, damit einerseits eine Unterstützung durch Peers erfolgen kann und andererseits Betroffene selbst ihre Bedürfnisse in der Öffentlichkeit vertreten können. Ebenso scheint es erforderlich, dass medizinische, juristische und psychologische Fachkräfte umfassend über diese Familienbildung informiert sind, sodass sie Ratsuchende und Patienten fundiert und wertfrei beraten können. Darüber hinaus können diese sowohl fach- als auch Laienliteratur erstellen, die in der Fachwelt rezipiert wird, und Interessenten damit schriftliches Informationsmaterial zur Verfügung stellt. Auf der Makroebene sind gesetzliche Änderungen erforderlich, die die Prinzipien von Fairness, Autonomie und den Grundsatz ,primum nil nocere“ für alle Beteiligten respektieren. Nur ein derart konzertiertes Vorgehen ist erfolgreich, damit Veränderungen auf einer Ebene nicht durch andere Kräfte unterminiert werden, sondern sich gegenseitig bei dem Prozess der Entstigmatisierung unterstützen.

98 Gottlieb/Lalos/Lindblad (Fußn. 95). 



\title{
Donor insemination and the dilemma of the "unknown father"
}

\author{
Eric Blyth
}

As has been the pattern worldwide, the provision of donor insemination (DI) - and subsequently other forms of donor conception - in the UK has traditionally been characterised by withholding the identities of donors and recipients and of donors and their donor-conceived offspring from each other. Furthermore, recipients of donor gametes were frequently advised not to tell anyone, including their children, of their recourse to donor conception. ${ }^{1}$ Inter-related factors promoting such practices include:

- the dubious legal and moral status of donor conception (including explicit disapproval from several major world religions) - with consequent concerns about the impact on the child and other family members if knowledge of donor conception became "common knowledge". ${ }^{2}$

- suspicions about sperm donors' personality and motivation. ${ }^{3}$

- recipients' ambivalence about the use of donor conception - especially DI. ${ }^{4}$

1 Royal College of Obstetricians and Gynaecologists, Donor Insemination, 1987.

2 Bielawska-Batorowicz, Poland: provision and guidelines for third party assisted conception, in: Blyth/Landau (eds.), Third Party Assisted Conception Across Cultures: Social, Legal and Ethical Perspectives, 2004, pp. 168 ff.

3 Feversham Committee, Report of the Departmental Committee on Human Artificial Insemination, 1960, Cmnd. 1105; Johnston, in: Wood/Leeton/Kovacs (eds.), Artificial Insemination, 1980.

${ }^{4}$ Brewaeys/Golombok/Naaktgeboren/deBruyn/v.Hall, Donor insemination: Dutch parents' opinions about confidentiality and donor anonymity and the emotional adjustment of their children, Human Reproduction 1997/12, $1591 \mathrm{ff}$. 
- uncertainties about the impact on the child of knowledge of the circumstances of their conception (or assumptions that such knowledge would be damaging) ${ }^{5}$

- uncertainties about the donor's legal or financial responsibilities towards the child and to protect them from unintended/undesired contact or responsibilities. ${ }^{6}$

- uncertain legal status of the child - and fears of prosecution/conviction for falsification of birth registration information. ${ }^{7}$

- stigma associated with male infertility in particular and families desires - and the opportunity - to "pass as normal". ${ }^{8}$

- potential consequences of the parents' imbalanced genetic relationship with the child and fears of the child's rejection of the non-genetic parent. ${ }^{9}$

- fears of unwanted intrusion by the donor in family affairs..$^{10}$

- initial reliance on fresh sperm, requiring management of the geographical and temporal proximity of donor and recipient. ${ }^{11}$

- parents' uncertainties about the timing and method of telling their child and the lack of information to give the child, making "not telling" - at least superficially - an easier option. ${ }^{12}$

- physicians' concerns about their potential exposure to critical external scrutiny, including risk of claims of unprofessional/illegal practice. ${ }^{13}$

- need to maintain donor services in the face of the assumed adverse impact of disclosure on supply.14

\footnotetext{
${ }^{5}$ Braude/Johnson/Aiken, The Human Fertilisation and Embryology bill goes to report stage, British Medical Journal 1990/300, 1410 ff.; Haimes, Gamete donation and the management of genetic origins in: Stacey (ed.), Gamete Donation and the Management of Genetic Origins. Changing Human Reproduction, 1992.

${ }^{6}$ Winston, This foolish threat to the gift of life, Daily Mail 1999/10; Vanfraussen/Ponjaert Kristoffersen/Brewaeys, An attempt to reconstruct children's donor concept: A comparison between children's and lesbian parents' attitudes towards donor anonymity, Human Reproduction 2001/16, 2019 ff.

${ }^{7}$ Department of Health and Social Security, Report of the Committee of Inquiry into Human Fertilisation and Embryology (The Warnock Report), 1984, Cmnd. 9314.

${ }^{8}$ Haimes, Secrecy: what can artificial reproduction learn from adoption?, International Journal of Law and the Family 1988/2, 46 ff.

9 Tarrant, State secrets: Access to information under the Human Reproductive Technology Act 1991 (WA), Journal of Law and Medicine 2002/9, $336 \mathrm{ff}$.

${ }^{10}$ Department of Health and Social Security (n. 7).

11 Petersen, The rights of donor-conceived children to know the identity of their donor: The problem of the known unknowns and the unknown unknowns, in: Bennett/Tomossy (eds.), Globalization and Health: Challenges for Health Law and Bioethics, 2006, pp. $151 \mathrm{ff}$.

12 Vanfraussen/Ponjaert-Kristoffersen/Brewaeys (n. 6), 2019 ff.; Lasker/Borg, In Search of Parenthood: Coping with Infertility and High-Tech Conception, Pandora Press 1989.

13 Petersen, in: Bennett/Tomossy (n. 11), pp. 151 ff.; Haimes, Do clinicians benefit from gamete donor anonymity?, Human Reproduction 1993/8, 1518 ff.

${ }_{14}$ Craft/Thornbill, Will removal of anonymity influence the recruitment of egg donors? A survey of past donors and recipients, Reproductive Biomedicine Online 2005/10, $325 \mathrm{ff}$.
} 


\section{Legislation in the United Kingdom}

When the United Kingdom (UK) first introduced legislation in 1991 to regulate assisted conception, the Human Fertilisation and Embryology Act 1990 (hereafter "the Act"), prevailing wisdom concerning donor anonymity was accepted, ${ }^{15}$ although the government did not rule out the possibility of donor-conceived people being able to learn the identity of their donor in the future. ${ }^{16}$ The Warnock Committee (on whose report the Act drew significantly) had already begun to encroach upon existing practices espousing secrecy. While the Committee explicitly endorsed anonymity (to "protect" all parties from legal complications and emotional difficulties ${ }^{17}$ and to avoid the "invasion of the third party into the family", it was less ready to endorse deception of donor-conceived people about their origins. ${ }^{18}$ The Committee expressed concerns that secrets in families could "undermine the whole network of family relationships" and believed that it was "wrong to deceive children about their origins", ${ }^{19}$ and proposed that, on reaching the age of 18, a donor-conceived person should have a legal right of access to "basic information about the donor's ethnic origins and genetic health". ${ }^{20}$ In order to ensure a donorconceived person's ability learn the circumstances of their conception, the Committee further proposed that, when registering the child's birth, parents should be able to add the text "by donation", 21 although this proposal was not pursued by UK lawmakers. ${ }^{22}$

With the exception of the Committee's proposals regarding birth registration, its recommendations regarding donor conception were generally encapsulated in the Act. A statutory regulatory body, the Human Fertilisation and Embryology Authority (HFEA), was established. Among the HFEA's responsibilities is maintaining a Register of Information recording information of all donor procedures performed by UK clinics, including details of donors, recipients of donated gametes or embryos and any children born as a result of a donor procedure. Basic social and health details of donors were also recorded and donors were also invited to provide brief further details that could be shared with any child or their parents. However, the information recorded by donors was of variable content and quality;

\footnotetext{
15 Except for powers given to a court to order disclosure of a donor's identity in the "interests of justice" or in connection with any legal proceedings resulting from the birth of a child with a congenital disability.

16 Clarke, House of Commons Debates. Official Report, 20.6.1990, col.. 1009, http://www.publications.parliament.uk/pa/cm198990/cmhansrd/1990-06-20/Debate-19.html.

17 Department of Health and Social Security (n. 7), 3.2.

18 Department of Health and Social Security (n. 7), 4.22.

${ }^{19}$ Department of Health and Social Security (n. 7), 4.12.

${ }^{20}$ Department of Health and Social Security (n. 7), 4.21.

${ }^{21}$ Department of Health and Social Security (n. 7), 4.25, 6.8.

${ }^{22}$ House of Commons Standing Committee B, House of Commons: Official Report, 15.5.1990, cols. 184 ff.
} 
some donors treating the request for information facetiously, providing comments likely to cause distress and offence to any offspring reading them. ${ }^{23}$

The Act provided that at the age of 18 any individual could ascertain if the HFEA Register of Information indicated that they had been conceived following donor conception. Of course the ability to access this information depends on the individual knowing - or at least suspecting - that they were conceived as a result of a reproductive procedure in the first place. Early evidence indicated that few parents told their donor-conceived children about their conception, ${ }^{24}$ and - up to 2004 - the HFEA merely advised centres "to take into account ... a child's potential need to know about their origins and whether or not the prospective parents are prepared for the questions which may arise while the child is growing up". 25 The Act provided for regulations to allow for the release of non-identifying donor information to a donor-conceived adult, although these were not drawn up and implemented until 2004. In addition, the Act permitted any two individuals contemplating marriage (i.e. aged at least 16 years) to ascertain whether the Register indicated the existence of a genetic relationship between them as a result of donor conception.

Challenges to legislative support for donor anonymity failed to counter combined medical and patient interests, concerned that a failure to protect donor anonymity would impact adversely on donor gamete supplies. ${ }^{26}$ Parliamentary support for anonymity drew on the alleged negative consequences for donor recruitment following Sweden's removal of anonymity in 1984, ${ }^{27}$ although this "evidence" was already out of date and no longer accurate ${ }^{28}$ - and what was overlooked entirely was the suggestion that the Swedish legislation had been actively undermined by physicians unsympathetic to it. ${ }^{29}$

23 Wincott/Crawshaw, From a social issue to policy: social work's advocacy for the rights of donor conceived people to genetic origins information in the United Kingdom, Social Work in Health Care 2006/43, $53 \mathrm{ff}$.

${ }^{24}$ Cook/Golombok/Bish/Murray, Disclosure of donor insemination: parental attitudes, American Journal of Orthopsychiatry 1995, 549 ff.;

Golombok/Brewaeys/Cook/Giavaz:i/Guerra/Mantoyani/v.Hall/Crosignani/Dexeus, The European study of assisted conception families: family functioning and child development, Human Reproduction 1996/17, $2324 \mathrm{ff}$

25 Human Fertilisation and Embryology Authority, Code of Practice, 6. edn. 2004, p. 13, http://www.hfea.gov.uk/docs/Code_of_Practice_Sixth_Edition_-_final.pdf. (my emphasis).

${ }^{26} \mathrm{Wincott} /$ Crawhaw (n. 23), $53 \mathrm{ff}$.

27 Primarolo, House of Commons Debates, 20.6.1990, cols. 989-990, http://www.publications.parliament.uk/pa/cm198990/cmhansrd/1990-06-20/Debate-17.html.

28 Jonsson, Artificial Insemination in Sweden. In: Gouvernement du Québec, Sortir la Maternité du Laboratoire Conseil du Statut de la Femme, 1988; Daniels/Lalos, The Swedish Insemination Act and the availability of donors, Human Reproduction 1995/10, $1871 \mathrm{ff}$.

29 Daniels, The Swedish Insemination Act and its impact, Australian and New Zealand Journal of Obstetrics and Gynaecology 1994/34, 437 ff. 


\section{Pressure for legislative change}

Despite the setback posed by the Act for opponents of donor anonymity, an increasingly concerted campaign evolved to use the government's promised introduction of regulations to determine the information that would be made available to donor-conceived people as a means to revisit the question of anonymity. The government's disinclination for urgency was perhaps understandable given that the first donor-conceived people to whom any such regulations would apply would not come of age until 2010. However, this stance failed to take account of the developing evidence, as noted above, that the limited information being collected for the HFEA Register of Information continued to compromise whatever rights to information a donor-conceived person would eventually receive and that without external pressure, the HFEA regime at the time showed no intention of changing its practices.

In 1995 a government minister indicated the government's intention to launch a consultation exercise to gauge public opinion "in due course" and a number of developments helped to progress the debate. ${ }^{30}$ First, accounts of the experiences of donor-conceived people, in the UK and other countries, that had not previously been available to the Warnock Committee and British lawmakers, indicated hitherto unacknowledged problems of anonymity. ${ }^{31}$ Without exception, they were severely critical of their inability to learn about their full biographical and genetic history and of the ways in which some had learned about their conception. As part of a global trend, support groups for donor-conceived families were also established (DI Network - subsequently the Donor Conception Network - and the Daisy Network), and at least some parents of donor-conceived children were proposing the abolition of anonymity in the light of first-hand evidence of its impact on their children. ${ }^{32}$

A direct challenge to the UK's maintenance of donor anonymity came in 2000 by way of an application to the English High Court for judicial review of the policy

\footnotetext{
${ }^{30}$ Lord Fraser of Carmyllie, Official Report, 6.6.1995, cols. 5-6, http://www.publications.parliament.uk/pa/ld199495/ldhansrd/vo950606/text/50606 15.htm.

31 Donor Conception Support Group of Australia Inc, Let the Offspring Speak: Discussions on Donor Conception, 1997; Cordray, A survey of people conceived through donor insemination, DI Network News 1999-2000/14, 4-5; Franz/Allen, Report to Health Canada on The Offspring speak. An International Conference of Donor Offspring, 12.8.2000, 2001; Spencer, What is the Experience of Confronting the Reality of Being a Donor Offspring?, 2000; Turner/Coyle, What does it mean to be a donor offspring? The identity experiences of adults conceived by donor insemination and the implications for counselling and therapy, Human Reproduction 2000/15, 2041 ff.; Gollancz, Donor insemination: a question of rights, Human Fertility 2001, 164 ff.; Rose, Exploring the Caves: Reflections on the Problems and Ethics of Being Conceived from "Donor" Sperm (unpublished BA [Hons] dissertation), 2001; Stevens, Offspring, 2001; Anonymous, How it feels to be a child of donor insemination?, British Medical Journal 2002/324, 797 ff., http://www.bmj.com/cgi/content/full/324/7340/7 97/DC1; Hamilton, Donor-conceived adults challenge the ethics of anonymity, Journal of Fertility Counselling 2002/9, 33-34; Hewitt, Missing links: identity issues of donor-conceived people, Journal of Fertility Counselling 2002/9, 14 ff.

32 Donor Conception Support Group of Australia Inc (n. 31); Lorbach, Experiences of donor conception: parents, offspring and donors through the years, 2003.
} 
by Joanna Rose, a donor-conceived adult, and an unidentified 6-year-old girl, EM. Rose and EM claimed breach of their rights under Article 8 of the European Convention on Human Rights (respect for private and family life) and Article 14 (protection from discrimination) on the grounds of discrimination against donor-conceived people relative to adoptive people (who are able to access available information about their birth parents) and that donor-conceived people born before implementation of the Act (who had no rights to information), experienced discrimination relative to donor-conceived people born after its implementation (who did have a right to access some information about their donor). ${ }^{33}$ The judge determined that Article 8 was engaged:

"what the claimants are trying to obtain is information about their biological fathers, something that goes to the very heart of their identity, and to their make up as people". ${ }^{34}$

However, consideration of whether there had been a human rights breach was adjourned pending the outcome of the government's consultation, which had eventually been launched in December 2001.35

In October 2002, the United Nations Committee on the Rights of the Child issued a periodic report reviewing progress made in the UK on implementing the UN Convention on the Rights of the Child. For the first time, the Committee formally questioned the compatibility of donor anonymity with the UK's endorsement of the Convention, in particular Article 3 (the best interests of the child) and Article 7 (the child's right "as far as possible .... to know and be cared for by his or her parents"). ${ }^{36}$ In January 2003 the government made public both the sub-

33 Rose and Another versus Secretary of State for Health and Human Fertilisation and Embryology Authority (2002), EWHC 1593.

34 Ibid at 33 .

35 Since the consultation resulted in the 2004 legislation removing donor anonymity with effect from 1.5.2005, the case was subsequently withdrawn. Department of Health, Providing information about sperm, egg and embryo donors, Consultation Document, 20.12.2001.

http://www.dh.gov.uk/assetRoot/04/01/87/74/04018774.pdf.

36 The UN Committee has similarly criticised Denmark, France, Monaco and Norway in respect of donor anonymity. However, since many more signatories of the UN Convention also endorse donor anonymity, the rationale for the Committee's selective approach is unclear. United Nations Committee on the Rights of the Child, Consideration of Reports Submitted by States Parties Under Article 44 of the Convention: Concluding Observations: United Kingdom of Great Britain and Northern Ireland, CRC/C/15/Add.188, 9.10.2002, http://www.unhchr.ch/tbs/doc.nsf/(Symbol)/CRC.C.15.Add.188.En?Opendocument;

United Nations Committee on the Rights of the Child, Concluding Observations of the Committee on the Rights of the Child: Denmark, CRC/C/15/Add.33, 15.2.1995, http://www.unhchr.ch/ tbs/doc.nsf/(Symbol)/ebe7468a5e679788412561510060c3a2?Opendocument; United Nations Committee on the Rights of the Child, Concluding Observations of the Committee on the Rights of the Child: France, CRC/C/15/Add.20, 25.4.1994,

http://www.unhchr.ch/tbs/doc.nsf/(Symbol)/25a7d876cfb56b5d412561510038866c?Opendocument; United Nations Committee on the Rights of the Child, Concluding Observations of the Committee on the Rights of the Child: Monaco, CRC/C/15/Add.158, 8.6.2001,

http://www.unhchr.ch/tbs/doc.nsf/(Symbol)/29bc23ab7979df2fc1256a76004b206e? Open-

document; United Nations Committee on the Rights of the Child, Concluding Observations of the Commit- 
stance of the responses to its consultation and its initial proposals for reform. ${ }^{37}$ Almost $90 \%$ of the 237 respondents to the consultation supported the right of donor-conceived people to non-identifying donor information ${ }^{38}$ while $56 \%$ of respondents advocated the complete prohibition of donor anonymity, $30 \%$ were opposed to its removal and 10\% were undecided. ${ }^{39}$ The minister for public health, Hazel Blears, acknowledged the government's acceptance of "a strong argument in principle for children conceived using donated sperm, eggs or embryos being able to find out the identity of their donor". 40

However, given the relatively few responses to the consultation from clinics, and concerns about the possible impact on donor recruitment and donor services, Blears announced that a final decision on anonymity would be postponed, pending further "research and engagement" with clinics and donors. At the same time, she announced the establishment of a pilot voluntary information exchange and contact register for people involved in a donor procedure undertaken before implementation of the Act. ${ }^{41}$ The Department of Health subsequently undertook its own survey of clinics ${ }^{42}$ and commissioned a study of donors' attitudes towards disclosure of information to offspring. ${ }^{43}$

In the meantime, parliament passed the Human Fertilisation and Embryology (Deceased Fathers) Act 2003. While this was not directly concerned with donor anonymity per se, it was centrally focussed on issues relating to identity and genetic

tee on the Rights of the Child: Norway, CRC/C/15/Add.23, 25.4.1994,

http://www.unhchr.ch/tbs/doc.nsf/(Symbol)/9fa425c091d1b821412561510038866f?.

37 Blears, Speech at HFEA Annual Conference, 28.1.2003;

http://www.dh.gov.uk/en/News/Speeches/Speecheslist/DH_4031642; Department of Health, Donor information: providing information about gamete or embryo donors: Responses to consultation, 28.1.2003; http://www.dh.gov.uk/assetRoot/04/07/18/43/04071843.pdf.

38 Some respondents who did not support the provision of non-identifying information made it clear that they considered only disclosure of identifying information to be adequate.

39 Data are incomplete because 225 respondents only replied to this question.

40 Blears (n. 37).

41 Blears (n. 37) As the Rose case (supra) highlighted, prior to 1991 clinics and physicians providing donor services were not required to maintain records, any records relating to a donor procedure prior to this date have no legal protection, and an individual conceived from a donor procedure undertaken before 1991 has no legal right to any information about their donor. The UK voluntary contact register, UK Donor Link (www.ukdonorlink.org.uk) was formally launched in April 2004. This facilitates information exchange and contact by mutual consent between anyone over the age of 18 who was conceived using donated sperm or eggs, donated in the UK, or thinks that they may be biologically related to a donor-conceived person. Uniquely, this register uses DNA profiling to establish a genetic match. An individual conceived as a result of a donor procedure undertaken outside the state regulatory system since 1991 (i.e. where an informal arrangement has been made between a donor and recipient) has no right to access any information about their donor. However, neither do they fall within the remit of UK DonorLink.

42 Frith/Blyth/Farrand, UK gamete donors' reflections on the removal of anonymity: Implications for recruitment, Human Reproduction 2007/22, 1675 ff.

43 Daniels/Blyth/Crawshaw/Curson, Previous semen donors and their views regarding the sharing of information with offspring, Human Reproduction 2005/20, 1670 ff.; Crawshaw/Blyth/Daniels, Past semen donors' views about the use of a voluntary contact register, Reproductive BioMedicine Online $2007 / 14,411 \mathrm{ff}$. 
truth and both parliamentary debate and wider media and public discussion helped keep these issues alive in the minds of the public and legislators, as did an incident occurring at a fertility clinic in Leeds that received considerable media attention. ${ }^{44}$ Here, the eggs of a woman undergoing an ICSI (intracytoplasmic sperm injection) procedure and who subsequently gave birth to twins had been mistakenly fertilised using the sperm of another man and not that of her husband. A court hearing was necessitated to determine the twins' legal parentage, again highlighting issues relating to genetic truth and rights to information about biographical and genetic history.

In January 2004, Blears' successor as Minister for Public Health, Melanie Johnson, announced that all new donors would be required to agree to their identity being disclosed to any individual conceived as a result of their donation, if so requested, once they reached 18.45 The Human Fertilisation and Embryology Authority (Disclosure of Donor Information) Regulations 2004 gave effect to the government's two-stage change in donor-conceived people's access to information about their donor. First, and for the first time, the government prescribed the non-identifying donor information to which a person born from a donor procedure undertaken after 1991 would be entitled once they reached the age of 18:

- Year of birth

- Country of birth

- Ethnic group

- Height

- Weight

- Eye, hair and skin colour

- Whether the donor had any children

- Any other details the donor may have agreed to provide, e.g. occupation, interests, religion, skills.

In addition, from July 2004, the HFEA was required to collect additional nonidentifying donor information which could be made available to any offspring seeking this information on reaching the age of 18 :

- Marital status

- Whether adopted

- Parents' ethnic group

- Details of any screening tests

- Personal and family medical history

\footnotetext{
${ }^{44}$ Leeds Teaching Hospitals NHS Trust v. (1) Mr A, (2) Mrs A, (3) YA \& (4) ZA (by their Litigation Friend, the Official Solicitor) (5) The Human Fertilisation and Embryology Authority, (6) Mr B, (7) Mrs B, Case No.: HQ02X02475/FD02P00895, 26.2.2003, [2003] EWHC 259(QB).

45 Johnson, Donor Anonymity and Review Keynote address. Human Fertilisation and Embryology Authority Annual Conference, London, 21.1.2004,

http://www.dh.gov.uk/en/News/Speeches/Speecheslist/DH_4071490.
} 
- Number and sex of own children (if any)

From April 2005 (with the exception of specific transitional arrangements permitting continuing use of gametes from anonymous donors for a limited period) all new donors have been required to consent to their identity being made available to any offspring seeking this information who has reached the age of 18.46 At such time, in addition to non-identifying information, a donor-conceived person may request from the HFEA the following donor information:

- Full names (and any former names)

- Date of birth and town or district where born

- "Appearance" 47

- Last known postal address (or address recorded at time of registration)

Independently, the HFEA had undertaken its own review of the Act's welfare of the child requirement. ${ }^{48}$ In its revised policy on the welfare of child, the HFEA amended its long-standing, ostensibly neutral, stance regarding parental disclosure: "Clinics should encourage and prepare [donor conception] patients to be open with their children from an early age about the circumstances of their conception." 49

\section{Resistance to changes}

As the risks to donor anonymity became increasingly apparent, resistance focused on a dominant objection, that its removal would have a serious negative impact on donor supplies, with a supplementary claim that, paradoxically, increasing the rights of donor-conceived people to have information about their biographical and genetic history would have the reverse effect because fewer parents would tell their donor-conceived children about their conception in the first place. Claims that donor numbers could fall by as much as $80 \%$ were augmented by apocalyptical comments from service providers..$^{50}$ Paul Serbal predicted the total demise of egg

\footnotetext{
46 Any donor who initially donated as an anonymous donor between 1991 and 2005 may re-register as an identifiable donor.

47 "Appearance" here refers to any information about the donor's physical appearance held by the HFEA that it is unable to divulge as non-identifying information.

48 Human Fertilisation and Embryology Authority. Tomorrow's children: Report of the policy review of welfare of the child assessments in licensed assisted conception treatment:, 2005 http://www.hfea.gov.uk/cps/rde/xbcr/SID-3F57D79B 0FA672DF/hfea/TomorrowsChildren.pdf 49 Human Fertilisation and Embryology Authority, New guidance on welfare of the child assessments, $\mathrm{CH}(05) 04,2.11 .2005$, http://www.hfea.gov.uk/cps/rde/xbcr/SID-3F57D79B-0FA672DF/hfea/Revised_Guidance.pdf; Human Fertilisation and Embryology Authority, Code of Practice, 7. edn. 2007, G.5.4.6., http://cop.hfea.gov.uk/cop/pdf/CodeOfPracticeVR_3.pdf.

50 Pennings, Commentary on Craft and Thornhill: new ethical strategies to recruit gamete donors, Reproductive Biomedicine Online 2005/10, $307 \mathrm{ff}$.
} 
sharing. ${ }^{51}$ Gillian Lockwood claimed to speak for a majority of her colleagues: "It is the opinion of most of the clinics that this will have quite significant consequences on reducing the supply of sperm and egg donors". ${ }^{52}$

The British Medical Association, ${ }^{53}$ the British Fertility Society ${ }^{54}$ and the Royal College of Obstetricians and Gynaecologists ${ }^{55}$ used the review of the Act undertaken by the House of Commons Science and Technology Select Committee during 2004 and $2005^{56}$ to reinforce their objections to the removal of anonymity; the British Fertility Society urging reconsideration of its preferred option, the "double track" system, that allows a donor to choose to be either anonymous or identifiable to offspring and recipients to opt to use gametes from either an anonymous or identifiable donor. ${ }^{57}$ The House of Commons Science and Technology Select Committee, itself sceptical of the decision to remove anonymity, endorsed this proposal. ${ }^{58}$

Once the legislative change had taken effect, it continued to be called to account for a decline in the provision of donor services, as indicated by national newspaper headlines, such as the Times' "Releasing donors' names causes sperm bank crisis". ${ }^{59}$ One of the most persistent critics, Evan Harris MP, denounced the decision to remove donor anonymity as "stupid and misguided". ${ }^{00}$ Adam Balen, responsible for a major assisted conception programme in Leeds, stated: "The change in the law has decimated our donor service", ${ }^{61}$ while Ian Craft described the

51 Cited in: Henderson, Egg shortage threatens baby hopes of thousands, The Times 22.1.2004; http://www.timesonline.co.uk/tol/news/uk/article1000444.ece.

52 Cited in: Blakemore, Couples "facing donor trauma", Birmingham Post 22.1.2004, p. 10.

${ }_{53}$ British Medical Association, Memorandum from the British Medical Association, Appendix 12, Ev. 228, 2004, In House of Commons Science and Technology Committee, Human Reproductive Technologies and the Law. Fifth Report of Session 2004-05, vol. II, 2005, Oral and Written Evidence, http://www.publications.parliament.uk/pa/cm200405/cmselect/cmsctech/7/7ii.pdf.

${ }^{54} \mathrm{~A}$ multi-disciplinary association representing a range of professional interests connected with fertility services and research, but in which the medical profession is dominant; British Fertility Society, Memorandum from the British Fertility Society, Appendix 9, Ev. 216, 2004, In House of Commons Science and Technology Committee, 2005.

55 Royal College of Obstetricians and Gynaecologists, Memorandum from the Royal College of Obstetricians and Gynaecologists 2004, In House of Commons Science and Technology Select Committee, 2005.

56 House of Commons Science and Technology Committee, Human Reproductive Technologies and the Law. Fifth Report of Session 2004-05, vol. I, 2005,

http://www.publications.parliament.uk/pa/cm200405/cmselect/cmsctech/7/7i.pdf.

57 Pennings, The "double track" policy for donor anonymity, Human Reproduction 1997/20, $2839 \mathrm{ff}$.

58 House of Commons Science and Technology Committee, Human Reproductive Technologies and the Law. Fifth Report of Session 2004-05, vol. I, 2005, p. 158,

http://www.publications.parliament.uk/pa/cm200405/cmselect/cmsctech/7/7i.pdf.

${ }^{59}$ Henderson, Releasing donors' names causes sperm bank crisis, The Times 14.9.2006, http://www.timesonline.co.uk/tol/news/uk/article638427.ece.

${ }^{60}$ cited in: Woolf, Sperm: Your country needs you, The Independent 30.6.2006, http://news.independent.co.uk/uk/health_medical/article1204521.ece.

${ }^{61}$ Cited in: Curtis, Infertile couples hit by big fall in sperm donors, The Guardian 24.4.2006, http://www.guardian.co.uk/medicine/story/0,1759930,00.html. 
removal of donor anonymity as a "killer blow" to egg donation. ${ }^{62}$ The first and second chairs of the HFEA, Sir Colin Campbell63 and Baroness Ruth Deech ${ }^{64}$ called for the policy to be reversed. The BBC, in a survey of UK fertility clinics, reported that almost $70 \%$ of participant clinics claimed that they either had insufficient supplies of donor sperm or had no access to donor sperm at all, that there was a solitary registered donor in Scotland, and none at all in Northern Ireland. ${ }^{65}$ However, in a study undertaken in 2004 prior to the change in legislation, the HFEA commented on the difficulty of obtaining accurate statistics from clinics ${ }^{66}$ and more recently, the HFEA has provided longer-term data relating to donor recruitment and usage between 1991 and 2006.67 These show that registrations of sperm donors peaked in 1993 and then declined until 2004. Year-on-year comparisons during this time have shown both reductions and increases in registrations. Correspondingly, these data show DI births peaking in 1994. Of particular interest, given continuing claims about the precariousness of supplies donor sperm, is that for each of 2005 and 2006, the HFEA has recorded an increase in donor registrations. The situation regarding egg donation is somewhat different, however. The same HFEA sources indicate that recruitment of registered egg donors followed an upward trajectory until 2001, since when the recruitment of new donors has declined, so that the number of registered donors recorded for 2006 is around $40 \%$ lower than the number registered in 2001 and, unlike sperm donor recruitment, egg donor recruitment has not shown signs of recent recovery.

Interpretation of these data should be undertaken with some circumspection, although what is clear is that the decline in sperm donor recruitment began well before any significant discussion regarding donor anonymity in the UK. Similar trends are evident in other countries, including countries like France, where the protection of donor anonymity has only recently been openly questioned. ${ }^{68}$ It is also evident that, at least for heterosexual couples where the male partner has a fertility impairment, since the 1990 s ICSI has become a "treatment of choice" in

${ }^{62}$ Craft, What price a baby at any age?, Bionews 15.5. 2006,

http:// www.bionews.org.uk/ commentary.lasso?storyid=3028.

${ }_{63}$ Campbell, Donor identity rules and IVF, The Times 21.9.2006,

http://www.timesonline.co.uk/article/0,,59-2367462.html.

${ }^{64}$ Deech, Competing interests in sperm donor secrecy, The Times 23.9.2006,

http://www.timesonline.co.uk/tol/comment/letters/article1074373.ece.

${ }^{65} \mathrm{Q}$ and A: Donor sperm shortage, BBC 13.9.2006,

http://news.bbc.co.uk/1/hi/health/5343008.stm; Dreaper, IVF donor sperm shortage revealed, BBC, 13.9.2006, http://news.bbc.co.uk/1/hi/health/5341982.stm.

${ }^{66}$ Human Fertilisation and Embryology Authority, Sperm, Egg and Embryo Donation (SEED) Policy Review. Findings of the Clinic Survey, 2005, http://www.hfea.gov.uk/cps/rde/xbcr/SID-3F57D79B-722E20B0/hfea/seed_review.pdf.

67 Human Fertilisation and Embryology Authority, A Long-term Analysis of the HFEA Register Data 1991-2006, 2007,

http://www.hfea.gov.uk/docs/2007-06-01_Latest_long_term_data_analysis_report_front_cover.pdf; Human Fertilisation and Embryology Authority, personal communication, 22.4.2008.

${ }^{68}$ Blyth/Frith, The UK's gamete donor "crisis" - a critical analysis, Critical Social Policy 2008, $74 \mathrm{ff}$. 
preference to DI and contributing at least in part to a reduced demand for donor sperm. ${ }^{69}$ In contrast to the experiences of most UK clinics regarding sperm donor recruitment, ${ }^{70}$ some clinics have reported success in recruiting identifiable sperm donors. ${ }^{71}$ However, there have been fewer accounts of successful egg donor recruitment strategies, Foster providing a sole exception. ${ }^{72}$ It may be that with attention mostly focused on the potential problems of sperm donor recruitment, less attention has been given to egg donor recruitment.

The second concern relating to the removal of donor anonymity is that it might have the perverse effect of reducing donor-conceived people's access to information about their biographical and genetic histories because their parents will be less likely to tell their children about the nature of their conception. ${ }^{73}$ This concern was articulated by the House of Commons Science and Technology Committee, as:

"a powerful argument that the ending of anonymity would result in a greater likelihood of parents not telling the child because the fear (rational or not) of rejection in favour of an identifiable biological parent outweighs the advantage of having identifying information to transmit" - my emphasis. ${ }^{74}$

It has to be said that, despite the House of Commons Committee's assertion, this is neither a powerful nor even a plausible argument. David Gollancz, a donorconceived adult invited to give oral evidence to the Committee responded that the idea was "fantasy", 75 while Susan Golombok provided the Committee with its sole

${ }^{69}$ However, the relationship between use of ICSI and demand for DI is not straightforward since there is evidence of extensive use of ICSI in the absence of a diagnosed male fertility impairment. Centers for Disease Control and Prevention, 2005 Assisted reproductive technology success rates: National summary and fertility clinic reports, 2007,

http://www.cdc.gov/ART/ART2005/508PDF/2005ART508.pdf; Human Fertilisation and Embryology Authority (n. 67).

70 British Fertility Society, Working Party on Sperm Donation Services in the UK: Report and recommendations, 2008,

http://www.fertility.org.uk/news/documents/2008_02_Sperm\%20Donation\%20Services.pdf;

Wardle/Skew, The real impact of the removal of donor anonymity, BioNews 18.2.2008, http:/ / www.bionews.org.uk/commentary.lasso?storyid=3731.

71 Adams-Pease/Lieberman, Donor recruitment: change attitudes, not the law, BioNews 16.10.2006, http:/ / www.bionews.org.uk/commentary.lasso?storyid=3225;

Witjens/Morris, Your country needs you! NGDT opinion, BioNews 7.8.2006,

http://www.bionews.org.uk/commentary.lasso?storyid=3138; Abuja/Linara/Smith, Donor insemination in the post-anonymity world: one clinic's experiences, BioNews 13.1.2008, http:/ / www.bionews.org.uk/commentary.lasso?storyid=3686.

72 Foster, Aber-donors flock to answer fertility appeal, 2007, http:// news.scotsman.com/scotland.cfm?id=1919182007.

73 British Medical Association (n. 53); British Medical Association, Parliamentary Brief: Human Fertilisation and Embryology Bill. Second reading, House of Lords, 19.11.2007; Harris, in: House of Commons Science and Technology Select Committee 2004, Human Reproductive Technologies and the Law: Fifth Report of Session 2004-5, vol. II, 2005, Ev.130; Winston, Sperm donors and secrecy, The Times 22.9.2006, http:/ / www.timesonline.co.uk/article/0,,59-2368771,00.html.

${ }^{74}$ House of Commons Science and Technology Committee (n.58), p. 157.

75 Gollancz, in: House of Commons Science and Technology Select Committee 2004, Human Reproductive Technologies and the Law. Fifth Report of Session 2004-05, vol. II, 2005, Ev. 33. 
formal source of "evidence", although she made clear that this was "all very anecdotal". ${ }^{76}$ At all other times the Select Committee tended to disregard such evidence, ${ }^{77}$ and its conclusion that the argument was a powerful one certainly does not rest on the evidence. The only other potential source of "evidence", but not referred to by the Committee - so its potential influence on the Committee's findings is a matter of conjecture - is an abstract of an oral presentation given by the Select Committee's medical advisor, Gillian Lockwood at the 2004 Annual Meeting of the European Society for Human Reproduction and Embryology. ${ }^{78}$ Lockwood reported a survey she had undertaken of returning and prospective recipients of donated gametes at her clinic. The abstract provides insufficient information about the underlying research methodology and analysis to determine its academic rigour, and - to date - no substantive paper based on the survey has been published.

While the Committee continued to use Sweden as a reference point to criticise the removal of anonymity, ${ }^{79}$ it failed to utilise available Swedish evidence that casts serious doubts on its own views. ${ }^{80}$ A 1982 study (i.e. prior to the change in Swedish legislation), reported that only 1 of 92 couples interviewed intended to tell their child about their conception a result of DI. ${ }^{81}$ This study sets the benchmark regarding levels of parental disclosure for two subsequent studies comparing parental disclosure practices before and after the removal of donor anonymity among the same survey population. In the first of these, $826 \%$ of parents of children born between 1983 and 1986 (i.e. before the change in legislation) and 18\% of parents of children born after 1987 said that they had already told their children about the nature of their conception and a further $40 \%$ of the total group stated their intention to tell their child "later". ${ }^{83}$ When this same group of parents was interviewed a second time, $61 \%$ had already told their child about her or his conception, and a further $22 \%$, who had not yet told their child(ren), were still intending to do so. ${ }^{84}$

76 Golombok, 2004, ibid Ev. 136.

77 House of Commons Science and Technology Committee (n. 58), p. 156.

${ }^{78}$ Lockwood, Tyranny of the minority? Can we balance the interests of 'possible people' against those of donor-conceived offspring seeking identity? Human Reproduction. 2004/19, i67 ff.

${ }^{79}$ Harris, in: House of Commons Science and Technology Select Committee, Human Reproductive Technologies and the Law. Fifth Report of Session 2004-05, vol. II, 2005, op. cit. 19.12005, Ev. 190; House of Commons Science and Technology Committee (n. 58), pp. 153-154.

${ }^{80}$ Harris (n. 79), Ev. 182.

81 Milsom/Bergman, A study of parental attitudes after donor insemination, Acta Obstetrica et Gynecologica Scandinavia 1982/61, $125 \mathrm{ff}$.

82 Gottlieb/Lalos/Lindblad, Disclosure of donor insemination to the child: the impact of Swedish legislation on couples' attitudes, Human Reproduction 2000/15, 2052 ff.

83 Although only the 1982 and 2000 studies concerning Sweden were available to the Committee at the time of its report, these should have been sufficient to alert it to the precariousness of its claims.

${ }^{84}$ Lalos/Gottlieb/Lalos, Legislated right for donor-insemination children to know their genetic origin: a study of parental thinking, Human Reproduction 2007/22, $1759 \mathrm{ff}$. There have also been suggestions of increasing levels of disclosure among parents in the UK, although less firmly evidenced.; Hunter/Salter-Ling/Glover, Donor insemination: telling children about their origins, Child Psychology \& Psychiatry Review 2000/5, 156-157; Lycett/Daniels/Curson/Golombok, Offspring created as a result of donor insemination: a study of family relationships, child adjustment, and disclosure, 


\section{Proposed changes in Human Fertilisation and Embryology Bill $2007^{85}$}

As part of the wide-ranging reform of legislation regulating assisted conception and embryo research currently being debated by the UK parliament, a number of proposals relate to donor conception. These are supported by the government and since none appear to be subject to parliamentary opposition of any substance, it seems likely that they will pass into law. These will:

- enable a child conceived as a result of donor conception to have both their mother and another female "parent" recorded on their birth certificate (i.e. where the mother is in a civil partnership or, if she is not in a civil partnership, another woman formally agrees to be treated as the "second/other parent"). .86

- give the right to a cohabiting couple, a couple in a same sex relationship intending to form a civil partnership and those planning an "intimate relationship" to ascertain if any information on the Register indicates whether they may be related to each other as a result of donor conception, subject to the consent of each individual.

- give a donor-conceived person the right to ascertain the existence, number, sex and year of birth and (by mutual agreement) the identity of any donorconceived genetic half siblings.

- give a donor the right to access limited non-identifying information regarding any offspring and in "some circumstances" to allow a donor to be informed when their identifying details have been requested by any offspring.

- permit the HFEA to run the voluntary contact register for individuals genetically related through donor conception if conceived prior to the enactment of the 1990 Act - or to delegate the running of it.

- reducing to 16 years the age at which a donor-conceived person may access non-identifying donor information on the HFEA Register.

Fertility and Sterility 2004/82, 172 ff.; Lycett/Daniels/Curson/Golombok, School-aged children of donor insemination: a study of parents' disclosure pattern, Human Reproduction 2005/20, $810 \mathrm{ff}$.

85 Some of these proposals were contained in earlier draft legislation, the Human Tissue and Embryos (Draft) Bill. However, following criticism of key provisions in the Draft Bill by a parliamentary committee, this was withdrawn and replaced by the Human Fertilisation and Embryology Bill. House of Lords and House of Commons, Joint Committee on the Human Tissue and Embryos (Draft) Bill, vol. I, Report, 2007,

http://www.publications.parliament.uk/pa/jt200607/jtselect/jtembryos/169/169.pdf; The parliamentary committee also proposed measures relating to donor conception that have been incorporated into the Bill. Two of the committee's proposals that have not been accepted by the government relate to annotating birth certificates and reducing to 16 the age at which a donor-conceived person may learn the identity of their donor. These are discussed further below.

${ }^{86}$ The Bill also proposes to extend to civil partners and any "two persons who are living as partners in an enduring family relationship" the ability to apply for a Parental Order where a child has been conceived following a surrogacy arrangement. 


\section{Conclusions}

Assuming the provisions currently contained in the Human Fertilisation and Embryology Bill are approved by parliament, from a global perspective, donor-conceived people in the UK will become among the best-served regarding their ability to access information about their biographical and genetic history. However, recent debates have highlighted several key areas for further debate and possible reform. Word limitations mean that these can be only briefly discussed here.

\subsection{Increasing donor-conceived people's awareness of their status and hence of their ability to access relevant information}

Provisions enabling a donor-conceived person to access information about their biographical and genetic history are of value only if the individual knows of their status in the first place. Levels of parental disclosure and non-disclosure are therefore critical. As shown above, British and Swedish evidence suggests that levels of parental disclosure may increase over time, although the passage of time as an adequate means of promoting the interests of donor-conceived people is questionable. The current legislative review has prompted a number of proposals designed to promote donor-conceived people's awareness of their status. The most radical is for the clinic or the HFEA to notify directly the donor-conceived person when reaching a specific age, following advance warning of this action to the individual's parents. ${ }^{87}$ Other proposals relate to modifying birth registration arrangements, along with strengthened provisions for advice, information and support to those considering donor procedures ${ }^{88}$ However, at least some of these proposals have raised concerns relating to privacy, of unwarranted state interference into family life and of undermining parental authority. ${ }^{89}$ The government has indicated that it

${ }^{87}$ Lord Mackay of Clashfern, House of Lords Debates: Official Report, 10.12.2007, cols. 100-101.

88 House of Lords and House of Commons (n. 85); Barker, House of Lords Debates: Official Report, 10.12.2007, cols. 94-95,

http://www.publications.parliament.uk/pa/ld200708/ldhansrd/text/71210-0014.htm; Blyth, Donor conception: what to do about birth certificates, BioNews 17.12.2007,

http://bionews.org.uk/commentary.lasso?storyid=3676; Blyth, Donor conception and birth certificates - more steps in the right direction, BioNews 19.5.2008,

http://www.bionews.org.uk/commentary.lasso?storyid=3840; International Donor Offspring Alliance, Human Fertilisation and Embryology Bill. Birth Certificates: The Case for Reform. Briefing For Members of the House of Commons, 2008,

http://web.jaguarpaw.co.uk/ tom/080408-collation.pdf; Partnership Focus Group on the Rights and Life Long needs of people created by donor assisted conception, Briefing for the Human Fertilisation and Embryology Bill, April 2008, 2008; Rusbbrooke, Proposals to bring donor-conceived people's birth certification into line with that of all other UK citizens, Bulletin of Medical Ethics, 2008.

${ }^{89}$ Bakewell, Do we have the right to know our parentage? A birth certificate is not the place to make a matter that is personal and intimate into official diktat, The Independent 3.8.2007, http://comment.independent.co.uk/columnists_a_l/joan_bakewell/article2831067.ece;

Harris, cited in: BBC News. Call for children donor awareness, 1.8.2007, http://news.bbc.co.uk/1/hi/health/6924615.stm; Lyons, Branding parents deceitful, Spiked 2.8.2007, 
has no plans to institute such reforms at the present time, favouring the encouragement of parental disclosure through education, requiring clinics to provide information about the importance of disclosure to people considering donor conception and requiring the HFEA to produce guidance to clinics on providing such information. ${ }^{90}$ However, it has promised a future review of the role of birth certificates in recording donor conception.

\subsection{Age at which a donor-conceived person may learn of their donor's identity}

The age at which a donor-conceived person may formally learn the identity of their donor is currently 18, and the government rejected proposals to reduce this to 16 years or to apply a test of competence that is based on the individual's maturity and not simply on chronological age, although a "maturity" test is used for such purpose in Sweden, ${ }^{91}$ and also operates in the UK in other areas of child welfare. ${ }^{92}$ Several other jurisdictions that enable a donor-conceived person to learn the identity of their donor permit this at an earlier age: Austria (14 years), ${ }^{93}$ the Netherlands $^{94}$ and Western Australia ${ }^{95}$ (16 years). ${ }^{96}$ As it stands, granting access to this information only at the age of 18 would appear to undermine compliance with provisions of the United Nations Convention on the Rights of the Child. ${ }^{97}$

http://www.spiked-online.com/index.php?/site/article/3695/; Nathanson, More than a gene pool Informing children born from donated sperm or eggs would undermine the real job of parenting, The Guardian 2.8.2007; http:/ / www.guardian.co.uk/commentisfree/story/0,2139777,00.html;

Donor Conception Network, Human Fertilisation and Embryology Bill. second Reading House of Commons, May 2008: Briefing from the Donor Conception Network, 2008.

90 Department of Health, Government Response to the Report from the Joint Committee on the Human Tissue and Embryos (Draft) Bill, CM 7209, 8.10.2007, http://www.dh.gov.uk/en/Publicationsandstatistics/Publications/PublicationsPolicyAndGuidance/ dh_079127.

${ }^{91}$ Lalos/Daniels/Gottlieb/Lalos, Recruitment and motivation of semen providers in Sweden, Human Reproduction 2003/18, $212 \mathrm{ff}$.

92 A UK court ruling provides the benchmark for determining young people's capacity to consent: "parental rights yield to the child's right to make his own decision when he reaches a sufficient understanding and intelligence to be capable of making his own mind up on the matter in question". Lord Scarman cited in: Franklin, The New Handbook of Children's rights; Comparative Policy and Practice, 2002, p. 61; However, this approach, known colloquially as "Gillick" or "Fraser" competence, is applied only in certain circumstances in the UK.

93 Fortpflanzungsmedizingesetz, 1992.

94 Wet donorgegevens kunstmatige bevruchting, 2002.

95 Human Reproductive Technology Amendment Act, 2004.

96 In Western Australia a donor-conceived person younger than 16 years may learn their donor's identity where the donor consents and the parent(s) consent(s) on behalf of the child. In Victoria, a parent of a person aged under 18 may apply for this information on behalf of the child. And while the specified age of access to this information in New Zealand is 18, a donor-conceived person aged at least 16 may apply to the Family Court to be treated as an 18 year old, although the court will need to be satisfied that it is in the individual's best interests to receive the information requested.

97 Tobin, The Convention on the Rights of the Child: The Rights and Best Interests of Children Conceived through Assisted Reproduction, 2004, 


\subsection{Extending rights to information to donor-conceived people's own children}

Anecdotal evidence from some donor-conceived adults indicates their own children's interest in knowing about their biographical and genetic history, although the Australian state of Victoria is currently the sole jurisdiction that extends to the descendants of a donor-conceived person the right to learn the identity of their parent's donor. There seem to be no legitimate barriers to granting independent rights to access this information, which may be especially pertinent where the parent has obtained this information themselves but has not passed it on to their own child(ren) or where the parent never sought this information for themselves (most likely because they were unaware of their status) and is no longer able to do so because of incapacity or death.

\subsection{Reviewing limits on the number of offspring per donor}

Currently the HFEA restricts use of donor gametes to the birth of children in ten families, subject to any lower limit imposed by the donor. Such limits were drawn up within the context of donor anonymity primarily to avoid a consanguineous relationship between genetically-related individuals. The removal of donor anonymity and proposed extension of rights to information regarding donor half siblings means that these limits should be reviewed in the context of both increased contact between donor half-siblings and the potential implications of large kinship networks ${ }^{98}$ and proposals to increase the maximum limit in the face of pressure on supplies of donor gametes. ${ }^{99}$

\subsection{Retrospective access to donor identity}

No jurisdiction that has removed donor anonymity has applied such provisions retrospectively, instead choosing to extend as far as possible new rights via the establishment of "voluntary contact registers". ${ }^{100}$ There are self-evidently tensions between the interests of donor-conceived people in learning about their biographical and genetic history and donors' rights to privacy and respect for the original agreement to donate (however out of tune with current sentiment previous consent arrangements now appear to be). ${ }^{101}$ It is unlikely that this will be resolved in the absence of a test of the legal merits of the respective claims.

http://www.lawreform.vic.gov.au/wps/wcm/connect/Law+Reform/resources/file/eb1dca4e81c7d ab/Tobin $\% 20$ paper $\% 20$ FINAL.pdf.

98 Kirkman, "Saviours and satyrs: ambivalence in narrative meanings of sperm provision", Culture Health and Sexuality 2004/6, $319 \mathrm{ff}$.

${ }_{99}$ British Fertility Society (n. 70).

${ }^{100}$ Blyth/Speirs, Meeting the rights and needs of donor-conceived people: the contribution of a voluntary contact register, Nordisk Socialt Arbeid 2004/24, 318 ff.

101 British Medical Association (n. 53); Rushbrooke (n. 88). 
In conclusion, while existing and proposed measures in the UK promote the interests of a donor-conceived person in learning about their biographical and genetic history, such information is neither complete nor is access to it assured. These will require further reforms, such as those identified above. 


\title{
Eizellspende und Eizellhandel - Risiken und Belastungen für die betroffenen Frauen
}

\author{
Sigrid Graumann
}

Seit einigen Jahren hat sich in Europa ein Markt für grenzüberschreitende fortpflanzungsmedizinische Leistungen entwickelt. ${ }^{1}$ Das hat mehrere Gründe: Der wichtigste ist sicher, dass in vielen Ländern die Kosten für eine Kinderwunschbehandlung teilweise oder ganz privat getragen werden müssen. Auf Grund des Wohlstandsgefälles innerhalb von Europa ist die Behandlung in anderen Ländern oft deutlich preiswerter. ${ }^{2}$ Ein weiterer Grund dafür ist, dass sich die Palette an Behandlungsangeboten auf Grund unterschiedlicher gesetzlicher Regelungen von Land zu Land unterscheiden. So ist beispielsweise die Eizellspende in Ländern wie Deutschland und Italien verboten. In Internetforen tauschen sich Kinderwunschpatientinnen darüber aus, wo die Eizellspende legal möglich ist und fortpflanzungsmedizinische Zentren in Spanien oder Tschechien werben mit deutsch- und italienischsprachigen Internetauftritten um deutsche und italienische Kundinnen. Aber auch beispielsweise Britinnen und Französinnen, in deren Ländern die Eizellspende erlaubt ist, werden umworben. Das liegt in Großbritannien daran, dass es auf Grund der Knappheit von Eizellen nicht ohne weiteres möglich ist, gespendete Eizellen zu bekommen, aber auch daran, dass die ausländischen Angebote kostengünstiger sind. Die neue Regelung in Großbritannien und vielen anderen

\footnotetext{
1 Spiewak, Schwanger um jeden Preis, Die Zeit 2002/20; Brodde, Die Jagd nach den Eizellen, Greenpeace Magazin 2006/3.

2 Schindele/Zimmermann, Rohstoff für das Mutterglück, Die Zeit 2007/4.
} 
westeuropäischen Ländern, keine anonymen Keimzellspenden mehr zuzulassen, hat dazu geführt, dass Kliniken in Ländern, in denen anonyme Spenden nach wie vor erlaubt sind, mit der Anonymität der Spenden werben - und das, obwohl die europäische Geweberichtlinie anonyme Keimzellspenden aus gutem Grund untersagt: Man weiß heute, dass viele Kinder, die aus Keimzellspenden hervorgegangen sind, psychisch darunter leiden, wenn sie die Identität ihrer biologischen Eltern nicht erfahren können.

Auch Dienstleistungen, wie Ersatzmutterdienste („Leihmutterschaft"), die in den meisten europäischen Ländern verboten sind, werden über das Internet beworben. In Deutschland ist die Ersatzmutterschaft verboten. In Großbritannien ist sie grundsätzlich möglich, aber nur wenige Frauen sind dazu bereit, Ersatzmutterdienste zu erbringen. Nicht so in der Ukraine und Russland, wo viele sehr arme Frauen leben. Hier werben Kliniken in ihren mehrsprachigen Internetauftritten für Ersatzmutterdienste mit absoluter Diskretion und rechtlicher Sicherheit.

Die genannten Entwicklungen haben sich zunächst vollzogen, ohne größere öffentliche Aufmerksamkeit zu erregen. Das hat sich mittlerweile geändert.

Zum einen ist der Fall einer international operierenden Firma bekannt geworden, die für Kliniken in New York und London Eizellspenden in Rumänien organisiert hat. Für die gespendeten Eizellen mussten die Kinderwunschpatientinnen noch nicht einmal nach Bukarest reisen. Das Sperma des Mannes wurde kryokonserviert nach Bukarest geflogen, dort fand die Befruchtung der gespendeten Eizellen statt, die kryokonservierten Embryonen wurden anschließend zurückgeflogen und in die Gebärmutter der Kinderwunschpatientin eingesetzt. Zum Skandal wurde die Geschichte, weil in einer Praxis in Rumänien, die von der Firma unterhalten wird, junge, arme Frauen als Spenderinnen angeworben wurden. Mindestens zwei der Spenderinnen trugen schwere Gesundheitsschäden davon und niemand wollte für ihre Behandlung aufkommen. ${ }^{3}$

Zum anderen hat sich über den Bedarf an menschlichen Eizellen für die Klonforschung eine heftige ethische Debatte entwickelt. In dieser Debatte geht es vor allem um die Gesundheitsrisiken der Eizellspenden für die Frauen und die Bezahlung der Spenderinnen. ${ }^{4}$ Während manche für die Bezahlung mit dem Ausgleich der Gesundheitsrisiken argumentieren, halten andere dieses Argument für vorgeschoben; in Wirklichkeit sei das Interesse doch nur, mehr Spenderinnen zu rekrutieren. Ein Aspekt wird meist nicht genannt: Durch den Eizellbedarf der Forschung verschärft sich der „Eizellmangel“ für Kinderwunschpatientinnen, was den grenzüberschreitenden Eizellmarkt weiter befördern dürfte.

Hierzulande entwickelt sich ganz aktuell eine Debatte über das mittlerweile 18 Jahre alte Embryonenschutzgesetz. Von Seiten der Fortpflanzungsmediziner werden dessen Regelungen als zu restriktiv angesehen. Es wird auf die neuen Ent-

${ }^{3}$ Schindele, Die verkaufte Hoffnung, Brigitte 2005/20.

${ }^{4}$ Check, Ethicists and biologists ponder the price of eggs, Nature 2006/442, $606 \mathrm{f}$. 
wicklungen in der Fortpflanzungsmedizin hingewiesen, von denen deutsche Kinderwunschpatientinnen auf Grund der Gesetzeslage nur teilweise profitieren könnten. ${ }^{5}$ Dabei wird auch eine Aufhebung des Verbots der Eizellspende in Deutschland gefordert. ${ }^{6}$ Als ethische Probleme werden dabei zwar mögliche Belastungen des Kindes durch eine ,gespaltene Elternschaft“ genannt, nicht aber die Situation der Spenderinnen. ${ }^{7}$

Vor diesem Hintergrund möchte ich mich im Folgenden mit der Frage beschäftigen, ob und falls ja unter welchen Bedingungen die Eizellspende ethisch gerechtfertigt sein könnte. Dabei werde ich mich vor allem auf die bislang in der Diskussion weitgehend ausgeblendete Perspektive der Spenderinnen konzentrieren und auf ihre Gesundheitsrisiken eingehen. Ich werde anschließend die ethischen Kriterien, die bei der Beurteilung der Eizellspende berücksichtigt werden müssen, vorstellen, und ich werde versuchen, einen Überblick über die Praxis und Regulierung der Eizellspende und des europäischen Eizellspendemarkts zu geben. SchlieBen werde ich mit einer zusammenfassenden ethischen Beurteilung.

\section{Belastungen und Risiken für die Spenderin}

Die Hauptindikation für eine Eizellspende ist eine stark eingeschränkte oder vorzeitig erloschene Eierstockfunktion bei der Kinderwunschpatientin. Weitere Indikationen sind genetische oder autoimmunologische Krankheiten oder eine überstandene Krebstherapie. ${ }^{8}$ Für viele dieser Kinderwunschpatientinnen ist eine Eizellspende die einzige Chance für eine Schwangerschaft. Allerdings - und das wird oft vergessen, wenn Eizellspende und Samenspende in einem Atemzug genannt werden - ist die Spende von Eizellen mit erheblichen Risiken für die Spenderin verbunden.

Die Eizellspende ist ein invasiver Eingriff, der psychisch und physisch anstrengend, schmerzhaft und stressreich sein kann und nicht frei von gesundheitlichen Risiken ist. Die Belastungen und Risiken für die Spenderin hängen mit der Eizellgewinnung zusammen. Die Eizellspenderin muss sich einer zweiwöchigen Hormonbehandlung unterziehen, mit der bewirkt wird, dass nicht nur eine Eizelle, wie im natürlichen Zyklus, sondern mehrere Eizellen gleichzeitig reifen. Diese werden dann mit einem invasiven Eingriff „geerntet“ und im Labor mit dem Sperma des Mannes der Kinderwunschpatientin befruchtet.

Die Belastungen und Risiken der Eizellgewinnung reichen von schlechter Laune bis zu einem schweren Überstimulationssyndrom. Ein mildes Hyperstimu-

5 Dietrich/Griesinger/Behre/Felberbaum/Montag/v.d.Ven/Strowitzki/v.Otte, Neue Entwicklungen in der Reproduktionsmedizin, Dt. Ärzteblatt 2005, 587 ff.

${ }^{6}$ Kentenich, Interview: „Retortenbabys erwirtschaften mehr, als sie kosten“, Spiegel online, recherchiert am 26.5.2008.

7 Katzorke, Grenzbereiche der Reproduktionsmedizin. Samenspende - Eizellspende - Leihmutterschaft, Gynäkologie + Geburtshilfe 2007/4, 21 ff.

${ }^{8}$ Katzorke (Fußn. 7), 21 ff. 
lationssyndrom ist mit Kopfschmerzen, Übelkeit und Schwellung der Eierstöcke verbunden. Bei der schweren Form kommt es zu Flüssigkeitsansammlungen in Bauchraum und Lunge und im Extremfall zu Nierenversagen oder einem Schlaganfall. Die Häufigkeitsangaben des Hyperstimulationssyndroms unterscheiden sich von Studie zu Studie. Die britische Behörde gibt die Häufigkeit der schweren Form mit bis $2 \%,{ }^{9}$ das deutsche IVF-Register mit $0,41 \%$ an. ${ }^{10}$ Weitere Risiken sind Verletzungen und Blutungen in Folge der Eizellpunktion.

Das Risiko für ein Hyperstimulationssyndrom ist bei jüngeren Frauen und geringem Körpergewicht sowie bei stärkerer Stimulation erhöht. ${ }^{11}$ Eizellspenderinnen sind oft sehr junge Frauen, und das Interesse an möglichst vielen Eizellen könnte ein Anreiz für eine starke Stimulation sein. Die Daten, die zur Einschätzung der Risiken zur Verfügung stehen, stammen aber von Frauen mit einem durchschnittlich deutlich höheren Alter und oft eingeschränkter Fruchtbarkeit (wenn das Fruchtbarkeitsproblem nicht überwiegend beim Partner liegt). Sie sind deshalb nur bedingt für die Einschätzung der Risiken für jüngere Eizellspenderinnen übertragbar.

Das gilt auch für einen weiteren Risikofaktor, der allerdings seit Jahren kontrovers diskutiert wird. Es wurden immer wieder Studien vorgelegt, welche die Hormonbehandlung mit einem erhöhten Risiko für das Entstehen hormonabhängiger Karzinome in Verbindung bringen. Andere Studien konnten das nicht belegen. ${ }^{12}$ Die Daten in Bezug auf das langfristige Krebsrisiko können aber ebenfalls nicht unbedingt auf die Situation der Eizellspende übertragen werden. Das Krebsrisiko könnte für die jüngeren und gesünderen Eizellspenderinnen höher sein. Andere relevante Daten, wie die Auswirkung auf die eigene Fruchtbarkeit der Spenderin, sind bisher gar nicht erhoben worden. Aus Einzelfällen ist bekannt, dass vor allem mehrmalige Eizellpunktionen zur Vernarbung der Eierstöcke und in der Folge zu Eisprungproblemen führen können.

Das heißt, dass Studien, die valide Informationen über die langfristigen Risiken der Eizellspende für die Spenderinnen geben könnten, fehlen. Die Risikostudien, die es gibt, wurden mit Kinderwunschpatientinnen gemacht, deren Familienplanung in der Regel nach der Behandlung abgeschlossen ist und die durchschnittlich deutlich älter sind als die Eizellspenderinnen. Deshalb wird es wahrscheinlich noch Jahrzehnte dauern, bis wirklich Klarheit über die Risiken der Eizellspende besteht. ${ }^{13}$

Festgehalten werden kann, dass die Eizellspende mit erheblichen potenziellen Gesundheitsrisiken für die Spenderin einher geht, die - in seltenen Fällen - sehr

\footnotetext{
9 HFE A, HFEA report: "A long term analysis of the HFEA Register data 1991-2006", 2006.

${ }^{10}$ Deutsches IVF-Register, Jahrbuch 2006, 2007; abrufbar unter www.deutsches-ivf-register.de.

11 http://ivf.hez1-1.srv.hoster03.de/index.php?id=101 (letzter Zugriff am 26.8.2008).

12 Johnson, The medical ethics of paid egg sharing in the UK: Opinion, Human Reproduction 1999/14, $1912 \mathrm{ff}$.

13 Pearson, Health effects of egg donation may take decades to emerge, Nature 2006/422, $607 \mathrm{f}$.
} 
schwer und sogar lebensbedrohlich sein können. Ein wesentliches Problem ist aber, dass man den Eizellspenderinnen auf Grund fehlender einschlägiger Langzeitstudien heute nicht mit Sicherheit sagen kann, welche gesundheitlichen Langzeitrisiken sie eingehen.

\section{Eizellspende - ein medizinischer Eingriff zum Wohle Dritter}

Aus medizinethischer Sicht ist die Eizellspende vor allem deshalb problematisch, weil sie mit einem drittnützigen medizinischen Eingriff einhergeht.

Voraussetzungen für die ethische Legitimität einer medizinischen Behandlung sind, dass eine freie und informierte Einwilligung vorliegt, dass der Beitrag zum gesundheitlichen Wohlergehen voraussichtlich Risiken und Belastungen überwiegt und dass das Wohlergehen der Patientin im Mittelpunkt steht, und nicht Interessen Dritter oder das Gemeinwohl. Wenn einzelne Voraussetzungen nicht erfüllt sind, muss dies ethisch gut begründet werden.

Vor diesem Hintergrund weist die Eizellspende eine Reihe von Besonderheiten auf, die sie ethisch besonders begründungspflichtig sein lässt. Eizellspenden sind immer mit einer In-vitro-Fertilisation verbunden. Das hat Auswirkungen auf die Beurteilung der Behandlung der Kinderwunschpatientin und der Eizellspenderin.

Für die Kinderwunschpatientin sind die allgemeinen Besonderheiten bei der In-vitro-Fertilisation zu nennen. Dazu gehört, dass einem unerfüllten Kinderwunsch zwar eine Krankheit zu Grunde liegen kann, es sich bei ihm selbst aber nicht um eine Krankheit handelt. Viele Menschen verzichten freiwillig auf (eigene) Kinder oder schieben ihre Familienplanung lange auf, und sie würden sich deshalb nicht als krank bezeichnen. Damit soll keineswegs über das subjektive Leiden der betroffenen Frauen und Paare hinweg gegangen werden, das wirklich erheblich sein kann. Feststellen muss man aber dennoch, dass es sich bei einem unerfüllten Kinderwunsch um ein subjektives Leiden handelt, das nicht objektiv behandlungsbedürftig ist. Manche Frauen und Paare entscheiden sich für eine Kinderwunschbehandlung, andere ändern ihre Lebensziele - mit oder ohne therapeutische Hilfe. Ein weiterer Aspekt ist, dass viele Kinderwunsch-Behandlungen erfolglos bleiben. Die sogenannten „baby-take-home-rate“ liegt pro angefangenem Zyklus ungefähr bei $14 \%$. Nach drei abgeschlossenen Behandlungszyklen bleiben durchschnittlich ca. 60\% der Paare kinderlos, nach vier Zyklen durchschnittlich ca. 50\%.14

Eine der medizinethischen Forderungen, die ich genannt habe, ist, dass der Beitrag zum Wohlergehen der Patientin die voraussichtlichen Belastungen und Risiken überwiegt. Nun ist bei einer erfolglosen Behandlung aber der Beitrag zum Wohlergehen der Kinderwunschpatientin fraglich. ${ }^{15}$ Deshalb hängt die ethische

\footnotetext{
14 Wischmann, Unerfüllter Kinderwunsch - Stereotype und Fakten, Journal für Reproduktionsmedizin und Endokrinologie 2006, $220 \mathrm{ff}$.

15 Sonnenmoser, Reproduktionsmedizin: Psychosoziale Folgen unterschätzt, Dt. Ärzteblatt 2006, $461 \mathrm{f}$.
} 
Legitimität der In-vitro-Fertilisation vor allem am subjektiven Leiden unter dem unerfüllten Kinderwunsch und an der freien und informierten Einwilligung.

Dazu kommen nun noch die Besonderheiten bei der Eizellspende: Das ist einerseits, dass die Risiken und Belastungen der Eizellgewinnung die Spenderin trägt, der Beitrag zum Wohlergehen aber der Kinderwunschpatientin zu Gute kommt. Mit Blick auf das ärztliche Handeln ist das als Körperverletzung zu werten, die nicht durch einen Beitrag zum Wohlergehen der Person, die verletzt wird, aufgewogen werden kann. Die ethische Legitimität der Eizellgewinnung hängt daher alleine an der freiwilligen und informierten Einwilligung der Spenderin; das Leiden am unerfüllten Kinderwunsch der Kinderwunschpatientin ist dafür irrelevant

\section{Praxis und Regulierung der Eizellspende}

Die Praxis und die Regulierung der Eizellspende sind in verschiedenen europäischen Ländern sehr unterschiedlich. ${ }^{16}$ In Norwegen, Italien, Deutschland, Österreich, Schweiz ist die Eizellspende verboten. In Dänemark, Belgien, Estland, Finnland, Frankreich, Griechenland, Großbritannien, Lettland, Niederlande, Schweden, Slowenien, Slowakei, Spanien, Tschechien, Ungarn ist sie unter unterschiedlichen Bedingungen erlaubt. Manche Länder führen Register und erlauben die Eizellspende nur in lizensierten Zentren. Andere haben Voraussetzungen für die Spenderinnen wie z.B. gesundheitliche Eignung, bereits eigene Kinder sowie Aufklärung und Beratung vorgeschrieben. In Frankreich muss die Kinderwunschpatientin selbst eine Spenderin rekrutieren. In Dänemark, den Niederlande und in Israel ist Eizellspende auf „egg sharing“ begrenzt. Darunter ist zu verstehen, dass Frauen, die sich selbst in einer IVF-Behandung befinden, „überzählige“ Eizellen an andere Kinderwunschpatientinnen spenden können.

In Irland, Litauen, Luxemburg, Malta, Polen ist die Eizellspende nicht geregelt. Unterschiede zeigen sich auch in der Handhabung der Anonymität von Eizellspenden. Großbritannien und Schweden haben die Möglichkeit von anonymen Spenden als erste untersagt. Mittlerweile verbietet die europäische Geweberichtlinie für alle Vertragsstaaten die Praxis der anonymen Eizellspende, was allerdings in

\footnotetext{
16 Die folgenden Angaben habe ich entweder den beiden hier genannten Berichten entnommen oder es handelt sich um persönliche Informationen von Frauen- und Patientenorganisationen aus den genannten Ländern, die auf dem ost-west-europäischen Workshop „European Biopolitics. Connecting Civil Society - Implement Basic Values" vorgestellt wurden, den das Institut Mensch, Ethik und Wissenschaft in Kooperation mit der Heinrich-Böll-Stiftung vom 17.-19.3.2006 in Berlin durchgeführt hat; Nyboe-Andersen/Goossens/Ferraretti/Bhattacharya/Felberbaum/d.Mouzon/Nygren, Assisted reproductive technology in Europe, 2004: results generated from European registers by ESHRE, Human Reproduction 2008/23, 756 ff.; European Commission, Report on the Regulation of Reproductive Cell Donation in the European Union, 2006.
} 
vielen Ländern bislang nicht umgesetzt wird. ${ }^{17}$ Weitere Unterschiede zeigen sich in den Regelungen der Aufwandsentschädigung für die Spenderinnen. Die EUGeweberichtlinie schreibt vor, dass keine Bezahlung, sondern lediglich eine Aufwandsentschädigung für die Spenderin gewährt werden darf, um eine Kommerzialisierung zu verhindern. In Wirklichkeit sind die Aufwandsentschädigungen aber so hoch bemessen, dass sie den Hauptanreiz für eine Eizellspende ausmachen.

Im Folgenden möchte ich Unterschiede in Praxis und Regulierung der Eizellspende an drei Beispielen exemplarisch erläutern:

Mein erstes Beispiel ist Großbritannien:

Großbritannien zeichnet sich durch ein hoch reguliertes System der Reproduktionsmedizin überhaupt und damit auch der Eizellspende aus. Anonyme Eizellspende ist nicht mehr zulässig. Als Entschädigung für „altruistische Eizellspenden“ werden Entschädigungen pro „Ernte“ von 250 - 370 Pfund bezahlt. Im Jahr 2005 wurden aber nur 926 altruistische Spenden registriert. ${ }^{18}$ Der überwiegende Anteil der Eizellspenden entfallen auf das ,egg sharing“. Allerdings scheinen damit höhere Stimulation, höhere Risiken und geringere Erfolgsraten der eigenen Kinderwunschbehandlung für die Spenderin einher zu gehen. Die britische Praxis zeichnet sich außerdem durch Transparenz aus: die HFEA stellt Informationen über Risiken und Belastungen für Spenderinnen bereit, und es existiert eine öffentliche Debatte über Risiken und Belastungen der Eizellspenden sowie über das Recht der Kinder auf Kenntnis der eigenen Herkunft. Trotz der Zulässigkeit altruistischer Eizellspenden und trotz der Existenz der ,egg sharing“-Programme herrscht in Großbritannien Eizellmangel, und britische Kinderwunschpatientinnen treten als wichtige Kundinnengruppe für Eizellspenden im Ausland hervor. Durch die Zulässigkeit von Eizellspenden für die Klonforschung wird sich der Mangel an gespendeten Eizellen im Land voraussichtlich verschärfen.

Mein zweites Beispiel ist Deutschland:

In Deutschland ist die Eizellspende durch das Embryonenschutzgesetz von 1990 verboten. Informationen über Eizellspenden im Ausland werden aber über das Internet (z.B. über www.eizellspende.de) verbreitet. Deutsche Frauen sind den vielen deutschsprachigen Web-Auftritten spanischer und osteuropäischer Kliniken nach zu urteilen - begehrte Kundinnen für Eizellspende-Dienste. Die Zielländer der deutschen Frauen sind u. a. Spanien, Ukraine, Russland, Polen, Tschechien und Südafrika. Information, Beratung sowie die Vor- und Nachbehandlung findet häufig in deutschen Praxen statt.

Mein drittes Beispiel ist Spanien:

Spanien zeichnet sich durch eine liberale Gesetzeslage mit geringer Regulierung aus. In Spanien werden offenbar nach wie vor anonyme Eizellspenden ange-

${ }_{17}$ Z.B. ist die Eizellspende in Spanien und in den meisten osteuropäischen Staaten nach wie vor anonym.

18 HFE $A$ (Fußn. 9). 
boten. Die Spenderinnen erhalten eine Aufwandsentschädigung von 600 - 1.000 Euro pro „Ernte“. Sie werden beispielsweise über Radiowerbung aber auch an Universitäten rekrutiert. Dennoch ist das Thema Eizellspende in Spanien tabuisiert. Es findet praktisch keine öffentliche Debatte statt. Spanien hat die größte Zahl an Anwendungen der Eizellspende in Europa und gilt derzeit als die Nummer Eins für den europäischen „Eizelltourismus“.

Die drei Beispiele sollten verdeutlichen, dass der Zusammenhang zwischen Praxis und Regulierung deutlich komplexer ist als das gemeinhin angenommen wird. Die britische Situation zeigt, dass eine regulierte Zulässigkeit der Eizellspende im eigenen Land keineswegs einen „Eizelltourismus“ in andere Länder verhindert. Deutlich wird aber auch, dass ein Verbot der Eizellspende, wie es in Deutschland herrscht, durch Angebote aus dem Ausland leicht unterlaufen werden kann, zu Lasten der Frauen in den ärmeren Ländern. Die Situation in Spanien verdeutlicht, dass viele Spenderinnen dann zur Verfügung stehen, wenn wenig öffentlich diskutiert wird und wenn finanzielle Anreize geboten werden.

\section{$4 \quad$ Eizellspende und Eizellhandel}

Wenn man nun die grenzüberschreitenden Angebote der Fortpflanzungsmedizin in Europa betrachtet, kann man sich fragen, ob es sich hier um einen Kommerzialisierung der Eizellspende handelt, wie in den Medienberichten nahegelegt wird. ${ }^{19}$ Ich denke ja, und zwar aus den folgenden Gründen: Bei den grenzüberschreitenden Eizellspende-Angeboten geht es um viel Geld. Mit Werbekampagnen werden gezielt Eizell-Spenderinnen und Kinderwunschpatientinnen für die Eizellspenden gewonnen. In einigen Fällen finden organisierte Eizellspende-Reisen statt, beispielsweise von Italien nach Russland. In anderen Fällen lässt sich ein organisierter Eizellhandel feststellen, wie beispielsweise in dem oben genannten Beispiel zwischen Großbritannien und Rumänien, vermittelt über eine international operierende Firma und kontrolliert durch die HFEA.

Nun kann man berechtigterweise fragen, was an dem Eizellhandel grundsätzlich ethisch problematisch sein soll, abgesehen davon, dass systematisch das Kommerzialisierungsverbot der europäischen Geweberichtlinie unterlaufen wird und dass einzelne zweifelhafte Anbieter auf dem Markt sind. Aus meiner Sicht sind vor allem zwei Aspekte problematisch:

Zum einen begünstigt das Wohlstandsgefälle zwischen Nord- und Süd- sowie zwischen West- und Ost-Europa den Eizellhandel. Die Kundinnen für grenzüberschreitende Eizellspende-Dienste sind meist ältere Frauen der Mittelschichten aus reichen Ländern, wo die Eizellspende verboten oder reguliert ist. Die Spenderinnen dagegen sind meist sehr junge, sozial schlechter gestellte Frauen aus ärmeren Ländern mit geringer oder fehlender Regulierung. Damit entsteht eine neue Form

19 Spiewak (Fußn. 1); Brodde (Fußn. 1); Scbindele (Fußn. 3); Scbindele/Zimmermann (Fußn. 2). 
eines Ausbeutungsverhältnisses, das gängige Vorstellungen von sozialer Gerechtigkeit verletzt. ${ }^{20}$ Erst wenn sich die sozialen Unterschiede innerhalb und zwischen den europäischen Ländern deutlich flacher darstellen würden, wären grenzüberschreitende Eizellspende-Angebote ohne das Entstehen solcher Ausbeutungsverhältnisse denkbar.

Zum anderen können die finanziellen Interessen der Kinderwunsch-Praxen und Kliniken mit dem Schutz der Rechte der Spenderinnen in Konflikt geraten. Ich glaube nicht, dass eine angemessene Aufklärung und Beratung potenzieller Spenderinnen durch die Anbieter garantiert werden kann, wenn diese damit rechnen müssen, dass die Entscheidung gegen eine Spende fällt, sobald über die Risiken einschließlich der möglichen gesundheitlichen Langzeitfolgen ehrlich gesprochen wird. Aber selbst wenn der Anspruch einer guten Aufklärung und Beratung tatsächlich verfolgt wird, fehlen hierfür die notwendigen Informationen, so lange keine belastbaren Studien über die gesundheitlichen, psychischen und sozialen Langzeitfolgen für Eizellspenderinnen zur Verfügung stehen. Die Risiken und Belastungen der Eizellspende trägt die Spenderin, weshalb die ethische Legitimität der Eizellspende alleine auf ihrer freien und informierten Einwilligung beruht. Diese aber kann unter den heutigen Voraussetzungen gar nicht garantiert werden. Informiertheit könnte nur gewährleistet werden, wenn das notwendige Wissen über gesundheitliche, psychische und soziale Folgen für Spenderinnen überhaupt vorhanden wäre und wenn eine unabhängige Aufklärung und Beratung erfolgen würde. Außerdem ist die Freiwilligkeit einerseits durch die Abhängigkeit der Kinderwunschpatientinnen von Ärzten beim „egg sharing“ und andererseits durch das Armutsgefälle verbunden mit direkten oder indirekten finanziellen Anreizen bei der ,altruistischen Spende“ gefährdet - ohne solche Anreize spenden die Frauen nämlich offenbar nicht!

${ }^{20}$ Brodde (Fußn. 1). 



\title{
"What about my right to choose?" Young people with physical impairments exercising reproductive choice
}

\author{
Marilyn Crawshaw
}

Attitudes towards disability are changing. Although equality legislation on disability matters in the United Kingdom (UK) came much later than that for gender and ethnicity, it is now firmly on the statute books and its impact is felt in the fields of employment, education and leisure in particular. In 2007 the new UK Equality and Human Rights Commission replaced the single issue bodies of the Equal Opportunities Commission (gender), Race Relations Board (race) and Disability Rights Commission with the aim of ensuring that existing hierarchies of discrimination are dismantled and activity strengthened through combined effort. ${ }^{1}$

Contemporary disability-related debates within the field of reproductive medicine, however, remain focussed on the morality and/or social desirability of "allowing" people to give birth knowingly to children with impairments rather than being located within an equality framework. ${ }^{2}$ The dominant discourse is that the elimination of impairment is desirable, thus marginalising discussion of the value that disabled people (including disabled parents) may contribute to a society or ways in which their status is socially constrained. In a country whose relatively permissive laws around termination of pregnancy come under attack regularly from

${ }^{1}$ Cf. www.equalityandhumanrights.com.

2 See for example Taylor, Debating deafness and embryo selection, Bionews 2008/454, Commentary 21.4.2008, http://www.bionews.org.uk/commentary.lasso?storyid=3808, accessed on 4.7.2008. 
various quarters (including during the passage of the Human Fertilisation and Embryology Bill 2008), the legal right in the UK to have a termination at any stage of pregnancy where the foetus is diagnosed as carrying an impairment is rarely questioned except by pro-life religious lobbies. Indeed, the number of so-called "therapeutic" terminations carried out following detection of foetal abnormality has risen significantly in recent years and antenatal screening has come to be viewed by pregnant women and professionals alike as a routine activity rather than one requiring careful thought. ${ }^{3}$ The "right to choose" slogan of the pro-abortion lobbyists pre-dated the rise in such abortions and its positing of the right of the pregnant woman to exercise choice about becoming a mother was located firmly within a context of female autonomy rather than disablism. There remains an absence of debate about the rights of disabled people to choose to become parents.

It is against such a backdrop that this chapter considers how people with physical impairments and those around them view their potential to become parents. It starts with setting out two key models for conceptualising disability - the individual model and the social model. Using this as a framework for the rest of the chapter, it goes on to consider the ways in which these models influence the responses of professionals and services to disabled people as potential or actual parents. Finally, it draws on the experiences of young people growing up with physical impairments or chronic health conditions to consider how far their views of themselves as being able to exercise reproductive choices are influenced by society's attitudes towards disability.

The chapter does not include material in relation to people with learning difficulties or those coping with mental health or substance abuse problems. While many of the principles and ideas set out here apply to those groups, there are also unique features which warrant fuller exploration than can be managed within the constraints of a single chapter.

\section{Conceptualising disability}

The way that a society views its disabled members reflects and reinforces its socialising influences. Attitudes neither exist in a vacuum nor are always informed by facts. Social construction theory suggests that societies place differing values on their citizens according to the desirability of certain attributes, most commonly age, gender, sexual orientation, ethnicity, religion, social class - and disability. ${ }^{4}$ The values that are ascribed can influence the person's life chances and their impact may be reinforced or diluted by being present in different combinations. In other

\footnotetext{
${ }^{3} \mathrm{~W}$ ard, Considered Choices: the new genetics, prenatal testing and people with learning disabilities, 2001; Asch/Parens, in: Asch/Parens (eds.), Prenatal testing and disability rights, 2000; Shakespeare, Disability, genetics and eugenics, in: Swain/French (eds.), Disability on equal terms, 2008, pp. 21 ff.

${ }^{4}$ Moynihan, Theories of Masculinity, British Medical Journal 1998/317, 1072 ff.; Oliver, Theories of disability in health practice and research, British Medical Journal 1998/317, 1446 ff.
} 
words, the more attributes an individual holds from the lower reaches of the social desirability scale, the more disadvantaged they are likely to be. ${ }^{5}$

Disability writers and activists have identified two main approaches to conceptualising disability:

- The individual or "personal tragedy" model (sometimes also known rather misleadingly as the "medical model")

- The social model

They argue that the individual model is dominant in most contemporary societies but that a social model approach is required if people with impairments are to be enabled to become fully participating and respected citizens able to exercise their entitlement to human rights.

It is worth spending a little time defining these models more closely.

\subsection{The individual model}

Within an individual model approach, disability is characterised by suffering, deficiency, personal tragedy and abnormality. ${ }^{6}$ It is viewed as imposing inevitable limitations on 'affected' individuals who may find themselves further emasculated by being identified primarily in relation to their condition (e.g. "the amputee", "the wheelchair user"). Descriptions which appear on the surface to be positive may in fact be measured against negative portrayals of disability. Awards for courage to disabled people and plaudits to "wonderful" carers of disabled children or adults so prevalent in the popular media are premised on the assumed awfulness of the experience of being disabled and reinforce the assumed deficiency attached to the condition. ${ }^{7}$

The individual model sees the impairment as the "problem" and the search for solutions is thus also individualised. Disabled people themselves are expected to aspire to a "normality" based on a norm derived from the experiences of non-disabled people alone. In keeping with this, "normal independence" in adulthood is seen to equate to living with minimal support from others in terms of housing, income, personal care and so on. ${ }^{8}$

The conceptualisation of disability as a tragedy has been shown to permeate all strands of society so that it shapes our developing and ongoing understanding as we move through childhood and onwards. For those living with or acquiring impairment, the message may be internalised into a personal sense of deficiency. This informs and is reinforced further by Western unease with ageing, based in an un-

\footnotetext{
5 Thompson, Anti-Discriminatory Practice, 4. edn. 2006; Vernon, The dialectics of multiple identities and the disabled people's movement, Disability and Society 1999/14, 385 ff.

6 Swain/French, Disability on equal terms, 2008.

7 French/Swain, There but for fortune, in: Swain/French (eds.), Disability on equal terms, 2008, pp. $7 \mathrm{ff}$.

8 PascallHendey, Disability and the transition to adulthood: the politics of parenting, Critical Social Policy 2004, $165 \mathrm{ff}$.
} 
derlying fear of acquiring disability with age as well as marginal economic and social status. When considered in relation to parenthood, the individual model fuels the perceived "tragedy" of becoming the parent of a disabled child or the perceived ill-advisedness of a disabled person becoming a parent. The latter is returned to below.

\subsection{The social model}

The social model recognises that disabled people may experience some limitations in their functioning as a result of their impairment. For some, this limitation may be reduced by the provision of aids and adaptations: the person experiencing mobility difficulties may have the resulting limitations reduced by the provision of a wheelchair for example. However, if the society of which they are a member does not take account of their functional limitations in the way that it builds its houses and town centres, the way it provides its transport system and organises its schools and services and so on, any individualised solutions may prove inadequate as well as costly. The message is also sent out that such needs are not important enough to be recognised or taken into account. The needs of the non-disabled majority are given paramountcy and the place of the disabled member is set on the margins. By failing to acknowledge or give equal attention to the needs of disabled members, physical barriers are overlaid with attitudinal barriers that further reinforce the social undesirability of impairment and the likelihood of disabled people internalising a sense of inferiority or alienation. ${ }^{9}$

A social model conceptualisation of disability places physical and attitudinal barriers at centre stage. They are the problem not the impairment. Revisiting definitions of "normal" independence in adulthood cited above within such an approach opens up very different solutions. Many disabled people can achieve independence in housing, income and personal care through the provision of substantial levels of support, in other words a communal rather than an individual solution. By incorporating understanding of disabled people's pathway to independence into an inclusive "norm", definitions start to shift with the realisation that independence may be better defined as the degree to which one feels in control of one's life rather than a numerical formula based on quantified levels of support. ${ }^{10}$ Obstacles to independence are now more likely to be seen as failure of the community to provide adequate support structures than failure of individual will, aptitude or perseverance.

\footnotetext{
9 Oliver, Understanding disability: From theory to practice, 1996; Oliver, If I had a hammer: the social model in action, in: Swain/French/Barnes/Thomas (eds.), Disabling Barriers, Enabling Environments, 2004; Swain/French, Disability on equal terms, 2008; Thompson (n. 5).

${ }_{10}$ Hendey/Pascall, Moving into adulthood: Young disabled people moving into adulthood, 2002, https://www.jrf.org.uk/knowledge/findings/foundations/512.asp, accessed on 2.7.2008.
} 
The social model thus conceptualises disability within a social context approach which can be applied to thinking about disabled people becoming parents as follows:

- The barriers to effective parenting come from the disabling effect of being in a society geared predominantly to non-disabled people - i.e. the "problem" lies outside the disabled person and the impairment that they have.

- Society and services provided within that society should start from the assumption that disabled people are capable of parenting unless they are found lacking after appropriate support has been provided.

The social model was developed within the disabled people's movement, primarily by those with physical impairments although it has been extended to other groups in recent years. ${ }^{11}$ The development of the model has not stood still and debate remains lively about how effectively it encompasses the complexity of experiences..$^{22}$ Its key strength lies in the fact that it does not ignore the physical, emotional and social impact of impairment but locates it within a social and political context as well as a personal one.

In summary, the social model urges the use of the term "impairment" to acknowledge functional limitation and the use of the term "disabling" to describe the impact of being excluded from mainstream activities as a result of people with impairments being ascribed low social value.

\section{Capacity or support? Opposing tensions within responses to disabled people}

A social model approach argues that dominant attitudes, expectations and assumptions in a society negatively influence disabled people's experiences as prospective or actual parents. Given that disabled people are at risk of being socially excluded from parenthood, the question to be asked should be:

"Is there anything that should be in place to lower any socially constructed barriers to parenting for this disabled person?"

Once any barriers are lowered - both practical and attitudinal - and support is put in place, if they are then struggling to provide adequate parenting then their capacity to parent should be assessed and not before.

11 Oliver, in: Swain/French/Barnes/Thomas (n. 9); Swain/French/Barnes/Thomas, in: Swain/French/Barnes/Thomas (eds.), Disabling Barriers, Enabling Environments, 2004.

12 Review Symposium, Disability rights and wrongs?, Disability and Society 2007/22, 209 ff.; Swain/French (n. 6). 


\subsection{Approaches within practice and research}

Research suggests that both services and research approaches are imbued with „capacity” thinking within an individual model framework, reflecting the tendency of mainstream activities to reproduce the processes of social exclusion.

Professionals, health and support services working with disabled people either contemplating parenthood or already parents have been found more likely to base their interventions on assessment of "capacity" than "support". ${ }^{13}$ In other words, deciding whether or not someone is "fit to parent" or "capable of parenting" is considered ahead of assessing what support they might need to enable them to parent successfully through lowering any practical and attitudinal barriers arising from disablism. An assessment of capacity is both approached and experienced very differently to that for support needs. This may apply to determining whether people should be "allowed" to parent either as part of an assessment for them to become parents (e.g. assessment of suitability for assisted conception treatment; prebirth child protection assessments) or to decide whether they should be "allowed" to continue in their parenting role.

Disabled adults having their needs for support services being assessed find that the associated paperwork rarely includes reference to any potential or actual parenting-related needs ${ }^{14}$, despite policy guidance recommending that it should. ${ }^{15}$ There are also lively debates between service sectors about whether parenting-related matters should fall within the remit of children's or adults' services and these debates are only partly informed by pragmatism (i.e. whose budgets are most healthy). When placed into the arena of children's services, the likelihood of capacity-led assessments increases, given their primary focus on safeguarding children. ${ }^{16}$

Finally, services have been found to frame service needs around the caring tasks of the children of disabled parents and develop services for these so-called "young carers" rather than for the whole family or for peer support). ${ }^{17}$ Those adopting a social model approach have argued that this runs the danger of distort-

\footnotetext{
${ }^{13}$ Department of Health/Social Services Inspectorate, A jigsaw of services: Inspections of services to support disabled adults in their parenting, 2000; Olsen/Clarke, Parenting and disability: disabled people's experiences of raising children, 2003; Olsen/Tyers, Supporting disabled parents as parents, 2004, www.jrf.org.uk/knowledge/findings/socialcare/n34.asp; Wates, Supporting disabled parents in their parenting role, 2002.

${ }^{14}$ Crawshaw/Wates, Mind the gap - a case study for changing organisational responses to disabled parents and their families using evidence based practice, Research Policy and Planning 2005/23, $111 \mathrm{ff}$; Morris, They said what? Some common myths about disabled parents and community care legislation, 2004.

${ }^{15}$ Department of Health, Fair Access to Care Services Guidance; Paragraph 9, 2002; www.doh.gov.uk/scg/facs/.

16 Department of Health/Social Services Inspectorate (n. 13); Olsen/Clarke (n. 13).

${ }_{17}$ See for example Frank/Tatum/Tucker, On small shoulders: learning from the experience of former young carers, 1999.
} 
ing the parent-child relationship, reinforcing the view of disabled parents as "deficient" and obscuring the responsibility of communities to be more inclusive. ${ }^{18}$

Reviews of research that has looked at disabled people as parents have also found that the research questions (and hence the outcomes) clustered more around issues of capacity than support and more informed by individual than social models:

- The focus was overwhelmingly on specific impairments rather than common experiences across a range of impairments.

- Impairments were often conceptualised in the research design as "the problem" and research objectives framed in such a way as to look for negative outcomes to the "problems resulting from" the impairment rather than those resulting from external barriers.

- Recommendations arising from research findings were more likely to suggest solutions designed to reduce the impact of the impairment rather than that of external barriers.

- Attempts to disentangle the physical impairment itself from other variables that might be present in the situation including poverty, housing, gender, ethnicity were rare.

- Where other family members' experiences were studied or responded to, the focus was more on "carers (including "young carers")" than on "whole family" experiences.

- Where disabled parents were in receipt of services, this was more likely to be in a child protection context than the family support services arena. Research was also more likely to recruit disabled parents through services than elsewhere thus skewing the population being studied but rarely commenting on this. ${ }^{19}$

As Cavet has argued in relation to research with disabled children and young people: 20

This type of approach has been criticised for failing to take into account the different circumstances encountered by disabled children, thus tending to pathologise them and their families rather than explicitly recognising the disabling effect of their environment.

\footnotetext{
${ }^{18}$ Keith/Morris, Easy targets: a disability rights perspective on the children as carers debate, Critical Social Policy 1995, 36 ff.; Morris, A response to Aldridge and Becker - disability rights and the denial of young carers: the dangers of zero-sum arguments, Critical Social Policy 1997, 133 ff.; Newman, Young carers and disabled parents: time for a change of direction?, Disability and Society 2002/17, $613 \mathrm{ff}$.

19 Morris/Wates, Supporting disabled parents and parents with additional support needs: Adult Services Knowledge Review, 2006; available to download:

www.scie.org.uk/publications/knowledgereviews/kr11.pdf; Olsen/Wates, Disabled parents: examining research assumptions, 2003; available to download: www.rip.org.uk; Olsen/Clarke (n. 13).

${ }^{20}$ Cavet, Children and young people with a hidden disability: an examination of the social work role, British Journal of Social Work 2000, 619 (822).
} 
At the same time, research that focuses on the social context of either parenting per se or disability per se (i.e. rather than disabled parents per se) typically includes only scant reference to disabled parents. ${ }^{21}$ As a society, we do not expect disabled people to parent and appear to find it difficult to accept that they may do so unproblematically if appropriate levels of support are available.

\subsection{Policy approaches}

Policy approaches also appear more likely to be capacity- than support-led. As with professional practice and research, impairment is frequently identified in policy documents as "the problem". Attempts to disentangle the physical impairment itself from other potentially influential variables including poverty, housing, gender and ethnicity are rare. There is little evidence of attention to whole family thinking and disabled people are missing, by and large, from the family support policy agenda.

Although policy approaches are predominantly embedded in the "capacity" tradition, this can sometimes be masked by what on the surface appears to be a "support/citizenship" approach. However, further exploration shows that such "rights-based" approaches are invariably focussed on children's (young carers) or carers' rights rather than the rights of disabled people and/or families that contain disabled people. The linking of the language of rights to only one part of the family system - and to one that, as shown above, is conceptualised within an individual model approach - the citizenship and support needs that would be identified so differently through a social model approach are obscured or distorted and capacity thinking is more likely to dominate.

\section{So how does this match the experiences of disabled young people growing up?}

Most non-disabled young people grow up with the assumption that they are fertile and will probably become parents when they are adults, even if they do not express this explicitly. ${ }^{22}$ The picture may be more equivocal for disabled young people. They are less likely to have role models of disabled parents to aspire to and may be exposed to messages that challenge any inclusive normative views of parenthood that they might otherwise acquire. Additionally, the lack of clear information available that is specific to any fertility aspects associated with their impairment together with the lack of opportunity to build strategies for preparing themselves for

21 Wates, It shouldn't be down to luck: Results of a DPN Consultation on access to information services to support pare, 2003; available to download: www.DisabledParentsNetwork.org.uk.

22 Fraser/Balen/Fielding, The views of the next generation: an exploration of priorities for adulthood and the meaning of parenthood amongst 10-16 year olds, in: Balen/Crawshaw (eds.), Sexuality and Fertility Issues in Ill Health and Disability, 2006, pp. 33 ff. 
romantic relationships and parenthood may affect them disproportionately to their non-disabled peers. ${ }^{23}$

There is a growing body of evidence to suggest that professional attention to sex and fertility is largely missing in services for young people living with a range with impairments. ${ }^{24}$ In a study that I conducted with a colleague into the experience of growing up with cancer that may have put fertility at risk, there was a marked absence of professionals from any discipline making an opening for those affected to ask questions or discuss its implications. Even where professionals thought that they were inviting such a dialogue, it was sometimes done in such a way that the young people did not recognise the invitation. For example, ambiguous questions or statements from professionals were not taken up as the young people were fearful that they may have misread the cue whereas some young women, like the one quoted below, did not realise that questions asked about their menstrual cycle were in order to assess whether their fertility had been affected:

Every time I went they would always say, you know, "how are your periods?", because apparently for a lot of girls, they stop during chemotherapy anyway, but mine never did. It was often commented on "oh, you know that that's a good sign". And I always used to think that a good sign meant that the chemo was doing what it was meant to, instead of interrupting everything else...... but looking back now I sense that maybe a good sign is that, you know, it wasn't affecting my ovaries, but I mean you don't know unless [they make it clear to you $]. . . . .^{25}$

The dialogues to which young people are exposed within their social circles not only contribute to the formation of attitudes but also provide information that can contribute to their biological understanding - or misunderstanding. The relationship between sex and fertility itself can be blurred and incomplete or poorly understood information can impact further on this. Some disabled young people may be given information relating to their reproductive capacity in ways which can heighten the potential for confusion, as with this young man living with cystic fibrosis:

My mother told me that I couldn't have kids but at the time I wasn't sure what she meant, whether it was having kids or having sex. ${ }^{26}$

\footnotetext{
${ }^{23}$ For first hand narrative accounts of such experiences, see Wates/Jade, Bigger than the sky: disabled women and parenting, 1999; Finger, Past due - a story of disability, pregnancy and birth, 1991.

24 Andrews, Sexuality and sexual health throughout the childhood years, in: Balen/Crawshaw (eds.), Sexuality and Fertility Issues in Ill Health and Disability, 2006, pp. 19 ff.; Attwood, Making Sense of Sex: A forthright guide to puberty, sex and relationships for people with Asperger's, 2008; Contreras, Discussing sexuality with disabled girls, in: Wates/Jade (eds.), Bigger than the sky: disabled women and parenting, 1999, pp. 25 ff.; Morris/Wates (n. 19).

${ }_{25}$ Crawshaw/Sloper, A qualitative study of the experiences of teenagers and young adults when faced with possible or actual fertility impairment following cancer, 2006, p. 72.

${ }^{26}$ Farrant/Sanyer, Sexual and reproductive health in young people with cystic fibrosis: Hard to talk about but too important to ignore, in: Balen/Crawshaw (eds.), Sexuality and Fertility Issues in Ill Health and Disability, 2006, pp. $177 \mathrm{ff}$.
} 
While messages to non-disabled young people may be aimed clearly at pregnancy avoidance in the "here and now"; disabled young people may have to cope additionally with ambiguity either about whether or not they are capable of reproducing at all or about whether it is deemed desirable at any age.

For some, knowledge of their impairment being made available to their wider social circles may be difficult to manage, especially given the stigma associated with disability. In our study, some young people had little control over the disclosure of information about their cancer. They had more control over whom to tell about their fertility and some decided to tell no-one, even when this led to them feeling additionally isolated. On occasion, they even engaged in outward banter about people who were infertile in order to be "one of the crowd" while feeling inwardly bereft as it left them in no doubt about the social undesirability of (in)fertility adding to that of physical impairment. ${ }^{27}$ In another study, one young woman was told by her father that she was HIV positive in an attempt to encourage her to make greater efforts to avoid becoming pregnant. She was devastated by the impact of this being made public: In one situation, a father decided it was time to tell his 14 year old daughter that she was HIV positive. In response to the information she told her boyfriend, he panicked and told the principal, who panicked and called the police. Both children were suspended from school and suddenly everyone in the school knew that the child was HIV positive. ${ }^{28}$

The situation surrounding sex and fertility is not static across context or time. New challenges unfold as does the need for new information to be made available together with the opportunity to talk and think it through. What works in one space or time may prove less effective elsewhere. Both information sharing and management strategies have to be flexible enough to adjust to changing needs. In reflections on practice experiences with young women living with Turner Syndrome (a congenital condition that affects females and stops the production of mature eggs), a social worker described one young woman's somewhat fatalistic approach to coping. If and when her approach no longer worked well enough for her, so might her need to access additional information and help in developing new strategies:

It was hard for my parents and me when I had my ovaries removed and they had to tell me I couldn't have children.... I love playing with children and it would be fantastic if I could have children but that's an impractical want, some things are just meant to be. ${ }^{29}$

\footnotetext{
${ }^{27}$ Crawshaw/Sloper (n. 25).

${ }^{28} \mathrm{Cincotta} /$ Childs/Eichenfield, Sexuality and growing up HIV-positive: lessons from practice, in: Balen/Crawshaw (eds.), Sexuality and Fertility Issues in Ill Health and Disability, 2006, pp. 206 ff. (211).

${ }^{29}$ Loughlin, Infertility: an unspoken presence in the lives of teens and young women with Turner Syndrome, in: Balen/Crawshaw (eds.), Sexuality and Fertility Issues in Ill Health and Disability, 2006, pp. $159 \mathrm{ff}$.
} 
For some disabled females, the route to parenthood may entail the use of donated eggs or surrogacy (laws permitting) with all the additional physical and emotional complexity for the woman herself and any children (and the family unit) formed through such routes. Preparation for this can (and arguably should) start far ahead of any decision-making as to whether or not to go down this route. If the only - or the favoured - family composition that a young person is exposed to is one where the parent-child relationship is biological as well as social, then they are more likely to approach donor assisted conception decisions from a deficit standpoint. Similarly, if prior professional contact has led the young person to feel their experience is seen as medicalised rather than social and emotional as well, then staff in assisted conception centres may be faced with someone who has considerable unresolved issues, as with this woman living with the intersex condition called Congenital Adrenal Hyperplasia:

I felt totally medicalised, with a total lack of body ownership, until I got a third opinion on surgery at 31 years of age... I am now 37 years old.... who knows what the future will bring - the work to lift the taboo of intersex goes on. ${ }^{30}$

While females may have to contemplate the use of another woman's eggs or womb, men who find themselves with impaired fertility have to consider the use of donated sperm (laws permitting) or other assisted conception treatment such as intra-cytoplasmic sperm injection (ICSI). For some in this position, feelings of guilt towards their partner arising from not being able to conceive a child together are similar and transcend gender difference. However, males have the additional fact to accommodate that it is their female partner who has to undergo the medically assisted conception treatment not them:

I recall feeling guilt about being the cancer survivor yet subjecting my wife to invasive and discomforting procedures, being angry at cancer, eight years after diagnosis and treatment for subjecting both of us to these trying procedures. ${ }^{31}$

Once a pregnancy is achieved, the story is far from over for those affected directly or their family members. As well as managing any personal concerns about the pregnancy and delivery itself, those affected now have the face the scrutiny of antenatal and postnatal health services and, if they require any practical assistance, that of social services. While some are able to reflect on the resilience and strength that comes through the adversity of living in a disabling society, the need to prove capacity to parent can take its toll and present the kind of external challenge that is not routinely experienced by non-disabled parents-to-be.

The experience of Reesha Armstead, ${ }^{32}$ a woman who has cerebral palsy, sets out the challenge:

\footnotetext{
30 Cull, Dispelling the taboo of intersex conditions: we're only human like you, in: Balen/Crawshaw (eds.), Sexuality and Fertility Issues in Ill Health and Disability, 2006, pp. 201 ff. (205).

31 Zebrack, Young adult cancer survivors: shaken up, getting back, moving on, in: Balen/Crawshaw (eds.), Sexuality and Fertility Issues in Ill Health and Disability, 2006, pp. 221 ff. (229).
} 
Had my non-disabled husband married a non-disabled woman and announced that his wife was expecting a baby, he'd have been congratulated, the families would have celebrated, he'd have been patted on the back! But he wasn't and they didn't and they couldn't.... When we told everyone about our baby, he wasn't congratulated, he was told off. No one celebrated, they just worried. We were grown adults, but we were treated like children. We were so happy in ourselves, but no one else had ever talked about the possibility of me becoming a mother. It was different for my sister, our parents longed for her to produce grandchildren and when she did, everyone was happy for her. I was frightened but no one comforted me. No one had faith in me to care for my baby. Most people saw it as a problem. But I grew strong. My determination to have a baby and confidence in myself overtook and the fear became everyone else's.

She goes on to describe some of the ways she fought back:

I told the midwife in the hospital that she couldn't give my baby a bottle and went on to breastfeed her for a whole year. I told the doctor to cancel the appointment with the gynaecologist to discuss being sterilised because I wanted another baby. I told the child social worker that I couldn't accept her offer (of her child being cared for in a family centre away from her) and then fought adult services for a direct payment to enable me to employ my own personal assistants to enable me to be a wife and mother. It worked.

\section{Conclusion}

If we as a society are to consider disabled people as having the "right to choose" whether or not to become parents as an expression of their human rights as citizens, then there needs to be a paradigm shift in the conceptualisation of disability. While it remains considered only, or even primarily, within an individual model then the impact on the ability to be a parent for disabled people living in a society geared to its non-disabled members remains invisible. This chapter has argued that disabled people are part of relationship networks and environmental systems that erect barriers to the full participation of disabled members through ascribing negative attributes to them. On either side of the barrier, disabled and non-disabled people alike are exposed to such stigmatising thinking with the resulting internalisation of its message. The danger of disabled people viewing themselves and others viewing them as asexual and/or lacking the capacity to parent is heightened. Individualising the "problem" and hence the "solution" only serves to reinforce those barriers. Although services (including those of research) can replicate oppressive tendencies within the status quo of the communities they

32 Armstead, A Different kind of Parent' Speech given at Joseph Rowntree Foundation Taskforce Conference "The Right Support" 23.9.2003; available for download:

http://www.disabledparentsnetwork.org.uk/cgi-bin/site/site.cgi?page=site/different_parent, accessed on 28.6.2008. 
serve, they also have the potential to challenge thinking and prompt change. ${ }^{33}$ There have been some exciting alliances in recent years between disability-led organisations, practitioners and researchers within which social model approaches have been used withrigour and imagination. ${ }^{34}$ If disabled people are to achieve the social and political engagement that comes with social inclusion, then the "right to choose" parenting on a par with non-disabled peers is a key element of that process.

33 Gillman, In practice from the viewpoint of a social worker, in: Swain/French (eds.), Disability on Equal Terms, 2008, pp. 171 ff.; Strier, Anti-oppressive research in social work: a preliminary definition, British Journal of Social Work 2007, $857 \mathrm{ff}$.

${ }^{34}$ See for example, the Disabled Parents Network UK:

www.DisabledParentsNetwork.org.uk, accessed on 28.6.2008. 



\title{
Wenn der Partner an einer progredienten, potenziell lebensbedrohlichen Erkrankung leidet - Fragen zur Entscheidungsfindung für eine In-vitro- Fertilisation mit ICSI
}

\author{
Gisela Bockenheimer-Lucius, Timo Sauer, Falk Ochsendorf, Inka Wiegratr, \\ Aglaja Stirn, Yasar Toraman
}

Seit der Geburt des ersten „Retortenbabys“ im Jahre 1978 wurde die Indikation zur In-vitro-Fertilisation (IVF), nämlich der Einsatz bei Fertilitätsstörungen der Frau, vielfach erweitert. Besondere Bedeutung hat dabei die Möglichkeit, über eine intrazytoplasmatische Spermieninjektion (ICSI) auch bestimmte Formen männlicher Zeugungsunfähigkeit therapieren zu können. ${ }^{1}$ Trotz nach wie vor kontroverser Diskussionen um die künstliche Befruchtung ${ }^{2}$ werden dank dieser Verfahren in Deutschland jährlich etwa 6000 Kinder geboren, weltweit sind es seit Louise Browns Geburt ca. 3,5 Millionen Kinder. Dabei gehört die IVF mit ICSI heute zu den Standardverfahren und ist die am häufigsten praktizierte Methode der künstlichen Befruchtung.

\footnotetext{
${ }^{1}$ ICSI wird in Deutschland seit Anfang der 1990er Jahre durchgeführt, wobei ein einzelnes Spermium mittels einer Nadel in die Eizelle injiziert wird: Bertelsmann/Gomes/Mund/Bauer/Matthias, Fehlbildungsrisiko bei extrakorporaler Befruchtung, Arztebl. 2008, 11 ff. Vgl. auch den Beitrag von Michelmann, Reproduktionsmedizin im Jahre 2008: Probleme - Wünsche - Lösungsansätze, in diesem Band.

2 Vgl. dazu Krones/Neuwohner/El Ansari/Wissner/Richter, Kinderwunsch und Wunschkinder. Möglichkeiten und Grenzen der In-vitro-Fertilisations-Behandlung, Ethik in der Medizin 2006, 51 ff.
} 
In seltenen, aber regelmäßig wiederkehrenden Fällen treten Frauen oder Paare mit dem Wunsch nach einer IVF an die Reproduktionsmedizin heran, bei denen der männliche Partner lebensbedrohlich und unheilbar erkrankt ist oder sich gar in der terminalen Phase einer Erkrankung befindet. ${ }^{3}$ In diesen Fällen haben die Paare in der Regel erfahren, dass die Spermienqualität des erkrankten Partners für eine homologe Insemination nicht mehr ausreicht, da die Erkrankung die Samenproduktion und -qualität bereits deutlich beeinträchtigt hat. Zudem stehen zumeist therapeutische Maßnahmen wie Chemotherapie und Bestrahlungen an, die eine erhebliche Schädigung der Fertilität mit sich bringen. Dann ist eine Schwangerschaft weder auf natürlichem Wege, noch durch homologe Insemination oder durch eine alleinige IVF zu erreichen. Hoffnung auf Erfolg kann nur der Weg über eine IVF mit ICSI geben. In Einzelfällen kommt es auch zu dem Wunsch einer Frau, mit ihrem Partner ein gemeinsames Kind zu haben, nachdem der Mann im Rahmen eines Unfalls oder postanoxischen Geschehens eine schwere zerebrale Schädigung (z.B. ein apallisches Syndrom) erlitten hat.

In den Erstkontakten mit den Anfragenden tauchen allerdings im reproduktionsmedizinischen Team nicht selten Zweifel auf, ob der emotional durchaus nachvollziehbare Kinderwunsch tatsächlich von beiden Partnern gleichermaßen getragen wird, ob möglicherweise eine verzweifelte Situation zu einem spontanen, kaum hinterfragten Wunsch nach einem gemeinsamen Kind geführt hat und/oder ob einer der beiden Partner unter erheblichem moralischem Druck steht. Auch der größere Familienkreis kann versuchen, Einfluss zu nehmen. Angesichts derartiger Unsicherheiten und Ambivalenzen entstand in den vergangenen drei Jahren in der Uniklinik Frankfurt am Main einige Male Bedarf an Ethikberatung durch das Klinische Ethik-Komitee (KEK) mit der Frage nach der Verantwortung der Reproduktionsmedizin gegenüber den Elternteilen und gegenüber dem noch nicht gezeugten Kind.

\section{Ethische Fragen}

Konsens besteht mit Blick auf die reproduktive Autonomie des Paares. Es gehört zweifellos zu den in besonderer Weise geschützten Persönlichkeitsrechten, nicht

\footnotetext{
${ }^{3}$ Das Ansinnen von Partnerin, Eltern oder anderen Angehörigen bei einem nicht mehr einwilligungsfähigen Patienten z.B. im Falle eines Hirntodes Samen über eine Hodenbiopsie oder entsprechende Verfahren (Nebenhoden, ableitende Samenwege) zu entnehmen, um einen ehemals geäußerten Kinderwunsch noch zu realisieren, wird an dieser Stelle nicht weiter verfolgt, da ein derartiger Eingriff in die körperliche Integrität nicht ohne persönliche Einwilligung des Betroffenen erfolgen darf und sich daher ethisch wie rechtlich von vornherein verbietet. Prinzipiell wäre eine Vorausverfügung, die die Entnahme von Sperma erlaubt und einen ausdrücklichen Kinderwunsch belegt, mit einer Reihe weiterer Bedingungen für diesen Eingriff durchaus zu diskutieren (vgl. dazu Tash/Applegarth/Kerr/Fins/Rosenwaks/Schlegel, Post mortem sperm retrieval: The effect of instituting guidelines, The Journal of Urology 2003/170, 1922 ff.). In Deutschland verbietet jedoch das Embryonenschutzgesetz ohne Einschränkungen ein derartiges Vorgehen (ESchG \4, Abs. 3: Mit Freiheitsstrafe bis zu drei Jahren wird bestraft, wer wissentlich eine Eizelle mit dem Samen eines Mannes nach dessen Tode künstlich befruchtet).
} 
daran gehindert zu werden ein Kind zu zeugen. Unabhängig von der umstrittenen Frage, ob es auch ein positives Recht auf Erfüllung des Kinderwunsches gibt, ${ }^{4}$ kann und darf es im Falle eines Paares, das sich vor dem aller Wahrscheinlichkeit nach unvermeidbaren Tod des Mannes sehnlich noch ein Kind wünscht, nicht darum gehen, die artifizielle Reproduktion unhinterfragt zu verweigern.

Die spezifische Problematik liegt in der gebotenen Beratung eines jeden IVFPaares, die eben nicht nur den medizinischen Part abdecken darf: „Die medizinische Information soll von einer psychosozialen Beratung begleitet werden. Die Patientin bzw. das Paar sind in die Lage zu versetzen, unter Kenntnis der medizinischen Sachverhalte, der Risiken, die mit der Inanspruchnahme reproduktionsmedizinischer Verfahren verbunden sind, sowie der ethischen Aspekte, zu denen das Kindeswohl gehört, in eigener Verantwortung zu entscheiden, ob sie die Reproduktionsmedizin in Anspruch nehmen möchten und welche Verfahren der ärztlich assistierten Reproduktion es sind, von denen sie Gebrauch machen wollen." "5

Um folglich in der besonderen Belastung durch eine todbringende Erkrankung des potenziellen Vaters implizite ethische Fragen nicht unbeachtet zu lassen und zugleich eine zuverlässige Basis zur Entscheidungsfindung für das beratende und behandelnde Team wie auch für die Betroffenen zu haben, sollen mit dem vorliegenden Beitrag grundsätzliche ethische Überlegungen für die Reproduktionsmedizin zur beschriebenen Problematik dargestellt werden (1), ein Perspektivenwechsel auf die Sicht der potenziellen Eltern vorgenommen werden (2) und schließlich ein Leitfaden vorgestellt werden, der das Verfahren der Beratung und Entscheidungsfindung in der konkreten Situation strukturieren und erleichtern soll (3).

Die ethische Fragestellung ist wie so oft in der Medizin sehr komplex, weil mehrere Akteure in unterschiedlichem Ausmaß beteiligt sind und die jeweiligen Rechte und Pflichten gegeneinander abgewogen werden müssen. Die folgende Analyse basiert auf den von Beauchamp und Childress für die Medizinethik entwickelten vier Prinzipien „mittlerer Reichweite“, d.h. dem Verzicht auf ein oberstes moralisches Prinzip zugunsten einer möglichst großen Nähe zur Lebenswirklichkeit der Betroffenen und eines kohärenten Begründungsansatzes. ${ }^{6}$ Das dominierende Prinzip dieses Modells ist die Respektierung der Autonomie des Patienten. Dies ist für die vorliegende Fragestellung nach der verantwortbaren reproduktionsmedizinischen Maßnahme von besonderer Bedeutung. Es verlangt nämlich nicht nur jeden Verzicht auf äußeren Zwang und Manipulation, sondern beinhaltet zugleich die Pflicht des Arztes bzw. des Teams, die Autonomie derer, die sich ihm anvertrauen, durch Informationen und Förderung des Entscheidungsprozesses Wirk-

\footnotetext{
4 Vgl. dazu etwa Bundesärztekammer, (Muster-) Richtlinie zur Durchführung der assistierten Reproduktion, Novelle 2006, Dt. Ärzteblatt 2006, 1392 ff.; ebenso Krones/Neunvohner/El Ansari/Wissner/Richter (Fußn. 2); in rechtlicher Hinsicht s. Heun, Restriktionen assistierter Reproduktion aus verfassungsrechtlicher Sicht, in diesem Band.

${ }^{5}$ Bundesärztekammer (Fußn. 4), 1393.

${ }^{6}$ Beauchamp/Childress, Principles of Biomedical Ethics, 5. Aufl. 2001; vgl. dazu auch Marckmann, Was ist eigentlich prinzipienorientierte Medizinethik?, in: Wiesing (Hrsg.), Diesseits von Hippokrates, 2003, S. 325 ff.
} 
lichkeit werden zu lassen.7 Das Prinzip, nicht schaden zu dürfen sowie das Fürsorgeprinzip müssen jeweils skrupelhaft abgewogen werden, um dem Wohl des individuellen Patienten gerecht zu werden. Die Berücksichtigung des Prinzips der Gerechtigkeit schließlich stellt mit Blick auf die Mittelverteilung bzw. die jeweiligen finanziellen Lasten für den Einzelnen im Bereich der Reproduktionsmedizin ein hochaktuelles und heftig diskutiertes Problem dar. Der Perspektivenwechsel auf die moralische Herausforderung für die betroffenen potenziellen Eltern wird über die vier genannten Prinzipien hinaus im Weiteren die ethischen Erörterungen um die elterliche Verantwortung erweitern.

\subsection{Der Blick auf das Kind}

Bei allen Entscheidungen in der Medizin, bei denen außer der Frau (und ihrem Partner oder ihrer Partnerin) als Dritter auch ein Kind betroffen ist, kommt dem Kindeswohl ein besonders hohes $\mathrm{Maß}$ an Bedeutung zu. Dies gilt z.B. in der Humangenetik, und so werden in der Geburtshilfe beispielsweise Fragen der sog. „Wunschsectio“8 oder des Zwangs zum Kaiserschnitt gegen den Willen der Mutter immer auch unter dem Aspekt des Kindeswohls und der Garantenpflicht des Arztes für Mutter und Kind diskutiert. ${ }^{9}$ Für die Reproduktionsmedizin betont die Bundesärztekammer dies in der (Muster)-Richtlinie zur Durchführung der assistierten Reproduktion nachdrücklich: „Sofern im konkreten Fall die Anwendung reproduktionsmedizinischer Verfahren die Voraussetzung dafür ist, dass ein Kinderwunsch überhaupt verwirklicht wird, trägt die Ärztin/der Arzt für das Wohl des mit ihrer/seiner medizinischen Assistenz erzeugten Kindes eine besondere Verantwortung. Die ärztliche Pflicht, zum Wohl des Patienten zu handeln und Schaden zu vermeiden, bezieht sich auf die Muter und die erwünschten Kinder.“ 10

Die Perspektive des noch ungezeugten Kindes selbst ist jedoch sehr spekulativ, da man von einer hypothetischen Person ausgehen muss. Dennoch soll hier so verfahren werden, da das (noch) hypothetische Kind zu einem späteren Zeitpunkt faktisch Folgen zu tragen hat, die aus dem Entscheidungsprozess vor seiner Zeugung hervorgehen. ${ }^{11}$

\footnotetext{
7 Vgl. zu diesem Aspekt der Aufklärungspflicht ausführlich Peintinger, Therapeutische Partnerschaft: Aufklärung zwischen Patientenautonomie und ärztlicher Selbstbestimmung, 2003.

8 Vgl. dazu u.a. Bockenheimer, Zwischen „natürlicher Geburt“ und Wunschsectio, Zum Problem der Selbstbestimmtheit in der Geburtshilfe, Ethik in der Medizin 2002, $186 \mathrm{ff}$.

9 Vgl. dazu in jüngerer Zeit: Samuels/Minkoff/Feldman/Awonuga/Wilson, Obstetricians, health attorneys, and court-ordered cesarean sections, Women's Health Issues 2007/17, $107 \mathrm{ff}$.

10 Bundesärztekammer (Fußn. 4), 1393.

11 Zweifellos gibt es eine umfangreiche philosophische Diskussion über die Pflichten gegenüber einem noch nicht gezeugten Kind. Man denke etwa an die Verantwortung gegenüber zukünftigen Generationen. Auch die Abtreibungsdebatte und die Diskussion um den moralischen Status des Embryos knüpfen an diesen Fragen an. Vgl. etwa Schöne/Seifert, Zum moralischen Status von potentiellen Personen, in: Meggle/Fehige (Hrsg.), Zum moralischen Denken, Bd. II, 1995, S. 210 ff. Im vorliegenden Zusammenhang würde diese Erörterung aber den gegebenen Rahmen sprengen.
} 
Nichtschaden/Nonmaleficence: Vor dem Hintergrund des Nichtschadensgebotes sind drei moralisch relevante Aspekte zu diskutieren:

(a) Kann das IVF/ICSI-Verfahren somatische oder psychische Schäden bei den Kindern verursachen, die man vorab bedenken muss?

Dies ist im Rahmen der Aufklärung der potenziellen Eltern zweifellos von Bedeutung. Es rührt an die noch zu diskutierende Frage, ob Eltern bewusst und u.U. wider besseres Wissen ein Kind zeugen dürfen, das einem hohen Risiko von gesundheitlichen Schäden ausgesetzt ist. ICSI ist Teil des Leistungskatalogs der gesetzlichen Krankenversicherung und seit der Einführung „begleitet von der Sorge, dass das Fehlbildungsrisiko im Vergleich zu natürlich gezeugten oder mit IVFgezeugten Kindern erhöht sein könnte." ${ }^{2}$ Die Fehlbildungsraten der mit ICSI gezeugten Kinder wurden daher in zahlreichen Studien überprüft. Eine umfangreiche Metaanalyse dieser Studien zeigt, dass für zuverlässige Aussagen noch erheblicher Forschungsbedarf besteht. Es erscheint unwahrscheinlich, „dass die Zeugung durch ICSI das Fehlbildungsrisiko der Kinder im Vergleich zur IVF deutlich erhöht. Dies relativiert auch die Sorge eines methodenimmanenten Risikos der ICSI zur Induktion von Fehlbildungen durch Manipulationen an der Eizelle. Es kann jedoch nicht ausgeschlossen werden, dass die ICSI das Risiko für große Fehlbildungen gegenüber der natürlichen Zeugung erhöht. Das gilt jedoch in gleichem Maße auch für die IVF."13 Die ICSI-Follow-up-Studie II, eine deutschlandweite Multicenterstudie, führte eine Nachuntersuchung der Kinder im Alter von vier bis sechs Jahren durch. Reif geborene Einlingskinder nach ICSI entwickeln sich danach ebenso unauffällig wie spontan konzipierte Kinder. ${ }^{14}$ Das Problem des Fehlbildungsrisikos muss folglich Teil einer umfassenden Aufklärung im Rahmen der extrakorporalen Befruchtung sein.

Die Frage nach psychischen Schäden der Kinder hat die anfängliche Debatte um die künstliche Befruchtung stark geprägt. Inzwischen zeigen zahlreiche Langzeitstudien, dass die sog. „Retortenbabys“ sich unauffällig entwickeln. ${ }^{15}$

\footnotetext{
12 Bertelsmann/Gomes/Mund/Bauer/Matthias (Fußn. 1), 11.

13 Bertelsmann/Gomes/Mund/Bauer/Matthias (Fußn. 1), 16; vgl. auch M.Ludwig/Diedrich/A.Ludwig, Deutsche ICSI-Follow-up-Studie II, Frauenarzt 2008, 306 ff.

${ }_{14}$ M.Ludwig/Diedrich/A.Ludwig (Fußn. 13), 312; Zu beachtende Einschränkungen ergeben sich allerdings für Mehrlingsgeburten und mit Blick auf das generelle Risiko von Frühgeburten nach artifizieller Reproduktion.

15 Vgl. dazu Ludwig/Diedrich, In vitro Fertilisation und Intrazytoplasmatische Spermieninjektion. Gibt es ein Gesundheitsrisiko für die geborenen Kinder?, Arztebl. 1999, 2892 ff.; umfangreiche Studien liegen vor von Golombok/Brewaeys/Cook/Giavazzi/Guerra/Mantovani/v.Hall/Crosignani/Dexeus, The European study of assisted reproduction families: family functioning and child development, Human Reproduction 1996/11, 2324 ff.; Golombok/Brewaeys/Giavazzi/Guerra/MacCallum/Rust, The European study of assisted reproduction families: The transition into adolescence. Human Reproduction 2002/17, 830 ff.; Halliday, Outcomes of IVF conceptions: are they different? Best Practice \& Research Clinical Obstetrics \& Gynaecology, 2007/21, 67 ff.; auch die deutsche ICSI-Follow-upStudie II belegt für reif geborene Einlingskinder im Alter von 4-6 Jahren eine „,normale emotionale Entwicklung" und „normales Verhalten“", M.Ludwig/Diedrich/A.Ludwig (Fußn. 13), 312.
} 
In der hier zu betrachtenden Situation des Kinderwunschs bei todkrankem Partner haben die Fragen nach somatischen oder psychischen kindlichen Schäden nach IVF/ICSI jedoch keinen spezifisch höheren Stellenwert, da die potenzielle Mutter kein höheres Risiko eingeht als eine Frau mit gesundem Partner.

(b) Ist die Wahrscheinlichkeit, dass ein in-vitro erzeugtes Kind ggf. ohne Vater aufwachsen muss, Grund genug, das reproduktionsmedizinische Verfahren nicht durchzuführen?

Ob die Aussicht auf ein Leben ohne leiblichen ggf. auch sozialen Vater für das Kind als Schaden zu bewerten ist, hat für den zu diskutierenden Spezialfall entscheidende Relevanz. Aussagen darüber haben allerdings einen hohen spekulativen Charakter. Die (Muster)-Richtlinie zur Durchführung der assistierten Reproduktion (Bundesärztekammer, Novelle 2006) sieht vor, dass das IVF/ICSI-Verfahren nur bei verheirateten Paaren durchgeführt werden soll, oder bei nicht-verheirateten Paaren, die nachweisen können, in einer eheähnlichen Verbindung zu leben. ${ }^{16}$ Was zunächst als Einmischung in den Lebensstil der potenziellen Eltern wirkt, folgt der Intuition, dem hypothetischen Kind beste Lebensvoraussetzungen (Vorhandensein von Vater und Mutter, Stabilität der elterlichen Verbindung) garantieren zu können. In Analogie dazu könnte man zu dem Schluss kommen, von einer IVF bei einem todkranken Vater grundsätzlich abzusehen, da die Vaterlosigkeit hochwahrscheinlich ist und somit die Bedingungen für das Kind von vornherein schlecht seien.

Es gibt jedoch keinen empirischen Nachweis dafür, dass eine Kindheit ohne Vater nicht völlig unauffällig verlaufen kann. Langzeitbeobachtungen, die etwa eine psychisch signifikant ungünstige Entwicklung von Kindern von alleinerziehenden Müttern (oder auch Vätern) belegen, gibt es nicht oder sie sind hoch kontrovers. ${ }^{17}$ Auch die Frage, ob und wie weit Vaterlosigkeit etwaige psychische Belastungen ursächlich erklärt, ist nicht generell zu beantworten. Vieles spricht dafür, dass es für die emotionale Entwicklung eines Kindes von höherer Bedeutung ist, ,gewollt“ zu sein. ${ }^{18}$ Günstige Rahmenbedingungen, wie eine emotional zugewandte Mutter, familiäre Bindungen, ein stabiler Freundeskreis, soziale Kontakte und eine zufrieden stellende finanzielle Lebensgrundlage, dürften erheblich stärkere Bedeutung für die gesunde Entwicklung eines Kindes haben. $\mathrm{Zu}$ fragen wäre auch, ob das geplante Kind sein Schicksal mit einem bereits geborenen, aber nur wenig älteren Geschwisterkind teilen wird. Schließlich ist grundsätzlich nicht absehbar, ob das Kind nicht in einer glücklichen Familienatmosphäre mit einem neuen Partner der Mutter und fürsorglichen Vater sowie Geschwistern aufwachsen wird.

16 Bundesärztekammer (Fußn. 4), 1395.

17 Vgl. dazu ESHRE, Task Force on Ethics and Law 11: Posthumous assisted reproduction, Human Reproduction 2006/21, $3051 \mathrm{ff}$.

18 Vgl. dazu die umfangreiche Literatur zu Abtreibung, anonymer Geburt und Adoption, z.B. bei Swientek, Die Wiederentdeckung der Schande, 2001, u.v.a. 
Das Fehlen eines Vaters kann also allenfalls als schwaches Argument gewertet werden, ${ }^{19}$ für zuverlässige Aussagen wären aber empirische Untersuchungen zur Entwicklung betroffener Kinder wünschenswert. ${ }^{20}$

(c) Ist für das Kind mit einem psychosozialen Schaden durch eine psychisch dekompensierte Mutter zu rechnen? Welche moralische Bedeutung hätte dies?

Auch ein antizipierter psychosozialer Schaden des hypothetischen Kindes durch eine psychisch dekompensierte Mutter hat spekulativen Charakter, ist jedoch sehr viel nahe liegender als ein Schaden durch die eventuelle Vaterlosigkeit. In der Berücksichtigung derartiger möglicher Belastungen, die nicht zuletzt die kindliche Entwicklung während der Schwangerschaft der Mutter betreffen, liegt die Hauptverantwortung der Reproduktionsmedizin gegenüber dem ungezeugten Kind. Bei der Durchführung der IVF muss gewährleistet sein, dass die Mutter in der Lage ist, die außerordentlichen physischen und psychosozialen Belastungen zu tragen, so dass dem Kind durch eine psychische Dekompensation der Mutter kein Schaden droht. Allerdings sind die zu bedenkenden Folgen dennoch spekulativer Art, denn eine psychisch überforderte Mutter mag zwar kaum in der Lage sein, für optimale Bedingungen zu sorgen, jedoch ist es denkbar, dass das Kind aufgrund seiner individuellen psychischen Konstitution Bewältigungsstrategien entwickeln kann. Erfahrungen aus Kriegs- und Krisensituationen unterschiedlichster Art belegen, dass das „not worth living"-Argument nicht überzeugen kann.

Wobltun/Beneficence: Von einer Wohltat für das zukünftige Kind kann man sinnvoller Weise nur unter der Prämisse sprechen, dass Menschen in der Regel ihr Leben einer Nichtexistenz vorziehen. Hinzu kommt die Mutmaßung, dass die durch IVF gezeugten Menschen ebenso ihrer Existenz zustimmen wie natürlich gezeugte. Für ein Kind mag die Motivation seiner Eltern, durch seine Geburt die gemeinsame Liebe und das Vertrauen in die Zukunft weiter zu tragen, ein überzeugender Grund sein, seine eigene Existenz als besonders wertvoll zu empfinden.

Autonomie: Dem noch nicht gezeugten Kind kann man aus Gründen der Logik keine Autonomie zusprechen.

\subsection{Der Blick auf die Frau}

Nichtschaden/Nonmaleficence: Während die Beurteilung des Wohles eines noch nicht gezeugten Kindes mit vielfältigen Mutmaßungen einhergehen muss, sind die Be-

\footnotetext{
${ }^{19}$ Bei der Richtlinie der BÄK spielen offensichtlich implizite Wertvorstellungen über die „richtige“ Familienkonstellation eine Rolle. So ist im gleichen Passus die IVF für lesbische Paare ausgeschlossen. Interessant ist die Formulierung im Kommentar zur (Muster-) Richtlinie: „Aus diesem Grund ist eine heterologe Insemination zurzeit bei Frauen ausgeschlossen, die in keiner Partnerschaft oder in einer gleichgeschlechtlichen Partnerschaft leben.“ Das „zurzeit“ erweckt den deutlichen Eindruck, dass auch die BÄK davon ausgeht, dass die derzeitige Regelung in dieser Hinsicht inkonsistent ist oder, dass sich in absehbarer Zeit daran etwas ändern wird. Bundesärztekammer (Fußn. 4), 1400.

${ }^{20}$ Vgl. ESHRE (Fußn. 17), 3051.
} 
lastungen, die für die betroffene Frau entstehen können, sehr genau aufzuzeigen. Daher muss sie im Mittelpunkt der ärztlichen Verantwortung stehen. Die Frau ist diejenige, die alle Risiken des Verfahrens tragen muss.

Die in der Regel noch recht jungen Frauen der todkranken Partner sind gesund und zeugungsfähig. Der Eingriff der Reproduktionsmedizin ist nicht durch eine medizinische Indikation gerechtfertigt, die diese Frau selbst betrifft. Erst die gemeinsame wohldurchdachte und selbstbestimmte Entscheidung des Paares zu einer ärztlich assistierten Reproduktion erlaubt den Eingriff in die körperliche Integrität der Frau. Damit ist sie zunächst dem nicht unerheblichen physischen Risiko einer hormonellen Stimulationsbehandlung ausgesetzt. Darüber hinaus trägt sie die hohe psychische Belastung, die sich aus der Unsicherheit des Verfahrens ergibt: In nur ca. einem Drittel der Fälle kommt es zum erfolgreichen Eintritt einer Schwangerschaft und in etwa $15-20 \%$ tatsächlich zur Geburt eines gesunden Kindes. Starke emotionale Störungen bis hin zur Depression können die Folge von Therapie und Misserfolgen sein und das Wohlbefinden bedrohen. Bei einer IVF muss zudem auch nach erfolgreichem Embryotransfer mit einer leicht erhöhten Abortrate gerechnet werden, die Risiken von Mehrlingsschwangerschaften kommen schließlich hinzu. ${ }^{21}$

Für viele Frauen sind bereits diese grundsätzlichen Probleme der medizinisch assistierten Reproduktion entscheidend für eine äußerst kritische bis ablehnende Haltung. ${ }^{22}$ Jedoch muss man auch bei einer grundsätzlich positiven und bejahenden Einstellung zu dieser Technik berücksichtigen, dass Risiken nicht völlig vermeidbar sind und eine erhebliche Mehrbelastung der Frau durch die schwere Erkrankung des Partners die Situation verschärft. Im schlimmsten Fall können die körperlich belastenden Folgen der Hormonbehandlung mit der Enttäuschung oder Depression nach einer fehlgeschlagenen IVF und dem gleichzeitigen Verlust des Ehe- oder Lebenspartners zusammentreffen. Ebenso ist eine risikoreiche Mehrlingsschwangerschaft mit drohenden gesundheitlichen Schäden für Mutter und Kinder bei gleichzeitiger Trauer um den Partner denkbar. Derartige Situationen können das körperliche und geistige Wohl der Frau durchaus dauerhaft schädigen.

Ein intra-institutioneller Vorschlag aus den USA zu Richtlinien für eine postmortem Sperma-Entnahme berücksichtigt ausdrücklich die Notwendigkeit der Trauerarbeit und sieht nach dem Tode des Mannes bis zur Durchführung einer IVF eine einjährige Wartezeit für die Partnerin und potenzielle Mutter vor, um die Trauerphase und die psychische Anpassung möglich zu machen. Darüber hinaus sind ausführliche medizinische und psychologische Beratungen vorgesehen, sowie eine professionelle Einschätzung der psychischen, familiären und auch finanziellen Stabilität der betroffenen Frau. ${ }^{23}$

${ }^{21}$ Hepp, Höhergradige Mehrlingsschwangerschaft. Klinische und ethische Aspekte, Frauenarzt 2007, $440 \mathrm{ff}$.

22 Vgl. dazu die jüngst im Deutschen Ärzteblatt geführte Diskussion: Telus, Reproduktionsmedizin: Zwischen Trauma und Tabu, Arztebl. 2001, $3430 \mathrm{ff}$.

23 Tash/Applegarth/Kerr/Fins/Rosenwaks/Schlegel (Fußn. 3), 1923. 
Wobltun/Beneficence: Das Wohl für die Frau liegt in der Umsetzung ihres Kinderwunschs und der Hilfe, die gewünschte Schwangerschaft zu erreichen. Schwangerschaft als Wunsch oder auch als Ausdruck einer Lebenskonzeption lässt sich allerdings nur bedingt mit dem Wunsch nach der Genesung von einer Krankheit vergleichen. Ob Sterilität (bzw. Infertilität) eine im engen Sinne behandlungsbedürftige Krankheit ist, bleibt deshalb umstritten. ${ }^{24}$ Stöbel-Richter et al. zitieren psychologisch fundierte Analysen, die aufzeigen, dass ungewollte Kinderlosigkeit zu den schmerzhaftesten Lebensereignissen zählt. ${ }^{25}$ Zuweilen wird die IVF aber auch in die Nähe der „präferenzorientierten Medizin“ gestellt, da es zur Überwindung der Sterilität nur vor dem Hintergrund einer gewünschten Schwangerschaft eine medizinische Indikation gibt. ${ }^{26}$ Auf der anderen Seite ist der Kinderwunsch einer Frau nicht mit den Wunsch nach kosmetischer Chirurgie oder der Verbesserung der körperlichen Leistungsfähigkeit (Enhancement) gleichzusetzen, die u.U. ausschließlich mit lebensstilabhängigen Präferenzen korreliert.

Zum Wohl der Frau wird es aber auch gehören, der Bindung an den sterbenden Partner durch ein gemeinsames Kind Ausdruck zu verleihen. Damit schlägt sie für sich einen Bogen von ihrer vergangenen Lebensgeschichte in ihre zukünftige. Wie vorsichtig diese Versuche zu bewerten sind, bringen Tash et al. zum Ausdruck: „Sperm preservation could provide the false impression that the man will live on through his retrieved sperm and its fertility potential." ${ }^{\text {"27 }}$

Autonomie: Das Recht auf ein selbstbestimmtes Leben impliziert eine freie Lebens- und Familienplanung. Der Wunsch eines Paares nach einem leiblichen Kind und nach der Gründung einer Familie ist legitim. Wenn er sich im Falle der Sterilität bzw. Infertilität an die Medizin richtet, so ist er nicht zurück zuweisen. Welche Motive ein Paar dazu bewegen ein Kind zu wollen, ist in den meisten Fällen wohl kaum zu eruieren. Im Falle eines lebensbedrohend kranken Mannes sollte die Authentizität des Kinderwunschs jedoch erörtert werden. Wo könnten einschränkende Faktoren liegen?

Zunächst ist es denkbar, dass die betroffene Frau bis zum Zeitpunkt der Erkrankung ihres Partners keinen Kinderwunsch hatte und nun unter dem emotionalen Erleben seines drohenden Todes die Zeugung eines gemeinsamen Kindes als letzten Liebesakt empfindet. So sehr dies intuitiv nachvollziehbar ist, so sehr ist die Reproduktionsmedizin jedoch gehalten, der Frau gerade in dieser emotional äuBerst schwierigen Phase eine autonome - und das bedeutet auch authentische - Entscheidung überhaupt möglich zu machen.

\footnotetext{
${ }^{24}$ Vgl. hierzu Krones/Neuwobner/El Ansari/Wissner/Richter (Fußn. 2).

25 Stöbel-Ricbter/Weidner/Förster/Bräbler/Berth, Familiengründung in Deutschland. Wie geplant sind Kinderwunsch, Schwangerschaft und Kinderlosigkeit?, Gynäkologische Endokrinologie 2008, Online First.

${ }^{26}$ Die Bundesärztekammer spricht zwar voraussetzungslos von einer Indikation, gewährt dem Mediziner bzw. der Medizinerin jedoch gleichzeitig das Recht auf Zurückweisung der Durchführung des Verfahrens aus Gewissensgründen. Vgl. Bundesärztekammer (Fußn. 4), 1394 f.

${ }^{27}$ Tash/Applegarth/Kerr/Fins/Rosenwaks/Schlegel (Fußn. 3), 1924.
} 
Authentizität des Kinderwunsches läge auch dann nicht vor, wenn dieser einseitig vom Partner ausginge, und die Frau aufgrund seiner Gesundheitssituation von eigenen Wünschen absähe.

Schließlich ist der Einfluss durch Dritte nicht zu unterschätzen: Gerade Eltern eines todkranken Mannes können der Vorstellung anhängen, mit einem Enkelkind den Verlust des Sohnes überwinden zu können, und die Schwiegertochter zu einer Schwangerschaft drängen.

Zwar sind einschränkende Faktoren eines authentischen Kinderwunschs ebenso bei voll zeugungsfähigen Paaren denkbar, womit die Medizin jedoch nicht befasst wird. Anders sieht es aus, wenn der Wunsch nach Unterstützung der Fortpflanzung an sie herangetragen wird. Es ist nicht Aufgabe der Ärztinnen und Ärzte in die Privatheit einer Lebensbeziehung einzugreifen. Wenn von ihnen aber eine medizinisch-technische Intervention verlangt wird, dann haben sie das Recht, diese ggf. auch abzulehnen, wenn es hierfür gute Gründe gibt. ${ }^{28}$

Vor diesem Hintergrund wird klar, dass zur Prüfung, ob eine potenzielle Mutter auch die psychischen Voraussetzungen erfüllt und voraussichtlich der Belastung gewachsen sein wird, hinreichender Aufwand betrieben werden muss.

Allerdings sind Heteronomie und Autonomie oftmals schwer von einander abgrenzbar und zur Wahrung der reproduktiven Autonomie muss der Kinderwunsch einer Frau solange als authentisch unterstellt werden, bis berechtigte und schwerwiegende Zweifel oder Gegenbeweise vorliegen. Es bleibt der Grundsatz bestehen, dass nicht das Ausführen der legalen und legitimen Maßnahme moralisch zu rechtfertigen ist, sondern deren Ablehnung!

\subsection{Der Blick auf den Mann}

Nichtschaden/Nonmaleficence: Auch der Mann ist in der u.U. dramatischen Situation mit Blick auf seine individuellen Belastungen zu schützen. Schaden ist vor allem von ihm fernzuhalten, wenn erkennbar ist, dass sein konkretes physisches Leiden im Vordergrund steht und sein Wohlbefinden und seine Entscheidungs- und Handlungsbereitschaft erheblich einschränkt. Dennoch kann auch sein Kinderwunsch stark ausgeprägt sein und die Verhinderung seiner Fortpflanzung einen Schaden für ihn darstellen.

\footnotetext{
${ }^{28}$ Die (Muster-) Richtlinie der BÄK hält unter den „Berufsrechtlichen Voraussetzungen“ fest, dass „kein Arzt und keine Ärztin dazu verpflichtet werden“ kann, „entgegen ihrer/seine Gewissensüberzeugung Verfahren der assistierten Reproduktion durchzuführen“. Die BÄK gibt keine nähere Begründung für diese Freistellung des Arztes an, hat jedoch wohl die impliziten moralischen Kontroversen um die Verfahren im Auge; vgl. Bundesärstekammer (Fußn. 4), 1395. Vgl. auch Tash/Applegarth/Kerr/Fins/Rosenwaks/Schlegel (Fußn. 3), 1925, wonach sich ein Weigerungsrecht des Arztes auf mögliche negative Folgen bezieht. Demnach hat der Arzt das Recht von Post Mortem Sperm Retrieval Abstand zu nehmen, wenn er davon ausgehen muss, dass der Eingriff für die Betroffenen signifikant nachteilige Folgen haben wird. Analoges muss auch für eine Entscheidung angesichts des nahenden Todes gelten.
} 
Denkbar sind die folgenden Konstellationen:

(a) Ein Mann, der an einer malignen Erkrankung mit progredientem Verlauf leidet, gibt eine Samenspende für eine Schwangerschaft seiner Partnerin ab. Er selbst hofft dabei auf Genesung von seinem Leiden. Im Verlauf zeigt sich jedoch, dass die maligne Erkrankung nicht aufgehalten werden kann. In der terminalen Phase verlangt das Paar die künstliche Befruchtung.

(b) Ein Mann, der an einer malignen Erkrankung mit progredientem Verlauf leidet, entschließt sich zusammen mit seiner Partnerin während der terminalen Phase seiner Erkrankung, dass durch Hodenbiopsie Samen für eine IVF/ICSI gewonnen werden solle.

(c) Ein Mann befindet sich längere Zeit nach einem schweren Unfall im apallischen Syndrom. Seine Partnerin verlangt die Gewinnung von Samen durch eine Hodenbiopsie zum Zwecke einer IVF mit der Begründung, er habe vor seinem Unfall seinen Kinderwunsch zum Ausdruck gebracht.

Die Position des potenziellen Vaters ist im Fall (a) aus ethischer Perspektive unproblematisch. Da er bereits Samen gespendet hat, muss er keine weiteren Risiken tragen, die vor dem Hintergrund des Nichtschadensgebotes bedacht werden müssten.

Da Eltern als „reproduktive Einheit“ an die Reproduktionsmedizin herantreten, könnte man eine unterbleibende Schwangerschaft bzw. eine verhinderte Familiengründung als Schaden interpretieren. Es stellt sich also die Frage, ob das Prinzip des Nichtschadendürfens die Durchführung der assistierten Reproduktion verlangt bzw. ob sie durch das Fürsorgeprinzip geboten ist. Die Pflicht zum Wohltun gilt allerdings nicht ohne Einschränkungen, und hier muss zur Entscheidungsfindung offensichtlich die noch zu diskutierende Verantwortung der potenziellen Eltern hinzukommen.

Im Fall (b) ist die Sachlage insofern anders, als die Gewinnung des Samens ggf. nur noch durch eine Hodenbiopsie möglich ist. Da die Biopsie ein invasiver Eingriff ist, muss sie (im Sinne der IVF/ICSI) indiziert und durch den aktuellen Willen des Mannes autorisiert sein, was aufgrund seiner Einwilligungsfähigkeit möglich ist.

Der Fall (c) ist komplexer. Da kein Samen vorliegt, muss dieser über eine Hodenbiopsie gewonnen werden. Anders als beim Hirntod schiebt das Embryonenschutzgesetz hier keinen Riegel vor und die Authentizität des Kinderwunsches könnte zumindest über den mutmaßlichen Willen erkundet werden. Die Entnahme von Samen ist jedoch ein Eingriff in die körperliche Integrität, die nur nach vorheriger Einwilligung durchgeführt werden darf. Bei einem nicht einwilligungsfähigen Mann verbietet sich daher die Hodenbiopsie ebenso wie jeder andere Eingriff zur Samengewinnung aus ethischen wie rechtlichen Gründen. Rechtlich und ethisch wäre zu klären, ob eine schriftliche Vorausverfïgung, die explizit eine Einwilligung in 
Samengewinnung von Elternschaft gemeinsam mit der nachfragenden Frau, den Eingriff prinzipiell legitimieren könnte.

Wobltun/Beneficence: Ähnlich wie bei der Frau ist die Schwangerschaft mit der Aussicht auf ein gemeinsames Kind als Wohl des Mannes zu betrachten. Auch wenn er körperlich nicht involviert ist, hat er doch als Teil der elterlichen Gemeinschaft ein hoch zu bewertendes Interesse daran, eine Familie zu gründen. Dies muss grundsätzlich für alle dargestellten Fälle unterstellt werden.

Autonomie: Die Authentizität des Kinderwunschs des potenziellen Vaters spielt nur im Hinblick auf seine Vaterschaft, nicht jedoch im Hinblick auf die zu tragenden Risiken eine Rolle, da diese in erster Linie die Frau betreffen. In den Fällen (a) und (b) ist die Situation unzweifelhaft, da der Patient einwilligungsfähig ist. Im Fall (c) ist die Sachlage anders, da der Patient sich zu der anstehenden Entscheidung nicht mehr äußern kann.

Zudem liegt in Fall (c) kein unbestreitbares Indiz für einen Kinderwunsch (insbesondere mit der anfragenden Frau) vor. Möglicherweise würde der mutmaßliche Wille des potenziellen Vaters, sei es in Form einer Äußerung seiner Partnerin und/oder anderer naher Verwandter, er habe immer Kinder gewollt oder er habe bereits von der Möglichkeit einer IVF gesprochen, ausreichen, um eine autonome Entscheidung zu unterstellen. Für den invasiven Eingriff einer Hodenbiopsie und die in der Folge anzuerkennende Vaterschaft bedarf es aber zwingend einer expliziten Einwilligung des Betroffenen.

Schließlich darf nicht übersehen werden, dass eine autonome, authentische Entscheidung für oder gegen ein Kind auch bei dem potenziellen Vater durch erheblichen moralischen Druck von Seiten seiner Partnerin, der Familie oder des Freundeskreises unmöglich gemacht werden kann.

\subsection{Fragen der Gerechtigkeit}

Fragen der Gerechtigkeit waren bisher nicht Thema der vorliegenden Erörterung. Sie spielen im Bereich der Grundsatzdiskussionen jedoch eine wesentliche Rolle. Ist die IVF ein Verfahren, das von der Solidargesellschaft getragen werden muss oder nicht? Dies steht in engem Zusammenhang mit der Frage, ob es sich um eine Heilbehandlung handelt oder nicht. Trotz aller Kontroversen, die sich darum ranken, muss gefolgert werden, dass IVF/ICSI in den Katalog der gesetzlichen Krankenkassen aufgenommen sind. Wenn daher angesichts der hier skizzierten Problematik eines potenziellen Vaters mit infauster Prognose auf der Basis eines wohl durchdachten und begründeten Kinderwunsches die Entscheidung für eine reproduktionsmedizinische Therapie gefallen ist, dann müssen die entstehenden Kosten von der Solidargemeinschaft getragen werden. ${ }^{29}$

\footnotetext{
${ }^{29}$ Wie umstritten und problematisch die Finanzierungsfragen allerdings sind, belegt der Beitrag von Oliver Rauprich, Sollen Kinderwunschbehandlungen von den Krankenkassen finanziert werden? Ethische und rechtliche Aspekte, in diesem Band.
} 


\section{Perspektivenwechsel: Der Blick der potenziellen Eltern}

Die Analyse der ethischen Herausforderungen bei Kinderwunsch und gleichzeitiger maligner Erkrankung des Mannes an das Team eines reproduktionsmedizinischen Zentrums führt bei Beachtung der Prinzipien mittlerer Reichweite zu dem Ergebnis, dass es keine grundsätzlichen moralischen Einwände gibt, eine IVF auch dann durch zu führen, wenn der potenzielle Vater die Geburt des Kindes wahrscheinlich nicht erleben wird. Nimmt man die Anforderungen an die Reproduktionsmedizin ernst, so muss Schaden für das Kind vermieden werden. Eine verantwortungsvolle Befassung des reproduktionsmedizinischen Teams mit dem zu erzeugenden Kind zeigt, dass das so stark herausgehobene Wohl des Kindes zwar Beachtung finden muss, im Falle eines noch nicht gezeugten jedoch zu viele vage und spekulative Voraussetzungen beinhaltet, um daraus generelle Regeln für gebotene oder verbotene Maßnahmen abzuleiten. Der Mann hat als Teil der „reproduktiven Gemeinschaft" das Recht auf Fortpflanzung, daran ändert auch sein gesundheitlicher Zustand nichts, Einschränkungen ergeben sich bei Nichteinwilligungsfähigkeit. Die eigentlich wichtige Person ist jedoch die potenzielle Mutter. Auch von ihr muss Schaden ferngehalten werden. Eine etwaige physische und psychische Belastung kann so groß sein, dass die Durchführung der IVF/ICSI aus moralischen Gründen nicht zu rechtfertigen wäre. Gleichzeitig müssen jedoch die Gründe für eine Ablehnung hinreichend gut sein, damit die elterliche Autonomie mit ihrem legitimen Kinderwunsch nicht verletzt wird.

Kehrt man noch einmal an die entscheidende Ausgangsprämisse zurück, so dient die Reflexion der ethischen Fragen der unabdingbaren professionellen Beratung des Kinderwunsch-Paares, um angesichts einer spezifischen Problematik zu einer gemeinsamen und tragfähigen Entscheidung zu finden.

Die herausragende Bedeutung der elterlichen reproduktiven Autonomie ist zwingend verbunden mit der Frage der Eltern nach ihrer persönlichen Verantwortung für ein zu zeugendes Kind. ${ }^{30}$ Die geteilte Entscheidungsfindung der Beratenden und Behandelnden mit den Eltern kommt nicht ohne den Perspektivenwechsel zu den potenziellen Eltern aus. Dies darf nicht mit einem Verschieben der Verantwortung auf das Paar verwechselt werden. Es geht vielmehr darum, dass die potenziellen Eltern letztendlich die Verantwortung für ihre Elternschaft werden übernehmen wollen. Sie selbst müssen einschätzen oder einzuschätzen lernen, welche physischen und psychischen Ressourcen und Bewältigungsstrategien ihnen zur Verfügung stehen. Auch die in diesem Beitrag angesprochenen väterlichen Konstellationen sind moralisch nicht ,gut“ zu entscheiden, ohne die gemeinsame Verantwortung für das Wohl der zukünftigen Mutter und ihres Kindes unter den Bedingungen zu reflektieren, dass der Partner versterben oder bereits die Frau und das Kind zurückgelassen haben wird.

\footnotetext{
${ }^{30}$ Vgl. hierzu den Beitrag Wiesemann, Der Embryo im Kontext: Warum die Biopolitik die menschlichen Beziehungen nicht vergessen darf, in diesem Band; sowie Wiesemann, Von der Verantwortung, ein Kind zu bekommen. Eine Ethik der Elternschaft, 2006.
} 
Den vielleicht zukünftigen Eltern wird es ein besonderes Anliegen sein, dass das zu zeugende Kind eine glückliche Entwicklung nehmen kann. Sie werden sich fragen müssen, ob ihr Kind vaterlos aufwachsen soll. Zweifellos wird die Mutter in einem solchen Fall ein sehr positives, möglicherweise idealisiertes Bild des verstorbenen Vaters vermitteln und dem Kind eine positive Bindung an den unbekannten Vater ermöglichen. Zudem haben die Entwicklungspotenziale eines Kindes hin zu einem psychisch ausgeglichenen, bindungs- und liebensfähigen Erwachsenen eine außerordentlich große Bandbreite und sind bei weitem nicht primär von der Form der Familienbildung abhängig.

Dennoch werden die potenziellen Eltern mit der Frage konfrontiert sein, ob sie einem Kind durch die Zeugung Schaden zufügen und dies gegebenenfalls verantworten und kompensieren können. Die Frage, ob es ein Schaden sein kann gezeugt zu sein, hat eine umfangreiche und kontroverse philosophische Debatte hervorgerufen. Da der Akt des möglichen Schadens zugleich der Akt des Wohltuns ist, nämlich das Leben zu ermöglichen, scheiden sich die Geister bei der Beantwortung. ${ }^{31}$ Nicht von der Hand zu weisen ist jedoch, dass Eltern ihren Kindern durch egoistische oder leichtfertige Entscheidungen und Handlungen oder bewusst gegen besseres Wissen erhebliches Unrecht zufügen können. Auch die begründete Mutmaßung, dass Menschen leben wollen und Leben der Nichtexistenz vorziehen, kann die individuelle verantwortete Entscheidung der Eltern für ein Ja zu einem Kind nicht ersetzen und die Auseinandersetzung mit der eigenen Lebenswirklichkeit nicht vermeiden.

Das Erleben von Krankheit, Sterbenmüssen und dem Wunsch nach einem gemeinsamen Kind ist Teil der unverwechselbaren Lebensgeschichte des betroffenen Paares. Die Menschen im Team der Reproduktionsmedizin sind aufgefordert, die Verantwortung dafür zu übernehmen, dass die Entscheidung des Paares nicht zu einem Trauma in ihrer Geschichte wird. Dazu dient die sorgfältige Befassung mit dem individuellen Fall. Darüber hinaus muss das Paar die Chance und Hilfe bekommen, durch eine professionelle medizinische und psychosoziale Beratung autonom und in Eigenverantwortung eine Entscheidung treffen zu können.

\section{Der Frankfurter Arbeitsbogen für ein Entscheidungsverfahren zur IVF/ICSI bei Männern mit progredienten, potenziell lebensbedrohlichen Erkrankungen}

Um der Komplexität der Situation gerecht zu werden, wurde durch eine Arbeitsgruppe des Klinischen Ethik-Komitees des Universitätsklinikums Frankfurt am Main ein Verfahrensbogen entwickelt, der die systematische Reflexion erleichtern und den Prozess transparent gestalten soll. Um vor allem der psychosozialen Be-

31 Vgl. dazu die zahreichen von Strong analysierten philosophischen Beiträge und die dort ausgearbeitete Kritik, Strong, Harming by conceiving: A review of misconceptions and a new analysis, Journal of Medicine and Philosophy 2005/30, $491 \mathrm{ff}$. 
ratung einen erkennbar hohen Stellenwert zu geben, ist ausdrücklich eine psychologische Beratung mit einer entsprechenden Dokumentation vorgesehen. Der Frankfurter Bogen geht derzeit in die Praxis und wird nach einem gewissen Zeitraum der Erprobung überprüft und gegebenenfalls fortgeschrieben werden. Die Diskussion der speziellen Problematik hat gezeigt, dass die Einbindung eines Klinischen Ethik-Komitees hilfreich ist, um zu reflektierten und gemeinsam zu tragenden Entscheidungs- und Handlungsabläufen zu kommen.

\section{Arbeitsbogen für ein Entscheidungsverfahren zur IVF/ICSI bei Männern mit progredienten, potenziell lebensbedrohlichen Erkrankungen}

\section{Medizinische Problematik}

Sind alle medizinischen Fragen in Bezug auf die Grunderkrankung des Mannes geklärt?

ja nein

Gibt es medizinische Ausschlusskriterien in Bezug auf die Frau?

ja nein

2. Ist das Paar über die Risiken und Erfolgsaussichten des Verfahrens hinreichend aufgeklärt?

ja nein

Dokumentierte Gespräche:

\section{Authentizität des Kinderwunsches}

Worin besteht die Motivation zur Mutterschaft?

Worin besteht die Motivation zur Vaterschaft?

Seit wann besteht der Kinderwunsch?** 
Herrscht Einigkeit zwischen den Eheleuten/Lebenspartnern?

$$
\text { ja nein }
$$

Nehmen die Herkunftsfamilien Einfluss auf den Kinderwunsch des Paares? In welcher Weise?

Welche Rolle spielen gesellschaftliche Erwartungen?

Ist der Kinderwunsch des Paares insgesamt als authentisch einzustufen?*

Mutter:

ja nein

Vater:

ja nein

\section{Psychische Stabilität der Mutter*}

Siehe psychologisches Gutachten

erstellt am

durch

5. Bei Nichteinwilligungsfähig des erkrankten Partners: Gibt es klare Hinweise, die auf die Bereitschaft zur Vaterschaft hindeuten?

Liegt bereits eine Samenspende vor?*

$$
\text { ja nein }
$$

Wurde der kranke Partner wegen des Kinderwunsches bereits durch Mitarbeiter des Klinikums beraten?

$$
\text { ja nein }
$$

Übereinstimmende Angaben der Partnerin und der Angehörigen?

ja nein

\section{Soziales Umfeld**}

Gibt es während der Behandlung und nach der Geburt eines Kindes Unterstützung durch die Familie oder den Freundeskreis? 
Sind die Wohnverhältnisse zufrieden stellend?

Sind die finanziellen Verhältnisse zufrieden stellend?

\section{Sonstiges}

Ist eine Beratung des Behandlungsteams und/ oder der Betroffenen durch Mitglieder des Klinischen Ethik-Komitees notwendig?
ja
nein

Begründung:

- Die mit * gekennzeichneten Aspekte sind ggf. Ausschlusskriterien, die mit ** gekennzeichneten Aspekte sind keine Ausschlusskriterien 



\title{
Lesben mit Kinderwunsch: Eine ethische Herausforderung für die Reproduktionsmedizin?
}

\author{
Lisa Herrmann-Green
}

Der Traum von einem Leben mit Kindern wird heute nicht nur von heterosexuellen Paaren geträumt, sondern auch von gleichgeschlechtlichen Paaren geteilt. Zunehmend entscheiden sich lesbische Paare bewusst für die Familiengründung und Kindererziehung. Obwohl lesbische Familien häufig als neues Phänomen betrachtet werden, ist die Existenz lesbischer Mütter nicht neu. Tatsächlich gab es schon immer lesbische Mütter, die ihre Kinder in heterosexuellen Lebensphasen, in der Regel einer Ehe, bekommen haben. ${ }^{1}$ Neu ist allerdings, dass mehr und mehr Lesben sich in ihrer lesbischen Beziehung oder alleine für Kinder entscheiden. Inzwischen werden so viele Lesben Eltern, dass einige US Autoren von einem „lesbischen Babyboom“ oder einem „Gaybyboom“ sprechen. ${ }^{2}$

\footnotetext{
${ }^{1}$ Epstein, Breaking with Tradition, Healthsharing 1993/14, 18 ff.; Falk, Lesbian Mothers: Psychosocial Assumptions in Family Law, American Psychologist 1989, 941 ff.; Jacob, Concerns of single women and lesbian couples considering conception through assisted reproduction, in: Leiblum (ed.), Infertility: Psychological issues and counseling strategies, 1997, pp. $189 \mathrm{ff}$.

2 Patterson, Children of the lesbian baby boom: Behavioral adjustment, self-concepts, and sex role identity, in Greene/Herek (eds.), Psychological perspectives on lesbian and gay issues: vol. 1, Lesbian and gay psychology: Theory, research, and clinical applications, 1994, pp. 156 ff.; Pies, Considering parenthood, 2. edn. 1988.
} 
In den letzten 20 Jahren haben immer mehr lesbische Paare Familien durch Adoption, Pflegeelternschaft oder, am häufigsten, durch Schwangerschaft gegründet. In diesen Fällen spricht man von einer geplanten lesbischen Familie, da das lesbische Paar eine bewusste Entscheidung für Kinder trifft. Es gibt verschiedene Möglichkeiten für Lesben schwanger zu werden: durch donogene Insemination (DI) mit Sperma von einer Samenbank (unbekannte Spender) oder einem privaten Spender (bekannte Spender) oder, was seltener geschieht, durch heterosexuellen Beischlaf. Samenbanken bieten bis zu zwei Arten von unbekannten Spendern an: den anonymen Spender (auch Nein-Spender genannt), dessen Identität anonym bleibt, und den identity-release Spender (auch Ja-Spender genannt), dessen Identität für das Kind unter bestimmten Umständen, z.B. mit 18 Jahren, evtl. erfahrbar wird. Das Kind wird in all diesen Konstellationen in eine Familie hineingeboren, in der sich beide Mütter von Anfang an als Lesben identifizieren. Die austragende Mutter ist die Geburtsmutter des Kindes und die nicht-leibliche Mutter, die zur Herkunftsfamilie des Kindes gehört, ist die soziale Mutter des Kindes. ${ }^{3}$ Diese geplanten lesbischen Familien mit Kindern durch DI (fortan als lesbische Inseminationsfamilien oder LDI Familien benannt; die Kinder aus LDI Familien werden LDI Kinder benannt) sind charakterisiert durch die lesbische Identität der Mütter, ein hohes Maß an Planung der Elternschaft und in vielen Fällen durch die Abwesenheit des biologischen Erzeugers und eines sozialen Vaters. ${ }^{4}$ Dieser Trend war zuerst in skandinavischen Ländern und den USA zu beobachten. Er boomt inzwischen auch in Deutschland. Somit erhöht sich die Wahrscheinlichkeit, dass Reproduktionsmediziner in Deutschland eine Anfrage über eine Behandlung mit DI von einem lesbischen Paar erhalten könnten.

Stellt die Behandlung von lesbischen Frauen(-paaren) eine ethische Herausforderung für die Reproduktionsmedizin dar? Diese Frage wird im vorliegenden Papier anhand der bisherigen psychosozialen Forschungsergebnisse diskutiert. Im ersten Teil werden gesellschaftliche Entwicklungen und Forschungsergebnisse hinsichtlich der Auswirkungen der Hauptmerkmale einer LDI Familie vorgestellt. Im zweiten Teil werden drei Aspekte der LDI Familie näher beleuchtet, nämlich, die Motivation Mutter zu werden, die Wahl des Spenders und die alltägliche Realität der LDI Familie.

\footnotetext{
${ }^{3}$ In Abgrenzung dazu wird im Fall einer neuen Partnerin der Geburtsmutter, die später hinzukommt und den Prozess der Patchwork-Familienbildung mitmacht, diese Mutter als Co-Mutter bezeichnet.

${ }^{4}$ Golombok/Brewaeys/Cook/Giavazzi/Guerra/Tovani/v.Hall/Crosignani/Dexeus, The European study of assisted reproduction families: Family functioning and child development, Human Reproduction 1996/11, $2324 \mathrm{ff}$.
} 


\section{Die Sicht der Gesellschaft}

Ein wichtiger Schritt zur Beendigung der Pathologisierung lesbischer und schwuler sexueller Orientierung bestand darin, dass 1973 die American Psychiatric Association Homosexualität in ihrem „Diagnostic and Statistical Manual“ (DSM) von der Liste psychiatrischer Erkrankungen strich. Im Jahr 1993 wurde Homosexualität schließlich auch aus der International Classification of Diseases (ICD) der Weltgesundheitsorganisation (WHO) entfernt. Heute wird in Fachkreisen eine homosexuelle Orientierung als eine normale Variante des menschlichen Sexualverhaltens angesehen und interpretiert. ${ }^{5}$

Trotzdem erwiesen sich die Stigmatisierung und die Vorurteile gegenüber Lesben und Schwulen als nachhaltig. Bis heute erleben sie rechtliche Diskriminierungen und schwere Menschenrechtsverletzungen in den meisten Ländern der Welt (Amnesty International). ${ }^{6}$ Die westeuropäischen und skandinavischen Nationen gehören jedoch zu den tolerantesten Ländern, wobei die völlige Gleichstellung von Homosexuellen und Heterosexuellen nur in Belgien, Holland, Kanada, Spanien und Norwegen gegeben ist. ${ }^{7}$ In Deutschland wurde 2001 mit der eingetragenen Lebenspartnerschaft eine Möglichkeit der Institutionalisierung der lesbischen und schwulen Paarbeziehung mit allen Pflichten und einigen Rechten einer Ehe geschaffen. Im Januar 2005 wurden die Rechte von eingetragenen Lebenspartnern 8 um einige wichtige Rechte erweitert. Es gibt seitdem die Möglichkeit z.B. in LDI Familien, das durch die Lebenspartnerin ausgetragene Kind durch die soziale Mutter zu adoptieren, so dass die soziale Eltern-Kind Beziehung auch rechtlich begründet werden kann. Der positive Einfluss der Stiefkindadoption auf das Gefühl von Sicherheit für die soziale Mutter ist gut dokumentiert; ${ }^{9}$ im Falle einer Trennung erhöht sie auch die Wahrscheinlichkeit für ein gemeinsames Sorgerecht und stellt die Kontinuität der Kind-soziale Mutter-Bindung sicher. ${ }^{10}$

Bedenken hinsichtlich der Tauglichkeit einer Familie mit lesbischen Eltern betreffen die hervorstehenden Merkmale dieser Familienform: die lesbische Orientierung der Mutter und die (soziale) Vaterlosigkeit der Kinder. Diese zwei Variablen werfen folgende gesellschaftliche Fragen auf: Haben lesbische Frauen diesel-

\footnotetext{
${ }^{5}$ Dresher, http://www.community-muenchen.de/drescher.htm (letzter Zugriff am 26.8.2008). ${ }^{6} \mathrm{http}: / /$ www2.amnesty.de/internet/deall.nsf/3c7abab8e052c42fc1256eeb004ce861/54a9779793f11d 8ac12571620050b580? (Open Document, letzter Zugriff am 26.8.2008).

${ }^{7}$ Die Institution „Ehe“ steht sowohl heterosexuellen wie homosexuellen Paaren offen.

8 „(Eingetragene) Lebenspartner“ ist die Bezeichnung für lesbische und schwule Paare, die in eingetragener Lebenspartnerschaft leben, analog „Ehepartner“ für gegengeschlechtliche Paare, die verheiratet sind.

${ }_{9}$ Gartrell/Hamilton/Banks/Hamilton/Reed/Sparks/Bishop/Rodas, The national lesbian family study: 2. Interviews with mothers of toddlers, American Journal of Orthopsychiatry 1999, 362 ff.; McClellan, The "other" mother and second parent adoption, Journal of Gay \& Lesbian Social Services 2001/13, $1 \mathrm{ff}$.

10 Gartrell/Banks/Reed/Hamilton/Rodas/Deck, The national lesbian family study: 3. Interviews with mothers of five-year-olds, American Journal of Orthopsychiatry 2000, $542 \mathrm{ff}$.
} 
ben mütterlichen Fähigkeiten wie heterosexuelle Frauen? Haben die sexuelle Orientierung der Mütter und die vaterlose Familienstruktur eine negative Auswirkung auf die Kindesentwicklung? Erleiden die Kinder Diskriminierung? Werden sie stigmatisiert und gehänselt? Wie stellt sich die gesellschaftliche (Be-) Deutung einer Frauen-definierten Familienform dar?

Die erste psychologische Forschung zu lesbischer Mutterschaft wurde in den 1970er Jahren durch Sorgerechtskämpfe von verheirateten Lesben bei der Scheidung angeregt. Wenn die neue sexuelle Orientierung der Mutter in der Gerichtsverhandlung thematisiert wurde, wurde ihr das Kind mit hoher Wahrscheinlichkeit nicht zugesprochen. Dies stand in starkem Kontrast zu Fällen mit heterosexuellen Müttern, die fast ausschließlich das Sorgerecht für ihr Kind behielten. ${ }^{11}$ Es galt, die existierenden Vorurteile gegen lesbische Mütter empirisch zu überprüfen. Seit Anfang der 1990er wurden auch geplante lesbische Familien untersucht, da sie nicht von eventuell konfundierenden Faktoren betroffen sind, wie z.B. dem Aufwachsen in jüngster Kindheit mit gegengeschlechtlichen Eltern, dem Coming-out Prozess der Mutter, einer Scheidung und dem oft damit verbundenen sozioökonomischen Abstieg. Bei diesen Studien wurden die lesbischen und heterosexuellen Mütter hinsichtlich psychologischer Variablen, z.B. Mütterlichkeit und psychische Stabilität und Aspekte der Elternschaft, miteinander verglichen. Die Kinder lesbischer und heterosexueller Mütter wurden hinsichtlich ihrer sozialen, kognitiven und psychosexuellen Entwicklung sowie ihrer psychischen Gesundheit miteinander verglichen. Die Sorge war, dass Lesben sich als psychisch instabil und als Mutter ungeeignet erweisen würden, und dass ihre Kinder so sehr unter der Stigmatisierung ihrer Mütter zu leiden hätten, dass sie soziale Ausgrenzung erfahren würden, was wiederum ihre sozio-emotionale Entwicklung stark beeinträchtigen würde. ${ }^{12}$ Das nicht Vorhandensein einer männlichen Identifikationsfigur sollte die psychosexuelle Entwicklung vor allem von Jungen stören, so dass sie häufiger schwul werden würden. ${ }^{13}$

Was sagt die Forschung dazu?

Kritik an den unten aufgeführten Ergebnissen liegt im Bereich der Generalisierbarkeit, die aufgrund der Stichprobengewinnung und -zusammensetzung noch eingeschränkt ist. Die Gesamtgröße der Population der Menschen, die als LDI Familien zu kategorisieren sind, ist unbekannt. Es liegen nur Schätzungen vor, wonach von einer hohen Dunkelziffer auszugehen ist. Die Forschung in diesem Bereich steht daher bezüglich der Stichprobengewinnung großen methodischen

\footnotetext{
11 Golombok, Research on gay and lesbian parenting: A historical perspective across 30 years, in: Tasker/Bigner (eds.), Gay and Lesbian Parenting: New Directions, 2008.

12 Brewaeys/Ponjaert/v.Hall/Golombok, Donor insemination: Child development and family functioning in lesbian mother families, Human Reproduction 1997/12, 1349 ff.

${ }^{13}$ Die Beurteilung der Tauglichkeit lesbischer Eltern anhand ihrer Fähigkeit, heterosexuelle Kinder zu produzieren, ist natürlich heterosexistisch behaftet, diskriminierend und somit an sich in Frage zu stellen. Golombok/Tasker, Do parents influence the sexual orientation of their children? Findings from a longitudinal study of lesbian families, Developmental Psychology 1996/32, 3 ff.; Tasker/Golombok, Growing up in a lesbian family, 1997.
} 
Schwierigkeiten gegenüber. Sie ist oft auf die freiwillige Bereitschaft an einer Studie teilzunehmen angewiesen. Die Studienteilnehmerinnen werden häufig durch die Schneeball-Technik und durch Werbung in einschlägigen Publikationen gewonnen. Daher könnte es sein, dass die Stichprobe stärker durch besonders selbstbewusste und mit der Szene vertraute Lesben vertreten wird. Es ist nicht auszuschließen, dass Familien, die versteckt leben, andere Ergebnisse erzielen würden bzw. dass Familien, die weniger gut funktionieren, nicht bereit sind, an Forschungen teilzunehmen. Die andere Methode, um Teilnehmerinnen für Studien zu gewinnen, ist Klientinnen an Samenbanken oder reproduktionsmedizinischen Kliniken zu rekrutieren. Die Repräsentativität ist dadurch auch nicht gewährleistet, da viele Lesben mit der „Becher-Methode“ selbst inseminieren. Zudem ist es nur in den Ländern anwendbar, in denen Lesben offenen Zugang zu Samenbanken und Kliniken haben. Ein weiterer Punkt ist die Homogenität der LDI Stichproben. Die LDI Stichproben aus der internationalen Forschung (z.B. Neuseeland, USA, Kanada, Belgien, die Niederlande, Großbritannien und Deutschland) bestehen überwiegend aus weißen, hoch ausgebildeten Lesben mit hohem sozio-ökonomischem Status. Obwohl manche Autoren ihre Stichproben durch Fertilitätszentren gewinnen konnten, um eine eventuelle Verzerrung der Ergebnisse zu vermeiden und Golombok und Kollegen und Wainright und Kollegen eine fast repräsentative Stichprobe durch die Anbindung an nationale Erhebungen erreichen konnten, teilten ihre Stichproben ebenfalls diese Charakteristika. ${ }^{14}$ Green postuliert, dass die gesellschaftliche und institutionalisierte Privilegierung von heterosexuellen Paaren in Hinblick auf den Zugang zur DI vorselektiert, so dass es möglich ist, dass nur Personen mit besonders intensivem Kinderwunsch und den nötigen emotionalen und finanziellen Ressourcen überhaupt erfolgreiche LDI Eltern werden können. ${ }^{15}$ Diese Sicht stellt die evtl. Möglichkeit in den Raum, dass obwohl ethnische und sozioökonomisch gemischte Stichproben nicht erzielt werden, die Stichproben trotzdem für LDI Mütter repräsentativ sein könnten.

\subsection{Lesbische Mütter}

Die Ergebnisse der Forschung hinsichtlich lesbischer Mütter sind konsistent: Die oben genannten Vorbehalte gegen lesbische Mütter wurden wiederholt als Vorurteile entlarvt. ${ }^{16}$ Trotzdem wurden auch Unterschiede festgestellt. In manchen Stu-

${ }^{14}$ Golombok/Perry/Burston/Murray/Mooney-Somers/Stevens, Children with lesbian parents: A community study, Developmental Psychology 2003/39, 20 ff.; Wainright/Patterson, Delinquency, victimization and substance use among adolescents with female same-sex parents, Journal of Family Psychology 2006, $526 \mathrm{ff}$.

${ }^{15}$ Green, Unconventional conceptions: Family planning in lesbian-headed families created by donor insemination, 2006.

16 Bos/v.Balen/v.d.Boom, Lesbian families and family functioning, Patient Education and Counseling 2005/59, 263 ff.; Bos/v.Balen/v.d.Boom, Experience of parenthood, couple relationship, social support, and child-rearing goals in planned lesbian mother families, Journal of Child psychology and Psychiatry 2004, 755 ff.; Bos/v.Balen/v.d.Boom, Planned lesbian families: Their desire and motivation to have 
dien schneiden lesbische Paare hinsichtlich ihres Erziehungsverhaltens und ihrer Erziehungsfähigkeiten besser ab als heterosexuelle Paare. Die Autoren räumen ein, dass dieser Unterschied auf das schlechtere Ergebnis von Vätern aus Erhebungen von Erziehungsfähigkeit zurückzuführen ist, so dass vermutet wird, dass das Geschlecht und nicht die sexuelle Orientierung eine Rolle spielt. ${ }^{17}$ Ein weiterer Unterschied zwischen den beiden Lebensformen betrifft die Arbeitsaufteilung: lesbische Eltern teilen sich die Haushalts- und Erziehungsarbeit egalitärer auf als heterosexuelle Eltern, ${ }^{18}$ und lesbische soziale Mütter ${ }^{19}$ sind involvierter in der Kindeserziehung als Väter. ${ }^{20}$ Die partnerschaftliche Zufriedenheit von Lesbenpaaren mit Kindern ist gut und steht in einem positiven Zusammenhang mit einer egalitären Arbeitsaufteilung. ${ }^{21}$

Neuere Forschungsarbeiten beschäftigten sich u.a. mit der Erhebung von realistischen Profilen von LDI Müttern. ${ }^{22}$ Die Homogenität von LDI Mütter-

children, Human Reproduction 2003/18, 2216 ff.; Chan/Raboy/Patterson, Psychosocial adjustment among children conceived via donor insemination by lesbian and heterosexual mothers, Child Development 1998, 443 ff.; Golombok/Tasker/Murray, Children raised in fatherless families from infancy: Family relationships and the socioemotional development of children of lesbian and single mothers, Journal of Child Psychology and Psychiatry 1997, 783 ff.; Golombok/Perry/Burston/Murray/MooneySomers/Stevens (Fußn. 14); Shelley-Sireci/Ciano-Boyce, How did they become parents? Becoming and being lesbian adoptive and birth parents. Paper presented at the American Psychological Association Annual Convention, Boston, 1999; Siegenthaler/Bigner, The value of children to lesbian and nonlesbian mothers, Journal of Homosexuality 2000/39, 73 ff.; Patterson, Families of the lesbian baby boom: Maternal mental health and child adjustment, Journal of Gay and Lesbian Psychotherapy 2001/4, $91 \mathrm{ff}$.

$17 \mathrm{Bos} / v$.Balen/v.d.Boom, Child adjustment and parenting in planned lesbian-parent families, American Journal of Orthopsychiatry 2007, 38 ff.; Flaks/Ficher/Masterpasqua/Joseph, Lesbians choosing motherhood: A comparative study of lesbians and heterosexual parents and their children, Developmental Psychology 1995/31, $105 \mathrm{ff}$.

18 Bos/v.Balen/v.d.Boom (Fußn. 16); Brewaeys/Ponjaert/v.Hall/Golombok (Fußn. 12); Ciano-Boyce/ShellySireci, Who is mommy tonight? Lesbian parenting issues, Journal of Homosexuality 2002/43, 1 ff.; Shelley-Sireci/Ciano-Boyce (Fußn. 16); Tasker/Golombok (Fußn. 13).

${ }^{19}$ Eine lesbische soziale Mutter bezeichnet eine Frau, die gemeinsam mit der leiblichen Mutter das Kind bereits geplant hat. Sie ist also der nicht-gebärende Elternteil analog zu einem Vater in einer heterosexuellen Beziehung. Bos/v.Balen/v.d.Boom (Fußn. 16); Brewaeys/Ponjaert/v.Hall/Golombok (Fußn. 12); Ciano-Boyce/Shelly-Sireci (Fußn. 18); Shelley-Sireci/Ciano-Boyce (Fußn. 16); Tasker/Golombok (Fußn. 13).

20 Bos/v.Balen/v.d.Boom (Fußn. 16); Brewaeys/Ponjaert/v.Hall/Golombok (Fußn. 12); Patterson, Lesbian mothers and their children, in: Laird/Green (eds.), Lesbians and Gays in Couples and Families: A Handbook for Therapists, 1996, pp. $420 \mathrm{ff}$.

${ }^{21}$ Herrmann-Green/Gehring, The German lesbian family study: Planning for parenthood via donor insemination [Special Issue], Journal of Gay, Lesbian, Bisexual and Transgender Family Studies 2007/3, 351 ff.; Herrmann-Green/Gehring, The German lesbian family study: Planning for parenthood via donor insemination, in: Tasker/Bigner (eds.), Gay and Lesbian Parenting: New Directions, 2008, pp. 351 ff.; Krïger-Lebus/Rauchfleisch, Zufriedenheit von Frauen in gleichgeschlechtlichen Partnerschaften mit und ohne Kinder. [Level of contentment in same sex relationships with and without children.], System Familie 1999, 74 ff.; Bos/v.Balen/v.d.Boom (Fußn. 16); Patterson, Families of the lesbian baby boom: Parent's division of labor and children's adjustment, Developmental Psychology 1995/31, 115 ff.; Patterson (Fußn. 20).

22 Gartrell/Hamilton/Banks/Mosbacher/Reed/Sparks/Bishop, The national lesbian family study: 1. Interviews with prospective mothers, American Journal of Orthopsychiatry 1996, 272 ff.; Green (Fußn. 15); Herrmann-Green/Gehring (Fußn. 21). 
Stichproben über Studien in verschiedenen Ländern ist beeindruckend. ${ }^{23}$ LDI Mütter sind meist spät gebärend und haben einen hohen sozio-ökonomischen Status, d.h. sie sind überwiegend Hochschulabgängerinnen mit meist professionellen Anstellungen. ${ }^{24}$ Sie zeichnen sich durch eine solide, positive lesbische Identität aus. ${ }^{25}$ Sie gehen in ihrer Umwelt offen mit ihrer Identität um, weisen niedrige internalisierte Homophobiewerte auf und möchten ihre Kinder unterstützen, unabhängig davon welche sexuelle Orientierung sie später entwickeln. ${ }^{26}$ Sie suchen primär soziale Kontexte auf, die positiv auf sie reagieren und berichten von großer sozialer Unterstützung seitens der Freunde, Herkunftsfamilien und anderer Regenbogenfamilien, ${ }^{27}$ d.h. Familien mit schwul-lesbischen Eltern. ${ }^{28}$ Sie verfügen über effektive Coping-Strategien für den Umgang mit der heterosexuellen Umwelt, und es ist davon auszugehen, dass sie diese auch an ihre Kinder weitergeben werden. ${ }^{29}$

\subsection{Kindesentwicklung}

Die Forschung über Kinder von lesbischen Müttern untersuchte sowohl Kinder, die in einer heterosexuellen Lebensphase ihrer Mütter, z.B. Ehe, geboren wurden, wie auch LDI Kinder. Die LDI Kinder wurden mit (Beischlaf-) Kindern, DI Kindern und Adoptivkindern mit heterosexuellen Eltern und mit Adoptivkindern mit lesbischen Eltern ${ }^{30}$ hinsichtlich ihrer sozio-emotionalen Entwicklung bzw. psychi-

\footnotetext{
23 Green (Fußn. 15).

${ }^{24}$ Baetens, Councelling lesbian couples: requests for donor insemination on social grounds, Reproductive BioMedicine Online 2002/6, 75 ff.; Gartrell/Hamilton/Banks/Mosbacher/Reed/Sparks/Bishop (Fußn. 22).

${ }^{25}$ Gartrell/Hamilton/Banks/Mosbacher/Reed/Sparks/Bishop (Fußn. 22).

26 Baetens (Fußn. 24); Gartrell/Hamilton/Banks/Hamilton/Reed/Sparks/Bishop/Rodas (Fußn. 9); Herrmann-Green/Gebring, in: Tasker/Bigner (Fußn. 21); Gartrell/Banks/Reed/Hamilton/Rodas/Deck (Fußn. 10).

27 Regenbogenfamilie ist ein allgemeiner Begriff, der alle Familienformen mit mindestens einem lesbischen oder schwulen Elternteil erfasst, unabhängig von der Art der Familienbildung und unabhängig davon, wie die Kinder in die Familie aufgenommen worden sind.

${ }^{28}$ Gartrell/Hamilton/Banks/Hamilton/Reed/Sparks/Bishop/Rodas (Fußn. 9); Gartrell/Banks/Reed/Hamilton/Rodas/Deck (Fußn. 10); Gartrell/Hamilton/Banks/Mosbacher/Reed/Sparks/Bishop (Fußn. 22).

${ }^{29}$ Green (Fußn. 15).

30 Die Ausführlichkeit der Vergleichsgruppen hängt mit der methodischen Schwierigkeit zusammen, eine geeignete Kontrollgruppe für LDI Kinder zu finden. Jede Kontrollgruppe unterscheidet sich von LDI Familien hinsichtlich mehrerer Merkmale - [1] der sexuellen Orientierung der Eltern, [2] dem damit verbundenen soziokulturellen Kontext, in dem die Familie lebt, z.B. mit/ohne Stigmatisierung, rechtliche Benachteiligung, etc., [3] der Art und Weise, wie das Kind in die Familie kam, z.B. Geschlechtsverkehr, DI oder Adoption, [4] den damit verbundenen Herausforderungen oder Themen für die Eltern, z.B. soziale Erwartungen erfüllen für Eltern nach spontaner Zeugung, Infertilität für DI und Adoptiveltern, [5] den damit verbundenen Herausforderungen oder Themen für das Kind, z.B. „Verlassen werden“ für Adoptivkinder, „Gewünschtheit“ für DI Kinder, [6] dem Vorhandsein bzw. der Anzahl der dem Kind unbekannten genetischen Elternteile. Sollten gravierende Unterschiede festgestellt werden, wäre es aufgrund der Konfundierungen nicht möglich, sie allein auf die sexuelle Orientierung der Eltern zurückzuführen.
} 
schen Gesundheit, ${ }^{31}$ ihrer kognitiven und psychosexuellen Entwicklung verglichen. ${ }^{32}$ Diese Forschung über fast 30 Jahre hinweg konnte keinen Unterschied zwischen den Gruppen finden. ${ }^{33}$ Es ergaben sich durchweg mehr Ähnlichkeiten als Unterschiede, was die Schlussfolgerung unterstützt, dass die Qualität des Funktionierens der Familiendynamik nicht per se von der Familienstruktur abhängt. Stattdessen wird davon ausgegangen, dass Kinder lesbischer Mütter in ähnlicher Weise aufwachsen wie Kinder heterosexueller Eltern. ${ }^{34}$

Die Forschung hat auch einige Besonderheiten von Kindern lesbischer Mütter beleuchtet. Obwohl die erwachsenen Kinder lesbischer Mütter sich nicht öfter selber als homosexuell identifizierten als Kinder anderer Familienformen, ${ }^{35}$ beschrieben sie sich als offener gegenüber gleichgeschlechtlichen Beziehungen. ${ }^{36}$ Das Fehlen einer männlichen Identifikationsfigur in der Familie scheint die Entwicklung einer heterosexuellen Identität nicht empfindlich zu stören; ${ }^{37}$ stattdessen unterstützt dieses Ergebnis die Hypothese, dass Kinder lesbischer Eltern weniger homophobisch sind und alternativen Lebensmodellen toleranter gegenüber stehen als Kinder heterosexueller Eltern. ${ }^{38}$

Einige Faktoren wurden identifiziert, die die kindliche Entwicklung beeinflussen. Je jünger die Kinder von geschiedenen Lesben sind, wenn sie von der lesbischen Identität ihrer Mutter erfahren, desto leichter fällt es ihnen, dies anzunehmen. ${ }^{39}$ LDI Kinder dagegen erleben die sexuelle Orientierung ihrer Mütter als selbstverständlich, obwohl sie bereits im Kindergartenalter den Unterschied zwischen ihrer Familie und Familien mit heterosexuellen Eltern wahrnehmen. ${ }^{40}$ Kontakt zu anderen Regenbogenfamilien ist ebenfalls wichtig für die Kinder ${ }^{41}$ und ihre Eltern. ${ }^{42}$ In den Studien von Herrmann-Green/Gehring ${ }^{43}$ wünschten sich Dreiviertel der in Deutschland lebenden LDI Mütter mehr Kontakt zu anderen Regenbogen-

31 Bos/v.Balen/v.d.Boom (Fußn. 17); Brewaeys/Ponjaert/v.Hall/Golombok (Fußn. 12); Chan/Raboy/Patterson (Fußn. 16); Golombok/Perry/Burston/Murray/Mooney-Somers/Stevens (Fußn. 14); Patterson (Fußn. 2);

Golombok/Tasker/Murray (Fußn. 16).

32 Flaks/Ficher/Masterpasqua/Joseph (Fußn. 17); Brewaeys/Ponjaert/v.Hall/Golombok (Fußn. 12); Golombok/ Tasker (Fußn. 13); Tasker/Golobmok (Fußn. 13).

33 Tasker/Patterson, Research on gay and lesbian parenting: Retrospect and prospect, in: Tasker/Bigner (eds.), Gay and Lesbian Parenting: New Directions, 2008, pp. 9 ff.

${ }^{34} \mathrm{Bos} / v$.Balen/v.d.Boom (Fußn. 17).

35 Tasker/Patterson (Fußn. 33).

36 Tasker/Golombok (Fußn. 13).

${ }^{37}$ Golombok (Fußn. 11).

38 Herrmann-Green/Gehring, in: Tasker/Bigner (Fußn. 21).

39 Patterson, Children of Lesbian and Gay Parents, Child Development 1992, 1025 ff.

40 Gartrell/Banks/Reed/Hamilton/Rodas/Deck. (Fußn. 10).

${ }^{41}$ Vanfraussen/Ponjaert-Kristoffersen/Brewaeys, What does it mean for youngsters to grow up in a lesbian family created by means of donor insemination? Journal of Reproductive \& Infant Psychology 2002/20, $237 \mathrm{ff}$.

${ }^{42}$ Bei diesen Kontakten steht weniger die Herkunft der Kinder, sondern das gemeinsame Merkmal lesbisch-schwuler Eltern im Vordergrund.

${ }^{43}$ Herrmann-Green/Gehring, in:Tasker/Bigner (Fußn. 21). 
familien. Gershon und Kollegen berichten, dass das Selbstwertgefühl der Kinder lesbischer Eltern höher ist, wenn das empfundene Stigma niedrig ist, also scheint ein die Homosexualität akzeptierender sozialer Kontext die Kinder positiv zu beeinflussen. Je offener die Familienstruktur nach außen vertreten wird, desto mehr Akzeptanz und Toleranz erfährt die Familie in ihrem sozialen Umfeld. Patterson, Chan und Kollegen berichten, dass die egalitäre Arbeitsteilung der lesbischen Eltern sich positiv auf die partnerschaftliche Zufriedenheit des Paares und die psychische Gesundheit der Kinder auswirkt. ${ }^{44}$ Ebenfalls fanden Bos und Kollegen, sowie Wainright und Patterson heraus, dass die kindliche Entwicklung in Familien mit lesbischen versus heterosexuellen Eltern unabhängig von der Familienstruktur ist; sie wird vielmehr von der elterlichen Dynamik, z.B. Zufriedenheit mit der Partnerin bzw. dem Partner als Elternteil und Qualität der Eltern-Kind-Beziehung, beeinflusst. ${ }^{45}$ Daraus wird geschlussfolgert, dass nicht die Familienstruktur, z.B. Mutter-Mutter oder Mutter-Vater Familie, sondern der Familienprozess die Kindesentwicklung nachhaltig beeinflusst.

\subsection{Diskriminierung}

Aus gesellschaftlicher Sicht besteht darüber hinaus die Sorge, dass die Kinder lesbischer Eltern stigmatisiert werden, aufgrund der mütterlichen sexuellen Orientierung soziale Ausgrenzung erfahren und von Peers besonders gehänselt werden könnten. ${ }^{46}$ V anfraussen und Kollegen kommen zu dem Ergebnis, dass Kinder lesbischer Eltern nicht häufiger gehänselt werden als Kinder heterosexueller Eltern. ${ }^{47}$ Alle Kinder werden unabhängig von ihrer Familienform aufgrund ihrer äußerlichen Erscheinung, ihrer Intelligenz oder beispielsweise bei Verliebtheit gehänselt. Die Hänseleien, die Kinder lesbischer Eltern erlebten, waren auch familienbezogen (zwei Mütter, kein Vater, selber homosexuell).

Auch Lesben mit Kinderwunsch und lesbische Mütter sorgen sich, ob ihre Kinder besondere Diskriminierungen erleiden könnten und welche Auswirkungen die gesellschaftliche Homophobie und der Heterosexismus auf ihre Kinder haben werden. ${ }^{48}$ Sie beginnen bereits in der Familienplanungsphase, sich mit möglichen

\footnotetext{
${ }^{4}$ Patterson (Fußn. 21); Chan/Raboy/Patterson (Fußn. 16).

45 Bos/Hakvoort, Child adjustment and parenting in planned lesbian-parent families with known and as-yet unknown donors, Journal of Psychosomatic Obstetrics \& Gynaecology 2007/28, 121 ff.; Bos/v.Balen/v.d.Boom (Fußn. 17); Wainright/Patterson (Fußn. 14).

46 Interessanterweise werden mögliche zukünftige Diskriminierungserfahrungen, z.B. von Kindern religiöser oder ethnischer Minderheiten, gesellschaftlich nicht als Hinderungsgrund für eine Familienbildung interpretiert. Stattdessen wird die „Lösung“ in der Ankurbelung von gesellschaftlicher Toleranz gesehen.

${ }^{47}$ Vanfraussen/Ponjaert-Kristoffersen/Brewaeys (Fußn. 41).

48 Gartrell/Hamilton/Banks/Hamilton/Reed/Sparks/Bishop/Rodas (Fußn. 9);

Gartrell/Banks/Reed/Hamilton/Rodas/Deck (Fußn. 10); Gartrell/Deck/Rodas/Peyser/Banks, The national lesbian family study: 4. Interviews with the 10-year-old children, American Journal of Orthopsychiatry 2005, 518 ff.; Herrmann-Green/Gehring, in: Tasker/Bigner (Fußn. 21).
} 
Strategien auseinanderzusetzen, um ihre Kinder auf eventuelle homophobische Erfahrungen vorzubereiten und vor eventuellen negativen gesellschaftlichen Einflüssen zu schützen, z.B. offen lesbisch leben (out sein), Rollenspiele mit Kindern einüben, „gay pride“ der Kinder fördern, die Schule mit Wertlegung auf Vielfalt und Offenheit für Regenbogenfamilien und schwul-lesbischem Lehrpersonal wählen. Gartrell und Kollegen berichten, dass ungefähr die Hälfte ihrer Stichprobe die erste homophobische Erfahrung bis zum Alter von 10 Jahren erlebt hat. ${ }^{49}$ Die Autoren beschreiben sehr offensive und selbstbewusste kindliche Reaktionen auf die homophobische Äußerung. Die lesbischen Eltern gaben an, dass ihre Kinder gute akademische Leistungen erbrachten $(85 \%)$ und gute Peerbeziehungen $(81 \%)$ hatten. Vanfraussen und Kollegen berichten, dass die Kinder aus lesbischen Familien in ihrer Studie sich ebenso von Peers akzeptiert fühlten wie Kinder mit heterosexuellen Eltern und keine Bedenken hatten, Freunde nach Hause einzuladen. ${ }^{50}$

LDI Kinder haben nicht nur gleich guten Kontakt zu ihren Peers, sondern auch soziale Beziehungen zu anderen Erwachsenen. Lesbische Mütter unterstützen den Kontakt zwischen männlichen Bezugspersonen und ihren Kindern. ${ }^{51}$ LDI Mütter erreichen dies, indem sie entweder einen bekannten Spender oder einen Patenonkel für ihr Kind aussuchen. ${ }^{52}$ LDI Kinder haben auch regelmäßigen Kontakt zu ihren Großeltern ${ }^{53}$ und die Großeltern erkennen größtenteils die Kinder lesbischer Töchter als Enkel an. ${ }^{54} \mathrm{Zu}$ dem erweiterten Familienkreis einer lesbischen Familie gehören ebenso nicht-blutsverwandte Personen, die sowohl heterosexuell als auch homosexuell sein können. ${ }^{55}$ Die Kinder erleben somit eine Vielfalt an sozialen Beziehungen in ihrem erweiterten Familienkreis.

Institutionalisierte Diskriminierung stellt für Lesben und Schwule mit Kinderwunsch und Regenbogenfamilien die eigentliche Herausforderung dar. Sie findet überall dort statt, wo ein Unterschied zwischen heterosexuellen und homosexuellen Personen und Paaren aufgrund ihrer sexuellen Orientierung gemacht wird. In Deutschland hat seit 2001 eine starke Angleichung der Rechte von Lesben und Schwulen an heterosexuelle Personen stattgefunden. Trotzdem ist die eingetragene Lebenspartnerschaft der Ehe nicht komplett gleichgestellt. Diese Situation hat

\footnotetext{
${ }^{49}$ Gartrell/Hamilton/Banks/Hamilton/Reed/Sparks/Bishop/Rodas (Fußn. 9); Gartrell/Banks/Reed/Hamilton/Rodas/Deck (Fußn. 10); Gartrell/Deck/Rodas/Peyser/Banks (Fußn. 48); Herrmann-Green/Gehring, in: Tasker/Bigner (Fußn. 21).

${ }^{50}$ Vanfraussen/Ponjaert-Kristoffersen/Brewaeys (Fußn. 41).

51 Gartrell/Hamilton/Banks/Mosbacher/Reed/Sparks/Bishop (Fußn. 22).

52 Baetens (Fußn. 24); Gartrell/Banks/Reed/Hamilton/Rodas/Deck (Fußn. 10); Herrmann-Green/Gehring, in: Tasker/Bigner (Fußn. 21).

53 Fulcher/Chan/Raboy/Patterson, Contact with grandparents among children conceived via donor insemination by lesbian and heterosexual mothers, Parenting: Science and Practice 2002, 61 ff.; Patterson/Hurt/Mason, Families of the lesbian baby boom: Childrens' contact with grandparents and other adults, American Journal of Orthopsychiatry 1998, $390 \mathrm{ff}$.

${ }^{54}$ Gartrell/Hamilton/Banks/Hamilton/Reed/Sparks/Bishop/Rodas (Fußn. 9); Gartrell/Banks/Reed/Hamilton/Rodas/Deck (Fußn. 10); Gartrell/Deck/Rodas/Peyser/Banks (Fußn. 48); 55 Fulcher/Chan/Raboy/Patterson (Fußn. 53).
} 
besondere Auswirkung auf LDI Familien in der Phase der Familiengründung: (1) Die Möglichkeiten, eine Familie zu gründen, sind eingeschränkt, dies zeigt sich am erschwerten Zugang zu reproduktionsmedizinischen Behandlungen in Deutschland und am Fehlen eines gemeinsamen Adoptionsrechts. (2) Obwohl die Möglichkeit der Stiefkindadoption unbestreitbar bedeutsam für LDI Familien ist, gibt es einen Nachteil. Das gemeinsam geplante LDI Kind muss auch bei eingetragenen Lebenspartnerinnen nach der Geburt mittels Stiefkindadoption ${ }^{56}$ von der sozialen Mutter adoptiert werden, was eine Phase der Absicherungslücke für das Kind und eine emotionale Belastung für die soziale Mutter bedeutet. Das Anerkennungsverfahren für nicht-eheliche Väter ist dagegen deutlich einfacher. (3) Lesben sind Frauen, und viele Frauen arbeiten im psychosozialen Sektor des Arbeitsmarkts, der häufig kirchliche Träger hat. Im Arbeitsverhältnis bei einem katholischen Träger droht Lesben die Kündigung, wenn sie sich „outen“ und/oder eine eingetragene Lebenspartnerschaft eingehen, die u.a. als Absicherung ihrer Familie dient und Voraussetzung für die Stiefkindadoption ist. (4) Ungleiche Anwendung von steuerlichen Begünstigungen für Familien, z.B. das Ehegatten-Splitting, verursacht eine finanzielle Schlechterstellung von LDI Familien gegenüber Familien mit heterosexuellen Eltern.

\section{4 (Be-)Deutung einer Frauen definierten Familienform}

Die Forschung hat also über 30 Jahre hinweg keine Unterschiede zwischen Eltern und Kindern in lesbischen versus heterosexuellen Familien finden können, die bedenklich wären. Der größere Teil der Forschung geht zwar von einem defizitären Ansatz aus, war aber trotzdem wichtig, da erstmals heterosexistische Vorbehalte gegen lesbische Mütter als nicht zutreffende Vorurteile aufgezeigt werden konnten. Stattdessen dokumentieren die Studien die Tragfähigkeit einer Familie mit lesbischen Eltern. Damit müsste einer weiteren Stigmatisierung bzw. Diskriminierung von lesbischen Müttern und Lesben mit Kinderwunsch die Legitimation entzogen sein. Dass es offensichtlich dennoch ein Thema ist, beschäftigt Forscher der lesbisch, schwulen, bisexuellen und transgender Psychologie, da der Transfer der Forschungsergebnisse in den sozio-kulturellen Raum offensichtlich (noch) nicht gänzlich geglückt ist. ${ }^{57}$ Dennoch wurde der Weg frei für Forschungsarbeiten mit einem nicht-diskriminierenden und explorativen oder beschreibenden Ansatz.

\footnotetext{
56 Im Falle eines bekannten, privaten Spenders muss dieser der Adoption durch die soziale Mutter zustimmen. Dadurch erlischt seine rechtliche Vaterschaft. Im Falle eines unbekannten Spenders, d.h. Nein- und Ja-Spender, kann der Spender nicht einwilligen, da seine Identität nicht bekannt ist. In beiden Fällen erwirbt das LDI Kind durch die Stiefkindadoption einen zweiten gleichberechtigten rechtlichen Elternteil durch die soziale Mutter. In jedem Fall klärt die Stiefkindadoption ebenfalls den Status des Spenders, da jegliche (väterliche) Rechte und Pflichten erlöschen und auf die soziale Mutter übergehen.

${ }^{57}$ Dies ergibt der Austausch auf dem Listserver der Division 44 der American Psychological Association für die psychologische Untersuchung von Lesben, Schwulen, Bisexuellen und Transgendern.
} 
Insgesamt sprechen die Forschungsergebnisse dafür, dass (1) die sexuelle Orientierung der Mutter irrelevant ist und dass (2) nicht die Familienstruktur, sondern Familienprozesse für die Kindesentwicklung ausschlaggebend sind. Die Tragfähigkeit der Mutter-Mutter-Kind Familie ist insofern von gesellschaftlicher Bedeutung, indem sie heteronormative bzw. „traditionelle“ Vorstellungen von Familie (Familie $=$ Mutter + Vater + leibliches Kind) in Frage stellt. Trotzdem erleben sich die LDI Familien als ganz gewöhnliche Familien. ${ }^{58}$ Die überwiegende Wahl der LDI Mütter für ein Familienmodell von Mutter-Mutter-Kind(er) stellt eine Modifizierung des Kernfamilienkonzepts dar, um die lesbische Beziehung aufzunehmen. ${ }^{59}$

\section{Die Sicht der LDI Familie}

Neuere Forschungsarbeiten haben einen explorativen oder beschreibenden Ansatz, da sie die Einzigartigkeit einer LDI Familie entdecken wollten oder ihr Erleben zu beschreiben versuchten.

\subsection{Motivation Mutter zu werden}

Untersuchungen hinsichtlich des Kinderwunsches von Lesben sind begrenzt. Ergebnisse der Studien von Siegenthaler und Bigner und Bos, van Balen und van Boom legen nahe, dass lesbische und heterosexuelle Paare Elternschaft aus denselben Gründen anstreben wie z.B. erwartetes Glück und Erfüllung durch eine Elternschaft. ${ }^{60}$ Allerdings sind künftige lesbische Eltern weniger motiviert durch soziale Erwartungen der „Normalität“ oder Generativität und sie reflektieren ihren Kinderwunsch länger als heterosexuelle Eltern. Hermann-Green und Gehring berichten, dass die LDI Mütter in ihrer deutschen Stichprobe ihren Kinderwunsch im Schnitt 2 Jahre reflektierten, bevor sie die erste Insemination unternahmen. ${ }^{61}$ In dieser Zeit setzten sie sich sowohl mit allgemeinen als auch lesbisch-spezifischen Aspekten der Familienplanung auseinander. ${ }^{62}$ Die unterschiedliche Reflexionszeit wird anhand der unterschiedlichen soziokulturellen Kontexte, in denen lesbische und heterosexuelle Paare die Entscheidung zur Elternschaft treffen, erklärt: lesbische Eltern entscheiden sich für Kinder in einer Umgebung, die sie stigmatisiert, und müssen größere Anstrengungen auf sich nehmen, um schwanger zu werden als fertile heterosexuelle Paare. ${ }^{63}$

Die bisherigen Untersuchungen ergeben, dass es keinen eindeutigen Auslöser für den Kinderwunsch bei Lesben gibt. Viele lesbische Mütter können jedenfalls

\footnotetext{
${ }^{58}$ Herrmann-Green/Gehring, in: Tasker/Bigner (Fußn. 21).

${ }^{59}$ Herrmann-Green/Gebring, in: Tasker/Bigner (Fußn. 21).

${ }^{60} \mathrm{Bos} /$ v.Balen/v.d.Boom (Fußn. 16); Siegenthaler/Bigner (Fußn. 16).

${ }^{61}$ Herrmann-Green/Gehring, in: Tasker/Bigner (Fußn. 21).

${ }^{62}$ Green (Fußn. 15).

${ }^{63} \mathrm{Bos} /$ v.Balen/v.d.Boom (Fußn. 16); Siegenthaler/Bigner (Fußn. 16).
} 
keinen äußeren Anlass für ihren Kinderwunsch benennen, da er immer da war. ${ }^{64}$ Die Entscheidung für ein leibliches Kind anstelle einer Adoption oder der Aufnahme eines Pflegekinds beruht auf dem Wunsch, Schwangerschaft und Geburt zu erleben sowie einen Säugling zu erziehen. ${ }^{65}$ Daniels berichtet von denselben Beweggründen bei heterosexuellen Frauen. ${ }^{66}$ Die deutsche Studie von Herrmann-Green und Gehring ergab, dass Lesben die Herbeiführung einer Schwangerschaft durch Insemination dem Geschlechtsverkehr mit einem Mann vorziehen, weil sie in monogamen Beziehungen leben. ${ }^{67}$ Lesben realisieren ihren Kinderwunsch in auf Dauer angelegten Paarbeziehungen. Diese sind durch ein hohes Maß an intern wahrgenommener Verbindlichkeit und durch äußere Anzeichen von Verbindlichkeit charakterisiert, auch wenn das Paar nicht in eingetragener Lebenspartnerschaft lebt. Sexuelle Kontakte zu Dritten würden die Integrität der Beziehung verletzen, und als Lesben lehnen viele sexuelle Kontakte zu Männern für sich selbst ab.

Das Lesbenpaar mit Kinderwunsch hat die besondere Situation, dass es entscheiden muss, wer das (erste) Kind austragen soll bzw. darf. Die Entscheidung, welche der Partnerinnen (zuerst) schwanger wird, hängt davon ab, welche Frau den stärkeren Wunsch hat, Schwangerschaft und Geburt zu erleben. Wenn beide Frauen schwanger werden wollen, entscheidet in der Regel das Alter, d.h. die Ältere versucht als erstes schwanger zu werden. ${ }^{68}$ Bei der Planung vom ersten Kind wird häufig gleichzeitig ein zweites Kind geplant; in der Hälfte dieser Fälle soll es dabei einen Rollentausch geben, so dass die soziale Mutter Geburtsmutter vom Geschwisterkind wird. ${ }^{69}$ Unabhängig von der Rolle der Partnerinnen in Bezug auf das Kind, ob Geburtsmutter oder soziale Mutter, wird das Kind als ein ,eigenes, gemeinsames" Kind erlebt, wenn beide bei der Familienplanung beteiligt sind. ${ }^{70}$

${ }^{64}$ Baetens (Fußn. 24); Gartrell/Hamilton/Banks/Mosbacher/Reed/Sparks/Bishop (Fußn. 22); HerrmannGreen/Gehring, in: Tasker/Bigner (Fußn. 21).

65 Herrmann-Green/Gehring, in: Tasker/Bigner (Fußn. 21).

66 Daniels, Adoption and donor insemination: Factors influencing couples' choices, Child Welfare $1994 / 73,5 \mathrm{ff}$.

${ }^{67}$ Herrmann-Green/Gehring, in: Tasker/Bigner (Fußn. 21).

${ }^{6}$ Baetens (Fußn. 24); Herrmann-Green/Gebring, in: Tasker/Bigner (Fußn. 21).

${ }^{69}$ Herrmann-Green/Gebring, in: Tasker/Bigner (Fußn. 21).

70 Das Erleben der Partnerinnen, ein gemeinsames Kind zu bekommen, bedarf Berücksichtigung seitens des medizinischen Personals, die Lesbenpaare inseminieren. Nicht nur die Frau, die das Kind austragen wird, wird Mutter, sondern auch die nicht-austragende Frau. Partnerinnen ist es wichtig, dass das Verhalten ihres Arztes die Anerkennung dieser Tatsache widerspiegelt, z.B. indem beide Frauen angesprochen und gewürdigt werden. Green/Mitchell, Different storks for different folks: Gay and Lesbian Parents' experiences with alternative insemination and surrogacy, in: Tasker/Bigner (eds.); Golombok, Gay and Lesbian Parenting: New Directions, 2008, pp. 81 ff.; Rauchfleisch, Alternative Familienformen: Eineltern, gleichgeschlechtliche Paare, Hausmänner [Alternative family forms: Single parents, same-sex parents and stay-at-home dads], 1997. 


\subsection{Wahl des Spender(typ)s}

Lesben mit Kinderwunsch fehlt es in der Regel lediglich an Sperma, um ein Kind zu bekommen. Grundsätzlich können sie Sperma von einer Samenbank (unbekannter Spender) beziehen oder einen privaten Spender (bekannter Spender) suchen. Je nach Vorschrift des Landes oder der Klinik haben Samenbanken bis zu zwei Arten von unbekannten Spendern: anonyme bzw. Nein-Spender, deren Identität nicht erfahrbar ist, und „identity-release“ bzw. Ja-Spender, deren Identität für das daraus entstandene Kind unter bestimmten Umständen, z.B. mit 18 Jahren, erfahrbar wird. In Deutschland werden Lesben mit Kinderwunsch in ihrem Zugang zur Reproduktionsmedizin gehindert. Die Richtlinien der Bundesärztekammer empfehlen, DI nur bei verheirateten heterosexuellen Paaren durchzuführen. ${ }^{71}$ Zudem ist der rechtliche Status eines Spenders bezüglich eines Kindes, das außerehelich geboren wird, in Deutschland nicht eindeutig geregelt, so dass die meisten Ärzte die Behandlung von verheirateten Paaren vorziehen, ${ }^{72}$ obwohl die Stiefkindadoption durch die soziale Mutter den rechtlichen Status des Spenders nach der Geburt eindeutig klar stellt. ${ }^{73}$ In Deutschland lebende Lesben müssen sich daher entweder an ausländische Samenbanken und Kliniken wenden oder Selbstinsemination mit Sperma eines privaten Spenders oder importiertem gefrorenem Sperma durchführen. Die Selbstinsemination mit privatem/bekanntem Spender ist mit gesundheit-lichem Risiko für die zu inseminierende Frau verbunden, da die Spermaproben, die inseminiert werden, nicht auf sexuell übertragbare Krankheiten getestet sind. Herrmann-Green und Gehring untersuchten u.a. die Wahl zwischen drei Spendertypen in ihrer deutschen Stichprobe: Nein-Spender, Ja-Spender und privater/bekannter Spender. ${ }^{74}$ Für die lesbischen Eltern ist es eine wohlüberlegte Ent-

${ }^{71}$ Thorn/Wischmann, Eine kritische Würdigung der Novellierung der (Muster-) Richtlinie der Bundesärztekammer 2006 aus der Perspektive der psychosozialen Beratung, Journal für Reproduktionsmedizin und Endokrinologie 2008, 1 ff.

72 Thorn/Wischmann (Fußn. 71).

${ }^{73}$ Der eindeutige rechtliche Status als Nicht-Vater ist für einen Spender nur bei einer verheirateten Frau gegeben, da der Ehemann automatisch rechtlich Vater eines Kindes wird, das seine Frau während der Ehe oder innerhalb 270 Tagen nach der Scheidung gebärt, unabhängig davon, ob er auch der biologische Vater des Kindes ist. Bei Lebenspartnerinnen erfolgt keine automatische rechtliche Elternschaft für den sozialen Elternteil; sie kann dies nur durch die Stiefkindadoption erlangen. Die Dauer einer Stiefkindadoption ist außerhalb des direkten Einflussbereiches des Lesbenpaares - sie ist von der Auslastung und Mitarbeit der jeweiligen Jugendämter und Gerichte abhängig, und es kann mehrere Monate dauern, bis das Verfahren entschieden wird. Jedoch klärt die jetzige Stiefkindadoption durch die soziale Mutter den rechtlichen Status eines Spenders und ist als große rechtliche Errungenschaft für Lesbenpaare und ihre Kinder anzusehen.

${ }^{74}$ Untersuchungen über die Wahl des Spenders wurden bisher bei LDI Familien kaum vorgenommen, da die Studien in der Regel im Zusammenhang mit einem Reproduktionszentrum unternommen wurden, die nur einen Spendertyp anbieten. Eine Ausnahme bilden die Arbeiten von Scheib/Riordan/Shaver, Choosing between anonymous and identity-release sperm donors: Recipient and donor characteristics, Reproductive Technologies 2000/10, 50 ff.; und Brewaeys/d.Bruyn/Louve/Helmerhorst, Anonymous or identity-registered sperm donors? A study of Dutch recipients' choices, Human Reproduction 2005/20, 820 ff.: dies zumal The Spermbank of California und das Fertilitätszentrum der Universitätsklinik in Leiden (bis 2004) sowohl Nein-Spender als auch Ja-Spender anbieten und ausdrücklich offen für die Behandlung von alleinstehenden und lesbischen 
scheidung, ob sie einen bekannten oder unbekannten Spender wählen, die sie gründlich reflektieren. Das Lesbenpaar mit Kinderwunsch muss eine Entscheidung treffen über die Zukunft des Kindes mit dem Wissen, dass die Konsequenzen hauptsächlich das Kind tragen wird, ohne diese genau zu kennen. Das Paar muss darüber hinaus berücksichtigen, dass das Kind in einem sozialen Klima aufwächst, das annimmt, dass eine positive Kindesentwicklung am ehesten möglich ist, wenn es bei seinem biologischen Vater und seiner biologischen Mutter aufwächst. Zudem ist von der Wahl des Spendertyps abhängig, wie stark die Grenzen der zukünftigen Familie nach außen geschützt werden können.

Im Folgenden werden die Ergebnisse von Hermann-Green und Gehring hinsichtlich der Spendertypwahl beschrieben. ${ }^{75}$ Daten zu verschiedenen Phasen der LDI Familienbildung wurden retrospektiv von 105 in Deutschland lebenden LDI Müttern anhand eines strukturierten Fragenbogens erhoben. Das demografische Profil der Teilnehmerinnen entspricht denselben Charakteristika von LDI Müttern, die an psychologischer Forschung teilnehmen, und die Stichprobe unterliegt ebenfalls den gewöhnlichen Einschränkungen für das Erforschen unsichtbarer Populationen. Die retrospektive Datenerhebung lässt nicht ausschließen, dass die Angaben eventuell durch eine selektive Erinnerung bzw. veränderte Einstellungen aufgrund der Lebenserfahrung modifiziert sein könnten. Es ergaben sich keine signifikanten Unterschiede zwischen den Gruppen aufgrund der Mutterrolle (Geburtsmutter/soziale Mutter), Spendertyp (Nein-Spender/Ja-Spender/bekannter Spender) und Familienbildungsphase (vor-elterliche Phase/Alter des ersten DI Kindes 0-3 Jahre/3-6 Jahre/6+), es sei denn es wird explizit erwähnt.

Nein-Spender:

Lesben, die einen Nein-Spender wählten, waren der Meinung, dass das Geschlecht der Eltern nicht wesentlich für die Kindesentwicklung sei. Sie meinten, der Wunsch, seine biologischen Wurzeln zu kennen, sei eher ein Resultat des gesellschaftlichen Drucks als eine wirkliche biologische Notwendigkeit für jedes Individuum. Die wesentlichen positiven Aspekte dieser Wahl beinhalten Sicherheit (das Sperma wird auf HIV und sexuell übertragbare Krankheiten getestet) und das Vorhandensein einer klaren Familienstruktur (Geburtsmutter - soziale Mutter Kind), in welcher besonders die Beziehung zwischen sozialer Mutter und Kind am besten nach außen geschützt ist. ${ }^{76}$ Die Frauen, die einen Nein-Spender gewählt haben, waren sich über die Konsequenzen dieser Entscheidung für das Kind bewusst und sorgten sich, ob das Kind ihnen dies eines Tages übel nehmen könnte.

Frauen plädieren. Scheib und Brewaeys berichten, dass mehr Lesbenpaare als heterosexuelle Paare sich für Ja-Spender entscheiden, wobei beide ähnliche Beweggründe für die Wahl eines Ja-Spenders hatten, nämlich um dem Kind die Möglichkeit zu erhalten, die Identität des Spenders zu erfahren. Die Bereitschaft, das Kind über seine DI Herkunft aufzuklären, stand in positivem Zusammenhang mit der Wahl eines Ja-Spenders vs. einem Nein-Spender.

75 Herrmann-Green/Gehring, in: Tasker/Bigner (Fußn. 21).

76 Zum Zeitpunkt der Datenerhebung der Hermann-Green und Gebring Studie fehlte die heutige gesetzliche Regelung der Stiefkindadoption, was vor allem die Beziehung soziale Mutter-Kind vulnerabel machte. 
Die Frauen selber verspürten kein Bedürfnis, den Spender kennenzulernen, und haben wenig über ihn nachgedacht. Interessanterweise würden $88 \%$ der Frauen, die einen anonymen Spender gewählt haben, dem Wunsch zustimmen, dass ihre Kinder den Spender treffen könnten, wenn es denn möglich wäre und das Kind es sich wünschte.

\section{Privater/ bekannter Spender:}

Die Frauen aus der oben genannten Studie, die einen privaten/bekannten Spender gewählt hatten, waren der Meinung, dass es schädlich für ein Kind sei, seinen zweiten genetischen Elternteil nicht zu kennen. Sie sahen auch den Wunsch, die eigenen genetischen Wurzeln zu kennen, eher als Folge eines innewohnenden biologischen Triebs als ein Ergebnis von gesellschaftlichem Druck. Die wesentlichen positiven Aspekte dieser Spenderwahl waren, dass das Kind seinen anderen biologischen Elternteil kennen kann und dies zudem ermöglicht, kreative FamilienKonstellationen zu bilden, mit zwei, drei oder vier Elternfiguren. Trotzdem waren auch hier die Geburtsmutter und die soziale Mutter als Eltern des Kindes geplant. Während die Identität des biologischen Vaters dem Kind zwar bekannt sein sollte, war seine Rolle, wenn überhaupt, als „Freund der Familie“ oder „Onkel“ geplant, und nicht als sozialer Vater oder „Papa“. Die Hauptschwierigkeit mit diesem Spendertyp war es, einen Mann zu finden, der gewillt war, den Vorstellungen des lesbischen Paares von seiner Rolle in ihrer Familie zuzustimmen. Die gebräuchlichste Methode bestand darin, einen dem Paar bekannten Mann zu fragen oder über eine Anzeige Kontakt zu knüpfen. Interessanterweise stellte sich in dieser Untersuchung heraus, dass die Spender meistens Männer waren, die dem Paar vorher nicht bekannt waren. Auch wurden bevorzugt homosexuelle Männer ausgewählt. ${ }^{77}$ Die Frauen nannten als Motivation des Spenders dessen eigenen Kinderwunsch. Trotz des Wunsches, dass das Kind seinen Spender kennen kann, wurde die Familie definiert als das lesbische Paar und das Kind. Es wurde weder geplant, den Spender in die Geburtsurkunde einzutragen, was juristische und finanzielle Folgen hätte, noch wurde er mit finanzieller Verantwortung, Entscheidungsbefugnis oder Verantwortung in der Kinderbetreuung belegt. Insgesamt scheinen die Vereinbarungen mit bekannten Spendern locker zu sein: es waren wenig Absprachen erforderlich, die Rolle des Spenders zu definieren. Eine Einigung für den Umgang mit einer eventuellen Trennung der Lesben oder eine Vereinbarung, falls sich die Parteien Änderungen hinsichtlich der abgesprochenen ursprünglichen Rollenverteilung wünschten, gab es hingegen - wenn überhaupt nur mündlich. Mit einem bekannten Spender hatten die Frauen den größten Grad an Selbstbestimmung, nämlich der Auswahl des Spenders und der Möglichkeit der Selbstinsemination. Die wichtigsten Kriterien bei der Spenderwahl waren die Bereitschaft der gewollten Rolle in der LDI Familie zuzustimmen und Sympathie.

\footnotetext{
77 Dieser Aspekt ist besonders erwähnenswert, da diese Gruppe als Spender für Samenbanken aufgrund der Einstufung als „Risikogruppe“ ausscheidet, obwohl gefrorene Spermaproben hinreichend getestet werden können, während die frischen Samenproben, die hier inseminiert werden, überhaupt nicht getestet sind.
} 


\section{Ja-Spender:}

Frauen in dieser Studie, die Ja-Spender gewählt hatten, hatten ähnliche Überzeugungen wie Frauen, die einen bekannten Spender gewählt hatten, sie waren aber weniger stark ausgeprägt. Diese Frauen nannten als positiven Aspekt die Sicherheit durch den Gebrauch von getestetem Sperma und die klare Familienstruktur. Es gibt dem Kind die Möglichkeit (im Erwachsenenalter), die Identität des Spenders zu erfahren, falls dies für es wichtig werden sollte. Die Frauen selber verspürten überwiegend kein Bedürfnis, den Spender kennenzulernen. Wenn aber doch, wollten sie ihm gerne ihre Dankbarkeit mitteilen. Ein Nachteil dieser Spenderwahl war die geringe Verfügbarkeit. Zudem bestand die Sorge, dass das Kind Erwartungen bezüglich des Spenders aufbaut, die sich in der Realität nicht erfüllen lassen.

Konsequenzen der Spendertypen:

Die unterschiedlichen Spendertypen bieten unterschiedliche Sicherheiten und unterschiedliche Herausforderungen bzw. Angriffsflächen für die LDI Familien. Den Müttern, die Nein-Spender hatten, war es wichtig, die Grenzen der LDI Familie durch die Nicht-Involviertheit des Spenders zu schützen. Außerdem wollten sie dem Kind eine eventuelle Enttäuschung ersparen, sollte der Spender nicht auffindbar sein oder keinen Kontakt zum Kind wollen. Sie müssen ihre Entscheidung eventuell häufiger verteidigen, da ihre Spenderwahl den deutlichsten Bruch zu heteronormativen Vorstellungen von Familie darstellt; ihre Entscheidung könnte von außen interpretiert werden als „den Kindern einen Vater verweigern“ oder als „Väter sind unwichtig“. Mütter, die die Möglichkeit eines Ja-Spenders nutzten, achteten auch auf den Schutz der LDI Familiengrenze durch Sicherstellung der Nicht-Involviertheit des Spenders, jedoch wollten sie dem Kind den Zugang zur Identität des Spenders ermöglichen, wenn das Kind es wünschte; eine Einstellung, die mehr mit heteronormativen Vorstellungen übereinstimmt und deshalb wahrscheinlich weniger Rechtfertigung erfordert. Die Herausforderung eines Ja-Spenders könnte sein, das Kind während der Phase um eine bevorstehende und tatsächliche Spenderidentitätsfreigabe zu begleiten und als Familie mit den unvorhersehbaren Konsequenzen zurechtzukommen. Für Mütter, die bekannte Spender hatten, war das Kennen der Spenderidentität gesichert. Diese Konstellation kommt heteronormativen Vorstellungen von Familie am nächsten, besonders, wenn es sich um einen involvierten Spender handelt; jedoch könnte die dauerhafte Regulierung der Beteiligung des Spenders, so dass es für alle Beteiligten stimmig ist, einer der anspruchsvollsten Aspekte dieser Spenderwahl werden.

Obwohl ihre Studie drei unterschiedliche Typen von Spendern betrachtet, räumen Herrmann-Green und Gehring ein, dass dies nicht notwendigerweise impliziert, dass lesbische Frauen die freie Wahl haben. ${ }^{78}$ Eine wirklich freie Wahl wäre gegeben, wenn sie unabhängig von Finanzen, der sexuellen Orientierung und der Verfügbarkeit einen Spendertyp aussuchen könnten. Für einige Frauen in dieser Stichprobe war dies nicht der Fall. Die meisten Frauen, die einen Ja-Spender hat-

${ }^{78}$ Herrmann-Green/Gehring, in: Tasker/Bigner (Fußn. 21). 
ten, konnten frei zwischen „ja“ und „nein“ Spendern wählen, wohingegen die Frauen, die einen anonymen Spender hatten, nur zu 5\% diese Wahlmöglichkeit besaßen. Außerdem gaben einige Frauen, die schließlich einen anonymen Spender wählten, an, dass sie auch die Möglichkeit eines bekannten Spenders verfolgt hatten, beziehungsweise es vorgezogen hätten, die Identität des Spenders zu kennen. Green stellt die Hypothese auf, dass die Spender-Präferenz durch die Überzeugungen bezüglich der Rolle eines biologischen Vaters beeinflusst wird, und die „Wahl“ durch die Verfügbarkeit modifiziert wird. ${ }^{79}$ Dennoch waren alle Teilnehmerinnen in der Lage, Aspekte ihres Spenders zu benennen, die so positiv waren, dass sie ihn wählten, obwohl sie potenzielle Probleme sahen. Schlussfolgernd gibt es keine umfassende Lösung für alle, sondern nur Lösungen für einzelne Paare.

Gartrell und Kollegen berichten, dass sich die kindliche Entwicklung von Kindern mit bekanntem und unbekanntem Spender nicht unterscheidet. ${ }^{80}$ Bos und Kollegen geben dagegen an, dass Kinder in ihrer Stichprobe mit privatem/bekanntem Spender häufiger verhaltensauffällig seien als Kinder mit unbekanntem Spender ${ }^{81}$ Sicherlich sind mehr Langzeitstudien notwendig, um die tatsächlichen Konsequenzen eines Spendertyps für das Kind und seine Familie zu identifizieren.

Männer im Leben des Kindes:

Herrmann-Green und Gebring berichten ferner, dass sich Lesben mit Kinderwunsch bei der Wahl des Spendertyps gleichzeitig mit der Bedeutung von Männern im Leben ihres Kindes auseinander setzen. ${ }^{82}$ Lesben mit Kinderwunsch müssen entscheiden ob, in welchem Ausmaß und in welcher Weise sie beabsichtigen, Männer in das Leben ihres Kindes einzubeziehen. Die Teilnehmerinnen beabsichtigten, dass ihre Kinder auch männliche Bezugspersonen haben werden und waren sich einig, dass dies nicht unbedingt der leibliche Vater des Kindes sein muss. Die Frauen, die mit bekanntem Spender inseminierten, hatten diesen Spendertyp gewählt, damit ihr Kind seinen biologischen Vater kennen kann und sahen in ihm ein mögliches Rollenmodell. Viele Frauen mit unbekannten Spendern, d.h. NeinSpender und Ja-Spender, planten, dass ein nicht-verwandter Mann das Rollenmodell des Kindes werden sollte, indem sie einen guten Freund fragten, ob er „Pate“ für das Kind werden wolle. Die anderen fanden es unnötig, da das Kind seine eigenen Modelle suchen und finden würde.

\subsection{Familiengestaltung}

Wie sieht die Alltagsrealität von LDI Familien und ihrem Familienkonzept aus? Herrmann-Green und Gehring berichten, LDI Familien, Eltern wie Kinder, erleben

\footnotetext{
${ }^{79}$ Green (Fußn. 15).

${ }^{80}$ Gartrell/Deck/Rodas/Peyser/Banks (Fußn. 48).

${ }^{81} \mathrm{Bos} /$ Hakvoort (Fußn. 45).

${ }^{82}$ Herrmann-Green/Gebring, in: Tasker/Bigner (Fußn. 21).
} 
ihre Familie als etwas ganz Selbstverständliches. ${ }^{83}$ Sie leben überwiegend eine modifizierte Kernfamilie und definieren die Familie als Geburtsmutter - soziale Mutter - Kind. Auch wenn der leibliche Vater ein bekannter Spender ist und Kontakt zu ihm bestehen soll, soll der Lebensmittelpunkt des Kindes in den meisten Fällen bei den Müttern sein. Ganz typisch für LDI Elternschaft ist eine Wertlegung auf eine gleichberechtigte Mutterschaft und eine egalitäre Arbeitsaufteilung. Die Gleichwertigkeit der Mutterrollen spiegelt sich häufig in der Wahl der Benennungen der Mütter, z.B. Mama für eine Mutter und Mami für die andere. Dies soll die Rolle beider Frauen als Mutter nach außen transparent machen und trotzdem eine Differenzierung zwischen den Frauen ermöglichen. Beide Merkmale können auch als Strategie interpretiert werden, um einer biologisch(-rechtlich)en Asymmetrie in der Elternschaft entgegenzuwirken. In der LDI Familie wird der Spender als biologischer Vater, jedoch nicht als „Papa“ wahrgenommen. ${ }^{84}$ Der Begriff „Papa“ spricht für LDI Mütter die sozialen und emotionalen Aspekte einer Elternschaft an, die ein unbekannter, häufig aber auch bekannter Spender nicht erfüllt. Jedoch ist die Rolle des Spenders positiv besetzt; er wird als ein netter, toller Mann, der dem Paar ihr Kind geschenkt hat, konzeptualisiert. LDI Mütter klären ihre Kinder über ihre DI Herkunft auf, und Gespräche über den Spender sind nicht tabu. Die Aufklärung ihrer Kinder ist nicht als einmaliges Ereignis zu verstehen, sondern stellt eher einen Prozess dar. Die Informationen, die die Kinder erhalten, werden ihrem Alter und ihren kognitiven Möglichkeiten angepasst. ${ }^{85}$ Die Aufklärung der Umwelt über die Familienform, dass es zwei Mütter gibt und keinen Vater und wie das möglich ist, ist auch ein stetiger Prozess und wird mit der Zeit als lästig oder indiskret erlebt. ${ }^{86}$ Üblicherweise übernehmen diese Aufgabe die Eltern, z.B. im Kindergarten und in der Grundschule, während ältere Kinder gerne selber darüber bestimmen wollen, wer was weiß. ${ }^{87}$ Das Sichtbarwerden von Regenbogenfamilien wird von den Familien als wichtig angesehen; hierzu wäre es wünschenswert, dass Schule und Kindergarten die Vielfalt an familiären Lebensformen offen thematisierten und dass sich dies im Schulmaterial spiegeln würde.

\footnotetext{
83 Herrmann-Green/Gebring, in: Tasker/Bigner (Fußn. 21).

${ }^{84}$ Eine Ausnahme bildet eine Subgruppe von LDI Familien, die sich als Queer Family bezeichnet. Queer Family bedeutet, dass das Lesbenpaar mit einem Schwulen(paar) zusammen ein Kind bekommen und aufziehen möchte. Beabsichtigt ist dann eine Familie mit drei bzw. vier Elternteilen, wobei der Spender und evtl. dessen schwuler Lebenspartner besonders aktiv soziale Vaterschaft leben werden.

${ }^{85}$ Green/Mitchell (Fußn. 70); Mitchell, The birds, the bees...and the sperm banks: How lesbian mothers talk with their children about sex and reproduction, American Journal of Orthopsychiatry 1998, $400 \mathrm{ff}$.

${ }^{86}$ Green/Mitchell (Fußn. 70).

87 Green/Mitchell, in: Tasker/Bigner (Fußn. 70); Golombok, in: Tasker/Bigner (Fußn. 11); Tasker/Patterson, in: Tasker/Bigner (Fußn. 21).
} 


\section{Schlussfolgerung}

Sich als Lesbe für ein Kind zu entscheiden, ist das Ergebnis einer mehrjährigen Auseinandersetzung mit dem eigenen Kinderwunsch. Lesbische Frauen unterscheiden sich nicht von heterosexuellen Frauen in ihrer Motivation, Mutter zu werden. Negative Vorbehalte gegen lesbische Mütter und Befürchtungen für ihre Kinder lassen sich in der Realität nicht bestätigen, so dass anzunehmen ist, dass die sexuelle Orientierung von Eltern nicht für das Funktionieren der Familiendynamik ausschlaggebend ist. Es spricht bisher alles dafür, dass LDI Mütter sowohl persönliche, soziale und finanzielle Ressourcen als auch effektive Coping-Strategien haben, um sich in der heterosexuell-geprägten Umwelt gut zu behaupten, und dass sie diese an ihre Kinder weitergeben können. Kinder von Lesben entwickeln sich und gedeihen genauso gut wie Kinder von heterosexuellen Eltern. Sie werden nicht öfter homosexuell, aber toleranter. Die Kinder von Lesben werden auch nicht öfters gehänselt als andere Kinder. LDI Kinder erleben eine Vielfalt an sozialen Beziehungen im erweiterten Familienkreis und haben einen guten Kontakt zu ihren Peers. Lesbische Mütter gehen offen mit der DI Herkunft ihrer Kinder um und klären ihre Kinder altersgerecht auf. Die Wahl eines anonymen Spenders oder JaSpenders oder privaten Spenders scheint mit Überzeugungen hinsichtlich „Väter“ und Verfügbarkeit zusammenzuhängen. Lesben sind insgesamt offen für Möglichkeiten ihrer Kinder, Beziehungen zu „besonderen Männern“ aufzubauen und die Identität des Spenders zu kennen. Wenn sie selber auswählen dürfen, bevorzugen sie schwule Männer als Spender.

Auch wenn die Generalisierbarkeit der Forschungsergebnisse noch nicht durchgehend möglich ist, sprechen die momentanen Ergebnisse der psychologischen Literatur eine klare Sprache: Sie sprechen für die Adäquatheit der LDI Familien. Aus psychosozialer Sicht spricht nichts gegen eine reproduktionsmedizinische Behandlung von lesbischen Frauen. Aus Sicht der Lesben mit Kinderwunsch bzw. der LDI Familien wären daher der Zugang zu reproduktionsmedizinischer Behandlung für alleinstehende Frauen und Lesbenpaare in Deutschland, die Möglichkeit einer freien Wahl zwischen anonymem Spender und Ja-Spender, die Möglichkeit auch schwule Männer als Spender zu haben und eine rechtliche Entkoppelung eines Spenders von der rechtlichen Vaterschaft, unabhängig von dem Familienstand der austragenden Mutter wünschenswert. Die derzeitigen Einschränkungen verletzen das Selbstbestimmungsrecht von Lesben erheblich und verhindern die gebührende Unterstützung für ihre Lebensentscheidungen. Unter ethischen Aspekten wäre die reproduktive Autonomie lesbischer Frauen also nicht einzuschränken, sondern es müssten ihnen ähnliche Möglichkeiten offen stehen wie heterosexuellen Paaren. Da zudem die Forschung dafür spricht, dass die so gezeugten Kinder keine Nachteile erfahren, wird auch weder gegen das Prinzip der Fairness noch gegen das Nichtschadensgebot verstoßen.

Insofern können Lesben mit Kinderwunsch nicht als eine ethische Herausforderung für die Reproduktionsmedizin interpretiert werden. Die Herausforderung 
besteht eher darin, die konsistenten Ergebnisse in den soziokulturellen und rechtlichen Raum zu transportieren, so dass z.B. die Gesetzgebung und die ärztlichen Richtlinien diese Erkenntnisse reflektieren. Eine weitere Herausforderung besteht darin, ein Bewusstsein für subtile Erscheinungsformen von Homophobie und Heterosexismus zu schaffen, so dass diese abgebaut werden können. 



\title{
Die Eizellspende - eine Chance für wen?
}

\author{
Giselind Berg
}

Die Einführung der In-vitro-Fertilisation (IVF) im Jahr 1978 eröffnete Paaren eine Chance auf Elternschaft, die bis dahin nicht in der Lage waren, ihren Wunsch nach einem eigenen Kind zu realisieren. Sie war zunächst vorgesehen, um Frauen mit undurchlässigen oder fehlenden Eileitern zu einem Kind zu verhelfen. Neue Techniken sowie eine stetige Erweiterung der ursprünglichen Indikation, darunter auch die Indikation zur Eizellspende, führten zu einem enormen Anstieg der Behandlungen und der IVF-Zentren.

\section{Die Indikationen}

Als Indikationen für die Eizellspende, die seit über 20 Jahren durchgeführt wird, galten ursprünglich ovarielle Störungen durch Bestrahlung der Eierstöcke nach Krebs oder eine vorzeitige Menopause. Inzwischen sind der Kinderwunsch postmenopausaler Frauen sowie fehlgeschlagene IVF-Versuche als wesentliche Anwendungsbereiche hinzugekommen. Außerdem hat der Aufschub der Familiengründung die altersbedingte Unfruchtbarkeit von Frauen anwachsen lassen und der Einsatz fremder Eizellen - von jungen Spenderinnen - verspricht in diesen Fällen eine bessere Erfolgsquote. Seit kurzem gibt es eine neue Indikation außerhalb der IVF: den Einsatz humaner Eizellen für die embryonale Stammzellforschung und die Klonforschung (sog. „therapeutisches Klonen“). Die Human Fertilisation and 
Embryology Authority (HFEA) hat unlängst erlaubt, dass Engländerinnen ihre Eizellen altruistisch für diese Forschungsprojekte bereitstellen.

\section{Der Prozess}

Die Eizellspende kann als Teil einer IVF-Behandlung (egg-sharing) oder außerhalb eines solchen Programms stattfinden. Beim egg-sharing handelt es sich um ein Arrangement, bei dem eine Frau die Möglichkeit einer kostenlosen bzw. kostenreduzierten IVF erhält und als Gegenwert einen Teil ihrer Eizellen abgibt, wie dies in Belgien, Großbritannien oder den USA möglich ist. Als Alternative zur kommerzialisierten Eizellspende gedacht, soll so dem Mangel an Eizellen begegnet werden. ${ }^{1}$ Die Befürworter sehen darin die ideale Form der Eizellspende, da auf diese Weise keine Frau zusätzlichen gesundheitlichen Risiken ausgesetzt wird. Aus diesem Grund beschränken einige Länder wie Dänemark, Holland, Kanada und Israel die Eizellspende auf jene Frauen, die sich ohnehin einer IVF unterziehen. Dennoch ist egg-sharing nicht unumstritten. Es wird befürchtet, dass die Chancen auf eine Schwangerschaft bei der Spenderin geringer sind oder ihr durch die Aufteilung der Eizellen unter Umständen keine Embryonen mehr für weitere eigene Zyklen zur Verfügung stehen. Schwierig ist es auch, wenn die Empfängerin schwanger wird, die Spenderin jedoch ohne Kind bleibt.

Nimmt die Eizellspenderin nicht an einem IVF-Programm teil, findet zunächst eine ausführliche Anamnese der Spenderin und ihrer Familie statt. Anschließend folgen medizinische Untersuchungen: gynäkologisch und auf Infektionen, genetische Tests sollen die Übertragung von Krankheiten verhindern. Darüber hinaus interessiert der psychologische Hintergrund, dazu wird in Ländern wie den USA oder Spanien ein psychologisches Screening durchgeführt. ${ }^{2}$ Für die Spenderin beginnt die Behandlung mit der Einnahme eines Kontrazeptivums, um ihren Menstruationszyklus mit dem der Empfängerin zu synchronisieren. Dann erhält sie eine Hormonspritze mit der ihr Zyklus ausgesetzt wird, um nach zwei Wochen durch weitere Hormongaben ihre Eizellproduktion erneut anzuregen. Die Entnahme der Follikel wird mit Hilfe einer Kanüle durch die Scheidenwand vorgenommen. Vor Beginn der Behandlung ist der ,informed consent“ der Spenderin einzuholen, dem eine entsprechende Aufklärung vorausgehen soll. In einigen europäischen Ländern, z.B. in Belgien, England oder Frankreich, gibt es eine Beratungspflicht, um Spenderin wie Empfängerin neben den medizinischen auch über die mittelfristigen bzw. längerfristigen psychologischen Risiken sowie die komplexe soziale und rechtliche

\footnotetext{
1 Simons/Abuja, Egg-sharing: an evidence based solution to donor egg shortages, The Obstetrician and Gynaecologist 2005/7, $112 \mathrm{ff}$.

${ }^{2}$ Garrido/Zuquarregui/Meseguer/Simón/Remohy/Pellicer, Sperm and oocyte donor selection and management: experience of a 10 year follow-up of more than 2100 candidates, Human Reproduction $2002 / 17,3142 \mathrm{ff}$.
} 
Situation zu informieren. Die Beratung soll neben Behandlungsablauf und Risiken ausführlich über die Konsequenzen für das weitere persönliche Leben aufklären. Wie einige aktuelle Berichte zeigen, geschieht dies nicht überall bzw. nicht in ausreichender Form. Der erforderliche „informed consent" soll freiwillig und ohne finanziellen oder sozialen Zwang gegeben werden. Über die verschiedenen psychologischen Dimensionen, die im Kontext der Eizellspende von Bedeutung sind, geben die Richtlinien zur Infertilitätsberatung der European Society of Human Reproduction and Embryology (ESHRE) Auskunft. So kann für eine finanziell benachteiligte Spenderin in einem egg-sharing Programm die Freiwilligkeit ihres „,informed consent" eingeschränkt sein, denn bilden sich nicht genügend Eizellen, hat sie die Schwierigkeit, dass ihre eigene IVF nicht finanziert ist. In Fällen, in denen die Paare sich selbst um eine Spenderin kümmern, wie etwa in Frankreich, kann es zu Problemen der Spenderin mit ihrer Rolle gegenüber dem Kind kommen. Wird die genetische Herkunft dem Kind gegenüber offen gelegt, sind mögliche Folgen für die Spenderin wie ihre Familie zu berücksichtigen. Die Empfängerin der Eizellen ist auf die Möglichkeit hinzuweisen, dass der Transfer unter Umständen nicht stattfinden kann, etwa wenn die Spenderin die Behandlung kurz vor oder während der Stimulation abbricht. Ein weiteres wichtiges Thema in der Beratung ist darüber hinaus die Frage, wie die künftigen Eltern mit der Gametenspende gegenüber dem Kind wie auch ihrem sozialen Umfeld umgehen wollen. ${ }^{3}$

\section{Die Rechtliche Situation in Europa}

Während die Eizellspende in einigen Ländern Europas verboten ist (Österreich, Norwegen, Schweiz, Italien, Deutschland) ist sie in der Mehrheit der Länder erlaubt (Belgien, Dänemark, Estland, Finnland, Frankreich, Griechenland, Großbritannien, Lettland, Niederlande, Schweden, Slowenien, Slowakei, Spanien, Tschechien, Ungarn). Einige Länder, wie Irland, Litauen, Luxemburg, Malta oder Polen, haben keine Regelung. ${ }^{4}$ Die konkreten Anwendungsbedingungen unterscheiden sich erheblich. Je nach den Gegebenheiten des Landes kann die Eizellspende im Rahmen eines egg-sharing Programms durchgeführt werden, sie kann in einem altruistischen oder einem kommerziellen Modell erfolgen oder die Spenderin kann durch die Wunscheltern rekrutiert werden. Mitunter existieren mehrere Formen nebeneinander. Gemäß der jeweiligen Rechtslage kann die Spende anonym bzw. nicht anonym stattfinden. Für die EU gilt die Geweberichtlinie, die eine freiwillige und unentgeltliche Spende von Gewebe und Zellen vorschreibt. Über eine Kom-

\footnotetext{
${ }^{3}$ European Society of Human Reproduction and Embryology (Special Interest group), ,Psychology and Counseling"; Guidelines for Counseling in Infertility, Human Reproduchtion 2001/16, 1301 ff., http://www.eshre.com/emc.asp?pageId=372.

${ }^{4}$ European Commission, Report on the Regulation of reproductive Cell Donation in the European Union. Results of Survey. Directorate C - Public Health and Risk Assessment. C6 Health Measures, 2006.
} 
pensation für Ausgaben und Unannehmlichkeiten können die Länder entscheiden. ${ }^{5}$ Obwohl die Eizellspende im Prinzip freiwillig, altruistisch und nicht-kommerziell erfolgen soll, lassen sich in verschiedenen Ländern vielfältige Arten der Kostenerstattung feststellen. ${ }^{6}$ Selbst zwischen europäischen Ländern mit permissiver Gesetzgebung variiert die Praxis der Eizellspende erheblich, sei es in der Art der Anwendung oder auch der Präsentation des Themas in der Öffentlichkeit. Auf der einen Seite sehen wir England, ein hochgradig reguliertes System mit einer nichtanonymen Keimzellspende, wo Risiken und technische Entwicklungen öffentlich diskutiert werden. Auf der anderen Seite steht Spanien mit einem liberalen Recht und einer anonymen Spendenpraxis, wo die Eizellspende zwar überall öffentlich präsent ist, aber bis dato keine gesellschaftliche Debatte stattfindet. ${ }^{7}$

\section{Der Reproduktionstourismus}

Die steigende Nachfrage und der zunehmende Mangel an Eizellen in Industrieländern haben zu einem internationalen Eizellhandel und einem Fruchtbarkeitstourismus geführt. ${ }^{8}$ Es lassen sich mehrere Typen beobachten. Spenderinnen aus meist weniger wohlhabenden Ländern stellen ihre Eizellen in lokalen IVF-Zentren zur Verfügung, die unter Umständen von dort in andere Länder verschickt werden. Paare aus Industrieländern fahren in Fertilitätszentren süd/osteuropäischer bzw. außereuropäischer Länder. Es handelt sich dabei um eine der verschiedenen Formen des Medizintourismus, der dadurch gekennzeichnet ist, dass Menschen aus ihrer Heimatregion an einen anderen Ort reisen, um dort eine medizinische Behandlung durchführen zu lassen. Jones und Keith unterscheiden dabei zwei Typen: eine notwendige und eine freiwillige Form. ${ }^{9}$ Die erste tritt auf, wenn eine Behandlung am Herkunftsort nicht zugänglich oder illegal ist. Die zweite bezieht sich auf Behandlungen, die in der Heimat vorhanden sind, aber in einem anderen Land schneller oder billiger zu erlangen sind. Eine wachsende Zahl von Patienten aus Industrieländern reist inzwischen für medizinische Therapien in ärmere Länder. Dieser Trend kann Schwellenländer dazu veranlassen, auf Kosten der Basisgesundheitsversorgung ihrer Bürger für ausländische Patienten technologieintensive Angebote bereitzustellen. Außerdem kann auf diese Weise die Abwanderung von

\footnotetext{
${ }^{5}$ Richtlinie 2004/23/EG des europäischen Parlaments und des Rates zur Festlegung von Qualitätsund Sicherheitsstandards für die Spende, Beschaffung, Testung, Verarbeitung, Konservierung, Lagerung und Verteilung von menschlichen Geweben und Zellen v. 31.3.2004, Abl. Nr. L. 102/48.

${ }^{6}$ Heng, Legal and ethical issues in the international transaction of donor sperm and eggs, Journal of Assisted Reproduction and Genetics 2007/24, 107 ff.

7 Alkorta-Idiaker, Egg donation in Spain. European Biopolitics Conference. Connecting Civil Society - Implementing Basic Values, Berlin, 17.-19.3.2006.

8 Heng, The advent of the international "mail-to-order" egg donation, British Journal of Obstetrics and Gynaecology 2006/113, 1225 ff.

9 Jones/Keith, Medical tourism and reproductive outsourcing: the dawning of a new pradigm for healthcare, International Journal of Fertility and Women's Medicine 2006/51, 251 ff.
} 
Fachkräften vom öffentlichen in den privaten Gesundheitssektor verstärkt werden, wodurch sich die Zugangschancen für die lokale Bevölkerung vermindern. Wegen der nachfragebedingt steigenden Preise werden lokale Patienten von Behandlungen ausgeschlossen. Beispiele in verschiedenen Schwellenländern, z.B. Thailand oder Indien, zeigen bereits die verzerrenden Effekte dieses Trends und stellen die Frage nach sozialer Gleichheit bei der Verteilung der knappen Ressourcen. ${ }^{10}$

Mittlerweile haben verschiedene IVF-Zentren Programme entwickelt, für die sie Eizellen aus armen Ländern beziehen. ${ }^{11}$ Umgekehrt reisen deutsche, österreichische oder italienische Frauen nach Tschechien, Spanien, in osteuropäische oder baltische Länder sowie nach Südafrika. Ähnliches gilt auch für Frauen aus Großbritannien, wo zwar die Eizellspende erlaubt ist, aber aufgrund der Aufhebung der Spenderanonymität ein dramatischer Rückgang der Spendebereitschaft befürchtet wird. ${ }^{12}$ Auch französische Paare gehen wegen Eizellmangels ins Ausland, obwohl die Verwendung fremder Eizellen möglich ist. Spanien - mit 203 autorisierten IVF-Kliniken (2003) - hat die größte Zahl von IVF-Zentren in Europa. Spenderinnen in privaten Kliniken, das sind ungefähr $80 \%$ aller spanischen Zentren, erhalten ein Entgelt von etwa $€$ 900. Lediglich 7 der 38 öffentlichen IVFZentren bieten die unentgeltliche Eizellspende an, ihre Wartelisten reichen bis zu drei Jahren. ${ }^{13}$ Um die Nachfrage nach hellhäutigen Spenderinnen zu befriedigen, versuchen spanische IVF-Zentren diesen Typus aus der Gruppe polnischer oder russischer Immigrantinnen zu rekrutieren. Osteuropäische IVF-Zentren, z.B. auf Zypern, versuchen Patientinnen aus westlichen Ländern anzusprechen, indem sie neben der Fruchtbarkeitsbehandlung noch einen klinikeigenen Strand offerieren. Junge Frauen aus der Ukraine werden eingeladen, Eizellen gegen eine Aufwandsentschädigung in Höhe von $\$ 500 \mathrm{zu}$ spenden. ${ }^{14}$ In den Vereinigten Staaten sind alle reproduktionsmedizinischen Angebote erlaubt, es ist das Land, in dem weltweit die meisten Behandlungen mit fremden Eizellen durchgeführt werden. Dennoch reisen viele Amerikanerinnen zur Behandlung ins Ausland, nach Südafrika, Spanien oder Griechenland, wo die Kosten nur etwa 10\% der heimischen Preise ausmachen. Paare, die dunkelhäutige Spenderinnen suchen, fliegen nach Kamerun oder Burkina Faso. ${ }^{15}$ Obwohl nach amerikanischer Rechtslage der Verkauf menschlicher Körperteile verboten ist, hat sich der Handel mit Eizellen zu einem blühenden Markt entwickelt, der es erlaubt, Spenderinnen zu suchen, die spezifischen Wünschen entsprechen. Gemäß dem Prinzip von Angebot und Nachfrage

\footnotetext{
${ }^{10}$ de Arellano, Patients without borders, International Journal of Health Services 2007/37, 193 ff.

${ }^{11}$ Heng (Fußn. 8).

12 Frith/Blyth/Farrand, UK gamete donors' reflections on the removal of anonymity: implications for recruitment, Human Reproduction 2007/22, 1675 ff.

13 Alkorta-Idiakez (Fußn. 7).

${ }^{14}$ Barnett/Smith, Cruel cost of the human egg trade, The Observer 30.4.2006; http://observer.guardian.co.uk/uk_news/story/0,1764680,00.html.

15 Withrow, Trade in the human eggs thrives as infertile couples seek donors, International Herald Tribune 29.1.2007.
} 
hat der Mangel von Spenderinnen aus spezifischen ethnischen Gruppen (Ostasiaten, Juden) zu entsprechend höheren Vergütungen geführt. Als Eigenschaften, die den Marktpreis für Eizellen bestimmen, haben sich für die Spenderinnen die ethnische Herkunft, die Ausbildung, ihre Beschäftigung sowie das Aussehen herausgestellt. ${ }^{16}$

Die Entwicklung des Reproduktionstourismus hat zum einen mit einer Erweiterung der Angebotspalette zu tun, zum anderen kommen vor allem die strukturellen und ökonomischen Unterschiede zwischen den Ländern bzw. Regionen zum Tragen. Das zeigt sich einerseits in den USA, wo nahezu keine Beschränkungen existieren und andererseits in Frankreich, wo ein Mangel an Eizellen herrscht, obwohl die Eizellspende zugelassen ist. Besonders schwierig ist die Situation in Ländern ohne gesetzliche Regelung und somit quasi einem rechtsfreien Raum.

\section{Die Motivation}

Als gewünschte Motivation für eine Gametenspende gilt im Prinzip eine altruistische Orientierung. ${ }^{17}$ Die Bereitschaft Eizellen zu spenden, ist offensichtlich auch kulturell determiniert: Frauen asiatischer Herkunft zeigten sich im Vergleich mit Europäerinnen weniger dazu bereit, wobei keine Beziehung von Altruismus und Spendenbereitschaft besteht. ${ }^{18}$ Eigentlich soll eine Eizellspende auch in den USA unentgeltlich, mit einer Kompensation der Unkosten, erfolgen. Amerikanische Studien lassen jedoch Zweifel an den viel bekundeten altruistischen Motiven aufkommen. Es zeigte sich, dass auch Frauen, die sich für ihre Eizellspende an Agenturen gewandt haben, erklären „helfen zu wollen“. Vor ihrer Aufnahme in die Spenderinnenkartei müssen sie Fragebögen ausfüllen, die auf der Homepage des Institutes präsentiert werden. In einer Studie, die diesen Prozess in Agenturen (für Ei- bzw. Samenspende) analysiert hat, trat zu Tage, dass das Personal der Agenturen beim Ausfüllen der Formulare großen Einfluss nimmt. Im Hinblick auf die geschlechtspezifischen Erwartungen ihrer Kunden vermitteln sie an die Spenderin, sich nicht finanziell interessiert zu zeigen. Bei Samenspendern wird es akzeptiert, wenn sie ihre finanziellen Interessen offen legen. ${ }^{19}$

Zunehmend kommen auch monetäre Gründe zur Sprache. Um genügend Spenderinnen zu haben, hatten amerikanische IVF-Zentren ihre Kompensation von \$ 2.500 auf \$ 5.000 erhöht. In der Folge gaben deutlich mehr Frauen an, ihre

\footnotetext{
16 Heng, Factors influencing the reimbursement of egg donation with a competitive free-market system, Reproductive Biomedicine Online 2007/15, $16 \mathrm{ff}$.

${ }^{17}$ Fielding, Motivation, attitudes and experience of donation: a follow-up of women donating eggs in assisted conception treatment, Journal of Community and Applied Social Psychology 1998/8, 273 ff.

18 Purewal/v.d.Akker, British women's attitudes towards oocyte donation: ethnic differences and altruism, Patient Education and Counseling 2006/64, $43 \mathrm{ff}$.

19 Ameling, "Why do you want to be a donor?": gender and the production of altruism in egg and sperm donation, New Genetics and Society 2006/25, 143 ff.
} 
Eizellen aus finanziellen Motiven abzugeben. ${ }^{20}$ Es wird allerdings befürchtet, dass diese Summe für junge Frauen eine Versuchung darstellen kann, dass sie möglicherweise nicht in der Lage sind, die Risiken von Überstimulation und Eizellpunktion gegen diese Vergütung angemessen abzuwägen. ${ }^{21}$ In Zeitungen und Universitätsmagazinen bieten Agenturen mitunter $\$ 50.000$ bis $\$ 80.000$ für Eizellen von attraktiven, sportlichen oder musikalischen Harvardstudentinnen. ${ }^{22}$ Es darf bezweifelt werden, dass diese Preise jemals bezahlt werden, vermutlich sind es Publicity Aktionen, um Spenderinnen anzulocken. Frauen mit Abschlüssen amerikanischer Eliteuniversitäten, z.B. Harvard, Princeton oder Yale, sollen für ihre Eizellen \$ 10.000 und mehr erzielen können. ${ }^{23}$ Die amerikanische Gesellschaft für Reproduktionsmedizin hat eine Untersuchung durchführen lassen, in der die Programme ihrer Mitglieder nach der Kompensation für ihre Eizellspenderinnen befragt wurden. In 207 der 394 Kliniken wurde eine Standardkompensation von durchschnittlich ca. \$5.000 ermittelt. Regional zeigten sich erhebliche Abweichungen, einzelne Kliniken zahlten deutlich mehr: von \$ $10.000 \mathrm{im}$ Nordosten bis zu \$ $15.000 \mathrm{im}$ Westen der Vereinigten Staaten. ${ }^{24} \mathrm{Ob}$ und inwieweit finanzielle Anreize auch für die Motivation der zahlreichen jungen spanischen Spenderinnen, überwiegend Studentinnen, eine Rolle spielen, ist nicht untersucht.

Seit menschliche Eizellen - von jungen und gesunden Frauen - für Stammzellforschung und „therapeutisches Klonen“ gefordert werden, wurden die Bedingungen der Eizellspende neu ins öffentliche Bewusstsein gerückt, es geht allerdings dabei hauptsächlich um eine Frage, woher die gewünschten Eizellen kommen sollen. Es ist eine heftige Kontroverse darüber entbrannt, wie die damit verbundenen Risken für die Frauen einzuschätzen sind und was als adäquate Entlohnung gelten kann. Soll, wie bisher, eine Aufwandsentschädigung für die Unkosten gezahlt werden oder ist eher eine Kompensation für Zeit, Unannehmlichkeiten und Beschwerden angemessen, wie dies die Internationale Gesellschaft für Stammzellforschung (ICSCR) fordert. ${ }^{25}$ Gegen diesen Vorschlag wird eingewendet, dass auf diese Weise ein unangemessener Anreiz geschaffen wird, durch den insbesondere arme Frauen sich genötigt sehen könnten, Eizellen abzugeben. ${ }^{26}$

\footnotetext{
${ }^{20} \mathrm{Klock} /$ Stout/Davidson, Psychological characteristics and factors related to willingness to donate again among anonymous oocyte donors, Fertility and Sterility 2003/79, $1312 \mathrm{ff}$.

${ }^{21}$ Lindheim/Chase/Sauer, Assessing the influence of payment on motivations of women participating as oocyte donors. Gynecologic Obstetric Investigation 2001/52, $89 \mathrm{ff}$.

22 Steinbock, Payment for egg donation and surrogacy, The Mount Sinai Journal of Medicine 2004/71, $255 \mathrm{ff}$.

23 Spar, The egg trade - making sense of the market for human oocytes, New England Journal of Medicine 2007/13, $1289 \mathrm{ff}$.

${ }^{24}$ Covington/Gibbons, For the Society for Assisted Reproductive Technology. What is happening to the price of eggs? Fertility and Sterility 2007/5, 1001 ff.

${ }^{25}$ Hyun, Fair payment or undue inducement?, Nature 2006/442, 629-630.

${ }^{26}$ Check, Ethicists and biologists ponder the price of eggs, Nature 2006/442, 606-607.
} 


\section{Risiken der Eizellspende}

„Die Eizellspende ist - in den Worten der englischen Regulierungsbehörde HFEA - ein medizinischer und operativ invasiver Prozess, der körperlich anstrengend, schmerzhaft, stressreich sein kann und als Teil einer IVF-Behandlung nicht frei von realen und potenziellen gesundheitlichen Risiken ist. Dies gilt vor allem für das Überstimulationssyndrom, das zwischen 1-10\% variiert und in der schweren Form bis zu 2\% der IVF-Behandlungen auftritt" ${ }^{27}$ nach Meinung anderer Autoren kann es bei bis zu 5\% der Fälle zum schweren Überstimulationssyndrom kommen. ${ }^{28}$ Lebensbedrohliche Flüssigkeitsansammlungen in Bauchraum und Lunge können schwere Funktionsstörungen zur Folge haben, die von Atemnot bis zu Nierenversagen reichen können. ${ }^{29}$ Sie machen eine intensivmedizinische Behandlung erforderlich, aus England wird über fünf Todesfälle berichtet, die im Rahmen einer Eizellspende aufgetreten sind. ${ }^{30}$ Wird in Folge eines schweren Überstimulationssyndroms ein operativer Eingriff notwendig, kann dieser die zukünftige Fruchtbarkeit der Spenderin beeinträchtigen. Bei der operativen Entnahme der Eizellen kann es zu Blutungen, Organverletzungen oder Infektionen kommen.

Schwangere nach Eizellspende haben - auch im Vergleich zu sonstigen IVF Patientinnen - ein erhöhtes Risiko für einen schwangerschaftsinduzierten Bluthochdruck und für Präeklampsie, nicht nur bei den ohnehin erhöhten Mehrlingschwangerschaften. Diese stellen wegen der überdurchschnittlichen Kaiserschnittrate, den häufigen Frühgeburten mit untergewichtigen Kindern ein Risiko für Mutter und Kind dar, ${ }^{31}$ das unabhängig vom Alter der Mutter besteht. ${ }^{32}$ Ungeklärt ist bisher die Frage nach möglichen Langzeitfolgen der Medikamente, die Entstehung hormonabhängiger Karzinome wird nach wie vor kontrovers diskutiert. Nach derzeitigem Kenntnisstand soll für Frauen, die mit ovulationsauslösenden Medikamenten behandelt wurden, kein erhöhtes Krebsrisiko bestehen. Die Analysen zeigten jedoch bei infertilen, mit Clomifen behandelten Frauen, ein erhöhtes Risiko für Brustkrebs, ${ }^{33}$ Eierstock- oder Gebärmutterkrebs, sowie weitere

27 O'Toole, Procurement of Gametes and Embryos for Research, Human Fertilisation and Embryology Authority. Authority Paper, 15.2.2006, 294, pp. 4-5.

${ }^{28}$ Delvigne/Rozenberg, Epidemiology and prevention of ovarian hyperstimulation syndrome (OHSS): a review, Human Reproduction 2002/8, 559 ff.

29 Pearson, Health effects of egg donation may take decades to emerge, Nature 2006/442, 607-608.

30 Spar (Fußn. 23).

31 Wiggins/Main, Outcomes of pregnancies achieved by donor egg in vitro fertilization - a comparison with standard in vitro fertilization pregnancies, American Journal of Obstetrics and Gynecology 2005/6, $2002 \mathrm{ff}$.

32 Sheffer-Mimouni/Mashiach/Dor/Levra/Seidman, Factors influencing the obstetric and perinatal outcome after oocyte donation, Human Reproduction 2002/17, 2636.

${ }_{33}$ Lerner-Geva/Keinan-Boker/Blumstein/Boyko/Olmar/Masbiach/Rabinovici/Potashnik/Lunenfeld/Schenker/ Shushan/Fisbman/Coben/Vagman/Lunenfeld, Infertility, ovulation induction treatments and the incidence of breast cancer - a historical prospective cohort of Israeli women, Breast Cancer Research and Treatment 2006/100, $201 \mathrm{ff}$. 
Krebsformen. Die Studien sind allerdings inkonsistent. ${ }^{34}$ Ein weiteres Problem besteht darin, dass einige der zur Stimulation eingesetzten Medikamente für diese Verwendung nicht zugelassen und hinsichtlich ihrer Langzeitwirkungen nicht adäquat untersucht sind. ${ }^{35}$

\section{$7 \quad$ Risikoinformation der Spenderin}

Hinsichtlich der Risikoinformation der Spenderinnen ist die Situation in verschiedenen Ländern nicht zufrieden stellend. Aus amerikanischen Studien geht hervor, dass vor allem im kommerziellen System die Aufklärung unzureichend ist. Bei telefonischen Anfragen entsprechender Programme wurden unvollständige und/oder ungenaue Angaben gemacht. ${ }^{36}$ Dies bestätigen auch ehemalige Spenderinnen, die darüber hinaus weitere Aspekte des Rekrutierungsprozesses, die Bedeutung des Geldes oder der medizinischen Betreuung kritisieren. ${ }^{37}$ Voraussetzung für einen ,informed consent" ist jedoch eine umfassende und akkurate Information über kurz- und langfristige Risiken der Stimulation sowie der Eizellentnahme. ${ }^{38}$ So berichten junge Frauen, z.B. russische Immigrantinnen, die nach Zypern zur Eizellspende kommen, dass sie weder über die medizinischen Risiken aufgeklärt wurden, noch ihnen jemand psychologische Beratung angeboten habe. Als Motiv nennen sie Geld; einige Frauen, die den Prozess mehrfach durchlaufen haben, betrachten die Eizellspende als ihre Haupteinnahmequelle. ${ }^{39}$

Unlängst gab es Beispiele aus Rumänien, wo junge Frauen in Folge einer Eizellspende schwere gesundheitliche Schäden davon getragen haben und von der Klinik (GlobalArt), in der die Entnahme vorgenommen wurde, keine medizinische Hilfe erhalten haben. Sie erklärten, dass sie nicht entsprechend aufgeklärt wurden. Mit dem Erlös (\$ 100 - \$250) wollten sie die Lebensbedingungen für sich und ihre Familien aufbessern. In Rumänien ist die Eizellspende nicht gesetzlich geregelt. Dementsprechend ist auch die Frage ungeklärt, wer für etwaige gesundheitliche Folgekosten, z.B. nach einem schweren Überstimulationssyndrom, aufkommt, wenn, wie in diesem Fall, die Frauen dazu nicht in der Lage sind. Die Eizellen waren im Rahmen eines Kooperationsprojektes für Frauen in London bestimmt, die dortige (Bridge) Klinik arbeitet inzwischen mit einem Zentrum in Kiew zusammen.

\footnotetext{
${ }^{34}$ Brinton/Westhoff/Scoccia/Lamb/Althuis/Mabie/Moghissi, Causes of infertility as predictors of subsequent cancer risk, Epidemiology 2005/16, 500 ff.

35 Beeson/Lippman, Egg harvesting for stem cell research: medical risks and ethical problems, Reproductive Biomedicine Online 2006/13, 573 ff; Pearson (Fußn. 29).

36 Gurmakin, Risk information provided to prospective oocyte donors in a preliminary phone call, American Journal of Bioethics 2001/1, 3 ff.

37 Kalfoglou/Gittelson, A qualitative follow-up study of women's experiences with oocyte donation, Human Reproduction 2000/15, 798.

38 Schneider, Oocyte donation for reproduction and research cloning - the perils of commodification and the need for European and international regulation, Law and the human genome Review 2006/25, 205 ff.; Spar (Fußn. 23).

39 Barnett/Smith (Fußn. 14).
} 
Dieses zahlt den Spenderinnen eine Aufwandentschädigung von etwa $€$ 300, während britische Paare für die Behandlung bis zu $£, 16.000$ aufwenden müssen. ${ }^{40}$ GlobalArt stellte Eizellen aus Rumänien auch für israelische und amerikanische Zentren bereit. Dazu wird das Sperma zuvor in den USA kryokonserviert nach Rumänien geschickt, um damit die gespendeten Eizellen zu fertilisieren. Die daraus resultierenden Embryos werden eingefroren und kommen per Luftpost in die USA zurück, wo sie der Empfängerin implantiert werden. Der Zyklus wird in diesem Fall mit \$ 13.650 berechnet. ${ }^{41}$ Andere Frauen, denen auf Zypern Eizellen entnommen wurden, berichteten von einer erfolgsabhängigen Entlohnung. Je größer die Zahl der Eizellen, umso größer der Betrag, £ 420 für einen Zyklus mit 12 Eizellen, wenn sie mehr Eizellen produzierten, wurden $£ 500$ bezahlt. Damit wurde ein Anreiz geschaffen, möglichst viele Eizellen bereitzustellen, wodurch die Frauen dem Risiko eines Überstimulationssyndroms ausgesetzt wurden. Frauen, die von Westeuropa für eine Eizellspende nach Zypern fahren, haben dafür $\$ 5.000 \mathrm{zu}$ entrichten. Dort befragte Ärzte betonen, dass ihre Patientinnen alle Informationen erhalten und ihre Spenderinnen aus altruistischen Motiven handeln. Ein Reproduktionsmediziner eines ukrainischen Zentrums in Kiew weist darauf hin, dass in seiner Klinik die Spenderinnen nicht bezahlt werden, sondern, wie in westeuropäischen Ländern, eine Kompensation (\$300) für ihre Zeit erhalten. ${ }^{42}$

\section{Die Akteure}

In der wissenschaftlichen Darstellung haben die Akteure im Prozess der Eizellspende unterschiedliches Gewicht. Zunächst die Spenderinnen, mit ihnen beschäftigt sich eine Fülle von kleineren Studien. Dabei geht es - neben ihren psychologischen Charakteristika - um ihre Einstellungen zu Spendenbereitschaft, Anonymität, und Offenlegung der Abstammung oder um ihre Zufriedenheit mit dem Behandlungsverlauf.43 Seltener spielen Beschwerden, Schmerzen oder Ängste und negative Emotionen, psychiatrische Symptome bzw. langfristige Nebenwirkungen nach Abschluss der Eizellspende eine Rolle. ${ }^{44}$ Von Seiten der Spenderin-

\footnotetext{
40 Schindele/Zimmermann, Rohstoff für das Mutterglück, Die Zeit 2007/4.

41 Buying babies, bit by bit. An international guide to baby-making, The Economist 19.12.2006, http://www.economist.com/science/PrinterFriendly.cfm?story_id=8345513.

42 Barnett/Smith (Fußn. 14)

${ }^{43} \mathrm{Hahn} / \mathrm{Craft}$-Rosenberg, The disclosure decisions of parents who conceive children using donor eggs, Journal of Obstetric, Gynecologic and Neonatal Nursing 2002/31, 283 ff.; Jordan, Anonymous oocyte donation: a follow-up analysis of donors' experiences, Journal of Psychosomatic Obstetrics and Gynecology 2004/25, 145 ff.

${ }^{44}$ Jordan (Fußn. 43); Klock/Stout/Davidson (Fußn. 20); Abuja/Mostyn/Simons, Egg sharing and egg donation: attitudes of British egg donors and recipients, Human Reproduction 1997/12, 2845 ff.
} 
nen besteht großes Interesse zu erfahren, zu welchem Ergebnis ihre Spende geführt hat, ob also mit Hilfe ihrer Eizellen eine andere Frau ein Kind geboren hat. ${ }^{45}$

Paare - als weitere Akteure -, die sich ihren Kinderwunsch mit fremden Eizellen erfüllen wollen, interessieren sich für mehrere Themen: zunächst für die Gesundheit der Spenderin, um die Übertragung infektiöser oder genetischer Krankheiten zu vermeiden. Hier kann sich unter Umständen die Frage nach einer möglichen Haftung von Spenderin oder Arzt stellen, falls das Kind mit einer vorher bekannten, angeborenen Krankheit zur Welt kommt. ${ }^{46}$ Das Interesse der Eltern in spe richtet sich darüber hinaus auf den kulturellen und sozialen Hintergrund, das psychische Profil der Spenderin. Aber auch ihre eigene Beziehung zum künftigen Kind ist für sie ein sehr wichtiges Thema. ${ }^{47}$ Hier nimmt die Frage, wie mit der Offenlegung der Konzeptionsmethode umgegangen werden soll, eine zentrale Stellung ein, sei es gegenüber dem Kind oder im Familien- und Bekanntenkreis. ${ }^{48}$ Dass dies in der Praxis ein schwieriges und komplexes Thema ist, macht eine französische Studie deutlich, in der Eltern befragt wurden, wieweit sie ihre Kinder über die Eizellspende informiert haben. Innerhalb von 10 Jahren hatte die Mehrheit (70\%) der Paare zwar über die IVF, aber nicht über die Eizellspende gesprochen, dabei kamen die Hälfte der „symbolischen“ Spenderinnen, wie man sie nennt, aus der Familie (Schwester, Schwägerin, Cousine), ein weiteres Drittel aus dem Freundeskreis, nur in 10\% der Fälle stammte die Eizelle von fremden Frauen. Nach der Geburt des Kindes hatte ein Viertel der Familien keinen Kontakt zur Spenderin, 41\% der Familien haben sie zur Patin des Kindes erklärt und in $15 \%$ der Fälle wurde die Spenderin als Erste über die Geburt des Kindes informiert. ${ }^{49}$

Zwischen Spenderinnen und Empfängerinnen der Eizellen befindet sich die Gruppe der professionellen Akteure, in erster Linie Mediziner, Embryologen und Biologen. Je nach der lokalen Situation können auch Vermittlungsagenturen und Juristen involviert sein. Sind medizinische Professionelle an Rekrutierung, Beratung oder Kostenerstattung von Spenderinnen beteiligt, kann es zu einem Interessenkonflikt kommen. Um mehr Patienten zu gewinnen, könnte privaten Kliniken daran gelegen sein, Eizellspenderinnen zu gewinnen, um ein attraktives Angebot zu

45 Fielding (Fußn. 17); Kalfoglou/Geller, A follow-up study with oocyte donors exploring their experiences, knowledge, and attitudes about the use of their oocytes and the outcome of the donation, Fertility and Sterility 2002/74, $660 \mathrm{ff}$.

46 Carey, Wrongful life and wrongful birth: legal aspects of failed genetic testing in oocyte donation, Penn Bioethics Journal 2005/2, 1 ff.

47 Greenfield/Klock, Parents' knowledge about the donors and their attitudes toward disclosure in oocyte donation, Human Reproduction 2004/19, 1575 ff.; Garrido/Zuzuarregui/Meseguer/Simón/ Remohy/Pellicer (Fußn. 2); Hershberger, Recipients of oocyte donation: an integrative review, Journal of Obstetric, Gynecologic and Neonatal Nursing 2004/33, 610 ff.

48 v.d.Akker, A review of family donor constructs: current research and future directions, Human Reproduction 2006/12, 91 ff.; Hershberger/Klock/Barnes, Disclosure decisions among pregnant women who received donor oocytes: a phenomenological study, Fertility and Sterility 2007/87, $288 \mathrm{ff}$.

${ }^{49} \mathrm{Karpel} /$ Flis-Treves/Blanchet/Olivennes/Frydman, Oocyte donation: parents's secrets and lies. European Journal of Obstetrics \& Gynecology and Reproductive Biology 2005/34, 557 ff. 
haben. Hier besteht die Gefahr, dass das Wohl der Spenderin den Interessen der Empfängerin untergeordnet wird. Um entsprechende Konflikte zu vermeiden, wird vorgeschlagen, die Infertilitätsbehandlung von der Rekrutierung der Spenderinnen zu trennen und diese an unabhängige Institutionen zu übertragen, wie es in England oder Frankreich geschieht. ${ }^{50}$

Angesichts der umfangreichen Literatur zur Eizellspende, nehmen sich Zahl und Samplegröße der Studien über die Entwicklung der daraus hervorgegangenen Kinder bescheiden aus. Es wird ihnen eine normale körperliche und emotionale Entwicklung bescheinigt, ${ }^{51}$ sowie eine gutes Verhältnis zu ihren Eltern, es ist sogar besser, als die Beziehung von natürlich gezeugten Kindern zu ihren Eltern. ${ }^{2}$ Kein Entwicklungsunterschied zeigte sich bei (17) Zwölfjährigen, die aus einer Eizellspende entstanden sind und einer Vergleichsgruppe von IVF-Kindern. ${ }^{53}$ In den Familien wurde allerdings eine geringe Bereitschaft beobachtet, die Herkunft der Kinder offen zu legen. ${ }^{54}$ Ausgehend von Erfahrungen aus der Adoptionsforschung wird im Hinblick auf die Identitätsfindung des Kindes angenommen, dass seine Entwicklung durch das Zurückhalten von Informationen über seine Entstehung ungünstig beeinflusst wird und Geheimnisse in der Familie nachteilige Wirkungen auf die Kinder haben können. ${ }^{55}$ Für Kinder nach Gametenspende ist die Frage nach der Bedeutung von Offenheit bzw. Geheimhaltung nicht untersucht. ${ }^{56} \mathrm{Um}-$ in Übereinstimmung mit der UN-Kinderrechtskonvention - das Recht von Kindern auf Kenntnis ihrer Herkunft umzusetzen, haben einige Länder - Schweden, Niederlande, Norwegen, Österreich und die Schweiz - die anonyme Gametenspende rechtlich ausgeschlossen. Angesichts der Datenlage werden zur Einschätzung des Kindeswohls Langzeitstudien gefordert. ${ }^{57}$ Trotz einer 20jährigen Praxis mit mehr als 100.000 Behandlungszyklen gibt es bisher noch keine aussage-

${ }^{50}$ Heng, Should fertility doctors and clinical embryologists be involved in the recruitment, counselling and reimbursement of egg donors? Journal of Medical Ethics 2008/34, 414; Kalfoglou/Geller, Navigating conflict of interest in oocyte donation: A analysis of donors' experiences, Womens Health Issues 2000/10, $226 \mathrm{ff}$.

51 Applegarth/Goldberg/Cholst/McGoff/Fantini/Zellers/Black/Rosenwaks, Families created through ovum donation: a preliminary investigation of obstetrical outcome and psychosocial adjustment, Journal of Assisted Reproduction and Genetics 1995/12, 574 ff.; Söderström-Anttila/Sajaniemi/Tiitinen/Hovatta, Health and development of children born after oocyte donation compared with that of those born after in-vitro fertilization, and parents' attitudes regarding secrecy, Human Reproduction 1998/13, $2009 \mathrm{ff}$.

${ }^{52}$ Golombok/Lycett/MacCallum/Murray/Rust/Abdalla/Jenkins/Margara, Parenting infants conceived by gamete donation, Journal of Family Psychology 2004/18, 443 ff.

53 Murray/MacCallum/Golombok, Egg donation parents and their children: follow-up at age 12 years, Fertility and Sterility 2006/85, $610 \mathrm{ff}$.

54 Murray/Golombok, To tell or not to tell: the decision-making process of egg-donation parents, Human Fertility 2003/6, 89 ff.

55 Golombok, Parenting and secrecy issues related to children of assisted reproduction, Journal of Assisted Reproduction and Genetics 1997/14, 375 ff.

56 European Society of Human Reproduction and Embryology (Special Interest group) (Fußn. 3).

57 Söderström-Anttila/Sajaniemi/Tiitinen/Hovatta (Fußn. 51). 
fähigen Studien zu den Langzeiteffekten der Eizellspende auf Spenderinnen wie Empfängerinnen, die Kinder und die Familien. ${ }^{58}$

\section{Welche Chancen, welche Risiken werden erkennbar?}

Betrachtet man die Akteure im Feld, vermittelt die Eizellspende zunächst Frauen oder Paaren die Hoffnung, ein biologisch oder genetisch zumindest mit einem Elternteil verwandtes Kind bekommen zu können. Zur Umsetzung ihres Zieles sind sie auf die Expertise der Reproduktionsmedizin angewiesen, wie auch auf die Bereitschaft anderer Frauen, ihre Eizellen abzugeben. Wie für die Paare steht auch für die professionelle Seite der Wunsch der Eltern in spe nach einem eigenen Kind im Vordergrund ihrer Handlungsperspektive. Darüber hinaus bleibt noch die Frage nach dem sog. „besten Interesse des Kindes“, sie ist sehr viel schwerer zu beantworten, wenn sie denn überhaupt gestellt wird. ${ }^{59}$ In der Literatur findet sich dazu wenig, vor allem, wenn man das Interesse über die kurz- und mittelfristigen Ergebnisse reproduktionsmedizinischer Behandlungen hinaus ausdehnt und nach Erkenntnissen zur weiteren Entwicklung dieser Kinder als Erwachsene sucht. ${ }^{60}$

Lassen sich die Vorteile der Eizellspende vor allem auf Seiten der Paare mit Kinderwunsch und der Professionellen ausmachen, liegen die Risiken überwiegend bei den Frauen als realen und potenziellen Eizellspenderinnen, auch wenn dies keinesfalls für alle Fälle zutreffend sein muss. Neben den gesundheitlichen Belastungen für die einzelne Spenderin sind mögliche Konsequenzen auf gesellschaftlicher Ebene in Betracht zu ziehen. Frauen, die Eizellen - für Reproduktion oder Forschung - spenden, werden gesundheitlichen Risiken ausgesetzt. Besonders altruistische Spenderinnen ohne eigene IVF nehmen Risiken in Kauf ohne einen eigenen therapeutischen Vorteil. Man kann also fragen, ob eine solche Intervention zugunsten einer dritten Person, wenn keine lebensbedrohliche Situation vorliegt, nicht im Widerspruch zum medizinischen Prinzip steht, „nicht zu schaden“.61 Frauen zur Spende aufzurufen, ist daher eine ethisch umstrittene Praxis, nicht zuletzt, da bisher für das „therapeutische Klonen“ noch jeglicher Nachweis einer Therapie aussteht. ${ }^{62}$ Befürworter der Stammzellforschung sehen in der Höhe der

\footnotetext{
58 Sauer/Kavic, Oocyte and embryo donation 2006: reviewing two decades of innovation and controversy, Reproductive Biomedicine Online 2006/12, $153 \mathrm{ff}$.

${ }^{59} \mathrm{Blyth} /$ Cameron, The welfare of the child. An emerging issue in the regulation of assisted conception, Human Reproduction 1998/13, 2339 ff.; Daniels/Blyth/Hall/Hanson, The best interests of the child in assisted human reproduction: the interplay between the state, professionals, and parents. Politics and the Life Science 2000, $33 \mathrm{ff}$.

${ }^{60}$ Turner/Coyle, What does it mean to be a donor offspring? The identity experiences of adults conceived by donor insemination and the implications for counselling and therapy, Human Reproduction 2000/15, $2041 \mathrm{ff}$.

${ }^{61}$ Schneider (Fußn. 38).

${ }^{62} \mathrm{Heng} /$ Tong/Stojkovic, The egg-sharing model for human therapeutic cloning research: managing donor selection criteria, the proportion of shared oocytes allocated to research, and amount of financial subsidy given to the donor, Medical Hypotheses 2006/66, $1022 \mathrm{ff}$.
} 
Kompensation keinen Anreiz, der den „informed consent“ der Frauen in Frage stellen könnte. ${ }^{63}$ Aus kritischer Perspektive wird es für problematisch gehalten, Eizellen für die Forschung zu entnehmen, da hier Fragen von Gesundheit und Wohlbefinden der Frauen sowie ihres gesellschaftlichen Status zur Debatte stehen. In diesem Kontext wird die Bedeutung des „informed consent“ skeptisch betrachtet. Bei der Abwägung von Kosten und Nutzen wird die Frage gestellt, ob in einem Klima starker ökonomischer und sozialer Einflüsse, die Risiken für die Frauen zunehmend als notwendiger Ausgleich für den wissenschaftlichen Fortschritt angesehen werden. ${ }^{64}$ Frauen aus weniger wohlhabenden, armen oder den ärmsten Ländern geben die Eizellen ab, um ihren Lebensunterhalt aufzubessern. Das betrifft Spenderinnen aus südosteuropäischen Ländern, den GUS-Staaten, Russland oder dem Baltikum ebenso wie arme Frauen aus Lateinamerika oder Afrika. Ökonomisch abhängige Frauen ermöglichen so zum einen Frauen in reichen oder reicheren Ländern mit ihrer Eizellspende die Chance auf ein Kind, jedoch keineswegs allen, die eine Eizellspende benötigen, sondern den finanziell Privilegierten. Auf diese Weise wird die Ungleichheit unter Frauen vertieft und die Ausbeutung armer und abhängiger Frauen verstärkt. Verschärft wird dieses Problem noch angesichts der wachsenden Nachfrage nach Eizellen für die Forschung. Sie wird wohl kaum durch altruistische Spenden zu decken sein. Ob der Mangel an Eizellen längerfristig durch technische Entwicklungen wie die In Vitro Maturation oder die erfolgreiche Kryokonservierung von Eizellen zu beheben sein wird, lässt sich derzeit noch nicht absehen. Von verschiedenen Seiten, Regierungen, Institutionen oder Forschern, wird die Frage gestellt, woher die Eizellen kommen sollen. Es wird für wahrscheinlich gehalten, dass es sich dabei vornehmlich um arme Frauen oder Frauen aus Entwicklungsländern handeln wird, die das Rohmaterial bereitstellen, um damit die Krankheiten der industrialisierten Welt zu behandeln. Selbst wenn die in Aussicht gestellten Therapien zur Anwendung kommen sollten, wird jedoch die Bevölkerungsmehrheit der armen Länder - wie auch bedürftige Gruppen in den Industrieländern - davon kaum profitieren. ${ }^{65}$ Schließlich verkaufen sie ihr reproduktives Material auf Grund ihrer ökonomischen Notlage, wäre ihre Situation nicht so verzweifelt, würden sie es kaum tun. ${ }^{66}$ Auf gesellschaftlicher Ebene zeigt der Handel mit den Eizellen - wegen der Entfremdung der Eizellen aus dem reproduktiven Kontext der Frau - dass sie zur Ware werden und somit der Kommerzialisierung des weiblichen Körpers Vorschub geleistet wird. ${ }^{67}$

\footnotetext{
${ }^{63}$ Robertson, Compensation for donation research, Fertility and Sterility 2006/86, $1573 \mathrm{ff}$.

${ }^{64}$ George, What about the women? Ethical and policy aspects of egg supply for cloning research, Reproductive Biomedicine Online 2007/15, 127 ff.

${ }^{65}$ Cregan, Ethics in medicine. Ethical and social issues of embryonic stem cell technology, International Medicine Journal 2005/2, $126 \mathrm{f}$.

66 Fox, Paying for particulars in people-to-be: commercialisation, commodification and commensurability in human reproduction, Journal of Medical Ethics 2008/5, $162 \mathrm{ff}$.

67 Awaya, Common ethical issues in regenerative medicine, International Journal of Bioethics 2005/16, 192-193.
} 


\section{Was ist also zu tun?}

Der Prozess der Eizellspende bedarf einer genauen wissenschaftlichen Überprüfung, wobei neben den medizinischen und psychologischen auch die sozialen Folgen für Spenderin wie Empfängerin der Eizellen zu berücksichtigen sind. Erforderlich sind langfristig angelegte Studien, die die Wirkungen der unterschiedlichen Medikamente ebenso wie die Gesundheit der Spenderinnen untersuchen. ${ }^{68}$ Wo noch keine vorhanden sind - wie in den USA - Standards für den ,informed consent" zu entwickeln. Dazu sind genaue, umfassende und unabhängige Informationen erforderlich, um sie den Interessentinnen zur Verfügung zu stellen. ${ }^{69}$ In den vertraglichen Vereinbarungen sind Regelungen für etwaige Folgekosten zu treffen, wenn sich die Eizellspenderin einer medizinischen Behandlung unterziehen muss, deren Kosten sind von den Wunscheltern oder einer Versicherung zu übernehmen. Im Hinblick auf die „besten Interessen des Kindes“ sollten Ei- und Samenspende nicht anonym durchgeführt werden. Angesichts der diversen Risiken und der Anforderungen der komplexen Situation im Rahmen einer Eizellspende ist es wichtig, in diesem Prozess neben den Interessen der Anbieter und der Eltern in spe deutlicher die Situation der Spenderinnen und vor allem der Kinder in den Blick zu nehmen. Nur dann lässt sich im Zusammenhang mit der Eizellspende von Chancen sprechen.

68 Stein, More long-term studies of oocyte donors are needed, Fertility and Sterility 2007/88, 756.

69 Schneider (Fußn. 38). 



\title{
Autorenverzeichnis
}

\author{
Dr. Giselind Berg
}

Giselind Berg arbeitet am Institut für Ökologie der Technischen Universität Berlin. Sie hat Soziologie und politische Wissenschaften mit den Schwerpunkten Medizinsoziologie und Soziologie der Entwicklungsländer studiert. Ihre Arbeitsgebiete sind Public Health/Gesundheitswissenschaften sowie Migration und Gesundheit (Promotion). Einer ihrer weiteren Forschungsschwerpunkte liegt in den unterschiedlichen Aspekten der menschlichen Reproduktion und Fragen zur Schwangerenversorgung sowie zur Geburtshilfe bzw. dem unerfüllten Kinderwunsch und seiner Bewältigung mit Hilfe neuer Reproduktionstechniken.

\section{Prof. Eric Blyth, CQSW BA MA PhD}

Eric Blyth is Professor of Social Work at the University of Huddersfield, England and visiting professor at the Department of Applied Social Sciences at Hong Kong Polytechnic University. He is Co-Chair of the British Association of Social Workers Project Group on Assisted Reproduction (PROGAR). He has researched and published extensively in the area of infertility and assisted conception and has presented conference papers in several European countries, Australia, Canada, Hong Kong, Israel, Japan, New Zealand, Taiwan and the United States.

\section{Dr. Gisela Bockenheimer}

Gisela Bockenheimer-Lucius ist approbierte Ärztin. Sie betreut seit 1989 die Redaktion und koordinierende Schriftleitung der Zeitschrift Ethik in der Medizin (Organ der Akademie für Ethik in der Medizin). Seit 1999 ist sie Wissenschaftliche Mitarbeiterin am Senckenbergischen Institut für Geschichte und Ethik der Medizin in Frankfurt am Main, Geschäftsführerin des Klinischen Ethik-Komitees am Universitätsklinikum, Mitglied und im Vorstand der Zentralen Ethik-Kommission der Bundesärztekammer (ZEKO), sowie Mitglied der IVF-Kommission der Landesärztekammer Hessen. Sie hat unter anderem zu moralischen Themen des Lebensbeginns und der Reproduktionsmedizin sowie zu Problemen der Ethikberatung in Altersheimen publiziert.

\section{Marilyn Crawshaw, BSc MA CQSW PhD}

Marilyn Crawshaw is a Senior Lecturer in Social Work at the University of York and has a long standing professional and academic interest in the impact of reproductive health, disability and the social context on individuals and their relation- 
ships. As well as practising as a social worker in this field for many years, she has researched into related areas including the experiences of fertility impairment associated with cancer, donor conception and adoption. She has been an Inspector and Adviser to the UK Human Fertilisation and Embryology Authority and is currently Co-Chair (with Professor Eric Blyth) of the multi-agency UK Project Group on Assisted Reproduction and national Adviser to UK DonorLink, the government funded voluntary information exchange and contact register for adults genetically related through donor conception.

\section{Dr. Sigrid Graumann}

Sigrid Grauman studierte Biologie und Philosophie an der Universität Tübingen. Nach einem dreijährigen Promotionsstipendium der DFG arbeitete sie seit 1997 als wissenschaftliche Angestellte an dem der Universität Tübingen zugehörigen Interfakultären Zentrum für Ethik in den Wissenschaften. Sie promovierte 1999 mit einer Arbeit zur Somatischen Gentherapie im Fach Humangenetik. Von 1997 bis 1999 betreute sie als wissenschaftliche Koordinatorin das "European Network for Biomedical Ethics: Ethical Problems of In Vitro Fertilisation (IVF) with Particular Regard to its Connections with Genetic Diagnosis and Therapy”. Von 1999 bis 2002 bearbeitete sie das DFG-Forschungsprojekt „In-vitro-Techniken am Beginn des menschlichen Lebens: ein Vergleich von Folgenanalysen und Bewertungsdiskursen“. Seit April 2002 ist sie wissenschaftliche Mitarbeiterin am Institut Mensch, Ethik und Wissenschaft Berlin.

\section{Dr. Lisa Herrmann-Green}

Lisa Herrmann-Green ist Angehörige der Universität Konstanz und promovierte Psychologin. Ihr Forschungsschwerpunkt gilt Regenbogenfamilien, insbesondere lesbischen Familien mit Wunschkindern durch eine Samenspende (LDI Familien). Mit ihrer Partnerin und ihren gemeinsamen Kindern lebt sie seit 18 Jahren in Deutschland und ist seit über zehn Jahren in der Beratung von Lesben mit Kinderwunsch tätig. Sie ist Vorstandsmitglied im Verband lesbischer Psychologinnen und schwuler Psychologen e.V. und Mitbegründerin von LesKids und LesFam.

\section{Prof. Dr. Werner Heun}

Werner Heun ist seit 1990 Professor an der Rechtswissenschaftlichen Fakultät der Universität Göttingen und Direktor des Instituts für Allgemeine Staatslehre und Politische Wissenschaften und Autor zahlreicher wissenschaftlicher Publikationen. Seine Forschungsschwerpunkte umfassen unter anderem deutsches und amerikanisches Verfassungsrecht, europäische und amerikanische Verfassungsgeschichte, Finanzrecht, Kirchenrecht, Staatstheorie und Medizinrecht. 


\section{Prof. Dr. Thomas Katzorke}

Thomas Katzorke ist approbierter Arzt und seit nunmehr dreißig Jahren als Facharzt für Frauenheilkunde und Geburtshilfe tätig. Er ist derzeit ärztlicher Leiter des Zentrums für Reproduktionsmedizin Essen. Seit 1996 ist er Vorstandsmitglied des Bundesverbandes Reproduktionsmedizinischer Zentren (BRZ), wurde 2003 zum außerplanmäßigen Professor für Gynäkologie und Geburtshilfe mit Schwerpunkt Endokrinologie und Reproduktionsmedizin an der Universität Padua berufen und ist seit 2006 als Vorsitzender des Arbeitskreises donogene Insemination tätig.

\section{Mag. Eva-Maria Knoll}

Eva-Maria Knoll absolvierte das Studium der Völkerkunde und ist derzeit wissenschaftliche Mitarbeiterin der Forschungsstelle Sozialanthropologie am Zentrum Asienwissenschaften und Sozialanthropologie der Österreichischen Akademie der Wissenschaften. Ihre Forschungsschwerpunkte umfassen Tourismus-, Geschlechter- und Technologieforschung (v.a. Reproduktionstechnologien). Sie ist Mitglied der American Anthropological Association (AAA) und der Ethnomedizinischen Gesellschaft Wien und zudem als Lehrende und Vortragende im universitären und außeruniversitären Bereich (u.a. in Wien, Göttingen und Ljubljana) tätig. Derzeit schließt sie ihr Dissertationsprojekt zum Thema „Fortpflanzungstourismus in Europa" ab.

\section{PD Dr. Tanja Krones}

Tanja Krones ist Dozentin für Medizinethik am Fachbereich Medizin der PhilippsUniversität Marburg und in der Geschäftsführung der Forschungs-Ethikkommission tätig. Sie ist Mitglied der Zentralen Ethikkommission der Bundesärztekammer, gemeinsam mit Prof. Uwe Körner Vorsitzende der AG Embryonenschutz und Reproduktionsmedizin der Akademie für Ethik in der Medizin, sowie gemeinsam mit Daniel Strech Vorsitzende des Fachbereichs Evidenzbasierte Medizin und Ethik des Deutschen Netzwerks evidenzbasierter Medizin. Sie hat Medizin, Soziologie, Psychologie und Politologie studiert, promovierte 2000 und habilitierte sich 2006 für das Fach Ethik in der Medizin an der Universität Marburg.

\section{Prof. Dr. Hans-Wilhelm Michelmann}

Hans Wilhelm Michelmann ist Leiter der reproduktionsmedizinischen Labore der Arbeitsgruppe Reproduktionsmedizin an der Universitätsfrauenklinik Göttingen. Er hat in Göttingen Agarwissenschaften studiert, wurde 1975 promoviert und habilitierte sich nach einem zweijährigen Forschungsaufenthalt in den USA in den Fächern Fortpflanzungsbiologie und Zytogenetik. Seit 1988 ist er Professor für 
Reproduktionsbiologie an der Universitätsfrauenklinik Göttingen. 1993 erhielt er den Doktor Honoris Causa der Universität Bukarest. Er war mehr als 10 Jahre lang sowohl im Vorstand der Deutschen Gesellschaft für Gynäkologische Endokrinologie und Fortpflanzungsmedizin als auch der Deutschen Gesellschaft für Reproduktionsmedizin tätig. Seit 1993 ist er Mitglied der Akademie Ethik in der Medizin.

\section{Prof. Dr. Falk Ochsendorf}

Falk Ochsendorf ist Arzt für Dermatologie und Venerologie, Andrologie, Allergologie und "Master of Medical Education". Er ist seit 1984 am Zentrum Dermatologie und Venerologie (Direktor Prof. Dr. R. Kaufmann) des Klinikums der J. W. Goethe-Universität Frankfurt am Main tätig und habilitierte sich 1996 mit einem andrologischen Thema. Derzeit ist er leitender Oberarzt und leitet dort die andrologische Ambulanz. Neben der klinischen Dermatologie und der Andrologie ist die medizinische Ausbildung sein Hauptarbeitsgebiet.

\section{Dr. Oliver Rauprich}

Oliver Rauprich ist Leiter der BMBF-Nachwuchsgruppe „Gerechtigkeit in der modernen Medizin“ am Institut für Medizinische Ethik und Geschichte der Medizin der Ruhr-Universität Bochum. Er hat Biologie und Philosophie in München und Chicago studiert und wurde an der Universität München in Philosophie promoviert. Als Gastwissenschaftler war er an der Georgetown University in Washington DC sowie an der Harvard University in Boston. Seine Forschungsschwerpunkte sind biomedizinische Ethik, Theorien der Gerechtigkeit sowie Evolutionäre Ethik.

\section{Timo Sauer, $M A$}

Timo Sauer absolvierte das Studium der Philosophie, Soziologie und Geschichte an der J. W. Goethe-Universität Frankfurt am Main und an der Université de Picardie Jules Vernes Amiens, Frankreich. Er ist examinierter Krankenpfleger und wissenschaftlicher Mitarbeiter im Klinischen Ethik-Komitee der Universitätsklinik Frankfurt am Main. Sein Forschungsschwerpunkt liegt in der Analyse spezifischer Probleme einer interdisziplinären Ethik und Ethikberatung mit besonderem Blick auf die Pflege. 


\section{PD Dr. Aglaja Stim}

Aglaja Stirn ist Leiterin des Funktionsbereiches Psychosomatik der J. W. GoetheUniversitätsklinik Frankfurt am Main, Fachärztin für Psychosomatische Medizin, Psychotherapeutin, Psychoanalytikerin, Lehranalytikerin, Gruppenanalytikerin. Aufenthalte in den USA und im Max-Planck-Institut für Hirnforschung Frankfurt am Main. Sie ist Reviewerin für mehrere Zeitschriften und Autorin zahlreicher Publikationen zu Körperbild und Körperveränderungen, Essstörungen, aber auch zu Depression, Zwang und selbstverletzendem Verhalten, darüber hinaus auch zu buddhistischer Klosterkunst und indischen Ethnien.

\section{Dr. Petra Thorn}

Petra Thorn ist Sozialarbeiterin, Sozialtherapeutin und Familientherapeutin DGSF. Sie arbeitet seit 1994 in der Beratung bei Kinderwunsch. Ihr klinischer und wissenschaftlicher Schwerpunkt ist die Familienbildung mit Gametenspende. Hierzu hat sie zahlreich publiziert. Sie ist Vorsitzende des Beratungsnetzwerks Kinderwunsch Deutschland e.V. und der Special Interest Group Psychology \& Counselling der ESHRE sowie u.a. Vorstandsmitglied des Arbeitskreises für donogene Insemination e.V. und der International Infertility Counselling Organisation.

\section{Yasar Toraman}

Yasar Toraman ist Dipl. Biologin und hat die Leitung und Organisation des biologischen IVF-Labors der J. W. Goethe-Universitätsklinik Frankfurt am Main inne. Ihr Aufgabenbereich umfasst die Vorbereitung und Durchführung sämtlicher im Rahmen des biologischen IVF-Labors notwendiger Maßnahmen wie IVF und ICSI, Kryokonservierung von pronucleus-Stadien, Durchführung des Embryotransfers, Beurteilung und Auswertung von Nativspermiogrammen, Aufbereitung des Spermas über Dichtegradienten oder Swim up Methode für IUI, IVF oder ICSI, Wartung aller Gerätschaften, die für ein funktionierendes IVF-Labor benötigt werden. Sie hat über die „Konservierung von Ovarialgewebe und Vitalitätsanalyse nach dem Auftauen" promoviert.

\section{Prof. Dr. Christiane Wendehorst, LLM}

Christiane Wendehorst ist seit März 2008 Professorin für Zivilrecht an der Rechtswissenschaftlichen Fakultät der Universität Wien. Sie absolvierte das Studium der Rechtswissenschaften, wurde 1994 promoviert und 1998 habilitiert. Von 1999 bis 2008 war sie Professorin (C4) für Bürgerliches Recht, Medizinrecht, Internationales Privatrecht und Rechtsvergleichung, an der Universität Göttingen. Von 1999 bis 2005 war sie Direktorin der Abteilung für Arzt- und Arzneimittel- 
recht der Juristischen Fakultät und von 2006 bis 2008 im Vorstand des Zentrums für Medizinrecht. In den Jahren 2001 bis 2008 war sie Mitglied der Ethik-Kommission am Universitätsklinikum Göttingen. Seit 2001 ist sie Mitglied der Arbeitsgruppe Reproduktionsmedizin in der Akademie für Ethik in der Medizin.

\section{PD Dr. Inka Wiegratr.}

Inka Wiegratz ist Fachärztin für Gynäkologie und Geburtshilfe und seit 1990 an der Universitätsfrauenklinik Frankfurt tätig, wo sie seit 2007 die komissarische Leitung des Schwerpunkts gynäkologische Endokrinologie und Reproduktionsmedizin inne hat. Sie verfügt über langjährige Erfahrung in der Arbeit mit Patientinnen, die Probleme aus dem Gebiet der gynäkologischen Endokrinologie aufweisen und befasst sich mit der Therapie von Patientinnen mit primärer und sekundärer Sterilität, einschließlich Stimulationstherapie, IUI, IVF und ICSI-Therapie. Sie habilitierte sich 2005.

\section{Prof. Dr. Claudia Wiesemann}

Claudia Wiesemann ist Direktorin des Instituts für Ethik und Geschichte der Medizin an der Medizinischen Fakultät der Universität Göttingen und Präsidentin der Akademie für Ethik in der Medizin e.V., Mitglied der Zentralen Ethikkommission für Stammzellforschung, sowie Stellvertretende Vorsitzende der Ethikkommission an der Universität Göttingen. Sie hat Medizin, Philosophie und Geschichte studiert und wurde in Medizingeschichte an der Universität Münster promoviert. An der Universität Erlangen-Nürnberg habilitierte sie sich 1996 für Geschichte und Ethik der Medizin. Thre Forschungsschwerpunkte sind Medizingeschichte und Medizinethik aus kulturwissenschaftlicher Perspektive, Geschichte der Todesfeststellung im 20. Jahrhundert und die Ethik der Elternschaft.

\section{Prof. Dr. Barbara Zoll}

Barbara Zoll absolvierte das Studium der Medizin und durchlief die Ausbildung zur Fachärztin für Humangenetik mit Fachkunde für genetische Beratung, Zytogenetik und Molekulargenetik. Sie habilitierte sich im Fach Humangenetik und leitet als approbierte Oberärztin die genetische Beratungsstelle am Institut für Humangenetik der Universitätsmedizin Göttingen. Ihre Arbeitsschwerpunkte umfassen unter anderem die Genetische Beratung, Syndromologie und Entwicklungsgenetik. 
A uch 30 Jahre nach der Geburt des ersten „Retortenbabys“ Louise Brown im Jahr 1978 bleibt für viele Paare der Wunsch nach einem eigenen Kind unerfüllt, und ihre Hoffnungen richten sich auf moderne Techniken assistierter Reproduktion. Die Reproduktionsmedizin hat seitdem immense Fortschritte gemacht und neue Chancen eröffnet. Mit diesen Chancen ist indessen zugleich eine Fülle neuer Herausforderungen verbunden, deren moralische und rechtliche Implikationen erheblich sind.

Der vorliegende Band vereint Beiträge zweier Tagungen, die im Jahr 2007 vom »Forum für Ethik in der Medizin Frankfurt am Main e.V.« gemeinsam mit der »Arbeitsgruppe Reproduktionsmedizin und Embryonenschutz in der Akademie für Ethik in der Medizin « sowie vom »Zentrum für Medizinrecht« der Universität Göttingen veranstaltet wurden und welche die aktuelle Debatte um die Reproduktionsmedizin und ihre Möglichkeiten aufgreifen. Sie wenden sich den Errungenschaften und Problemen assistierter Fortpflanzung im Allgemeinen zu, widmen jedoch ihr Augenmerk speziell donogenen Techniken (Eizellspende, Samenspende, Embryospende) sowie dem Kinderwunsch in besonderen Situationen (etwa körperliche Behinderung, letale Erkrankung eines Partners, gleichgeschlechtliche Lebensgemeinschaften). 